THESES SIS/LIBRARY

R.G. MENZIES LIBRARY BUILDING NO:2

THE AUSTRALIAN NATIONAL UNIVERSITY

CANBERRA ACT 0200 AUSTRALIA
TELEPHONE: +61261254631

FACSIMILE: +61261254063

EMAIL: library.theses@anu.edu.au

\title{
USE OF THESES
}

This copy is supplied for purposes

of private study and research only.

Passages from the thesis may not be

copied or closely paraphrased without the

written consent of the author. 


\section{Testing Realistic Forensic Speaker Identification In Japanese: A Likelihood Ratio Based Approach Using Formants}

\section{Yuko KINOSHITA}


Unless otherwise acknowledged in the text, this thesis represents the original research of the author.

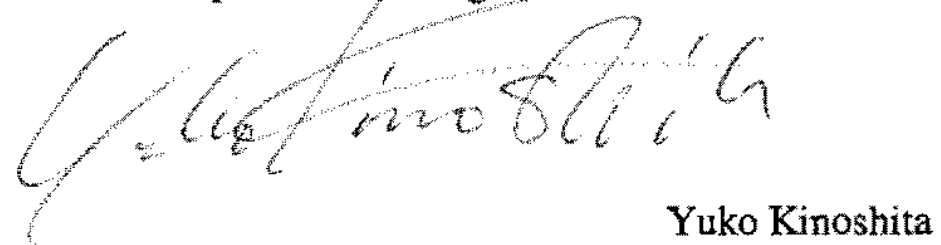




\section{ACKNOWLEDGEMENTS}

I would like to thank my supervisor, Dr. Phil Rose, for providing me with detailed comments, encouragement and support throughout my $\mathrm{PhD}$. Without his patient guidance, this research could not have happened. I hope that he was not annoyed that I kept hanging around and asking questions (I have been doing this for around 6 years so far, including when I was doing my Masters degree), as I expect him to remain as my mentor for as long as I remain working in phonetics.

I also would like to thank John Maindonald at the Statistical Consulting Unit of the Graduate School, ANU. He spent so much time with me, explaining statistical concepts, patiently answering countless very fundamental questions, and performing some statistical analyses. I know that this was a very painful process for him, as we really struggled to communicate due to my poor understanding of statistics.

I must thank all of the lecturers in the Linguistics department of ANU for everything that I have learnt from them and the administrator, Carolin Thomas, for her administrative and moral support. It was a wonderful experience to be here as a postgraduate student.

I also need to thank my co-workers at the Japan Centre of the ANU, especially Dr. Duck Young Lee and Shun Ishihara. Teaching classes while working on a thesis was not always an easy task, but their understanding and encouragement helped me greatly.

My thanks also go to the 13 informants who participated in this study. They kindly agreed to come down all the way to the recording studio at the ANU twice, despite their busy schedules. This study would not have been possible without their generous cooperation. 
I would also like to thank Drs. 'Spike' Barlow and Frantz Clermont of the Australian Defence Force Academy for giving me many useful comments in seminars.

I must thank my proofreaders, Sue Novak and Andrew Lim who went through bits of my earlier draft, Pascale Jacq who helped me with the final check of my English usage, references, tables and figures etc, and Ingrid Singh who has been reading my extremely messy drafts over four years from the earliest stage of this research. Ingrid was so generous as to lie to me that she enjoyed reading the drafts and was eagerly waiting for more. What a wonderful friend.

Last but not least, I would like to thank my family and friends who provided moral support, which was invaluable at various stages during the construction of this $\mathrm{PhD}$ thesis. I would like to thank my mum, dad and sister, Shoko, for being there for me at the toughest time (so far!) in my life and backing me up in their own individual very different ways. I am also very grateful to my grandmother in Japan and grandfather somewhere up there for their love and understanding. The fact that they feel pride for me, instead of disappointment for staying at university for all these years has given me a great deal of strength.

All my friends, both in Japan and Australia, have been great. Everyone did a wonderful job of making me laugh, cheering me up, and calming me down when I panicked, but I would especially like to thank Andrew and Ingrid. Andrew made me smile (and laugh) at least ten times a day, even when I was exhausted and miserable, which is impressive work. Ingrid has been there for me throughout entire my $\mathrm{PhD}$ period. I believe that my life as a $\mathrm{PhD}$ candidate would have been so much tougher without her friendship. 


\section{ABSTRACT}

This thesis sets out to investigate whether or not forensic speaker identification can be performed, using forensically realistic data, which is natural and non-contemporaneous speech. To date, there is no forensic phonetic research which tests how accurately speakers can be discriminated on the basis of their voice under forensically realistic conditions, despite the fact that the use of speech recordings for forensic investigation or as a part of evidence in court is not an unusual practice today. This research thus aims to provide the first test of the accuracy of realistic forensic speaker identification using centre frequencies of formants, which are today the most commonly used acoustic parameter in actual forensic speaker identification today.

The current state of forensic speaker identification in Japan also signifies this research. Forensic speaker identification in Japan has relied on visual examination of spectrograms and occasional use of the automatic speaker recognition technique. The research on forensic speaker identification also concentrates on the application of the automatic speaker recognition technique, and no linguistic analysis or interpretation of speech data has been included. This thesis therefore will serve as the first linguistic analysis in Japanese forensic speaker identification research.

This thesis firstly examines what segment / formant combinations are more promising as the speaker identification parameter. Those parameters are then incorporated, and how accurately they discriminate two speech samples is tested. For the testing, three different statistical approaches are presented and examined. As result, the distance based approach using likelihood ratio as the score for discrimination test (likelihood ratio-based distance method) was found to be most effective. The results of this testing showed that speakers can indeed be discriminated on the basis of their formant frequencies, as long as enough number of parameters are incorporated. With this approach incorporating six parameters, the successful discrimination 
rates were found to be approximately $96.7 \%$ for positive discrimination (discriminating two different speakers) and $90 \%$ for negative discrimination (identifying the same speaker), when the threshold was set at likelihood ratio 1. 


\section{TABLE OF CONTENTS}

INTRODUCTION 1

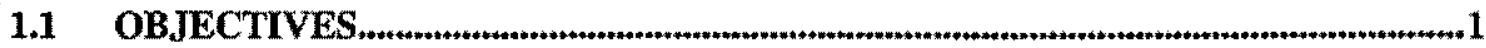

1.2 SPEAKER RECOGNITION AND SPEECH RECOGNITION ...............................4

1.3 TYPES OF SPEAKER RECOGNITION .................................................................7

1.3.1 FORENSIC SPEAKER RECOGNITION AND OTHER APPLICATIONS .........................7

1.3.2 TYPES OF SPEAKER RECOGNITION - TWOFOLD OR THREEFOLD? ........................

1.3.2.1 Threefold distinction ? SRL, SRM, and SR S ......................................................... 8

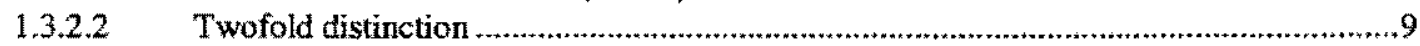

1.3.3 THREE TECHNIQUES IN FORENSIC SPEAKER IDENTIFICATION ......................10

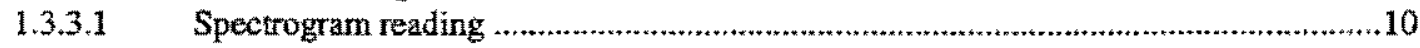

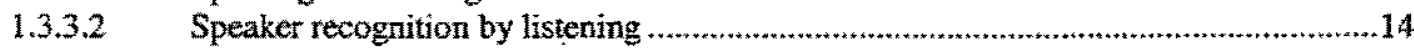

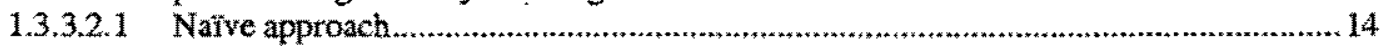

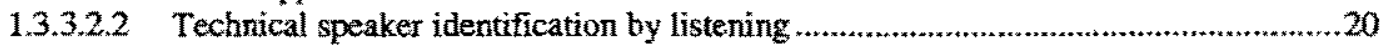

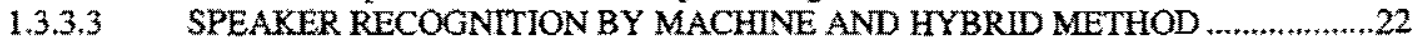

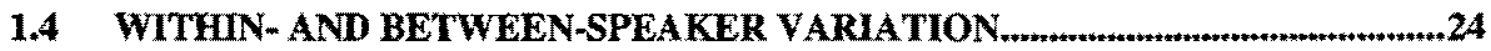

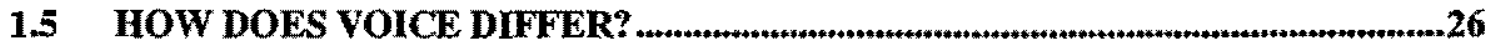

1.6 LIMITATIONS IN FORENSIC SPEAKER IDENTIFICATION …….......................30

1.7 PRESENTATION OF ANALYSIS AND BAYES' APPROACH................................32

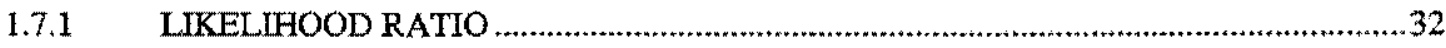

1.7.2 BAYESIAN APPROACH ...............................................................................

1.8 TASKS INVOLVED IN FORENSIC PHONETICS.....................................................33

1.9 FORENSIC SPEAKER RECOGNITION IN JAPANESE ….........................................35 
2.1 OBJECTIVE

2.2 MORA AND SYLLABLE $\ldots \ldots \ldots \ldots \ldots$

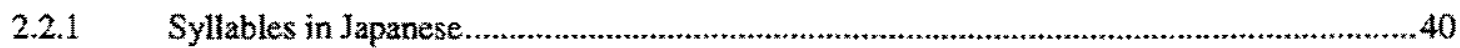

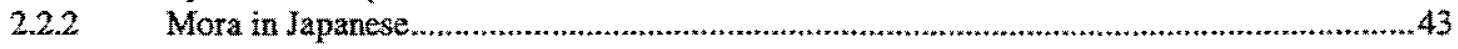

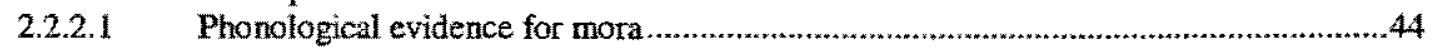

2.2.2.1.1 Evidence from haiku and tanka ..........................................................................44

2.2.2.1.2 Evidence from song lyrics .................................................................................44

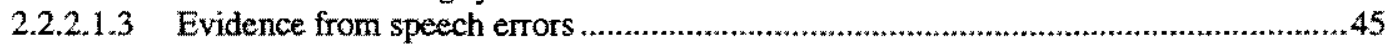

2.2.2.1.4 Evidence from the location of pitch accent ..........................................................46

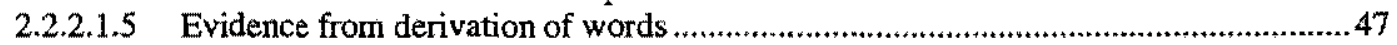

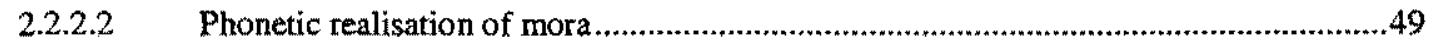

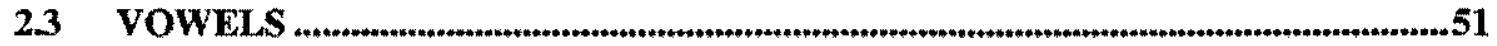

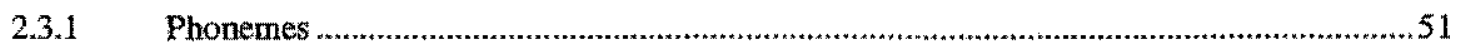

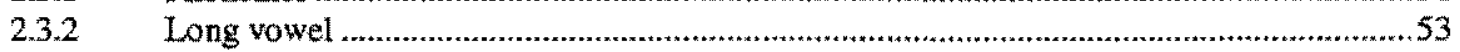

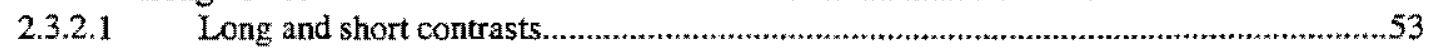

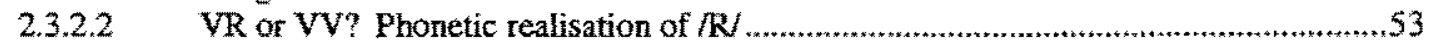

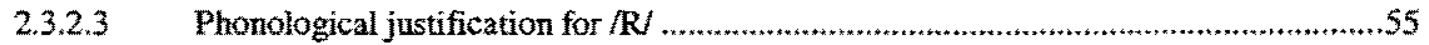

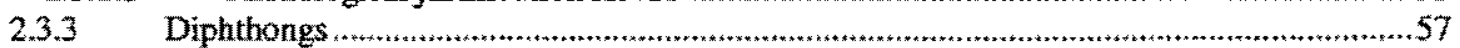

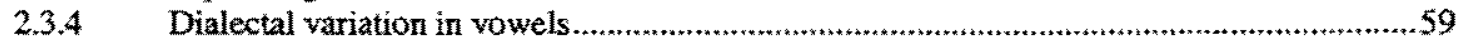

2.4 CONSONANTS

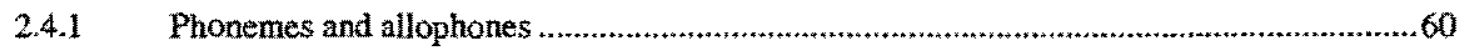

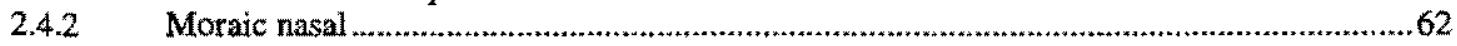

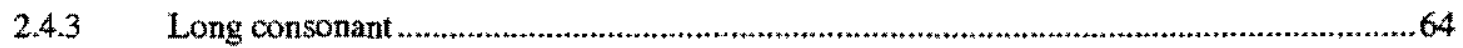

2,5 ACCENT

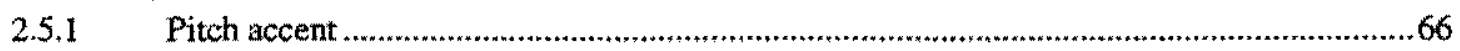

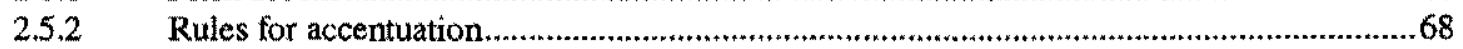

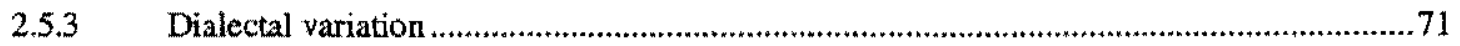

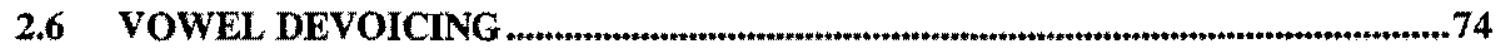

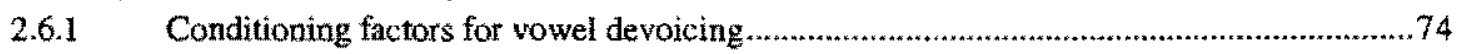

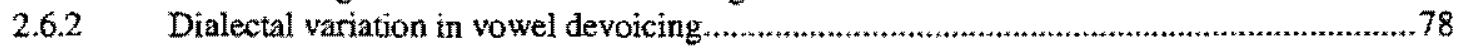

2.7 INTONATION AND WORD ACCENT

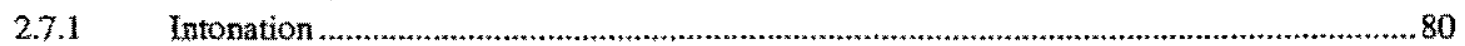

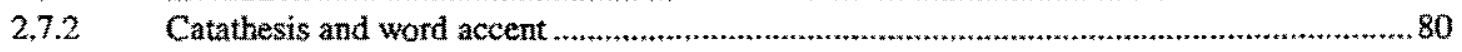

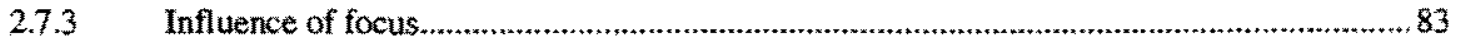

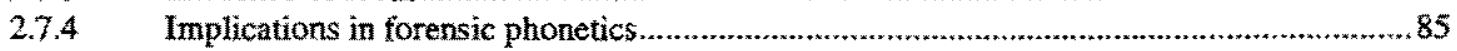

2.8 DIALECTAL VARIATION AND FORENSIC PHONETICS IN JAPANESE 
3.1 OBJECTIVES

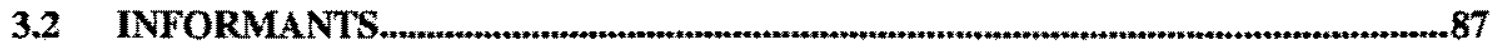

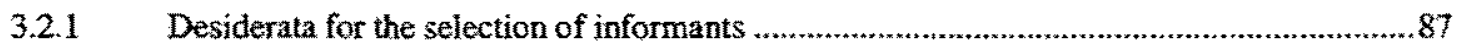

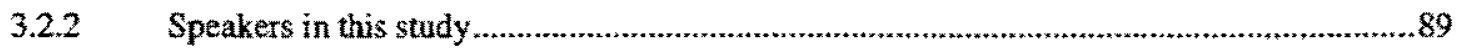

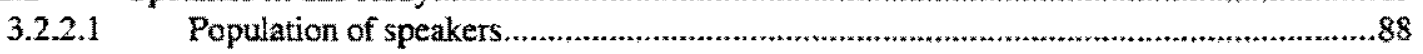

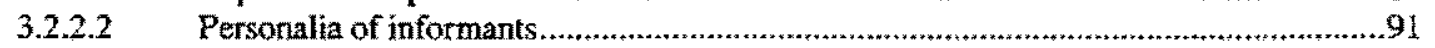

3.3 PARAMETERS FOR SPEAKER IDENTIFICATION

3.3.1 Criteria for speaker recognition parameters ........................................................................93

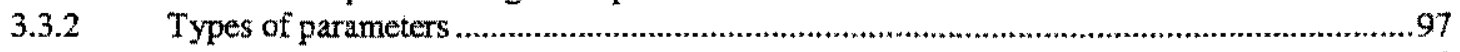

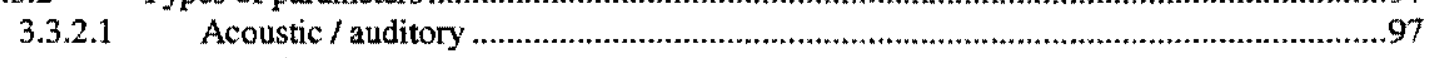

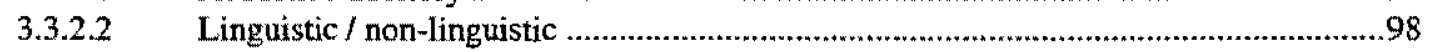

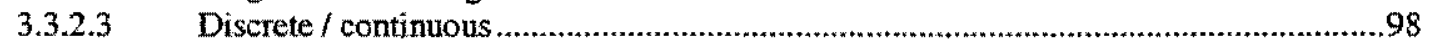

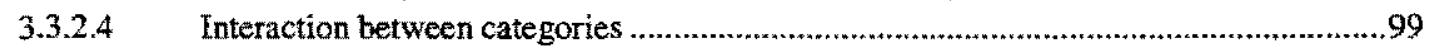

3.3.3 Commonly used acoustic parameters in forensic speaker identification...............................99

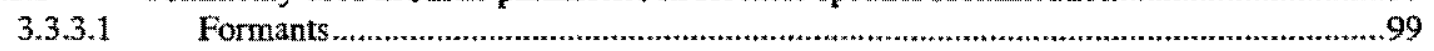

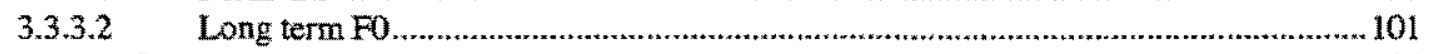

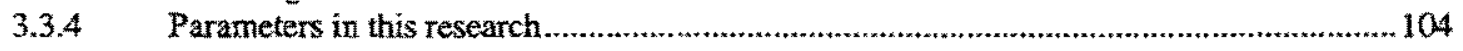

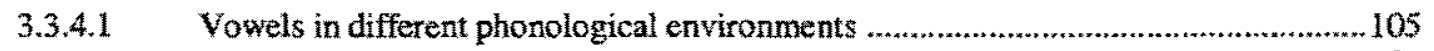

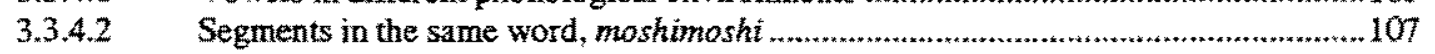

3.3.5 Between-languages differences in forensic speaker identification....................................... 109

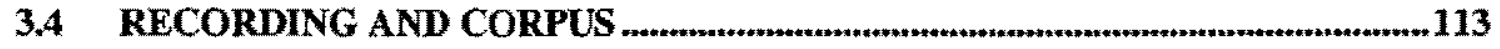

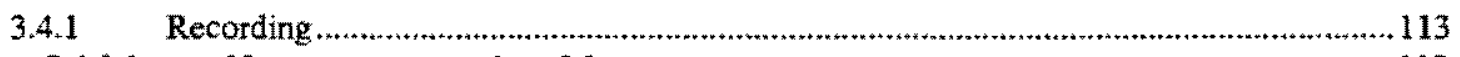

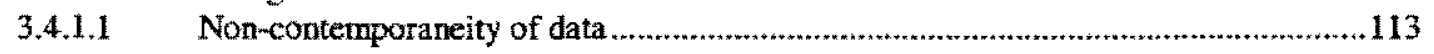

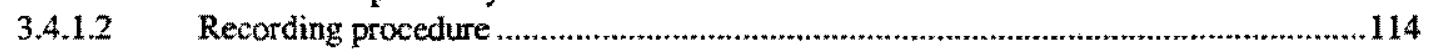

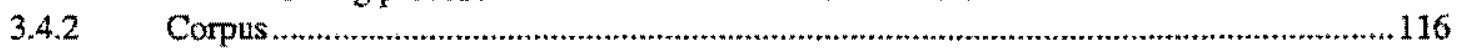

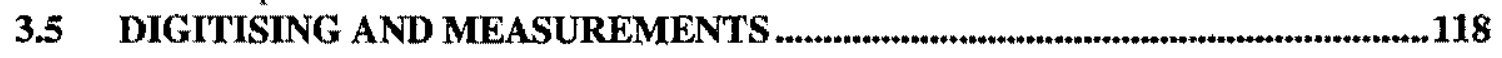

3.5.1 Digitising recorded samples..............................................................................................118

3.5.2 Vowels in different contexts (Chapter 4) ....................................................................... 118

3.5.3 Moshimoshi (Chapter 5) .................................................................................................. 123

3.6 STATISTICAL PROCEDURE 


\section{DETERMINING EFFICIENT FORMANT PARAMETERS FOR}

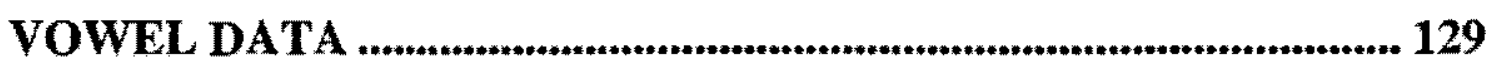

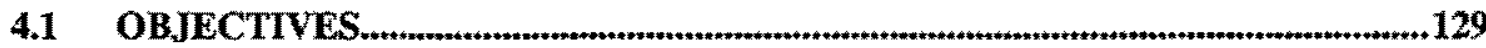

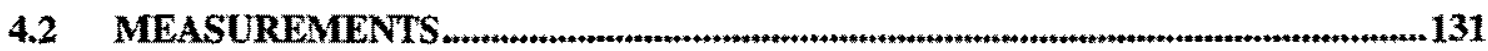

4.3 ILLEGAL VOWEL DEVOICING

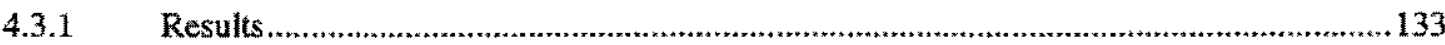

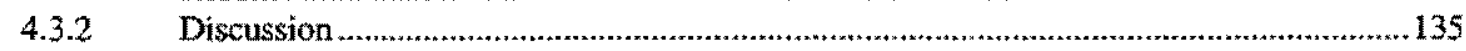

4.3.2.1 Within-speaker variation in vowel devoicing ..........................................................135

4.3.2.2 Between speaker variation in vowel devoicing ........................................................137

4.3.2.2.1 Incidence of illegal vowel devoicing..................................................................137

4.3.2.2.2 Consistency of vowel devoicing within a speaker................................................... 141

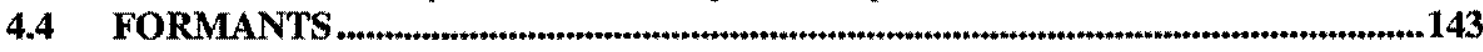

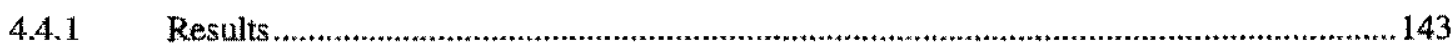

4.4.2 Contemporaneous/ non-contemporaneous comparisons .................................................150

4.4.3 Between-Speaker Variation in formant patterns ............................................................... 152

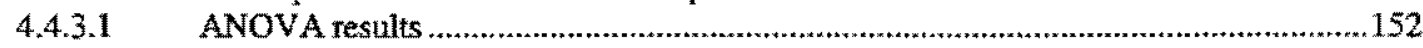

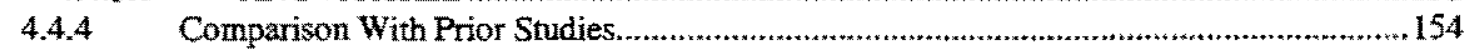

4.5 EFFECTIVE F2

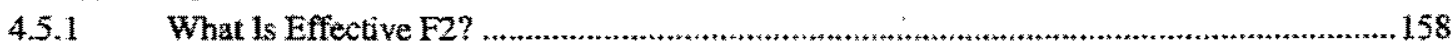

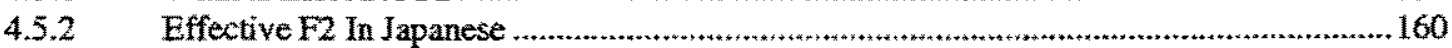

4.5.3 Suitability of Effective F2 As A Speaker Identification Parameter ....................................165

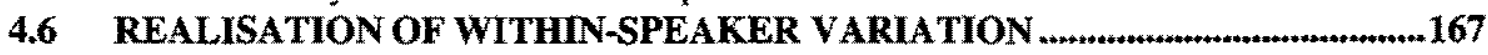

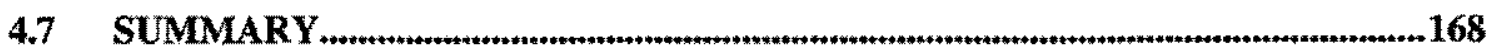




\section{DETERMINING EFFICIENT FORMANT PARAMETERS FOR}

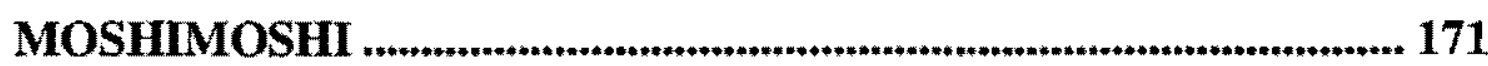

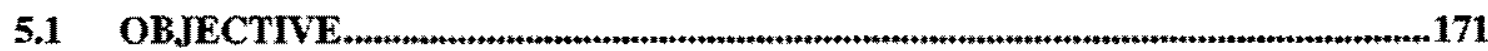

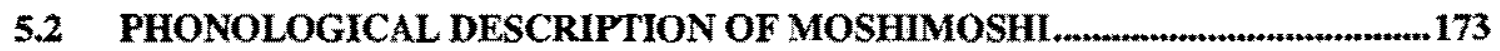

5.3 DESCRIPTION OF CAREFULLY CITED MOSHIMOSHI AND BOSHIBOSHI

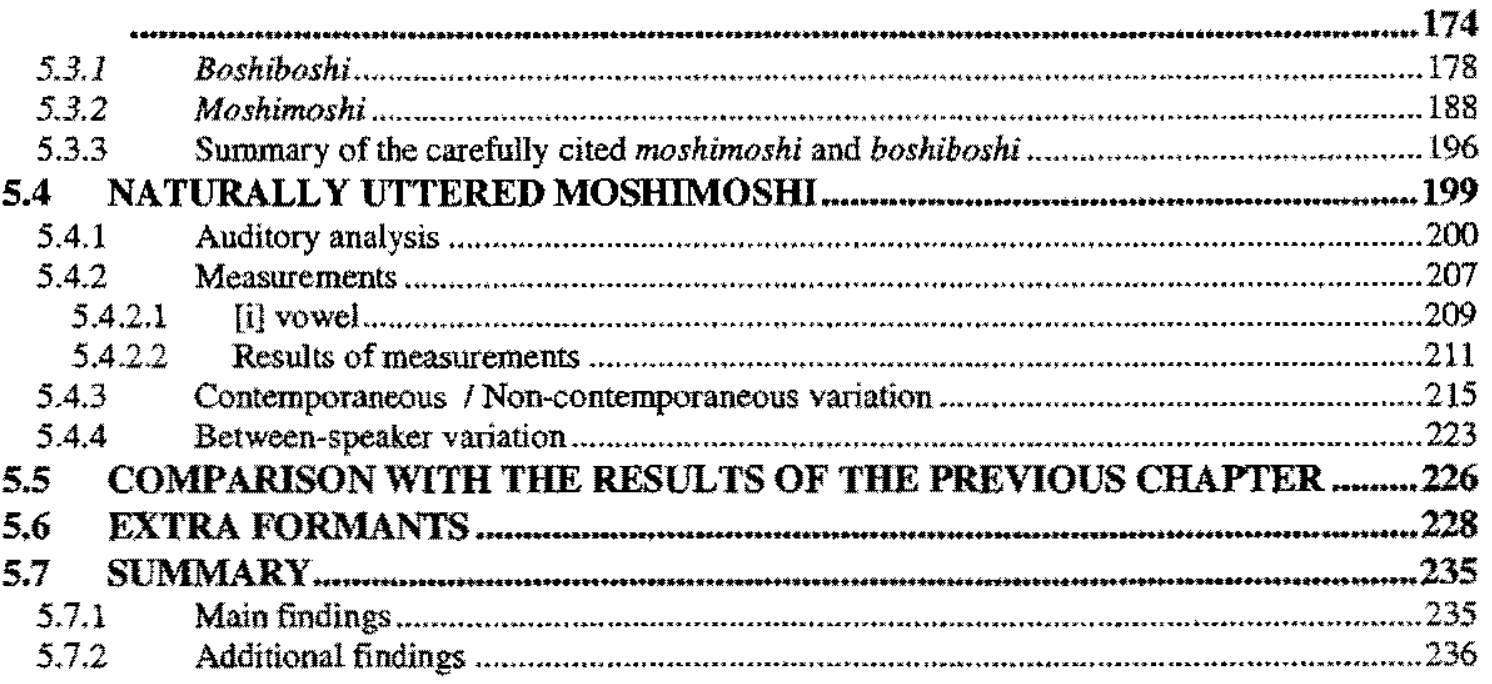


6.1 OBJECTIVES

6.2 DISCRIMINATION OR IDENTIFICATION?

6.3 STATISTICAL APPROACHES TESTED IN THIS STUDY

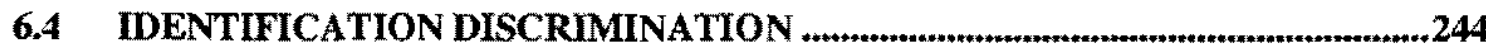

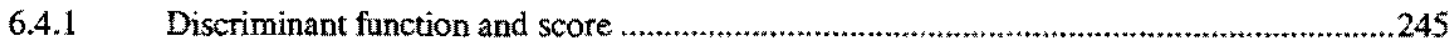

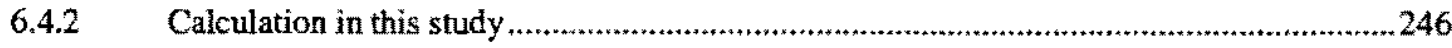

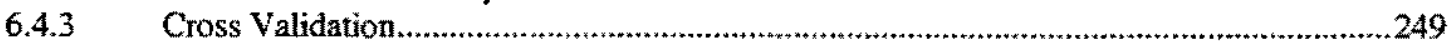

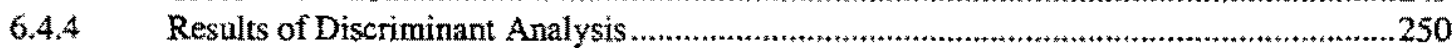

6.4.5 Problems with the discriminant analysis .................................................................251

6.5 FORENSIC DISCRIMINATIONः DISCRIMINANT SCORE-BASED DISTANCE

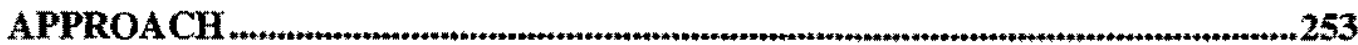

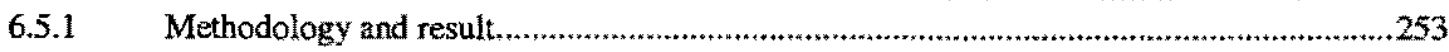

6.5.2 Problems with discriminant score-Based Distance Method ................................................261

6.6 FORENSIC DISCRIMINATION: LIKELIHOOD RATIO-BASED DISTANCE

APPROACH

6.6.1 Likelihood ratio and Bayesian approach ……................................................................263

6.6.1.1 What do likelihood ratio and Bayesian approach evaluate? .......................................263

6.6.1.2 Likelihood ratio calculation..............................................................................26

6.6.1.3 Problems in Aitkin fs for ml a and an att a nai ve appracht ot hs st ud ......................268

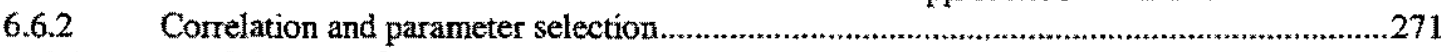

6.6.2.1 Selection of parameters ......................................................................................271

6.6.2.2 Effect of between-parameter correlation on statistics ................................................273

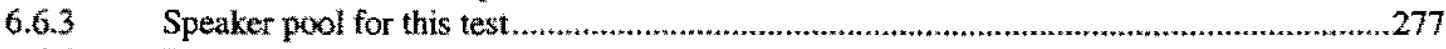

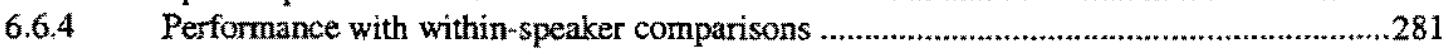

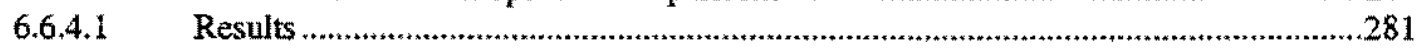

6.6.4.2 Causes of high / low posterior likelihood ratios …...................................................290

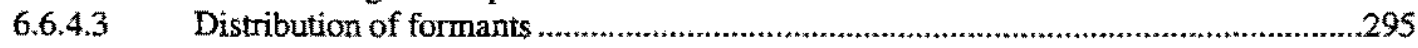

6.6.5 Performance with between-speaker comparisons ......................................................297

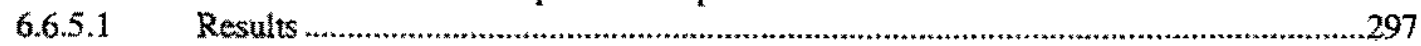

6.6.5.2 Details of unsuccessful discrimination ..................................................................308

6.6.5.3 Causes for unsuccessful discrimination..................................................................311

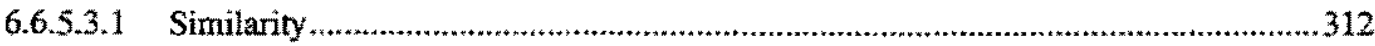

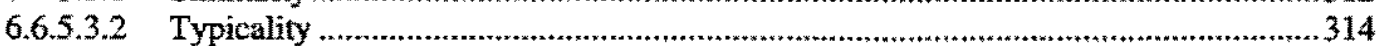

6.6.6 Numbers of parameters and discrimination ...............................................................316

6.6.7 Equal error rate and production of likelihood ratio for the test ..........................................324

6.6.8 Problems with likelihood ratio-based distance approach …...................................................328

6.7 SUMMARY 
7.1 ANSWERS TO THE RESEARCH QUESTIONS OF THIS THESIS ......................334

7.1.1 Answers to the main questions ..............................................................................335

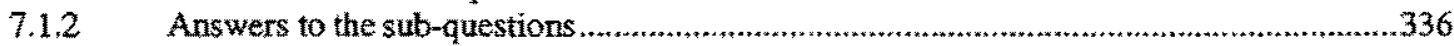

7.1.2.1 What are the optimal parameters? ........................................................................336

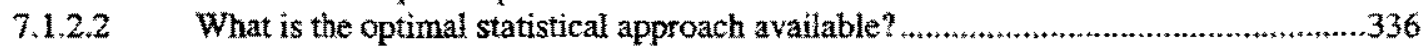

7.1.2.3 Are there any language specific differences?? ..............................................................339

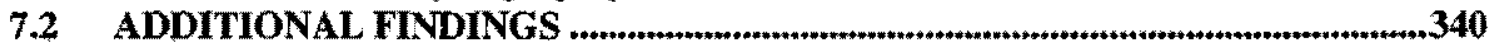

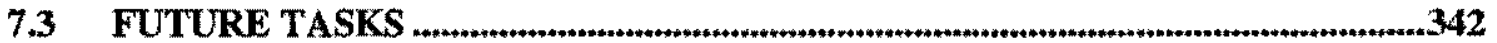




\section{TABLES}

Table 2.1 List of phonemes in standard Japanese. .......................................................................61

Table 2.2 Phonetic transcription of all phones that occur in standard Japanese. ........................61

Table 2.3 Phonemes and allophonic variation in consonants......................................................62

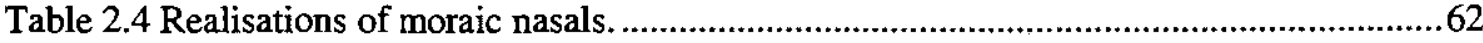

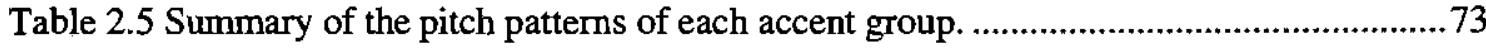

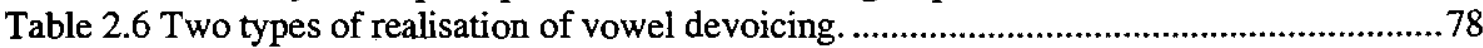

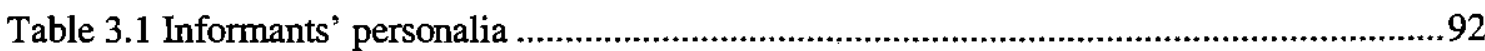

Table 3.2 Correlations between acoustic parameters for a single speaker (HA)........................96

Table 3.3 $\mathrm{FO}(\mathrm{Hz})$ of accented /a/ vowels and their following vowels, and the F0 difference

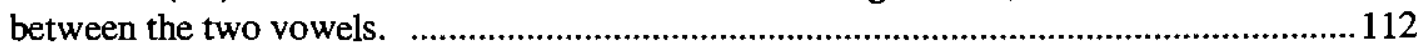

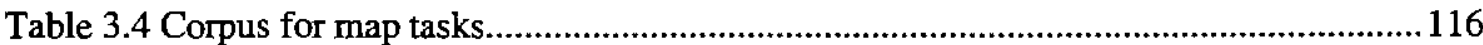

Table 3.5 Numbers of tokens which were recorded for each speaker.........................................117

Table 4.1 Incidence of illegal vowel devoicing of tokushima 'Tokushima (name)' and doobutsuen 'zoo' as function of speaker, recording session, and repeat.

Table 4.2 Summary of how frequently the illegal vowel devoicing occurred in two recording sessions.

Table 4.3 Summary of the between-speaker comparison in incidence of illegal vowel devoicing

Table 4.4 Means, standard deviations, and sample size for vowel formant centre frequency in 11 male Japanese speakers' five accented short vowels.

Table 4.5 Means and standard deviations across all 11 speakers for each vowel / formant combination.

Table 4.6 Results of one-way repeated ANOVA.

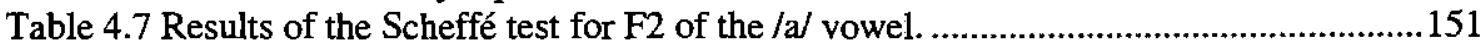

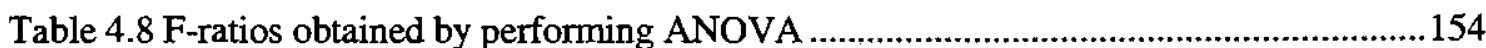

Table 4.9 Mean and standard deviation of effective F2 for the short vowels of 11 Japanese males.

Table 4.10 Mean of standard deviations of the raw F2 and effective F2 .................................165

Table 4.11 F-ratios for all vowel / formant combinations............................................................

Table 5.1 Means and standard deviations for the all measuring points in boshiboshi. ............182

Table 5.2 Means and standard deviations of F1 to F4 of each segment in moshimoshi. .........192

Table 5.3 Summary of the observation of the formant structures of carefully sited boshiboshi and moshimoshi.

Table 5.4 A broad classification of the interlocutors and informants' choices of their attitudes towards those interlocutors.

Table 5.5 Summary of the duration and F0 of speaker MM's moshimoshi from the blunt and normal tokens.

Table 5.6 Numbers of moshimoshi recorded for each speaker.

Table 5.7 Incidence of $/ \mathrm{i}$ devoicing in moshimosh $i$ as function of speaker, recording session, and position in word.

Table 5.8 Individual speaker's means and standard deviations for F-pattem in moshimoshi...214

Table 5.9 Mean F-pattern in moshimoshi across all 13 speakers for each measuring point. ....214

Table 5.10 Results of a t-test on F-pattern in non-contemporaneous moshimoshis. 
Table 5.11 Results of the t-test, all speakers into a single data set.

220

Table 5.12 F-ratios for between-speaker variation and the number of measurements made for each segment.

Table 5.13 Extra formants observed in moshimoshi for all speakers

Table 5.14 Summary of the higher rates ( $75 \%$ and above) of the incidence of extra formants. 233

Table 6.1 Summary of the four possible judgements made in a speaker discrimination test. .241

Table 6.2 Discriminant scores calculated for each word for each speaker.

Table 6.3 Results of the discriminant analysis.

Table 6.4 Mean within-speaker distances calculated by comparing five tokens of each speaker.

Table 6.5 Mean distances of linear discriminant functions between speakers.

Table 6.6 Summary of the results of the classification tests with the thresholds $2.5,2.53$, and 2.5 .

Table 6.7 Rates of the illegal vowel devoicing incidence.

Table 6.8 Correlation between parameters and the numbers of comparisons made for each parameter combination.

Table 6.9 Means and standard deviations used for the likelihood calculations. ......................279

Table 6.10 Summary of each speaker's likelihood ratios and posterior odds. ...........................286

Table 6.11 Means of the posterior likelihood ratios...............................................................28

Table 6.12 Summary of the observation of the likelihood ratios produced by speaker with extreme posterior odds.

Table 6.13 Means and standard deviations for the parameters which produced particularly high or low likelihood ratios.

Table 6.14 Difference between two recording sessions within a speaker in the means for the speaker / parameter combinations in question.

Table 6.15 Results of the likelihood ratio calculations.

Table 6.16 Summary of the between-speaker comparisons which produced likelihood ratios over 1 (false negative discrimination).

Table 6.17 Likelihood ratios and the posterior odds for the between-speaker combinations which were not discriminated correctly.

Table 6.18 Means of the datasets which the Bayesian approach failed to discriminate. ..........313

Table 6,19 Absolute values of the subtraction of one dataset from the other.

Table 6.20 Means, standard deviations, minimum and maximum values of the difference between 2 speakers.

Table 6.21 Total numbers, means, standard deviations, and percentages of successful discrimination for each numbers of parameters incorporated in discrimination tests........320

Table 6.22 Parameter combinations which produced best and worst successful discrimination rates.

Table 6.23 Each speaker's mean likelihood ratios and standard deviations for each parameter.

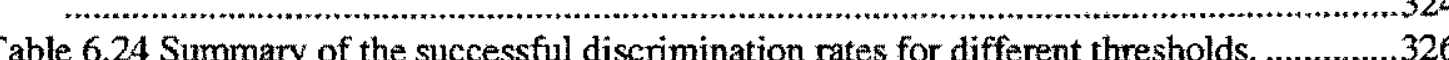

Table 6.25 Example of effects of rounding on calculation of posterior odds. ..........................330 


\section{FIGURES}

Figure 1.1 Model of the distribution of within - and between-speaker variation..........................25

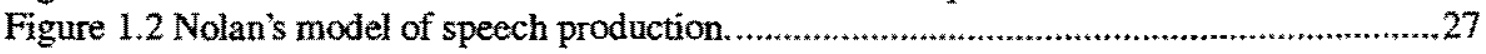

Figure 1.3 Decision making tasks in speaker recognition.........................................................35

Figure 2.1 Hierarchical phonological structure of Japanese word hottokeeki 'pancake. "............39

Figure 2.2 Spectrograms and sound wave forms of sato + oya 'foster parent" and satoo + ya

'sugar vendor."

Figure 2.3 Types of suprasegmentals in world languages........................................................67

Figure 2.4 Distribution of the accent groups in Japan. ............................................................ 72

Figure 2.5 Examples of voiced and devoiced $/ \mathrm{u} / \mathrm{uttered}$ by the same speaker........................... 75

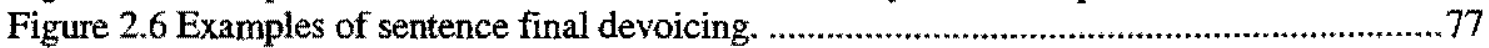

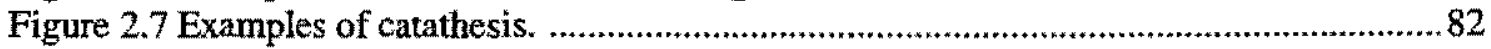

Figure 2.8 Example of the influence of focus. . .............................................................. 84

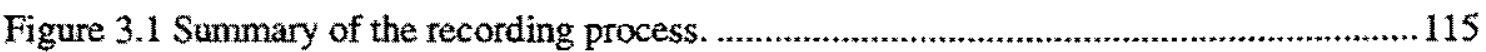

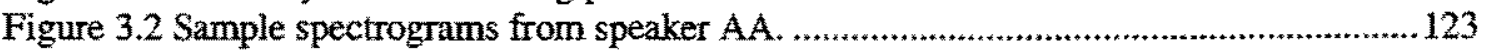

Figure 3.3 Example of measurement of F-pattern in moshimoshi. ................................... 125

Figure 4.1 Numbers of tokens recorded for each vowel for each speaker. ..............................132

Figure 4.2 Example of within-speaker variation in vowel devoicing. . .................................... 137

Figure 4,3 Audio waveforms and spectrograms of tokushima [tokuçima] e Tokus h m (na ne)

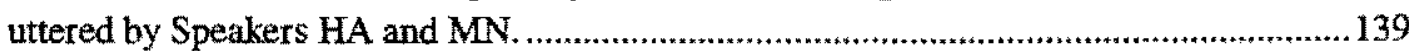

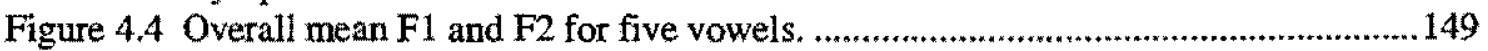

Figure 4.5 Scattergram for the mean F1 and F2 of Japanese and American English vowels... 156

Figure 4.6 Scattergrams which plotted F1 against F2 (on right) and F1 against the effective F2 (on left).

Figure 4.7 Scattergrams of mean F1 against mean F2 (on left) and mean effective F2 (on right)

for each speaker $f s$ fi ve vo vel s............................................................................................. 164

Figure 5.1 Phonological structure of moshimoshi.....................................................................173

Figure 5.2 Models of vocal tract configuration of the nasal consonant [m] and the nasalised vowel [0].

Figure 5.3 A set of four boshiboshis carefully uttered by a male Japanese speaker................ 181

Figure 5.4 A set of four moshimoshis carefully uttered by a male Japanese speaker. .............191

Figure 5.5 Mean F-patterns for boshiboshi and moshimoshi compared................................. 198

Figure 5.6 Example of the F0 range differences between normal speech and blunt speech of Speaker MM.

Figure 5.7 Plotted $\mathrm{Fl}$ and $\mathrm{F} 2$ for the first and the second /o/ of Speaker MM's blunt utterance.

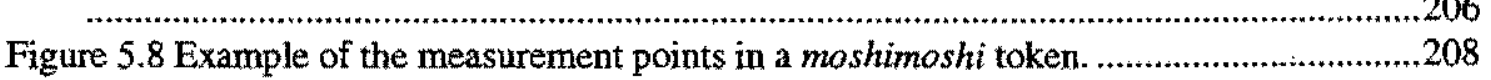

Figure 5.9 Mean F-pattern for moshimoshi as function of measurement points......................215

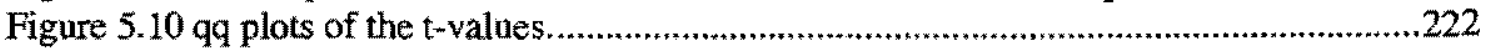


Figure 6.1 Summary of the three statistical measures tested in this chapter.

Figure 6.2 Scatterplots of the five linear discriminant scores for each of the 11 speakers. ......253

Figure 6.3 Model of the locations of thresholds.................................................................258

Figure 6.4 Summary of the comparison which each speaker combination comprised. ....,......280

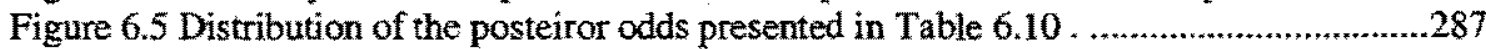

Figure 6.6 Frequency distribution of each parameter. ....................................................294

Figure 6.7 Distribution of olmid with three different bin sizes, 10,15, and 20. .....................296

Figure 6.8 Distribution of the log of the posteiror odds presented in Table $6.15 \ldots \ldots \ldots \ldots \ldots \ldots . . . .306$

Figure 6.9 Distribution of the population and the locations of Speakers TN and KA, who could not be distinguished despite the large difference between their means. ............................315

Figure 6.10 Distribution of olmid of the data sets, KA I and TN 2 .....................................316

Figure 6.11 Numbers of incorporated parameters in discrimination tests and improvement in successful discrimination rates for the worst and best parameter combinations and means.

\section{APPENDICES}

Appendix 3 Map and information sheet for task.

Appendix 4.1 Between-speaker comparisons of the rate of illegal devoicing incidence. ........ 345

Appendix 4.2 Each speaker's raw measurements for vowel formants. ..................................... 349

Appendix 5.1 F-pattem measurements for each speaker. ...............................................................359

Appendix 5.2 Summary of the numbers of tokens measured for each sampling point.............. 372

Appendix 6.1 Likelihood ratios for each parameter and the posterior odds for the speaker combinations which were not discriminated correctly for the calculation using effective $F 2$.

Appendix 6.2 The mean values of the datasets for the calculation using effective $F 2$ which the Bayesian approach failed to discriminate.

Appendix 6.3 Absolute values of the subtraction of one dataset from the other for the calculation using effective $\mathrm{F} 2$.

Appendix 6.4 The mean, standard deviation, minimum and maximum values of the difference between 2 speakers for the calculation using effective $\mathrm{F} 2$. 


\section{Chapter 1}

\section{INTRODUCTION}

\subsection{OBJECTIVES}

The use of speech recordings for forensic investigation or as a part of evidence in court is not an unusual practice today. As a recent example from Japan, following a previous decision made in the Osaka district court, on $27^{\text {th }}$ of March 2001 the Osaka high court sentenced a man to death for murdering five women and abandoning their bodies. The court, however, added another charge of a ransom demand to the previous sentence, stating that

\footnotetext{
「電話の犯人と鎌田被告の双方の言い回しに同じ特徴的な癖がある。 『同一人物』とする警察の鑑定も信用できる」
}

"The speaker of the phone call and the defendant share the same peculiarity in speech style, and the police testimony claiming that the speaker in question is the same individual as the defendant is reliable."

(Asahi newspaper, 28 of March 2001, author's emphasis and translation.)

Although the appropriateness of this police testimony is very arguable (the presentation of evidence will be discussed later in section 1.7 ), this article clearly shows that voice evidence is now a part of the judicial system in Japan.

Despite the growing demand and public interest in forensic phonetics, as we recognise from the fact that some institutes started to offer courses in this area (for instance, a summer school at the University of Birmingham in September 2001), forensic speaker identification remains a very challenging task, even now when automatic speaker recognition is already in commercial 
use. In fact, some researchers have a very pessimistic view of the prospect of forensic speaker identification (for instance, Boë 2000), because of the limitations a situation can impose upon forensic speaker identification. The fact that automatic speaker recognition is so successful, at least in the laboratory environment, seems to indicate, however, that people can be discriminated from each other on the basis of their voices.

This thesis sets out to investigate whether or not forensic speaker identification can be performed, using forensically realistic data, which is natural and non-contemporaneous speech. The research questions to be answered in this thesis are:

Two main questions:

1) Do formants discriminate speakers?

2) If they do, how well do they perform?

In addition, the following three secondary questions:

3) What are the optimal parameters?

4) What is the optimal statistical approach available now?

5) Is there any language specific difference in forensic speaker identification?

The importance of the two main questions of this research is illuminated by an article by Monastersky (1998) on the U.S. court decision on the treatment of scientific evidence. In the case of Daubert v. Merrell Dow Pharmaceuticals, Inc., U.S. Supreme Court has held that judges must assess the scientific reliability and relevance of the reasoning or methodology underlying any proposed testimony. The Daubert decision offered a list of factors which needs to be considered in the use of scientific evidence. One of these factors is "whether a theory or technique can be, and has been, tested." 
This seems nothing more than a reasonable and just condition, considering the significance of the effect which the evidence might cast upon the defendant's life. Given the current state of affairs, however, following this court decision would result in not admitting voice evidence to the court. To date, there is no forensic phonetic research which tests how accurately speakers can be discriminated on the basis of their voice under forensically realistic conditions. Obviously this situation must be rectified, as the demand for forensic speaker identification has been, and will continue to be, strong. This research thus aims to provide the first test of the accuracy of realistic forensic speaker identification.

Further, it should be noted that this study is based on centre frequencies of formants, which are the most commonly used acoustic parameter in actual forensic speaker identification today. As mentioned, automatic speaker recognition reports high successful identification rates. Most research in automatic speaker recognition today uses cepstrum as a parameter, however, and it is still not certain how powerful formant patterns can be in discriminating speakers.

As to its general structure, this thesis can be divided into three parts. The first part provides the necessary background information for the discussion in this research. Chapters 1, 2, and 3 are the chapters included in this first part of the thesis. Chapter 1 discusses the nature of forensic speaker identification and its relevant issues, Chapter 2 introduces the sound structure of Japanese, and Chapter 3 describes the detail of the procedures of experiments in this study. In Chapter 3, the selection of parameters for speaker identification is also discussed in detail, including the justification for the use of centre frequencies of formants as a parameter for forensic speaker identification.

The second part of this thesis searches for the promising candidates for the speaker identification parameter, and Chapters 4 and 5 are included here. These two chapters study vowels embedded in different phonological contexts, and a Japanese word moshimoshi (which is "hello" in English) focusing on which segment / formant combinations have larger between- 
to within-speaker variation ratios. In these chapters, the effect of non-contemporaneity of datasets is also examined.

The final part of this thesis, which is Chapter 6, seeks the answers to the two main research questions of this thesis. Two questions, whether or not formant patterns can discriminate speakers and, if they can, how well they perform, are investigated by carrying out discrimination tests. The parameters which are used for the discrimination tests are selected on the basis of the results produced in Chapters 4 and 5 .

In addition to the three main parts described above, finally Chapter 7 presents the conclusion of this study. This chapter summaries the findings of this research and also discusses future tasks for further research in forensic speaker identification.

\subsection{SPEAKER RECOGNITION AND SPEECH RECOGNITION}

Speech recognition and speaker recognition are sometimes confused. They are both processes which we perform in the course of our every day speech communication and they even sound similar. Their goals are quite distinct, however, as speech recognition aims to process the content of speech, whereas speaker recognition aims to identify who is speaking. The acoustic differences in speech signals reflect both the linguistic differences and the speaker-dependent differences, which derive from physiology and articulations of the given speaker. In order to communicate with other people at all we need to promptly identify the linguistically important acoustic characteristics as we receive speech signals. The task of extracting the linguistically significant differences and therefore understanding the contents of the utterance constitutes speech recognition. 
In speaker recognition, on the other hand, the task which listeners perform is the extraction of the non-linguistically-significant, speaker-dependent characteristics. Speech recognition and speaker recognition are thus complementary processes working on different aspects of speech signals. A simple example can be given using the difference in fundamental frequency (F0). Assume that there are two male speakers, both saying "hello" in a very similar way with similar psychological states. Speaker A has a rather high pitched voice. His mean F0 is around $160 \mathrm{~Hz}$. Speaker B has, on the other hand, a low voice and his mean F0 turns out to be around $90 \mathrm{~Hz}$. In this case, the answer to the question whether or not they are the same speaker would be obvious to any listener. There are of course many other cues, such as formant structures, for listeners to perceive the difference in these two speakers' voices, however there is no doubt that the large difference between two speakers' fundamental frequencies would provide a strong clue. F0 is perceived as a cue for speaker recognition here.

Now, imagine that these are Thai speakers. Thai phonology comprises five tone types: low, mid, high, falling, and rising. Differences in F0 heights and contour distinguish lexical meanings of words. For instance, the segmental sequence [naa] can mean something totally different depending on its F0. The meaning and tone are listed as below.

\begin{tabular}{lll} 
Tone & \multicolumn{2}{c}{ Meaning } \\
naa & low tone & 'a nickname' \\
naa & mid tone & 'rice paddy' \\
naa & high tone & 'young maternal uncle or aunt' \\
naa & falling tone & 'face' \\
naa & rising tone & 'thick'
\end{tabular}

As mentioned above, Speakers A and B have $70 \mathrm{~Hz}$ mean F0 difference. Naturally their F0s of the word "naa" reflect this difference, so that it is quite possible that F0 of Speaker A's low 
tone is in fact higher than that of Speaker B's high tone. In actual communication, however, such confusion hardly occurs. Listeners understand the meaning of word correctly. This is because listeners constantly normalise the absolute height of F0 they hear in order to extract the linguistic content of the received speech signals. In this process, listeners ignore the absolute height of F0 and focus on the relative height differences within a speaker, performing speech recognition.

Out of these two processes of human perception, this study focuses on speaker recognition. The experiments and discussion are thus concentrated on the speaker-dependent acoustic characteristics in this thesis. Before continuing the discussion though, I present how speaker recognition has been defined in preceding studies. Two examples are presented below:

\footnotetext{
"Speaker recognition is the analysis of speech to infer the identity of the speaker to check a claimed identity (speaker verification)" (Crystal 1991:323).

"... any decision-making process that uses some features of the speech signals to determine if a particular person is the speaker of a given utterance which will include tasks such as identification, verification, discrimination, and authentication of speakers" (Atal 1976:460 f1).
}

The definition by Crystal concisely sums up the nature of speaker recognition, but it is too concise in the sense that it mentions only speaker verification, which is only one part of the various decision making tasks involved in speaker recognition. Atal's definition, on the other hand, mentions all of these tasks. The differences in the decision making tasks have significant influence on the design of the test and statistical procedures, so they should not be ignored. The difference in tasks will be discussed in the later section of this chapter (1.8). 


\subsection{TYPES OF SPEAKER RECOGNITION \\ 1.3.1 FORENSIC SPEAKER RECOGNITION AND OTHER APPLICATIONS}

The technique of speaker recognition is applied for different purposes. Voice activated security systems or telephone banking are well known. Künzel (1995:68) calls these areas of application 'commercial speaker recognition', opposed to 'forensic speaker recognition'. Forensic speaker recognition, which is the topic of this research, and commercial speaker recognition obviously share some techniques in areas such as the extraction of features of speakers or measurements of acoustic parameters. It cannot be emphasized enough, however, that commercial and forensic speaker recognition are very different in terms of the challenges they face. Künzel (1995:68) already said that commercial application in 1995 did not have many scientific or technological problems. For this Künzel's comment, there are objections claiming that speaker recognition outside the laboratory still needs much improvement (Michael Barlow, Frantz Clermont p.c.). It seems, however, that forensic speaker recognition still has far more difficulties and unsolved problems than automatic speaker identification. What makes these two types of application so different, then?

The major difference between forensic and non-forensic speaker recognition lies in the control over data. For commercial speaker recognition, good control over the recording conditions is possible. The contents and the length of speech samples will be highly comparable, since the phrases uttered can be prearranged. Although, it is practically impossible for anyone to say anything in exactly the same way twice (Bricker and Pruzansky 1976:300), this minimises the difficulty in comparison. In forensic situations, however, we do not have much control at all over the speech materials. It is not realistic to expect to have two recordings of two forensic speech samples which include the same words uttered in a similar context, intonation and/or psychological states. 
Also, the samples obtained from a crime scene are often poor in their recording quality. They might contain a lot of background noise, or they might be distorted due to the poor quality of a phone line or recording equipment, such as a security video camera. Moreover, recordings through an analogue telephone line introduce a problem of a different sort: telephone line attenuates signals outside of a certain frequency range. The signal is typically bandpassed at 300-3400 Hertz with a telephone line. Thus, low-frequency components containing the first formant (F1) of some segments and the fundamental frequency are severely distorted in telephone speech (Rose and Simmons 1996), and high frequency components, such as fricative noise from $/ \mathrm{s} /$ or $/ \mathrm{J} /$, which possibly contains highly speaker-specific features, become unobservable (Künzel 1995:72). Given the fact that recording over telephone is one of the most common situations in forensic cases, such as bomb threats or ransom demands following abduction, this is a serious issue.

Attitudes of the speakers are another significant difference between these two types of speaker recognition. Speakers can be expected to be compliant in commercial recognition, whereas, in forensic scenes, speakers normally do not wish to be identified. This difference in speakers' attitudes often manifests itself as a voice disguise in forensic situations. Speakers might lower or raise their F0, whisper, attempt to put on a foreign accent, or cover their mouth in order to alter the aural impression of their voices, when they are aware of the possibility of interception. This makes it impossible to measure some or even most of the acoustic parameters and, thus makes the process of forensic speaker identification even more complicated.

\subsubsection{TYPES OF SPEAKER RECOGNITION - TWOFOLD OR THREEFOLD?}

\subsubsection{Threefold distinction ? SRL, SRM, and SRS}

Speaker recognition involves various techniques. Bricker and Pruzansky (1976) divided speaker recognition into three types, according to the techniques applied in the analysis of 
speech samples. They talk about speaker recognition by listening (SRL), by machine (SRM), and by visual inspection of spectrograms (SRS). The concise summary of each category is provided by Nolan (1983):

\begin{abstract}
"... SRL involves the study of how human listeners achieve the task of associating a particular voice with a particular individual or group, and indeed to what extent such a task can be performed. SRM encompasses the attempts to develop automatic and semi-automatic strategies, standardly computer-based, for associating voices with speakers; SRM is therefore often thought of as 'objective' in comparison with SRL because of its relative freedom from human decision-making. The third category, SRS, comprises efforts to make decisions on the identity or non-identity of voices on the basis of visual examination of speech spectrograms by trained observers (p.6)."
\end{abstract}

\title{
1.3.2.2 Twofold distinction
}

Nolan (1983:7), however, does not agree with Bricker and Pruzansky's categorisation, and he proposes a twofold division based on different criteria. The point of his counter-argument is concerned with SRL. He claims that the main difference between SRL and the other two approaches lies in the fact that SRL is most often performed by untrained individuals who witnessed crime scenes, but not by trained experts. SRM and SRS are, on the other hand, always performed by experts, using analytic techniques and solid knowledge of acoustic and linguistic phonetics. Nolan claims that this difference between naïve listeners and experts is far more important than the differences in technique and, thus proposes the twofold division technical and naïve recognition.

Nolan provides further justification for favouring the twofold division. Firstly, SRS and SRM are not always two different analyses, but they are often contingent. That is often the case that spectrogram analysis is incorporated in the process of SRM. Secondly, in Bricker and Pruzansky's three-way division, there is no place for technical speaker recognition by listening. SRL by trained phoneticians (technical speaker recognition by listening in Nolan's classification) is to be distinguished from SRL by naive listeners. Auditory analysis by 
phoneticians employs techniques which are acquired through phonetic training, and the judgments made in this process are based on the knowledge of phonetics and phonological structure of the given language, as opposed to that of naive listeners who rely solely on their auditory impressions.

Furthermore, technical auditory analysis is the basis of all the acoustic analyses. In the forensic situation in particular, it is careful listening which tells experts whether or not the given speech recordings are in fact comparable or even usable and, if they are comparable, which phrases or words are suitable for comparison.

It is thus not possible to categorise the styles of speaker recognition into three by the techniques involved as Bricker proposes. The following sections discuss each technique spectrogram reading, auditory, and machine - separately. This classification is, however, mere classification of the available techniques, and not the categorisation of the types of speaker recognition. The discussion of the auditory method is divided further into two, technical and naive recognition, since they differ considerably in the technique used, although they both are based on auditory impression of samples.

\subsubsection{THREE TECHNIQUES IN FORENSIC SPEAKER IDENTIFICATION}

\subsubsection{Spectrogram reading}

The visual interpretation of ordinary broadband spectrograms as a measure for forensic speaker identification was first proposed by Kersta (1962), introducing the new term 'voiceprint.' A spectrogram can be visually presented in courts as evidence, as opposed to speaker recognition by listening where juries rely solely on the statements made by an expert or non-expert witness. This made the visual inspection of spectrograms look more scientific than what it is in reality, and forensic speaker recognition by this method was claimed to be objective. 
According to Künzel (1995) this approach was widely employed in the United States, parts of Europe, Israel and other countries, during the late 1960s and 1970s. It has, however, lost ground today in some countries like the United States and it was completely abandoned in Germany. The FBI in the United States is using it only for investigative purposes, but not as evidence (Koenig 1986). Although it is still in use in countries such as Israel, Italy, Spain and Colombia, the credibility of this procedure is now almost discredited (e.g. Künzel 1995; Nolan 1990; Nolan 1996; Reich et al. 1976; Stevens 1971).

So, what then are the problems of visual inspection of spectrograms?

The answer to this question lies in the fundamental concept underpinning this approach. Kersta (1962), who proposed the term 'voiceprint identification,' claims that 'people can be identified by a spectrographic examination of their voice." He continues:

"Closely analogous to fingerprint identification, which uses the unique features found in people's fingerprints, voiceprint identification uses the unique features found in their utterance. (p. 1253)"

Clearly, Kersta considers that spectrographic images of human voice are comparable to fingerprints. The difference between these two is substantial, however, as pointed out by Nolan (1990:3). The most significant difference between a fingerprint and a voiceprint lies in the plasticity of human voice. A fingerprint is a direct and stable record of human anatomy which varies from person to person. A voice is, on the other hand, "an indirect record of a variable acoustic artefact produced by a highly plastic organic mechanism (Nolan 1990:3)." The voice quality of an individual can be altered both intentionally and non-intentionally in various directions with various causes. A voice may be changed by the physiological state, such as a speaker's health state or fatigue, or by linguistic reasons, such as differences in speech styles or accents. There is also the possibility of attempts to disguise speech sample.

Nolan's claims on the flexibility of human voice are clearly supported by Reich et al. 's (1976:919) study. Reich et. al. report that spectrographic speaker identification is vulnerable to 
voice disguise, as opposed to the prior report claiming that the voiceprint speaker identification is unaffected by disguise. The speech spectrograms of a normal and a disguised voice reveal strong variations in formant-bar structure. Reich also reports that spectrograms of the same speaker recorded over periods up to 29 years show significant variation in formant-bar position and mean fundamental frequency.

Furthermore, the effectiveness of voiceprint method of speaker identification is not consistent across different studies. In Kersta (1962)'s experiment, he trained high-school girls to read spectrograms of monosyllables. The girls were provided with spectrograms and requested to sort them out into the piles of appropriate speakers. He altered the number of speakers from five to nine and 12 speakers, and the successful identification rate for this experiment was $99.6 \%, 99.2 \%$, and $99.0 \%$ respectively.

These astonishingly high successful identification rates were, however, not replicated by others. For instance, Young and Campbell (1967) report much lower success rate, 78.4\% using similar methodology. Moreover this success rate was achieved under relatively favourable conditions, when phrases were uttered in isolation. When the word was elicited from sentence context, the identification rate dropped even further to as low as $37.3 \%$.

Hazan (1973) questions Kersta's methodology which uses read-out speech. He experimented with speaker recognition by spectrogram using readings of naturally uttered phrases. The successful identification rate he obtained as a result was nothing like Kersta's. When the test word came from the same context as that of the reference sample, the success rate was $57.4 \%$. When the test sample are elicited from the different context, the success rate turned out to be only $16.8 \%$. From these results, Hazen concludes that it is not possible to identify speakers solely on spectrograms.

Tosi et al. (1972) also report much lower accuracy in their experiments under the more realistic conditions. In their experiments, the clue words were produced in various contexts, two 
samples to be matched were recorded non-contemporaneously (separated by a month), and the reference samples may or may not have included the hypothetical criminal. As a result, approximately $18 \%$ of the matches attempted by the subjects were incorrect, and this error rate is far too large to be performed as evidence in courts.

Stevens (1971:223-224) explains the discrepancy between experiments as differences in the condition of the experiments. The set of ideal voiceprints for speaker identification would be the spectrograms of a number of clue words spoken in isolation; words to be matched are produced on the same occasion as the reference words, and this is what Kersta used. This experimental condition is, however, far from realistic. In forensic situations, it is unlikely that such data is obtained, and high accuracy achieved under such ideal conditions does not justify the use of voiceprint, although it is not impossible to identify speakers with these ideal spectrographic images under closed tests (cf. Simmons 1997).

Thus, voiceprint identification, which was once acclaimed to be highly scientific and an exciting application of phonetics, is now in disrepute. Although it still plays an important role in the course of the acoustic analysis of speech samples, no decision on speakers' identity should be made based solely on spectrograms. As for Japan, however, an official from the National Research Institute of Police Science reported that this spectrogram reading still is the prime methodology for forensic speaker identification (Osanai et al. 1995; Suzuki et al. 1994) even now in 2001 (Takashi Osanai p.c.), with occasional use of the automatic speaker recognition technique. This is a rather disconcerting report. 


\subsubsection{Speaker recognition by listening}

\subsubsection{Naive approach}

As it has been briefly addressed in the previous section, speaker recognition by listening can be classified into two types: naïve and technical. Here, the terminology 'naïve' does not only address listeners in the sense that they are not trained in phonetics or speaker identification. The term 'naïve' characterises the techniques which are involved in the judgement making process. In the technical approach, listeners are allowed to listen to both incriminating recordings and the suspects as many times as they wish and perform analytic observation using their knowledge of phonetics and linguistics. Any cases which deny the access to these techniques are to be considered naive, regardless of the qualification or training level of the listeners.

Naïve auditory speaker identification is requested when cases involve an earwitness as a part of evidence. An earwitness is called for voice line-up and asked whether he /she recognises any of the voices presented as the voice they heard at the crime scene. How objective and accurate naïve auditory speaker identification has been questioned by many (for instance, see Goggin et al. 1991; Goldstein et al. 1981; Hollien 1990; Hollien et al. 1995; Köster and Schiller 1996; Köster and Schiller 1997; Köster and Schiller 1998; Künzel 1994; Künzel 1995; Ladefoged 1978; Ladefoged and Ladefoged 1980; Laubstein 1997; Markham 1999; Nolan 1990; Rose and Duncan 1995; Schiller et al. 1997).

Künzel (1995:75) points out that the information which earwitnesses provide is a valuable tool for crime investigations under the circumstances where a live recording of incriminating speech does not exist. Naïve auditory speaker identification thus undoubtedly has its own significance. There are many experiments, however, which urge cautions in the application of earwitnesses' testimony, reporting that the performance of a caution can vary greatly depending on the condition of identification. 
First of all, performances in speaker identification vary depending on listeners' familiarity to the voice. Rose and Duncan's (1995) experiment on naïve auditory identification reports that even naïve listeners can achieve a high identification rate with familiar voices. In this experiment, although Rose and Duncan used speakers with very similar voice samples from close family members, such as a father and his son, the person who is familiar with the voice (also close family members, such as a mother / a wife of the speakers) could identify the voice successfully. From these results, they claims that

\footnotetext{
"...even under these conditions of superimposed difficulty, as long as the utterance duration is above a certain threshold, listeners can successfully recognise the voice of people they know well, and that the accuracy of their judgments is reflected by their confidence (p.15)."
}

Schmidt-Nielsen and Stem (1985) carried out an experiment, examining the relationship between the listener's familiarity to the stimuli and the identification rate. In their experiment, 24 listeners performed speaker identification on 24 speakers, who were their co-workers. He reports that familiarity ratings with the given voices by the listeners were significantly correlated with correct identifications. Thus, although Rose and Duncan report a high identification rate with familiar speakers' voices, the accuracy is expected to vary according to the degree of the familiarity. The establishment of the objective scale for the degree of familiarity is obviously difficult. Evaluation of how reliable a particular ear witness is with the given voice is therefore not a simple task.

Furthermore, forensic cases involve identification with unfamiliar voices, and the accuracy of identification with an unfamiliar voice is not great. There are studies which claim the cognitive processes for speaker identification differ depending on whether the given voice is familiar or not to the listener. Lancker et al. (1985) report that the task for identification of familiar voices is something like pattern recognition. In identification of unfamiliar voice, on the other hand, listeners need to perform feature analyses of the given voice rather than pattern recognition, since listeners do not have the acoustic cues for the given voice stored as patterns in their memory. The two types of stimuli are processed possibly even using the different 
cerebral hemispheres of brain. Identification of a familiar voice, which is essentially a pattern recognition, is carried out in the right hemisphere (Lancker et al. 1985:34), whereas identification of an unfamiliar voice would probably be processed in left hemisphere, which is in charge of sequential/analytic processes.

There is also listener-to-listener difference in listening ability. For instance, Köster et al. (1998) reports that, as well as experts in speaker identification, people with musicality or musical training perform better in speaker recognition. In forensic situations, it is not possible to have control over those abilities of the earwitnesses. Although it may be possible to weigh testimonies made by earwitnesses, as Köster et al, (1998) report that the ability to recognise speakers correlates with the results of Pahn and Pahn's (1991) test on speech sensitivity, much further research is necessary for the establishment of a methodology of weighting.

The duration of time which elapses between the commission of the crime and the voice line-up also influences the accuracy of speaker identification. Under normal forensic circumstances, it usually takes a certain amount of time for the arrest of suspects. This can lead to serious problems in the process of speaker recognition by listening. McGhee's $(1937,1944)$ experiments showed that the recognition rates dropped sharply with increasing time lag between the first acquaintance with a voice and the recognition task. In fact, the recognition rate drops down to the level of chance in under six months.

A study by Huntley Bahr and Pass (1996) demonstrates that speaker's utterance types have a significant impact on identification judgments. They presented voice samples representing casual, extemporaneous and formal conditions which were elicited from five African-American males. The results showed that comparison between two different speech registers seriously degrades listeners' identification performance. Hence, they concluded, "...code switching, as induced by changes in the social situation and recording environment, may result in the misidentification of a particular speaker (p.24)." 
The knowledge of the language is another factor which affects auditory speaker identification. The knowledge of a given language helps listeners to distinguish the characteristics of the particular speaker from the linguistic or phonetic features of the language. Using Taiwanese, white Americans, and black Americans as speakers and white Americans as listeners, Goldstein et al. (1981) investigated the effect of foreign accents on speaker recognition. The results showed that correct identification rates do not differ for each accent group, although once the duration of stimuli was shortened, the performance level on the identification of Taiwanese accented English dropped. They also compared speaker identification by white American listeners using heavy Spanish-accented English and Spanish stimuli. Listener's performance on these two languages did not differ, and they conclude: "Practically speaking, voice recognition is just as good (or as poor) for foreign voices as it is for native voices." However, the credibility of Goldstein et. al.'s study deserves criticism for two reasons. Firstly the retention time between the exposure to the stimuli and the selection of the speaker was only 10 minutes in this study, which is unrealistic in the forensic situation. As time degrades the memory of voice significantly, their results are not very reliable. Secondly, in the experiment of identification using incomprehensible foreign language as stimuli, they compared the results just with heavily accented English, but not with the listener's native language, white American English. The fact that the listeners identified speakers as well (or as poorly) in both types of stimuli thus seems not enough to justify their claim, since English with heavy Spanish accent may be very foreign to white American listeners.

In fact, there are studies which claim that the knowledge of the language affects the listener's ability to identify speakers (Goggin et al. 1991; Köster and Schiller 1996, 1997; Schiller et al. 1997). Goggin et al,'s (1991) experiments demonstrated monolingual listeners identified bilingual speakers' voices much better when the speakers spoke in the listeners' native language. They also demonstrated the importance of linguistic knowledge in speaker identification by presenting systematically deformed English by rearranging words and syllables, and reversing normal text. Voice recognition rates deteriorate as the spoken passage 
becomes more unintelligible. They claim that these results confirm that language familiarity plays an important role in voice identification.

Köster and Schiller (1996) tested three groups of listeners - American English listeners with some knowledge of German, American English listeners without knowledge of German, and native German listeners, and German was used as the stimuli language. The results showed that there is statistically significant difference between English listeners without knowledge of German and native German listeners in their speaker identification performance.

Köster and Schiller (1997) replicated the same experiment with Spanish and Chinese listeners, with and without the knowledge of German. The results showed that Spanish and Chinese listeners with knowledge of German performed significantly better than their compatriots with no knowledge of the target language. Further, even Spanish and Chinese listeners with knowledge of German perform significantly worse than native Germans.

From the studies discussed above, Schiller et al. (1997) considered that listeners with different native-language backgrounds should perform similarly in identifying a target speaker, when linguistic information is removed from the presented stimulus materials. They removed most linguistic information from the speech of a German speaker and presented it to three groups of listeners; native English listeners without knowledge of German, native English listeners with some knowledge of German, and native German listeners. The results show that without linguistic information, the performance levels in speaker identification do not differ significantly among these three groups. Listeners' knowledge of the given language, thus, seems to affect speaker identification considerably.

Psychological factors are also known to have a large effect on human's perception. For instance, Ladefoged (1978) reports that even highly skilled and experienced phoneticians are heavily affected by psychological factors, when requested to identify speakers in a naïve 
manner. In his experiments, phoneticians were asked to listen to some voices and to identify the speakers if they recognised the given voices. In general, they performed well. Nine out of ten listeners correctly identified all the eleven speakers they knew well. When they came across an African American speaker who was not familiar to them, however, they showed rather worrying responses. All of the listeners realised that one of the speakers they heard was African American, but they wrongly identified him as a co-worker of theirs, who is also an African American, presumably because of their expectations. This indicates that even trained phoneticians cannot be totally objective when judging speakers' identity, if they are not allowed technical analyses. Ladefoged and Ladefoged (1980:47) claim that people tend to identify a voice as a given person's when they are expecting to hear that person's voice. It is thus perfectly possible that an innocent suspect is identified as a criminal, without any malice being involved, because of the witness's quite understandable enthusiasm to identify the criminal.

Hollien et al. (1995:149) point out that listeners' awareness of the importance of what they hear also can affect their performance in voice line-ups. In real forensic cases, unlike in the experimental conditions, earwitnesses may or may not know the need to remember the voice they are hearing. This can affect the accuracy of speaker identification, as those who are aware of the necessity would perform better than those who are not. This listeners' awareness is, however, not a controllable factor in real cases.

Hollien et al. (1995:149) also address a problem associated with the methodology of voice lineups. They claim the design of voice line-ups can affect the listeners' performance greatly. Accuracy of identification can be influenced by whether or not the target voice is included in the line-up. The use of too many voices in a line-up is also a problem, as witnesses' memory can be weakened by being exposed to too many voices at one time. Any alterations in the suspect's voice or sample presentation mode may confuse the witness and result in false 
positive identification. Telephone transmission and poor recording quality may degrade the witnesses' performance in identifying the target voice.

Laubstein (1997) examines the validity of the transcript method, which is a commonly used method in the construction of the foils for voice line-ups. He employed two types of foils, actors and police officers, and provided them with the transcript of the phrases which the subject uttered. Through a series of questionnaires Laubstein demonstrated that listeners' responses to the suspect's speech were quite different from those to the foils' speech, and therefore he concludes the transcript method is likely to bias the voice line-ups. Taking note of many preceding studies on aural voice identification, he sums up:

\footnotetext{
"The whole area of aural perceptual voice identification is just beginning to be investigated, and the enormous lack of knowledge in this area makes the use of the voice line-up to identify suspects at best problematic and at worst dangerous. (p.276)"
}

\subsection{Technical speaker identification by listening}

The problems and concerns with earwitness testimonies made by naïve listeners have been extensively discussed above. However, these do not deny the validity of aural speaker identification altogether. As mentioned briefly in the previous section, the technical approach by experts stands on a totally different ground from that of its counter part, the naïve approach, and technical speaker identification by listening is well credited as evidence. In fact, the speech evidence in United Kingdom up until 1990 has been based mostly on careful analytic listening of the speech samples rather than anything else (Nolan 1990:4).

The difference between these two approaches firstly lies in the skill that the listeners have. The person involved in technical auditory analysis must be an expert. Phoneticians (or speech experts) are trained to listen to the subtle phonetic differences in speech and analyse these systematically. They also have knowledge of linguistics and phonetics, which provides the 
essential basis for observation and judgment making. Knowledge in these areas enables experts to extract speaker-specific characteristics and to evaluate how common or unusual the voice quality of the given speech sample is.

As to the actual process in usual practice, experts carefully listen to the given speech samples many times and transcribe, if it is necessary. While listening, experts make notes on what seem to be characteristics for the speaker. If the speaker has speech defects, for instance, it can provide useful information on the speaker's identity. In most cases, however, experts need to work on more subtle differences, such as the realisation of certain vowels. After careful listening, these features are compared across the recordings. In technical auditory identification, the whole process is thus strictly based on specialised skills which experts have, and observations are made in more analytic and objective manners than in the natve approach.

In addition to the difference between the two types of aural identification in the skills and the methodologies, there is another factor which favours the technical approach over the naive one. That is, experts were not there when the crime broke out. Since they are not the people who have actually been affected by the crime, they are less likely to be influenced by psychological factors, such as fear or anger. This will also reduce the chance of being biased.

However, it does not necessarily mean that technical speaker recognition by listening is fully reliable by itself as evidence. Quoting an actual bank fraud case, Nolan (1990) demonstrates that even experts using the technical approach can make a wrong judgement on speaker's identity if they make the decision based exclusively on auditory analyses. In this case, two experts were called for by the prosecution and were given the suspect's telephone conversation recordings. The experts performed auditory analyses and produced very similar diagnoses. Both experts thought the given two samples were from the same speaker, ie. favouring the hypothesis that the suspect is guilty. Nolan, being called for by the defence, carried out auditory analysis and, on the basis of that, he also performed acoustic analysis. The result of 
acoustic analysis contradicted the testimony made by the two prosecution experts. The acoustic analysis revealed more characteristics which suggested that those two speakers were different individuals. Although, only the suspect and the real criminal of this case (supposing that the suspect is innocent) knows the truth in theory, this and other pieces of evidence made prosecution drop the case.

The studies reviewed above thus show that the auditory approach is not powerful enough by itself to offer conclusive evidence to identify someone as a criminal. This does not mean, that the auditory technique is not useful. On the contrary, careful listening to the speech samples is essential, as too is the application of the acoustic analysis of speech by machine which is to be discussed in next section. Some peculiarities in voice quality and speech style are often identified more easily by careful listening rather than acoustic analysis. More importantly, it is by auditory analyses that experts decide whether or not the given speech samples are indeed comparable. Disguise, background noise, and poor recording quality sometimes make the comparison between samples impossible. The difference in situations, emotional states and / or interlocutors can also make large differences in their acoustic output. There is no use comparing an aggressive yelling voice at a bank robbery and an intimidated nervous voice talking at the police interview. Auditory analyses by experts are the most effective method to evaluate the comparability of the data.

\subsubsection{SPEAKER RECOGNITION BY MACHINE AND HYBRID METHOD}

The approach finally mentioned here, speaker recognition by machine, has a significant advantage over auditory approaches mentioned above. Speaker recognition by machine involves acoustic analysis of speech signals, and allows us to quantify data. Quantification of data using statistics is indispensable for objective assessment of data. Although quantification 
of the results of auditory analysis is also possible by counting and using non-parametric statistics, continuous data produced by acoustic analysis is usually more easily quantified.

Given the importance of objectivity and faimess in forensic speaker identification, the advantage of the machine-aided method in quantification is extremely variable. For the effective use of machine as an aid for the forensic speaker identification, however, we need to also incorporate the other two approaches discussed above (Majewski and Basztura 1996). In fact, speaker identification based purely on machine is not possible. Speaker identification by machine in forensic context thus really requires a hybrid method.

As discussed in the previous section, auditory analyses by human ears are indispensable in the selection of the suitable targets for comparison and the evaluation of the comparability between the speech samples. The samples from a crime scene and from police interrogation with suspects are quite possibly uttered in different psychological states, social context or even dialects. It is also quite possible that the two samples were recorded with different equipment, resulting in different recording quality of the samples. These differences are reflected in the acoustic signals, but these acoustic differences here are nothing to do with the speaker's identity. As auditory analysis excels over machine-oriented methods in the identification of factors which are unrelated to speakers' identity, auditory analysis should always be incorporated in speaker recognition.

The hybrid method thus incorporates the advantages of the approaches which are available to us now. This does, however, also introduce a different problem from an approach where speaker identification purely based machine, such as automatic speaker recognition. That is, subjectivity. In order to incorporate auditory analyses, which are absolutely necessary in forensic speaker identification, human judgement is also integrated into the analysis. Thus, unlike in automatic speaker verification, the assumption that the machine aided approach equals objectivity is an oversimplification in forensic speaker identification, as Nolan 
(1997:763) points out. Currently no forensic speaker identification can be free from subjectivity.

As a final point, the implication of the use of speech analysis software should also be noted. Digitisation and measurement of speech samples with a computer program facilitates acoustic analysis immensely. The significance of the role played by speech analysis software packages in modern acoustic phonetics research is thus inarguable. It should not be forgotten, however, that what we are dealing with in acoustic analysis are signals transformed by speech processing software (Computer Speech Laboratory in this study), as opposed to direct recordings of the acoustic energy. The values measured by particular sound analysis software are thus a secondary form of acoustic output, and there is always a possibility that the tools bias the measurements. It is thus very important to be consistent with the use of speech analysis software in any acoustic analysis. Comparing the data sampled using different software may introduce the variation arising from the differences in the software, but not from the speech samples themselves.

\subsection{WITHIN-AND BETWEEN-SPEAKER VARIATION}

Speaker identification stems from the idea of within- and between- speaker variation. The high plasticity of the human speech mechanism allows us to have a certain range of variation in our speech. In fact, even if we try our hardest, we can never say anything twice in exactly the same acoustic quality. This variation which is produced by a single speaker is called within-speaker variation. Between-speaker variation means, simply, the variation between different speakers.

Speaker identification is based on the idea that between-speaker variation is larger than the within-speaker variation. Nolan (1997) describes this within-speaker variation using a term 'speaker space,' which is a "multi-dimensional space comprised of the dimensions along which 
speakers are differentiated (p.743)." In this view, each speaker occupies an area, not a point, because of the plasticity of the human speech mechanism. The size of speaker space possibly differs from speaker to speaker. Speakers also can share some space (i.e. they share a part of the acoustic output of their speech sounds). The speakers sharing larger space are those with similar voices. Although some speakers share certain areas, the distance between different speakers is assumed generally to be larger than within-speaker variation. Speakers A and B may share some quality in their speech, but each speaker has his or her own speaker space, and this space does not accommodate the entire space of the other speaker. If it does, then, these two people are indistinguishable in terms of the acoustic quality of their voice. The conceptualised distribution of within- and between-speaker variation is presented below in Figure 1.1.

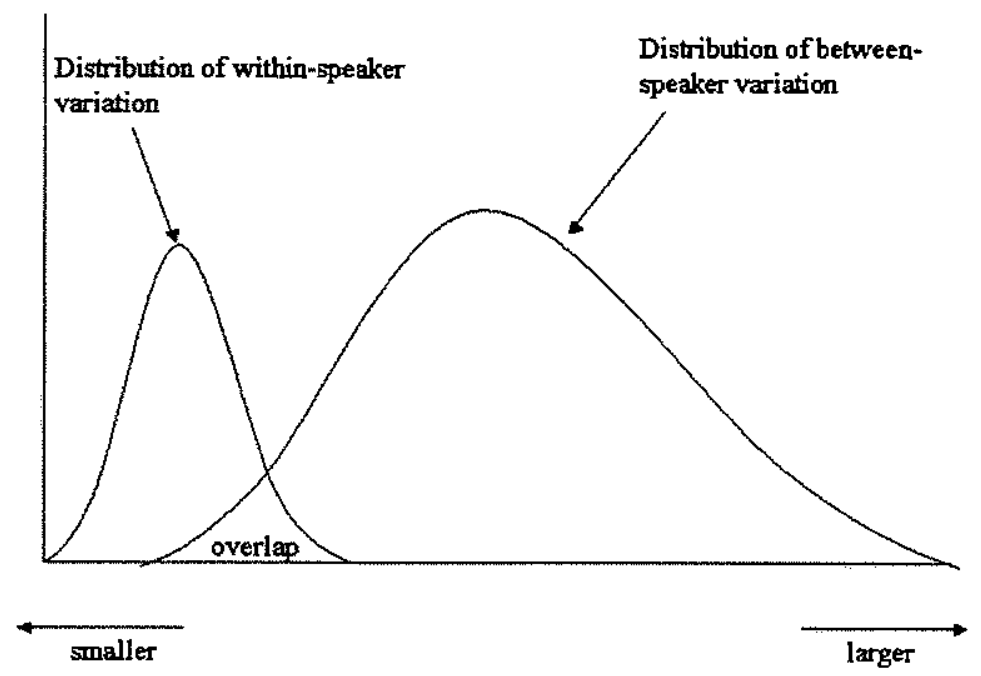

Figure 1.1 Model of the distribution of within- and between-speaker variation.

As seen in Figure 1.1, within-speaker comparison supposedly produces smaller variation than between-speaker comparison. There are some speakers who have similar voice quality to each other. The difference between these speakers can in some cases be smaller than the difference between two samples taken from a single speaker. Overlapping area between two sets of variation is produced under such conditions. The task in speaker identification is to find out 
where the threshold should be set in order to maximally distinguish within- and betweenspeaker variation and also to determine the enror rate produced using the threshold.

\title{
1.5 HOW DOES VOICE DIFFER?
}

Before going into any further detail of forensic speaker identification, what causes differences in human voice should be discussed. There are an enormous number of factors which can differentiate voices. The sources of the differences are traditionally divided into two categories; organic and learned differences (cf. Atal 1976; Bricker and Pruzansky 1976; Garvin and Ladefoged 1963; Glenn and Kleiner 1968; Stevens 1971; Tosi et al. 1972; Wolf 1972) Wolf (1972) describes these two categories as follows:

\begin{abstract}
"Differences in voice stem from two broad bases: organic and learned differences. Organic differences are the result of variations in the sizes and shapes of the components of the vocal tract: larynx, pharynx, tongue, teeth, and the oral and nasal cavities. ... Learned differences are the result of differences in the patterns of coordinated neural commands to the separate articulators learned by each individual. Such differences give rise to variations in the dynamics of the vocal tract such as the rate of formant transitions and coarticulation effects." (p. 2045)
\end{abstract}

This 'organic / learned' dichotomy also has a variation. For instance, Garvin and Ladefoged (1963:194) divide 'leaned' differences further into two categories: 1) "individual variation within a particular single group pattern" and 2) "idiosyncratic speech patterns due to the use of a mixture of social and/or regional varieties of speech by a given individual."

Nolan (1997), however, criticises the adequacy of this organic versus learned dichotomy. Although he admits that the 'organic/ learned' classification is a good approximation to grasp the nature of the source of the individuality of human speech, he claims that, the complex nature of the human speech mechanism makes it impossible to classify the source of people's individuality of their voices simply into organic or learned differences. For instance, formant frequencies, which are the acoustic parameter used in this research, can be determined by both anatomical differences in the vocal tract size and shape and also the phonetic quality of the 
segments which are to be produced. The idiosyncrasy of the vocal apparatus is thus not directly reflected in speech sound, but just limits the acoustical range of speech sounds which a speaker can produce (Nolan 1983:32).

Instead of following the traditional organic / leamed dichotomy, Nolan $(1983,1996,1997)$ suggests to approach the sources of the differences in speech, taking whole speech mechanism into account. Nolan (1996:3 of the second handout) presents a model of the variables which construct acoustic differences in speech. Figure 1.2 below is the reproduction of Nolan's model.

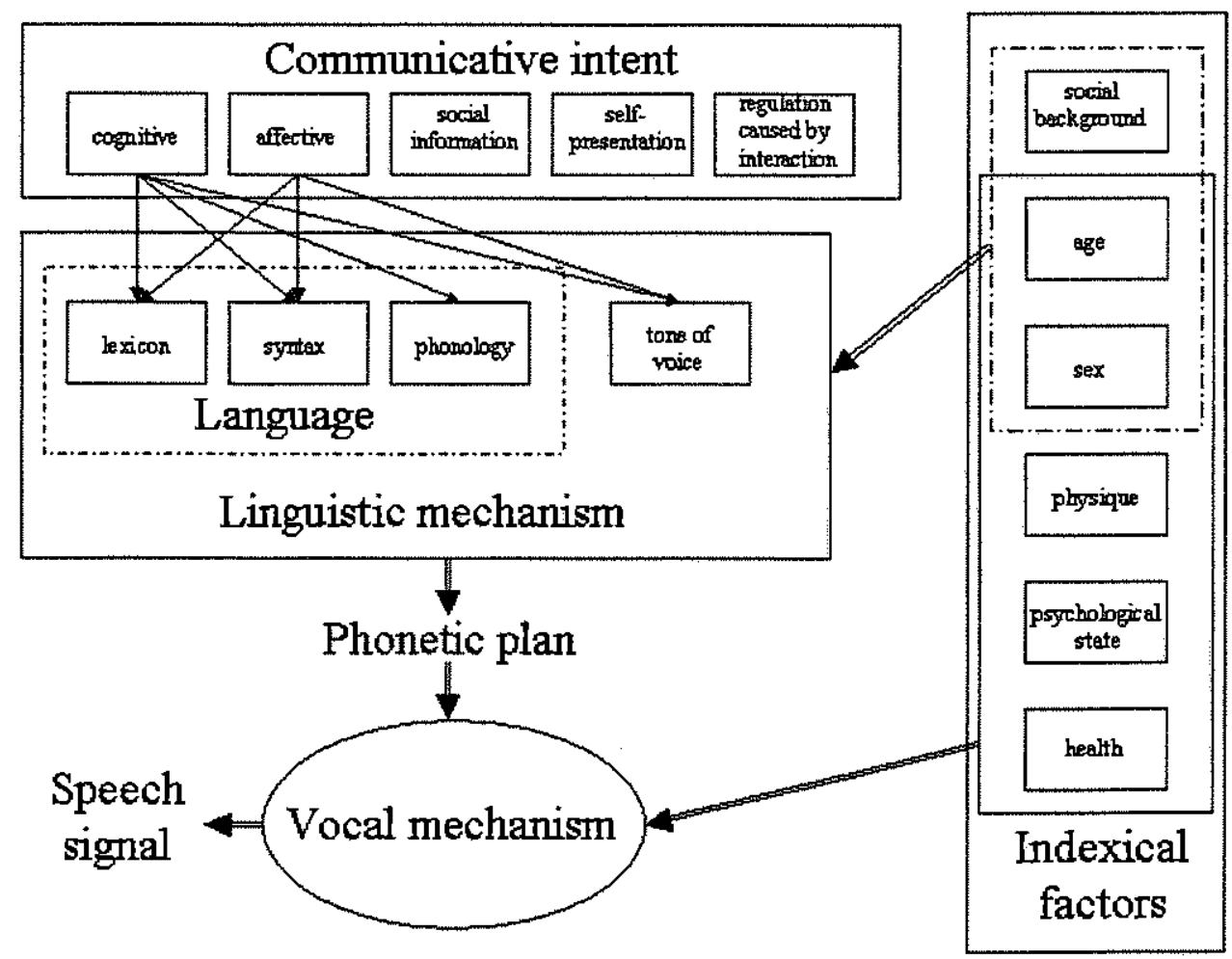

Figure 1.2 Nolan's model of speech production.

Nolan's model of the human speech mechanism shows a speaker firstly has a communicative intent. This communicative intent should be distinguished from the simplistic cognitive content of an utterance, since the term 'communicative intent' includes all aspects of the information which a speaker intends to convey. This consists of affective, social, self- 
presentational information, and regulation of an interaction, as well as cognitive information, which is the literal meaning of their speech, and each component of this communicative intent forms the first set of sources of variance in speech. The affective part of communicative intent carries the information of a speaker's emotions and / or attitude. It is common knowledge that emotions and attitudes both have influence on voices; we often describe people's voice as happy / depressed, angry / kind, or warm / arrogant tones of voice. Social intent comprises speech style switching, for instance, casual to formal, or dialect to standardised language according to context. In this switching, speakers may choose one style over the other to converge or diverge in their speech styles to assimilate or distinguish themselves into / from the other speakers. Self-presentational information is projected with their speech on the basis of the speaker's self-image of their personality. A person who wants to be recognised as a socially and economically powerful person, a boy who wishes to sound the "coolest" among his mates in school, or a sales person who tries to sound sincere to sell his products, all try to project their self-images with their speech. These different self-images naturally contribute to the differences in their speech. The regulation caused by interaction refers to the speech behaviours associated with the way in which a verbal interaction should be structured. For instance, certain changes in F0 and /or intensity are expected to occur at the timing of turn taking in a conversation. The acoustic realisation of speech is thus regulated by the rule of interaction as well.

To convey communicative intents, the intents have to be mapped onto the phonetic realisation. The communicative intents discussed above have to be realised within the limit of the linguistic mechanism of whatever language the speaker is using. A linguistic mechanism, the second set of sources of variance in speech, comprises lexicon, syntax, phonology of a given language, and tone of voice. Tone of voice here refers to "non-phonological manipulation of voice quality and pitch." (Nolan 1996: 2 of handout 2) Tone of voice has a slightly different nature from other components of the linguistic mechanism, in the sense that the realisation of a tone of voice has more general characteristics across the world's languages than language 
specific characteristics. For instance, even people without knowledge of English would be able to tell enthusiastic "yes!" from very sceptical "yes," showing that tone of voice is a very effective means to convey the paralinguistic information.

Cornmunicative intents, the first set of sources of variation, heavily influences this second set, linguistic mechanism. Speakers choose different lexicon, sentence structures and /or tones of voice from their linguistic mechanisms, depending on their communicative intents. Obviously, people speak differently when they are happy or when they are angry; or when they are talking to friends or to a stranger. The interaction between communicative intent and linguistic mechanism is very complex and how they interact differs greatly from speaker to speaker, and from situation to situation. Some people select different sets of lexicon, syntax, or phonology when they are angry, and other people do not (although their tone of voice may be different). People who refrain from expressing their anger verbally while they are at their work place may start swearing where they consider doing so is acceptable, perhaps in a car driving by themselves.

In addition to communicative intent and linguistic mechanism discussed so far, there is another set of sources of variation which also contribute to the difference in speech: indexical factors. Indexical factors consist of social background of the speaker: age, sex, physique, physical state, and health. These are the factors which physically limit the possible range of phonetic production of each speaker. Indexical factors do not affect just the vocal mechanism, however. Some of the factors have a strong interaction with parts of the linguistic mechanism as well. Social background, age and sex are those which influence the linguistic mechanism, and they affect a speaker's choices of lexicon syntax and phonology. An old highly respected man does not speak in the same way as a five year old girl does. 
In the final stage of the speech sounds production, phonetic plans which are produced as a result of the interaction of communicative intent, linguistic mechanism and indexical factors are implemented at the vocal mechanism, which indexical factors also conditions.

The discussion above shows that there are a substantial number of factors which condition human speech. Moreover, it reveals that, these factors do not always function independently but influence each other considerably. The sources of variance in speech is thus far too complicated to be described with the simple 'organic / leamed' dichotomy.

\subsection{LIMITATIONS IN FORENSIC SPEAKER IDENTIFICATION}

Robertson and Vignaux (1995:12) define an ideal piece of evidence as "something that always occurs when what we are trying to prove is true and never occurs otherwise." They also note that it is very hard to come across with the evidence which meets their definition, and most evidence is something that is more likely to occur when the given hypothesis is true than when it is not. The difference between speech samples, which is the interest of this thesis, is certainly not ideal evidence. This section discusses the limitations in forensic speaker identification, referring to the factors which prevent speech samples from being ideal evidence.

First of all, it has to be noted that speech is not a dinect record of human anatomy unlike fingerprints which provide an individual's unique anatomical characteristics, as mentioned earlier in the discussion of the spectrogram reading method (1.3.3.1). Though anatomy of the vocal tract may be analogous to the fingerprint, one-to-one matching of speaker's anatomy cannot be established from voice samples, since the acoustic output of human speech is a complex interaction of many factors. Even very similar acoustic values can be produced by different speakers with different anatomy using different configuration of their vocal mechanism. 
The flexibility of speech further leads us to the second point: within-speaker variation. Speech can vary extensively within a single speaker. A person's blood type will not change across the different occasions of examination, but a voice does. Identification of an individual based on blood stain is therefore much more straightforward. If the suspect's blood type is different from the criminal's blood stain found at the crime scene, the blood stain evidence indicates they are different individuals. It does not, however, work like that with speech, since a single speaker is capable of producing very different acoustic outputs from occasion to occasion.

Within-speaker variation can be caused by many factors. Speaker's physical conditions, such as fatigue, stress, intoxication (Hollien and Martin 1996) and the diumal cycle in the short term have a great relevance in forensic speaker identification. As an example of more drastic changes, having a cold often change one's voice very much in one night. It may affect the resonances of the nasal cavity; it may change the mode of vibration of the vocal cords; or it may cause some serious laryngeal pathology. Not very often, but occasionally, the long term factor such as aging can also influence identification process.

Speech style also has an effect on the variation of speech output. The word 'speech style' here includes speaker's psychological state, formality, and communicative intents, basically anything other than the factors determined by the physical conditions. The speech style can have a large effect on human voice. Loudness, durational factors, mean pitch, pitch range, and phonation type are all affected by this, and auditory impression can also be affected. Thus the comparison of speech data which are in different speech styles (which is often the case in forensic speaker identification) have to deal with these additional variables, making the comparison between speech samples even more complicated.

The discussions presented above show that it is technically impossible to arrive at a conclusive judgement on a speaker's identity in real forensic speaker identification. Instead, what is possible is to estimate how likely it is to observe the particular difference between the samples 
when the hypothesis is true, rather than when it is not, like it has been done in the analysis of most other types of evidence. This estimate can be expressed in the form of a likelihood ratio, which is going to be discussed in next section.

\subsection{PRESENTATION OF ANALYSIS AND BAYES $f$ APPOACH 1.7.1 LIKELIHOOD RATIO}

As described in the previous sections, majority of evidence is only indicative rather than determinative. With most evidence, therefore, expert cannot come to the conclusion that a hypothesis is true (or not true), as the evidence merely shows what is more LIKELY to be true, meaning that there is always a chance that the evidence is observed under different circumstances. For this reason and also for the fact that an expert is not in the position to make a decision on whether or not the defendant is guilty, Robertson and Vignaux (1995:21) claim that the presentation of expert evidence should be restricted to the likelihood ratio. Likelihood ratio is the ratio of the probability supposing that the evidence for the given hypothesis is true to the probability supposing that the evidence for the given hypothesis is not true (Robertson and Vignaux 1995:17). This form of presentation of evidence is now becoming more common in many areas of scientific evidence, such as DNA or blood types. Forensic speaker identification is, however, slow to bring the use of likelihood ratio to the same line as other scientific evidence.

\subsubsection{BAYESIAN APPROACH}

Bayesian approach is the method used to incorporate multiple pieces of independent evidence into a single likelihood ratio by simple multiplication of individual likelihood ratios. The simple incorporation of multiple pieces of evidence is an attractive feature in forensic phonetics. Since it is not possible to reach any reliable decision on the speaker's identity by 
relying on just one acoustic parameter, incorporating multiple pieces of evidence is essential in forensic speaker identification.

This research uses the likelihood ratio and Bayesian approach in a later chapter (Chapter 6). The way they are used is not conventional. They are used as the tool for discrimination tests between two speech samples. The details are extensively discussed in Chapter 6 .

\subsection{TASKS INVOLVED IN FORENSIC PHONETICS}

The decision-making tasks on speaker's identity are often called speaker identification. Strictly speaking, however, the term 'speaker identification' is not always used precisely. In forensic phonetics especially, the task involved is not speaker identification in the strict sense.

Nolan (1983:8) classifies speaker recognition tasks into two categories; speaker verification and speaker identification. Nolan characterises speaker verification as the process whereby:

\footnotetext{
"an identity claim by an individual is accepted or rejected by comparing a sample of his speech against a stored reference sample spoken by the individual whose identity he is claiming, and making a decision on the basis of a predetermined similarity threshold."
}

Speaker verification involves the comparison of one speaker to the other, and a decision is made according to a preset similarity threshold. This process usually yields one of four possible judgments: correct acceptance; correct rejection; false acceptance; false rejection; or no decision.

In speaker identification, an utterance from an unknown speaker has to be classified, or not, into one of a group of known speakers (Nolan 1983:9). Speaker identification is divided into a further three subcategories according to the type of the test: closed tests, open tests, and discrimination tests. In a closed test, we are certain that the speaker to be identified is included 
in the reference population. In an open test, on the other hand, the speaker to be identified may or may not be included in the speaker pool. In a discrimination test, the decision has to be made whether or not two samples of speech are similar enough to be judged as being from the same speaker.

It is noticeable that the discrimination test itself closely resembles speaker verification, as both tasks evaluate a test sample and reference sample to determine whether or not these two samples are from the one speaker, according to an acceptance threshold. This being so, then, what are the differences between speaker verification and speaker identification?

Nolan (1983:9) admits that they are the same process as far as the nature of the decision problem is concerned - typically an incriminating sample has to be attributed, or not, to a suspect. He claims, however, that the difference lies in the circumstantial characteristics in these two tasks. The underlying assumption for speaker verification tasks is that both test and reference samples will be from cooperative speakers. Vocal mimicry by an impostor is possible, but vocal disguise on the part of the true speaker is not expected. This is clearly not the case for forensic situations. Figure1.3 below summarises the types of decision-making tasks in speaker recognition. 


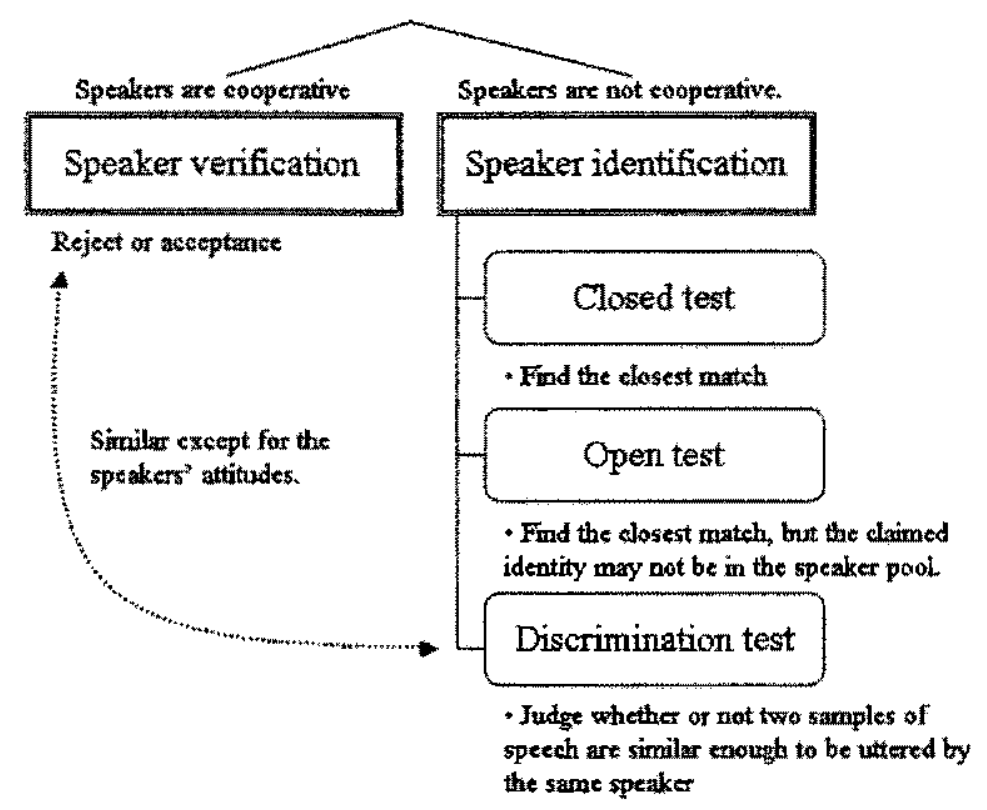

Figurel.3 Decision making tasks in speaker recognition.

In most cases, experts of forensic phonetics are requested to compare and analyse two speech samples with respect to how likely the recordings are to have originated from the same individual. Thus most of the actual tasks in forensic speaker identification are, precisely speaking, speaker discrimination, rather than speaker identification, and the tests performed in this research are no exception. The general decision-making process in forensic phonetic contexts, however, is going to be addressed as forensic speaker identification in this thesis, as it is the most common term used to express the process.

\subsection{FORENSIC SPEAKER RECOGNITION IN JAPANESE}

From the 1960's onwards, a substantial amount of research on Japanese speaker recognition has been done. Although these studies are mostly on automatic speaker identification, there have been studies on Japanese forensic phonetics as well, mostly by the National Research Institute of Police Science. Earlier studies go back to 1970 's, the targets of the investigation at this period were mostly concemed with the acoustic qualities of the various voices, such as 
voices under disguise (Suzuki 1973b; Suzuki and Odagawa 1976; Yamane 1973), and formant shifts of adolescent's voice change (Suzuki 1978), or female voice (Suzuki 1973a). There are also a few studies on F0. Suzuki (1973b) studied the effect on the F0 change in spectrograms, and the potential use of the distribution of the long term F0 for the speaker identification has been researched by Suzuki and Inomata (1975). These studies are all with significant forensic relevance, and there is no doubt that they serve as foundation of the forensic phonetic research in Japanese. However, there was a long way to go before these results were applied to the actual forensic speaker identification in a systematic manner, as no actual identification (or discrimination) tests were performed in those studies. The number of subjects was very small in all studies (one to ten speakers were used in the studies mentioned above), and they were all forensically fairly unrealistic data (in the sense that all studies were based on the words or vowels which were uttered in isolation).

The research in forensic speaker identification in Japan, then, headed off in a totally different direction from that of countries like Germany, England, or Australia from 1980's. In order to overcome the subjectivity of the spectrogram reading approach, Japanese forensic speaker identification research chose the application of the automatic speaker recognition system in forensic context, instead of incorporating linguistic acoustic analysis (Noda 1992; Noda et al. 1981; Noda et al. 1982; Osanai and Ozeki 1995; Osanai et al. 1995; Suzuki et al. 1994), and since then the parameters for the research became almost exclusively LPC (linear predictive coding) cepstrum. Most of these studies use many speakers unlike the studies in 1970's, some of them use even over 500 speakers. They also use non-contemporaneous recordings, separated by three to four months. The successful identification reported by these studies is mostly over $80 \%$, Osanai and Ozeki's (1995) study reports over $90 \%$ successful verification rates, using multiple words for testing. This all sounds very exciting and promising, there is, however, a large problem in these studies. That is, the nature of their speech samples. 
Despite the improvement in the number of speakers and the realism of recording occasions, none of those studies used natural speech. The speech data used by them are still words or vowels uttered in isolation. This suggests that it is very probable that these high successful identification rates reported by them will not be replicated in real forensic speaker identification.

Further, the use of automatic speaker recognition system in forensic speaker identification itself seems questionable. The complete lack of the consideration of linguistic factors in those studies is very worrying. It is known that often linguistic parameters are far more powerful indicators of the speaker's peculiarity than some of the acoustic parameters. The parameter or words which are very powerful indicators of the individuality of speech may not be very useful under different linguistic conditions, such as in different intonation, different positions in the sentence, and so forth.

Despite these apprehensions with the current research direction in Japanese forensic speaker identification research, forensic speaker identification is currently practiced in Japan, as shown at the beginning of this chapter. This thesis thus intends to contribute to the development of forensic speaker identification research in Japan, by performing the linguistically based forensic speaker identification test in the Japanese language for the first time. 


\title{
Chapter 2
}

\section{SOUND STRUCTURE OF}

\author{
JAPANESE
}

\subsection{OBJECTIVE}

This chapter describes issues relevant to Japanese phonology, referring also to some previous research in phonetics. The phonological structures of languages have a significant influence on the design of experiments of the type presented in this thesis and the interpretation of their results. A basic knowledge of Japanese sound structure is therefore indispensable for the current research. In consideration of this fact, this chapter discusses syllable and mora structures, phonemes and allophones, accentuation system, intonation and devoicing. The discussions are mostly based on modern standard Japanese, although some descriptions of regional dialects are also included.

In this chapter, first of all the structures of syllable and mora are discussed. Identification of both syllable and mora are necessary in the discussion of Japanese phonology. Japanese word structure consists of syllables. These syllables consist of a mora or two, and the morae comprise of segments.

Figure 2.1 below is a model of the phonological structure of the Japanese word, hottokeeki 'pancake.' 


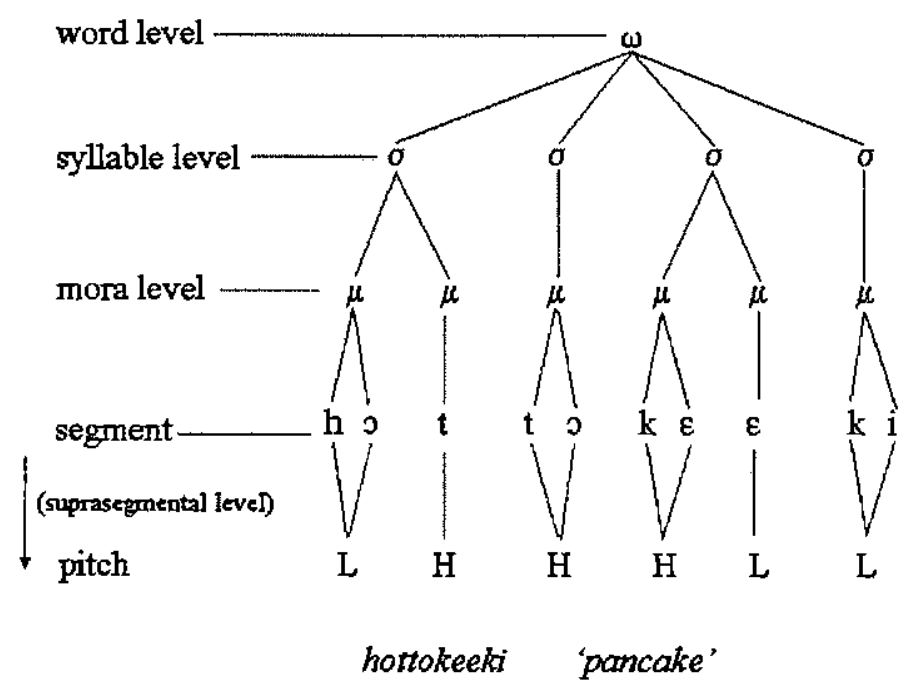

Figure 2.1 Hierarchical phonological structure of Japanese word hottokeeki 'pancake.'

Figure 2.1 shows that this word consists of four syllables, hot. to. kee. ki. The number of morae in this word is, however, six not four, as the first and third syllables of this word, namely hot and kee, are comprised of two morae.

In subsequent sections, the vowel and consonantal systems in Japanese are described. This section includes the description of what Japanese linguists call special morae (tokushuhaku in Japanese), as well as descriptions of phonemes and allophones. Special morae refer to those which cannot stand at the syllable initial position. A special mora always occurs as the second mora of a syllable, and is the only mora which can occur in this position. There are three types of special morae in Japanese, the second half of a long vowel, the first half of a long consonant, and the moraic nasal, phonemically represented as $/ \mathrm{R}, / \mathrm{Q} /$, and $/ \mathrm{N} /$ respectively.

Accentuation in Japanese is another topic which is discussed in this chapter. Japanese has a pitch accent system, and the rules on the accent placement and its realisation are portrayed. The effect of intonation on the realisation of pitch accent is described as well. The phonetic realisation of pitch accent in Japanese is severely conditioned by intonation. 
Vowel devoicing, which is a rather unusual phonological phenomenon, is also discussed in this chapter. Vowel devoicing in Japanese usually occurs when a high vowel is adjacent to voiceless segments. It is often said that the realisation of vowel devoicing reflects differences in speakers' regional accent. If that is the case, the importance of this parameter in forensic phonetics is evident. There are also other factors which condition the realisation of devoicing. Those factors will be illustrated in this chapter too.

Finally this chapter also briefly addresses the topic of using dialectal features as a forensic speaker identification parameter in Japan today.

\subsection{MORA AND SYLLABLE 2.2.1 SYLLABLES IN JAPANESE}

Guessenhoven and Jacobs (1998:160) describe a mora as "the intermediate level of structure" of a syllable, and syllable can be defined as a light (monomoraic) or a heavy (bimoraic) syllable according to the number of morae which the syllable contains. The number of morae that one syllable can contain is language specific.

Regarding the relationship between mora and syllable in Japanese, McCawley (1977) says: "The only reasonable definition of 'mora' that has been proposed is: 'something of which a
long syllable consists of two and a short syllable consists of one.' (p. 262)"

Japanese generally prefers a light open syllable, such as (C)V. In Japanese, there are only three phonemes which can be added to the end of this (C)V structure to create a heavy syllable, as briefly mentioned in the previous section. They are $/ R /, / Q /$, and $/ N /: / R /$ represents the second half of long vowels, $/ \mathrm{N} /$ represents moraic nasals, and $/ Q /$ represents the first half of the long consonants. Words like men (CVN) 'noodle' or ryoo (CyVR) 'dormitory' thus consist of two morae, but these two morae belong to one syllable. The possible Japanese syllable structures 
for each type of syllable are presented in Example 1 below. ' $y$ ' represents a palatal glide, and the mora and syllable boundaries are indicated by a space and ' $\because$, respectively.

\section{Example 1}

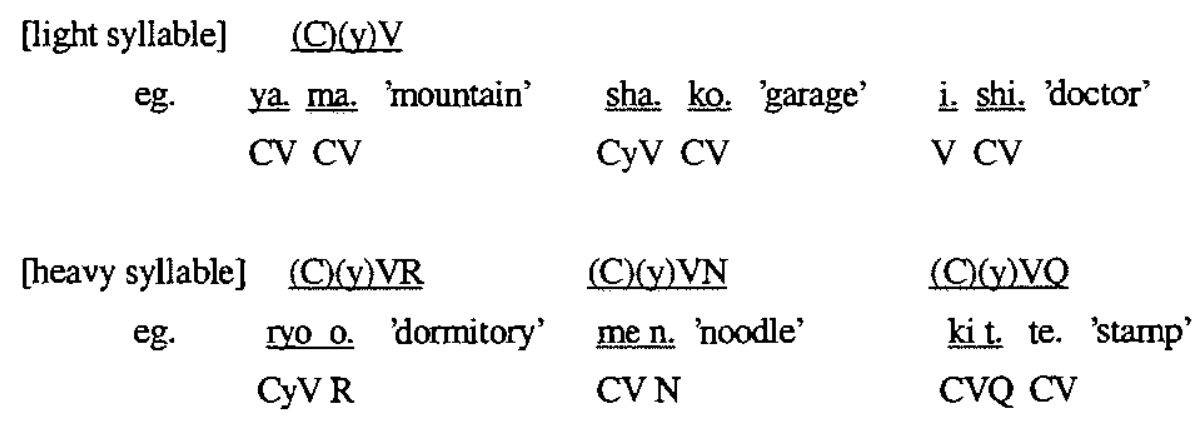

Languages which have three degrees of vowel duration, such as Dinka, or languages which allow a geminate after a long vowel, such as Tamil, have trimoraic syllables. This trimoraic syllable is called a superheavy syllable, and the languages which allow superheavy syllables are quite rare (Guessenhoven and Jacobs 1998:162). Although its distribution is limited to foreign origin words, compound words or onomatopoeia, Japanese has quite a few words with syllables which appear to be superheavy, as seen in Example 2 below.

\section{Example 2}

$\begin{array}{llll}\text { [super heavy syllable] } \quad(\mathrm{C})(\mathrm{y}) \mathrm{VRN}, & (\mathrm{C})(\mathrm{y}) \mathrm{VVN}, \quad(\mathrm{C})(\mathrm{y}) \mathrm{VRQ}\end{array}$

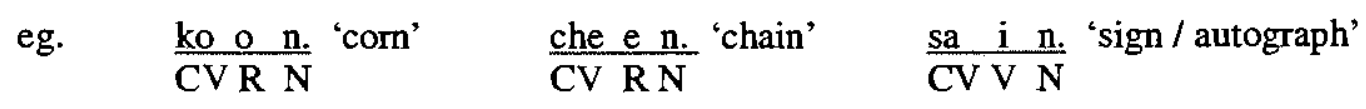
to o. kyo o k. ko. 'a person who was born and brought up in Tokyo' CyV R Q

Prima facie, these words seem to include superheavy syllables, but whether or not there really are three morae in the same syllable is questionable. Kubozono and Ota (1998:158) dispute this syllable structure on the grounds of accentuation shift in the case of derivation. There is a rule for accentuation of compounded words. That is, only the first mora of the syllable can carry an accent. A superheavy syllable word like che'en 'chain' changes the location of accent 
from the first mora to the second mora, ie. che'en to chee'n, when it is compounded with the word ten 'shop.' This accent shift suggests that the word cheen is divided into two syllables che and $e n$, so that the second ' $e$ ' of cheen is in fact the first mora of the syllable $e n$. See Example 3 below, showing the shift location of the word accent.

\section{Example 3}

$$
\begin{gathered}
\text { che'en 'chain' + ten 'shop' } \Rightarrow \text { che } e^{\prime} n \text { ten 'franchise' } \\
s a \text { 'in 'autograph' }+k a i \text { 'event' } \Rightarrow \text { sa } i \text { 'n } k a i \text { 'event to get an autograph of a } \\
\text { famous person' }
\end{gathered}
$$

Structures which involve a long vowel and geminate (CVRQ), such as tookyokko, meaning 'a person born and brought up in Tokyo', may be interpreted in three different ways. It has been reported that, when the word is pronounced normally, either the $R$ or $Q$ in the CVRQ sequence of syllables is frequently omitted. Thus the CVRQ trimoraic structure turns into a CVQ or CVR bimoraic structure. When this word is spoken with care, all the constituents of a superheavy syllable are retained. The syllable in question, CVRQ is, however, pronounced more like two syllables, CV. VQ. Thus, for example, it can be interpreted that kyook of tookyookko is actually made from two syllables which consist of the mora kyo and a two morae syllable $o k(\mathrm{~V}$ Q). It is therefore possible to consider Japanese has light and heavy syllables, but not superheavy syllables. Example 4 presents the three ways of syllabification of tookyookko, according to its phonetic realisation.

\section{Example 4}

$$
\begin{aligned}
& \text { to } \circ \text { kyook ko } \Rightarrow \quad \text { a) to o. kyok. ko. } \\
& \text { CVRQ CVQ } \\
& \text { b) to o. kyo o. ko. } \\
& \text { CV R } \\
& \text { c) to o. kyo. ok. ko. } \\
& \mathrm{CV} \frac{\mathrm{VQ}}{\mathrm{VQ}}
\end{aligned}
$$




\subsubsection{MORA IN JAPANESE}

Traditionally, the mora was considered as a unit of timing. Ladefoged (1993:251) describes the mora as a unit which "takes about the same length of time to say." The mora is usually not paid much attention to in the phonology of other languages. In Japanese, however, the mora is known to play an important role as a rhythmic unit.

There are four types of morae in Japanese. The possible segmental structures of morae in Japanese are represented as:

$\begin{array}{ll}\text { Type 1 } & (\mathrm{C})(\mathrm{y}) \mathrm{V} \\ \text { Type 2 } & \mathrm{R} \\ \text { Type 3 } & \mathrm{N} \\ \text { Type 4 } & \mathrm{Q}\end{array}$

Here, $/ \mathrm{R} /$ represents the second half of long vowels, $/ \mathrm{N} /$ represents moraic nasals, and $/ \mathrm{Q} /$ represents the first half of the long consonant, as mentioned earlier. $/ \mathrm{R} /, \mathrm{N} /$, and $/ \mathrm{Q} /$ are traditionally called special morae in Japanese phonology, as opposed to the regular (C)(y)V mora. It is known that native speakers of Japanese intuitively regard mora as the unit for counting sounds. This claim can be justified by many phonological phenomena, such as speech rhythm, song lyrics, speech errors, accentuation, and word formation (Kubozono and Ota 1998). The following sections present examples of these phonological phenomena, following Kubozono and Ota's discussion. 


\subsubsection{Phonological evidence for mora}

\subsection{Evidence from haiku and tanka}

Traditional styles of Japanese poetry, haiku (俳句) and tanka (短歌), are often presented as examples of how Japanese speakers intuitively count 'sounds.' Haiku and tanka both have strict rules for their rhythm. Unlike English poems in which the rhythm is determined by the location of stress, the rhythm structures of haiku and tanka are determined by the numbers of morae which each line comprises. Each line must be composed with 5, 7, and 5 morae for haiku, or of $5,7,5,7$, and 7 morae for tanka. In these styles of poem, the special morae, $/ \mathrm{R} /$, $/ \mathrm{Q} /$, and $/ \mathrm{N} /$, are regarded as single morae, as in the following example. The boundary of 5,7 , 5 is indicated by ' $"$ '. The second part has 7 morae, including a special mora $/ Q /$. See Example 5 below.

Example 5

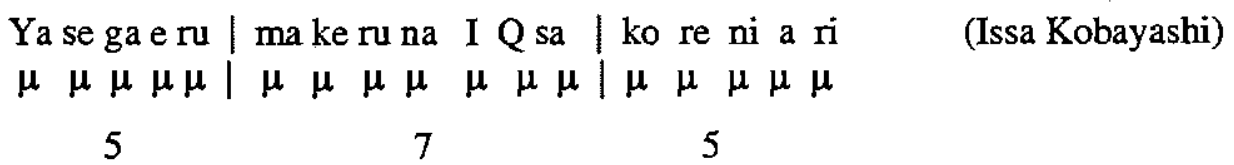

(Examples quoted from Kubozono 1998:25)

"Skinny frog, don't give up. I, Issa, am here for you"

\subsection{Evidence from song lyrics}

Similar things can be said about song lyrics. In English, the basic unit for assigning a note is a syllable. This can be seen for example in the well-known example 6, where each syllable carries a note. 


\section{Example 6}

twin kle twin kle litt le star

In Japanese, however, the mora, not the syllable, is the basic unit for assigning a note. This can be seen in example 7, which is an excerpt from a Japanese children"s song, "Genkotsu yama no tanuki san (Mr. Racoon of Mt. Genkotsu)". In this example, a special mora is also assigned to one note, just like the normal mora as shown above.

\section{Example 7}

ge $\mathrm{N}$ ko tsu ya ma no ta nu ki sa $\mathrm{N}$

genkotsu 'fist,' yama 'mountain,' no 'of,' tanuki 'racoon,' san 'Mr'

"Mr. Racoon of Mi. Genkotsu"

\subsection{Evidence from speech errors}

Speech errors are another indicator of how a unit of sound is defined by linguistically naive Japanese speakers. Kubozono and Ota (1998: 33) point out the fact that speech errors in Japanese mostly manifest as substituting or exchanging one mora with another. These are called substitution and transposition errors respectively, whereas speech errors in English generally substitute or exchange only onsets or rhyme of syllables, but not the following vowel. See Example 8. The first examples are substitution errors, in which one segment replaces another segment, and the second ones are the transposition errors, in which two segments are exchanged ( $\because$ ' indicates syllable boundaries). 


\section{Example 8}

Correct forms

\section{Speech errors}

[Japanese]

Substitution: ka a. ta a da i. to o. ryo o. $\Rightarrow \mathrm{ka}$ i. ta i. da i. to 0 . ryo o.

'President Carter'

Transposition: a. ra. bu. zi n.

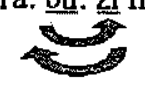

$\Rightarrow$ a. ra ji. bu N.

'Arabian people'

[English]

Substitution: a. rea. ding. list

$\Rightarrow$ a. lea. ding. rist

'a reading list'

Transposition: Chom. sky. and. Hal. le $\Rightarrow$ Hom. sky and Chal. le

'Chomsky and Halle'

(Kubozono and Ota, 1998: 32-33)

According to Kubozono and Ota, stutters also show that the mora is an important rhythmic unit in Japanese. Where a stutter occurs, the first mora tends to be repeated in Japanese, as opposed to in English, where only the onset of the first syllable is repeated (Example 9).

\section{Example 9}

Japanese

$$
\begin{array}{ll}
\text { sa-sa-sa-sakana } & \text { 'fish' } \\
\text { to-to-to-toNbo } & \text { 'dragonfly' }
\end{array}
$$

English

s-s-s-six 'six'

t-t-t-ten 'ten' (Kubozono and Ota 1998:33)

\subsection{Evidence from the location of pitch accent}

Morae play an important role in the assignment of accentuation as well. In Japanese, the syllable is the unit which carries the pitch accent (for detail on accentuation, see 2.5 below). 
The pitch change occurs, however, at the boundaries of mora and not at the syllable boundary. Example 10 presents words which feature a pitch change within a syllable. ' $H$ ' and ' $L$ ' indicate high and low tones, respectively. The moraic boundaries within the syllables are shown by a space.

\section{Example 10}

ho N. 'book' shi N. bu N. shi 'newsprint'

H L L H. H L. L

The pitch change in hoN (HL) occurs between two morae in the same syllable, ho and $N$. The pitch changes of shiNbuNshi (LHHLL) occur at two places, one is between shi and the first $N$, due to initial lowering and the other is $b u$ and the second $N$. In both examples, the pitch changes occur at the moraic boundaries, but not at the syllabic boundaries.

\subsection{Evidence from derivation of words}

Further, some phenomena in word formation are described better with mora than with syllables. When foreign words are transformed to fit into Japanese phonology, vowels are inserted between consonant clusters, since Japanese prefers open syllables. As a result, the borrowed words tend to be longer than in their original forms, and the words formed by compounding two foreign words often become too long. To rectify the situation, these are then shortened. The most common method for this abbreviation is to take the first two morae from each word and combine them, as shown in Example 11. 


\section{Example 11}
a) ri. mo R. to. + ko N. to. ro R. ra R. ('remote' + 'controller') $\Rightarrow$ ri. mo. koN 'remote controller'
b) fa. mi. ri R. + ko N. pyu R. ta R ("family" + "computer") $\Rightarrow$ fa. mi. koN $\quad$ video game'
c) do N.to + ma. i N. do ('don't' + 'mind') $\Rightarrow$ do N.ma i $\quad$ 'don't mind"

(Kubozono and Ota 1998:29-30)

The first two morae of the second components of Example 11 a) and b) ("ko $N$ to ro $R$ ra $R$, and 'ko N pyu $R$ ta $R$ ') and of the first component of Example $11 \mathrm{c}$ ) ('do $N$ to') are contained in one syllable, In Example 11 a), the second and the third morae 'mo' and ' $R$ ' of the first component ' $r$ i mo $R$ to' also form a single syllable. This syllable is, however, split into $/ \mathrm{m} /+$ $/ R /$, so that the first and the second morae are extracted to compose a new word. The same can be said with Example $11 \mathrm{c}$ ), where /i/ and $/ \mathrm{N} /$ are separated to extract the first 2 morae for the derivation. These examples clearly show that the mora, and not the syllable, functions as the unit for counting sound in Japanese word formations.

Morae play an important role in derivational morphology in Japanese as well. In the derivation of new words, word length is determined by the number of morae. The first few morae of the first component and the second component are often combined. The length of the newly formed word will have the same number of morae as the second component of the word, regardless of the number of syllables in those words.

Example 12 shows how new words can thus be formed. The numbers under [mora] and [syllable] show how many morae or syllables the original words consisted of, and the numbers after ' $\Rightarrow$ ' show how many morae and syllables the newly derived words have. For instance, 
example b) shows that 'kyg be. tsu' and 'ni $N . z i N^{\prime}$ ' had 3 morae and 4 morae, respectively. The newly derived word from these two words, 'kya. be. zi $N$,' has 4 morae.

\section{Example 12}

[mora] [syllable]

a) go. ri. ra 'gorilla'/ ku. zi ra 'whale'

$\Rightarrow$ go. zi.ra $\quad 3 / 3 \Rightarrow 3 \quad 3 / 3 \Rightarrow 3$

'Godzilla"

b) kya. be. tsu. 'cabbage' / ni N. zi N. 'carrot'

$\Rightarrow$ kya. be. zi N.

$3 / 4 \Rightarrow 4 \quad 3 / 2 \Rightarrow 3$

'(name of medicine)'

c) da. su. to. "dust' / zo R. ki N "wiper"

$\Rightarrow$ da. su. kiN $\quad 3 / 4 \Rightarrow 4 \quad 3 / 2 \Rightarrow 3$

'(name of commercial wiper)"

d) ro Q.te "(name of company)"/ka. fe. te.ri. a "cafeteria"

$\Rightarrow$ ro Q. te. ri. a $\quad 3 / 5 \Rightarrow 5 \quad 2 / 5 \Rightarrow 4$

'(name of a fast-food chain)'

(Examples are quoted from Kubozono 1998:29-30)

The relation between the length of the original words and the combined words is obvious if the number of morae in each word is analysed. The number of syllables, on the other hand, does not reveal any constant relationship. This also suggests that mora plays important roles as a rhythmic unit in Japanese phonology.

\subsubsection{Phonetic realisation of mora}

Whether a phonological idea is supported by phonetic data is another matter, however. In the past, all morae were considered to have a similar duration, regardless of their composition. 
Given the importance of mora as a rhythmic unit, if all morae are shown to have a similar duration, this will be strong phonological evidence of the mora. Acoustic experiments have, however, revealed that this is not the case. Beckman (1982) carried out an experiment on the effects of adjacent segments on mora duration. She hypothesized that 1) if special morae have phonetic reality, they have similar duration to other morae, and 2) the segment duration would vary to compensate for the difference in the intrinsic durations of adjacent segments. For instance, voiceless fricatives, such as $/ \mathrm{s} /$, have a longer intrinsic duration than their voiced counterpart. If the mora duration is meant to be similar regardless of its constituents, the vowel duration should vary to compensate for the difference in the duration of the preceding consonant. This hypothesis was not confirmed and Beckman therefore questions the phonetic reality of mora.

Sugito $(1989: 169)$ also reports that special morae are shorter than normal morae. The comparison between words which are either with or without special morae, such as koto 'harp' and kon 'navy blue' or koo 'back/ shell,' revealed that the compensation for the durational difference of mora does not occur when these words are measured in isolation. The phonetic reality of mora in Japanese is thus not confirmed, despite the extensive evidence of its psychological reality. The phonetic reality of mora is not completely denied either, however, as Sugito reports that the duration of a word correlates with the number of morae rather than syllables in a word. Further, a durational compensation for special morae is observed, when words containing special morae are planted into the same frame sentence and compared. This means that, although the compensation for the mora duration does not take place within a word, it does occur within a larger unit, such as a sentence. 


\subsection{VOWELS}

\subsubsection{PHONEMES}

Modern standard Japanese has 5 contrastive vowel phonemes. They are $/ a /, / \mathrm{J} / \mathrm{w} / \mathrm{de}$, and $/ \mathrm{o} / \mathrm{.}$

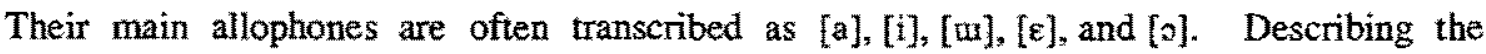
allophones of the five Japanese vowel phonemes in comparison to the cardinal vowels, Vance (1987:11) claims that, although the Japanese /i/ corresponds to the cardinal vowel 1 , the allophones of other phonemes do not correspond closely to the cardinal vowels. He characterises Japanese $/ \mathrm{a} /$ as a sound between $[0]$ and $[\mathrm{a}], / \mathrm{o} /$ in between $[0]$ and $[0]$, and $/ \mathrm{e} /$ as in between [e] and $[\varepsilon]$. Furthermore, Japanese $/ w /$ is much more front than cardinal vowel [u] and also it is unrounded. The list of words in Example 13 is a minimal quintuplet demonstrating the contrast between the five Japanese vowel phonemes. It can be seen that they are preceded by the same consonant $/ \mathrm{k} /$, but they all represent different meanings.

\section{Example 13}

$\begin{array}{ll}\text { ka } & \text { "mosquito" } \\ \text { ki } & \text { "tree" } \\ \text { ku "phrase" } \\ \text { ke "hair" } \\ \text { ko "child" }\end{array}$

(Examples quoted from Vance 1987: 9)

The realization of the vowel phonemes can be conditional upon the context. Akamatsu (2000:71) lists three possible conditions. The vowels often have nasalised allophones when they follow a nasal consonant. Also, the vowels are followed or preceded by glottal stops when they are located before or after a pause. Finally, some vowels often have devoiced allophones under specific circumstances. This will be discussed in detail in the section 2.6 below. See Example 14. 


\section{Example 14}

[Nasalisation]

/sakaya/ [sakaja] "bottle shop" but, /sakana/ [sakanã] "fish"

[Insertion of glottal stops]

/kiku/ [kikur?] "chrysanthemum" but, /ki'/ [ki2] "tree",

$$
\text { /i/ [?i2] "stomach" }
$$

(in case where these words are pronounced in isolation)

[Devoicing /i/]

/himo/ [hims] "rope"

but, /hito/ [hito] "person"

[Devoicing $/ \mathrm{u} / \mathrm{]}$

/sumi"/ [surmi] "charcol" but, /sushi'/ [sụci] "sushi"

As well as the variation discussed above, there is a wide range of dialectal variation in vowel quality (Imaishi and Mitsuwa 1989; Shibatani 1990). These dialectal differences are discussed in a later section (2.3.4).

\subsubsection{LONG VOWEL}

\subsubsection{Long and short contrasts}

The previous section demonstrated the phonemic contrast in five vowel qualities in Japanese. All the examples were presented as short vowels. Japanese, however, has surface contrasts between long vowels (represented by two vowels in orthography) versus short vowels, where the durational differences in vowels are contrastive. The pairs of words in Example 15 below are identical except for the difference in duration of the underlined vowels, and yet lexically they have totally different meanings. 


\section{Example 15}

\begin{tabular}{llll} 
& [short vowel] & \multicolumn{2}{c}{ [long vowel] } \\
hato & "pigeon" & haato & "heart" \\
i & "stomach" & ii & "good" \\
kukki & "stem" & kuuki & "atmosphere" \\
tekki & "enemy" & teeki & "transport pass" \\
toki & "time" & tooki & "pottery"
\end{tabular}

The view that Japanese long vowels have a different nature from, for instance, English long vowels, such as [i:] in the word "feel," is widely accepted. There are disagreements on the phonological representation of the Japanese phonetically long vowel, however. The following sections review the different approaches to Japanese long vowels.

\subsubsection{VR or VV? Phonetic realisation of $/ R /$}

In Japanese phonology, a phonetically long vowel is usually expressed using $/ \mathrm{R}$ for the second half of the long vowel. $/ \mathrm{R}$ is a phoneme which represents the second half of a long vowel, originally proposed by Kindaichi (1967). His view was that Japanese has two types of "long vowels": true long vowels and geminate vowels which arise as a result of compounding or suffixation. Kindaichi provides two words, sato + oya ('village' + 'parent' $\rightarrow$ 'foster parent') and satoo $+y a$ ('sugar' + 'shop' $\rightarrow$ 'sugar shop') as examples of these two types. In his view, sato + oya 'foster parent' has two identical vowels, $/ \mathrm{O} /$, in succession, and this $/ o 0 /$ is not a long vowel, as these two vowels belong to the two different morphemes. In satoo $+y a$ 'sugar shop,' conversely, the long vowel belongs to a single morpheme, therefore this $/ 00 /$ is a long vowel. He claims that a true long vowel should be transcribed as VR, whereas the two identical vowels should be transcribed as VV to represent these two types of structure correctly. 
The phonetic reality of this distinction is suspect. Shibatani (1990:162) is not convinced by McCawley's claim (McCawley 1977) that the morpheme boundary between two identical vowels is realized as a glottal constriction. He still refers, however, to Sugito's observation as a part justification for classification of long vowels into two categories. Sugito observed that during the articulation of a geminate vowel, there is a slight narrowing of the lips at the morpheme boundary. This claim is not supported by acoustic evidence, however, at least when the vowel is uttered at normal speed without careful articulation. Figure 2.2 below presents spectrograms of sato + oya 'foster parents' and satoo + ya 'sugar shop' spoken by the author at the speed of normal conversation, in order to see whether or not there is acoustic evidence of lip narrowing.

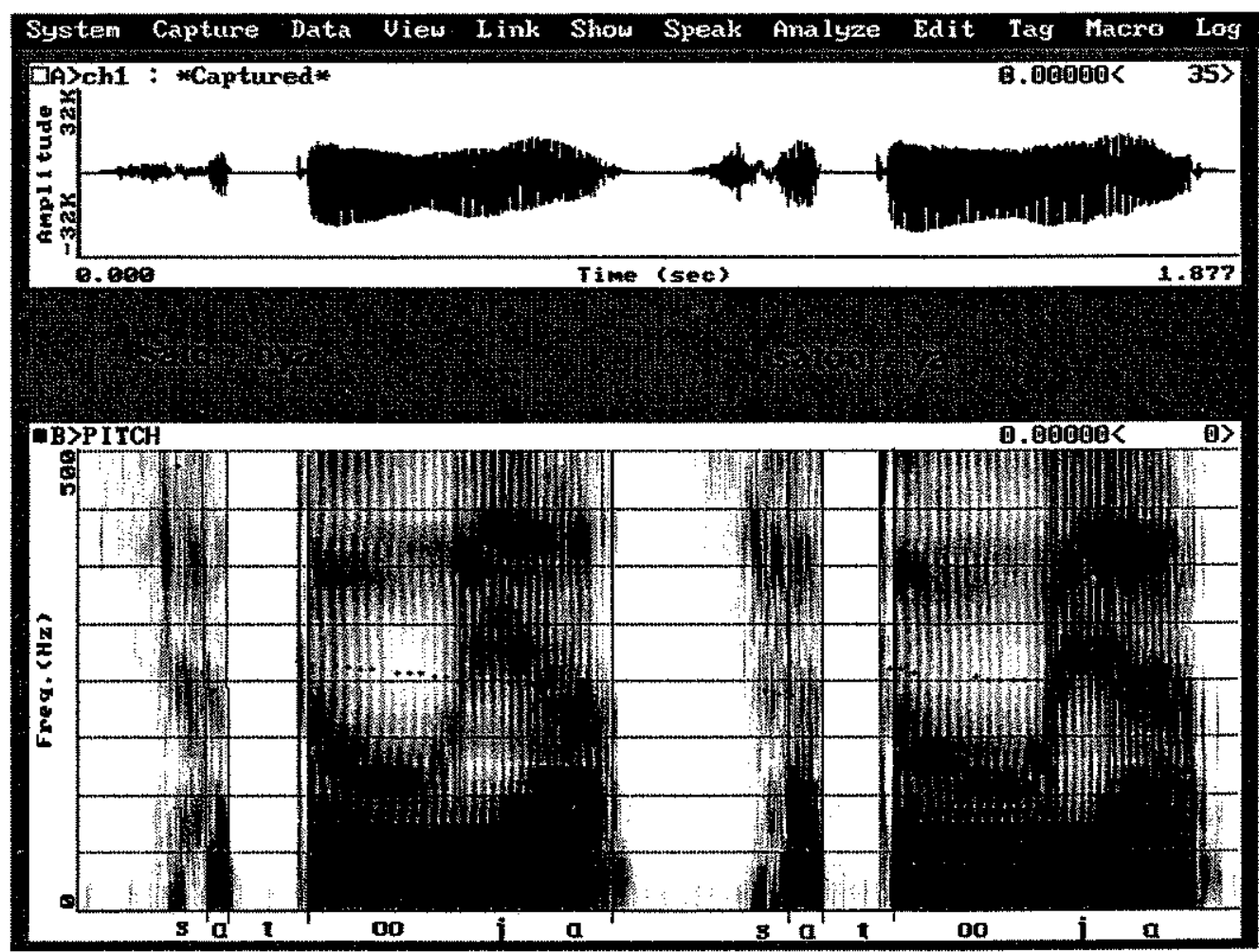

Figure 2.2 Spectrograms and sound wave forms of sato + oya 'foster parent' and satoo + ya 'sugar vendor.' The first half is sato + oya, and the latter is and satoo + ya. The dotted lines on the spectrograms indicate the pitch change of these words.

Despite Sugito's observation, the spectrogram presented above shows no obvious difference between these two words. In the first spectrogram, sato + oya 'foster parents', the acoustic reflection of some articulatory gesture, such as lip rounding, should be observed between the 
first and the second $/ \%$. Acoustically, lip rounding is known to manifest as lowered F2 and F3 for back vowels (Stevens 1998). No visible sign of such changes, however, is observed in Figure 2.2. In fact, it is indistinguishable from the $/ 00$ in satoo + ya 'sugar shop,' which is supposedly the true long vowel. The acoustic difference between $/ \mathrm{oo} /$ and $/ \mathrm{oR} /$ is thus not attested, at least in this particular speaker's case.

\subsubsection{Phonological justification for $/ \mathrm{R} /$}

Although acoustically the differences between VR and VV sequences are not attested, phonologically it is worth establishing $/ \mathrm{R} /$ as a phoneme, instead of just transcribing as VV. There is a clear difference in the status between the first and the second components of long vowels. The second component of the long vowel is independent enough to be in a different pitch from the first component, as mentioned above in 2.2.2.1.4, but the second component cannot carry an accent. Kubozono and Ota (1998:38) present examples of the accentuation rules of newly introduced foreign words. Normally the accent of the loan word is located at the third from the last mora. Example 16 presents some loan words, where accented morae are marked by bold case, and mora and syllable boundaries are shown by $\mu$ and ' $?$, respectively.

\section{Example 16}

$\begin{array}{llllllll}\text { o } & \text { o. } & \text { su. } & \text { to. } & \text { ri. } & \text { a. } & & \text { 'Austria" } \\ \mu & \mu & \mu & \mu & \mu & \mu & \\ & & & & & \\ \text { o } & \text { o. } & \text { su. } & \text { to. } & \text { ra. } & \text { ri. a. } & \text { 'Australia' } \\ \mu & \mu & \mu & \mu & \mu & \mu & \mu & \\ & & & & & & \\ \text { ro. } & \text { sa } & \text { N. } & \text { ze. } & \text { ru. } & \text { su. } & \text { 'Los Angeles' } \\ \mu & \mu & \mu & \mu & \mu & \mu & \end{array}$

This rule, however, cannot be applied to the words which have special morae as the third mora from the last. Special morae include the first component of a consonant cluster, the second 
component of the long vowel, and moraic nasals, as mentioned earlier. The accent is moved to the preceding mora when the third mora from the last is a special mora. Thus the rule of the accent placement for the foreign origin words is: "the syllable which contains the third mora from the last carries an accent."

Example 17 presents some foreign origin words whose third mora from the last is a special mora.

\section{Example 17}

1) bu. ryu Q. se. ru. 'Brussels'

$\mu \quad \mu \quad \mu \quad \mu \quad \mu$

2) wa. shi N. to N. Washington"

$\mu \quad \mu \quad \mu \quad \mu \mu$

3) ko. pe N. ha R. ge N. 'Copenhagen'

$\begin{array}{lllllll}\mu & \mu & \mu & \mu & \mu & \mu & \mu\end{array}$

Thus, a long vowel in Japanese is not seen phonologically as two consecutive vowels, as the second component of a long vowel is independent enough to be in a different pitch from the first half. The second component does not behave in the same way as the other vowels, since it cannot carry an accent by itself. For the reasons above, a phoneme $/ \mathrm{R} /$ which represents the second component of a long vowel is useful for the description of Japanese phonology, and it is assumed more appropriate to transcribe long vowels as $N R$, rather than $N \mathrm{~V} /$ or $N:$, at least phonologically.

\subsubsection{DIPHTHONGS}

Non-identical vowel sequences, such as [iu], [ei] or [ou], are treated as diphthongs in many languages. Diphthongs are defined as vowels with two articulatory targets within a single 
syllable. Japanese also has non-identical vowel sequences. A few examples are listed in Example 18.

Example 18

$\begin{array}{llll}\text { tai } & \text { 'snapper' } & \underline{\text { ie }} & \text { 'house' } \\ \underline{\text { au }} & \text { 'to meet' } & \text { mei } & \text { 'niece' } \\ \underline{\text { ao }} & \text { 'blue' } & \text { toi } & \text { 'question' }\end{array}$

Diphthongs in Japanese have very different characteristics from those in other languages, such as English. They consist of two morae, and the mora is the unit for timing in Japanese. They thus always have a distinctively longer duration than ordmary single mora vowels. This is one reason that the majority of linguistically naïve native Japanese speakers recognise diphthongs as two sounds rather than a single unit.

The differences between English diphthongs and Japanese vowel sequences are found in their articulations too. Imai (1980:34) points out that the two vowel components in English diphthongs are not articulated with equal importance. One component, which is normally the first one, is the main vowel and the other becomes something like a sub-vowel. With these two, only the main vowel achieves its articulatory aim indicated by the IPA. The two phonetic symbols for English diphthongs do not indicate the starting point and the goal of the diphthongs, but they indicate the starting point and the destination for the tongue to head toward. In Japanese, on the other hand, the target is achieved to the same extent for both vowels, if other conditions are equal. Tsukada's study (Tsukada 2000) on the second language acquisition of a diphthong /ai/ in English and Japanese also confirms Imai's observation. In the course of acoustic analysis of native and non-native speakers' /ai/ in Japanese and English, she revealed that Fl and F2 plots for two phonetic targets of the diphthong were more separated from each other in Japanese than they were in English, when it was produced by native speakers. This means that the two targets are articulated more distinctively in Japanese than in English. 
Despite all these differences from English diphthongs, there are a couple of phonological reasons to consider these vowel sequences as diphthongs. Firstly, if the two vowels are within the same morpheme, only the first vowel can carry a pitch accent, but not the second one, just like the long vowels, which have been discussed in the previous section (2.3.2.3). This suggests that those two components have different status within a syllable; the first component is the primary quality, and the second component is subordinate to the first component. The second reason is derived from the development of variations in regional dialects. In many dialects, [ai] or [ji] in the standard Japanese has developed into various forms of a long vowel, such as [£:]or [æ:]. This implies the closeness of the relationship between these two vowels (Maekawa 1997b:16).

Does Japanese then have diphthongs? If we follow the definition given at the beginning of this section: "vowels which have two acoustic targets within a syllable", the answer must be yes. The characteristics of vowel sequences in Japanese discussed above suggest that these should be considered as diphthongs. It is true that Japanese vowel sequences have very different acoustic characteristics from diphthongs in other languages, such as English. The fact that the second component is not independent from the first component in terms of accentuation, however, suggests that these two vowels form a single linguistic unit, the syllable. Furthermore, the acoustic difference between English and Japanese diphthongs is explained by the difference in other phonological characteristics of these two languages. That is, English is a syllable-timed language, and Japanese is a mora-timed language. Due to this difference in timing, Japanese diphthongs can take a much longer time to achieve their two articulatory goals within the syllable. It should be thus much easier to articulate the second target of diphthongs accurately in Japanese than in English.

\subsubsection{DIALECTAL VARIATION IN VOWELS}

The Japanese language has many dialects, which vary to a great extent in their phonology. There are a few regional dialects which do not have a five-vowel-phoneme system like 
standard Japanese does. For instance, Okinawa and Tohoku dialects have three and four vowel phonemes respectively, whereas Amami dialect has seven and Nagoya dialect has eight vowel phonemes (Sugito 1996c:6).

Even among dialects which appear to have a similar five-vowel phoneme system, phonetic realisation varies. One of the most commonly known dialectal variations is probably the lip rounding of $/ \mathrm{v} /$. It has been pointed out by many that the $/ \mathrm{v} /$ vowel is pronounced with more lip protrusion in the Western part of Japan than it is in the rest of Japan (c.f. Shibatani 1990:161; Kawakami 1977:23; Sugito 1996c:6). Imaishi and Mitsuwa (1989) also report that speakers of different dialects have different vowel qualities, even when the corresponding five vowel phonemes are compared.

Dialectal variety in Japanese is becoming less distinct now largely due to the daily exposure to the media and use of a standard orthography, which is based on the standard five vowel phonemes. There is, however, still considerable variation in the realisation of the Japanese vowel phonemes. Careful and systematic auditory analysis of this variation is very important in forensic speaker identification, as the variation in the realisation of phonemes can serve as the linguistic cue in forensic speaker identification.

Following Wells's (1982) classification, Nolan (1983) proposes a framework for the analysis of between-speaker variation, categorising the variation into four types: systemic, phonotactic, incidental, and realisational. This framework for the analysis of between-speaker variation is also applicable to Japanese. It has already been mentioned that different regional dialects in Japan have a different number of phonemes. For instance, the speakers of Okinawa dialect and Amami dialect have three and seven vowel phonemes respectively. This difference in the number of phonemes can produce systemic differences between speakers. If only three vowel phonemes are observed in incriminating evidence whereas there are seven in the suspect's recordings, it is likely that the two speakers are different individuals. Furthermore, vowel 
devoicing in Tokyo Japanese (and modern standard Japanese) is supposed to occur where a high vowel does not have any voiced segments adjacent to it. It is known, however, that Kansai dialect speakers tend not to devoice high vowels in the same environment. This shows that speakers of Tokyo style Japanese and the Kansai dialect have realisational difference.

The dialectal variation discussed above is more easily detected by careful auditory analysis. The systematic analysis of the dialectal differences by careful listening can be performed systematically, using Wells's framework. Although they are mostly categorical data, the quantification and statistical examination is also possible if enough data are available. The observation of dialectal differences between two speech samples can provide clues for forensic speaker identification.

\subsection{CONSONANTS \\ 2.4.1 PHONEMES AND ALLOPHONES}

Japanese has a typologically unremarkable consonant system. Lass (1984:147-159) lists the common features for phonemic systems across world languages, and his list shows that the phonemes in Japanese are generally typical. For instance, stops in Japanese consist of three stops, $/ \mathrm{p} /, / \mathrm{t} /$, and $/ \mathrm{k} /$, and according to Lass, these are the most universally distributed stops. For fricatives, the most commonly found phoneme is $/ \mathrm{s} /$, and this phoneme also exists in the Japanese consonant system. Regarding the voice / voiceless contrast, Lass says the number of voiced obstruents does not exceed that of voiceless counterparts in most languages. This is also true in Japanese. As for nasals, Japanese is again very typical. The most common nasal in the world languages is $/ \mathrm{m} /$, followed by $/ \mathrm{n} /$ and $/ \mathrm{g} /$, and $/ \mathrm{m} /$ and $/ \mathrm{n} /$ are part of the Japanese consonantal phoneme system. With regard to approximants, Lass reports that $86 \%$ of languages have $/ \mathrm{j} /$ and $75 \%$ have $/ \mathrm{w} /$, and other types of approximant are rare. Japanese also has $/ \mathrm{j} /$ and $/ \mathrm{w} /$, the two most common approximants. 
There are, however, a few aspects of the Japanese consonantal system which are not quite as typical. Japanese does not have affricates as phonemes, although phonetically they exist in the system. With sonorants, the absence of any lateral phoneme should be noted. Japanese has one liquid, $/ \mathrm{s} /$, which has the most common place of articulation for liquids. The manner of articulation is, however, not lateral, as is most commonly found (according to Lass, more than $79 \%$ of languages have one or more laterals), but tap.

The consonantal phonemes in Japanese are listed as below in Table 2.1.

\begin{tabular}{|c|c|c|c|c|c|}
\hline & bilabial & alveolar & palatal & velar & glottal \\
\hline stop & $\mathrm{p} \mathrm{b}$ & $\mathrm{t} \mathrm{d}$ & & $\mathrm{kg}$ & \\
nasal & $\mathrm{m}$ & $\mathrm{n}$ & & & \\
tap & & $\mathrm{r}$ & & & $\mathrm{h}$ \\
fricative & & $\mathrm{s} \mathrm{z}$ & & & \\
approximant & $\mathrm{w}$ & & $\mathrm{j}$ & & \\
\hline
\end{tabular}

[moraic nasal $/ \mathrm{N} /]^{1}$

Table 2.1 List of consonantal phonemes in standard Japanese.

The major consonantal allophones are shown in Table 2.2 .

\begin{tabular}{|c|c|c|c|c|c|}
\hline & bilabial & alveolar & palato-alveolar & palatal & velar \\
\hline stop & $\mathrm{pb}$ & $t d$ & & & $\mathrm{~kg}$ \\
\hline nasal & $\mathrm{m}$ & $\mathrm{n}$ & & $\mathrm{n}$ & I \\
\hline tap & & r & & & \\
\hline fricative & $\Phi$ & $s \mathbf{z}$ & 67 & 乌 & h \\
\hline affricate & & ts $\mathrm{dz}$ & t6 $\mathrm{dz}$ & & \\
\hline approximant & $\mathbf{w}$ & & & $\mathrm{j}$ & \\
\hline
\end{tabular}

Table 2.2 Phonetic transcription of all consonantal phones that occur in standard Japanese.

The allophonic variations of consonants occur mainly when the phonemes are followed by high vowels /i/ or / $/ \mathrm{l}$. Generally speaking, place of articulation of consonants has a tendency to be assimilated by the articulation of following vowels. The allophonic variations are summarised and presented with some examples below in Table 2.3. The consonants which have allophonic variations are indicated by bold case.

\footnotetext{
${ }^{1}$ See section 2.4.2. for the discussion of moraic nasals.
} 


\begin{tabular}{|c|c|c|c|c|}
\hline phoneme & & elsewhere & followed by /i/ & followed by $/ \mathrm{w} /$ \\
\hline \multirow[t]{2}{*}{$t d$} & realisation & $\mathrm{td}$ & tc $\mathrm{d} z$ & ts $\mathrm{dz}$ \\
\hline & examples & $\begin{array}{l}\text { [hato] 'pigeon' } \\
\text { [dzữwa] 'phone' }\end{array}$ & $\begin{array}{l}\text { [hatcii] 'eight' } \\
\text { [dzimsữ 'land' }\end{array}$ & $\begin{array}{l}\text { [kutsur] 'shoes' } \\
\text { [dzwno:] 'brain' }\end{array}$ \\
\hline \multirow[t]{2}{*}{$s z$} & realisation & $s z$ & 67 & \\
\hline & examples & $\begin{array}{l}\text { [same] 'shark' } \\
\text { [aza] 'bruise' }\end{array}$ & $\begin{array}{l}\text { [cimoun] 'fingerprint' } \\
\text { [azi] 'taste' }\end{array}$ & \\
\hline \multirow[t]{2}{*}{ h } & realisation & h & $\mathrm{c}$ & $\Phi$ \\
\hline & examples & [hana] 'flower' & [çina] 'chick' & [фun $\varepsilon]$ \\
\hline
\end{tabular}

Table 2.3 Phonemes and allophonic variations in consonants.

\subsubsection{MORAIC NASAL}

Japanese consonants include two types of nasal phonemes. One is the consonantal phoneme, namely $/ \mathrm{m} /$ and $/ \mathrm{n} /$, and the other is the moraic nasal. A moraic nasal is often represented as $\mathbb{N} /$ to be distinguished from other nasal consonants. Moraic nasals differ from consonantal nasal phonemes in two aspects.

First, the place of articulation (and therefore the acoustic quality) of a moraic nasal changes depending on the segment that follows it. A moraic nasal assimilates to the place of articulation of the segment which immediately follows it. The assimilation process is summarized in Table 2.4. The first row indicates the place of articulation of the following segment, the second row shows the examples of the following phonemes, and the bottom row indicates the phonetic realisation of the moraic nasals' place of articulation. Being followed by a velar consonant $/ \mathrm{k} /$, for example, a moraic nasal is realised as [ $\mathrm{g}]$.

\begin{tabular}{|l|c|c|c|c|}
\hline place of articulation & bilabial & (palato-) alveolar & velar / glottal & vowels / glides \\
\hline Following segments & $\mathrm{p}, \mathrm{b}, \mathrm{m}$ & $\mathrm{t}, \mathrm{d}, \mathrm{s}, \mathrm{z}, \mathrm{n}, \mathrm{r}$ & $\mathrm{k}, \mathrm{g}, \mathrm{h}$ & $\mathrm{j}, \mathrm{w}$, vowels \\
\hline Realisation & $\mathrm{m}$ & $\mathrm{n}$ & $\mathrm{g}$ & $\mathrm{ux}$ \\
\hline
\end{tabular}

Table 2.4 Realisations of moraic nasals. 
Secondly, a moraic nasal stands as one mora by itself at the syllable final position, whereas consonantal nasals cannot. Example 19 are examples of words which contain two different types of nasals. The moraic boundaries within words are indicated by spacing.

\section{Example 19}
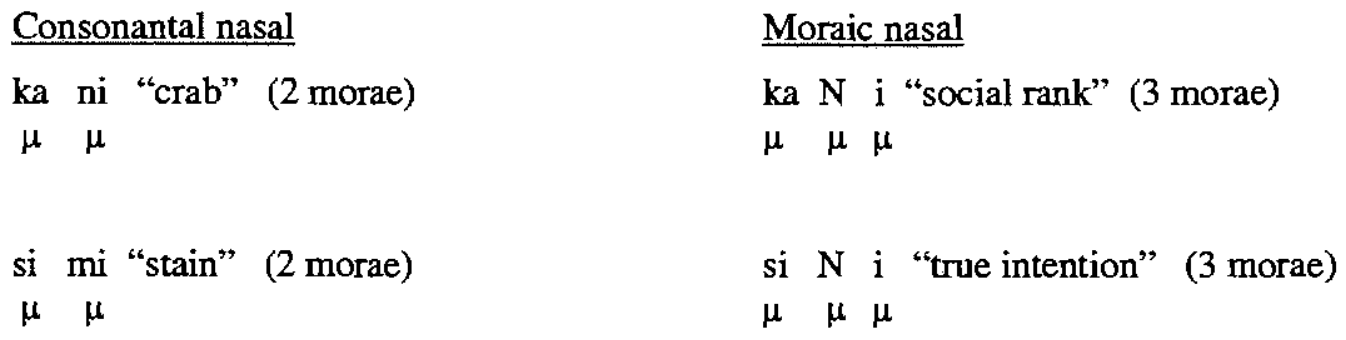

Although both types of words consist of two syllables, they differ in their numbers of morae. The words which contain a normal nasal consonant have two morae, whereas the ones with a moraic nasal $/ \mathrm{N} /$ have three morae. This is because the moraic nasal cannot stand as a syllable by itself, as has been discussed earlier in this chapter (see 2.2.2). Moraic nasals can only occur syllable finally, forming a heavy syllable. Moraic nasals, thus, are subordinate to the first mora of a syllable. Pitch changes can occur in between the first mora and the moraic nasal of a syllable, but an accent cannot be placed on the moraic nasal, this is similar to other special morae like long vowels and diphthongs.

As well as the differences in phonological status, there are some phonetic differences between the two nasals. Mora is considered a unit for timing. Although it has been reported that a moraic nasal /N/ is not as long as a (C)V mora (Sugito 1989:165), the experiments by Sato (1993) demonstrate that $/ \mathrm{N} /$ is consistently longer than nasal consonants which are located at the syllable initial position, such as a $\mathrm{C}$ in $\mathrm{CV}$ syllable structure. Moraic nasals thus differ from consonantal nasals, not only phonologically, but also phonetically. 


\subsubsection{LONG CONSONANT}

In Japanese, the duration of some consonants can also be contrastive. See Example 20 for pairs of words which have identical segmental structures except for the duration of consonants. The moraic boundaries in the example below are shown by spacing.

\section{Example 20}

Normal consonant si ta "tongue" (2 morae) $\mu \mu$

se ka i "world" (3 morae) $\mu \mu \mu$

\section{Long consonant}

si $t$ ta "(past plain form of) to know" (3 morae)

$\mu \mu \mu$

se $\underline{\mathrm{k}} \mathrm{ka} \mathrm{i}$ "lime" (4 morae)

$\mu \mu \mu \mu$

In conventional phonotactic description of Japanese, the first half of the long consonant is represented as $/ Q /$. Thus sitta 'to know (past)' and sekkai 'lime' are phonologically represented as /siQta/ and /seQkai/, as opposed to their single consonant counterparts sita 'tongue' and sekai 'world,' represented /sita/ and /sekai/. Just like /R/ or $/ N /$, which have been discussed above, establishing $/ Q /$ as a phoneme is crucial for the discussion of the Japanese syllable structure and the placement of pitch accent.

Long consonants in Japanese, i.e. $/ \mathrm{Q} /+/ \mathrm{C} /$, can only be voiceless consonants, namely $/ \mathrm{p}, \mathrm{t}, \mathrm{k}, \mathrm{s}$, h/. Many are found in onomatopoeia, the morpheme boundaries of Chinese origin words, or in so-called loan words that were introduced from foreign languages, such as Dutch, Portuguese, German, French, or English. In native Japanese words, consonant clusters are found most frequently in conjugated verbs, as in the example sitta 'to know (past)' presented in Example 20 above.

Example 21 presents words with long consonants occurring with each voiceless consonant phoneme /p, t, k, s, h/. 


\section{Example 21}

haQpa "leaf" $\quad$ kiQte "stamp" huQkatsu "revival"
zaQshi "magazine" baQha "Bach"

Finally, the phonetic characteristics of the long consonant in Japanese should be mentioned. Although the first half of the long consonant is considered to stand as an independent mora, its duration is not quite as long as that of a /(C)V/ mora, just like /N/ (Sugito 1989). It seems, however, to possess long enough duration to be recognized as one mora by native speakers.

Phonologically, a language which contrasts the duration of consonants is not that unusual. Languages, such as Kannada, Tamil, Telugu, Hausa, Italian, Icelandic, Norwegian, Finnish, Hungarian, Arabic, Shilha, Amharic, Galla, Dogri, Bengali, Sinhalese, and Rembrrnga, are all known to contrast the duration of consonants. Phonetically speaking, however, long consonants in such languages have a very different nature from Japanese long consonants (Maddieson 1985; Maekawa 1997a). Japanese is distinguished from these languages by its lack of implementing closed syllable vowel shortening. In the syllabification of a consonantal sequence, the first component of the sequence becomes a coda, attached to the preceding vowel. In many languages, vowel duration is known to become significantly shorter when the syllable consists of coda, that is, when the syllable is a closed syllable, rather than when the syllable is an open syllable. The languages listed at the beginning of this paragraph are all reported to have closed syllable vowel shortening (Maddieson 1985). In Japanese, however, it has been reported that the vowel shortening occurs rarely or, even when it occurs, it tends to be fairly slight (Maekawa 1997a). Japanese long consonants thus have a phonetically different nature from those of other languages. 


\subsection{ACCENT \\ 2.5.1 PITCH ACCENT}

World languages are prosodically typologised into two categories, tone languages and accent languages, according to several, but not necessary mutually exclusive, suprasegmental parameters. Accent languages are distinguished from tone languages on the basis of their culminative nature. That is, in accent languages, each word can consist of only one prominent syllable, known as the word accent. The prominence is usually signalled by pitch. Not all accented languages are culminative in the strict sense, as some languages, such as Japanese, also allow the absence of accent in a word. According to Hymann (1977: 38), tone languages, on the other hand, "can allow a high tone to occur on more than one syllable of a word." The differences between tone and accent languages are thus in the predictability of the prosody of words. The pitch prosody of a word in accent languages can be predicted, once the location of the accent is known. The suprasegmental analysis of a word in tone languages, on the other hand, generally has to be assigned syllable by syllable.

Accent languages are classified further into stress accented and pitch accented languages. English is a stress-accented language, and Japanese is an example of a pitch-accented language. Stress languages mark an accented syllable acoustically with a combination of factors, such as change of fundamental frequency, higher intensity, and duration (Hymann 1977; Shibatani et al. 1981:70), F0 being the most important factor. Japanese, on the other hand, primarily uses the fall of FO to accentuate a syllable. The types of suprasegmental systems are thus summarised as in Figure 2.3. 


\section{Types of suprasegmental system}

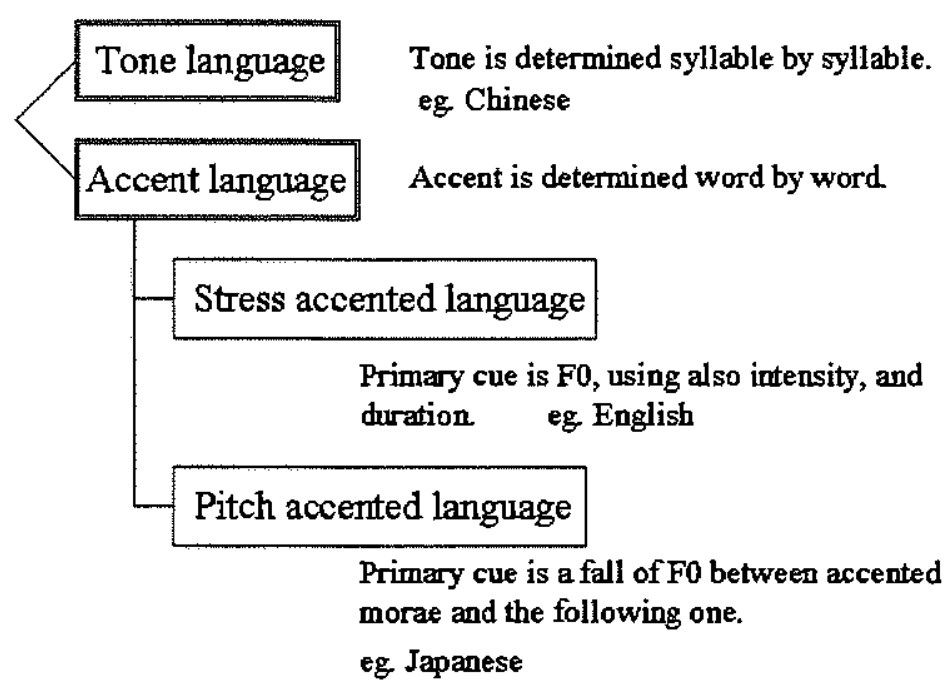

Figure 2.3 Types of suprasegmentals in world languages.

Japanese syllables contrast high and low pitch, and an accented syllable is marked by the shift of the pitch from high to low between the accented syllable and the following one. The difference in the location of an accent is lexically contrastive in Japanese as well. Example 22 shows examples from standard Japanese. These words are exactly the same in terms of their segments, their accentual difference is the only clue for the distinction of meaning.

\section{Example 22}
a me "candy"
a me "rain"
$\mathrm{L} \mathrm{H}$
$\mathrm{H} \mathrm{L}$
ha shi "bridge"
L $\mathbf{H}$
ha shi "chopstick"
H L

In addition to the acoustic realisation of accented syllables, the Japanese accentuation system differs from that of English in the respect that Japanese allows the absence of an accent in a word. This does not, however, count as a difference between pitch and stress accented languages, as Hymann (1977:38) points out that there are languages which allow the absence of accent in stress languages as well, such as Kitsai, Saho, and Seneca. 
Japanese pitch accent also seems to differ from stress in English in tense of the awareness of speakers. Average speakers of English would be able to identify the location of a stress accent in a word with no problem. It is, however, much more difficult for linguistically naïve Japanese speakers to identify the location of a word accent. The notion of pitch accent qua prominence is thus suspect.

\subsubsection{RULES FOR ACCENTUATION}

In this section, the rules for the location of accent and realisation of pitch pattern in standard Japanese are discussed. As previously mentioned, Japanese marks word accent by the contrast of two types of pitch; high and low. The rule for the placement of a word accent varies greatly depending on which word class a word belongs to. Verbs and adjectives, for instance, generally feature regularity in the location of accent. We discuss the rules for accentuation of nouns here, of which the location of accent is lexically determined and cannot be predicted.

Nouns in Japanese can be accented or unaccented, and if a noun is accented, the accent can be placed anywhere. The pitch patterns of the whole words are, however, easily predicted once the location of the accent is known, since standard Japanese has systematic rules to govern the realisation of pitch patterns from accent placement. The rules for pitch determination are summarized as follows.

1) A word can consist of no more than one accent and it can also have no accent.

2) Pitch falls after the accented syllable. Once it falls, it must stay low.

3) The first two morae must have different pitch; the initial mora is always low, unless the first syllable is accented. (This phenomenon is called initial lowering.) 
From these rules and the location of accents, the pitch pattems of words can be easily predicted. So, the possible pitch patterns for nouns, including the possibility that the word is unaccented, is equivalent to the number of syllables +1 (Vance 1987:80). Example 23 lists trisyllabic words with four $(3+1)$ possible pitch patterns. The location of the accent is indicated by "”.

\section{Example 23}

$\begin{array}{lll}\text { Accented on the first syllable } & \begin{array}{l}\text { i' no chi } \\ \text { H L L 'life' }\end{array} \\ \text { Accented on the second syllable } & \text { ko ko' ro } \\ & \text { L H L 'heart' } \\ \text { Accented on the third syllable } & \text { a ta ma' 'head' } \\ & \text { L H H } \\ \text { Unaccented } & \text { mi ya ko' 'capital city' } \\ & \text { L H H }\end{array}$

(Examples quoted from Shibatani et al. 1981:247)

The examples above show that atama' 'head' and miyako 'capital city' have the same pitch pattern, although one is accented on the final syllable and the other is unaccented. In fact, it is not possible to distinguish an unaccented word from a word that has an accent on the final syllable of the word, when that word is uttered in isolation. However, once they are followed by particles, such as ga (subject marker) or wa (topic marker), the difference between these two is clear, see Example 24. 
Example 24

\section{Accented}

$$
\begin{aligned}
& \text { atama' } \\
& \text { LHH }
\end{aligned} \quad \begin{aligned}
& \text { atama' ga } \\
& \text { LHHL }
\end{aligned} \quad \text { atama' 'head' city' }
$$

\section{Unaccented}

$$
\text { miyako } \Rightarrow \text { miyako ga miyako 'capital }
$$$$
\text { LHH L LH H }
$$

The difference comes out as the pitch difference in the following particle as marked in bold case above. In atama 'head', the accent on the final syllable triggers the fall of pitch in the same way as any other accented syllables do, and the fall is reflected on the following particle beyond the morpheme boundary. The pitch of particle following minako "capital city' stays high, on the other hand, as there is no accent on the preceding vowel that causes a fall of the pitch.

The syllables of the words in Example 24 are all light, that is, the syllables contain only one mora. Similar rules to those above generally apply to the words that consist of heavy syllables, except for the case of the third rule, of initial lowering. Initial lowering is becoming a less strictly applied rule, in words where the first syllable is long, than the words with light syllable. See Example 25 quoted from Vance (1987: 80). The location of the accent is indicated by " 


\section{Example 25}
a) /ko'okoo/
H L L L
'filial piety'
b) /kookoo/
L H H H $\quad(\sim \mathrm{HHHH})$
c) /ke'Ndoo/
HL L L
'Japanese fencing'
d) /keNto'o/
L H HL ( HHHL)
e) $/ \mathrm{keNtoo} /$
L H H H ( HHHL)
'high school'
'guess'
'examination'

(Examples quoted from Vance 1987:80)

As a), c), and d) in Example 25 show, the pitch fall from high to low occurs at the boundary of the mora, but not at the syllable boundary. That prompts a question as to the status of the syllable as an accent carrier. It is, however, also noticeable that there is no example of accentuation on the second mora of long syllables. This suggests that a syllable is the unit which determines the location of accent, but that actual pitch changes occur at the mora boundary. For the rules of accentuation of long syllables, we should add that the accent is always on the first mora of the syllable, so that the pitch difference is observed between the first and the second morae in the syllable. This exemplifies of the necessity for analysis of both mora and syllable in Japanese phonology.

\subsubsection{DIALECTAL VARIATION}

The placement of word accents is not consistent across the dialects in Japan. The location of accents varies from one dialect to another, and the rules of how to determine the pitch patterns of words also differ greatly. The accentuation systems of the regional dialects can be classified 
into five groups, according to the number of the contrasts made and the pitch pattern (Shibatani 1990:212). The distribution of each group is presented in the map in Figure 2.4.

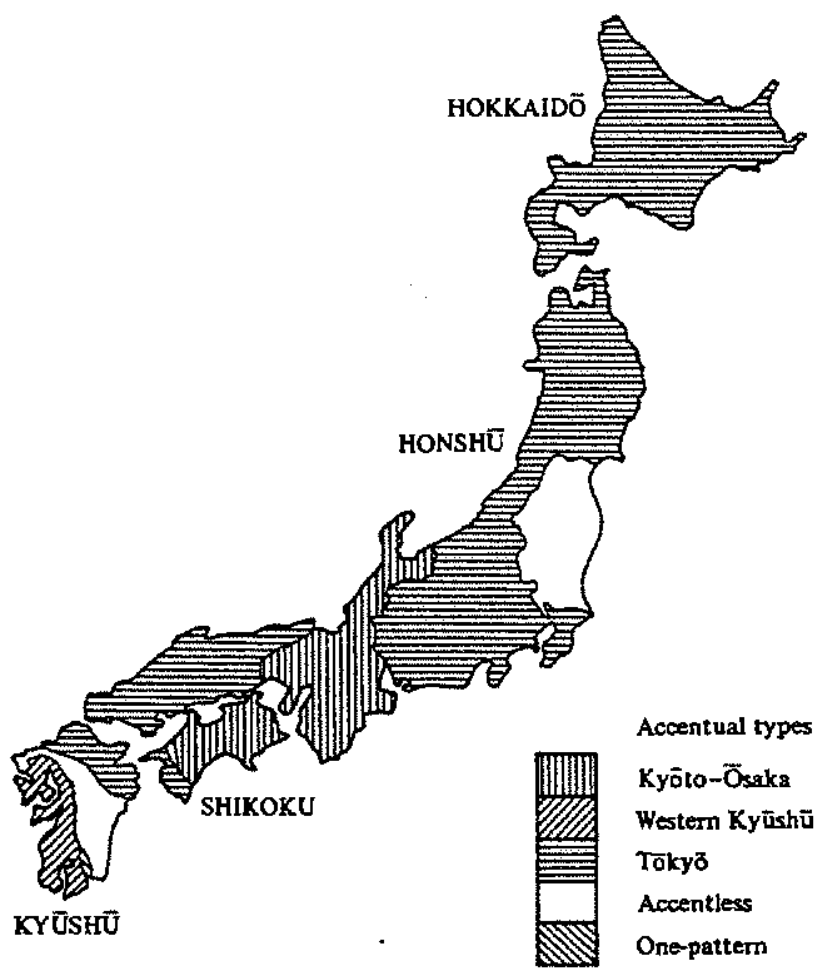

Figure 2.4 Distribution of the accent groups in Japan. Sourced from Shibatani (1990: 211)

Modern standard Japanese is based on the Tokyo dialect. The standard Japanese which has been discussed so far has the same accentuation system as the Tokyo style. Given a two syllable word combined with a subject marking particle $g a$, the possible pitch patterns in Tokyo accent are three: $H L(L), L H(L)$, and $L H(H)$.

The Osaka-Kyoto dialect is another accent group. This dialect has a pitch pattem $\mathrm{HH}(\mathrm{H})$, in addition to the three pitch patterns of Tokyo dialect.

The third group is the western Kyushu dialects, such as Kagoshima dialect, which has a twopattern accent. In this group of dialect, there are only two patterns in the location of pitch accent: word final or penultimate syllables. 
The fourth accent type is a one pattern system. In this group of dialects, only one pitch pattern is allowed, accented on the final syllable.

The fifth group comprise the accentless dialects. The dialects, such as Sendai (Miyagi prefecture), Kumamoto (Kumamoto prefecture) or Shirakawa (Fukushima prefecture), do not have a system of accentuation in their phonology. In those dialects, pitch change within a word is not determined lexically, but postlexically (ie. intonation). The words differing in pitch, such as hashi LH 'bridge' and hashi $\mathrm{HL}$ 'chopstick', are not found in these dialects. Hashi, meaning chopstick, can be pronounced in both HL and LH (Shibata 1955/1980:405). Table 2.5 summarises the discussion above.

\begin{tabular}{|c|l|l|}
\hline Accent types & Pitch patterns for '2 syllable word $+g a^{\prime}$ & \multicolumn{1}{|c|}{ Examples of dialects } \\
\hline Tokyo & HL(L), LH(L), LH(H) & Tokyo \\
\hline Osaka-Kyoto & HH(H),HL(L),LH(L), LH(H) & Osaka, Nara, Kyoto, Kobe \\
\hline Two-pattern & LH(L), LL(H) & Kagoshima, Nagasaki \\
\hline One-pattern & LL(H) & Miyakonojoo \\
\hline Accentless & Post lexical & Sendai, Kumamoto, Shirakawa \\
\hline
\end{tabular}

Table 2.5 Summary of the pitch patterns of each accent groups.

When speakers of regional dialects other than Tokyo dialect speak standard Japanese, often the accent patterns of their original dialect appear in their standard Japanese. Although this will result in extensive between-speaker variations of pitch pattern, the speakers of different dialects can normally communicate without much difficulty. The location of the accent determines lexical meanings of words when the words are uttered in isolation. The meaning that the speaker intends to convey is, however, usually understood from the context, so pitch accents are not necessarily a crucial component of communication in Japanese. The pitch pattern which does not fit in the listener's phonology is noticed as a 'strange' or 'foreign' accent, however.

The difference in pitch accent is thus clearly audible, and trained experts can analyse speaker's pitch accent relatively easily by listening. It is possible for a perpetrator to disguise his original pitch accent by putting on a different style of pitch accent, since the difference in pitch 
accent is so easily detected. However, as Nolan (1990:5) noted it is unlikely that an individual can achieve both consistent and comprehensive imitation of foreign accent. Speakers' styles of pitch accent therefore may assist the discrimination of two speech samples in some cases.

\subsection{VOWEL DEVOICING}

\subsubsection{CONDITIONING FACTORS FOR VOWEL DEVOICING}

Vowel devoicing in Japanese is characterised as a phenomenon where high vowels are devoiced under certain conditions. Japanese vowel devoicing is very complicated, because of the various conditioning factors and the different realisation of the devoicing. Shibatani (1990) summarises the conditions as follows.

(1) /i/ and / $/$ / will only devoice if not contiguous to a voiced sound

(2) $/ \mathrm{i} /$ and $/ \mathrm{w} / \mathrm{do}$ not devoice when they are word initial sounds even followed by a voiceless sounds.

(3) Accented $/ \mathrm{i} /$ and $/ \mathrm{u} / \mathrm{do}$ not devoice even if flanked by the voiceless consonants. The phenomenon also depends on the speech tempo: in slow deliberate speech, devoicing is less frequent. (p.161)

Two words, hakusai 'Chinese cabbage' and hakumai 'white rice' can be given as examples. The high vowel $/ \mathrm{w}$ in the first word hakusai 'Chinese cabbage' is devoiced since the $/ \mathrm{u} /$ vowel is between two voiceless consonants and is not accented. The / $/ /$ vowel in the second word hakumai 'white rice' is, on the other hand, not devoiced, as it is followed by the voiced consonant $/ \mathrm{m} /$. Figure 2.5 shows spectrograms of these two words. It presents examples of voiced and devoiced $/ \mathrm{u} /$ spoken by a single male speaker of Japanese. 


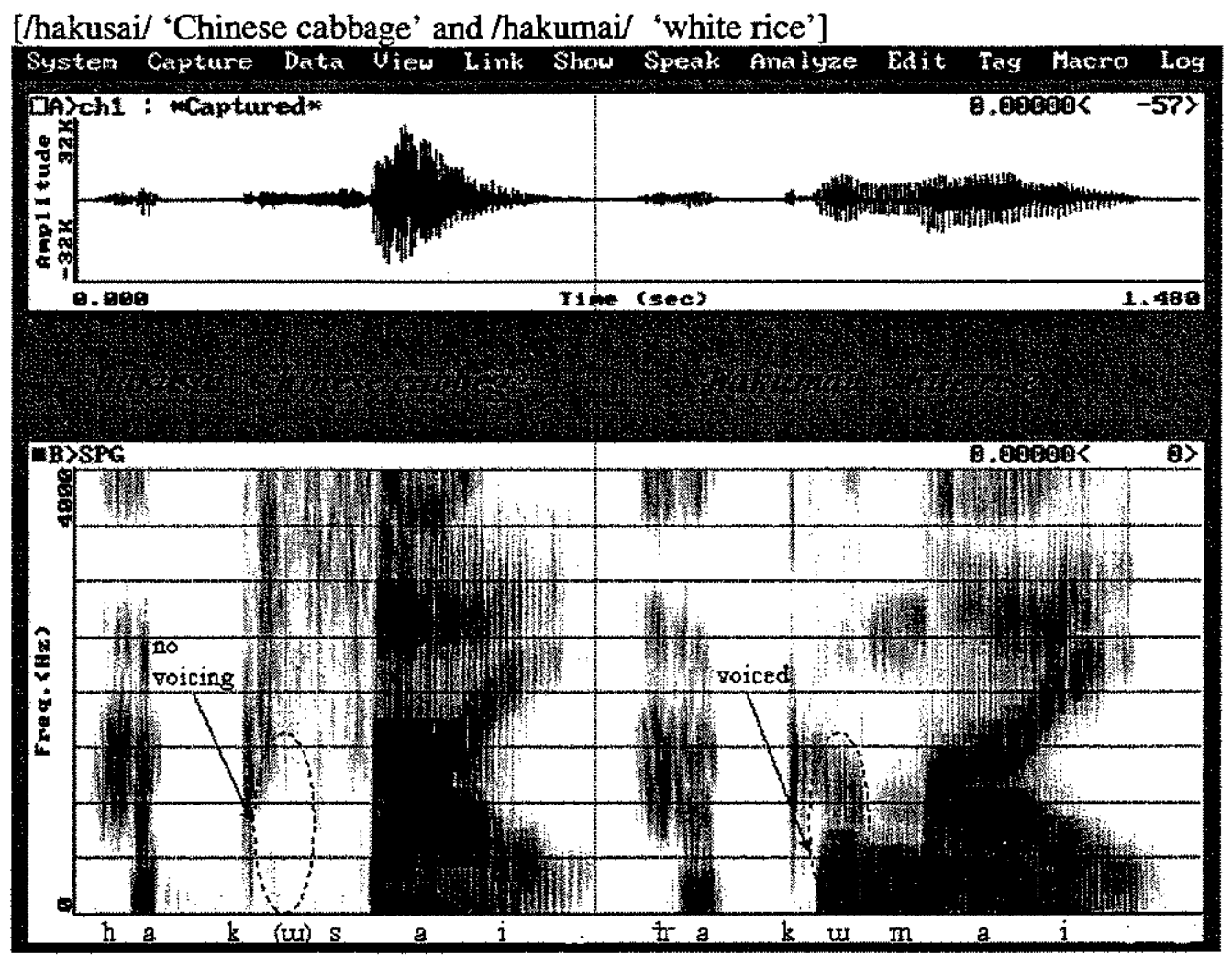

Figure 2.5 Examples of voiced and devoiced $/ w$ uttered by the same speaker. In the first word (hakusai 'Chinese cabbage'), $/ \mathrm{w}$ / was devoiced, whereas $/ \mathrm{w} /$ was fully voiced in the second word (hakumai "white rice').

The / $\mathrm{u} /$ vowel of hakusai in Figure 2.5 is transcribed in parentheses, as it is not clear if there was really a devoiced $[u]$ in the position of phonemic $/ w /$ in this token. It seems as if the $/ u /$ was totally omitted, phonetically realised as [haksai]. The phonetic realisation of devoicing is discussed later in this section.

Furthermore, there are observations that in faster and more casual speech, even non-high vowels are devoiced when they have no voiced segments around them. On the other hand, in slow deliberate speech, vowel devoicing is sometimes avoided regardless of the phonological environment the vowel is in.

There is also a general tendency to avoid devoicing an accented vowel. There are two ways of inhibiting the devoicing; accent shifting or voicing the vowel. Accent shifting is observed in verbs and adjectives, which, unlike nouns, have general rules for the location of accent. When 
an accent is located on a vowel prone to devoicing, the accentuation rule is overridden and the accent is located on the next voiced vowel. McCawley (1977: 266) provides examples of adjectives. In general, adjectives have an accent on the last syllable of the stem in non-past form. In the past tense form, the adjective's accent is located on the penultimate syllable of the stem. However, the accent is relocated when this rule assigns a vowel which is in the devoicing environment as an accent carrier (Example 26). The stems of the words are separated from the suffixes by " $\because$. The accented syllables are indicated by "'.

\section{Example 26}

$\begin{array}{lll}\text { 'high' } & \begin{array}{l}\text { Non past } \\ \text { taka' - i } \\ \text { (stem) }\end{array} & \begin{array}{l}\text { Past } \\ \text { ta'ka - katta }\end{array} \\ \text { 'dark' } & \begin{array}{l}\text { kura' - } \\ \text { (stem) }\end{array} & \text { ku'ra - katta } \\ \text { 'deep' } & \begin{array}{l}\text { fuka' - } \\ \text { (stem) }\end{array} & \text { fuka' - katta *fu'ka-katta (accent is shifted.) } \\ \text { 'near' } & \begin{array}{l}\text { chika' - i } \\ \text { (stem) }\end{array} & \text { chika' - katta *chi'ka-katta (accent is shifted.) }\end{array}$

In the above examples, all four words are adjectives. With fukai 'deep' and chikai 'near', the accentuation rule assigns the syllables with high vowels which are in between two voiceless consonants as accent carriers. In order to avoid placing an accent on the devoiced vowel, the accent is relocated to the following vowel.

In addition to the conditions listed above, Vance (1987:49-51) reports that intonation also affects devoicing. Normally, in standard Japanese at least, the final high vowel of a sentence is devoiced when it follows a voiceless consonant. It is not devoiced, however, when the sentence's final syllable carries a high boundary tone indicating a question. This is quite understandable, as the meaning of an utterance may not be marked by its grammatical structure but rather by its intonation in Japanese. For instance, a sentence like Nani ka miemasu 'something + can see (literally)' has two possible meanings depending on the boundary tones of the final syllable. It can mean "I can see something" where a low boundary tone L\% is 
present, but it also can mean "Can you see something?" if the high boundary tone H\% is placed on its final syllable. In this situation, the important final syllable cannot be devoiced, as it would obscure the meaning of utterance quite significantly. See Figure 2.6 for examples.

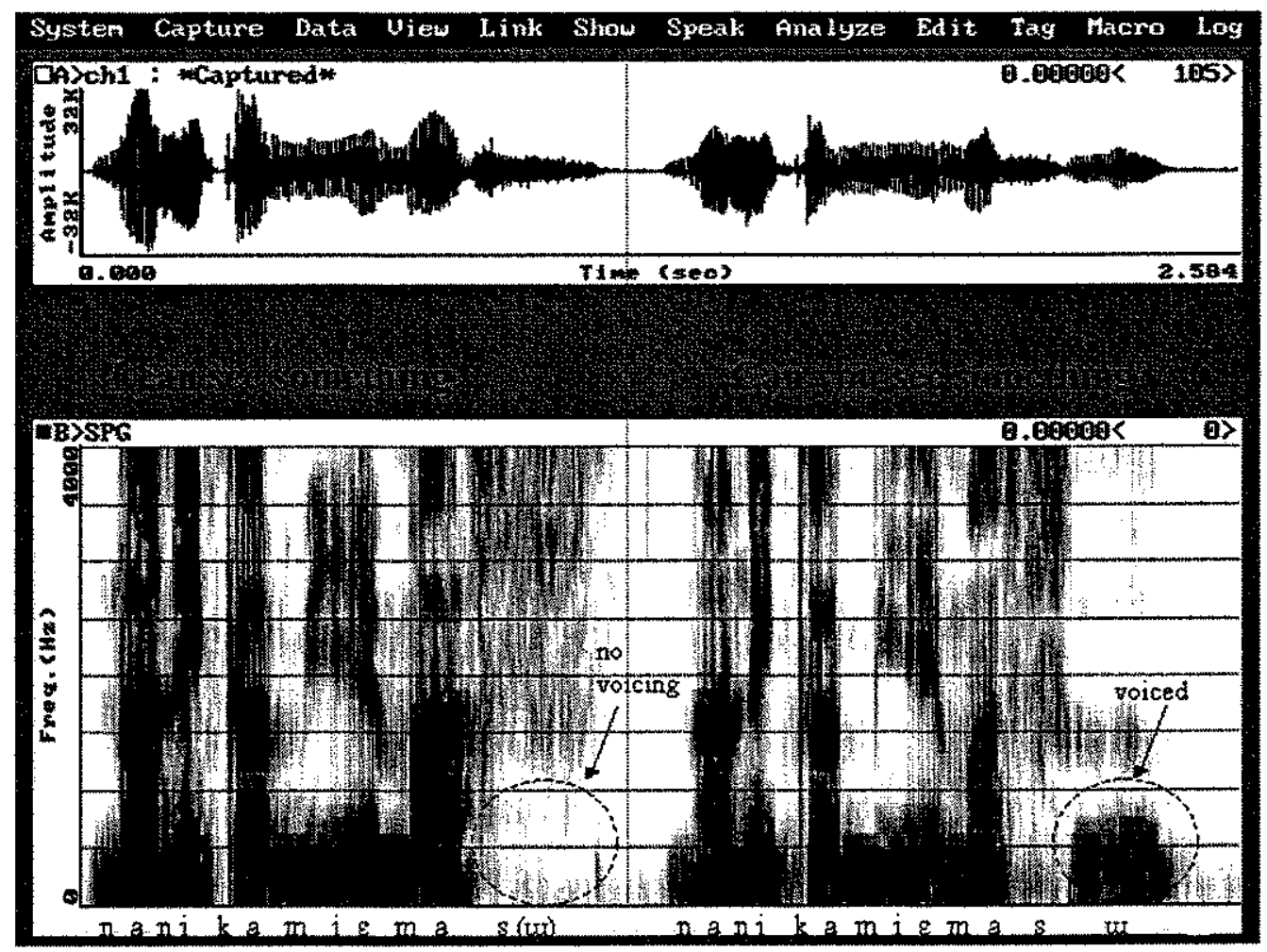

Figure 2.6 Examples of sentence final devoicing. The first sentence is a statement nani ka miemasu/ "I can see something," and the second sentence is a question nani ka miemasu/ ${ }^{\text {H\% }}$ "Can you see something?"

The realisation of vowel devoicing is the outcome of a concurrence of many complex factors. Thus, it cannot be predicted automatically from segmental phonological conditions surrounding vowels. Even if the phonological environment meets the three criteria for vowel devoicing listed at the beginning of this section, vowels may still not be devoiced. Alternatively, vowels may be devoiced without meeting the criteria. Maekawa (1989:144) reports that some segments trigger vowel devoicing more frequently than others. According to him, devoicing occurs with greater probability when a vowel follows a fricative than when it follows other consonants. When a vowel follows a voiceless consonant and precedes a voiced consonant, then vowel devoicing is not supposed to occur, according to Shibatani's criteria. However, even in this environment, devoicing can occur from time to time. It happens more 
often when the following consonant is a nasal than when it is an oral voiced consonant. Maekawa also points out that the segment that is not directly adjacent to the vowel can also affect devoicing, reporting that devoicing occurs more frequently when the following syllable consists of an open vowel, such as $/ \mathrm{a}, \mathrm{lel}$, or $/ 0 /$.

Another level of complexity is that the phonetic realisation of devoiced vowels also varies. Kawakami (1977:26) points out that in sorne cases, the vowels are devoiced and, in other cases, they are omitted. Devoiced vowels are distinguished from the omitted ones by the fact that there is some sign that a vowel originally existed there. A devoiced vowel naturally does not show fundamental frequency. Noise-excited formants are normally still observable, however. His report is summarised as below in Table 2.6 .

\begin{tabular}{|c|c|c|}
\hline & Devoiced & Omitited \\
\hline Followed by $/ \mathrm{i} /$ & $/ \mathrm{ki} / / \mathrm{pu} /$ & Isi thit thit \\
\hline Followed by / w & /ku//pu/ /syu//tyu/ & $/ \mathrm{su} / \mathrm{tw} / \mathrm{hu} /$ \\
\hline
\end{tabular}

Table 2.6 Two types of realisation of vowel devoicing.

Kawakami claims that when the syllable $/ \mathrm{ku} /$ is devoiced, phonetically it is realised as [k], rather than [km]. Figure 2.5 above, however, shows that the /u/ vowel in /hakusai/ was omitted rather than devoiced, as we cannot observe other formants of the / $\mathrm{w}$ vowel in the figure. No trace of the vowel is left. His observation is thus obviously not applicable to every speaker or to all occasions, though it may show a general tendency.

\subsubsection{DIALECTAL VARIATION IN VOWEL DEVOICING}

Finally, dialectal variance in the realisation of devoicing should be mentioned. It is well known that Kansai dialect speakers (including Osaka, Kobe, Kyoto, and Nara areas) tend to avoid devoicing. Sugito's (1996a) experiment reported that Tokyo dialect speakers devoice three times more often than Osaka dialect speakers. Furthermore, comparing eight major 
dialects, Sugito (1996b) reports that there are great differences in the frequency of devoicing depending on the dialect of speakers.

An additional complicating factor in this connection is age. Constant exposure to standard Japanese through the media seems to be promoting vowel devoicing in areas where vowels were not devoiced originally, and the younger generation is more likely to be subjected to these influences.

In this thesis, I shall investigate the realisation of vowel devoicing as a candidate for a forensic speaker identification parameter, in addition to the formant patterns. Sugito's observation mentioned above suggests that the simple presence or absence of vowel devoicing in a recording may not provide clear-cut information on speakers* identity or dialect area. Nevertheless, the possibility of the use of vowel devoicing as a parameter for forensic speaker identification will be researched in Chapters 4 and 5 .

\subsection{INTONATION AND WORD ACCENT}

The FO in a small section of speech, such as the size of the pitch change from high to low related to the word accent appears to be an attractive potential parameter for speaker identification in a pitch accented language. It is, however, optimistic to assume so. The acoustic realisation of pitch accent is the outcome of a complex mixture of factors, such as intonation and/or the location of focus. A large within-speaker variation is therefore inevitable. In this section, the complexity of the realisation in pitch patterns of words is demonstrated in relation to intonation and focus. 


\subsubsection{INTONATION}

Ladd (1996:6) defines intonation as “...suprasegmental phonetic features to convey 'postlexical' or sentence-level pragmatic meanings in a linguistically structured way." Traditional studies on intonation concentrate on suprasegmental features. He claims that there are two additional but equally important features that define intonation. Information conveyed by intonation has to possess sentence-level pragmatic meaning, ie. "meanings that apply to phrases or utterances as a whole, such as sentence type or speech act, or focus and information structure (p.7)", but not to individual words. This characteristic distinguishes intonation from other suprasegmental properties, such as stress, tone or accent. Ladd also claims that intonational features must be organised by categorically distinct entities, like low or high tone, or boundary rise, and also in relation to the context (eg. stronger or weaker than...). Continuously variable physical parameters (eg. tempo or loudness), which reflect paralinguistic features, such as speakers' emotional states, are excluded from the definition of intonation. An easy example of the function of intonation has already been shown in Figure 2.6 presented in 2.6.1. Here, two segmentally identical sentences are distinguished as statement and question, purely based on their intonation ( $\mathrm{H} \% / \mathrm{L} \%$ boundary tone).

\subsubsection{CATATHESIS AND WORD ACCENT}

Utterances normally have a gradual pitch declination in standard Japanese (Maekawa 1997b:41). In most cases, the original pitch pattern of each word is still retained within this gradual falling intonation. The range of pitch fall, however, can become narrower due to catathesis (or downstep). Catathesis is a phenomenon whereby the pitch of the following words is significantly lowered after a fall of pitch triggered by an accented word in an utterance (Pierrehumbert and Beckman 1988). This catathesis mainly affects the upper limit of the pitch range of the utterance rather than the lower limit, so consequently it reduces the size 
of the fall of pitch of the following words considerably. As an example of catathesis, two sentences uttered by one of the speaker recorded for the experiments in this study (Speaker JN) are shown in Figure 2.7. Those sentences are:

$\begin{array}{cccccc}\text { Awai } & \text { moji } & \text { wa } & \text { mienikui } & \text { desu } & \begin{array}{c}\text { yo } \\ \text { pale }\end{array} \\ \text { character } & \text { (topic) } & \text { hard to see } & \text { is } & \text { (ending) }\end{array}$

"Pale characters are difficult to see."

Aoi moji wa mienikui desu yo blue character (topic) hard to see is (ending)

"Blue characters are difficult to see."

Glosses shown in parentheses are particles. As can be seen, these two sentences are identical except for the first word awai (LHH) 'pale' and aoi (LHL) 'blue.' The word awai is an unaccented word, whereas aoi carries an accent on the second mora $/ \mathrm{o}$, therefore catathesis is expected to influence the words following aoi, but not awai. It should be noted here that there was no contrastive effect in the focus for these two sentences, as the two sentences were uttered separately. See Figure 2.7 below. 


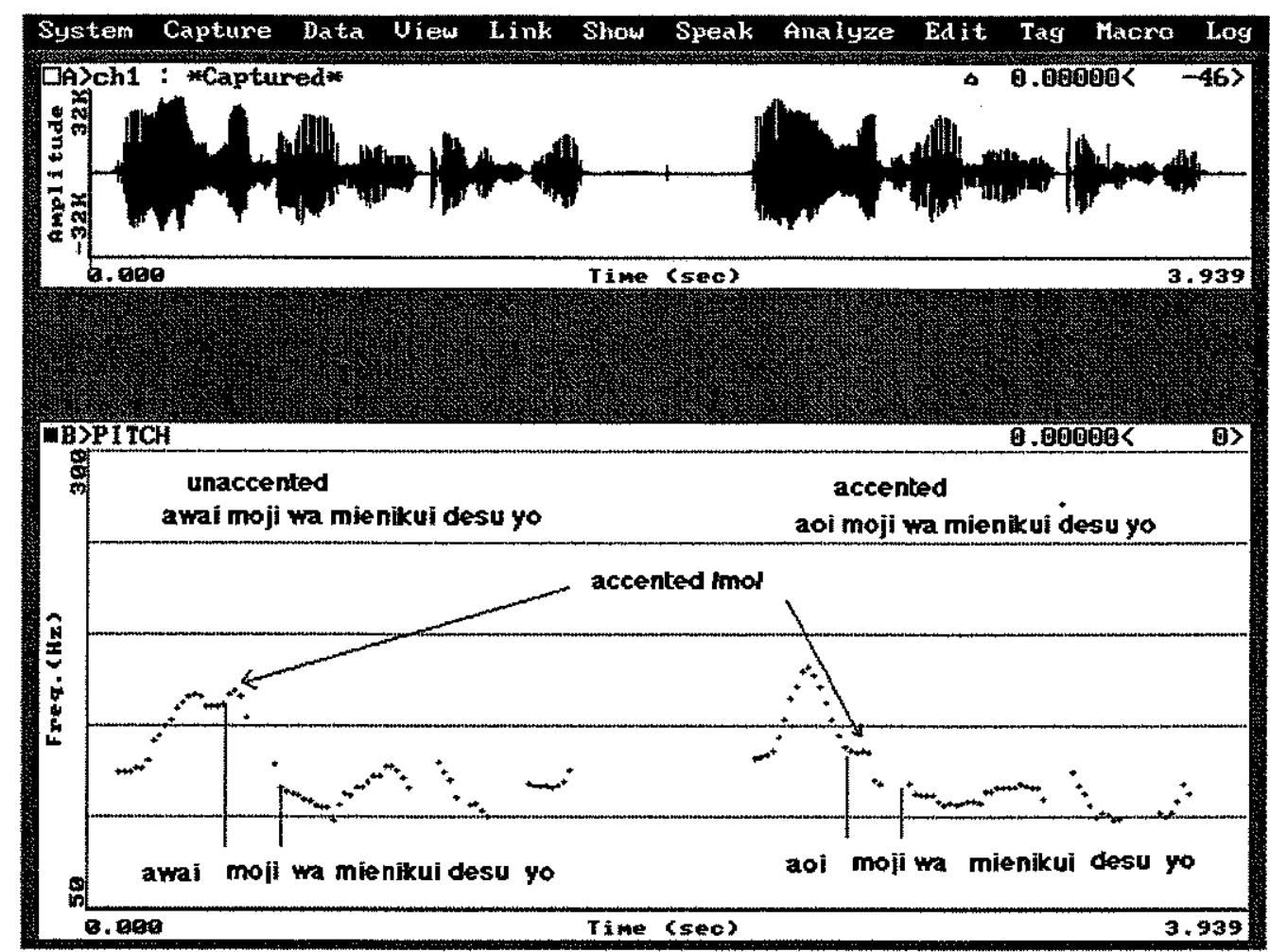

Figure 2.7 Examples of catathesis. F0 patterns of two sentences Awai moji wa mienikui desu yo "Pale characters are difficult to see' and Aoi moji wa mienikui desu yo 'Blue characters are difficult to see.'

The upper limit for the pitch accent of the word moji 'characters' (accented on the first mora $/ \mathrm{mo} /$ ) was considerably lower when the preceding word was accented aoi 'blue' than when it was the unaccented awai 'pale.' The F0 height of the accented $/ \mathrm{mo}$ / between the two sentences clearly differs.

The influence of catathesis is also found on the word mienikui 'difficult to see', although it is not as conspicuous as the difference in the pitch of moji 'character'. The word mienikui has an accent on the fourth mora $/ \mathrm{ku} /$. The pitch pattern of the word is thus predicted as LHHHL. The rise in F0 from the first low pitch to high is realised very differently between the two sentences. The rise is hardly recognisable in the second sentence, where the first word carries a pitch accent. 


\subsubsection{INFLUENCE OF FOCUS}

Catathesis can be interrupted by the placement of 'focus'. Focus is the most important information for the speaker to convey in the given utterance. A larger F0 range often marks the essential part of information conveyed in standard Japanese. The upper limit of the FO of a word is raised greatly when the word carries the focus of an utterance; catathesis thus does not appear with a focused word even the word follows an accented word. Two utterances of the same sentence with the exactly the same segmental structure are presented here. This sentences is (glosses shown in parentheses are particles):

$\begin{array}{llcccc}\text { Yamashita } & \text { san } & g a & \text { goji } & \text { ni } & \text { kaeri mashita } \\ \text { Yamashita } & \text { Mr. } & \text { (subj) } & 5 \text { o }^{\prime} \text { clock } & \text { (at) } & \text { went home }\end{array}$

"Mr. Yamashita went home at five o"clock."

This sentence was spoken by a male speaker (Speaker JN, who is one of the informants of this study) with two different focus locations. The first utterance is the response to the question "Everyone was supposed to stay until 6:00 today! Who went home at five o'clock?" The second one is the response to the question "(situation: Mr. Yamashita always works hard and usually stays until around eight o'clock.) Did anything unusual happen today?" This will elicit focus on yamashita san 'Mr. Yamashita' in the first utterance and on goji 'five o'clock' in the second utterance. See Figure 2.8 for the Fo change in the two different focus locations. 


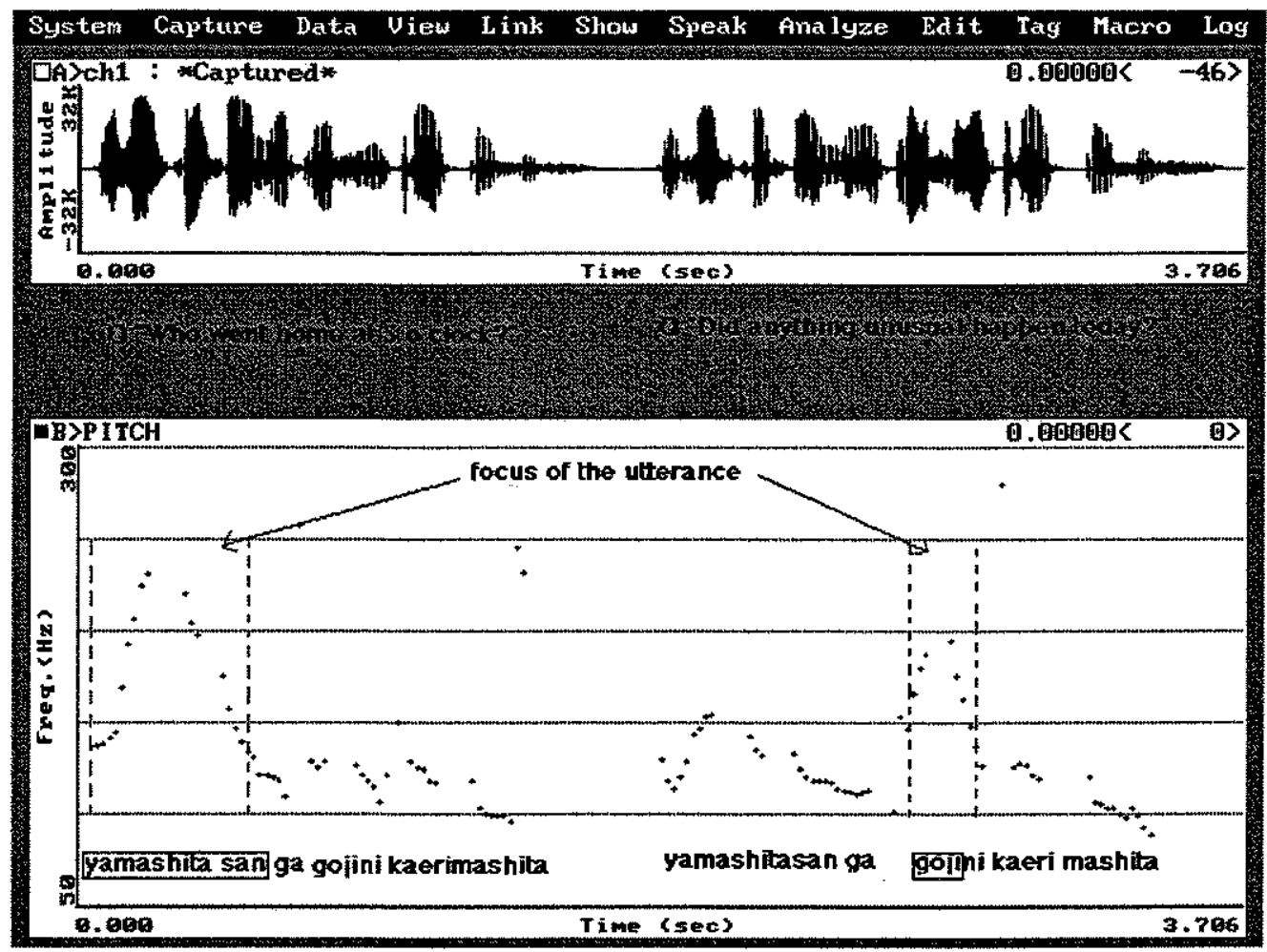

Figure 2.8 Example of the influence of focus. The sentence is Yamashita san ga goji ni kaeri mashita 'Mr. Yamashita went home at five o'clock,' spoken with two different focus locations.

The first word of the sentence yamashita 'Yamashita' has an accent on the second mora $/ \mathrm{ma}$. In the first sentence, the F0 of the accent of the following word is kept low despite the accent on the first mora /go/, because of the catathesis caused by the accent of the preceding word yamashita. In the second sentence, on the other hand, the word goji has a clear F0 rise. As the speaker has focused on the word goji, the catathesis on this word has therefore been overridden.

Additionally, Figure 2.8 also shows that focus amplifies the change in F0. Yamashita in the first sentence has much higher F0 than that in the second sentence. This is presumably caused by the fact that yamashita in the first sentence carried a focus, whereas that in the second sentence did not. 


\subsubsection{IMPLICATIONS IN FORENSIC PHONETICS}

The discussion above has shown that the actual realisation of FO in Japanese is the result of complex interactions among many factors. It is far more than something which can be described exhaustively by a simple $\mathrm{H}$ and $\mathrm{L}$ pitch accent system. Although it appears to be an attractive potential parameter for speaker identification in a pitch language, the $\mathrm{F} 0$ in a small section of speech, such as the size of the $\mathrm{HL}$ or $\mathrm{LH}$ pitch change relative to the word accent, would not be much use in forensic speaker identification due to the large within-speaker variation. Furthermore, establishing the comparability between samples is just too difficult for short term F0 change, especially in a real-life forensic situation. The use of F0 for speaker identification seems, in Japanese as well, to be limited to long term F0 parameters like mean and standard deviation.

\subsection{DIALECTAL VARIATION AND FORENSIC PHONETICS IN JAPANESE}

Finally, the possibility of the use of the regional dialects for forensic speaker identification in Japanese is briefly addressed. In some languages, such as British English, the characteristics associated with regional dialects may have very strong diagnostic power. It is, however, very difficult to detect a speaker's place of origin on the basis of the dialectal characteristics in Japanese. When the speaker is old (over 70 years old) and does not have a high educational background, occasionally it is possible to determine the speaker's place of origin in detail, as in up to X city, Y prefecture. It becomes suddenly far more difficult, however, when the speaker is younger than 50 years old. With the speaker under 30 years old, it is very rare to be able to identify the speaker's place of origin in that detail (Kikuo Maekawa p.c.).

It does not, however, mean that dialectal characteristics are valueless in Japanese forensic speaker identification. Although it is true that dialectal characteristics in Japanese do not have 
a strong enough diagnostic power to identify the detail of the speaker's regional background, usually it is still possible with the speaker over middle age to detect the regional feature on a larger scale (such as north east part of Japan, Tokyo area, or middle-west Japan which is called Kansai, and so on). Using a careful and systematic auditory analysis, it may thus be possible to discriminate speakers of two speech samples on the basis of the difference in their regional characteristics.

Having described the relevant issues in phonetics and phonology of Japanese language, I now go on to the description of the experiments carried out in this study. The next chapter will extensively describe the procedures of the experiments as well as the selection of the parameters for forensic speaker identification. 


\section{Chapter 3}

\section{PROCEDURE}

\subsection{OBJECTIVES}

This chapter describes and justifies the experiments carried out in this thesis. The selection of informants, parameters and corpus, the process of recording, and how the measurements were made are all discussed here in detail.

\subsection{INFORMANTS}

\subsubsection{DESIDERATA FOR THE SELECTION OF INFORMANTS}

As will be shown in detail below, the goal for forensic speaker identification is basically to estimate how many times more probable it is that the given speech samples came from the same individual than from different individuals. To estimate this probability, knowledge of the distributions of between-speaker variation and within-speaker variation are indispensable. This is because how much more likely that the difference between speech samples is due to withinspeaker variation rather than between-speaker variation is estimated by comparison to these distributions. In order to obtain reliable results, the reference population for forensic phonetic experiments thus needs to be large enough to accurately reflect the between-speaker and within-speaker variation of the population at large. 
The question which then arises here is: "How many speakers are enough?" Clearly, the more is the better for the aim of obtaining accurate pictures of within- and between-speaker variations of a general population. This is not, however, a realistic condition for phonetic experiments. In addition, the ways people differ from each other or differ within themselves are not infinite. Thus once the number of speakers used for data collection reaches a certain size, the distribution of the sample parameter becomes asymptotic to the distribution of the general population. In such cases, extra speakers would not add more information on between- and within-speaker variation. It is just gilding the lily.

However, it has to be noted that the reference population from which a suspect is chosen in forensic cases is not necessarily the entire general population. There are three factors that determine the size of reference population in a forensic situation. First of all, in forensic situations, the population of suspects is normally much smaller than the general population. It has to be remembered that if the two voices sound clearly different, they probably would not be compared in first place. For instance, no one compares a male voice and a female voice or an old person's voice and a teenager's voice, trying to claim that those two voices are from the same individual. The voices compared in forensic speaker identification are thus expected to be similar to some extent, and the distribution of between-speaker variation among people with a similar voice will be much less widely spread than that of the general population of the world or of speakers of the same language.

There is usually some prior information known which is used to narrow down the possible perpetrator, such as a young male, or a young male who speaks broad Australian English and who was also at a certain place at a certain time. In such cases, then, it is not necessary to include as many speakers as possible to profile between- and within-speaker variations of this particular population. This, together with the points made above, limit the population of the possible perpetrator. 
Finally the defence hypothesis can also condition the size of the reference population, as the reference population is determined by the number of people who could possibly be the criminal. For instance, if the defence hypothesis is "The offender is not my client, it is someone else", the population of the possible perpetrator will not be narrowed down by the denial. If the defence hypothesis is "This is not my client, this is someone else with a similar voice to my client", however, the population of the possible perpetrators is reduced significantly. Given that it is sensible to think that there would not be, say, 30 people with similar voices at the location where the crime was committed, the population with which the forensic phonetic investigation deals in reality does not have to be very large. The hypothesis could even be "This is not my client, this is the brother of my client." The population then becomes even smaller, to the extent that a closed set for comparison then exists.

The discussions so far also lead us to another important desideratum for the speaker selection in forensic phonetic research. That is, homogeneity of the speakers. It has already been pointed out that the voices compared in forensic speaker identification are usually similar or, at least, they do not differ very obviously. To contribute to real life forensic investigation, it is necessary to be able to perform discrimination among a homogeneous population with reasonably similar sounding voices.

\subsubsection{SPEAKERS IN THIS STUDY}

\subsubsection{Population of speakers}

In this study, the number of the speakers used varied slightly depending on the experiments, as it was possible to add three more speakers after the first experiment on vowel formants. For the investigation of vowel formants embedded in different contexts (Chapter 4), 11 speakers were recorded. After this experiment, three more speakers were added to the data, making the total number of the informants up to 14 . Speaker $\mathrm{KH}$, however, had to be excluded from the 
data in the analysis of moshimoshi in Chapter 5. Speaker KH is the informant who was recorded first. It was decided to repeat tasks after the recording of this speaker, which meant that there were not enough samples of moshimoshi to perform a statistical analysis of his recording. The number of informants for the experiments on the phrase moshimoshi in Chapter 5, thus, became 13. For the discrimination testing in Chapter 6 , only the speakers who were measured in both Chapter 4 and 5 were included. As result, the discrimination test was carried out with ten speakers.

In order to profile between- and within-speaker variation, 11 and 13 speakers may seem a rather small sample. The time constraints for this study, however, did not allow for any large population. To appreciate this, it can be noted that it usually took approximately 33 hours in total to process one speaker, including recording, digitising, segmentation and measurements on all measuring points. For all speakers, it thus took around 430 hours. This means that even if eight hours a day were spent solely on this processing (which is of course not practically possible), it would take about 54 days.

The number of the speakers for this study is thus admittedly not large. This, however, does not necessarily mean that the results of this study are not reliable. For instance, ten speakers in the discrimination test produce 45 different speaker combinations (i.e. AA/HA, AA/JN, AA/KA ... and so on). Further, each speaker's dataset consisted of two parts, the data from the first recording session and data from the second recording session (details of recording are described later in this chapter, at section 3.4). Each speaker combination, therefore, produced six different comparisons as below:

2 within-speaker comparisons - Session 1 vs. 2 within a single speaker * 2 speakers 4 between-speaker comparisons - Speaker A's session 1 vs. Speaker B's session 1, Speaker A's session 2 vs. Speaker B's session 2, Speaker A's session 1 vs. Speaker B's session 2, Speaker A's session 2 vs. Speaker B's session 1 
This means that 90 within-speaker non-contemporaneous, different-session comparisons and 180 between-speaker comparisons can be made for the discrimination test. These numbers seem adequate for discussing the performance of the parameters.

Furthermore, this study uses a homogeneous population, discussed in the following section (3.2.2.2). 11 and 13 speakers are certainly not enough to obtain a picture of between- and within-speaker variation of the general population. The ways for speakers to vary from each other are not infinite, however, and variations among a homogeneous population are assumed to be narrower than for the general population. If what to be investigated is between-speaker variation in a particular group of people who share a reasonably homogeneous speech style, the sampling size of this study may be just enough to produce a general picture of this particular population. Ultimately, of course, the only way to check this is by comparison with a homogeneous sample of greater size. It is not clear whether such a sample of forensic realistic Japanese speech exists. In the next section, informants' personalia are described in greater detail.

\subsubsection{Personalia of informants}

The informants of this study are young male native Japanese speakers with a relatively homogeneous social background.

Male speakers were chosen over females for this study considering the fact that males feature more frequently in practical forensic analysis. It is far more common for bomb threats or abductions, indeed to crime as a whole be committed by males than by females.

The informants are all young and educated. They were all studying at the Australian National University at the time of the recordings, and their ages ranged from $21-36$ years. Neither of them has audible speech defects. 
The ages and the dialectal backgrounds of the informants are summarized in Table 3.1. The far left column identifies individuals. In the column headed "Past Residence", the places where the speakers had lived in the past are presented. The numbers in the parentheses show the ages of the informants when they left each place. Informant MN was, for example, 24 years old at the time of the recording. He had lived in Kobe until he became 10 years old, then he moved to Himeji. He left Himeji for Osaka when he was 18 and, finally, he came to Australia at the age of 23 .

\begin{tabular}{|c|l|l|}
\hline Speaker & Age & \multicolumn{1}{|c|}{ Past Residence } \\
\hline AA & 27 & Tokyo (25) - Australia \\
\hline HA & 30 & Tokyo (30) - Australia \\
\hline JN & 28 & Tokyo (27) - Australia \\
\hline KA & 22 & Chiba (3) - Switzerland (5) - Saudi Arabia (8) - Kanagawa (14) - Malaysia (17) - Australia \\
\hline KF & 28 & Yokohama (25) - Australia \\
\hline KH & 23 & Tottori (18) - Yokohama (22) - Australia \\
\hline KO & 21 & Fukuoka (2) - Osaka (20) - Australia \\
\hline MM & 26 & Kyoto (15) - Tokyo (23) - Osaka (28) - Australia \\
\hline MN & 24 & Kobe (10) - Himeji (18) - Osaka (23) - Australia \\
\hline MO & 28 & Nara (18) - Osaka (20) - Australia \\
\hline TN & 34 & Yamanashi (18) - Tokyo (33) - Australia \\
\hline TS & 26 & Shizuoka (18) - Australia \\
\hline TY & 33 & Tokyo (30) - Australia \\
\hline YH & 36 & $\begin{array}{l}\text { Okinawa (8) - Nagoya (26) - America (28) - Tokyo (31) - Mexico - (33) - Nagoya (36) - } \\
\text { Australia }\end{array}$ \\
\hline
\end{tabular}

Table 3.1 Informants' personalia

The informants come from areas where different dialects of Japanese are spoken, as seen in Table 3.1 above. They are, however, judged as competent Standard Japanese speakers by the author. The fact that they performed tasks and conversation with the author in standard Japanese even without being requested to do so also clearly indicates that standard Japanese exists as a part of their language system as well as their regional dialects. An auditory analysis prior to the measurements showed that none of the speakers stands out in terms of their voice quality or vowel and consonantal qualities, although there is detectable phonetic influence of their dialect in their pitch pattern of words, particularly in Speakers MN and MO. These are Kansai dialect speakers, who are known to more persistently retain their speech styles or prosody in Standard Japanese. 


\subsection{PARAMETERS FOR SPEAKER IDENTIFICATION 3.3.1 CRITERIA FOR SPEAKER RECOGNITION PARAMETERS}

This section discusses the selection of parameters for forensic speaker identification. As described in Chapter 1, the human voice consists of many components. The number of acoustic features that may contribute to individual characteristics is almost countless. When the practical application of speaker identification is considered, however, severe limitations are imposed on the selection of potential parameters.

The criteria for establishing the speaker identification parameters need to be practical. Previous studies such as Wolf (1972:2044) and Nolan (1983:13) summarise the necessary criteria for speaker identification parameters. These proposed criteria are applicable to both forensic and non-forensic speaker recognition applications. The degree of restriction imposed by these criteria, however, differs greatly between the two types of application. Nolan's list of the criteria for parameters for speaker identification includes the following:
1) High between-speaker variability
2) Low within-speaker variability
3) Resistance to attempted disguise or mimicry
4) Availability
5) Robustness
6) Measurability

Nolan lists high between-speaker variability and low within-speaker variability as the first and the second criteria for the selection of parameters. These are essential criteria for parameters for speaker identification and discrimination to be possible. Speaker identification can only work when between-speaker variation is larger than within-speaker variation. The variation which a single speaker exhibits must be smaller than the difference between two different speakers. Unless this is the case, the parameter cannot discriminate between speakers. On the 
other hand, if within-speaker variation is small and the between-speaker variation is large, classification of speakers becomes easier and more reliable.

Nolan's third criterion is resistance against disguise or mimicry. Disguise is mainly found in forensic speaker identification, whereas mimicry can also occur in other areas of speaker recognition, such as voice recognition security systems. In forensic situations, criminal speech often involves voice disguise, when criminals are aware of the possibilities of being recorded, such as in bomb threats or ransom demands over the telephone. Although no parameter is known to be completely resistant against disguise, some are more resistant than others. Parameters which are very susceptible to disguise will not, therefore, be of much use in some forensic phonetic investigations.

The fourth and the fifth criteria, "availability" and "robustness' respectively, are of much more serious concern to forensic phonetics than other applications of speaker recognition. It is impossible to exercise any control over incriminating speech, with respect to both amount and content. Very occasionally it may be possible to exert some control over a suspect's speech, perhaps by requesting the suspect to say certain phrases, but even this amount of control is, however, not always available or advisable, as the difference between natural and spontaneous speech can affect the size of within-speaker variation (Kinoshita 1998). The acoustic parameters analysed in forensic investigations must therefore occur frequently in normal speech, so that it is likely that they will be found in sufficient quantity in a limited speech sample.

The parameters must also be sufficiently robust so as to be extracted from a sample of medium recording quality. Speech recordings in forensic phonetic investigations often contain background noise and are often recorded with poor sound quality recording equipment and/or over telephone lines with compromised transmission. Acoustic parameters for use in forensic 
speaker identification must be sufficiently robust to be resistant to these unfavourable conditions.

The final criterion listed, measurability, is a crucial condition for objective analysis of speech samples. Whilst there may be many acoustic features that characterise an individual, the use of these features in forensic speaker identification is restricted significantly unless they can be measured and statistically evaluated. The prime purpose of forensic speaker identification is to provide a court with an objective evaluation of given speech samples. Since the results of forensic phonetic analysis can significantly influence the lives of the people involved in such cases, the importance of objectivity in analyses and presentation of the results is high. Acoustic measurements are one of the ways to increase this objectivity. Complete objectivity can never be achieved, however, even with acoustic measurements, since human judgments are required at many stages of the acoustic analysis, as discussed in Chapter 1. Despite this, acoustic analysis based on careful measurement and statistical quantification remains the best method available to phoneticians. This technique is also absolutely necessary in forensic phonetics.

In addition to Nolan's criteria, there is another parameter which needs to be taken into consideration. That is independence between parameters, where independence means that the parameters are maximally uncorrelated. For instance, there is no point in comparing incriminating speech and suspect's speech at, say, $F 2$ of the middle point of $/ \mathrm{V} /$ and $F 2$ of the same vowel at the point $10 \mathrm{~ms}$ later and treat them as separate comparisons. F2 measured at these two points cannot be treated as two separate parameters, as the $F 2$ values at these two points are obviously very strongly correlated. The analyses based on the two strongly correlated measuring points should be considered as one piece of evidence, not two. When multiple parameters are incorporated for estimation of the probability, those parameters should not be correlated to each other in order to produce valid estimation. 
In fact, it is a sensible question to ask whether there are any acoustic parameters, except perhaps for some temporal factors, which are not related. After all, all acoustic parameters produced by a speaker are produced by a single vocal tract. It seems, however, that all acoustic parameters are not necessarily strongly correlated. Examination of the correlation among acoustic parameters within a speaker shows that not all acoustic parameters have a strong correlation with each other. This can be quickly illustrated by how much correlation one of the typical informants of this study (Speaker HA) showed in seven measuring points. The correlations between parameters are presented in Table 3.2, in the form of the Pearson's rvalue and degree of freedom (in parentheses) for each combination of sampling points. Values which reached a $95 \%$ level of significance are shown with shading. The labels $\mathrm{iF} 2, \mathrm{eF} 2, \mathrm{eF}$, $\mathrm{eF} 2$, o1 mid, $\mathrm{m} 2$, and s2 indicate F2 of the $/ \mathrm{V} /$ vowel, $\mathrm{F} 2$ of the /e/ vowel, F3 of the /e/ vowel, effective $\mathrm{F} 2$ of the /e/ vowel, $\mathrm{F} 3$ of the first /o/ in the word moshimoshi, F3 of the second $/ \mathrm{m} /$ in the word moshimoshi, and F3 of the first /s/ in the word moshimoshi, respectively.

\begin{tabular}{|c|c|c|c|c|c|c|c|c|c|c|c|}
\hline & $i F 2$ & & $e F 2$ & & $e F 3$ & & $e F 2$ & & olmid & $m 2$ & $s 2$ \\
\hline $\mathrm{iF} 2$ & 1 & & & & & & & & & & \\
\hline $\mathrm{eF} 2$ & 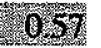 & 19 & 1 & & & & & & & & \\
\hline $\mathrm{eF3}$ & 0.01 & 18 & 0.32 & 19 & 1 & & & & & & \\
\hline $\mathrm{eF} 2$ & 0.52 & 18 & 099 & 19 & 0.43 & 19 & 1 & & & & \\
\hline olmid & -0.16 & 14 & -0.1 & 14 & -0.39 & 13 & -0.13 & 13 & 1 & & \\
\hline $\mathrm{m} 2$ & 0.14 & 14 & -0.15 & 14 & 0.33 & 13 & -0.1 & 13 & $0.05 \quad 14$ & 1 & \\
\hline $\mathrm{s} 2$ & 0.3 & 13 & 0.35 & 13 & -0.05 & 12 & 0.43 & 12 & $0.06 \quad 13$ & $-0.24 \quad 13$ & 1 \\
\hline
\end{tabular}

Table 3.2 Correlations between acoustic parameters for a single speaker (HA).

The table shows that it is clearly not the case that the majority of acoustic parameters are strongly correlated. Out of 21 pairs of comparison, only three parameter pairs were found to be significantly correlated. It should thus be possible to select relatively independent parameters for forensic speaker identification.

The criteria for parameter selection for speaker identification have been discussed in detail and the importance of each criterion established. The question before us now is whether or not parameters that fulfil all these criteria exist in reality. The answer is "No", at least to our 
knowledge today. Since perfect parameters either do not exist or are yet to be discovered, we must look for promising parameters which produce small within-speaker variation and large between-speaker variation and also meet some of these criteria and attempt to combine the results of their analysis. The possible acoustic parameters are discussed in a later section of this chapter (see 3.3.3).

\subsubsection{TYPES OF PARAMETERS}

Because of the complex nature of human speech, there are many ways in which speakers can differ. Rose (MS: 4) proposes three features by which the parameters for speaker identification can be categorised.

\subsubsection{Acoustic/ auditory}

Firstly, speaker identification parameters can be classified depending on whether they are acoustic or auditory. Both are equally important parameters in forensic speaker identification, as the characteristics which can be easily detected by analysing one type of parameter may not be evident in the other types of parameter. For instance, whether or not a speaker is in stress is much more easily detected by auditory analysis, but not by acoustic analysis. On the other hand, characteristics in formant structures, such as a singer's resonance, are observed easily by acoustic analysis, but not by auditory examination. In addition, auditory analyses must always be carried out prior to any acoustic analysis in order to find out whether or not the given two recordings are really comparable and, if they are, what features are to be compared. 


\subsubsection{Linguistic / non-linguistic}

Types of parameters can also be categorised on the basis of whether or not the parameter is a linguistic parameter. In forensic speaker identification, the auditory analyses of the linguistic features can be strongly diagnostic. For instance, if two samples differ in the pronunciation of the word 'either', as in [i:ða] in one recording and [aiða] in the other, the chances may be increased that the sample comes from a different individual.

Non-linguistic characteristics are, on the other hand, the features which do not contribute to the linguistic meaning of the speech, such as overall level of F0. High or low F0 of voices can characterises a speaker, but this overall FO does not affect the linguistic meaning of utterances. It should be noted that linguistic and non-linguistic parameters can be analysed not only by auditory observation, but also by acoustic analysis. Acoustic analysis will usually quantify the auditory observation of these parameters.

\subsubsection{Discrete / continuous}

The final aspect to categorise speaker identification parameters is whether the parameter is discrete or continuous. Continuous parameters are, as Rose (MS: 4) defines, "parameters on which values can be expressed in terms of any number and quantified to any degree of precision (although often within a particular range)." Usually, acoustic parameters are continuous. Frequencies of $\mathrm{FO}$ or formants, for example, can be measured in a continuous scale.

Discrete parameters are, on the other hand, expressed only by a fixed number of values. This fixed number can be very small. If one would like to quantify the realisation of a certain phoneme, for instance, the observation at each sample can be quantified in terms of the 
incidence of the feature. The incidence of a certain phoneme can be quantified by counting how many times the phoneme in question appears. Auditory parameters are usually discrete.

\subsubsection{Interaction between categories}

Now, three different dichotomies of parameter types have been discussed. As shown in the course of the introduction of each aspect, those three aspects are not mutually exclusive. Speaker identification parameters need all three aspects to be categorised. For instance, if someone is characterised by a particularly high-pitched voice, a non-linguistic parameter is being used. This is also a discrete auditory parameter. On the other hand, when this person's high pitched voice can be quantified in the form of $\mathrm{FO}$ through acoustic analysis, this parameter then becomes a non-linguistic acoustic continuous parameter. One phonetic event can thus belong to multiple categories.

\subsubsection{COMMONLY USED ACOUSTIC PARAMETERS IN FORENSIC SPEAKER IDENTIFICATION}

\subsubsection{Formants}

Although forensic and automatic speaker identifications have the same criteria for parameters, these two applications of speaker identification usually employ different parameters. Automatic speaker identification often uses parameters such as cepstrum or long-term spectra. In forensic speaker identification, these parameters are very rarely used. Instead, formant frequency and long-term average FO are two favoured parameters.

It is well known that formant structures contain a considerable amount of information of a speaker's identity. Formants are, in fact, one of the most widely used parameters in forensic speaker identification (e.g. Greisbach et al. 1995, Jessen 1997, Nolan 1983, Rose 1999a; 1999b). The formant pattern is the acoustic correlate of the supralaryngeal vocal tract 
configuration. The size and shape of the supralaryngeal vocal tract are determined by two factors: the phonetic targets of segments being produced, and the speaker's anatomical features. Differences in formant patterns of phonologically identical segments are considered to mainly reflect speakers' anatomical differences, although it is known that coarticulation can also affect the acoustic quality of segments. Formant pattems are thus one possible parameter for speaker identification.

Centre frequencies of formants are actually one of the most typically measured parameters in real forensic cases (Rose 1999b:4). There are other parameters which are known to be more powerful to distinguish speakers among the researchers of automatic speaker identification. For example, cepstrum. The use of cepstrum has been restricted to use in automatic speaker identification. It is only recently that the possibility of the use of the cepstrum in forensic speaker identification has been investigated (Mewuley and Drigajo 2001; Nakasone and Beck 2001; Rose and Clermont 2001). Rose and Clermont, for example, report that a cepstrumbased parameter (band-selective cepstral distance) performs better than formants in the discrimination of randomly selected similar sounding speech samples. Although the difference in performance level shown in their study is slight, they correctly emphasise the potential of cepstrum in forensic situations, pointing out that cepstrum is obtained relatively more easily than formants (for formants, the identification of the location is sometimes very difficult).

Despite the preference for the cepstrum over formants in automatic speaker recognition, in which higher rates of successful speaker discrimination one found, there are two reasons why formant pattern is a preferred parameter in forensic situations. Firstly, formant patterns (or at least the lowest three formants) can be interpreted in relation to auditory features relatively easily. Since auditory analyses are indispensable in both the evaluation of the comparability of samples and the selection of segments for comparison in forensic phonetics, this is a very important consideration. An analysis of formant pattems enables us to quantify and discuss the results of the auditory analysis acoustically, whereas it is much more difficult to relate the 
automatic recognition parameters to auditory impression. Although Clermont and Itahashi (2000) report that it is possible to relate the second and third Mel-cepstrum coefficients to the articulatory features, front or back, high or low, respectively, to a certain extent, formant patterns are still better than the cepstrum in the interpretation relating to the auditory analysis.

The other argument in favour of formants is that parameters for automatic recognition are based on mathematically very much more complex notions than formant patterns, making them much more difficult for juries and / or judges to understand. In forensic situations, the results of an analysis must be comprehensible by juries and / or judges, who are unlikely to have a phonetics background. The comprehensibility of the results should not be neglected in forensic speaker identification.

Provided they can be identified, formants are also relatively easily measurable. Formants can be measured using standard speech analysis packages which are readily available to phoneticians and no additional special tools are required. Considering the potential application to forensic investigation, this is an important advantage, especially since it is phoneticians who are usually engaged in forensic speaker identification, although with sorne segments identifying an accurate location of formants can be very difficult.

\subsubsection{Long term F0}

Long term $\mathrm{FO}$ is another acclaimed parameter for speaker identification. One of the early studies on this parameter is Atal's work (Atal 1972). In this research, a $97 \%$ successful identification rate is reported using the long term $\mathrm{FO}$ as a parameter. It is unlikely that this high success rate would be replicated in real forensic situations, however. This is firstly because Atal used a text reading method for data collection. This means that speech samples in Atal's study were much more comparable to each other than the incriminating and suspect speech 
samples in real life forensic situations would be. Moreover, the sentence used in his experiment was a very unusual one: "May we all learn a yellow lion roar." This sentence was composed to avoid any voiceless segments, so that F0 could be tracked continuously. The meaning of this sentence is, however, very obscure and there is no way that speakers can utter this sentence naturally. It is unclear how this unnatural sentence affected the size of withinand between-speaker variation. The fact that Atal used read-out speech predicts within-speaker variation in his study to be smaller, resulting in a higher successful identification rate than reality. It is, however, also possible that this unnatural sentence induced more within* and between-speaker variation as the speakers were not sure about the meaning of sentence. If that was the case, the speaker identification with long-term F0 can perform even better than $97 \%$. In any case, it is undoubtedly difficult to compare the average F0 in Atal's data and that of a realistic situation.

There are other, more realistic studies, however, which also report favourably on long term F0 as a speaker identification parameter. For instance, Sambur (1975) testifies that long term F0 is quite effective in classifying speakers into three categories; speakers with lower F0 (around $100 \mathrm{~Hz}$ ), those with a typical range of $\mathrm{F} 0$ (around $125 \mathrm{~Hz}$ ), and those with a relatively high range of F0 (around $160 \mathrm{~Hz}$ ). Although the F0 of a single speaker can vary radically across sessions, Sambur says, speakers rarely move from one group to another.

Jiang (1996) also investigated Fo based speaker identification, using three different tools, namely FFI (Fundamental Frequency Indicator), CSL, and cepstrum, to extract FO. In this experiment, Jiang tested the performance of $\mathrm{F} 0$ as a speaker identification parameter, using the Euclidian distance. The test set included ten foils and three speech samples made by a single target speaker. The target speaker's three samples were named as U1 (unknown 1), U2 (unknown 2), and $\mathrm{K}$ (known). He calculated Euclidian distance between U1 against each of $\mathrm{U} 2, \mathrm{~K}$ and the ten foils, in terms of their mean F0. Then he normalised the distances so that the minimal score was 1 and the maximum was 10 . The speaker is considered to be successfully 
identified, when the distance between $\mathrm{U} 1$ and $\mathrm{U} 2$ and between $\mathrm{U} 1$ and $\mathrm{K}$ are either 1 or the next smallest to one among the 12 Euclidean distances calculated. He also rotated the role to which the samples were assigned, so that in the sample which was $\mathrm{U} 1$ became $\mathrm{U} 2$, the sample which was $\mathrm{U} 2$ became $\mathrm{K}$, and the sample which was $\mathrm{K}$ became $\mathrm{U} 1$, and he carried out the same test again. Then he rotated the samples again, and tested as described. Jiang repeated the whole process using 25 different speakers as the target speakers. He then calculated the successful identification rate, examining whether or not the distance between the withinspeaker variations is smaller than the between-speaker variation (ie. the distance between $\mathrm{U} 1$ and $\mathrm{U} 2$ and between $\mathrm{U} 1$ and $\mathrm{K}$ is smaller than the distance between $\mathrm{U} 1$ and the 10 foils). $\mathrm{He}$ also averaged the results for the each speaker. As result, he found out that the speaker could be correctly identified at $80 \%, 76 \%$, and $90 \%$ success rate for high quality, noise-added and telephone bandpassed recordings, respectively, when FFI for F0 extraction was used. Although the experiments were based not on spontaneously uttered speech, but on 25 second speech samples trimmed from one-minute passage readings, this is nevertheless a good indication of the potential of long-term fundamental frequency as a cue for speaker identification.

There are some situations, however, where F0 cannot be used as a parameter. F0 is highly susceptible to circumstances in which a speaker is at the time of recording. The speaker's emotional state, intoxication due to drug or alcohol use, whether or not the speaker is on the phone, and background noise, can all affect FO severely (French 1994; Hirson et al. 1995; Maekawa 1998; Watanabe 1998). Boss (1996)'s comparison between F0s measured using real forensic data provides a good example. The incriminating recording was made from a security camera which taped a hold up at a petrol station, whilst the compared recording was from an interview at a police station. The speaker's average F0 measured from the recording at the crime scene was as high as $228 \mathrm{~Hz}$, whereas the average F0 in the police interview was $140 \mathrm{~Hz}$. The speakers of the two recordings were found to be the same individual from other pieces of evidence, such as self-confession as well as the same clothes and submachine gun used in the crime found in his apartment. The $88 \mathrm{~Hz}$ difference in average $F O$ is very large for a single 
speaker. Had these two F0 measurements been made from two comparable datasets, in which the speakers of both recordings are in the similar emotional state and speaking under similar circumstances, it would have strongly suggested that these speakers were two different individuals. The enormous $88 \mathrm{~Hz}$ difference in the long term F0 in this case, however, was caused by the obvious differences in the circumstances under which the recordings were made, including the speaker's emotional states, how noisy the surroundings were, who he was talking to, and so on.

Elliott (2000) also investigated that F0 difference between shouted and natural speech, using two general Australian English speakers. As a result, she found that these two speakers' F0 values were increased by $31 \%$ and $39 \%$ respectively, when the speakers shouted, compared to speaking normally. The F0 difference between the two speech styles was also found to be statistically significant.

In the application of long term F0 as a parameter, then, as with all other parameters, a careful examination of the comparability between data sets with respect to the criteria described above is necessary.

\subsubsection{PARAMETERS IN THIS RESEARCH}

Since they are one of the commonly used parameters in forensic phonetics, the acoustic analysis of this research focuses on formant patterns. Two different types of formant measurements are made in this research. One is the formant pattern of the same vowels which occurred in phonological contexts, such as /o/ in kino'shita 'Kinoshita (personal name)' and tosho'kan 'library.' The other is that of segments which are in the same phonological context by virtue of occurring in the same word, moshimoshi 'hello' (as in an opening phrase of telephone conversations). Both these types are common in forensic phonetic investigation. 


\subsubsection{Vowels in different phonological environments}

The first type of measurement, the measurement of the same vowels embedded in different phonological contexts, is attractive, particularly in forensic speaker identification. A wider choice of segments is available for measurements if the target segments are not restricted to occur in identical environments. On the other hand, it is a truism that vowels which are phonologically the same may not necessarily be phonetically equivalent (Broad 1976). Comparability of two vowels is phonologically at its best when the words in which the vowels are embedded are segmentally and prosodically identical, and a segment occurs in the same position in repetitions of the same word. These conditions are optimal for determining the nature of within- and between-speaker variation (Rose 1998:4). As mentioned in Chapter 1, forensic speaker identification, however, often suffers from a shortage of data for comparison. It is very likely that a sufficiently large number of suitable words for comparison is not available in both the incriminating speech and suspect's speech. In such circumstances, vowels in different phonological contexts but in the same prosodic positions are the next best choice for comparison. Although exactly the same words may not be found, it is still highly likely that those datasets contain at least the same vowels in comparable positions.

Actually, a research on five Japanese vowels has already been done with this assumption. Suzuki et al. (1994) investigated the performance of Japanese vowels in speaker verification using 523 male speakers, and report that $/ \mathrm{i} /$ and $/ \mathrm{e} /$ are the two vowels which produce smaller within-speaker variation and larger between-speaker variation. Their study is, however, based on LPC Cepstrum but not formants, so that the current research, which is based on centre formants frequencies, may produce different results. Furthermore, and more importantly, although 523 speakers sound very impressive, their experimental condition was forensically unrealistic. The vowels used in Suzuki et al.'s study were all produced in isolation. The importance of the use of natural speech in forensic phonetic research has already been discussed in Chapter 1. The investigation conducted here in this research is thus important, 
although it is still of interest to see whether or not the results turned out to be consistent with Suzuki et al.'s study.

Although the formant patterns of vowels embedded in different words seem to be a worthwhile parameter for investigation, there are obvious weaknesses as well. The most significant weakness is coarticulation. It is a well known fact that segments are coarticulated with their surrounding phonological environment, because of the continuous nature of speech production (Farnetani 1997). For instance, /a/ in soba'ya 'noodle shop' may become more front because of anticipation of the following palatal glide [i], and the same /a/ in sa'kata 'Sakata (personal name)' may become more back due to the coarticulation with the following velar consonant [k]. Coarticulation thus can introduce extra factors unrelated to the speakers' identity and, consequently, increase within-speaker variation. The larger ratio of within-speaker variation against between-speaker variation makes speaker identification more difficult, as seen by the fact that a small within-speaker variation and large between-speaker variation is part of the criteria for speaker identification parameters (see 3.3.1). This is, however, inevitable with this type of realistic comparison. The important thing is to find out to what extent it impairs speaker recognition.

It is also true that coarticulation does not necessarily only have unwanted effects on speaker identification. This is seen in Nolan (1983), where he shows that there is a potential for coarticulation (between $N /$ and the following vowel in British English in Nolan's research) to serve as a speaker identification parameter.

Nevertheless investigation on whether or not vowel formants can be useful for speaker identification regardless of their phonological environments will be highly beneficial in the advance of forensic phonetic research. The F-patterns of vowels in different contexts are thus researched and discussed extensively in Chapter 4. 


\subsubsection{Segments in the same word, moshimoshi}

Although the acoustic features that can be extracted regardless of context are useful, the results of analysis will be undoubtedly more reliable if the samples can be compared in a more controlled situation, such as in excerpts from the same word. This research utilises the phrase, moshimoshi, as the target word for analysis. The direct translation of this phrase is "hello", but it is used specifically in Japanese telephone conversations, where it is the most commonly used phrase to open a telephone conversation regardless of the speaker's gender, age or social status. Since a large proportion of forensic speaker identification cases involve telephone conversations (over 90\%, according to Hirson et al. (1995:230)), the availability of this particular phrase in forensic speech samples is expected to be reasonably high.

The expected high incidence is not the only advantage that this phrase offers. The segments included in this phrase, namely [m], [G], and [i], are known to contain a large amount of speaker specific information. The nasal consonants and the fricatives are, in particular, known to contain a large amount of information regarding the speaker's anatomical differences (Johnson 1997:122, 157).

Nasals are often claimed to be useful segments in forensic investigation. Although it is possible to modify a nasal apertures by shifting the position of velum or flaring the nostrils, it is impossible to modify the shape of the nasal cavity with a conscious effort. The flexibility of the shape of nasal cavity is, therefore, very limited. This means that the acoustic properties of nasals reflect an individual's anatomy more directly than oral segments (Nolan 1997; Stevens 1971). Glenn and Kleiner (1968), for instance, report that the power spectra of nasals revealed a $93 \%$ successful identification rate for 30 speakers. As identified already, this high accuracy will not be replicated in real forensic phonetic situations, as this experiment was based on data collected by card reading. Accuracy as high as $93 \%$, however, still seems very high. 
It has to be noted here that the acoustic properties of nasals can be severely affected by the condition of the speaker's health. If a speaker had a blocked nose due to a cold or hay fever, for instance, it is not clear that their nasals would be of any use for identification. Nevertheless, so long as the case does not involve speakers who are in poor health, nasals provide us with useful clues regarding the speaker's anatomical characteristics. Nasals should therefore definitely be closely analysed in the research of speaker identification.

The bandwidth of nasal consonants is usually greater than that of oral segments. This is due to a heavier acoustic damping, as the nasal cavity has a larger, more absorbent surface area. The formant above this lowest formant is usually produced by the main system: nasal tube from nostrils to glottis. Side cavities also play an important role in determining formant patterns of nasal consonants. In the case of $/ \mathrm{m} /$, it is the oral cavity, which extends from the closed lips to the velopharyngeal port, which functions as a side branch resonator. The resonances produced at this sub branch system become anti-resonances, and absorb the acoustic energy nearby. The other side branching systems, sinuses, also create an antiformant. The frequency of this antiformant is determined by the size of sinus cavities, and the size and the openings into them vary considerably from individual to individual, making nasals good segments for speaker identification. In addition to the absorption of nearby energy, an antiformant also has the effect of reducing the amplitudes of the formants in its vicinity. Consequently the acoustic energy of nasal segments is concentrated in the low frequency region (Johnson 1997:151). Thus nasals are not likely to provide much information on their higher formants.

The spectral shape of the palatoalveolar fricative consonant [ [] is determined mainly by the anatomical details of the regions around and forward of the alveolar ridge (Wolf 1971:2050). Even relatively minor individual differences, such as differences in dentition, can affect anterior tongue placement and fricative airflow in front oral fricatives (Beck 1997:257). This consonant is thus also expected to be a powerful parameter for speaker identification. The first formant of palatoalveolar voiceless fricatives is a Helmholtz resonance, although they usually 
do not have acoustic energy enough to be visible in spectrograms. The second formant frequency of this consonant is determined by the half-wavelength resonance of the back cavity (Stevens 1998: 406). However, the phonetic realisation of Japanese /si/ is alveo-palatal [6i] and not palato-alveolar [ji]. Ladefoged and Maddieson (1996:164) distinguish alveo-palatal [6] from palato-alveolar [S] by the fact that the part of the tongue immediately behind the constriction is raised more for [G] than that of the []]. The description above is thus applicable in Japanese, except that constriction area of [६] is a little longer than, say, English [f], resulting in slightly shorter back cavity. The second formant for this consonant is thus expected to be somewhat higher than the typical F2 for [f], $1900 \mathrm{~Hz}$.

The high vowel [i] has also been reported to be one of the vowels which have a strong tendency to be differentiated between speakers. Sambur (1975) reports, through experiments on American English speakers, that the F2 of front vowels, as well as the F3 of back vowels, reflect a speaker f identity well. Mokhtari and Clermont (1996) also report the strong speaker discriminating power of the F2 of front vowels and the F3 of back vowels. [i], therefore, seems to be a worthwhile vowel for investigation. The formant structures of the phrase moshimoshi will be studied in Chapter 5 .

\subsubsection{BETWEEN-LANGUAGES DIFFERENCES IN FORENSIC SPEAKER IDENTIFICATION}

Except for automatic speaker recognition, none of the research on forensic speaker identification parameters deals with Japanese. Japanese has a considerably different phonology from the languages on which the majority of the research in this area is based, such as English and German. (Because of this, a detailed discussion of the Japanese sound system has been given in Chapter 2). Although it is not unthinkable that forensic speaker identification is under the influence of language-to-language differences in their phonology or 
linguistic system, no discussion has been made on the potential of language specific characteristics in forensic speaker identification. In addition to the main research question, relating to which formants work, and how well, to distinguish speakers in Japanese, this study will also briefly investigate whether there are any language specific characteristics in the realisation of within- and between-speaker variation.

Between-language differences can be realised in two different ways. First of all, different languages may produce different results for the same or similar parameters. For instance, a certain vowel formant which is a very powerful cue for the speaker's identity in one language may not perform as well in other languages, possibly due to the differences in their phonology. Secondly, the parameters which are available to the languages can also vary from language to language. A phonological feature of one language may function as a powerful speaker identification cue, but the cue may not be available in other languages, as their phonological systems do not include the particular phonological feature. The realisation of Japanese vowel devoicing is one of these examples. Broadly speaking, vowel devoicing in Japanese is a phenomenon in which high vowels are devoiced when they do not have any adjacent voiced segments. It is known that there are regional variations in the realisation of vowel devoicing in Japanese. It is therefore possible that this vowel devoicing can provide a linguistic parameter for speaker identification, with respect to the speaker's place of origin. If this is found to be the case, the realisation of vowel devoicing is recognised as a speaker identification parameter which is specific to Japanese. In both Chapters 4 and 5 the realisation of devoicing is examined in detail.

As has been described in Chapter 2, Japanese has a pitch accentuation system. Local F0 may be another promising parameter for speaker identification in Japanese (local F0 here means the F0 value for a short period of time, as opposed to long term F0). As pointed out by Rose (1996:308), the variability of F0 is much more easily quantified in languages which have specific targets for the F0 realisation. In languages which do not employ F0 to mark the lexical 
meanings of words, such as English, the realisation of F0 can vary almost indefinitely even within an utterance made by a single speaker. In those languages, it is almost impossible to establish the comparability between speech samples in terms of local F0. Japanese, on the other hand, phonologically marks only two types of pitch: high and low (for detail, see Chapter 2). Japanese phonology, thus, imposes some restriction on the realisation of local F0, as high and low pitches have to be distinguished by the listener to convey the lexical meanings of words.

Long term F0 may also serve more effectively as a speaker identification parameter in Japanese than, for instance, in tone languages, because of the phonological system of Japanese. In Japanese, speakers have more freedom to vary from each other in the acoustic realisation of pitch than in tone languages, which require more complex manipulation of pitch. In other words, Japanese phonology seems to impose some restriction on the realisation of F0, which makes F0 of words more easily comparable, allowing enough freedom for speakers to differ from each other. The variation in F0 associated with the placement of the accents may thus reflect individual characteristics, and if it does, it could be used as a speaker identification parameter, which is not available to many other languages.

In reality, however, there are many factors that affect F0 other than the characteristics of individual speakers, as has been discussed extensively in the previous chapter (Chapter 2). Speakers show a large amount of within-speaker variation in F0. To illustrate this, Table 3.3 presents F0 of ten accented /a/ vowels and their following vowels, together with the F0 difference between these two vowels, which indicates the size of the pitch fall associated with the pitch accent. In the table, the results from Speaker AA's two sessions and Speaker KF's first session are presented. 


\begin{tabular}{|r|r|r|r|r|r|r|r|r|r|}
\hline & \multicolumn{3}{|c|}{ AA's session 1 } & \multicolumn{3}{c|}{ AA's session 2 } & \multicolumn{3}{|c|}{ KF's session 1 } \\
\cline { 2 - 11 } & \multicolumn{1}{|c|}{ High } & \multicolumn{1}{|c|}{ Low } & \multicolumn{1}{|c|}{ H-L } & High & Low & H-L & High & Low & H-L \\
\hline 1 & 109 & 89 & 20 & 147 & 104 & 43 & 108 & 79 & 29 \\
\hline 2 & 148 & 107 & 41 & 193 & 96 & 97 & 174 & 130 & 44 \\
\hline 3 & 155 & 121 & 34 & 179 & 115 & 64 & 229 & 213 & 16 \\
\hline 4 & 134 & 104 & 30 & 151 & 124 & 27 & 156 & 98 & 58 \\
\hline 5 & 124 & 120 & 4 & 174 & 98 & 76 & 117 & 99 & 18 \\
\hline 6 & 115 & 101 & 14 & 157 & 110 & 47 & 105 & 90 & 15 \\
\hline 7 & 176 & 112 & 64 & 219 & 101 & 118 & 110 & 104 & 6 \\
\hline 8 & 155 & 125 & 30 & 198 & 129 & 69 & 152 & 137 & 15 \\
\hline 9 & 124 & 113 & 11 & 174 & 110 & 64 & 137 & 115 & 22 \\
\hline 10 & 144 & 126 & 18 & 190 & 119 & 71 & 138 & 116 & 22 \\
\hline mean & 138.4 & 111.8 & 26.6 & 178.2 & 110.6 & 67.6 & 142.6 & 118.1 & 24.5 \\
\hline sd. & 20.91 & 11.78 & 17.37 & 22.64 & 11.14 & 26.23 & 38.15 & 37.72 & 15.54 \\
\hline
\end{tabular}

Table 3.3 FO $(\mathrm{Hz})$ of accented $/ \mathrm{a} / \mathrm{s}$ and their following vowels, and the F0 difference between the two vowels. The data are collected from Speaker AA's two recording sessions and Speaker KF's first recording session.

Table 3.3 clearly demonstrates that the between-session difference of speaker AA is much larger than the difference between the data of Speaker AA's first recording session and Speaker KF's data. The means of Speaker AA's first and second recording sessions are $26.6 \mathrm{~Hz}$ and $67.6 \mathrm{~Hz}$ respectively, producing $41 \mathrm{~Hz}$ difference between them, whereas the mean obtained from the KF's first recording session is $24.5 \mathrm{~Hz}$, from which the mean of Speaker AA's first recording session differs only $2.1 \mathrm{~Hz}$.

The values of F0 for accented syllables are heavily influenced by the location of the syllables in sentences. A naturally uttered sentence generally reveals a gradual falling intonation, and catathesis is expected to occur. As has been demonstrated in Chapter 2, catathesis is the phonological phenomenon where the pitch range of the words following an accented word is automatically suppressed. The F0 peak of an accented syllable thus can vary depending on the relationship with the location of other accented words in the same sentence. In addition, the F0 of a single syllable is far more susceptible to the placement of focus than long-term F0. Focus is reported to cause the F0 range within a word to increase considerably (Maekawa 1997:46). Identifying comparable words is an extremely complicated task, making the establishment of comparability of short term F0 difficult, and obtaining sufficient reliable data to perform statistical analysis is undoubtedly even more difficult. Furthermore, the data presented here 
are collected under very similar conditions (for the detail on the recording, see 3.4 in this chapter). If other differences are caused by external conditions such as a speaker's psychological state, the comparison will be even more difficult. Although it appeared attractive at first glance, local $\mathrm{F} 0$ is thus probably not very useful.

Another phonological characteristic of Japanese which may be useful in profiling speakers is moraic nasals. As explained in Chapter 2, the mora is a duration based (or timing) unit and it is a part of the phonological competence of Japanese. Moraic nasals are nasal consonants that can stand as morae without being accompanied by vowels. As moraic nasals have a longer duration than normal nasal consonants, the determination of an individual's features, which nasals are known to be able to provide, may be achieved more easily. In addition, moraic nasals appear with high frequency of occurrence in spoken language. So they are another instance of potential language specific forensic phonetic features. Unfortunately, they could not be included in the investigation in this study, due to time constraints.

\subsection{RECORDING AND CORPUS 3.4.1 RECORDING}

\subsubsection{Non-contemporaneity of data}

Two recording sessions, separated by two weeks, were held for the collection of forensically realistic data for this study. In real life forensic phonetic investigation, a suspect's speech and incriminating speech are bound to be recorded on different occasions. There is thus always some time gap between recordings to be compared in forensic speaker identification. The suspect's speech is usually recorded after the arrest of the suspect, a few days to weeks after the crime. Rose (1999b) reports that within-speaker variation increases with time, comparing the within-speaker variation in the data recorded within a minute to the within-speaker variation in the data collected over at least a year. Given that speaker identification stems from the ratio of within- and between-speaker variation, and that small within-speaker variation and 
large between-speaker variation are the criteria for speaker identification parameters, the results of an experiment which deals only with speech recorded in one occasion are unrealistic and optimistic. Any study which aims to simulate a forensically realistic investigation must take this time factor into consideration. Now, the question is how long the recording session should be separated to produce forensically realistic data. The time separating criminal and suspect samples varies from case to case. In addition, the exact correlation between the size of within-speaker variation and the separation period between recording sessions is uncertain. It is thus impossible to determine what is the optimal separation between recording sessions for forensic phonetic research. The answer to this question may depend on many factors, and so a wide range of separation periods should be tested. This study, however, selected two weeks as one possible realistic separation time, as multiple separation periods could not be achieved due to the time constraints.

\subsubsection{Recording procedure}

The recording sessions were carried out in the sound proof recording studio at the Australian National University. Each recording session took approximately 20 minutes.

The recording procedure for this research consisted of four parts: a short informal conversation with the author, tasks; card reading; and questions on telephone conversation. The recordings made through tasks and questions on telephone conversation were included in the data for the current research. The short conversation was recorded in between the adjustment of the recording equipment and the formal tasks. This conversation was held to relax the informants and familiarise them with speaking in front of the microphone. The conversation was initiated by the author asking about recent incidents, the subjects' work or trips. The total duration and formality of the conversation varied from speaker to speaker. 
After this conversation, speakers were requested to perform tasks, the details of which are discussed in the following section. This task was followed by flash card reading. This was to investigate the effect of the difference between natural and read-out speech styles, which is another important forensic phonetic topic, since suspects are sometimes asked to read out materials. The recordings of this read-out speech are have not been included in this study, but are found in Kinoshita (1998), where it was shown that read-out speech produces smaller within-speaker variation than natural speech does.

Speakers were then requested to repeat the task and card reading again.

Finally, the opening phrase for a telephone conversation, mashimoshi 'hello,' was recorded. This phrase was elicited by the author asking the informants questions about how they would make a phone call to various people. This conversation was also recorded with microphone, without using a telephone.

The whole process of recording is summarised in Figure 3.1.

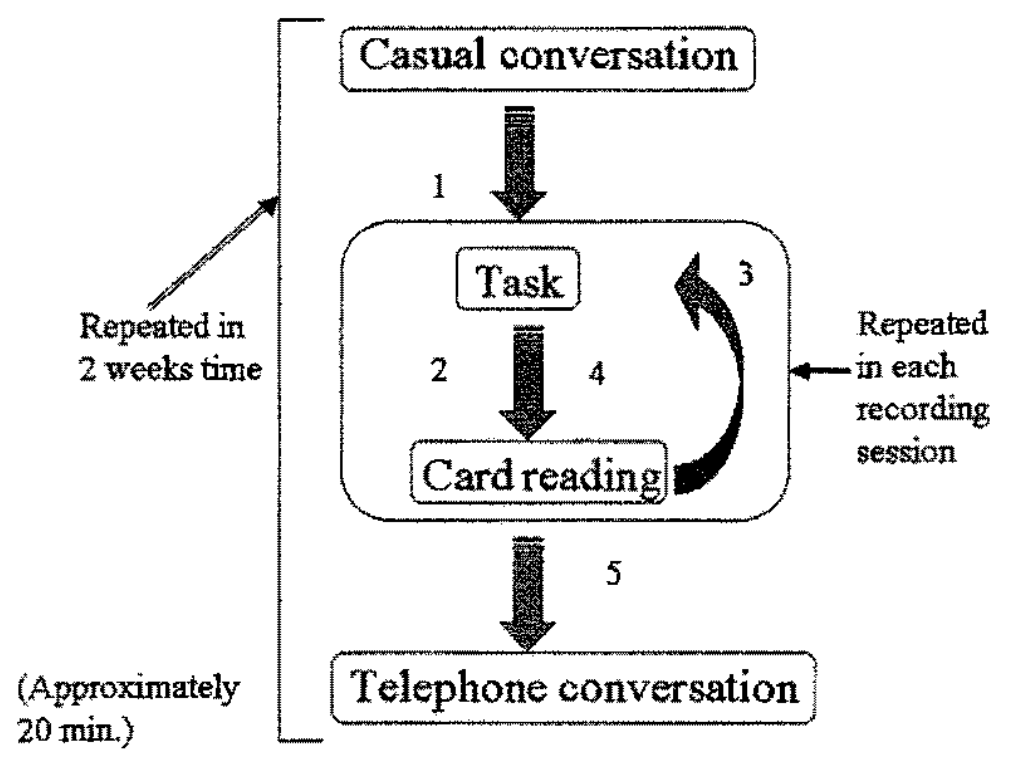

Figure 3.1 Summary of the recording process. 


\subsubsection{CORPUS}

The tasks in this study were designed to elicit natural speech. In the forensic situation, recordings from crime scenes are almost always natural speech, not read-out speech. It has been reported that natural speech has a larger within-speaker variation (Kinoshita 1998), as one might expect. Having natural speech samples as data is, therefore, one of the desiderata in forensic speaker identification experiments.

The informants were provided with a map and an information sheet. The map contained three bus routes and the names of shops and buildings. The information sheet consisted of four people's jobs, personality, and favourite foods. The informants were asked questions such as "Where does the route A bus stop?" or "What kind of job does person A do?" and were requested to answer the questions referring to the provided materials. For the map and information sheet, see the Appendix 3.1.

The corpus was constructed in order to elicit all five pitch-accented Japanese short vowel phonemes (with the exception of jibika 'otolaryngology' which is unaccented). The linguistic content of the corpus for each vowel contained five words in which the vowels occur at the accented position. The words used in the tasks are listed in Table 3.4, and the target vowels for measurements and analyses are underlined.

\begin{tabular}{|c|l|}
\hline Vowels & \multicolumn{1}{|c|}{ Words included in corpus } \\
\hline /a/ & $\begin{array}{l}\text { hanaya 'florist', panya 'bakery', sakata '(name)', sobaya 'noodle shop', } \\
\text { panyano 'of bakery' }\end{array}$ \\
\hline /i & $\begin{array}{l}\text { ïnja 'shrine', jibika 'otolaryngology', kobijutsu 'antique', } \\
\text { sushiya 'sushi bar', sanwaginkoo 'Sanwa bank' }\end{array}$ \\
\hline /w & $\begin{array}{l}\text { nikuya 'butcher', tokushima '(name)', kaguten 'furniture shop', } \\
\text { doobutsuen 'zoo', kurita '(name)' }\end{array}$ \\
\hline /e/ & $\begin{array}{l}\text { Nemoto '(name)', terebi 'TV', kitadeguchi 'north exit', } \\
\text { kitadeguchi 'north exit', minamideguchi 'south exit' }\end{array}$ \\
\hline /o/ & $\begin{array}{l}\text { Kinoshita '(name)', toshokan 'library', hoteru 'hotel', } \\
\text { honya 'book shop', toposu '(name of shop)' }\end{array}$ \\
\hline
\end{tabular}

Table 3.4 Corpus for map tasks. 
As shown above in Figure 3.1, informants were asked to repeat the tasks. Since each vowel occurred in five words, each speaker's dataset consists of ten tokens (five words * two repeats) in total for each vowel. Furthermore, two recording sessions were held for each speaker, so 20 tokens (five words * two repeats * two recording sessions) per vowel were elicited in this study.

To record the naturally occurring moshimoshi, the informants were asked the question, "What would you say when you call SOMEONE?" several times, altering SOMEONE in each scenario, such as the speaker's home, grandparents, a university lecturer in Japan, a close friend from the speaker's high school and so on. Examples of common openings for a telephone conversation are "Moshimoshi Tanaka san no otaku deshoo ka? (Hello, is that Tanaka's?)," or when answering the phone, "Hai, moshimoshi Satoo desu. (Hello, this is Sato's.)"

The hypothetical interlocutors vary in their closeness to the speaker and also in the level of formality that the speaker is expected to select. The variety in interlocutors was included in the question in order to force informants to pay attention to the choice of appropriate language depending on the type of interlocutor. The different interlocutors were chosen to obscure the intention of the question and avoid unnaturally clear pronunciation, as is often the case with text readings. This method was also found to be useful to induce more within-speaker variation making the data more realistic. Three to six samples of moshimoshi for each speaker were recorded in each recording session. The number of tokens which were actually recorded varied from speaker to speaker, because some speakers did not open their conversations with the phrase moshimoshi in some situations. Table 3.5 presents the numbers of moshimoshi recorded for each speaker.

\begin{tabular}{|c|c|c|c|c|c|c|c|c|c|c|c|c|c|}
\hline Speaker & AA & HA & JN & KA & KF & KO & MM & MN & MO & TN & TS & TY & YH \\
\hline Samples & 12 & 12 & 11 & 8 & 12 & 6 & 12 & 15 & 12 & 12 & 12 & 12 & 12 \\
\hline
\end{tabular}

Table 3.5 Numbers of tokens which were recorded for each speaker. 


\subsection{DIGITISING AND MEASUREMENTS 3.5.1 DIGITISING RECORDED SAMPLES}

Digitisation and measurements were carried out with CSL (Computer Speech Laboratory). The recordings were all digitised at a sampling rate of $16 \mathrm{kHz}$. This sampling rate was selected on the basis of the study by Rose and Simmons (1996), which reports that, in the use of CSL, the lowest acceptable order for adequate performance of the LPC (Linear Predictive Coding) analysis is 20. As the filter order is supposed to be $4 \mathrm{kHz}$ higher than sampling rate, $16 \mathrm{kHz}$ seemed the minimal sampling rate for adequate formant tracking with this software, (at the risk of introducing some spurious peaks with the higher filter order). The digitised speech samples were examined in the form of audio waveforms, and then the words which included target vowels were extracted and stored in separate files.

\subsubsection{VOWELS IN DIFFERENT CONTEXTS (CHAPTER 4)}

The first four formants of the five short accented vowels were measured at the midpoint of the vowel duration. Both the onset and offset of the vowels' F-patterns are expected to be directly influenced by adjacent segments. It was assumed that the middle point would be least affected by the adjacent segments Ladefoged and Maddieson (1996:287).

It is true that some vowel formants may not achieve their frequency height at the mid-point of their duration. This study, however, included five different words for each vowel, and it was extremely difficult to establish the most comparable points across speakers, word by word. The midpoint of the vowel duration was thus chosen, as it seems a relatively stable point across different words, although identifying the middle point was sometimes difficult, as there are of course no acoustic discontinuities which signal potential segment boundaries. This occurs, for instance, with hanaya 'florist' or sushiya 'sushi restaurant' between $/ \mathrm{a} /$ and $/ \mathrm{j} /$ or $\mathrm{f} / \mathrm{and} / \mathrm{j} /$. The examples of the difficulties in segmentation can be seen in Figure 3.2 presented at the end 
of this section (p.123). In this study, these vowels and semi-vowels were segmented at the point where the changes in the amplitude in the soundwave forms and / or formant transitions were clearly observed.

In order to measure the formants of target segments, firstly the audio waveform and spectrogram were generated. Frame length for the spectrogram was set at $100 \mathrm{~ms}$. Then, the LPC formant history was imposed on the spectrogram. This LPC function was produced with a frame length of $25 \mathrm{~ms}$ and a filter order of 20 . This traces the centre frequencies of the formants.

Measurements were made by identifying boundaries, then placing the cursor on the formant trace and reading off the values at the $50 \%$ point of the vowel duration. The spectra were also referred to when necessary. The spectra were not used as the prime source for the formant measurements, however, as the LPC formant function may not always pick up all the formants present, and this could easily result in the misidentification of formants. For instance, the third formant may be identified as the second formant when the real second formant is too weak to be picked up at the point of measurement. The measurement from the formant history projected on the spectrogram allows us to compare the formant traces and the formant structures seen in spectrograms, making the identification of formants more reliable.

Measurements in this study were limited to below $4000 \mathrm{~Hz}$. It is unlikely that acoustic information over $4000 \mathrm{~Hz}$ would be available in forensic situations, making the practical application of formants above $4000 \mathrm{~Hz}$ very limited. In addition, in actual forensic phonetic investigations incriminating samples are often recorded over telephone lines which normally utilise a signal that is bandpassed in the $300 \mathrm{~Hz}$ to $3400 \mathrm{~Hz}$ range (Künzel 1995). Even when incriminating recordings are not from telephone lines, they are often of poor recording quality, due to background noise and / or low quality recording equipment. Higher formants are more susceptible to these external factors. This makes analysis based on higher formants of little 
practical use in actual forensic phonetic investigations, although these formants may contain more information relevant to a speaker's identity.

Examples from the recording of speaker AA's first performance in the first recording session are now presented in Figure 3.2. The words presented here are shown below. (The target vowels are underlined.)

$$
\begin{array}{ll}
\text { /a/ } & \text { hanaya "florist" } \\
\text { /i/ } & \text { sushịya "sushi restaurant" } \\
\text { /u/ } & \text { nikuyya "butcher" } \\
\text { lel } & \text { nemoto "Nemoto (person's name)" } \\
\text { /o/ } & \text { grandohoteru "Grand Hotel" }
\end{array}
$$

The topmost panels of the figures contain audio waveform of whole words; the middle panels show the spectrum at the midpoint of the vowel duration, and the panels at the bottom are the spectrograms of whole words. 
[hanaya 'florist']

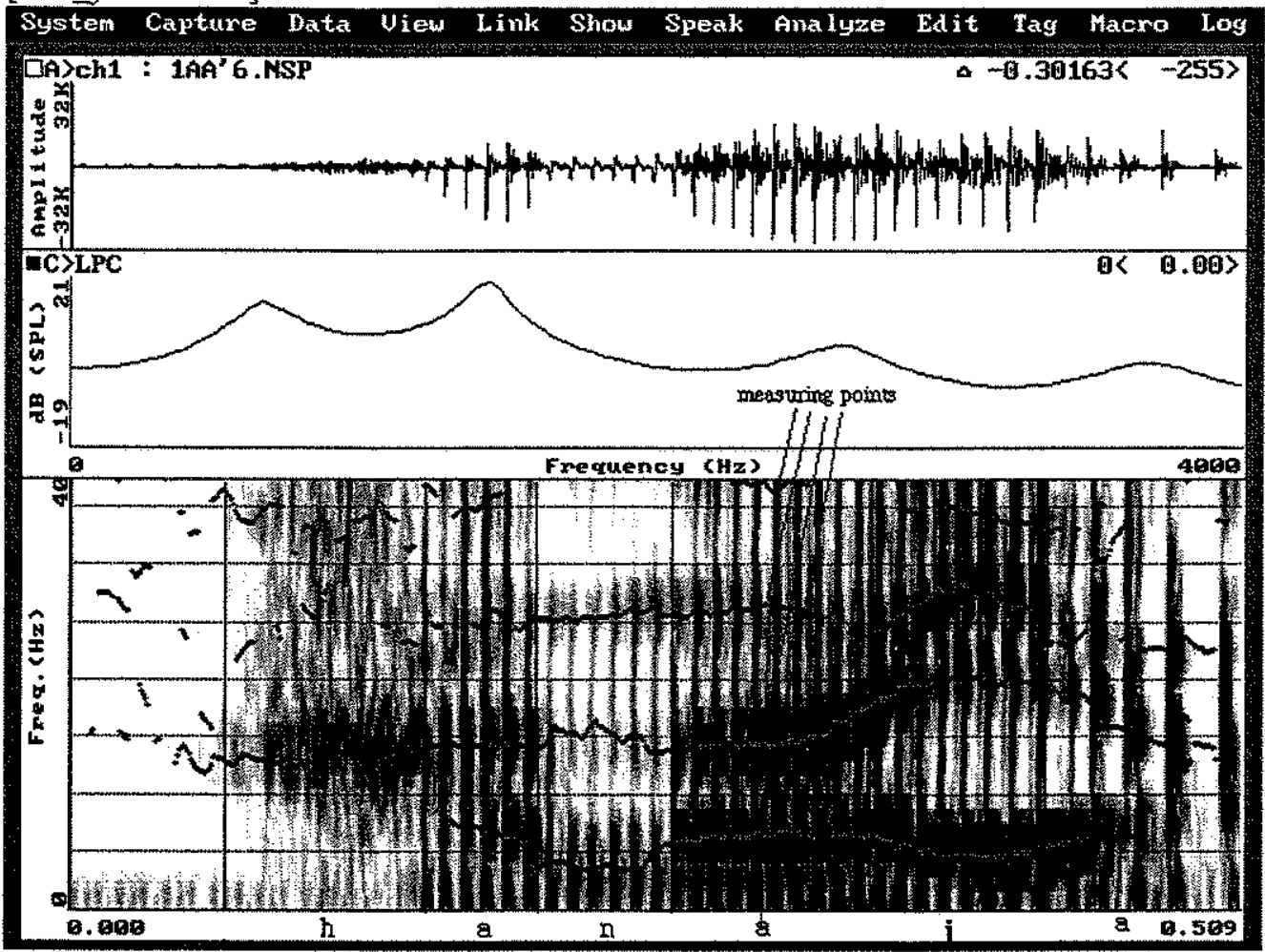

[sushiya 'sushi restaurant']

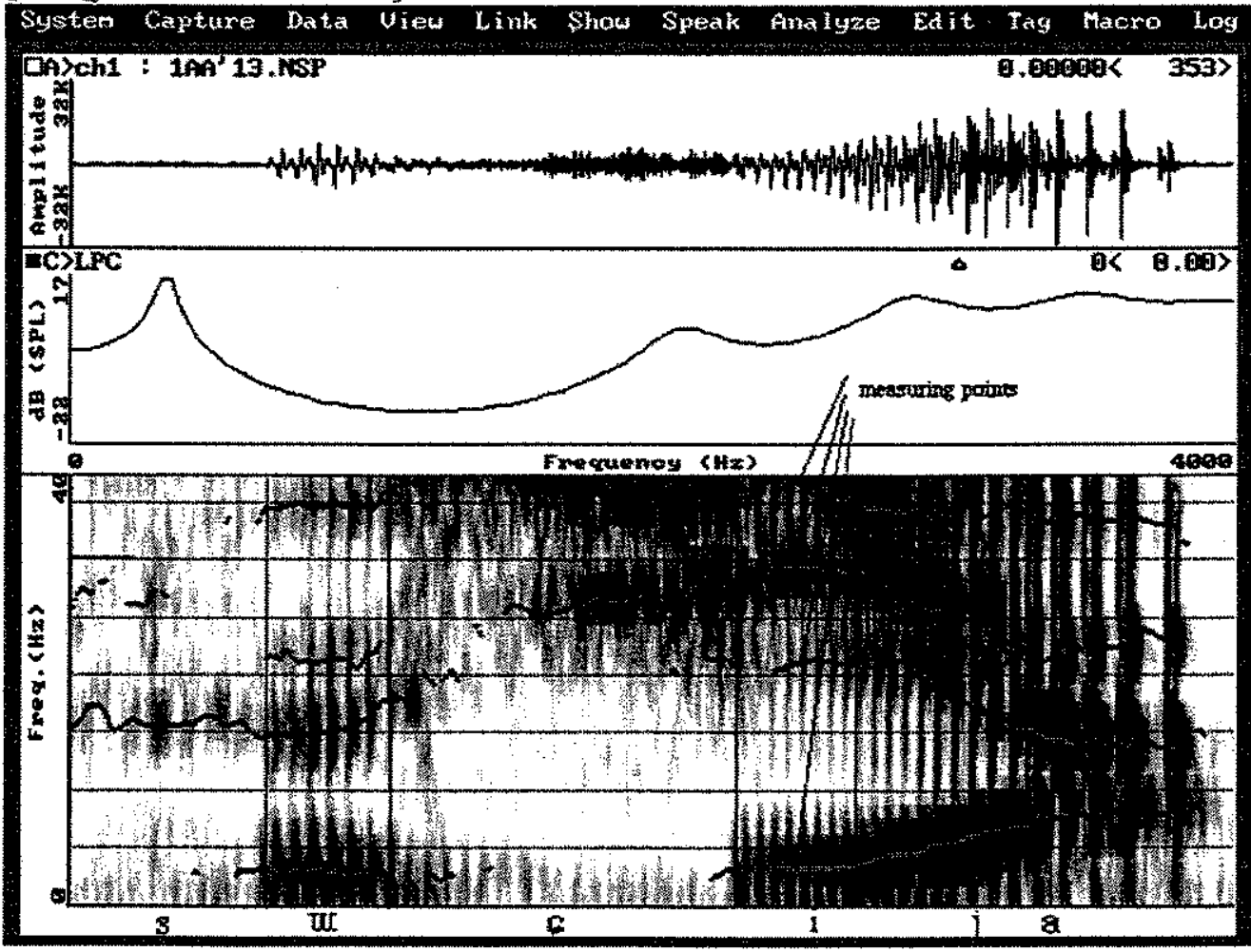



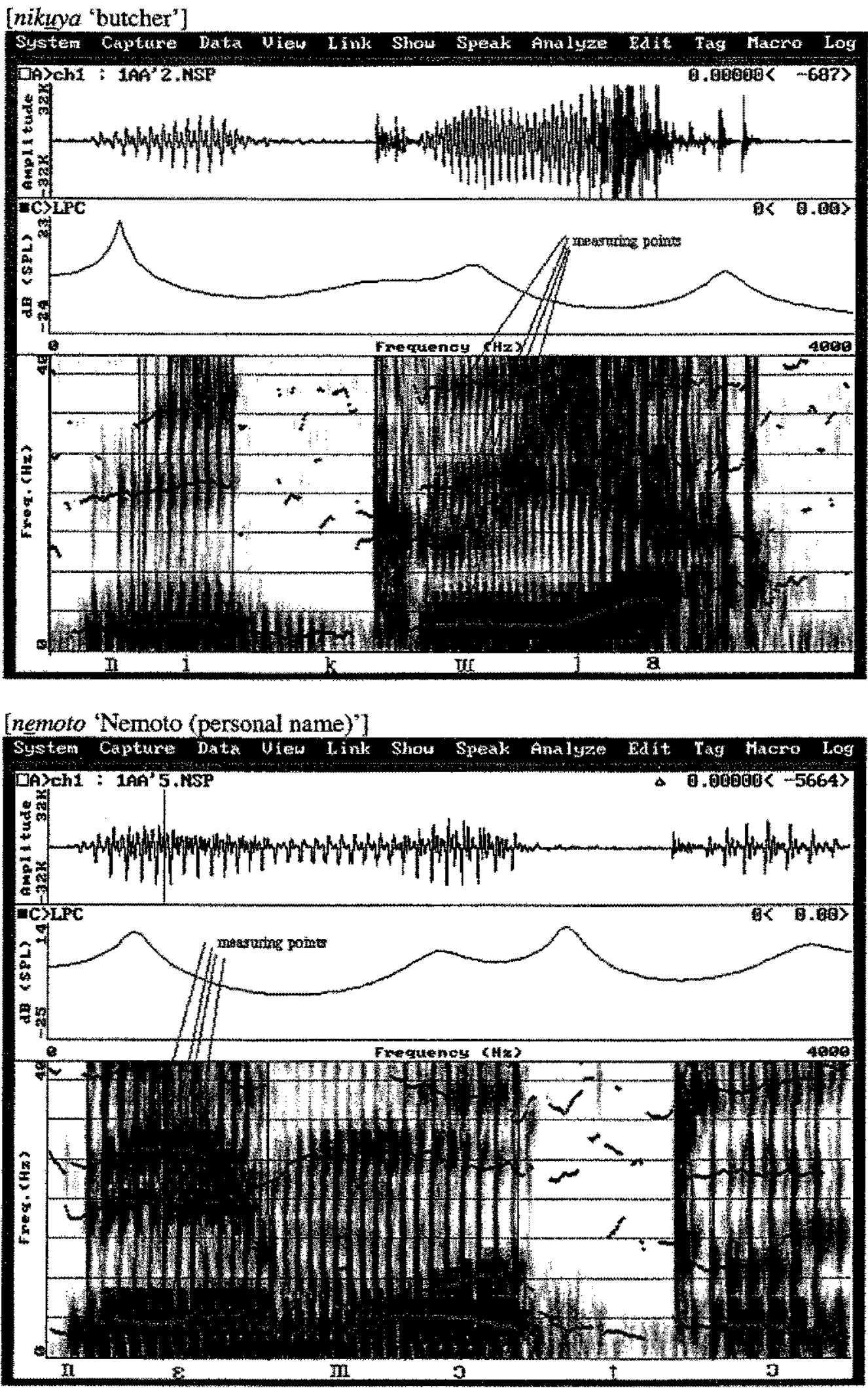


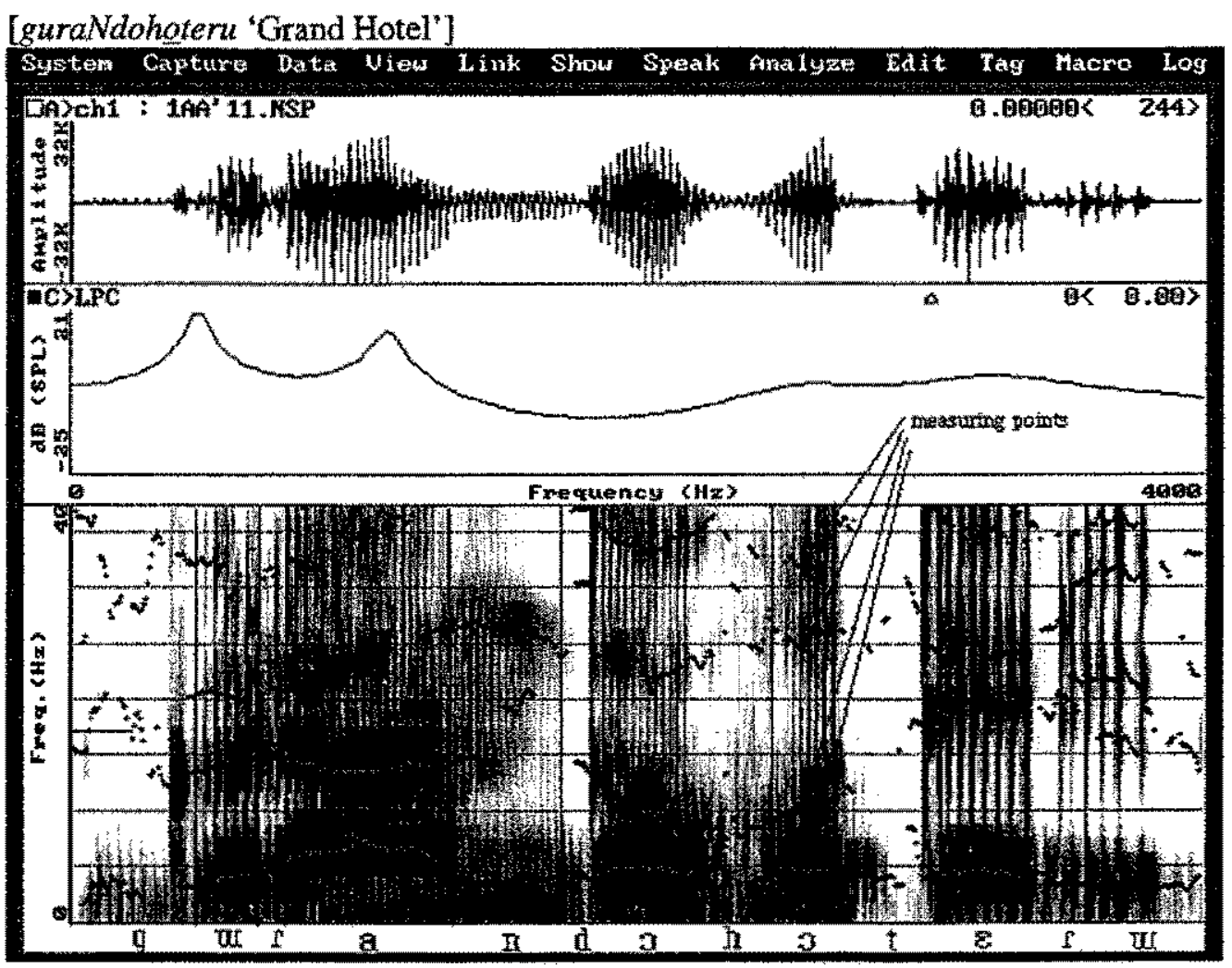

Figure 3.2 Sample spectrograms from speaker AA showing points at which vowel F-pattern was measured.

\subsubsection{MOSHIMOSHI (CHAPTER 5)}

For the investigation of the word moshimoshi in Chapter 5 , the following 12 sampling points were identified:

1) middle point of the first [m]

2) onset of the first [o]

3) middle point of the first [0]

4) affset of the first [0]

5) middle point of the first [c]

6) middle point of the first [i]

7) middle point of the second [m] 

8) onset of the second [o]
9) middle point of the second [0]
10) offset of the second [0]
11) middle point of the second [6]
12) middle point of the second [i]

The measurements were taken in the middle point of each of the 8 segments in moshimoshi, and at the onset and offset of both the first and the second $/ \mathrm{o} /$. The onset and offset of $/ \mathrm{o} /$ were included in the measurements to capture the rising F2 contour of this vowel caused by coarticulation. The /o/ vowels in this phrase have a rising $F 2$, due to the anticipating for the following consonant $/ \mathrm{s} /$ (which phonetically realises as [6]). As the degree and realisation of coarticulation can present some individual characteristics (Nolan 1983), this rising contour was included in the investigation.

Measurements were made in the same way as for the vowels in different words, by generating LPC function, placing the cursor at the 12 measuring points listed above, and reading off directly from the formant traces imposed on the spectrogram. For the measurements of this phrase as well, the spectra were not used as the prime source for the formant measurements, although they were referred to when necessary. The visual examination of the location of formants on the spectrogram became even more important in the measurements of moshimoshi, as the LPC formant history function seems often to be confused when tracking the formants of nasal segments.

Figure 3.3 shows, from top to bottom, an example of soundwave form, spectra, and spectrogram of moshimoshi. The measuring points are indicated by the white dots in the spectrogram, two measuring points for /i/ vowels are not shown here, however, as this speaker 
devoiced these two $/ \mathrm{i} /$ vowels. The spectra were taken at the middle point of the second $/ \mathrm{m} /$ and $/ \mathrm{o} /$.

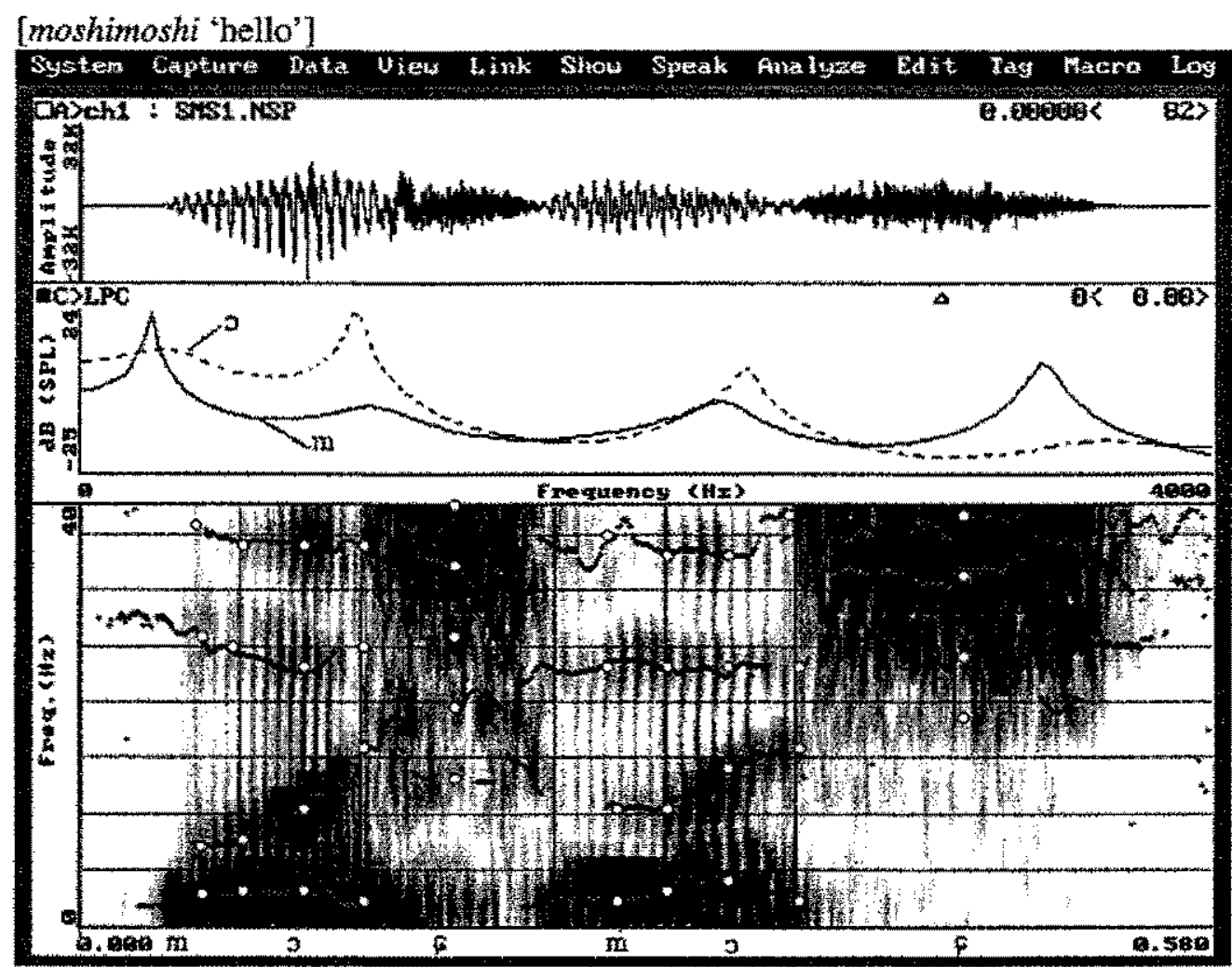

Figure 3.3 Example of measurement of F-pattern in moshimoshi. White dots indicate F-pattern measurements taken.

Figure 3.3 shows that the formant structure of mashimoshi is very complicated. First of all, it is noticeable that both $/ \mathrm{V} /$ vowels in moshimoshi were devoiced. This was the case for many of the speakers. The devoicing found here is, more precisely, the omission of a vowel. As mentioned in the discussion of vowel devoicing in Chapter 2 , it has been reported by Kawakami (1977:26) that the devoicing of /si/ syllable is phonetically realised as [6:], rather than [Gị. Figure 3.3 above confirms Kawakami's observation.

Figure 3.3 also shows that there is strong low frequency energy in the first voiceless consonant $/ s /$, especially at the first half of this consonant. It looks as if there is a residual voicing from the preceding segments. The vocal cords were vibrating for the [o] vowel just before the 
production of the consonant. The vocal cords need to be wide apart for production of the voiceless fricative, but maybe the vocal cords could not be opened widely and quickly enough, resulting in the residual voicing at the earlier part of the fricative consonant. It is, however, difficult to tell whether or not it is periodic from the spectrogram. The cause thus remains unclear.

In addition to the low frequency energy, the formants of the first /s/ are not easily traceable. The formant tracking could not trace the formants as continuing smoothly with the adjacent segments. This made the measurement of moshimoshi a more complicated task, since it was sometimes difficult to identify which formant was F1, F2, and so on. In those situations, the formants were identified on the basis of contingency with adjacent segments and with the formant structures of the second $/ \mathrm{s}$ ( as the second /o/ had clearer formant traces), as well as the expected formant values from the acoustic theories.

\subsection{STATISTICAL PROCEDURE}

After the raw figures obtained for each segment / formant combination were transferred to spreadsheets, they were processed statistically. The measurements were made for 20 vowel I formant combinations in Chapter 4 (four formants * five vowels) and 48 segment / formant combinations in Chapter 5 (four formants * 12 sampling points), producing 68 potential parameter for speaker discrimination test. Given that some parameters will not be as useful as the others, it is not practical to incorporate all 68 parameters. Chapters 4 and 5 therefore investigate which segment / formant combinations are more promising parameters for forensic speaker identification. In order to estimate discriminating power of the parameters, this study used F-ratio, following Wolf's (1972) study. Wolf performed ANOVA (Analysis of variance) in order to produce F-ratio, supposing that the parameter with larger F-ratio is the better speaker identification parameter. Since F-ratio is calculated as a between-group variation to 
within-group variation ratio (in this study, it can be paraphrased as the between-speaker variation to within-speaker variation ratio), a larger F-ratio indicates the stronger discriminating power of the parameter. The F-ratios are calculated by performing ANOVA. The software used for the statistics was R and Statview.

The parameters selected by ANOVA are then statistically tested in terms of how well they perform in the discrimination (or identification) of two sets of data. In Chapter 6, therefore, the parameters which produced high F-ratios are combined for discrimination tests between two randomly chosen speech samples. For the results of research on forensic speaker identification to be truly useful in practice, it is essential to know not only which parameters are powerful, but also to know the reliability of the discrimination test based on those parameters. Some parameters may discriminate between speakers with a $90 \%$ success rate, whereas others may achieve only a $60 \%$ success rate. This affects the strength of the evidence significantly, and it is thus not appropriate to apply parameters in the actual forensic investigation without the knowledge of potential error rates which the parameters incur. In order to investigate the performance of formants, three statistical approaches are examined in this study. The first method used in this study is discriminant analysis. This type of statistical analysis is frequently used in experiments on speaker identification (see Ingram et al. 1996; Jassem 1995). The second and third approaches use what is tentatively named the "distance based method" in this study. One distance used is discriminant scores; the other distance is the likelihood ratio.

On the basis of these tests, it is determined to what extent formants can be used in forensically realistic speech data to tell whether the samples are from the same or different speakers.

The next chapter will study the formant pattems of the vowels embedded in the different words. As described in this chapter, the investigation focuses on finding out what are the 
promising parameters for forensic speaker identification. The Japanese-specific linguistic parameter, vowel devoicing, is also looked into. 


\section{Chapter 4}

\section{DETERMINING EFFICIENT}

\section{FORMANT PARAMETERS FOR}

\section{VOWEL DATA}

\subsection{OBJECTIVES}

In the previous chapters, it has been explained that this thesis is going to investigate the performance of formants in forensic speaker identification. As a prior step for the discrimination test, which is going to be performed in Chapter 6 of this thesis, this chapter studies the centre formant frequency of vowels, focusing on centre formant frequencies of accented short vowels. It is evident that comparison of vowels in the same word is more ideal in quantifying between-speaker variation. In real forensic situations, however, it is often the case that not enough numbers of comparable words are available. The vowels studied in this chapter are thus embedded in different phonological contexts, although they are prosodically comparable.

The investigations in this chapter primarily aim to reveal which vowel / formant combinations may be more suitable for use as speaker identification parameters, but also look into the effect of the non-contemporaneity of the datasets, vowel devoicing and effective F2. 
Following the methodology used by Wolf (1972), the search for the effective parameter was carried out on the basis of F-ratios. Since the F-ratio is the ratio of the between-speaker variation to the within-speaker variation, the vowel / formant combinations which produce large F-ratios are considered to have better potential as a speaker identification parameter.

The non-contemporaneity of the datasets is investigated by comparing datasets which are recorded on different occasions. As described in Chapter 3, two recording sessions separated by two weeks were held for the data collection in this study in order to achieve forensic realism. Furthermore, speakers repeated the tasks on each recording session, consequently producing four sets of data which were recorded at the two different recording sessions. In this study, two types of comparison, which will be called contemporaneous (recorded at the same recording session) and non-contemporaneous datasets (recorded at two different recording sessions) are compared, and the effect of the time which elapses between the recordings is discussed.

As for vowel devoicing and effective F2, no study has been done on either of them in the context of forensic speaker identification. Certain characteristics of these parameters indicate that they may serve as useful clues for speaker identification. This chapter thus studies the possibility of using them as a forensic speaker identification parameter. 


\subsection{MEASUREMENTS}

Chapter 3 described how Japanese words naturally uttered by 11 male Japanese speakers were recorded for the experiment in this chapter. Tasks were designed to produce a recording of five different words for each vowel, with exception of the words for $/ e /$, which included four different words with one repeated. The speakers were asked to repeat the tasks immediately after their first performance of them. As a result, for each vowel, ten tokens per speaker were recorded at each recording session.

As has been described in Chapter 3, the recordings were then processed using speech analysis software, C(omputer) S(peech) L(aboratory). All samples were digitised at $16 \mathrm{kHz}$. Formant measurements were made at the middle point of the vowel duration, and the measurements were limited to below $4000 \mathrm{~Hz}$, considering the reality of forensic speaker identification where any information above $4000 \mathrm{~Hz}$ is unlikely to be usable. As a result, F1 to F4 were measured for most speakers.

The numbers of tokens for each vowel are summarised in Figure 4.1. 


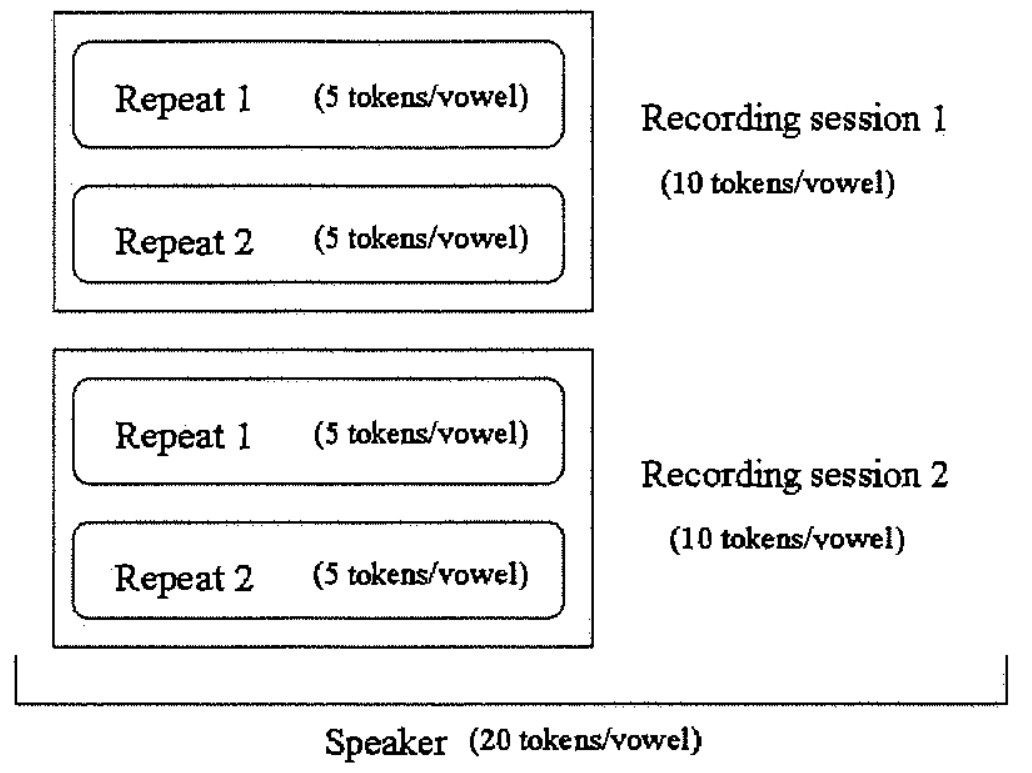

Figure 4.1 Numbers of tokens recorded for each vowel for each speaker.

It has to be noted, however, that not all speakers' ten tokens were measurable for all formants or all five vowels at each recording session, since some formants could not be observed and some of the /w/ vowels were devoiced. Devoiced vowels do not have a voice-excited energy, but their noiseexcited formants still should be observable. In most of the devoiced tokens for the / $\mathrm{w} /$ vowel in this study, segmentation of devoiced vowels was, however, very difficult. Suzuki et al.'s (1978) study on whispered and normal voices also showed that the change in phonation causes significant shift in formants. As devoiced vowels and whispered vowels are considered to be phonetically similar in the sense that they lack a voice-oriented energy, it was concluded that the inclusion of formants obtained from the devoiced vowels might be likely to distort the data. The formant measurements of the devoiced $/ \mathrm{u} /$ vowels were therefore not included in the data.

This devoicing of the / $\mathrm{w} /$ vowels was actually unexpected, since the vowels in question were accented and accented vowels are not supposed to be devoiced. Considering that the realisation of 
vowel devoicing varies according to the variety of the regional dialects (see Chapter 2), however, it is possible that vowel devoicing might also constitute a linguistic parameter in forensic speaker identification. It was thus decided that this illegal realisation of vowel devoicing was significant to this investigation, and this is done in the next section, prior to the main analysis of this chapter.

\subsection{ILLEGAL VOWEL DEVOICING 4.3.1 RESULTS}

The $/ \mathrm{u} /$ vowel in two words, tokushima [tokwçima] 'Tokushima (name)' and doobutsuen [do:burtsureũ] 'zoo' were devoiced by many speakers. This is intriguing, as the corpus in this study was designed to exclude vowel devoicing. According to the traditional devoicing rules (for instance, Shibatani (1990:16)), devoicing is expected to be avoided under the phonological environment in which these two $/ \mathrm{u} /$ vowels are embedded. It is true that $/ \mathrm{w} /$ in tokushima 'Tokushima (name)' is located between the two voiceless consonants [k] and [G], and $/ \mathrm{J} /$ in doobutsuen 'zoo' is preceded by a voiceless consonant [ts]. In both words, however, /u/ carried a pitch accent, and this supposed to prevent the vowel from being devoiced. Furthermore, the / $u$ / vowel in doobutsuen 'zoo' is followed by a voiced segment, $[\varepsilon]$ which one would expect would also interfere in the devoicing of $/ \mathrm{u} /$. In the subsequent discussions, this unexpected devoicing is referred to as 'illegal devoicing.'

If this unexpected devoicing consistently occurs in the data of particular speakers but not in the others', vowel devoicing can be considered a possible indicator of speakers' identity. The incidence of the devoicing in the speakers' data is summarised in Table 4.1. The left column indicates the speakers. 'Session 1 and 2' on the top row shows the recording session from which the data were collected. 'Repeat 1 and 2 ' in the next row shows which of the two repeats the data 
were collected from. ' $\mathrm{t}$ ' and ' $\mathrm{d}$ ' in the table represent two words, tokushima 'Tokushima (name)' and doobutsuen 'zoo', respectively. The devoiced tokens are marked by shading. For most speakers, both words were recorded a total of four times (two recording sessions * two repeats) each, so there are four tokens for each word. It has to be noted that speaker KO in the first recording session and speaker $\mathrm{KH}$ in both recording sessions did not repeat the tasks. This is because these two speakers were the first two informants who were recorded for this study, and it was decided to repeat tasks after speaker $\mathrm{KH}^{\prime} \mathrm{s}$ both recording sessions and Speaker KO's first recording session. This explains the missing values for these two speakers' results.

\begin{tabular}{|c|c|c|c|c|c|c|c|c|}
\hline & \multicolumn{4}{|c|}{ Session 1} & \multicolumn{4}{|c|}{ Session 2} \\
\hline & \multicolumn{2}{|c|}{ repeat 1} & \multicolumn{2}{|c|}{ repeat 2} & \multicolumn{2}{|c|}{ repeat 1} & \multicolumn{2}{|c|}{ repeat 2} \\
\hline $\mathrm{AA}$ & se & $\mathrm{d}$ & $t$ & $\mathrm{~d}$ & $t$ & 要 & $t$ & $d$ \\
\hline HA & 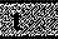 & 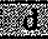 & 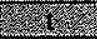 & 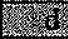 & S & (1) & 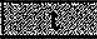 & 管 \\
\hline $\mathrm{JN}$ & (3) & (5) & 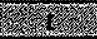 & Wy- & 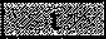 & 20 & 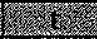 & $d$ \\
\hline $\mathrm{KA}$ & 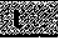 & (2) & 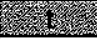 & W & 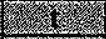 & 2 & $y_{1}$ & $d$ \\
\hline $\mathrm{KF}$ & $t$ & 自 & $t$ & 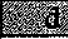 & 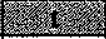 & d & 1) & 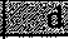 \\
\hline $\mathrm{KH}$ & $t$ & $d$ & - & - & $t$ & d & - & - \\
\hline $\mathrm{KO}$ & (x) & $\mathrm{d}$ & $=$ & $=$ & $t$ & $d$ & 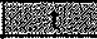 & 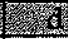 \\
\hline $\mathrm{MN}$ & $t$ & $\mathrm{~d}$ & $\mathrm{t}$ & d & $t$ & $\mathrm{~d}$ & $t$ & $\mathrm{~d}$ \\
\hline $\mathrm{TN}$ & 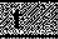 & 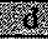 & SW & $\mathrm{d}$ & $t$ & 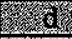 & ty & (1) \\
\hline $\mathrm{TS}$ & 实 & (2) & 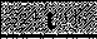 & 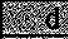 & $t$ & ris & 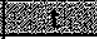 & 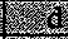 \\
\hline TY & Sy & d & 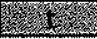 & (3) & 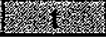 & 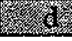 & $t$ & 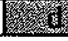 \\
\hline
\end{tabular}

Table 4.1 Incidence of illegal vowel devoicing of tokushima 'Tokushima (name)' and doobutsuen 'zoo' as function of speaker, recording session, and repeat. ' $t$ ' and ' $\mathrm{d}$ ' indicate tokushima and doobutsuen respectively, and devoiced tokens are shown in the shaded columns.

Table 4.1 shows that illegal vowel devoicing was found not to be constant even across the repeats made by a single speaker at the single recording session. A speaker who devoiced the vowels in the first utterance did not always devoice when he repeated the tasks. For instance, Speaker AA devoiced the word tokushima in the first repeat of the first recording session. He also devoiced doobutsuen in the first repeat of the second recording session, but not in the other tokens.

The data presented in Table 4.1 are further quantified in terms of their incidence at each recording session. Table 4.2 shows the how frequently illegal vowel devoicing was observed for each 
speaker's two recording sessions. Percentages were calculated by counting how many out of four $/ \mathrm{u} / \mathrm{s}$ were illegally devoiced.

\begin{tabular}{|r|r|r|}
\hline & Session 1 & Session 2 \\
\hline AA & $25 \%$ & $25 \%$ \\
\hline HA & $100 \%$ & $100 \%$ \\
\hline JN & $100 \%$ & $75 \%$ \\
\hline $\mathrm{KA}$ & $100 \%$ & $75 \%$ \\
\hline $\mathrm{KF}$ & $50 \%$ & $75 \%$ \\
\hline $\mathrm{KH}$ & $0 \%$ & $0 \%$ \\
\hline $\mathrm{KO}$ & $50 \%$ & $50 \%$ \\
\hline $\mathrm{MN}$ & $0 \%$ & $0 \%$ \\
\hline $\mathrm{TN}$ & $75 \%$ & $75 \%$ \\
\hline $\mathrm{TS}$ & $100 \%$ & $75 \%$ \\
\hline $\mathrm{TY}$ & $75 \%$ & $75 \%$ \\
\hline
\end{tabular}

Table 4.2 Summary of how frequently the illegal vowel devoicing occurred in two recording sessions.

Table 4.2 indicates that, although speakers vary within a single recording session with respect to whether or not they devoice the / $\mathrm{u} /$ vowel, there seems to be a consistency in terms of how often they devoice the vowel across sessions. Seven out of 11 speakers have the identical rates of illegal devoicing incidence. Even with the speakers who produced different rates across sessions, the differences between two sessions were not large.

\subsubsection{DISCUSSION}

\subsubsection{Within-speaker variation in vowel devoicing}

Table 4.1 in the previous section suggests that the realisation of illegal devoicing varies even within a speaker, as we see that some speakers devoiced on one occasion, but did not when they repeated the task at the same recording session. Figure 4.2 presents examples of within-speaker variation in the realisation of vowel devoicing. The figures consist of the audio waveforms and spectrograms of tokushima 'Tokushima (name)' uttered by Speaker TS. In the first recording session, he devoiced the $/ \mathrm{w} /$ vowel in this word in both repeats, but he produced the same vowel fully voiced in his first 
repeat at the second recording session. The word was segmented and phonetically transcribed, except for the devoiced / $\mathrm{w} /$ vowel in the first figure. The precise segmentation of the devoiced vowel was difficult. One cursor has, however, been positioned at the voice onset time in the second figure where / $\mathrm{w} /$ was not devoiced, and the other cursor has been located at the place where noise excited F2 appears to flatten out around $2000 \mathrm{~Hz}$.

[Speaker TS's second tokushima 'Tokushima (name)' in the first recording session] System Capture Data View Link Show Speak Analyze Edit Tag Macro Los
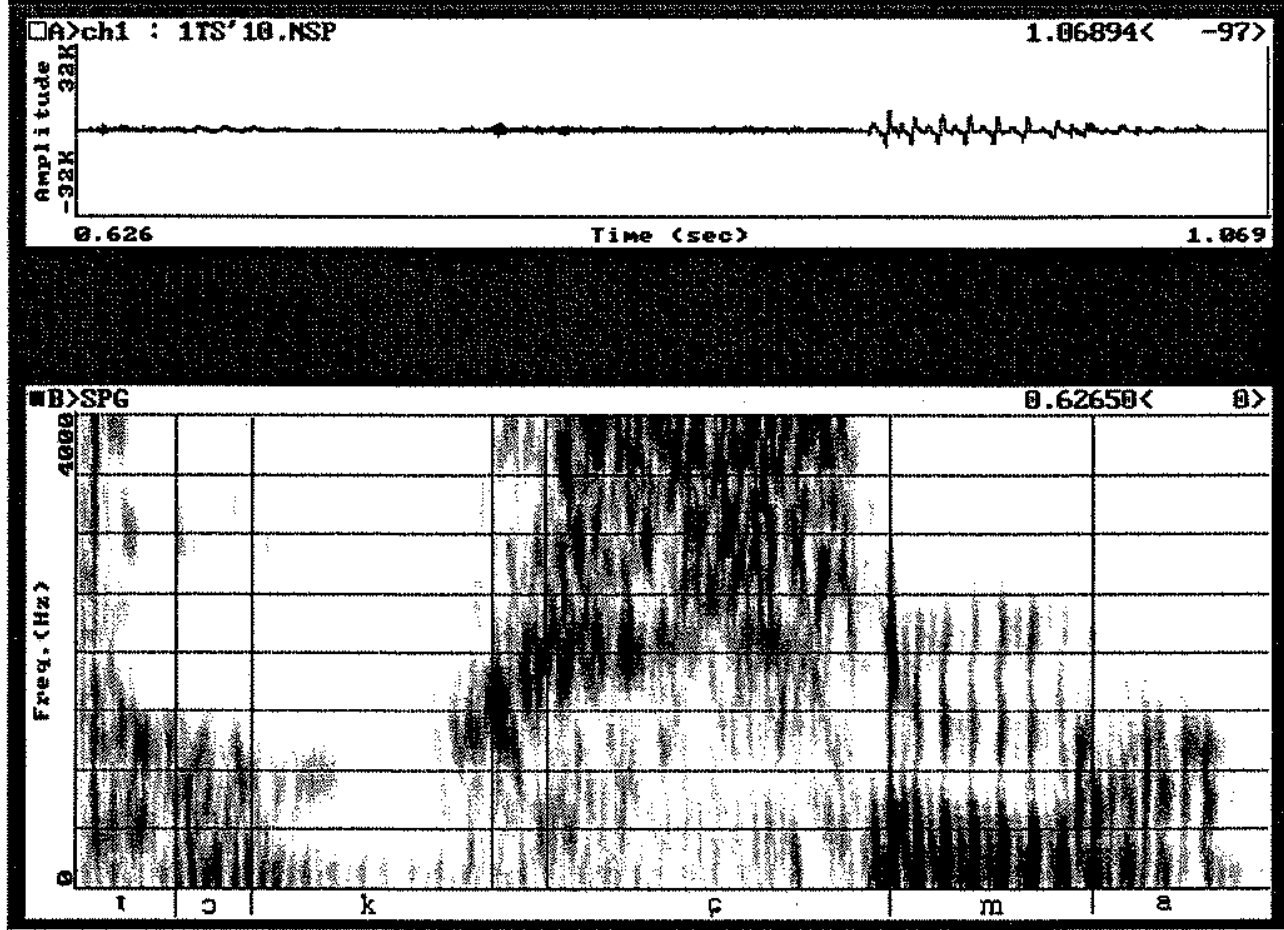
[Speaker TS's second tokushima 'Tokushima (name)' in the second recording session]
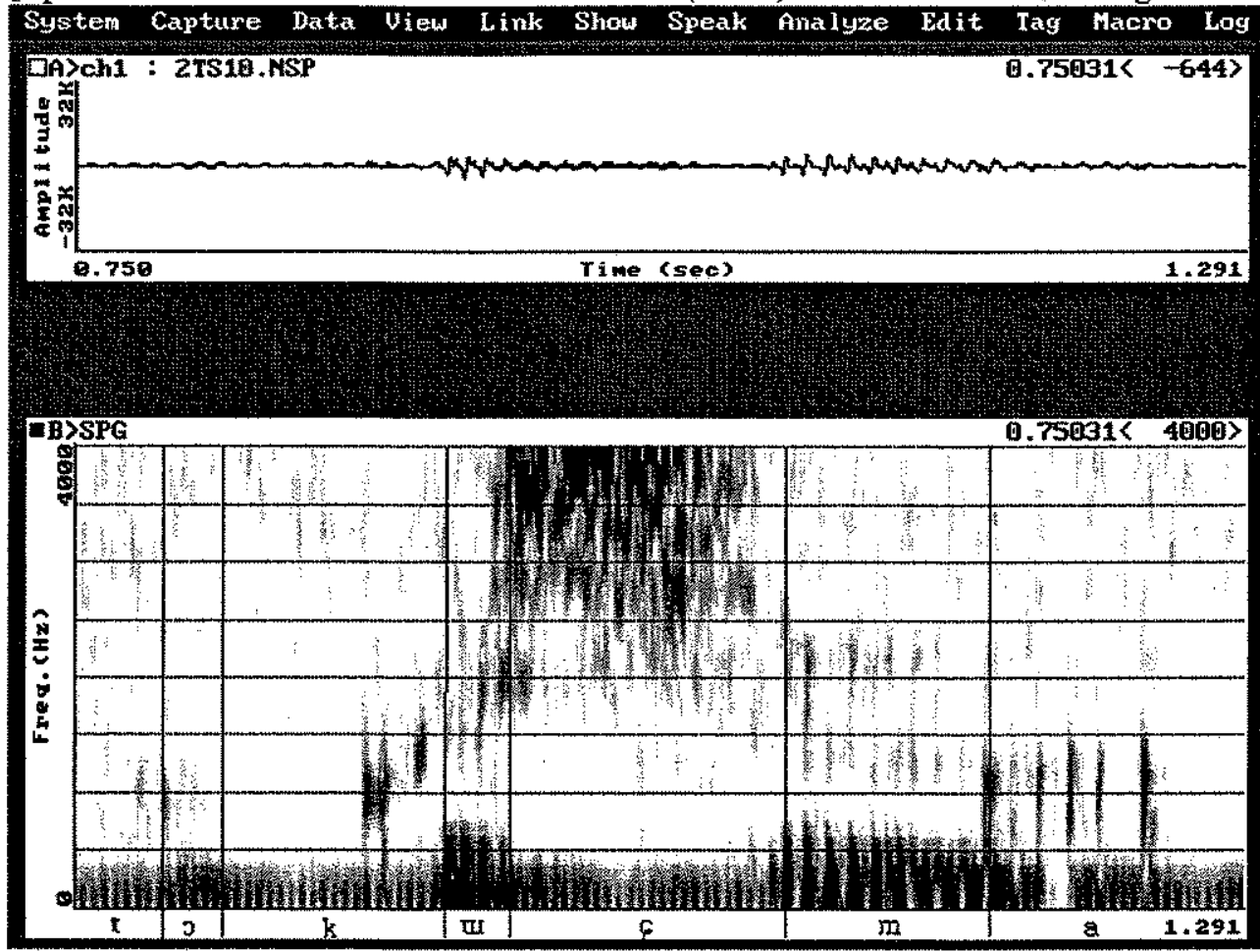

Figure 4.2 Example of within-speaker variation in vowel devoicing. The top figure shows an example of Speaker TS's devoiced / $\mathrm{u} /$, and bottom figure shows his fully voiced $/ \mathrm{u} /$.

The result summarised in Table 4.2 in previous section, however, shows that each speaker's rate of incidence of devoicing seems quite stable across the two different recording sessions. This suggests that how often the illegal vowel devoicing occurs can be an indicator of the speaker's identity, if between-speaker differences occur in the rates of the vowel devoicing incidence.

\subsubsection{Between-speaker variation in vowel devoicing}

\subsection{Incidence of illegal vowel devoicing}

The previous sections demonstrated that whether or not the illegal vowel devoicing occurs varies considerably even within a single speaker. This suggests that the realisation of vowel devoicing itself is not a useful parameter for forensic speaker identification. There is, however, an interesting 
finding in the results produced from data collected from Speakers HA and MN. As can be seen in Table 4.1 , Speaker HA devoiced all $8 / \mathrm{w} /$ vowels (4 in tokushima 'Tokushima (name) and 4 in doobutsuen 'zoo'), whereas speaker MN devoiced none of them. Figure 4.3 presents the audio waveform and spectrogram of tokushima uttered by Speakers HA and MN.

[Speaker HA's tokushima 'Tokushima (name)']
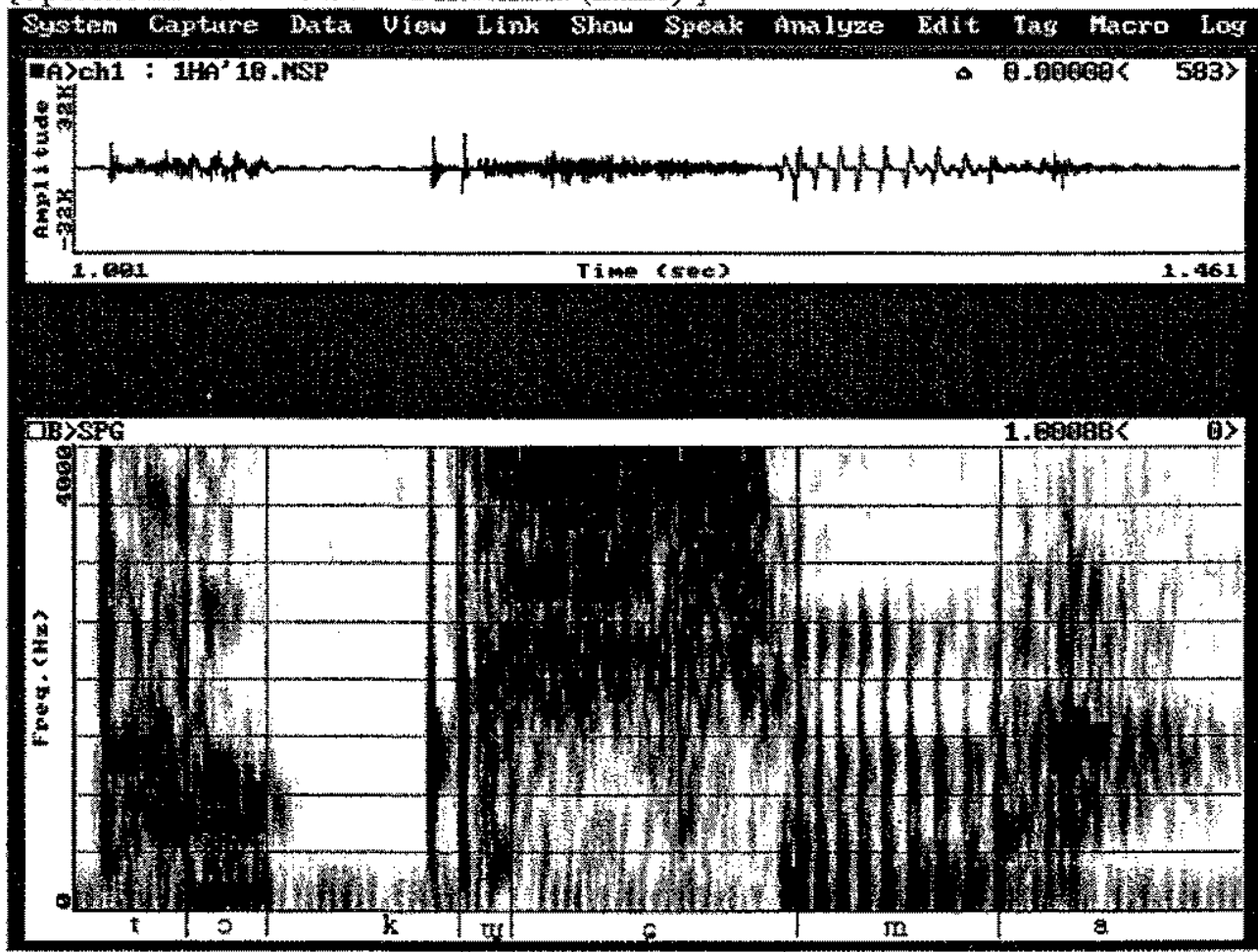


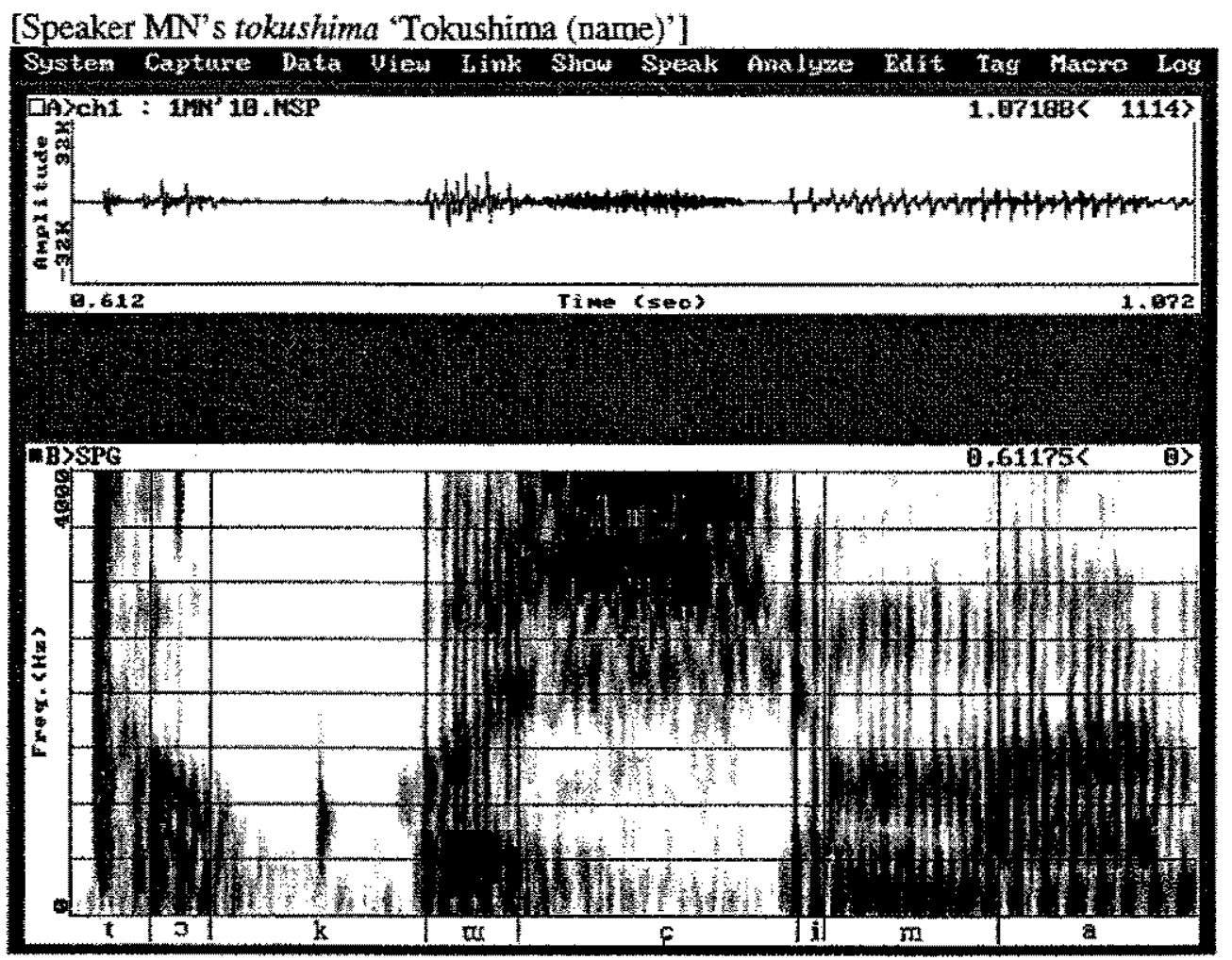

Figure 4.3 Audio waveforms and spectrograms of tokushima [tokmcima] 'Tokushima (name)' uttered by Speakers HA and MN.

These two speakers differ in their original dialect; Speaker HA is a Tokyo dialect speaker, and Speaker MN is a speaker of Kansai dialect. They both are regarded as typical speakers of those dialects, since neither of them has lived outside of their dialectal areas in Japan and, consequently their exposure to other dialects is assumed to have been fairly limited. Speaker HA's constant devoicing and speaker MN's complete absence of devoicing agree with the received knowledge that Tokyo dialect speakers devoice high voweis (although the vowels are not accented ones), whereas Kansai dialect speakers do not. These two speakers' results, thus, seem to suggest that what is observed here is a tendency associated with the regional differences.

It cannot be concluded, however, that the difference between these two speakers was caused by the dialectal differences between Tokyo and Kansai styles, as there are speakers who present counter evidence. Other speakers from Tokyo (and its surrounding area, such as Yokohama, which has 
similar dialectal features to Tokyo) did not devoice the / $\mathrm{u} /$ vowel as constantly as Speaker HA did. For instance, Speaker AA, who was born and brought up in Tokyo just like speaker HA, devoiced only two out of the eight / $\mathrm{w}$ / vowels. Furthermore, Speaker KO, who is a Kansai dialect speaker like Speaker MN, devoiced those words half the time. (Although Speaker KO was born in Fukuoka where devoicing is preferred, he moved to Osaka when he was 2 years old. He is thus regarded as a native Kansai dialect speaker in this study.)

Sugitoo (1996) reports that the realisation of vowel devoicing is not "all-or-nothing". She compared three Tokyo dialect speakers, three Osaka dialect speakers, and three Osaka dialect speakers who have some exposure to the Tokyo dialect with respect to their vowel devoicing. As results, Sugitoo reports that Tokyo and Osaka dialect speakers devoice high vowels on average 55\% and $34 \%$, respectively, of the total of 165 high vowels in the position where the traditional phonological description predicts the incidence of vowel devoicing. In other words, even Tokyo speakers sometimes produced voiced high vowels in the conditions where devoicing is preferred, and Osaka speakers sometimes devoiced high vowels. This agrees with the results of Speakers AA and $\mathrm{KO}$ in this study, suggesting that Speakers $\mathrm{HA}$ and $\mathrm{MN}$ are rather rare speakers.

The rareness of the consistent absence found in Speaker MN's data is illuminated by the Sugito's (1996) comments on the change in vowel devoicing in modern Japanese. Sugito reports that high vowel devoicing is gradually spreading to the areas where it did not originally occur, on the basis of her observation of the younger generation in the Osaka area. If that is the case, the consistent absence of devoicing will increase its value as a clue to speakers' identities, especially among the younger population.

The observation made above suggests that whether or not vowel devoicing occurs can serve as a broad indicator of the speaker's identity in some cases. However, the application of the incidence 
of devoicing as a parameter must be strictly limited to the cases where both incriminating evidence and suspect's samples show a very constant presence or absence of vowel devoicing. Furthermore, in order to apply to forensic speaker identification, what constitutes a very high or low rate of devoicing has to be researched first.

\subsection{Consistency of vowel devoicing within a speaker}

The previous section has demonstrated that whether or not a speaker devoices certain vowels itself does not serve as clue for the individuality. The other aspect of vowel devoicing, namely how consistently a speaker devoices the vowel, however, may provide more useful information. Table 4.2 in the previous section showed that the speakers are reasonably constant across two recording sessions in terms of their rate of vowel devoicing incidence. Categorical data like these cannot produce F-ratio, which is used in this study as the indicator of effectiveness as a speaker identification parameter. If the between-speaker variation in the rate of vowel devoicing incidence is found to be larger than the degree of within-speaker variation, this rate should be able to serve as the speaker identification parameter. How often the rates of the illegal devoicing incidence match across different speakers were thus examined in this section. The total number of the comparisons made was 220 , as 11 speakers made 55 possible combinations (as in Speakers AA vs. HA; Speakers AA vs. JN ... and so on), and two recording sessions produced four different between-speaker comparisons per speaker combination (eg. Speaker AA's session 1 vs. Speaker HA's session 1; Speaker AA's session 1 vs. Speaker HA's session 2; Speaker AA's session 2 vs. Speaker HA's session 1; Speaker AA's session 2 vs. Speaker HA's session 2). The combinations which showed no between-speaker variation (ie. produced matching devoicing rates, although they were produced by different individuals) are summarised in Table 4.3 (for the details of the comparison, see Appendix 4.1). 


\begin{tabular}{|c|r|r|r|}
\hline Comparison & Same rates & Total no. & \multicolumn{2}{|c|}{$\%$} \\
\hline Session1/1 & 8 & 55 & $15 \%$ \\
\hline Session1/2 & 4 & 55 & $7 \%$ \\
\hline Session2/1 & 9 & 55 & $16 \%$ \\
\hline Session2/2 & 13 & 55 & $24 \%$ \\
\hline TOTAL & 34 & 220 & $15 \%$ \\
\hline
\end{tabular}

Table 4.3 Summary of the between-speaker comparison in incidence of illegal vowel devoicing, showing number of the comparisons which did not show the between-speaker variation, total number of comparison, and percentage.

The same speaker comparison presented in Table 4.2 in the previous section showed that seven out of 11 speakers had consistent illegal devoicing incidence rates across two recording sessions. This is about $64 \%$. The matching rate $64 \%$ seems rather small, it is, however, still larger than the matching rate across different speaker pairs. Table 4.3 above shows that only $15 \%$ of the between speaker comparisons have matching devoicing rates. Thus there seems more between-speaker variation than within-speaker variation in the rate of illegal devoicing incidence, suggesting the possible use of this feature as a speaker identification parameter.

The proper evaluation of the effectiveness of the feature as a forensic phonetic parameter can be made by calculating likelihood ratios with the Bayesian approach. The Bayesian approach is extensively discussed in Chapter 6 of this thesis, where the strength of evidence of the vowel devoicing is quantified.

A point to be emphasised in this section is that the linguistic description of devoicing does not necessarily reflect phonetic reality. The two words, tokushima 'Tokushima (name)' and doobutsuen 'zoo', are not supposed to be devoiced according to the traditional phonological description of vowel devoicing (cf. Shibatani 1990). The results presented above remind us that linguistic descriptions reflect competence, but these descriptions do not necessarily reflect the actual phonetic performance. 
It is true that linguistic descriptions offer variable information on where speakers might possibly vary or what sort of similarity or dissimilarity may reflect individual characteristics, but not all the characteristics of the particular language. What it shows us is, however, just a typical behaviour found in the language.

\subsection{FORMANTS}

4.4.1 RESULTS

Table 4.4 presents the results (both mean and standard deviation) of the measurements taken of the centre frequencies of vowel formants. The results for each of the vowels are listed separately. The letters in the left most column represent each speaker. The first row shows formants, and the second row indicates the four different recording occasions in which the data was collected. ' $s 1$ ' and ' $\mathrm{s} 2$ ' indicate the recording sessions 1 and 2 respectively, ' $\mathrm{r} 1$ ' and ' $\mathrm{r} 2$ ' indicate the first and the second repeats within a recording session. For example, Speaker AA's mean F2 of his /a/ vowel recorded at the first repeat of the first recording session is $1242 \mathrm{~Hz}$, the standard deviation is 123.2 , and the sample size was five. (The actual individual measurements for each token are given in Appendix 4.2.) 


\begin{tabular}{|c|c|c|c|c|c|c|c|c|c|c|c|c|c|c|}
\hline \multirow[b]{2}{*}{ al } & \multicolumn{3}{|c|}{ F1 } & \multicolumn{4}{|c|}{ F2 } & \multicolumn{3}{|c|}{ F3 } & \multicolumn{4}{|c|}{$F$} \\
\hline & sir1 & $s 1 \times 2$ & \begin{tabular}{|l|l|l|}
$s 2 r 1$ & $s 2 \times 2$ \\
\end{tabular} & sir1 & $\sin 2$ & $\mathrm{~s} 2 \mathrm{rl}$ & $\mathrm{s} 2 \mathrm{r} 2$ & s1r1 & $\mathrm{s} 1 \mathrm{r} 2$ & \begin{tabular}{|l|l|}
$\mathrm{s} 2 \mathrm{r} 1$ & $\mathrm{~s} 2 \mathrm{r} 2$ \\
\end{tabular} & slr1 & $s 1+2$ & $\mathrm{~s} 2 \mathrm{rl}$ & $s 2+2$ \\
\hline \multirow{2}{*}{ AA mean } & 693.6 & 669.8 & 656.2676 .6 & 1242 & 1273 & 1361 & 1364 & 2485 & 2498 & 25372600 & 3567 & 3560 & 3451 & 3632 \\
\hline & 30.41 & 35.25 & 35.25 & 123.2 & 99.42 & 88.33 & 102.3 & 145.8 & 154.6 & \begin{tabular}{|l|l|l|}
237.765 .8 \\
\end{tabular} & 163.3 & 147.4 & & 136.3 \\
\hline no. & 5 & & 5 & 5 & 5 & 5 & & 3 & 5 & \begin{tabular}{|l|l|}
5 & 5 \\
\end{tabular} & 7 & 5 & & \\
\hline \multirow{2}{*}{$\mathrm{HA}$ mean } & 700.4 & 724.4 & \begin{tabular}{|l|l|}
700.4724 .4 \\
\end{tabular} & 1395 & 1460 & 1395 & 1460 & 2429 & 2706 & \begin{tabular}{|l|l|}
2429 & 2706 \\
\end{tabular} & 3665 & 3710 & 3665 & 3710 \\
\hline & 52.95 & 117.9 & \begin{tabular}{|l|l|l|}
52.95 & 117.9 \\
\end{tabular} & 213 & 163.2 & 213 & 163.2 & 255 & 428 & $\begin{array}{lll}255 & 428 \\
\end{array}$ & 154.3 & 24.04 & 154.3 & 24.04 \\
\hline no. & 5 & 5 & \begin{tabular}{r|r|}
5 & 5 \\
\end{tabular} & 5 & 5 & 5 & & 4 & 5 & 4 & 3 & 2 & 3 & 2 \\
\hline \multirow[t]{3}{*}{$\sqrt{N} n$} & 656.2 & 710.6 & \begin{tabular}{|l|l|}
669.8 & 629 \\
\end{tabular} & 1269 & 1320 & 1293 & 1290 & 2454 & 2382 & 24892342 & 3608 & 3428 & 3744 & 3638 \\
\hline & 152.3 & 86.18 & \begin{tabular}{|l|l|}
55.87 & 57.65 \\
\end{tabular} & 137.4 & 137.4 & 75.07 & 157.4 & 194 & 61.7 & \begin{tabular}{|l|l|}
178 & 298 \\
\end{tabular} & 48.08 & 95.87 & 158.1 & 179.6 \\
\hline & 5 & 5 & 5 & 5 & 5 & 5 & & 5 & 3 & 4 & 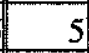 & 5 & & \\
\hline $\mathrm{KA}$ mean & 618.8 & 608.6 & 632.4 & 1504 & 1559 & 1535 & 1549 & 2232 & 2205 & \begin{tabular}{|l|l|}
2372 & 2229 \\
\end{tabular} & 3805 & 3665 & 3710 & 3799 \\
\hline sd. & 50.43 & & 74.493 & 92.37 & 147.6 & 201.3 & 185.6 & 123.4 & 146.5 & \begin{tabular}{|l|l|}
97.81 & 194.1 \\
\end{tabular} & 152.3 & 161.6 & 118.6 & 152.9 \\
\hline no. & 5 & & 5 & 5 & 5 & 5 & & 5 & 5 & \begin{tabular}{|l|l}
5 & 5 \\
\end{tabular} & 1 & 3 & 4 & \\
\hline $\mathrm{KF}$ mean & 591.6 & 618.8 & $\begin{array}{lll}611 & 624.8 \\
\end{array}$ & 1528 & 1606 & 1540 & 1545 & 2706 & 2597 & \begin{tabular}{|l|l|}
2723 & 2723 \\
\end{tabular} & 3723 & 3855 & 3696 & 3818 \\
\hline sd. & 143.4. & 53.22 & \begin{tabular}{l|l}
14.02 & 32.18 \\
\end{tabular} & 182.4 & 157.4 ? & 226.5 & 198.3 & 153.9 & 226.8 & 232.9232 .9 & 170.8 & 109.7 & 145.1 & 120.6 \\
\hline no. & 5 & 5 & & 5 & 5 & 5 & 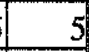 & 5 & 5 & 5 & 4 & 4 & & \\
\hline $\mathrm{KH}$ mean & 676.6 & & 690.2 & 1426 & & 1484 & & 2468 & & 2611 & 3443 & & 3826 & \\
\hline sd. & 42.33 & & 99.56 & 143.4 & & 167.1 & & 196.1 & & 286.2 & 315.4 & & 134.1 & \\
\hline no. & 5 & & 5 & 5 & & 5 & & 5 & & 5 & 5 & & 5 & \\
\hline KO mean & 707.4 & & \begin{tabular}{|l|l|}
707.639 .2 \\
\end{tabular} & 1446 & & 1422 & 1426 & 2546 & & \begin{tabular}{|l|l|}
2519 & 2508 \\
\end{tabular} & 3679 & & 3696 & 3693 \\
\hline sd. & 102.8 & & \begin{tabular}{|l|l|}
214.7 & 124.7 \\
\end{tabular} & 123.6 & & 180.4 & 150.2 & 312 & & 115.3249 .8 & 182.1 & & 145.9 & 108.2 \\
\hline no. & 5 & & \begin{tabular}{r|r}
5 & 5 \\
\end{tabular} & 5 & & 5 & 5 & 5 & & 5 & 5 & & 5 & 5 \\
\hline $\mathrm{MN}$ mean & 769.5 & 707.2 & 768.6802 .6 & 1385 & 1436 & 1450 & 1416 & 2873 & 2937 & \begin{tabular}{|l|l|}
2788 & 2651 \\
\end{tabular} & 3774 & 3833 & 3782 & 3872 \\
\hline sd. & 49.26 & 45.93 & \begin{tabular}{|l|l|}
47.52 & 42.71 \\
\end{tabular} & 90.36 & 172.7 & 179.7 & 191 & 161.9 & 343 & 104.4244 .1 & 56.17 & 80.19 & 108.7 & 9.44 \\
\hline no. & 4 & & 5 & 5 & 5 & 5 & 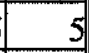 & 5 & 5 & 5 & 4 & & 4 & \\
\hline TN mean & 639.2 & 652.8 & \begin{tabular}{|l|l|}
625.6686 .8 \\
\end{tabular} & 1593 & 1582 & 1569 & 1487 & 2645 & 2580 & \begin{tabular}{|l|l|}
2648 & 2631 \\
\end{tabular} & 3642 & 3720 & 3758 & 3639 \\
\hline sd. & 84.66 & 30.88 & 67.3679 .37 & 195.3 & 120.6 & 122.1 & 144.5 & 253.2 & 109.3 & \begin{tabular}{|l|l|}
115.7 & 177.7 \\
\end{tabular} & 217.3 & 219.9 & 85.34 & 163.7 \\
\hline no. & 5 & 5 & 5 & 5 & 5 & 5 & 5 & 5 & 5 & 5 & 4 & 5 & 5 & 5 \\
\hline \multirow[b]{4}{*}{ Imeal } & 615.4 & 632.4 & 565.3599 .3 & 1252 & 1276 & 1307 & 1317 & 2672 & 2635 & \begin{tabular}{|l|l|}
2675 & 2566 \\
\end{tabular} & 3750 & 3744 & 3867 & 3755 \\
\hline & 14.22 & 76.41 & \begin{tabular}{|l|l|}
29.03 & 56.17 \\
\end{tabular} & 158.8 & 178.3 & 253.5 & 232.1 & 130 & 138.8 & 134.6168 .4 & 110.6 & 266.2 & 67.11 & 221.9 \\
\hline & 5 & & \begin{tabular}{|l|l}
4 & 4 \\
\end{tabular} & 5 & 5 & 5 & 5 & 5 & 5 & \begin{tabular}{|l|l|}
5 & 5 \\
\end{tabular} & 3 & 4 & 4 & 3 \\
\hline & 656.2 & 710.6 & \begin{tabular}{|l|l|}
669.8 & 629 \\
\end{tabular} & 1269 & 1320 & 1293 & 1290 & 2232 & 2205 & \begin{tabular}{|l|l|}
2372 & 2229 \\
\end{tabular} & 3608 & 3428 & 3744 & 3638 \\
\hline \multirow[t]{2}{*}{$\overline{\text { sd. }}$} & 152.3 & 86.18 & \begin{tabular}{|l|l}
55.87 & 57.65 \\
\end{tabular} & 137.4 & 137.4 & 75.07 & 157.4 & 123.4 & 146.5 & \begin{tabular}{|l|l|}
97.81 & 194.1 \\
\end{tabular} & 48.08 & 95.87 & 158.1 & 9.6 \\
\hline & 5 & & 5 & 5 & 5 & & & & 5 & \begin{tabular}{|l|l|}
5 & 5 \\
\end{tabular} & 5 & 5 & & \\
\hline
\end{tabular}




\begin{tabular}{|c|c|c|c|c|c|c|c|c|c|c|c|c|c|c|c|c|}
\hline \multirow[b]{2}{*}{ i/ } & \multicolumn{4}{|c|}{ F1 } & \multicolumn{4}{|c|}{$F 2$} & \multicolumn{4}{|c|}{ F3 } & \multicolumn{4}{|c|}{ F4 } \\
\hline & $\operatorname{sir} 1$ & $\mathrm{~s} 1 \mathrm{n} 2$ & $\mathrm{~s} 2 \mathrm{r} 1$ & $s 2 \times 2$ & tr1 & $\sin 2$ & $\mathrm{~s} 2 \mathrm{r} 1$ & $\mathrm{~s} 2 \mathrm{r} 2$ & slr1 & slr2 & $\mathrm{s} 2 \times 1$ & $s 2 \mathrm{r} 2$ & $\operatorname{sir} 1$ & sir 2 & $\mathrm{~s} 2 \mathrm{r} 1$ & $s 2 \times 2$ \\
\hline \multirow{3}{*}{ AA me } & 289 & 346.8 & 326.4 & 333.2 & 2001 & 2079 & 2093 & 2079 & 2856 & 2939 & 2944 & 3012 & 3458 & $\begin{array}{l}3594 \\
\end{array}$ & $\begin{array}{l}43549 \\
4\end{array}$ & \begin{tabular}{l|l|}
9737 \\
\end{tabular} \\
\hline & 34 & 58.4 & 50.14 & 53.22 & 104.5 & 66.28 & 60.5 & 142.9 & 198 & 131.6 & 242.8 & 225.9 & 152.7 & 159.2 & 2165.9 & 9216.9 \\
\hline & 5 & 5 & 5 & & \begin{tabular}{|l|}
5 \\
\end{tabular} & 5 & & & 5 & 5 & 5 & 5 & 5 & 5 & & $5 \quad 5$ \\
\hline \multirow[t]{3}{*}{$\mathrm{m}$} & 353.6 & 294.6 & 309.4 & 316.2 & 2454 & 2543 & 2532 & 2421 & 3200 & 3090 & 3021 & 3073 & 3770 & 3767 & 73812 & 23835 \\
\hline & 62.92 & 56.19 & 25.22 & 48.98 & 233.7 & 130.1 & 228.7 & 111 & 168 & 109 & 470 & 170 & 90.49 & 9.815 & 524.04 & 485.31 \\
\hline & 5 & 5 & & & \begin{tabular}{|l|}
5 \\
\end{tabular} & 5 & & & 3 & 5 & 2 & & 4 & & ? & \\
\hline \multirow{3}{*}{$\mathrm{IN}$ me } & 306 & 295.8 & 299.2 & 302.6 & 1933 & 2008 & 1954 & 2011 & 3025 & 3167 & 3005 & 2883 & 3373 & 3363 & $3 \quad 3435$ & \begin{tabular}{|l|l}
5 & 3577 \\
\end{tabular} \\
\hline & 17 & 19.38 & 25.78 & 42.33 & 191.4 & 128.9 & 84.49 & 81 & 213 & 235 & 222 & 191 & 149.7 & 45.93 & 3152.2 & 2154.6 \\
\hline & 5 & 5 & 5 & & 5 & 5 & & & & 5 & 5 & & & & & \\
\hline \multirow{3}{*}{$\mathrm{KA} \mid \mathrm{m}$} & 326.4 & 326.4 & 289 & 289 & 2151 & 2001 & 2195 & 2212 & 2434 & 2630 & 2604 & 2716 & 3821 & 3693 & 33692 & 23646 \\
\hline & 72.52 & 47.17 & 36.06 & 24.04 & 125.8 & 257.6 & 122 & 196.2 & 126.9 & 32.55 & 246.8 & 195.9 & 143.2 & 277.5 & 186.9 & 9229.9 \\
\hline & 5 & & 5 & & 5 & 5 & & & 4 & 4 & 5 & & 4 & 5 & 5 & 5 \\
\hline \multirow[t]{3}{*}{$\mathrm{KF}=\mathrm{m}$} & 312.8 & 292.4 & 258.4 & 241.4 & 2161 & 2467 & 2239 & 2290 & 2995 & 2900 & 2859 & 2886 & 3706 & 3852 & 23758 & 83787 \\
\hline & 51.84 & 32.7 & 18.62 & 22.17 & 185.6 & 271.5 & 85.51 & 117.3 & 219 & 347.7 & 197.9 & 204.2 & 218.3 & 211.2 & 2156.5 & 5104.3 \\
\hline & 5 & 5 & 5 & & 4 & & 5 & 5 & 5 & 5 & 5 & & 4 & 3 & \begin{tabular}{|l|l|}
3 & 5 \\
\end{tabular} & \begin{tabular}{|l|l|}
5 & 4 \\
\end{tabular} \\
\hline $\mathrm{KH}$ mean & 255 & & 306 & & 2052 & & 2165 & & 2764 & & 2947 & & 3665 & & 3849 & \\
\hline sd. & 20.82 & & 20.82 & & 67.57 & & 171.9 & & 74.68 & & 143.9 & & 222.7 & & 76.41 & \\
\hline no. & 5 & & 5 & & 5 & & & & 5 & & 5 & & & & 5 & \\
\hline $\mathrm{KO}$ mean & 323 & & 306 & 346.8 & 2022 & & 1846 & 2042 & 2757 & & 2725 & 2856 & 3673 & & 3650 & 3676 \\
\hline sd. & 38.01 & & 24.04 & 30.88 & 138.8 & & 289.1 & 136 & 166.6 & & 177.81 & 114.9 & 107.1 & & 203.9 & 94.65 \\
\hline no. & 5 & & 5 & & 5 & & & & 5 & & $\pi$ & & 4 & & 5 & \\
\hline $\mathrm{MN}$ mean & 329.8 & 350.2 & 367.2 & 397.2 & 2469 & 2463 & 2359 & 2325 & 3128 & 3155 & 3019 & 3145 & 3731 & 3574 & 3485 & 3498 \\
\hline sd. & 25.78 & 15.21 & 54.56 & 63.98 & 159.8 & 105.4 & 136.7 & 208.3 & 208.1 & 132.5 & 221.7 & 95.96 & 192.5 & 158.3 & \begin{tabular}{|l|}
3 \\
\end{tabular} & 5108.2 \\
\hline no. & 5 & 5 & 5 & & 5 & 5 & 5 & 5 & 5 & 5 & 5 & & 4 & 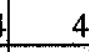 & $4 \quad 4$ & $\begin{array}{l}4 \quad 2 \\
\end{array}$ \\
\hline TN mean & 241.4 & 285.6 & 258.4 & 244.8 & 2151 & 2226 & 2202 & 2205 & 2818 & 2759 & 2784 & 2958 & 3805 & 3428 & 3690 & 3679 \\
\hline sd. & 67.36 & 99.42 & 36.86 & 54.56 & 161.1 & 86.18 & 124.1 & 62 & 264.6 & 260.3 & 182.5 & 237.3 & 103.8 & 325.7 & 7192.9 & 9192.9 \\
\hline no. & 5 & 5 & 5 & & 5 & & 5 & 7 & 5 & 5 & 5 & & 5 & & 5 & $5 \quad 5$ \\
\hline TS mean & 323 & 336.6 & 251.6 & 278.8 & 2188 & 2243 & 2188 & 2161 & 3039 & 2975 & 2903 & 2886 & 3804 & 3774 & 43816 & 63863 \\
\hline sd. & 31.8 & 40.59 & 30.41 & 148.98 & 111.9 & 76.41 & 40.94 & 110.2 & 210.4 & 184.4 & 139.5 & 121.8 & 143.2 & 80.19 & 156.7 & 795.16 \\
\hline no. & 5 & 5 & 5 & & 5 & 5 & 5 & 5 & 5 & 5 & 5 & & 4 & 4 & 4 & 4 \\
\hline TY mean & 306 & 295.8 & 299.2 & 302.6 & 1933 & 2008 & 1954 & 2011 & 2434 & 2630 & 2604 & 2716 & 3373 & 3363 & 3435 & 5577 \\
\hline sd. & 17. & 19.38 & 25.78 & 42.33 & 191.4 & 128.9 & 84.49 & 81 & 126.9 & 32.55 & 246.8 & 195.9 & 149.7 & 45.93 & 152.2 & 154.6 \\
\hline no. & 5 & 5 & 5 & & 5 & 5 & 5 & 5 & 4 & 4 & 5 & 5 & 5 & 5 & 5 & \\
\hline
\end{tabular}




\begin{tabular}{|c|c|c|c|c|c|c|c|c|c|c|c|c|c|c|}
\hline \multirow[b]{2}{*}{$/ 4 /$} & \multicolumn{3}{|c|}{$F 1$} & \multicolumn{4}{|c|}{$F_{2}$} & \multicolumn{3}{|c|}{ F3 } & \multicolumn{4}{|c|}{ F4 } \\
\hline & $\sin 1$ & $\sin 2$ & $\mathrm{~s} 2 \mathrm{rl} \mid \mathrm{s} 2 \mathrm{r} 2$ & slrl & $\sin 2$ & $52 \mathrm{rl}$ & $s 2 r 2$ & slr1 & $\sin 2$ & $\$ 2 r 1 \quad \$ 2 r 2$ & slr1 & slr2 & $s 2 \mathrm{r} 1$ & $\$ 2 \times 2$ \\
\hline \multirow[t]{3}{*}{ AAn } & 301.8 & 329.8 & 306333,2 & 1350 & 1415 & 1612 & 1541 & 2195 & 2185 & $2 2 5 5 \longdiv { 2 2 3 6 }$ & 3298 & 3322 & 3315 & 3390 \\
\hline & 29.03 & 37.25 & 31.0444 .33 & 171.1 & 65.18 & 156.5 & 42.67 & 171.7 & 35.25 & 81.5333 .14 & 147 & 81.88 & 371.29 & 154.1 \\
\hline & 4 & 5 & 4 & 3 & 5 & 4 & & 4 & 5 & 4 & 4 & 5 & & \\
\hline \multirow[t]{3}{*}{$\mathrm{HAm}$} & 382.5 & 328.7 & 3627317.3 & 1651 & 1662 & 1736 & 1475 & 2646 & 2819 & 25082587 & 3659 & & 2910 & 3863 \\
\hline & 12.02 & 42.78 & 25.9754 .6 & & 168.3 & & 332.8 & 275.8 & 2112 & $93.63274,1$ & 408.7 & & & 48.08 \\
\hline & 2 & 3 & $3 \quad 3$ & 1 & 3 & 1 & & 2 & & \begin{tabular}{|l|l}
3 & 3 \\
\end{tabular} & 2 & & & \\
\hline \multirow[t]{3}{*}{$\mathrm{IN} \mathrm{n}$} & 357 & 362.7 & 368.3 & 1387 & 1338 & 1248 & 1438 & 2411 & 2757 & 20592087 & 3585 & 3634 & 43574 & 3540 \\
\hline & 24.04 & 35.39 & 25.9736 .72 & 147.9 & 142.6 & 216.6 & 141.9 & 374 & & 74.193 .6 & 221,9 & 444.8 & 2.5 & 96.17 \\
\hline & 4 & 3 & $\begin{array}{l}34 \\
\end{array}$ & $A$ & 3 & & & 3 & & 3 & 3 & & & \\
\hline \multirow[t]{3}{*}{$A$} & 362.7 & 340 & \begin{tabular}{l|l}
340 & 340 \\
\end{tabular} & 1725 & 1696 & 1634 & 1724 & 2127 & 2240 & 22352305 & 3670 & & 3807 & 3702 \\
\hline & 35.39 & 34 & 51 & 110.6 & 143.1 & 133.4 & 171.6 & 156.4 & 93.63 & \begin{tabular}{l|l|l|l}
172.5 & 107,7 \\
\end{tabular} & 320.9 & & 205.4 & 108.2 \\
\hline & 3 & 3 & 3 & 3 & 3 & 3 & & 4 & & 3 & 3 & 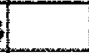 & 3 & 2 \\
\hline \multirow{3}{*}{$\mathrm{KF}$} & 335.8 & 297.5 & 297.5260 .7 & 1753 & 1553 & 1608 & 1787 & 2365 & 2337 & 23542291 & 3330 & 3736 & 5485 & 3676 \\
\hline & 32.18 & 51 & 35.3951 .94 & 162.6 & 356.9 & 208.5 & 106.2 & 95.1 & 35.39 & 64.3685 .56 & 437.7 & 236.6 & 231.6 & 192.3 \\
\hline & 4 & 4 & 4 & 3 & 4 & 4 & & 3 & 3 & 3 & 3 & 4 & & \\
\hline \multirow{3}{*}{$\mathrm{KHT}$} & 329.8 & & 340 & 1589 & & 1595 & & 2529 & & 2468 & 3506 & & 3614 & \\
\hline & 45.93 & & 13.88 & 117.4 & & 287.4 & & 122.7 & & 89.32 & 43.34 & & 83.86 & \\
\hline & 5 & & 4 & 5 & & 4 & & 5 & & 4 & 5 & & 3. & \\
\hline KO mean & 344.3 & & 3402294.7 & 1676 & & 1830 & 1827 & 2498 & & 21872257 & 3595 & & 3182 & 3188 \\
\hline sd. & 29.03 & & 035.39 & 281.7 & & 36.06 & 76.66 & 277.1 & & 12.0278 .52 & 100.5 & & 24.04 & 52.37 \\
\hline no. & 4 & & $\begin{array}{ll}2 & 3 \\
\end{array}$ & 4 & & 2 & & 4 & & 23 & 4 & & -4 & 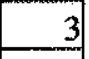 \\
\hline MNImean & 404.6 & 411.4 & 397.8421 .6 & 1371 & 1480 & 1524 & 1497 & 2639 & 2726 & 26792635 & 3652 & 3544 & 43550 & 3533 \\
\hline sd. & 14.22 & 32.7 & 48.98477 .17 & 151.4 & 120.6 & 306.5 & 278.5 & 246.4 & 167.2 & 65.470 .5 & 281.6 & 71.29 & 9166.8 & 271.1 \\
\hline no. & 피 & 5 & 5 & 5 & 5 & 5 & & 5 & 5 & 5 & 5 & 4 & 5 & \\
\hline $\mathrm{TN}$ mean & 204 & 331.5 & 233.8260 .7 & 1656 & 1451 & 1442 & 1560 & 2428 & 2468 & 24642399 & 3540 & 3307 & 3723 & 3523 \\
\hline sd. & 17) & 147.9 & 21.3935 .39 & 434.7 & 406.7 & 481.7 & 139 & 86.03 & 340.5 & 174.551 .5 & 68 & 338 & 118.9 & 253.9 \\
\hline no. & 3 & 4 & 4 & 3 & 4 & & 3 & 3 & 3 & \begin{tabular}{|l|l|} 
& 3 \\
\end{tabular} & 3 & & 3 & 3 \\
\hline Ts mean & 345.7 & 340 & $279.3 \quad 289$ & 1435 & 1583 & 1693 & 1638 & 2507 & 2451 & 25022698 & & 3704 & 43676 & 3727 \\
\hline sd. & 19.63 & & 31.3244 .98 & 125.3 & 90.52 & 142.4 & 167.6 & 193.7 & 45.54 & 25384.15 & & 137.4 & 465.11 & 120.2 \\
\hline no. & 3 & 3 & 43 & 3 & 3 & 4 & & 3 & 3 & 42 & & & \begin{tabular}{|l|l|} 
& 4 \\
\end{tabular} & \\
\hline TY mean & 357 & 362.7 & \begin{tabular}{|l|l|}
368.3 & 357 \\
\end{tabular} & 1387 & 1338 & 1248 & 1438 & 2127 & 2240 & 22352305 & 3585 & 3634 & 43574 & 3540 \\
\hline sd. & 24.04 & 35.39 & 25.9736 .72 & 147.9 & 142.6 & 216.6 & 141.9 & 156.4 & 93.63 & 172.5107 .7 & 221.9 & 444.8 & 312.5 & 96.17 \\
\hline no. & 4 & 3 & 3 & & & & & & 3 & $34 \quad 4$ & & & & \\
\hline
\end{tabular}




\begin{tabular}{|c|c|c|c|c|c|c|c|c|c|c|c|c|c|c|}
\hline \multirow[b]{2}{*}{ lel } & \multicolumn{3}{|c|}{ F1 } & \multicolumn{4}{|c|}{$\mathrm{F} 2$} & \multicolumn{3}{|c|}{ F3 } & \multicolumn{4}{|c|}{$\mathrm{F} 4$} \\
\hline & slr1 & $\sin 2$ & \begin{tabular}{|l|l}
$\mathrm{s} 2 \mathrm{r} 1$ & $\mathrm{~s} 2 \mathrm{r} 2$ \\
\end{tabular} & slrt & $\sin 2$ & $\mathrm{~s} 2 \mathrm{r} 1$ & $\mathrm{~s} 2 \mathrm{r} 2$ & slr1 & $s 1 \times 2$ & \begin{tabular}{|l|l}
$\mathrm{s} 2 \mathrm{r} 1$ & $\mathrm{~s} 2 \mathrm{r} 2$ \\
\end{tabular} & slr1 & $\operatorname{sir} 2$ & $\mathrm{~s} 2 \mathrm{r} 1$ & $\mathrm{~s} 2 \mathrm{r} 2$ \\
\hline $\mathrm{AA}$ mean & 707.6 & 741.6 & \begin{tabular}{|l|l|}
728 & 758.6 \\
\end{tabular} & 1961 & 2042 & 2080 & 2107 & 2665 & 2788 & \begin{tabular}{|l|l|l|}
2839 \\
\end{tabular} & 3430 & 3510 & 3668 & 3659 \\
\hline sd. & 62 & 43.3 & \begin{tabular}{|l|l|}
67.4 & 27.9 \\
\end{tabular} & 111 & 47.2 & 82.1 & 31.8 & 57.2 & 69.5 & \begin{tabular}{|l|l|}
88.7 & 79.2 \\
\end{tabular} & 164 & 139 & 155 & 79.7 \\
\hline no. & 5 & 5 & 5 & 5 & 5 & & & 5 & & 7 & & & & \\
\hline \multirow{2}{*}{ HA mean } & 452.2 & 442 & \begin{tabular}{|l|l|}
445.4 & 445.4 \\
\end{tabular} & 2212 & 2236 & 2175 & 2154 & 2965 & 2953 & \begin{tabular}{|l|l|}
2893 & 2937 \\
\end{tabular} & & 3922 & 3922 & 3926 \\
\hline & 81.17 & 36.06 & \begin{tabular}{|l|l|}
38.77 & 32.7 \\
\end{tabular} & 167 & 115.7 & 87.02 & 64.29 & 62.16 & 49.07 & 43.3439 .14 & & 67.9 & 67.9 & 113.5 \\
\hline no. & 5 & 5 & 5 & 5 & 5 & 5 & & 5 & & 5 & & 4 & & \\
\hline JN mean & 411.4 & 421.6 & 391 & 1824 & 1821 & 1838 & 1886 & 2459 & 2736 & $2458 \quad 2423$ & 3702 & 3588 & 3753 & 3713 \\
\hline sd. & 38.77 & 45.62 & 31.839 .87 & 83.63 & & 73.12 & 84.49 & 125 & 157 & $39.5 \quad 41.4$ & 307.4 & 158.3 & 270 & 273.4 \\
\hline no. & 5 & 5 & 55 & 5 & 5 & & & 4 & & \begin{tabular}{l|l}
5 & 5 \\
\end{tabular} & 4 & & & \\
\hline $\mathrm{KA}$ mean & 394.4 & 418.2 & 418.2404 .6 & 2049 & 1991 & 2032 & 1967 & 2471 & 2444 & \begin{tabular}{|l|l|}
2457 & 2512 \\
\end{tabular} & 3775 & 3859 & 3982 & 23792 \\
\hline sd. & 32.7 & 62 & 66.567 .36 & 83.8 & 167 & 185.7 & 166.8 & 103.7 & 73.2 & \begin{tabular}{|l|l|}
93.25 & 54.56 \\
\end{tabular} & 261.8 & 76.52 & & 3.51 \\
\hline no. & 5 & 5 & \begin{tabular}{l|l}
5 & 5 \\
\end{tabular} & 5 & 5 & & & 5 & 5 & \begin{tabular}{|l|l|}
5 & 5 \\
\end{tabular} & 5 & & & \\
\hline $\mathrm{KF}$ mean & 353.6 & 391 & \begin{tabular}{|l|l|}
387.6 & 326.4 \\
\end{tabular} & 2178 & 2168 & 2011 & 2130 & 2631 & 2614 & \begin{tabular}{|l|l|}
2628 & 2652 \\
\end{tabular} & & 3693 & 3643 & \begin{tabular}{|l|l|}
3791 \\
\end{tabular} \\
\hline sd. & 27.93 & & \begin{tabular}{|l|l|l|}
30.41 & 34.84 \\
\end{tabular} & 77.9 & 100.3 & 89.47 & 56.89 & 181.4 & 206.4 & \begin{tabular}{|l|l}
124.1 & 62.92 \\
\end{tabular} & & 144.2 & 74.57 & 7) 140.4 \\
\hline no. & 5 & 5 & 5 & 5 & 5 & & & 5 & 5 & 5 & & 4 & & \\
\hline $\mathrm{KH}$ mean & 346.8 & & 448.8 & 1879 & & 1960 & & 2590 & & 2645 & 3649 & & 3890 & \\
\hline sd. & 68.63 & & 96.62 & 87.18 & & 36.86 & & 60.58 & & 89.63 & 16.2 & & 132 & \\
\hline no. & 5 & & 5 & 5 & & 3 & & 5 & & 5 & & & 5 & . \\
\hline KO mean & 408 & & \begin{tabular}{|l|l|}
431.8 & 445.4 \\
\end{tabular} & 1943 & & 1916 & 1909 & 2682 & & \begin{tabular}{|l|l|}
2689 & 2703 \\
\end{tabular} & 3645 & & 3666 & 365 \\
\hline sd. & 9.74 & & 25.7836 .86 & 71.95 & & 66.5 & 116.2 & 35.25 & & \begin{tabular}{|l|l|}
109.569 .47 \\
\end{tabular} & 139.9 & & 166 & 5159.2 \\
\hline no. & 5 & & 5 & 5 & & & & 5 & & \begin{tabular}{r|r}
5 & 5 \\
\end{tabular} & 5 & & 3 & 1 \\
\hline $\mathrm{MN}$ mean & 505.2 & 506.6 & 513.4 & 2229 & 2215 & 2083 & 2080 & 2956 & 2845 & $2750 \quad 2815$ & 3856 & 3666 & 3669 & 3727 \\
\hline sd. & 103.7 & 92.65 & \begin{tabular}{|l|l|}
59.38 & 85.85 \\
\end{tabular} & 163.5 & 154.1 & 121.2 & 84.01 & 72.95 & 50.14 & \begin{tabular}{|r|r|}
62 & 102.4 \\
\end{tabular} & 130.6 & 136.3 & 124.1 & \\
\hline no. & 5 & 5 & \begin{tabular}{|l|l|}
5 & 5 \\
\end{tabular} & 5 & 5 & 5 & & 5 & 3 & \begin{tabular}{|l|l|}
5 & 5 \\
\end{tabular} & 5 & 5 & 3 & \\
\hline TN mean & 326.4 & 503.2 & 377.4346 .8 & 2120 & 2147 & 2049 & 2059 & 2652 & 2624 & \begin{tabular}{|l|l|}
2611 & 2601 \\
\end{tabular} & 3853 & 3941 & 3822 & 23798 \\
\hline sd. & 56.89 & 65.4 & 51.5622 .81 & 100.3 & 130.8 & 85.51 & 78.83 & 55.61 & 55.61 & \begin{tabular}{l|l|}
127 & 81 \\
\end{tabular} & 48.98 & 41.3 & 79.37 & 742.33 \\
\hline no. & & 2 & 5 & 5 & 5 & 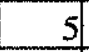 & & 5 & 5 & 5 & 5 & 5 & 5 & It \\
\hline TS mean & 445.4 & 442 & 428.4445 .4 & 1903 & 1892 & 1896 & 1831 & 2703 & 2642 & $2740 \quad 2781$ & 3931 & 3131 & 3761 & 13850 \\
\hline sd. & 22.17 & 12.02 & 40.5922 .17 & 81.88 & 100.1 & 45.93 & 90.44 & 237.8 & 203.2 & 53.7697 .36 & & & 34 & 435.05 \\
\hline no. & 5 & 5 & 5 & 5 & 5 & 5 & 5 & 5 & 5 & $\begin{array}{l}5 \\
\end{array}$ & 1 & 4 & 3 & 4 \\
\hline $\mathrm{TY}$ mean & 411.4 & 421.6 & 391 & 1824 & 1821 & 1838 & 1886 & 2471 & 2444 & $2457 \quad 2512$ & 3702 & 3588 & 3753 & 33713 \\
\hline sd. & 38.77 & 45.62 & 31.839 .87 & 83.63 & 85 & 73.12 & 84.49 & 103.7 & 73.2 & 93.2554 .56 & 307.4 & 158.3 & 270 & 273.4 \\
\hline no. & 5 & & 5 & 3 & ㄱ) & & & & & & 4 & & & \\
\hline
\end{tabular}




\begin{tabular}{|c|c|c|c|c|c|c|c|c|c|c|c|c|c|c|c|c|}
\hline \multirow[b]{2}{*}{$10 /$} & \multicolumn{4}{|c|}{ F1 } & \multicolumn{4}{|c|}{ F2 } & \multicolumn{4}{|c|}{ F3 } & \multicolumn{4}{|c|}{ F4 } \\
\hline & s1r1 & $\sin 2$ & $\mathrm{~s} 2 \mathrm{r} 1$ & $52 \mathrm{r} 2$ & rl & $\sin 2$ & $\mathrm{~s} 2 \mathrm{r} 1$ & $\mathrm{~s} 2 \times 2$ & slr1 & $\sin 2$ & $\mathrm{~s} 2 \mathrm{r} 1$ & $s 2 \times 2$ & slr1 & $\mathrm{s} 1 \mathrm{r} 2$ & $\mathrm{~s} 2 \mathrm{r} 1$ & $s 2 \times 2$ \\
\hline AA mean & 554.4 & \begin{tabular}{|l|}
779 \\
\end{tabular} & 581.6 & 622.4 & 1231 & 1434 & 252 & 1395 & 2420 & 2597 & 2570 & 2383 & 3136 & \begin{tabular}{|l|l|}
6 & 3276 \\
\end{tabular} & 53212 & 3247 \\
\hline sd. & 44.3 & 123 & 57.1 & 60.6 & 134 & 385 & 108 & 96.5 & 171 & 179 & 127 & 119 & 82.8 & \begin{tabular}{|l|l|}
8 & 98.7 \\
\end{tabular} & 117 & 267 \\
\hline no & 5 & & 5 & & 5 & & 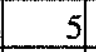 & & 5 & & & & & \begin{tabular}{|l|l|}
4 & 4 \\
\end{tabular} & & \\
\hline $\mathrm{HA}$ mean & 547 & 499.8 & 489.6 & 476 & 1079 & 1075 & 1031 & 1086 & 3050 & 2662 & 2447 & 2549 & 3880 & 03686 & 63638 & 3523 \\
\hline \multirow{3}{*}{\begin{tabular}{c|c} 
sd. \\
no. \\
\end{tabular}} & 166.2 & .15 & 52.95 & 68 & 168.2 & 138.8 & 120.6 & 116.8 & 100.2 & 213.9 & 212.2 & 282.7 & & 276.9 & 135.6 & 185.7 \\
\hline & 4 & 5 & & & 3 & 5 & 5 & & 4 & 5 & 5 & & 1 & \begin{tabular}{|l|l|}
1 & 5 \\
\end{tabular} & & \\
\hline & 418.2 & 428.4 & 411.4 & 425 & 991 & 991 & 976.4 & 966.2 & 2343 & 2462 & 2221 & 2212 & 3550 & 03564 & 3787 & 3615 \\
\hline & 42.67 & 32.7 & 34.84 & 26.88 & 151.5 & 151.5 & 224 & 191.5 & 264 & \begin{tabular}{|l|}
298 \\
\end{tabular} & \begin{tabular}{|l|} 
\\
\end{tabular} & 66.6 & 303.2 & 223.8 & 137.8 & 217.9 \\
\hline & 5 & & & & 4 & 4 & 5 & & 3 & & 4 & & 5 & & & \\
\hline KA mean & 445.4 & 435.2 & 479.4 & 476 & 1177 & 1014 & 1123 & 1120 & 2338 & 2267 & 2423 & 2362 & 3628 & $8 \mid 3412$ & 3478 & 3411 \\
\hline sd. & 7.93 & 45.93 & & 4.04 & 249.1 & 194.4 & 209.9 & 142.9 & \begin{tabular}{|l|}
141 \\
\end{tabular} & 166.8 & 158.4 & \begin{tabular}{|l|}
255 \\
\end{tabular} & 113.7 & 7323.2 & 76.66 & 235.6 \\
\hline no. & 5 & & 5 & & 5 & 5 & 5 & & 5 & \begin{tabular}{|l|}
4 \\
\end{tabular} & 5 & 5 & 5 & 4 & & \\
\hline $\mathrm{KF}$ mean & 384.2 & 377.4 & 333.2 & 346.8 & 1133 & 1154 & 1208 & 1225 & 2784 & 2681 & 2662 & 2396 & 3858 & 83948 & 3387 & 3724 \\
\hline sd. & 19.38 & 22.17 & 65.4 & 51.84 & 171.1 & 152.7 & 109.5 & 168.7 & 224.5 & 184.4 & 178.9 & 242 & 125.9 & & 232.2 & 204.1 \\
\hline no. & 5 & & 5 & & 5 & 5 & 5 & & 5 & 4 & 5 & 5 & 3 & 3 & & \\
\hline $\mathrm{KH}$ mean & 318.8 & & \begin{tabular}{|l|}
374 \\
\end{tabular} & & 1031 & & 1164 & & 2438 & & 2389 & & 3281 & & 3519 & \\
\hline \multirow{3}{*}{\begin{tabular}{|c|} 
sd. \\
no. \\
KO mean \\
\end{tabular}} & 93.18 & & 120.8 & & 153.3 & & 238.2 & & 22.4 & & 155.7 & & 178.8 & & 192.5 & \\
\hline & 5 & & 5 & & 5 & & 5 & & 4 & & 5 & & 4 & 4 & & \\
\hline & 503.2 & & 452.2 & 452.2 & 1150 & & 1103 & 1123 & 2679 & & 2553 & 2672 & 3486 & & 3462 & 340 \\
\hline \multirow{3}{*}{\begin{tabular}{|c|} 
sd. \\
no. \\
$\mathrm{MN}$ mean \\
\end{tabular}} & 171.1 & & 63.15 & 28.45 & 201.4 & & 185.2 & 181.5 & 243.6 & & 228.2 & 238.9 & 98.69 & & 92.8 & 260.9 \\
\hline & 5 & & 5 & & 5 & & 5 & & 5 & & 3 & & 5 & 5 & & \\
\hline & 547.4 & 557.6 & 527 & 567.8 & 1174 & 1167 & 1188 & 1150 & 2665 & 2512 & 2641 & 2601 & 3570 & 3484 & 3561 & 3587 \\
\hline \multirow{3}{*}{\begin{tabular}{|c|} 
sd. \\
no. \\
$T N$ \\
\end{tabular}} & 66.28 & 84.49 & 73.12 & 68.63 & 101.3 & 132 & 124 & 149.5 & 235.2 & 318.7 & 113 & 119.8 & 230.9 & 431.3 & 377.6 & 373.2 \\
\hline & 5 & 5 & & & 5 & 5 & 5 & & 5 & 5 & 5 & 5 & 5 & 5 & 4 & \\
\hline & 414.8 & 612 & 326.4 & 302.6 & 1174 & 1167 & 1188 & 1150 & 2526 & 2578 & 2584 & 2485 & 3659 & 3628 & 3598 & 3554 \\
\hline sd. & 50.43 & 43.34 & & 52.95 & 101.3 & 132 & 124 & 149.5 & 85.5 & 192 & 47.2 & 63.9 & 192 & \begin{tabular}{|l|}
2328 \\
\end{tabular} & 146 & 130 \\
\hline no. & 5 & 5 & 5 & & 5 & 5 & 5 & & 5 & 4 & 5 & 5 & 5 & 5 & If & 5 \\
\hline TS mean & 489.4 & 455.6 & 380.8 & 397.8 & 1293 & 1144 & 1011 & 1157 & 2529 & 2583 & 2621 & 2583 & 3587 & 3659 & 3489 & 3632 \\
\hline sd. & 98.98 & 14.22 & 35.25 & 105.2 & 255.6 & 197.9 & 182.5 & 129.5 & 307 & 293 & 345.6 & 195.9 & 149.1 & 1124.9 & 162.2 & 188 \\
\hline no. & 5 & 5 & & & 5 & 4 & 5 & & 5 & 4 & 3 & & 4 & 4 & 3 & \\
\hline $\mathrm{TY}$ mean & 418.2 & 428.4 & 411.4 & \begin{tabular}{|l|}
425 \\
\end{tabular} & 973 & 991. & 976 & 966 & 2338 & 2267 & 2423 & 2362 & 3550 & 3564 & 3787 & 3615 \\
\hline sd. & 67 & 32.7 & 34.84 & 26.88 & 245 & 152 & 224 & 192 & 141 & 166.8 & 158.4 & 255 & 303.2 & 223.8 & 137.8 & 217.9 \\
\hline & & & & & & & & & & & & & & & & \\
\hline
\end{tabular}

Table 4.4 Means, standard deviations, and sample size for vowel formant centre frequency in 11 male Japanese speakers' five accented short vowels.

On the basis of each speaker's means presented in Table 4.4, the overall means and standard deviations across all 11 speakers were also calculated. These are presented in Table 4.5. In this table, the far left column indicates the vowels, F1 to F4 in the first row indicate formants, and the 
second row indicates the four different recording occasions in which the data were collected. As before, "s1' and ' $\mathrm{s} 2$ ' indicate the recording sessions 1 and 2, ' $\mathrm{r} 1$ ' and ' $\mathrm{r} 2$ ' indicate the first and the second repeats within a recording session.

\begin{tabular}{|c|c|c|c|c|c|c|c|c|c|c|c|c|c|c|c|c|}
\hline & \multicolumn{4}{|c|}{$\mathrm{Fl}$} & \multicolumn{4}{|c|}{$\mathrm{F}_{2}$} & \multicolumn{4}{|c|}{$F_{3}$} & \multicolumn{4}{|c|}{$\mathrm{F} 4$} \\
\hline & slr1 & $\sin 2$ & $\$ 2 \times 1$ & $s 2+2$ & slr1 & $51 \times 2$ & $\$ 2 \pi 1$ & $s 2 \sqrt{2}$ & sir1 & $\sin 2$ & $52 \times 1$ & 5212 & sir1 & $\mathrm{s} 1 \mathrm{r} 2$ & $\$ 2 r 1$ & $52 \sqrt{2}$ \\
\hline a/mean & 665.9 & 670.6 & 663.4 & 662.4 & 1391.7 & 1425.8 & 1422.6 & 1414.4 & 2522.0 & 2527.2 & 2560.3 & 2518.5 & 3660.4 & 3660.3 & 3721.7 & 3719.4 \\
\hline $\mathrm{sd}$ & 50.7 & 44.4 & 54.8 & 62.4 & 121.6 & 134.2 & 101.9 & 975 & 195.2 & 236.8 & 140,4 & 187.1 & 103.9 & 157.6 & 107.7 & 87.4 \\
\hline$/ 1$ mean & 306.9 & 313.8 & 297.3 & 305.3 & 2137.7 & 2226.4 & 2157.0 & 2175.7 & 2859.1 & 29161 & 2855.9 & 2913.1 & 3652.6 & 3600.9 & 3651.9 & 3687.5 \\
\hline sd & 33.1 & 25.9 & 33.5 & 46.9 & 183.4 & 218.6 & 193.9 & 141.2 & 254.8 & 207.0 & 156.1 & 139.2 & 170.9 & 184.7 & 154.4 & 118.9 \\
\hline h/mean & 338.7 & 3449 & 330.3 & 323.1 & 1543,6 & 1501.8 & 1560.9 & 1592.5 & 2406.5 & 2469.2 & 2358.7 & 2380.0 & 3542.0 & 3554.4 & 3491.8 & 3568.2 \\
\hline sd & 52.1 & 31.7 & 47.3 & 49.8 & 157.7 & 130.8 & 185.2 & 1449 & 186.6 & 243.6 & 181,4 & 197.3 & 131.0 & 174.8 & 261.6 & 189.4 \\
\hline ef mean & 432,9 & 476.4 & 451.0 & 448.2 & 2011.1 & 20370 & 1988.9 & 2000.9 & 2658.6 & 2676.7 & 2651.5 & 2677.1 & 3727.0 & 3655.3 & 3775.4 & 3762.5 \\
\hline $\mathrm{sd}$ & 104.5 & 106.7 & 99.6 & 123.0 & 153.4 & 164.7 & 108.7 & 118.3 & 173.4 & 172.0 & 150.9 & 166.2 & 149.5 & 250.2 & 1153 & 85.3 \\
\hline \multirow[t]{2}{*}{$10 /$ mean } & 458.3 & 508.2 & 433.4 & 449.2 & 1127.8 & 1126.3 & 1110.9 & 1133.8 & 2555.5 & 2512.1 & 2503.1 & 2460.5 & 3562.3 & 3580.1 & 3538.0 & 3531,2 \\
\hline & 76.3 & 124.8 & 80.7 & 950 & 99.8 & 137.4 & 98.4 & 123.1 & 223.0 & 154.2 & 134.3 & 141.1 & 216.7 & 188.9 & 167.1 & 140.0 \\
\hline
\end{tabular}

Table 4.5 Means and standard deviations across all 11 speakers for each vowel / formant combination.

The overall mean F1 and F2 for the five vowels are plotted and presented in Figure 4.4.

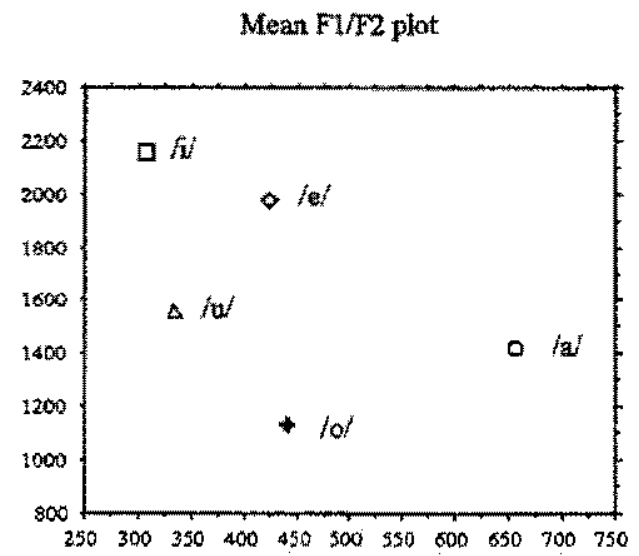

Figure 4.4 Overall mean $F 1$ and $F 2$ for five vowels. 


\subsubsection{CONTEMPORANEOUS / NON-CONTEMPORANEOUS COMPARISONS}

This section investigates contemporaneous and non-contemporaneous variation in formant frequencies. The research on the effect of the difference in recording sessions is very important, considering forensic reality. Two sets of recordings to be compared in real forensic speaker identification are always recorded on two different occasions. The research into the effect of time, which is caused by forensically realistic time difference, is thus necessary. Preceding studies, such as Rose (1999) and Nolan (1983), already have reported that difference in recording occasion can cause larger within-speaker variation. The size of time difference was three months in Nolan's study and, in Rose's experiment, the recording sessions were separated over one year. This amount of separation time, Rose's in particular, seems somewhat too long in order to apply to the real forensic situations. This section looks into the difference between contemporaneous and noncontemporaneous comparisons using a shorter, perhaps more realistic separation time of two weeks.

To evaluate the effect of time passing, the sets of data (recorded at two different sessions, repeating twice at each recording session) were compared using a repeated measures one-way ANOVA (Analysis of Variance). Repeated measures ANOVA is a modified form of ANOVA. In a regular ANOVA, the data sets to be compared have to be collected from different subjects. The repeated measures ANOVA, on the other hand, compares multiple data sets all collected from a single subject. When data is sampled from the same subjects, the variation within a dataset is expected to be smaller than that found within data sets that were sampled from different subjects. A repeated measures ANOVA takes this difference into account (Hatch and Lazaraton 1991).

In order to investigate the systematic effect of the time difference across speakers, the 11 speaker's individual means for the four different recording occasions were first calculated. These means for all speakers were then combined according to recording occasions, and these different occasions 
were then compared on the basis of those means. ANOVA was carried out for each vowel / formant combination separately. F-ratios and probabilities are presented in Table 4.6. The level of significance is set at $95 \%$, and formant / vowel combinations which reached the level of significance are indicated by shading.

\begin{tabular}{|c|c|c|c|c|c|c|c|c|}
\hline & \multicolumn{2}{|c|}{$\mathrm{F} 1$} & \multicolumn{2}{|c|}{$\mathrm{F} 2$} & \multicolumn{2}{|c|}{ F3 } & \multicolumn{2}{|c|}{ F4 } \\
\hline & F-ratio & p-value & F-ratio & p-value & F-ratio & $p$-value & F-ratio & p-value \\
\hline$/ \mathrm{a} /$ & 0.40 & 0.75 & 4 & 0.03 & 0.20 & 0.93 & 0.60 & 0.60 \\
\hline ii & 0.80 & 0.52 & 1.20 & 0.33 & 1.20 & 0.33 & 0.20 & 0.87 \\
\hline$/ 2 /$ & 2 & 0.15 & 0.50 & 0.67 & 1.30 & 0.31 & 0.60 & 0.62 \\
\hline lel & 0.90 & 0.45 & 1.20 & 0.35 & 0.50 & 0.69 & 1 & 0.43 \\
\hline $10 /$ & 1 & 0.40 & 1 & 0.42 & 3 & 0.06 & 1 & 0.40 \\
\hline
\end{tabular}

Table 4.6 Results of one-way repeated ANOVA. Shading shows the formant / vowel combination which has significant time effect at $95 \%$ confidence level.

Table 4.6 above shows that there is hardly any time effect which is consistent across speakers. There is not much variation over two weeks in vowel formant patterns. The only vowel / formant combination significantly affected by time factor was $F 2$ of the $/ a /$ vowel, although the F-ratio of the $\mathrm{F} 3$ of the /o/ vowel is also clearly large compared to the others. In order to examine the detail of this significant difference, ANOVA was followed by a Scheffé test. The result of the Scheffé test is summarised in Table 4.7 below. The comparison which reached the level of significance is indicated by shading.

\begin{tabular}{|c|c|c|c|}
\hline \multicolumn{3}{|c|}{ Comparison } & Scheffé F \\
\hline session 1 repeat 1 & vs. & session 1 repeat 2 & 1.9 \\
\hline session 1 repeat 1 & vs. & session 2 repeat 1 & 1.9 \\
\hline session ( repeat 1 & vis & session 2 repeat 2 & 3.6 \\
\hline session 1 repeat 2 & vs. & session 2 repeat 1 & 0.000079 \\
\hline session 1 repeat 2 & vs. & session 2 repeat 2 & 0.3 \\
\hline
\end{tabular}

Table 4.7 Results of the Scheffe test for F2 of the /a/ vowel.

The results above show that, out of 100 comparisons (five vowels * four formants * five possible comparisons of different occasions), only one comparison reached the level of significance. The 
significant difference was found in the $\mathrm{F} 2$ of the /a/ vowel, between the first repeat of the first recording session and the second repeat of the second recording session. Although this is a noncontemporaneous comparison, one significant difference is obviously not enough to conclude that the two weeks' time separation affects a speaker's acoustic output significantly.

The observations made in this section contradict preceding studies, such as Nolan (1983) and Rose (1999), which have reported that non-contemporaneous within-speaker variation is larger than contemporaneous within-speaker variation. The reason for this inconsistency may lie in the amount of time between two recording sessions. Nolan's two sets of recordings were separated by three months and Rose's data was recorded with one year of separation, which are much longer separation times than the two weeks used for this study.

Considering the results of the preceding research, it may be hypothesised that within-speaker variation is very small for two weeks separation but increases when the duration between the recording occasions becomes longer. Although further study is of course necessary to come to any firm conclusion on effect of the duration between recording occasions, it seems that two weeks of time difference might not cause much problem in forensic speaker identification.

\subsubsection{BETWEEN-SPEAKER VARIATION IN FORMANT PATTERNS 4.4.3.1 ANOVA results}

The main interest of this chapter is discussed here, namely, which vowel / formant combinations may serve as effective speaker identification parameters.

In order to find out which vowel / formant combinations produce a larger ratio of between-speaker variation to within-speaker variation, ANOVA was carried out. Following preceding studies (Nolan 
1983; Rose and Clermont 2000; Rose 1999a; Wolf 1972), one-way ANOVA was repeatedly performed for each vowel / formant combination in this section.

This method deserves some criticism. One obvious criticism is that repetition of ANOVA many times (20 times in this section, since four formants of five vowels were sampled) will increase the chance of producing significant values by chance. This particular part of the study is, however, interested only in the magnitude of F-ratio. Since whether or not the difference is significant is not the concern, having significant difference by chance will not cause problems here.

A second criticism is that performing ANOVA for each of the measuring points separately cannot take the interaction between vowel and formants into account, and therefore accurate comparison is not possible. The sizes of datasets for each measuring point are fairly similar in this study, however, and comparison of the sizes of F-ratios obtained by repeating ANOVA will still provide us with a rough estimation of what are the most useful measuring points for forensic speaker identification.

Although ANOVA can be criticised, it was decided to use it, because this is the method used in preceding research, specifically Wolf's study, which researched the effective acoustic parameters for speaker recognition, has been referred to by many and served as a basis of the parameter selection in this area. The fact that everyone uses it, of course, does not suggest it is an appropriate method for forensic investigation. The suitability of the method is ultimately guaranteed by the results of discrimination tests, and these were found to be satisfactory.

In this section, all 20 tokens per measuring point for each speaker (five words for each vowel * two repeats of the task * two recording sessions) are combined as one dataset, as it was demonstrated in section 4.4.2 that two weeks of time difference between recording sessions did not have a 
significant effect on acoustic output. The results of ANOVA are listed in Table 4.8. It should be noted that the number of data items for the $/ w /$ vowel is smaller than that of other vowels due to vowel devoicing, as discussed in section 4.3. The vowel / formant combination which showed larger F-ratios are indicated by shading.

\begin{tabular}{|c|c|c|c|c|}
\hline & F1 & F2 & F3 & F4 \\
\hline lal & 8 & 9 & 9 & 5 \\
\hline ii & 7 & 26 & 10 & 8 \\
\hline$/ \mathrm{W}$ & 8 & 3 & 10 & 3 \\
\hline lel & 11 & Mris & 27 & 8 \\
\hline $10 /$ & 12 & 3 & 6 & 7 \\
\hline
\end{tabular}

Table 4.8 F-ratios obtained by performing ANOVA

Table 4.8 above shows that $\mathrm{F} 2$ of $/ \mathrm{i} /, \mathrm{F} 2$ and $\mathrm{F} 3$ of /e/ with F-ratios of 26,31 , and 27 respectively, have considerably larger F-ratios than other vowel / formant combinations. It thus seems that these three vowel / formant combinations are the most promising candidates for speaker identification parameters.

\subsubsection{COMPARISON WITH PRIOR STUDIES}

First of all, despite the difference in the experimental conditions, the results of this study agree with Suzuki et al.'s (1994) findings that $/ 1 /$ and /e/ vowels are the two most useful vowels for speaker identification in Japanese.

The utilisation of vowel formants as possible speaker identification parameters has also been studied by others. For instance, Sambur (1975) reports that F2 of the front vowel and the F3 of the back vowel ho/ are the most speaker-discriminating parameters in experiments with American English. These results were further confirmed by Mokhtari and Clermont (1996) for Australian English. Mokhtari and Clermont (1996) carried out research on Australian English vowel 
formants, using a list of monosyllabic words. The words were in CVd context, where $\mathrm{C}=/ \mathrm{h}, \mathrm{b}, \mathrm{d}$, $\mathrm{g}, \mathrm{p}, \mathrm{t}, \mathrm{k} /$ and $\mathrm{V}=/ \mathrm{i}, \mathrm{I}, \varepsilon, \mathrm{a}, \mathfrak{\mathrm { x }}, \mathrm{o}, \mathrm{u}, \mathrm{v}, \partial, \mathrm{N} /(9$ Australian English vowels). Five random repetitions of each /CVd/ monosyllable were recorded. The results showed that between-speaker variability was most clearly observed in the spectral regions of the F2 of front vowels and the F3 of back vowels.

In the previous section, $\mathrm{F} 2$ of $/ \mathrm{i} /$, and $\mathrm{F} 2$ and $\mathrm{F} 3$ of /e/ were found to produce relatively high $\mathrm{F}$ ratios. This study therefore agrees with the finding by Sambur (1975) and Mokhtari and Clermont (1996) with respect to the fact that the F2 values of high vowels are potentially useful parameters for speaker identification. The current study does not entirely agree with the findings in those studies, however. Mokhtari and Clermont report that the F3 of back vowels also has a high F-ratio, whereas F3 of front vowel /e/ (and not back vowels), exhibited a large F-ratio in this study. The fact that $F 2$ of /e/ has a larger F-ratio than F2 of $/ 1$ in the current study also draws attention.

There are two possible reasons for why /u/ F3 did not produce high a F-ratio in this study. First of all, the $/ \mathrm{u} /$ vowels were devoiced by many speakers, so that the sampling number for this vowel was smaller than other vowels. This probably has affected the size of F-ratio for all formants of this vowel.

Secondly, the two studies had different methods for data collection. Sambur (1975) and Mokhtari and Clermont (1996) used a pre-prepared word list for data collection, whereas this research used natural speech. It is also possible that $\mathrm{F} 3$ of $/ \mathrm{w} /$ was in a context that was more susceptible to coarticulation than high front vowel $/ \mathrm{i} /$, because the vowels are all embedded in various phonological contexts, so that it could not produce a high F-ratio in this study. 
As to the reason for high F-ratio of /e/ here, linguistic differences may be the causing factor. English and Japanese differ in their phonological structure, including their phonemic vowel inventories. For a clear demonstration of this difference, F1 and F2 of Japanese and English vowels were plotted in Figure 4.5. The left figure plots the mean F1 and F2 of Japanese five vowels presented in Table 4.5, and the right figure plots the F1 and F2 of American English vowels. The data for American vowels are quoted from Backen (1996:358), which Backen himself has taken from the famous Peterson and Barney (1952) study. These data were collected from 33 male American English speakers reading out a list of ten words (heed, hid, head, had, hod, hawed, who'd, hud, and heard).
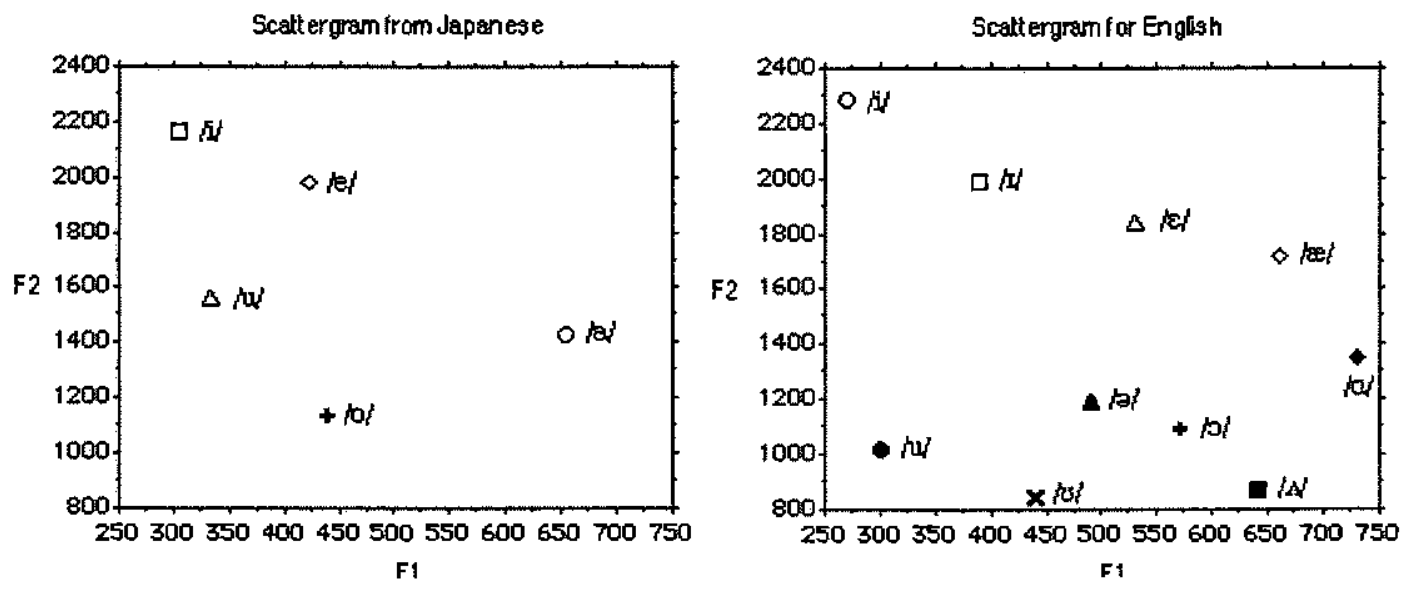

Figure 4.5 Scattergram for the mean F1 and F2 of Japanese and American English vowels. The data for Japanese vowels are the mean of all speakers in the current research, and the data for American English vowels are from Peterson and Barney (1952) quoted in Backen (1996).

Japanese clearly has a larger space for each vowel to spread than English does, as Japanese has a smaller set of vowel phonemes. The relatively large F-ratio for F2 of /e/ may thus possibly be attributed to the fact that /e/ in Japanese has more room for idiosyncrasy than the equivalent vowels in other languages with many vowel phonemes, such as English. Theoretically, a larger vowel space allows speakers more variation without confusing with other vowels, and this can result in both larger within- and between-speaker variation. As speakers cannot go beyond the physical 
limitation of their vocal range, however, the size of within-speaker variation is confined by the speaker's anatomy. The vowels produced by a single speaker will not, thus, be able to spread through the given vowel space as much as multiple speakers with different anatomy can do. If the larger vowel space influences between-speaker variation more than it influences within-speaker variation, the corresponding $F$-ratios will become larger consequently.

It must be noted here that the actual difference in vowel space between these two languages is assumed to be even larger, since Peterson and Bamey's English data were elicited from words uttered in isolation, as opposed to the Japanese data which are taken from natural speech. It is common knowledge that carefully uttered words are often associated with hyper articulations, maximally separating from each other in the vowel space, whereas naturally uttered vowels in running speech spread much less. The difference in vowel space of F2 of Japanese /e/ and English $/ \varepsilon /$ in natural speech would thus be expected to be even larger.

Altematively, the large F-ratio for /e/ vowel in Japanese may be attributed to the smaller effect of coarticulation on this vowel. Kinoshita and Maidonald (2000) have shown that $/ / /$ and /e/ vowels show less coarticulation with the phonetic environment in which they are placed. In other words, the context affects these vowels less. This characteristic may have contributed to the smaller within-speaker variation for these vowels and, consequently, to their larger F-ratios.

Finally, the high F-ratio of /e/ in Japanese in relation to that of /i/ should also be discussed. If the frontness of tongue articulation, which correlates with the position of $F 2$, is closely related to the size of within- or between-speaker variation, /i/ would be expected to produce a larger F-ratio than /el. However, the results of this study showed, otherwise. The high F-ratio of /e/ is perhaps explained as follows: first of all, articulatory gestures for the high front vowels / $/$ and /e/ equally produce less within-speaker variation than those for non-high vowels do. Then, with respect to 
between-speaker variation, /e/ has a larger vowel space than /i/ does, consequently producing the larger between-speaker variation. With regard to this size of within-and between-speaker variation, Suzuki et al.'s (1994) study on Japanese vowels showed between-speaker variation of $/ \mathrm{V} /$ to be larger than that of $/ e$, which contradicts the hypothesis presented above. However, their experimental conditions differ considerably from this study (ie. the use of vowels uttered in isolation, and analysis based on LPC Cepstrum), so perhaps this is the cause of the discrepancy. The examination of the corpus shows that there were three / / /s embedded in the same phonological environment, ie. $\mathrm{d} \_g$, whereas $\mathrm{i} / \mathrm{s}$ were embedded in five different environments. It is reasonable to think that the size of the within-speaker variation for $/ \mathrm{e} /$ was smaller for this reason. However, Kinoshita and Maindonald (2000) have demonstrated that the difference among three /e/ vowels embedded in d g enviromment did not differ much from the difference between vowels embedded in different words.

\subsection{EFFECTIVE F2 \\ 4.5.1 WHAT IS EFFECTIVE F2?}

This section discusses the possible use of effective $F 2$ as forensic phonetic parameters. Effective F2 is a formant-based acoustic parameter which approximates the auditory differences of vowels. What is attractive about effective F2 is the fact that it is formulated by incorporating the first three formants into a single figure. In other words, it serves as a dimensionality reducing function.

In the procedure of forensic speaker identification, formants are usually analysed individually, resulting in a separate evaluation for each formant. This method is, however, less than ideal. As has been discussed in Chapter 1, human speech production is a highly complicated process. Thus no single parameter known at present can distinguish speakers with strong confidence. As an evaluation based on a single formant can never be sufficient to make a statement on a given 
speaker's identity, multiple parameters must be taken into consideration. Comparing multiple values is, however, far more complex than comparing single values, and analyses based on multiple parameters are thus not an easy task. The reduction of dimensions is thus a very important process in speaker identification.

There are different approaches used to reduce dimensions. Effective F2 is a particularly attractive choice among them, as it is probably an easier concept for juries to understand than the abstract figures which are the result of complicated statistical procedures. As has been noted in the discussion of the preference towards formant patterns in Chapter 3, understandability for juries should not be neglected in forensic speaker identification.

For these reasons above, effective F2 seems to be a worthwhile parameter to investigate. This section therefore researches effective F2's candidature as a speaker identification parameter, by comparing its F-ratio with that of the conventional vowel formants.

Effective F2 was originally used as a perceptually based transformation for optimal separation of vowels in languages with high- and mid- front vowels, such as Swedish (Fant 1973). Experimenting on Swedish vowels, Fant reports that back vowels can be approximated quite well using only natural F1 and F2 but, for some other vowels (front-rounded vowels in particular) F3 and formants above are also relevant. Fant thus decided to take F3 into account for the approximation of vowel quality. The value which locates between natural F2 and F3 was thus formulated and proposed as effective $F 2$. The vowel mapping based on $F 1$ and effective $F 2$ was more successful in separating vowels, which overlap heavily in the natural F1 and F2 mapping. Fant's formula is shown as:

$$
F 2=F 2+\frac{F 3-F 2}{2} \times \frac{F 2-F 1}{F 3-F 1}
$$




\subsubsection{EFFECTIVE F2 IN JAPANESE}

Following the formula presented in the previous section, the effective F2 of the frve Japanese vowels was calculated. Each speaker's mean and standard deviations of effective $F 2$ are presented in Table 4.9. In the calculation of means and standard deviations, four different recording occasions were combined, as the earlier section (4.4.2) has demonstrated that there is hardly any significant difference between those occasions.

\begin{tabular}{|c|c|c|c|c|c|c|c|c|c|c|}
\hline \multirow[b]{2}{*}{ Speaker } & \multicolumn{2}{|c|}{ la } & \multicolumn{2}{|c|}{ fiv } & \multicolumn{2}{|c|}{$f u^{\prime}$} & \multicolumn{2}{|c|}{ el } & \multicolumn{2}{|c|}{ of } \\
\hline & mean & sd. & mean & sd. & mean & sd. & mean & sd. & mean & sd. \\
\hline $\mathrm{AA}$ & 1519.8 & 124.13 & 2352.8 & 115.62 & 1703.9 & 122.06 & 2037.7 & 486.04 & 1246.9 & 256.06 \\
\hline $\mathrm{HA}$ & 1613.1 & 189.65 & 2705.6 & 145.47 & 1885.4 & 233.06 & 2458 & 91.027 & 1271.9 & 143.62 \\
\hline $\mathbb{N}$ & 1674.4 & 109.56 & 2223.2 & 193.88 & 2270.3 & 1068.9 & 1996.6 & 81.295 & 1350,4 & 178.37 \\
\hline $\mathrm{KA}$ & 1782.4 & 161.55 & 2393.9 & 194.49 & 1906.8 & 103.67 & 2230.2 & 142.57 & 1339.2 & 232. \\
\hline $\mathrm{KF}$ & 1795.4 & 199.38 & 2541.2 & 155.48 & 1893.6 & 237.18 & 2337.4 & 98.615 & 1425.2 & 164.94 \\
\hline $\mathrm{KH}$ & 1674.6 & 170.12 & 2371.4 & 133.68 & 1845.9 & 179.19 & 2167.1 & 56.72 & 1421.9 & 327.79 \\
\hline KO & 1643.5 & 169.9 & 2242.8 & 191.94 & 1944.1 & 188.84 & 2166.7 & 69.081 & 1346.6 & 206.07 \\
\hline MN & 1656.4 & 181.09 & 2663.2 & 150.97 & 1738.1 & 214.47 & 2391.3 & 125.65 & 1384 & 139.95 \\
\hline $\mathrm{TN}$ & 1799.9 & 159.07 & 2431.9 & 135.94 & 1803.8 & 306.13 & 2291.4 & 71.765 & 1525 & 267.74 \\
\hline TS & 1508.7 & 223.75 & 2462.9 & 92.819 & 1846.1 & 152.37 & 2142.8 & 70.909 & 1406.2 & 227.4 \\
\hline$T Y$ & 1479.4 & 132.54 & 2186.2 & 128.02 & 1582.7 & 144.17 & 2061.2 & 72.717 & 1161.7 & 228.88 \\
\hline Mean & 1649.8 & 165.52 & 2415.9 & 148.94 & 1856.4 & 268.19 & 2207.3 & 124.22 & 1352.6 & 215.78 \\
\hline
\end{tabular}

Table 4.9 Mean and standard deviation of effective F2 for the short vowels of 11 Japanese males. Left-hand column shows speakers. Vowels are shown in the top row.

Plotting F1 against effective F2, Fant (1973:53) successfully distinguished 14 Swedish vowels, which had heavy overlaps when F1 was plotted against natural F2. It is of interest to see how effective F2 works in Japanese. Figure 4.6 shows each speaker's scattergram of the F1, F2 and effective F2 for five vowels. The natural F1 and F2 are plotted in the figure on left, and the natural $F 1$ and effective F2 are plotted in the figure on the right side. The unit for values on the $\mathrm{X}$ - and $\mathrm{Y}$ axes in the figures is $\mathrm{Hz}$. 
AA

[Natural F1 and natural F2]

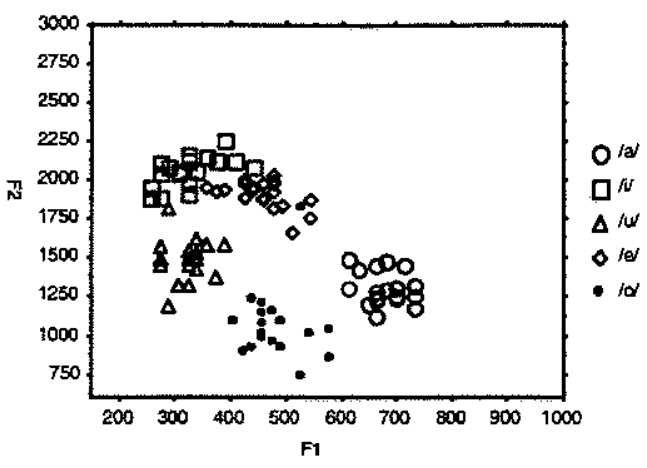

HA

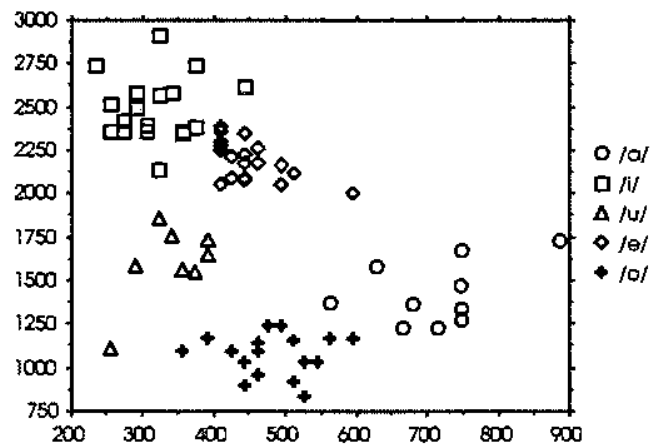

$\mathrm{JN}$

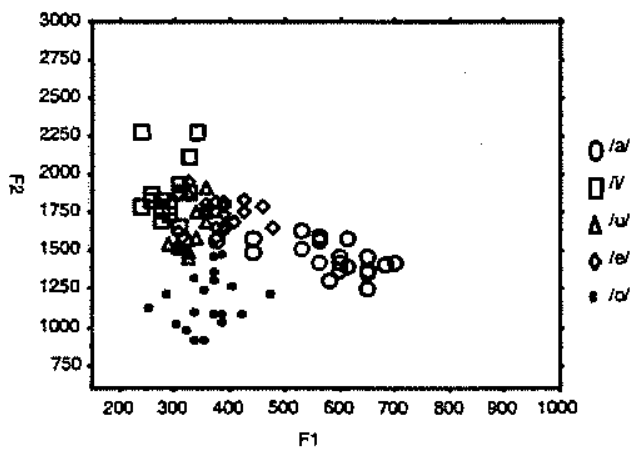

KA

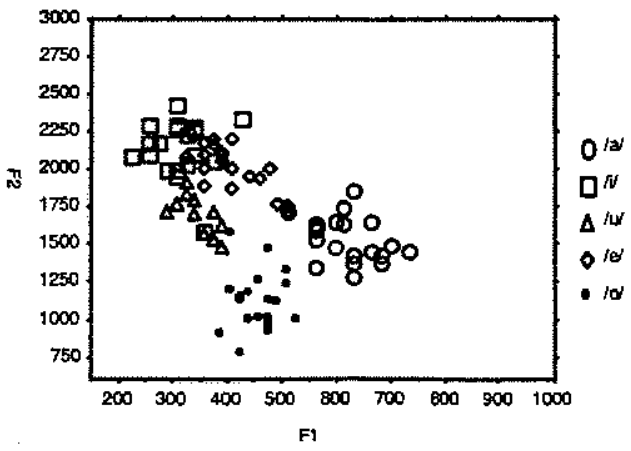

[Natural F1 and effective F2]
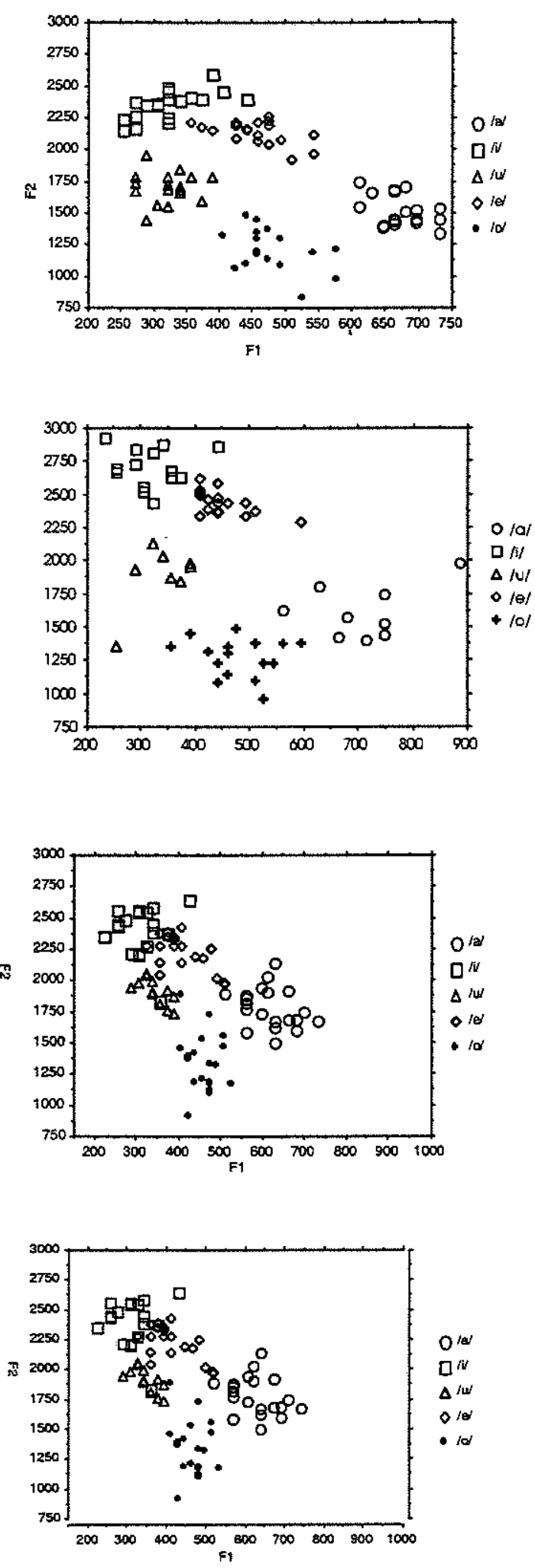


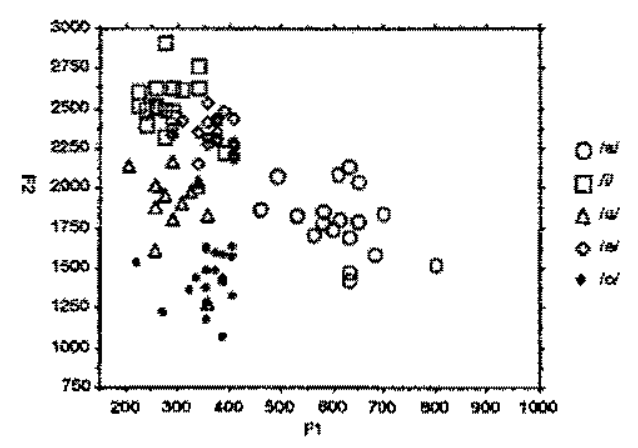

KH

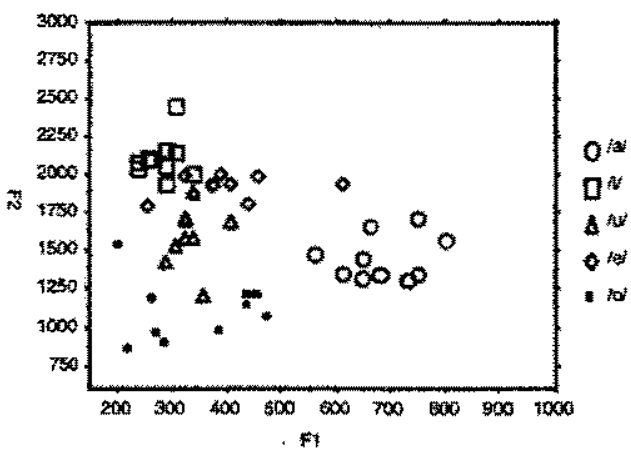

KO

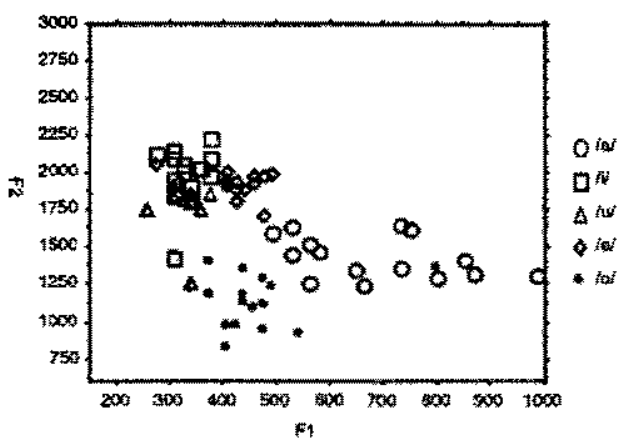

MN

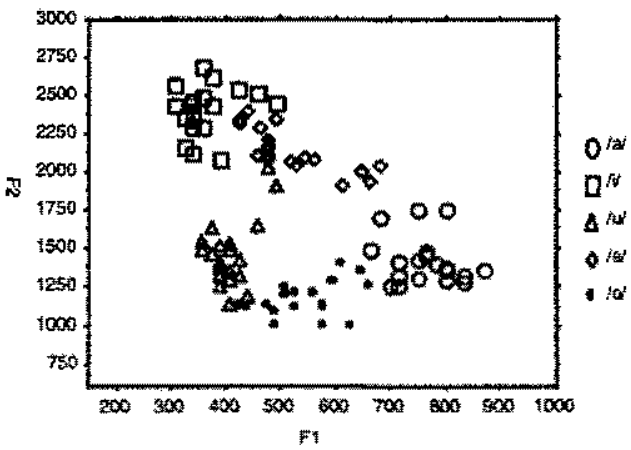

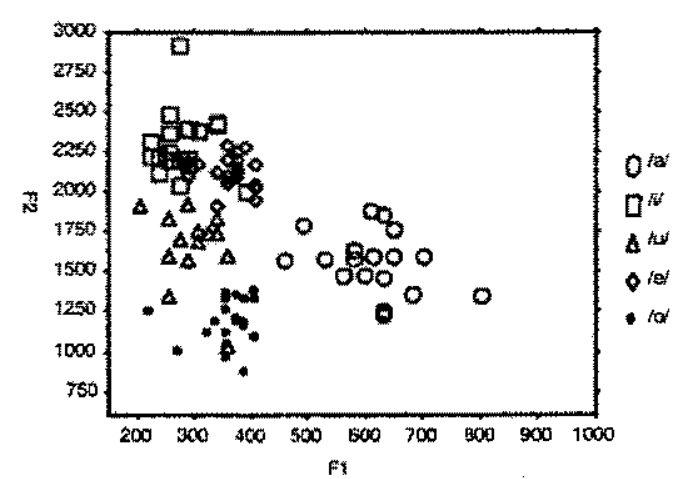
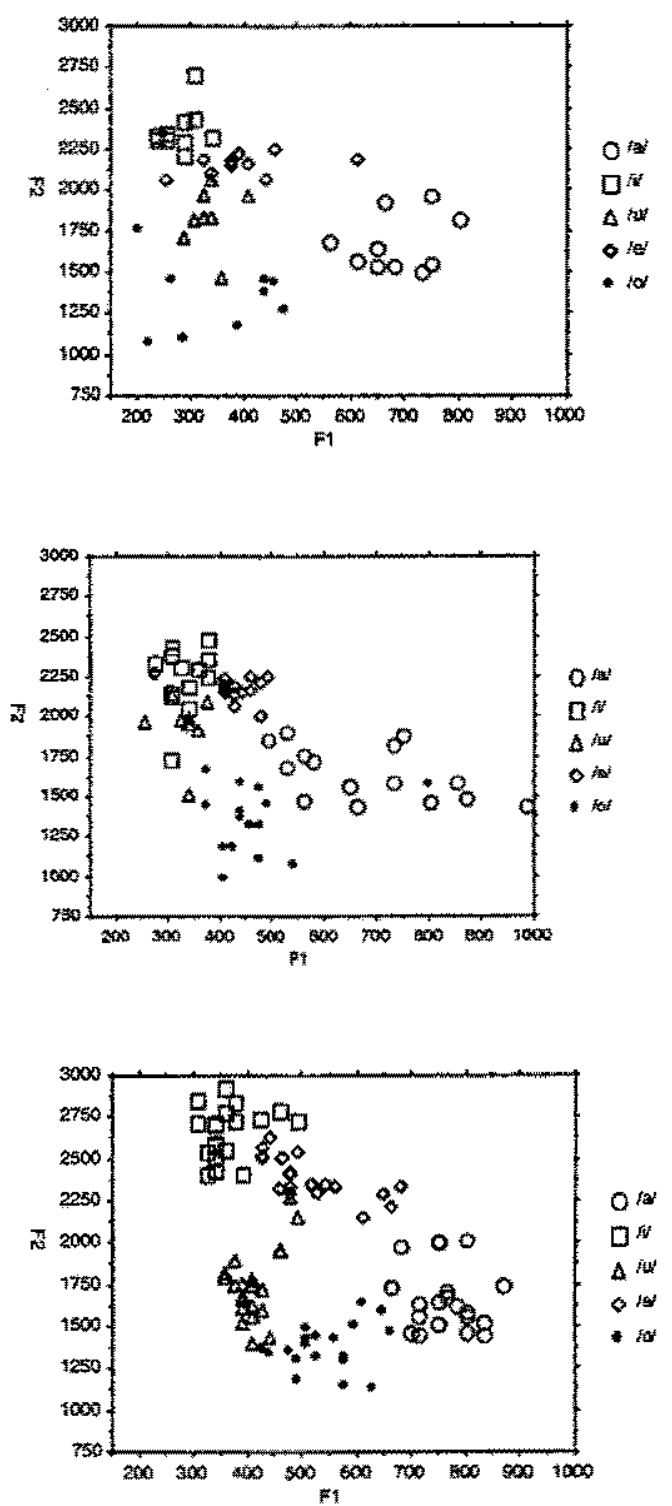
TN
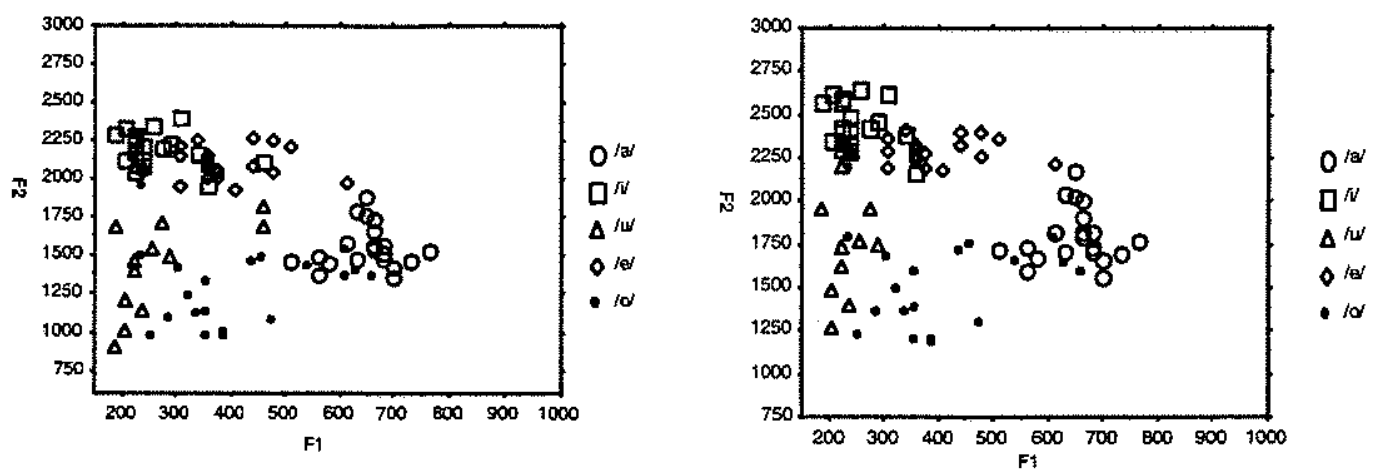

TS
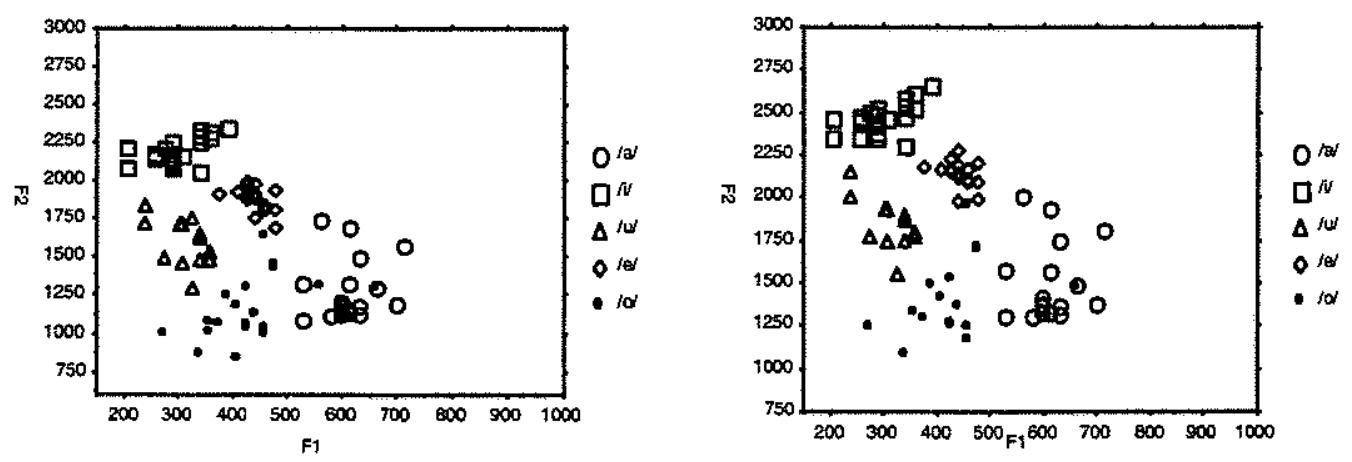

TY
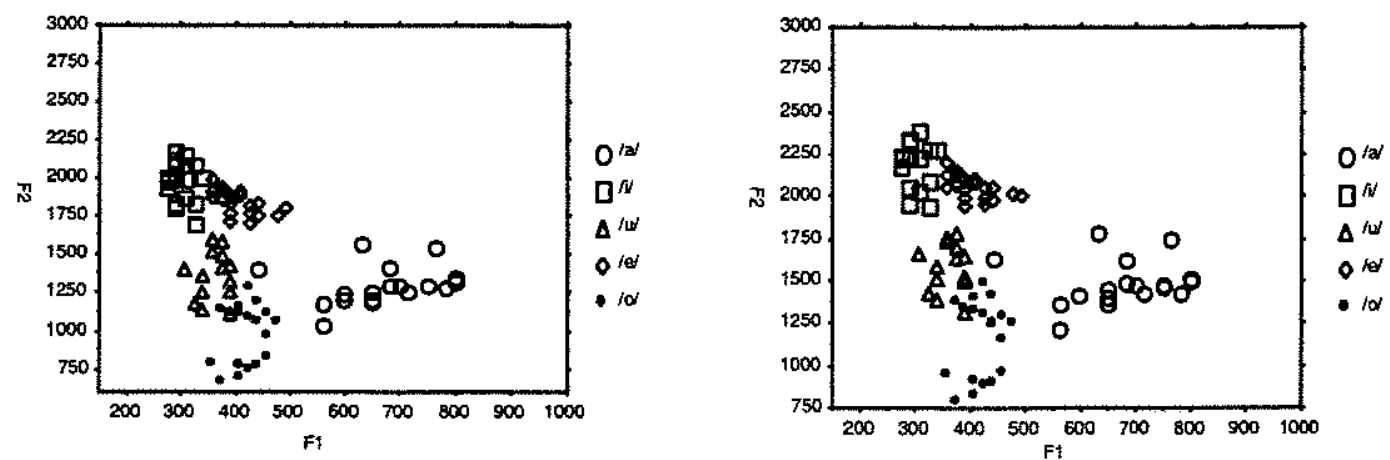

Figure 4.6 Scattergrams which plotted F1 against F2 (on right) and F1 against the effective F2 (on left), presenting the distribution of F1and F2 for all five vowels.

It is known that F1 and F2 are associated with vowel height and frontness respectively. Generally, vowels with low F1 are high vowels, and those with high F2 are front vowels. The same principle seems to apply to the scattergram with effective $\mathrm{F} 2$. 
Figure 4.6 shows that the vowels in Japanese are well separated even with natural F2. Generally speaking, however, effective F2 does seem to improve the separation between some vowels, namely $/ \mathrm{o} /$ and $/ \mathrm{w} /$ and $/ \mathrm{o} /$ and $/ \mathrm{a} /$. However, the degree of improvement from the use of effective $\mathrm{F} 2$ seems to differ slightly from speaker to speaker.

The difference between these two types of plotting is more evident when an individual's mean calculated across his 20 tokens was plotted. Figure 4.7 shows the scattergrams of each speaker's mean $F 1, F 2$ and effective $F 2$ for the five vowels investigated. The natural $F 1$ and $F 2$ values are shown in the left figure and the natural F1 and effective F2 are shown in the right figure.
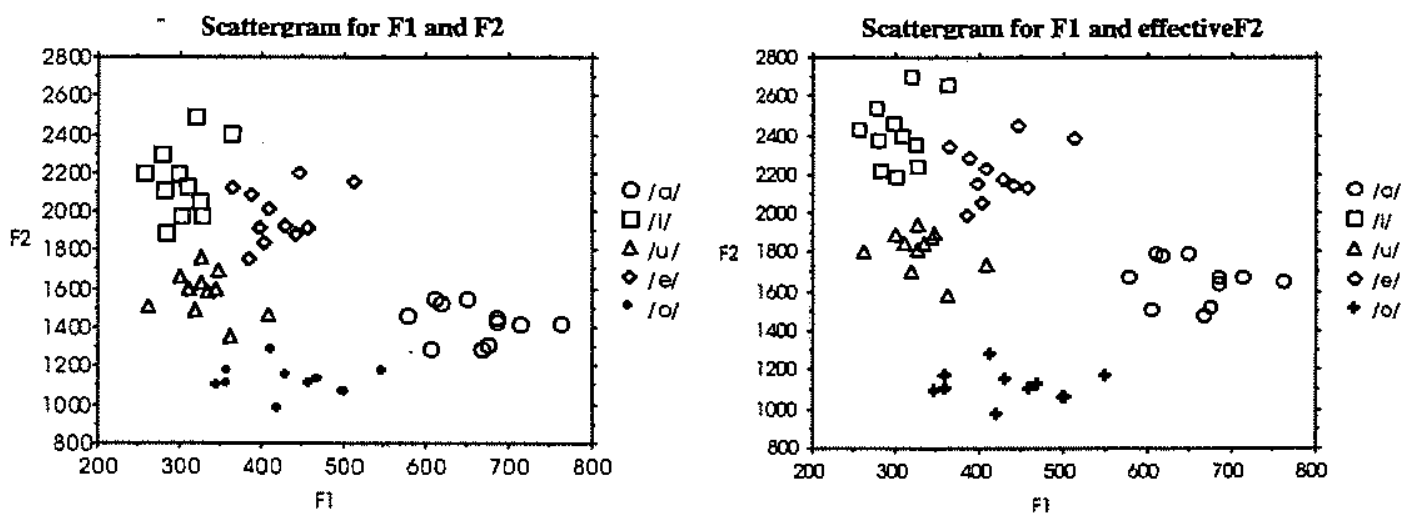

Figure 4.7 Scattergrams of mean F1 against mean F2 (on left) and mean effective F2 (on right) for each speaker's five vowels.

The differences between the two figures are not as dramatic as in Fant's Swedish results, as vowels in Japanese are fairly well separated with natural F1 and F2 in the first place. The fact that Japanese has a better separation of vowels than Swedish is hardly surprising, considering that Japanese has only 5 vowel phonemes, whereas Swedish has as many as 14 . If the vowels of these two languages have a similarly sized spread, Japanese is less likely to have overlaps, assuming that having a smaller phonemic vowel inventory does not necessarily mean that each vowel of the language will maximally utilise the given vowel space. 
A careful observation of Figure 4.7 , however, still shows that $/ \mathrm{w} /$ and $/ \mathrm{o} /$, and $/ \mathrm{o} /$ and $/ \mathrm{a} /$ are separated better in the figure plotted with effective F2. This is a noteworthy result, as Fant reports back vowels are well approximated just by natural F1 and F2, whereas front-rounded vowels require the use of effective $F 2$ for successful separation in Swedish.

Improvement in the separation of back vowels appears not to agree with Fant's observation. In Figure 4.7, the effective $F 2$ of $/ 0 /$, which is the only rounded vowel among them, seemed unchanged, whereas that of the other vowels $/ u /$ and $/ a /$ were raised. As has been noted in the discussion of the Japanese sound system (see Chapter 2), the / $/$ / vowel in Japanese is unrounded. So effective F2 maybe separates rounded vowels well in general, not just front-rounded vowels.

\subsubsection{SUITABILITY OF EFFECTIVE F2 AS A SPEAKER IDENTIFICATION PARAMETER}

The standard deviations were calculated for each speaker separately, and then the mean values across speakers were calculated in order to see the general size of within-speaker variation in effective F2. The comparison of mean standard deviations for effective F2 and natural F2 demonstrates that the effective $F 2$ of each vowel spreads in smaller space than natural $F 2$ values do. Table 4.10 shows the mean of standard deviations for raw F2 and effective F2.

\begin{tabular}{|c|c|c|c|c|c|}
\hline & $/ \mathrm{a} /$ & $/ \mathrm{i} /$ & $/ \mathrm{u} /$ & $/ \mathrm{e} /$ & $/ \mathrm{o} /$ \\
\hline Raw F2 & 165.52 & 148.94 & 268.19 & 124.22 & 215.78 \\
\hline Effective F2 & 154.93 & 144.92 & 221.30 & 86.16 & 178.28 \\
\hline
\end{tabular}

Table 4.10 Mean of standard deviations of the raw F2 and effective F2.

The fact that the standard deviation for effective $F 2$ values are smaller than that of natural $F 2$ in all vowels indicates that effective F2 has smaller within-speaker variation than natural F2. Since small 
within-speaker variation is one of the important criteria for speaker identification parameters, this is a promising result.

Then, in order to see the F-ratios of effective F2, one-way ANOVA was performed. The F-ratios of both natural formants and effective F2 are presented in Table 4.11 below. F-ratios over 20 are marked by snading.

\begin{tabular}{|c|c|c|c|c|c|}
\hline & $\mathrm{F} 1$ & F2 & 53 & F4 & $F^{2} 2$ \\
\hline$/ a /$ & 8 & 9 & 9 & 5 & 8.8 \\
\hline IV & 7 & 26 & 10 & 8 & 211 \\
\hline $\mathrm{ful}$ & 8 & 3 & 10 & 3 & 2.9 \\
\hline $\mathrm{fel}$ & 11 & 31 & 27 & 8 & 43.3 \\
\hline $1 \mathrm{~d}$ & 12 & 3 & 6 & 7 & 3.6 \\
\hline
\end{tabular}

Table 4,11. F-ratios for all vowel / formant combinations.

Table 4.11 shows that, as already revealed for natural formants, $F 2$ of $/ \mathrm{j}$, and $\mathrm{F} 2$ and $\mathrm{F} 3$ of $/ \mathrm{e} /$ have a higher F-ratio than the other formants $(26,31$, and 27 respectively). As for effective $F 2$, /e/ is shown to have a considerably larger F-ratio than any other formant. The use of the effective F2 did not improve the F-ratio of the other vowels, however. The other three vowels, namely $/ \mathrm{a} /, \mathrm{w} /$, and $10 /$, did not show a significant difference between the F-ratios of natural F2 and effective F2. As for the $/ \mathrm{i} /$ vowel, although the F-ratio of effective F2 (21.1) seems large, it is still notably smaller than that of natural F2. The generalisation "effective F2 is the better measure for obtaining high F-ratio" is, therefore, not valid.

F-ratio of 43.3 which the effective $F 2$ of /e/ produced was the highest F-ratio by far amongst all of the results collected so far. The le/ vowel was also found to be one of the promising vowels as a parameter for the study of the natural formants, and that seems to suggest more than just a fortuitous result. Table 4.11 shows that $/ \mathrm{e} /$ is the only vowel which has relatively high $\mathrm{F}$-ratios for $\mathrm{Fl}, \mathrm{F} 2$ and F3. Combining originally powerful parameters may have produced an even more powerful 
parameter. For the other vowels which did not have high F-ratios for raw F2 and F3, on the contrary, the effective F2 calculation did not improve the F-ratios. This may have been especially true for $/ \mathrm{i}$. Combining the strong parameter F2 (F-ratio 26) with less powerful parameters F3 (Fratio 10) may have resulted in a lower F-ratio (21.1). The performance of effective F2 in discrimination of speakers will be examined in Chapter 6 .

\subsection{REALISATION OF WITHIN-SPEAKER VARIATION}

Finally, this section briefly examines speaker specific characteristics in the realisation of withinspeaker variation, referring to the $F 1$ and $F 2$ distribution as an example. This is based on the idea that between-speaker variation includes not only the acoustic differences between speakers, but also includes the differences in how within-speaker variation is realised. In other words, the way that the speakers' within-speaker variation varies may serve as an effective parameter to classify speakers.

This between-speaker variation in the realisation of the within-speaker variation seems not to be a Japanese specific characteristic, and a very similar result has already been reported by Rose (1999). Rose studied long- and short-term within-speaker variation of hello uttered by the 6 general Australian speakers. As a result, he reports that, in both long- and short-term comparisons, there are between-speaker variations in terms of how many parameters produced a statistically significant between-recording session difference.

Furthermore, Suzuki et al.'s (1978) study on the whispered speech also reports between-speaker variation in the realisation of within-speaker variation. In their study, Suzuki et. al compared F1 to F4 in two different phonation types: normal and whispering. They report that formants shift when phonation changes, the size and direction of shifts, however, can vary from speaker to speaker. 
In the previous section, the scatterplots presented for demonstration of effective F2 (Figure 4.6) showed quite different patterns from speaker to speaker. This means that there are between-speaker differences in the distribution of F1 and F2. Although these differences are not quantified here, with an adequate supply of comparable vowels for both criminal and suspect's recordings, the way that within-speaker variation is realised may serve as an indicator of the speaker's identity.

To perform a comparison on the basis of this parameter, however, a substantial number of samples will be required, as the data must contain enough numbers of tokens to portray the speaker's characteristics in distribution. Thus, the practical application of this parameter may not be possible in most real forensic cases.

\subsection{SUMMARY}

This chapter investigated what is the useful vowel / formant combination for forensic speaker identification in Japanese. In addition to the natural vowel formants, effects of the time difference in recording occasions, realisation of vowel devoicing, effective $F 2$, and realisation of withinspeaker variation were also considered as possible candidates for speaker identification parameters.

As for the results of the main investigation, the search for the promising vowel $/$ formant combinations, it was revealed that the $F 2$ of $/ \mathrm{i} /$, the $\mathrm{F} 2$ and the $\mathrm{F} 3$ of / $/$ have the highest $\mathrm{F}$-ratios and are therefore suitable candidates for Japanese speaker identification.

Comparison with results of preceding research revealed disagreements in some respects. It was suggested that these disagreements might be attributed to two reasons; difference in the methodology of the experiments, and the linguistic differences between Japanese and English. 
These discrepancies imply the possibility of recording-style specific and/or language specific characteristics in speaker identification, consequently suggesting that it can be a dangerous practice for experts to base their conclusions on methods of read-out speech, or refer to experiments on languages different to those they are working on.

Additional investigations also provided us with some interesting findings. Firstly, in the analysis of the realisation of vowel devoicing, two possible ways of interpreting the realisation of vowel devoicing were suggested. The first one is a comparison in terms of the presence of vowel devoicing in the data. As a result of this comparison, the some weak regional variation was observed. The vowel devoicing, consistent absence of devoicing in particular, has a potential to contribute to the speaker identification process, although the situation where it can be used is very strictly limited, since vowel devoicing was realised in a very arbitrary way for majority of the speakers.

The other way of the interpreting vowel devoicing data is by comparing the rates of the incidence of vowel devoicing. Given that there is preceding research showing that the realisation of vowel devoicing is usually not 'all-or-nothing' phenomena (Sugito 1996), this is probably a sensible approach. The study in this chapter (section 4.3.2.2) showed that the rates of the incidence of vowel devoicing were more consistent within a speaker across sessions rather than between speaker comparisons. This suggests that the use of the rate of the incidence of vowel devoicing is a possible speaker identification parameter. The effectiveness of this parameter will be explored using Bayesian approach later in Chapter 6.

The effect of two weeks' time difference in recording occasions was also researched in this chapter. It was found largely not to cause significant differences in speakers' acoustic output. Although F2 
of $/ \mathrm{a} /$ and $\mathrm{F} 3$ of $/ \mathrm{o} /$ showed relatively large F-ratios, only one of the two non-contemporaneous comparisons of $\mathrm{F} 2$ of $/ \mathrm{a} /$ reached the $95 \%$ level of significance.

The experiments in this section suggested that effective F2 might also be useful in forensic speaker identification. The results of analysis using ANOVA showed that use of effective F2 could yield much higher F-ratios than natural formants do, although this is only the case for one vowel. In this study, only the /e/ vowel improved its F-ratio by calculation of the effective F2.

Finally, each speaker's scatterplot of F1 and F2 and effective F2 for vowels showed that three is speaker to speaker difference in the degree of within-speaker variation. Although the possibility for quantification of similarity (or dissimilarity) was not explored in this study, the potential application should be noted. Further research may be beneficial. 


\section{Chapter 5}

\section{DETERMINING EFFICIENT}

\section{FORMANT PARAMETERS FOR}

\section{MOSHIMOSHI}

\subsection{OBJECTIVE}

Similarly to Chapter 4, the main aim of this chapter is to search for possible speaker identification parameters. In this chapter, a Japanese word moshimoshi is going to be analysed. The word moshimoshi is usually translated as "hello" and is used exclusively in telephone conversations in modern Japanese. This is the most commonly used word to open a telephone conversation by both a caller and a receiver, regardless of the gender, age or social status of the speakers. As most forensic speaker identification cases involve telephone conversations (over 90\%, according to Hirson et al. 1995:230), the incidence of this word is expected to be high. The investigation of this particular word is thus considered to be highly useful.

Furthermore, the word moshimoshi includes three segments, [m], [6] and [i], which are expected to contain a significant amount of speaker specific information. Both nasal and fricative 
consonants contain a considerable amount of information about the speakers' anatomical differences (Johnson 1997: 122, 157) and the high vowel [i] has also been reported to be one of the most promising vowels to differentiate speakers (Mokhtari and Clermont 1996; Sambur 1975). The investigation in Chapter 4 also demonstrated that the $/ \mathrm{i} /$ vowel produces a high F-ratio in Japanese as well.

In addition, the comparison of segments in the same word enables the exclusion of bias caused by differences in phonological environments which may be introduced when segments in different contexts are compared, as has been reported in Kinoshita and Maindonald (2000). Speakers' identity is portrayed more effectively when the conditions outside of the investigation are held constant. The variables outside of the target of investigations will increase the range of withinspeaker variation and, consequently, the ratio of the between-speaker variation to within-speaker variation becomes smaller. This clearly inhibits the effectiveness of speaker discrimination.

The word moshimoshi is thus a potentially very useful word, containing a lot of speaker specific information and also occurring with reasonably high frequency.

This chapter also studies other aspects of this word. The effect of the two weeks' time difference between two recording sessions is investigated in this chapter again. Chapter 4 has already demonstrated that there are very few significant time effects on the acoustic output of vowels; it is still of interest to see whether the result is replicated with the comparison of moshimoshi.

Finally, the realisation of vowel devoicing and extra formants are also going to be discussed in this chapter. 


\subsection{PHONOLOGICAL DESCRIPTION OF MOSHIMOSHI}

The word moshimoshi is, as mentioned at the beginning of this chapter, an equivalent word to hello in English telephone conversations. Moshimoshi is transcribed phonemically as /mo'simosi/, carrying a pitch accent on the first syllable and in broad phonetics as [moçimoçi (HLLL)] (although the actual phonetic realisation of this word can vary speaker to speaker, as will be discussed in the later sections). As has been described extensively in Chapter 2, Japanese is a pitch accented language and can have only one pitch fall within a word. Also once pitch falls, the pitch is not allowed to rise again within a word. The pitch pattem of the whole moshimoshi is thus realised as HILL. Figure 5.1 below summarises the phonological structure of moshimoshi.

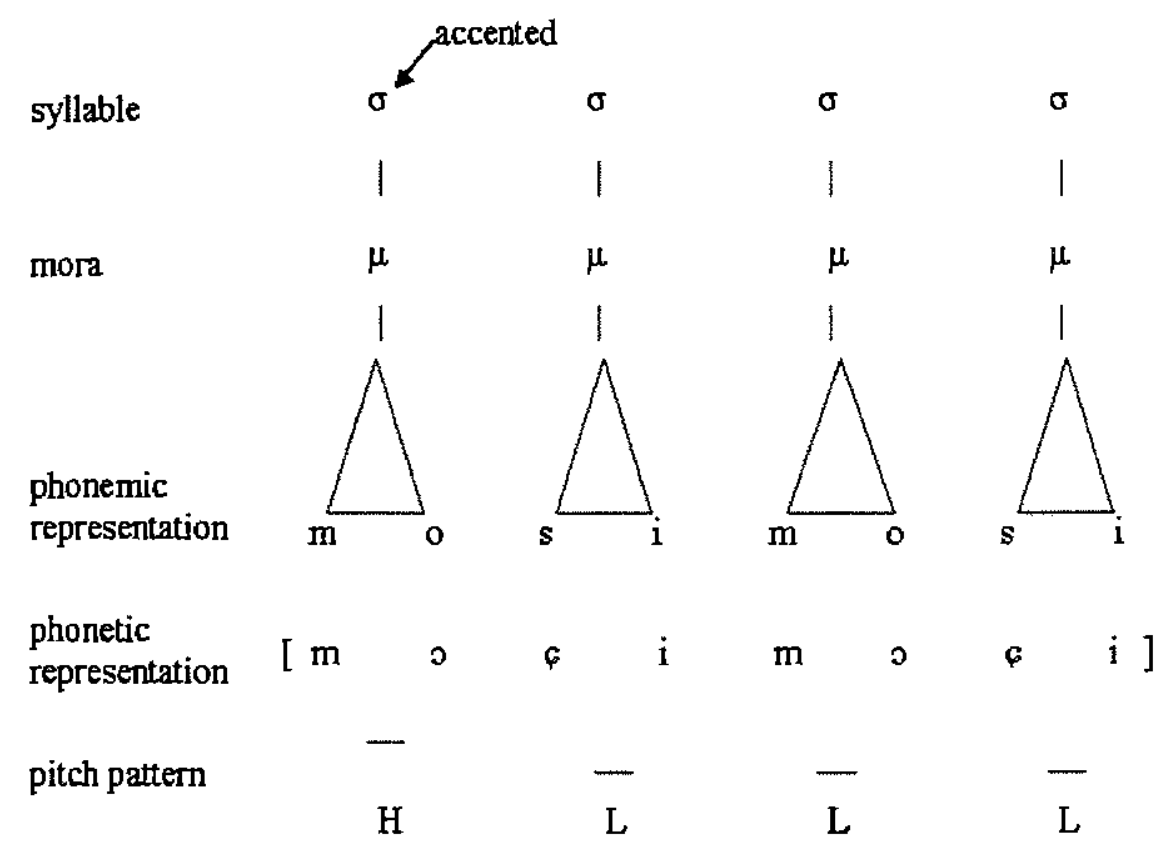

Figure 5.1 Phonological structure of moshimoshi. 


\subsection{DESCRIPTION OF CAREFULLY CITED MOSHIMOSHI AND BOSHIBOSHI}

Although researching moshimoshi is likely to be beneficial for forensic purposes, acoustic analysis of this word is not a simple task. As will be seen in the observation of carefully uttered moshimoshi in the later section of this chapter (Figure 5.4 in 5.3.2), the two nasal consonants influence the acoustic structure of other segments in the word considerably.

The main resonator of nasal consonants is the nasal cavity. The main articulatory system for nasal consonants stretches from the glottis to the nostrils. Nasal consonants, such as $/ m /$ and $/ \mathbf{n} /$, also have a branching subsystem, which is constituted by the oral cavity behind the articulatory closure (Hattori et al. 1958). The subsystem is coupled to the main system and consequently generates extra poles and antiformants which tend to attenuate the adjacent energy. The effects caused by the subsystem, therefore, can be realised as either an absence of energy or weakened energy, depending on the amplitude and the location of spectral peaks near the antiformant. In spectrograms, an antiformant would be observed as a white band if there were no formants around that region. If a formant exists in the vicinity, the antiformant will be realised as a weakened energy with a lighter colouring (Johnson 1997:151). These effects of the subsystem make the measurements of formants in nasal segments considerably more complicated than those of other segments.

The nasal consonant $/ \mathrm{m} /$ is not, however, the segment with the most complex formant structure in the word moshimoshi. Measurement of the following vowel / $/$ can be even more complicated. This vowel is, of course, phonologically not nasalised. Acoustically, however, /o/ is most likely to be nasalised, due to the influence of the preceding nasal consonant $/ \mathrm{m} /$. Johnson (1997) describes the articulation of nasalised vowels as "the most complicated configuration of the vocal tract found in speech" (p. 157). He attributes the reason for the complexity of the articulation of nasalised vowels to the fact that both the oral and the nasal cavities are open to the atmosphere at their ends. 
(In the production of nasal consonants, on the other hand, the oral cavity is closed at one end.) Figure 5.2 presents the model of the vocal tract configuration for the production of the nasal consonant [m] and the nasalised vowel [j].

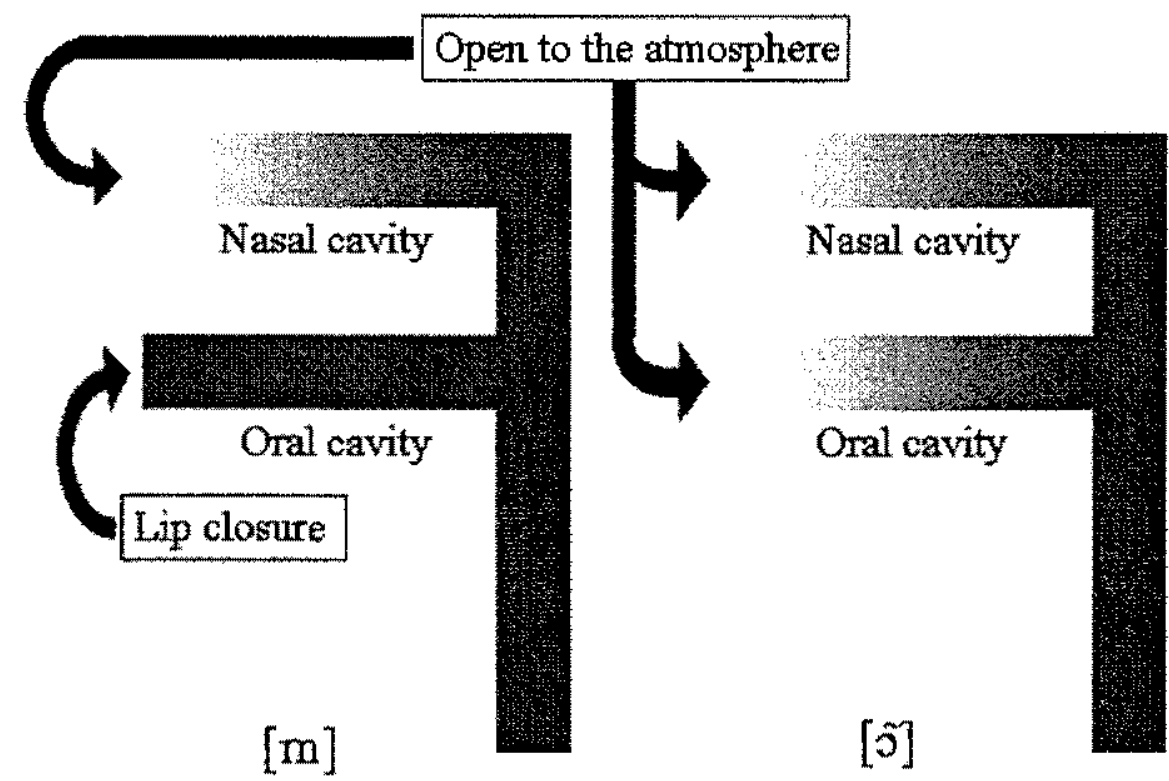

Figure 5.2 Models of vocal tract configuration of the nasal consonant [m] and the nasalised vowel [j].

The nasal cavity functions as the main resonator for nasals, and an oral cavity is a sub branching system which generates extra oral formants and antiformants. When nasalised vowels are produced, these two cavities exchange their functions. The oral cavity becomes the main cavity and the nasal cavity becomes the sub branch, since the acoustic coupling of mouth to the atmosphere is greater than that of nostrils. The frequencies of antiformants, which are generated from the nasal passage, are susceptible to the degree of coupling between the nasal cavity and the pharynx. When the coupling is weak, antiformants locate just slightly above the nasal formants. Once the coupling becomes stronger, as the velopharyngeal port opens wider, however, the 
frequencies of the antifarmants rise. It is therefore difficult to predict both the location of the antiformants and the kind of effects the antiformant will impose on the data. As a result, the measurement of nasalised vowel formants becomes a complicated task. Antiformants might obliterate an oral formant, for example.

The formant measurements of moshimoshi are thus anticipated to be very difficult. In fact a quick glance at the spectrograms of moshimoshi produced by CSL seem to confirm this apprehension. In order to overcome this difficulty in formant measurements, it was decided to include an extra process prior to the formant measurements of the collected data. That is an analysis of the nonnasal nonsense word, boshiboshi, and a carefully uttered moshimoshi, both of which were spoken by a male native speaker of Japanese.

Interpretation of formant structures of the carefully uttered moshimoshi is assumed to be easier than the casually and naturally uttered words in various contexts, as naturally uttered words may show paralinguistic factors, which are reflected as variations in intonation or intensity of the utterance. This undoubtedly makes the formant structure of the word more complicated.

The nonsense word boshiboshi was thus also included for the purpose of the controlled comparison with moshimoshi. The nonsense word, boshiboshi, is included in this study for two reasons. Boshiboshi has exactly the same segmental structure as moshimoshi, except for its lack of nasality of the bilabial consonants. The analysis of this word is thus expected to be useful in the separation of effects of the nasal consonant $/ \mathrm{m} /$ from the acoustic features of other segments, possibly providing a picture of the formant structures in the oral consonant and the vowels included in moshimoshi. Comparing the formant patterns of this nonsense word with that of moshimoshi will show where to expect the oral formants of $[0]$ and thus facilitate the identification of the effects of the nasal consonant. 
Furthermore, the comparison between boshiboshi and moshimoshi is assumed useful for examining the performance of the CSL formant tracing. There is a concern about the ability of CSL in accurately tracing the formants of nasal segments. Formant tracking in CSL is performed based on LPC analysis. For mathematical tractability, LPC analysis typically assumes that segments are characterised by resonances only and ignores any anti-resonances. This means that LPC analysis might not be suitable for the representation of the spectra of segments such as nasals, laterals, and some fricatives with which the spectral zeros and valleys play an important role, although it is effective for the analysis of non-nasalised vowels (Johnson 1997:87). In addition, LPC analysis looks for broad spectral peaks to identify formants. This suggests the possibility of them not being able to resolve formant peaks located close to each other and, as a result, treating those peaks as a single peak. As has been pointed out, the $/ 0 /$ vowel in moshimoshi is very likely to be acoustically nasalised. The mean $\mathrm{F} 1$ of the /o/ vowel showed in Chapter 4 was $451 \mathrm{~Hz}$ for the first recording session and $429 \mathrm{~Hz}$ for the second recording session. This is close to an expected nasal pole, there is thus a strong possibility that the LPC analysis is unable to extract these two formants correctly.

These potential shortcomings of formant estimation from LPC analysis can cause serious problems in forensic speaker identification. Given that nasals are known to contain much speaker specific information, it is pity to exclude nasal segments from analyses. How well LPC analysis works in the analysis of the nasalised segments is therefore a question with significant implication in forensic phonetic research - perhaps they are better analysed cepstrally. The comparison of the formant patterns of boshiboshi with that of moshimoshi will hopefully provide us with some ideas on how well LPC analysis picks up the formant patterns of nasalised segments and what sort of problems occur in the analysis of nasals with LPC analysis, as well as guides for the measurements of the paturally uttered moshimoshi. 
All moshimoshi and boshiboshi samples presented below were uttered carefully by a native Japanese male speaker who is not included in the data for the main experiments of this research. The recordings were digitised with a sampling rate of $16 \mathrm{kHz}$, using CSL. Audio waves, LPC power spectra, and spectrograms were produced for each sample and are presented in the following sections 5.3.1 and 5.3.2. Audio waveforms and spectrograms were produced for the entire words' duration. The LPC power spectrum for boshibosht was produced at the middle point of the duration of the first $/ \mathrm{o}$. For moshimoshi, the spectrum was made at two points: at the middle point of the second $/ \mathrm{o} /$ and second $/ \mathrm{m} /$.

\subsubsection{BOSHIBOSHI}

Four tokens of boshiboshi are presented and examined in this section. Figure 5.3 presents, from top to bottom, an audio waveform, an LPC power spectrum and a spectrogram, produced by CSL. The LPC power spectrum was produced at the middle point of the first /o/ of boshiboshi. 
[boshiboshi 1]

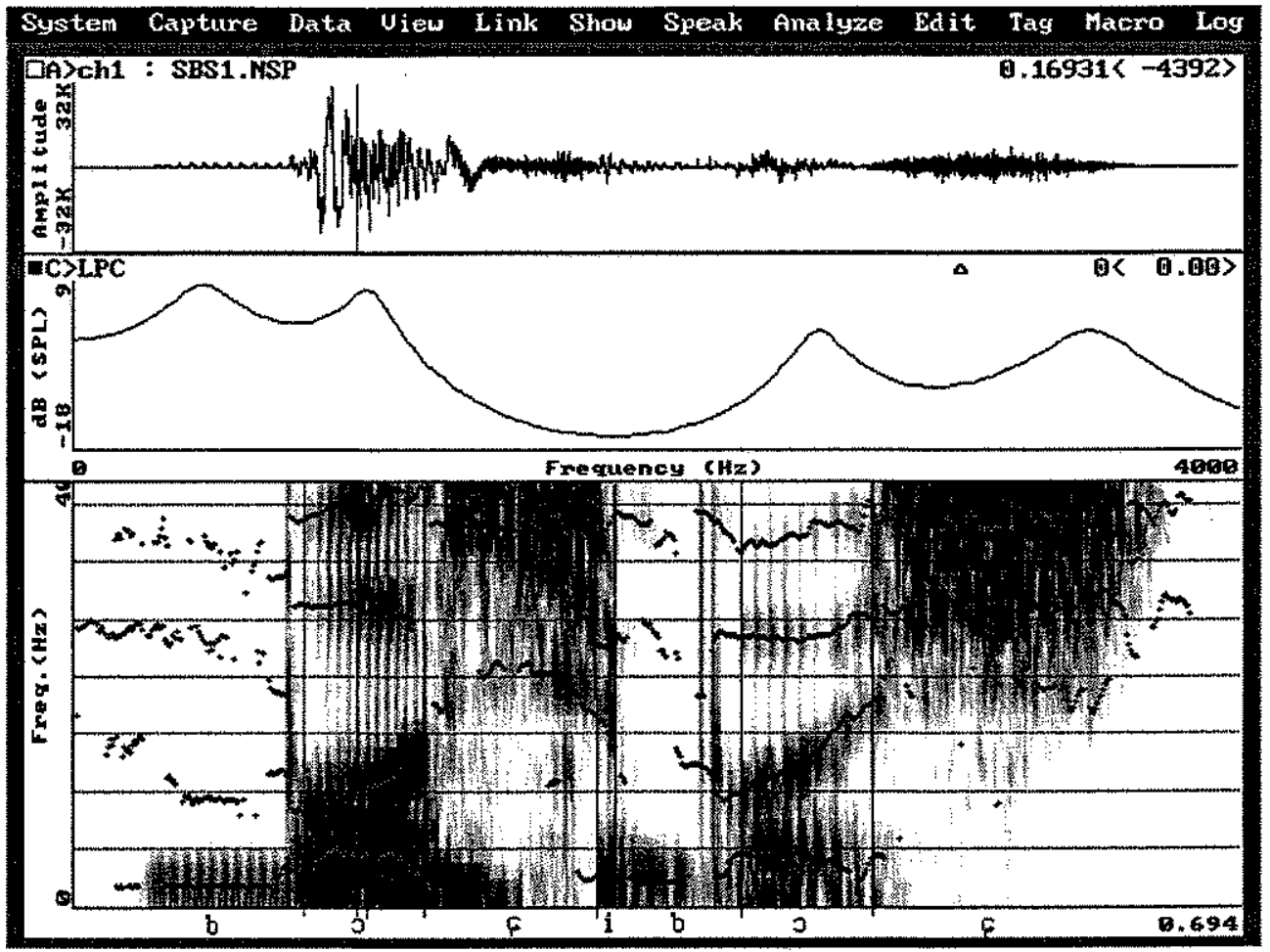

[boshiboshi 2]

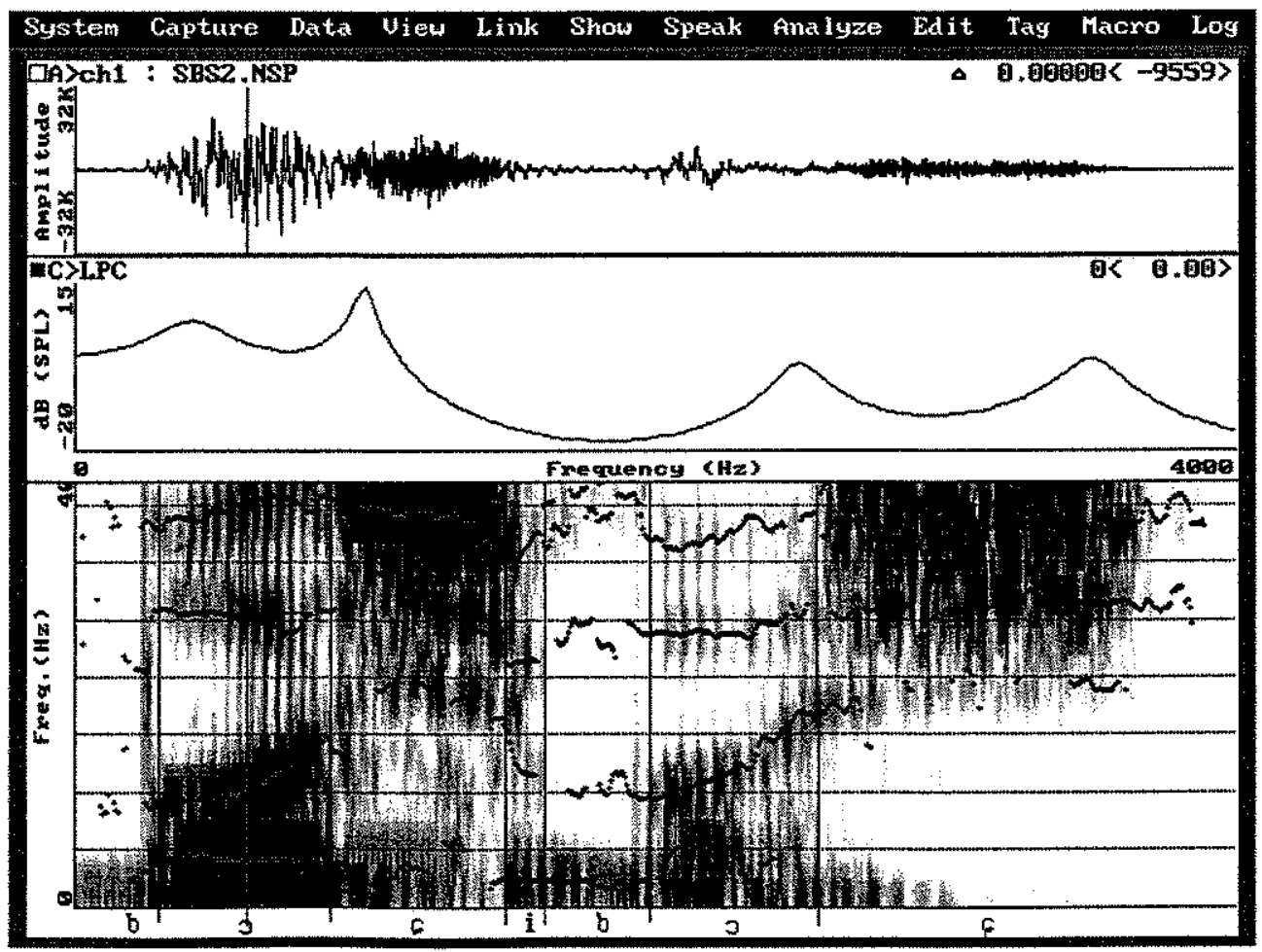


[boshiboshi 3]

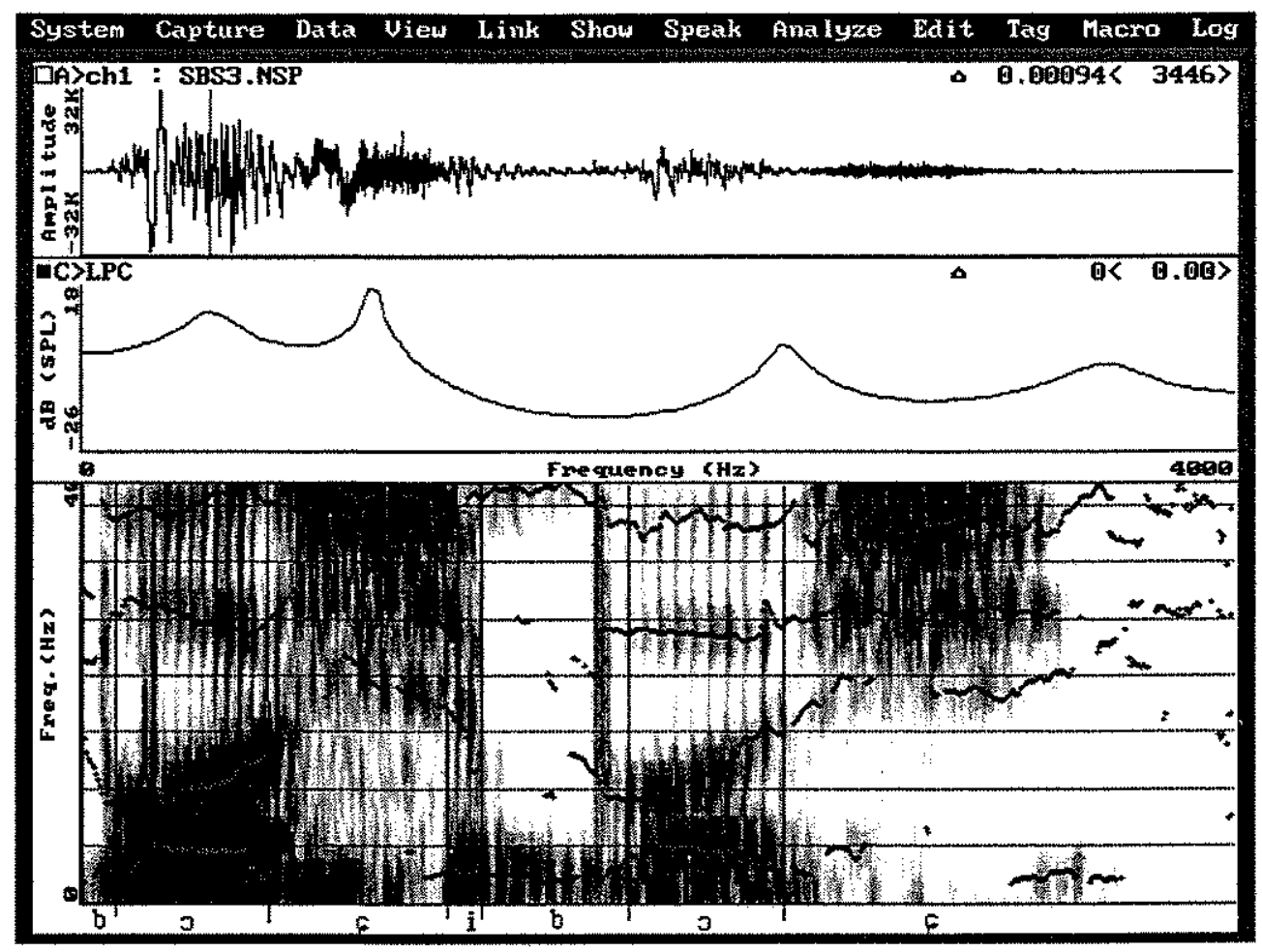




\section{[boshiboshi 4]}

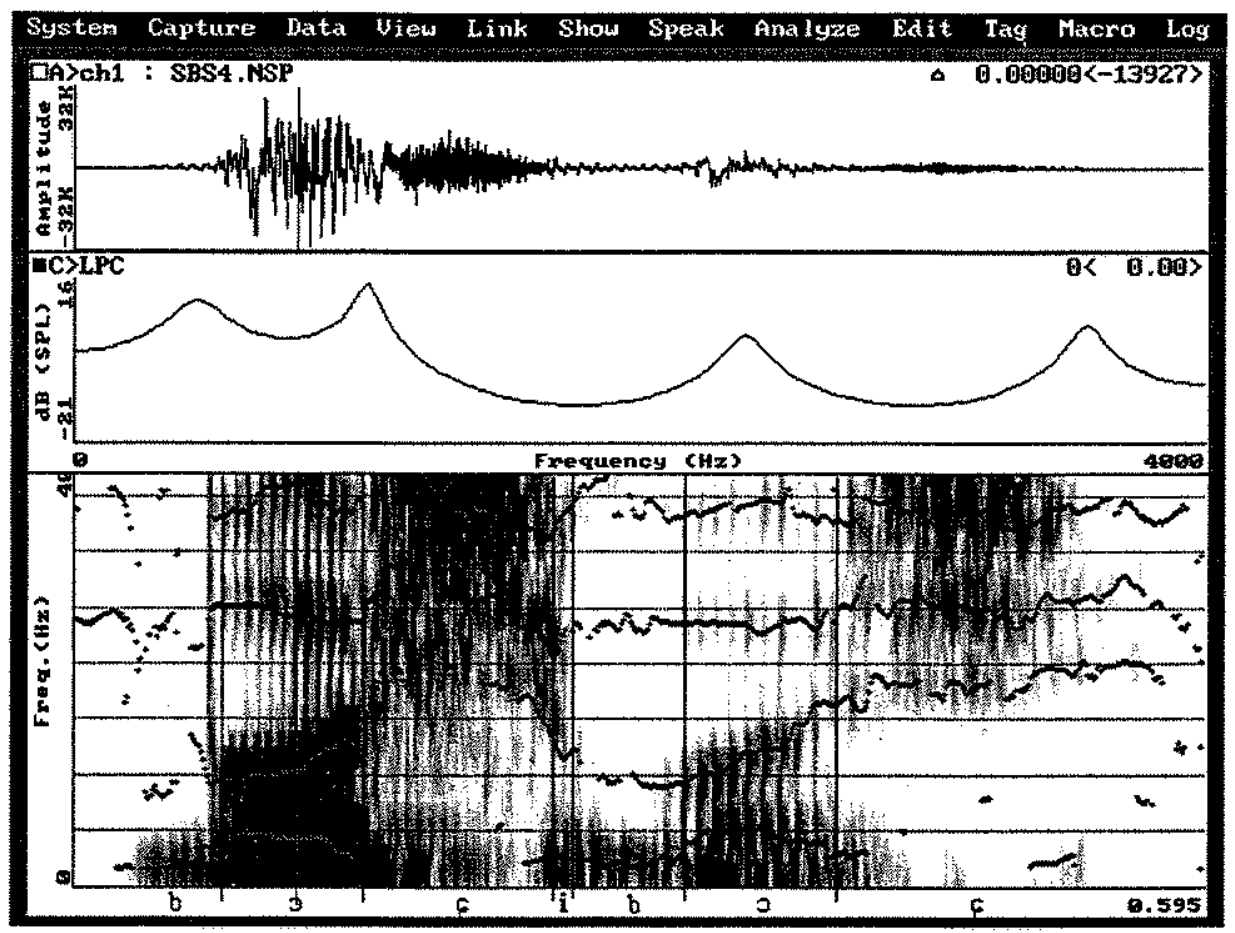

Figure 5.3 A set of four boshiboshis carefully uttered by a male Japanese speaker. The spectrum was taken at the middle point of the duration of the first $/ \mathrm{o}$.

The overall formant patterns of the whole word appear similar across all four boshiboshi samples. The first part of boshiboshi has a noticeably stronger intensity than the latter part of boshiboshi in all samples, as can be seen most clearly in the audio waveforms. This difference in intensity can be attributed to the location of the word accent. Although the word boshiboshi is a nonsense word, the speaker was requested to read it in the same manner as moshimoshi, and the accent of moshimoshi is, as mentioned, located on the first syllable, $/ \mathrm{mo} /$.

An interesting finding here is that, except for the first token (boshiboshi 1), the accent affects not only the intensity of the accented syllable, but also the intensity of the following syllable /si/ (phonetically realised as [6i]). As has been described in Chapter 2, Japanese is a pitch accented language, and the accented syllable is marked by a fall in the F0 from high to low. The fact that 
the following syllable /si/ as well as the accented syllable has a distinctively higher intensity in some boshiboshis but not all suggests that the intensity is not strictly controlled in the Japanese accent marking system. If it were a part of the accentuation marking system, the realisation of the intensity of the following /s/ should be consistent across all boshiboshis. Although the intensity of the accented syllable may be higher than the other syllables, this is not the result of the control of intensity itself, but of the manipulation of F0.

F1 to F4 of each segment in boshiboshi were measured at the midpoint of the segment's duration. The means and standard deviations are presented in Table 5.1.

\begin{tabular}{|c|c|r|r|r|r|}
\hline & & \multicolumn{1}{|c|}{ F1 } & \multicolumn{1}{|c|}{ F2 } & F3 & \multicolumn{1}{|c|}{ F4 } \\
\hline b1 & mean & 197.25 & 1078.50 & 2080.50 & 2916.67 \\
& sd & 65.08 & 207.06 & 150.68 & 196.95 \\
\hline o1 & mean & 431.00 & 1010.25 & 2500.25 & 3509.75 \\
& sd & 23.34 & 11.09 & 62.32 & 64.43 \\
\hline s1 & mean & & 2019.50 & 3009.00 & 3562.50 \\
& sd & & 133.64 & 156.23 & 196.20 \\
\hline 11 & mean & 272.00 & 1288.50 & 2132.00 & 3229.50 \\
& sd & 37.88 & 198.48 & 91.22 & 142.88 \\
\hline b2 & mean & 185.25 & 967.00 & 2230.00 & 3290.00 \\
& sd & 12.53 & 44.32 & 141.32 & 80.39 \\
\hline o2 & mean & 471.33 & 1135.50 & 2330.25 & 3311.00 \\
& sd & 44.74 & 133.43 & 44.78 & 99.83 \\
\hline s2 & mean & & 1959.50 & 2704.75 & 3377.75 \\
& sd & & 277.53 & 271.63 & 158.53 \\
\hline
\end{tabular}

Table 5.1 Means and standard deviations for the all measuring points in boshiboshi. Measurements were made at the middle point of the segment duration $(n=4)$.

Detailed observation of this word is presented for relevant segments separately.

$\mathrm{lo} /$ : The results of the mean measurements for $/ \mathrm{o} /$ in Chapter 4 were approximately $\mathrm{F} 1=440 \mathrm{~Hz}$, $\mathrm{F} 2=1130 \mathrm{~Hz}, \mathrm{~F} 3=2500 \mathrm{~Hz}$, and $\mathrm{F} 4=3540 \mathrm{~Hz}$. What we find in the observation of boshiboshi agrees mostly with these values. $\mathrm{F} 1$ to $\mathrm{F} 4$ in boshiboshi were found to be $\mathrm{F} 1=431 \mathrm{~Hz}, \mathrm{~F} 2=1010$ 
$\mathrm{Hz}, \mathrm{F} 3=2500 \mathrm{~Hz}$, and F4 $=3509 \mathrm{~Hz}$. The first and the second $/ \mathrm{o} /$ look similar except for their intensity.

The F-pattern configuration of $/ \mathrm{o} /$ is conditioned by its transition from the adjacent target $/ \mathrm{b} /$ to $/ \mathrm{s} /$, as well as its own phonetic target. In the first and the second /o/s of all four tokens, FI becomes lower towards the end of the vowel duration. This is assumed to be the effect of the following segment $/ \mathrm{s} /$. The closure of vocal tract will result in a decrease of $\mathrm{Fl}$, a transition from $/ \mathrm{o} / \mathrm{to} / \mathrm{s} /$ will thus lower F1. Furthermore, the tongue position for $/ \mathrm{s} /$ is high, as it is realised phonetically as [6], and an anticipation of $/ \mathrm{s} /$ also can lower $\mathrm{Fl}$, since the high tongue position is associated with low F1.

F2 in the $/ \mathrm{o} /$, on the other hand, becomes higher with time. In both the first and the second $/ 0 / \mathrm{s}$ of all four tokens, F2 starts around $800-1000 \mathrm{~Hz}$ and rises to around $1500 \mathrm{~Hz}$. This is also assumed to be the result of anticipation for $/ \mathrm{s} /$, which is phonetically an alveo-palatal fricative [c], since high $\mathrm{F} 2$ is known to be a reflection of frontness of the articulation. The contour of $\mathrm{F} 3$ differs between the first and the second $/ \mathrm{o} / \mathrm{s}$. The first $/ \mathrm{o} /$ has a slightly downward contour, whereas the second $/ 0 /$ has a flat contour. Their frequencies are also slightly different. The first $/ \mathrm{o} /$ has a slightly higher frequency than the second $/ \mathrm{o} /$ does. The first $/ \mathrm{o} /$ starts just above $2500 \mathrm{~Hz}$ and ends just below $2500 \mathrm{~Hz}$. The second / $/$ starts and stays below $2500 \mathrm{~Hz}$, around the $2300-2400 \mathrm{~Hz}$ region.

Similarly to F3, F4 of the first /o/ was about $150-200 \mathrm{~Hz}$ higher than the second /o/ across all tokens. The height difference between the first and the second /o/s in their F3 and F4 probably correlates with pitch accent. Since the word accent is located on the first syllable $/ \mathrm{bo} /, \mathrm{FO}$ of the first /bo/ is expected to be higher than the rest of the word. In order to produce higher F0, larynx is probably raised. This results in shortening of vocal tract length, with consequently increases frequency of higher formants. F3 is also known as a primary determinant of the vocal tract length, 
and the shorter vocal tract produces higher F3 (Rose 1999b:6). In fact, it has been reported by Suzuki (1973) that the formant pattern of the /o/ vowel is heavily affected by the pitch change. Through his experiments on the vowel uttered in isolation, he even reports that $/ \mathrm{o} /$ is the vowel which is particularly susceptible to the pitch change than others for a male speaker. As his study used only one male speaker and one female speaker, it is difficult to draw a generalisation from his results. Nevertheless the agreement with the current study should be noted.

/s/: F2 to F4 of palato-alveolar fricative [ [] is expected to be observed around $1900 \mathrm{~Hz}, 2500 \mathrm{~Hz}$, and $3500 \mathrm{~Hz}$, respectively (Stevens 1998:406). The fricative observed here is, however, alveopalatal fricative [G] phonetically, which raises more "of the part of tongue immediately behind the constriction" (Ladefoged and Maddieson 1996:164). This difference will result in a longer constriction area and smaller back cavity, thus F3, for which the back cavity is responsible (Johnson 1997:120), will be somewhat higher than that of [S], whereas F2 and F4 which are determined by the front cavity, are expected to have similar values.

The observation of $/ \mathrm{s} /$ in boshiboshi presented here showed F2 $=2019 \mathrm{~Hz}, \mathrm{~F} 3=3009 \mathrm{~Hz}$, and F4 $=$ $3562 \mathrm{~Hz}$ for the first $/ \mathrm{s} /$, and $\mathrm{F} 2=1959 \mathrm{~Hz}, \mathrm{~F} 3=2704 \mathrm{~Hz}$, and $\mathrm{F} 4=3377 \mathrm{~Hz}$ for the second $/ \mathrm{s} /$. The formants of the first /s/ agree with the expected values well, however the formants of the second $/ \mathrm{s} /$ are $50-300 \mathrm{~Hz}$ lower than that. Unlike $/ \mathrm{o} /$, the observation of $/ \mathrm{s} /$ shows that the first and the second $/ \mathrm{s} / \mathrm{s}$ have rather different formant patterns. Generally, formants are lower for the second $/ \mathrm{s} /$.

As for formant patterns, F2 to F4 of the first /s/ have a falling contour, whilst the contour of the second $/ \mathrm{s} /$ is flat in all tokens. This fall may be conditioned by the following $/ \mathrm{b} /$, as the duration of 
Ii/ is very short. Also a strong energy in the F1 region is obvious in the first $/ \mathrm{s} /$, but not in the second /st

The two /s/s show a constant durational difference, with the second $/ \mathrm{s} /$ being longer than the first. The durational difference can be explained by the position of these segments within the word. Although phonologically the /i/ vowel is supposed to follow the second $/ \mathrm{s} /$, no final /i/ was observed in this speaker"s data and, consequently, the second $/ \mathrm{s} /$ is phonetically in the final position of the word.

The second / $/$ was probably sustained longer to compensate for the duration, which the missing $/ \mathrm{t}$ was supposed to occupy. As Japanese is a mora-timed language (Shibatani 1990:159), /S may have been lengthened to keep the duration of the mora /si/ roughly the same as other morae. Boshiboshis 1 and 4, however, show that the second /s/ is considerably longer than the duration of the first $/ \mathrm{s} / \mathrm{i} / \mathrm{i}$. This suggests that compensation of the mora duration is probably not the reason for this longer duration for second $/ \mathrm{s} /$.

Strong energy in the low frequency area is observed for this speaker, which is unexpected for a voiceless fricative. Although not identified clearly by the formant tracking of CSL, it was observed that the first $/ \mathrm{s} /$ had strong energy at around $300 \mathrm{~Hz}$. The first formant of the alveopalatal configuration is a Helmholtz resonance (Stevens 1998:406), and the strong energy at around $300 \mathrm{~Hz}$ is presumed to be this $\mathrm{Fl}$. The intensity of F1 was high at the beginning and gradually decayed with time. The reason for this is not clear. In fact, it is quite unusual to have such strong energy in the low frequency region for $/ \mathrm{s} /$. One possible explanation is that the first $/ \mathrm{s} /$ was partly voiced at the onset due to the influence of the surrounding voiced segments. Boshiboshi 2 and 4 also had low energy but in a different frequency region. They showed a weaker energy at around 
$600 \mathrm{~Hz}$. This is assumed to be a tracheal resonance, since there should be substantial coupling between tracheal and supralaryngeal vocal tract for voiceless fricatives.

In the 1200 to $1300 \mathrm{~Hz}$ region, which is below F2, there seems to be another resonance. The X-ray tracings of the tongue position for $[6]$ in Ladefoged and Maddieson $(1996: 151,154,161)$ show that some speakers have a small pocket under the tongue when they produce alveo-palatal fricatives. This resonance is thus presumably a sublaminal resonance associated with this pocket. Although the energy level is not as strong as for the other formants, this resonance appeared consistently across all four tokens. This extra pole is found mainly in the first $/ \mathrm{s} /$. Except for boshiboshi 1 , this pole is found in none of the second $/ \mathrm{s} / \mathrm{s}$. In boshiboshi 1 , the sublaminal pole of the second $/ \mathrm{s} /$ appears, although it has a very short duration.

F2 and F3 of the first /s/ had a rise-and-fall contour, whilst the F2 and F3 contours of the second /s/ stayed flat. This is explained by the coarticulation with the following segments. The first $/ \mathrm{s} /$ is followed by $/ \mathrm{i} /$, whereas the second $/ \mathrm{s} /$ did not have a vowel due to devoicing. The formant structure of the first $/ \mathrm{s} /$ appears to be influenced by $/ b /$, which follows $/ \mathrm{i} /$, rather than $/ \mathrm{i} /$ itself. Roughly speaking, F2 starts at $1500 \mathrm{~Hz}$, rises to $2000 \mathrm{~Hz}$ and, then falls to $1700 \mathrm{~Hz}$. F3 starts at $2600 \mathrm{~Hz}$, rises to $3000 \mathrm{~Hz}$, and falls to $2400 \mathrm{~Hz}$. F2 and F3 of the second /s/ stay in the $1800-$ $2000 \mathrm{~Hz}$ region and $2500 \mathrm{~Hz}$ regions, respectively.

F4 of the first /s/ has a slight downward slope from around $3500 \mathrm{~Hz}$ to $3250 \mathrm{~Hz}$. The second /s/ stays roughly flat at just below $3500 \mathrm{~Hz}$, in the $3300-3400 \mathrm{~Hz}$ regions.

/i/: It is noticed that all word final $/ \mathrm{i} / \mathrm{s}$ were devoiced by this speaker. For the first $/ \mathrm{i} /$, on the other hand, there was a very short voiced segment which can be identified as the first $/ \mathrm{i} /$. The word intermal / $/$ / is fully voiced in all four samples, although they are very short in duration and very 
weak in their intensity. The measurements in Chapter 4 showed that the mean formant frequencies for $F 1$ to $F 4$ of $/ /$ are approximately $F 1=304 \mathrm{~Hz}, F 2=2167 \mathrm{~Hz}, F 3=2916 \mathrm{~Hz}$, and $F 4=3682 \mathrm{~Hz}$. Here those formants are found to be $\mathrm{F} 1=272 \mathrm{~Hz}, \mathrm{~F} 2=1288 \mathrm{~Hz}, \mathrm{~F} 3=2132 \mathrm{~Hz}$, and F4 $=3229 \mathrm{~Hz}$. These are very different results from what is expected. F2 in boshiboshi is much lower than F2 in Chapter 4. In fact, F3 in boshiboshi seems to be the equivalent to the F2 in Chapter 4. It seems that CSL has recognised the sublaminal formant, which was mentioned above in the analysis of $/ \mathrm{s} /$, as F2.

The differences in F3 may have been caused by a range of factors, such as this speaker's idiosyncrasies and coarticulation with the following segments. The $\mathrm{b} / \mathrm{s}$ are followed by a rounded vowel / $/$. This combination may have caused a slight lip protrusion in the articulation of this syliable, and the anticipation for this configuration may have consequently tuned down the formants of the preceding segment. It is unlikely that this happened to the $f /$ vowels in Chapter 4 , as the phonological environments where the /i/ vowels were embedded (in terms of a preceding

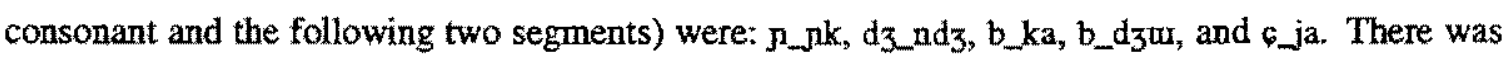
no instance of the vowel being followed by either a bilabial sound or rounded vowels.

It has been pointed out above that $/ \mathrm{N}$ in this word has a very short duration. The preceding consonant of this vowel, namely $/ \mathrm{s} /$, is a consonant with a longer intrinsic duration. Japanese tends to keep the duration of each mora similar, so the duration of the fi/ may be shortened to compensate for the long duration of the preceding consonant $/ \mathrm{s} /$. The comparison to the duration of the other mora /bo/ shows clearly that /i/did not need to be shortened to an extreme extent, however, as both $/$ bo/s had longer durations than /si/. An alternative explanation for this short duration of $/ \mathrm{i} /$ is aerodynamic. As $/ \mathrm{V} /$ has a narrow oral constriction, the volume velocity of the airflow is small. It therefore takes longer for the transglottal pressure differential to be achieved to allow voicing to 
start. This results in a delayed onset time for /i/ and, consequently, it shortens the duration of /i/ considerably.

Alternatively, it is also possible to interpret that / $\mathrm{j} /$ has been omitted. What appears to be $/ \mathrm{i} /$ is in fact just a transition period from $/ \mathrm{s} /$ to $/ \mathrm{b} /$, where the configuration of the articulator is closer to $/ \mathrm{s} /$ (which is similar to /i/) and yet the voicing for the following voiced consonant has already started. Given the extremely short duration of the voiced segment, this seems a more plausible interpretation. This also agrees with Kawakami's (1977:26) description of which consonant / vowel combinations are devoiced and which ones are omitted, as seen in Chapter 2 . He claims that /si/ is one of the combinations where the vowel is omitted, realising as [6:], instead of being devoiced as in [6i] ].

Having described the F-pattern in non-nasal boshiboshi, we can now move to the description of moshimoshi, which is the research target of this study. The next section describes the set of carefully uttered moshimoshis by the same speaker.

\subsubsection{MOSHIMOSHI}

This section compares four moshimoshis uttered by the same speaker. The observations made here are also going to be compared to the observation of boshiboshis, in the previous section. Figure 5.4 below shows the audio waveforms, power spectra and spectrograms of four carefully read-out moshimoshi samples. LPC spectra (the middle section of the figures) were made at the middle point of the second $/ \mathrm{m} /$ and the second $/ \mathrm{o} /$. In the LPC spectra, the solid line and the dotted line show the spectrums of $/ \mathrm{m} /$ and $/ \mathrm{o} /$, respectively. 
[moshimoshi 1]

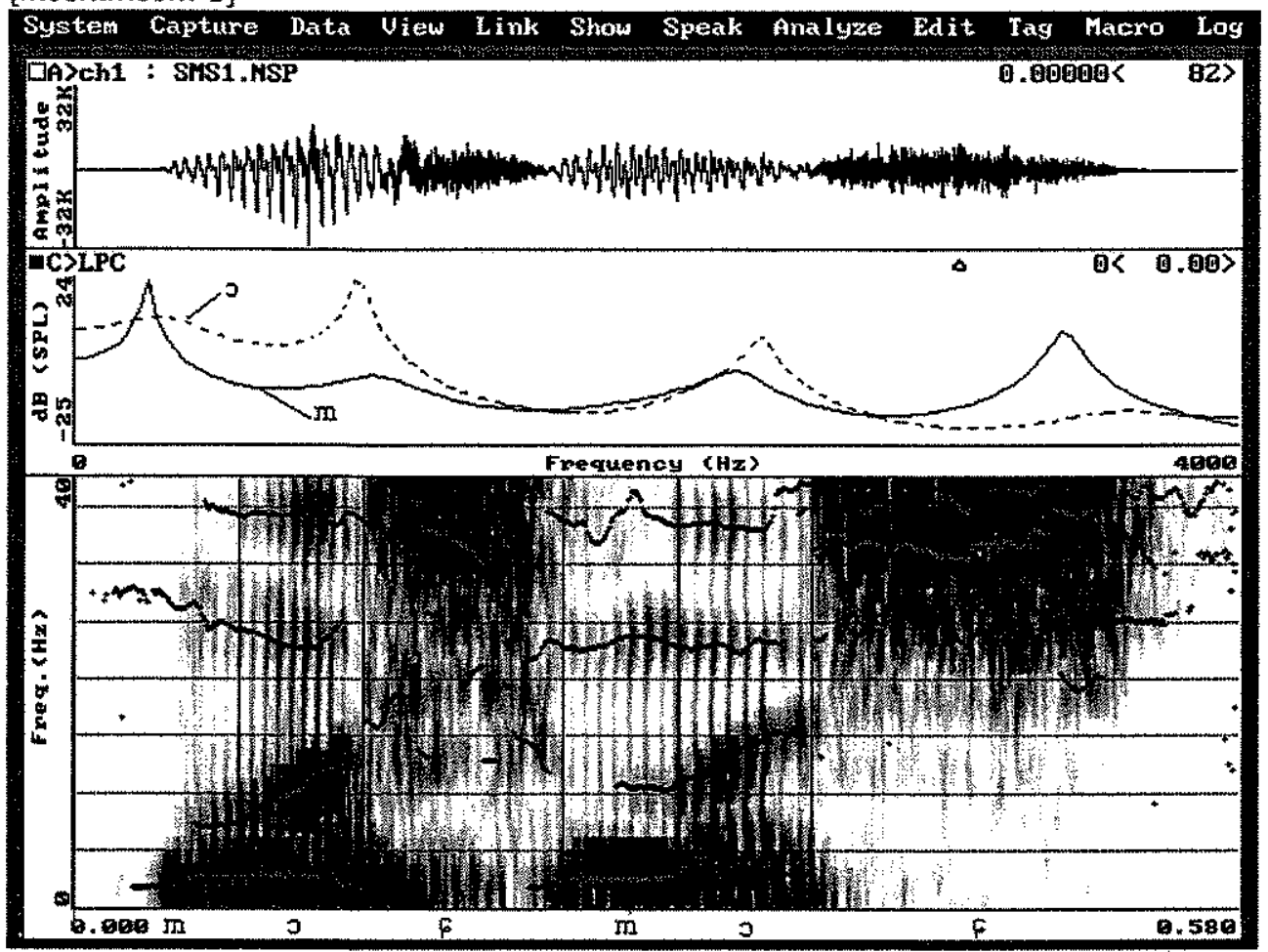

[moshimoshi 2]

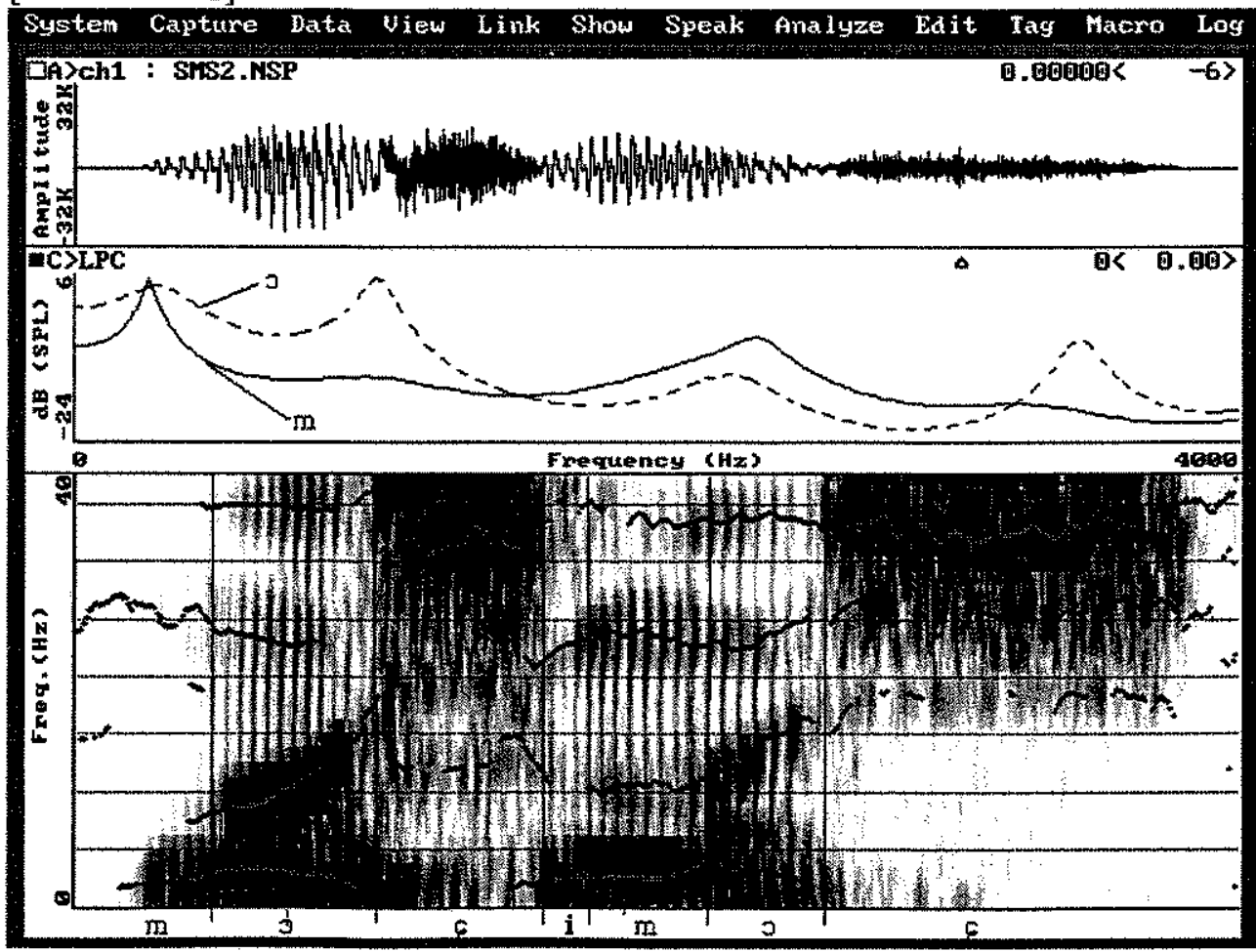


[moshimoshi 3]

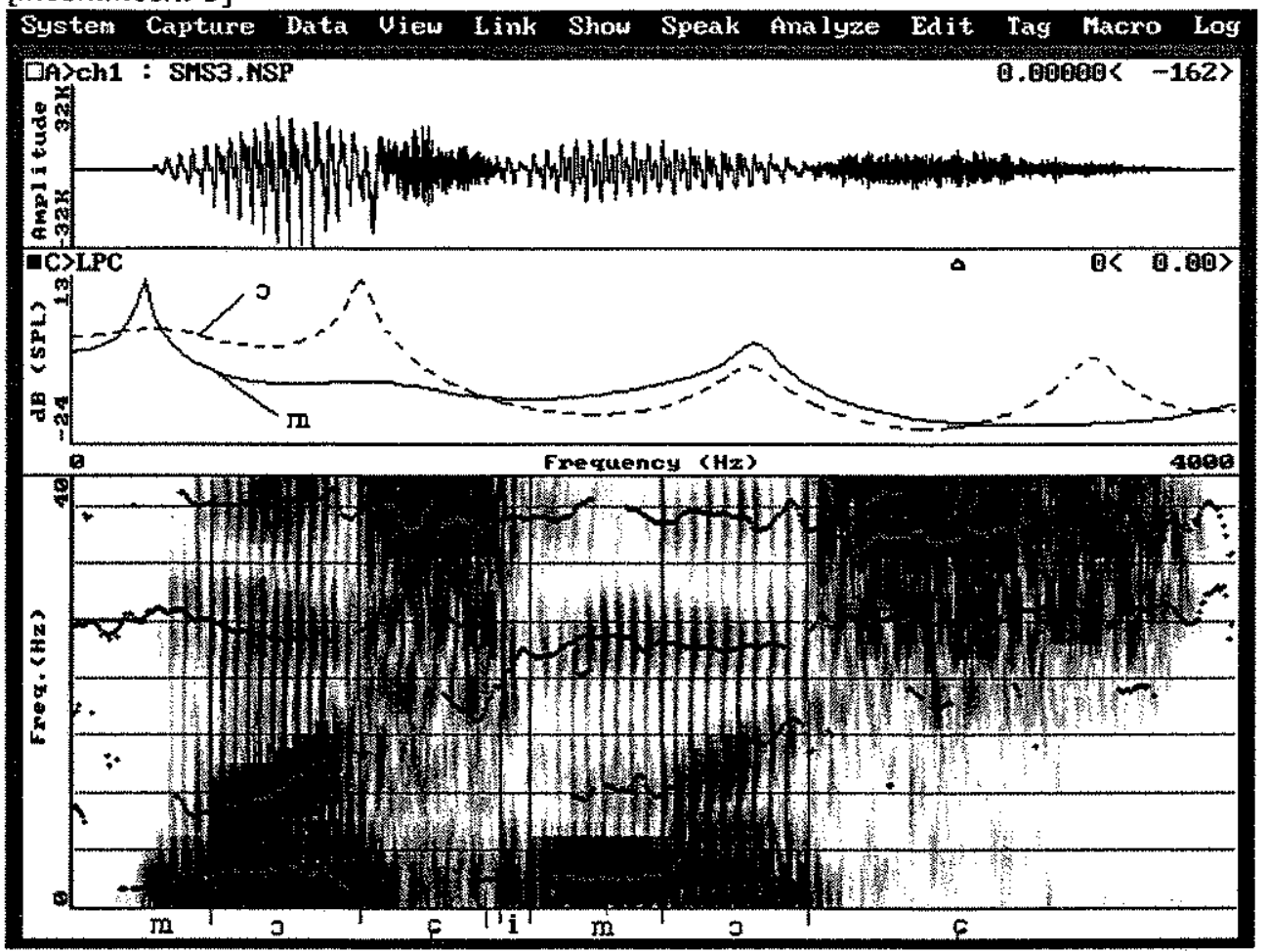




\section{[moshimoshi 4]}

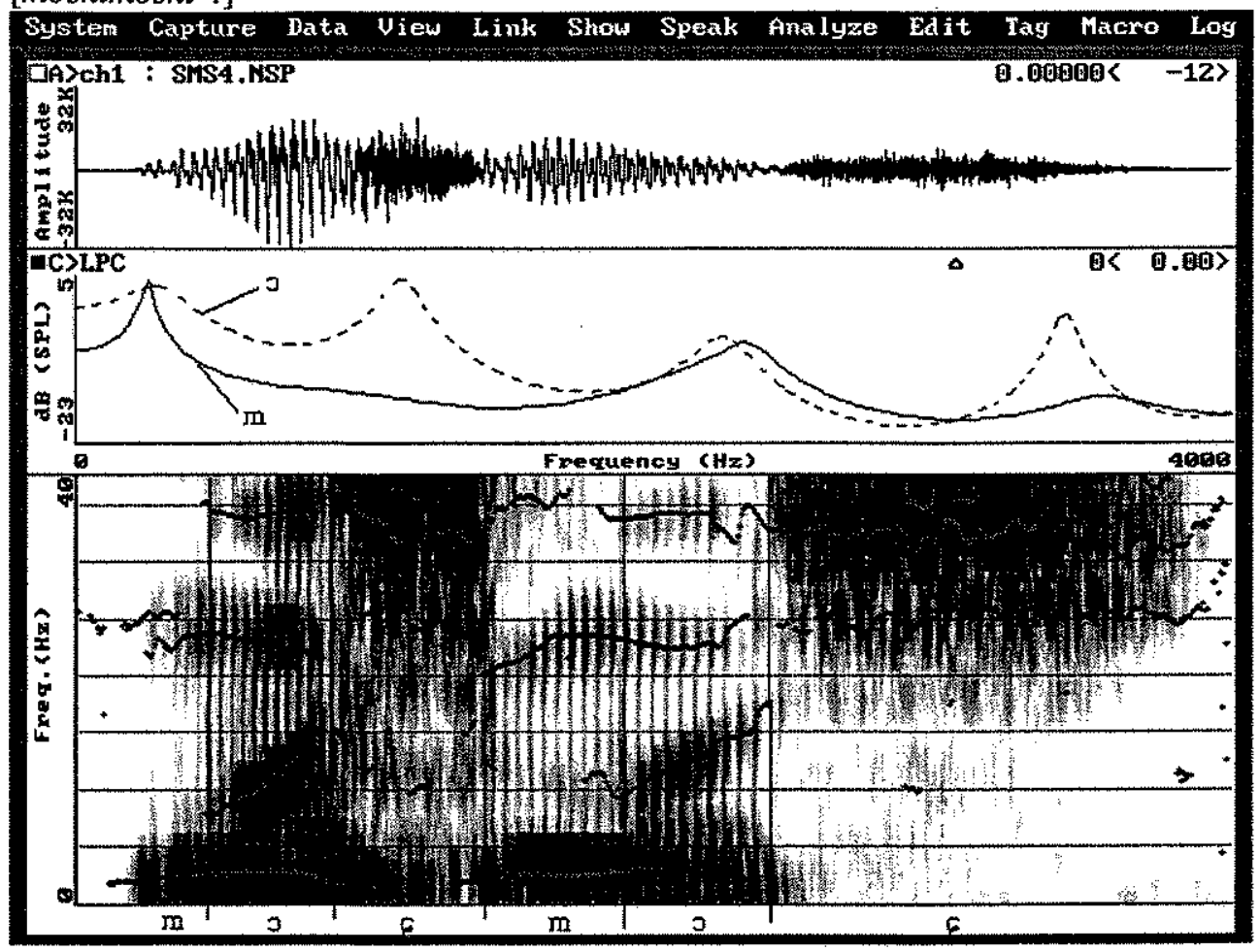

Figure 5.4 A set of four moshimoshis carefully uttered by a male Japanese speaker. In the LPC panel, the solid line shows the second $/ \mathrm{m} /$ and the dotted line shows the second $/ \mathrm{o} /$.

The first four formants of each segment of the four moshimoshis were measured at the middle point of segment duration, using the LPC power spectrum. The mean and standard deviations are summarised in Table 5.2 . 


\begin{tabular}{|c|c|r|r|r|r|}
\hline & & \multicolumn{1}{|c|}{$\mathrm{F} 1$} & \multicolumn{1}{|c|}{$\mathrm{F} 2$} & \multicolumn{1}{c|}{$\mathrm{F} 3$} & \multicolumn{1}{c|}{$\mathrm{F} 4$} \\
\hline $\mathrm{m} 1$ & mean & 213.75 & 1079.75 & 2722.25 & 3742.00 \\
& $\mathrm{sd}$ & 12.79 & 201.17 & 243.37 & 324.70 \\
\hline $\mathrm{ol}$ & mean & 224.50 & 1027.50 & 2279.50 & 3413.25 \\
& $\mathrm{sd}$ & 91.19 & 71.30 & 31.50 & 124.40 \\
\hline $\mathrm{s} 1$ & mean & & & 2409.25 & 3155.25 \\
& $\mathrm{sd}$ & & & 105.00 & 63.77 \\
\hline $\mathrm{i} 1$ & mean & 183.00 & 1205.00 & 2235.00 & 3195.00 \\
& $\mathrm{sd}$ & $\mathrm{N} / \mathrm{A}$ & $\mathrm{N} / \mathrm{A}$ & $\mathrm{N} / \mathrm{A}$ & $\mathrm{N} / \mathrm{A}$ \\
\hline $\mathrm{m} 2$ & mean & 257.25 & 1031.00 & 2310.75 & 3578.00 \\
& $\mathrm{sd}$ & 3.95 & 224.71 & 53.42 & 116.50 \\
\hline $\mathrm{o} 2$ & mean & 300.25 & 1321.25 & 2236.75 & 3368.50 \\
& $\mathrm{sd}$ & 158.35 & 102.61 & 47.79 & 37.76 \\
\hline $\mathrm{s} 2$ & mean & & 1916.00 & 2609.75 & 3276.75 \\
& $\mathrm{sd}$ & & 98.96 & 65.43 & 66.74 \\
\hline
\end{tabular}

Table 5.2 Means and standard deviations of F1 to F4 of each segment in moshimoshi. The measurements were made at the middle of the segment duration $(n=4)$.

The first interesting finding is that the first $/ \mathrm{i} /$ is observed only in moshimoshi 2 and 3 , despite the fact that the first $/ \mathrm{i} /$ is followed by a voiced segment $/ \mathrm{m} /$. Moreover, vowel devoicing is supposed to be less frequent in carefully uttered words, as was the case here. It is thus doubly unexpected to see that $/ \mathrm{i} /$ is not realised phonetically in this position. The second $/ \mathrm{i} /$ is not observed in any of the four samples. As has been demonstrated in Chapter 4, the realisation of devoicing is thus not stable within a speaker. The data for moshimoshi presented here is not only consistent with the results of Chapter 4 , but also shows that a single speaker's realisation of devoicing may not be constant even in carefully read-out speech.

Detailed discussion is presented on each segment separately.

$/ \mathrm{m} /$ : With the observation of $/ \mathrm{m} /$, firstly a strong low energy with wide bandwidth at around 250 to $300 \mathrm{~Hz}$ is expected to be observed (Fujimura 1962; Fujimura and Erickson 1997; Hattori et al. 1958; Stevens 1997; Stevens 1998). This wide-banded first resonance is the result of heavy damping, which is caused by the larger surface area and greater volume of the nasal cavity (Johnson 1997:143). The nasal consonant $/ \mathrm{m} /$ is also expected to have an antiformant in the area 
around 750-1250 $\mathrm{Hz}$ (Hattori et al. 1958:268), and the second formant is just above the antiformant. All four moshimoshis presented here show a strong low resonance with a wide bandwidth below the $200-250 \mathrm{~Hz}$ region, which is typical for nasal consonants.

F2 in both the first and the second $/ \mathrm{m} / \mathrm{s}$ was observed at approximately $1050 \mathrm{~Hz}$, which again agrees with the preceding research (for instance, Hattori et al. 1958). It is noticed that F2 cannot be seen at the onset of the consonant.

As Johnson (1997:151) points out, antiformants do not always appear as a total absence of energy in that area. In the spectrogram presented here, it is seen as weakened energy in the region, and this is assumed to be the effect of the antiformant. Theoretically the location of the antiformant also reflects the speaker's anatomical features, as the location of the antiformant is determined by the configuration of the subsystem, in this case, the oral cavity. It is, however, still difficult to identify the exact location of the antiformants for quantification or any form of statistical analysis. In addition, LPC is designed to recognise only peaks of formants but not antiformants. It is thus extremely difficult to determine the location of antiformant, and this is therefore not a practical choice to profile the characteristics of a speaker.

In all figures, F3 and F4 are lower for the second $/ \mathrm{m} /$ than for the first $/ \mathrm{m} /$. The mean F2 and F3 for the first $/ \mathrm{m} /$ are $2722 \mathrm{~Hz}$ and $3742 \mathrm{~Hz}$, and those for the second $/ \mathrm{m} /$ are $2310 \mathrm{~Hz}$ and $3578 \mathrm{~Hz}$ as seen in Table 5.2 (p.192).

A large durational difference between the first and the second $/ \mathrm{m} / \mathrm{s}$ is also noticed. The first $/ \mathrm{m} /$ is shorter than the second $/ \mathrm{m} /$ with the exception of moshimoshi 1 . One possible explanation for why the first $/ 0 /$ is longer than the second is the pitch accent on the first syllable. The pitch accent prolonged the duration of the first $/ \mathrm{O}$, and the durational difference between two $/ \mathrm{m} /$ may have 
occurred due to temporal compensation to keep the duration of morae reasonably constant. This idea must be discarded, however, as the durational difference between two $/ \mathrm{m} / \mathrm{s}$ is even larger than the difference between two $/ \mathrm{o} / \mathrm{s}$, and consequently the accented syllable (or mora) became considerably shorter than its unaccented counterpart.

The other reason for the shorter duration of the first $/ \mathrm{m} /$ may lie in the position of the segment within the word. The first $/ \mathrm{m} /$ was located at the word initial position, and the second $/ \mathrm{m} /$ was at the inter-vocalic position. This means that the vocal cords had been already vibrating prior to the production of the second $/ \mathrm{m} /$, whereas the vocal cords had to start vibrating for the production of the first $/ \mathrm{m} /$. In the production of the first $/ \mathrm{m} /$, there may have been some delay of the phonation, consequently making the duration of the first $/ \mathrm{m} /$ shorter.

/ol: $\mathrm{F} 1$ to $\mathrm{F} 4$ in boshiboshis in previous section were found to be $\mathrm{Fl}=431 \mathrm{~Hz}, \mathrm{~F} 2=1010 \mathrm{~Hz}, \mathrm{~F} 3=$ $2500 \mathrm{~Hz}, \mathrm{~F} 4=3509 \mathrm{~Hz} . \mathrm{F} 1$ to $\mathrm{F} 4$ of $/ \mathrm{o} /$ in moshimoshi are found to be generally very similar, except for $\mathrm{F} 1$.

Both of the first and the second/o/s in moshimoshi exhibited a strong resonance in the $200-300 \mathrm{~Hz}$ region (the means are $224 \mathrm{~Hz}$ for the first $/ 0 /$ and $300 \mathrm{~Hz}$ for the second $/ \mathrm{o}$ ) throughout the entire vowel duration. This is far lower than the Fl expected for the fo/ vowel, which was around $430 \mathrm{~Hz}$ in the observation of boshiboshi in previous section. This strong resonance is assumed to be the result of the nasalisation, caused by the preceding nasal consonant.

F2 for all tokens had a rising contour from $1000 \mathrm{~Hz}$ to $1500 \mathrm{~Hz}$. F3 and F4 had relatively flat contours, around $2250 \mathrm{~Hz}-2500 \mathrm{~Hz}$ and $3400 \mathrm{~Hz}-3500 \mathrm{~Hz}$, respectively, 
A close examination of the spectrograms reveals that there are actually two separate formants below $700 \mathrm{~Hz}$, although the LPC formant history function (the lines on the spectrograms) had tracked only one. As mentioned above, the lower formant, in the $250-300 \mathrm{~Hz}$ region is probably a nasal pole. The other formant located around $600-700 \mathrm{~Hz}$ region is presumably the first formant of the vowel, although $600-700 \mathrm{~Hz}$ is much higher than the $\mathrm{F} 1$ of the non-nasal /o/ in boshiboshi. The formant tracking for the second /o/ can be seen running in between the two formants, although this is not obvious for the first /o/. It thus appears that the formant history function of CSL has failed to separate the nasal pole and the first formant of the $/ 0 /$ vowel.

This observation leads us to question the reliability of the formant tracking system of CSL under certain conditions. Although most formants appear to be tracked appropriately, when there is more than one formant within a small frequency range, CSL seems not to distinguish those formants well. In the context of forensic phonetics, the observation made above means that the lower frequencies of nasalised segments are probably not a good parameter for reliably comparing data, as accurate measurements may be very difficult. The data in most experimental phonetics is not a direct record of human voice, and they are inevitably influenced by the mathematical process used by the analysis software package. What we can observe is thus conditioned by the equipment. The parameters which cannot be measured accurately by our equipment are going to be of much less use.

/s/: The observation of $/ \mathrm{s} /$ in boshiboshi presented here showed F2 $=2019 \mathrm{~Hz}, \mathrm{F3}=3009 \mathrm{~Hz}$, and $\mathrm{F} 4=3562 \mathrm{~Hz}$ for the first $/ \mathrm{s} /$, and F2 $=1959 \mathrm{~Hz}, \mathrm{~F} 3=2704 \mathrm{~Hz}$, and F4 $=3377 \mathrm{~Hz}$ for the second /s/. The LPC formant tracking did not pick up F2 of the first $/ \mathrm{s} /$, although the inspection of the spectrogram shows that there is resonance around the $2000 \mathrm{~Hz}$ region. The extra pole, which was assumingly introduced by the sublaminal pocket, was found around $1200 \mathrm{~Hz}-1300 \mathrm{~Hz}$, and it seems to have been confused by the software with the real $\mathrm{F} 2$, which is located around $1800-1900 \mathrm{~Hz}$. 
This sublaminal resonance was also observed in the tokens of boshiboshi. The sublaminal resonances of $/ \mathrm{s} /$ in moshimoshis, however, have stronger energy.

$\mathrm{F} 3$ and $\mathrm{F} 4 \mathrm{of} / \mathrm{s} /$ in moshimoshi are found to be very similar to the values of $/ \mathrm{s} /$ in boshiboshi.

It is also noticed that the first /s/ of this speaker had a remarkable amount of energy in the lower frequency region, around $250 \mathrm{~Hz}$, as seen in the comparing with of boshiboshi, especially at the onset. This is probably because the first $/ \mathrm{s} /$ had some residual voicing. The intensity of this low energy was much higher for moshimoshi than for boshiboshi, however. This may be the result of the nasality carried over from the nasal consonant $/ \mathrm{m} /$.

/i/: Only two of the first $/ \mathrm{i} / \mathrm{s}$ and none of the second $/ \mathrm{i} / \mathrm{were}$ observed in moshimoshi. These two /i/s are very short in their duration, similarly to boshiboshi in the previous section. In the observation of boshiboshi above, formants are found to be Fl $=272 \mathrm{~Hz}, \mathrm{~F} 2=1288 \mathrm{~Hz}, \mathrm{~F} 3=2132$ $\mathrm{Hz}$, and F4 $=3229 \mathrm{~Hz} . \mathrm{F} 1$ to $\mathrm{F} 4$ of $/ \mathrm{i} /$ in moshimoshi are $\mathrm{F} 1=201 \mathrm{~Hz}, \mathrm{~F} 2=1205 \mathrm{~Hz}, \mathrm{~F} 3=2217$ $\mathrm{Hz}$, and $\mathrm{F} 4=3302 \mathrm{~Hz}$. As pointed out in the observation of $/ \mathrm{i} / \mathrm{in}$ boshiboshi, $1205 \mathrm{~Hz}$ is probably a sublaminal resonance, rather than F2. Therefore the formants shown as F3 and F4 are probably $\mathrm{F} 2$ and $\mathrm{F} 3$.

\subsubsection{SUMMARY OF THE CAREFULLY CITED MOSHIMOSHI AND BOSHIBOSHI}

The observations made in the previous two sections are summarised in Table 5.4. The observations on boshiboshi are presented on the left and those on moshimoshi are presented on the right. As mentioned, each segment occurs twice in these words. When the first and the second 
occurrences of the segment show different formant contours, the observations for each segment are presented separately. The first and the second occurrences are indicated by "1:" and " $2: "$ respectively. Where the formant contours differ across tokens, the observations are presented according to the tokens. Tokens are represented as boshi 1-4 and moshi 1-4 in the table. It should be noted that the values shown in the table are all approximated. The formants produced at the tracts other than oral cavity are indicated with shading.

\begin{tabular}{|c|c|c|c|}
\hline & & boshiboshi & moshimoshi \\
\hline \multirow{3}{*}[\mathrm{m}]{} & F1 & \multirow{3}{*}{$\mathrm{NA}$} & $200-250 \mathrm{~Hz}$ \\
\hline & F3 & & $\begin{array}{l}\text { 1: } \mathrm{NA} \\
\text { 2: } 1000-1100 \mathrm{~Hz} \\
\text { 1: } \mathrm{NA} \\
\text { 2: } 2800 \mathrm{~Hz}\end{array}$ \\
\hline & F4 & & $\begin{array}{l}\text { 1: NA } \\
2: 3300-3400 \mathrm{~Hz}\end{array}$ \\
\hline \multirow{4}{*}{ [0] } & F1 & $470 \mathrm{~Hz}$ & 250 -300212 (nasa pola) \\
\hline & $\overline{F 2}$ & ap from $800-1000 \mathrm{~Hz}$ to $1500 \mathrm{~Hz}$ & up from 1000 to $1500 \mathrm{~Hz}$ \\
\hline & F3 & $\begin{array}{l}\text { 1: down from } 2600 \mathrm{~Hz} \text { to } 2400 \mathrm{~Hz} \\
2: 2400 \mathrm{~Hz}\end{array}$ & $2250-2300 \mathrm{~Hz}$ \\
\hline & F4 & $\begin{array}{l}\text { 1: down from } 3500-3600 \mathrm{~Hz} \text { to } 3200 \mathrm{~Hz} \\
\text { 2: } 3200-3300 \mathrm{~Hz}\end{array}$ & $3400-3500 \mathrm{~Hz}$ \\
\hline \multirow{4}{*}{ [द] } & F1 & 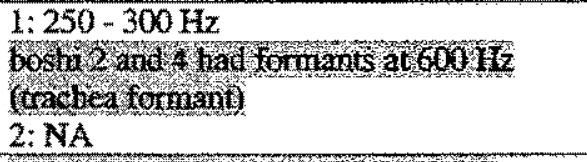 & $\begin{array}{l}1: 250-300 \mathrm{~Hz} \\
2: \mathrm{NA}\end{array}$ \\
\hline & $F_{2}$ & $\begin{array}{l}\text { 1200:1300 Az (sublaminal fornant) } \\
1: \text { up from } 1500 \text { to } 2000 \mathrm{~Hz} \text { and down to } \\
1700 \mathrm{~Hz} \\
2: 1800-2000 \mathrm{~Hz}\end{array}$ & $\begin{array}{l}1200-1300 \mathrm{~Hz} \text { (siblamiagl fow mann) } \\
1800-1900 \mathrm{~Hz}\end{array}$ \\
\hline & F3 & $\begin{array}{l}\text { 1: up from } 2600 \text { to } 3000 \mathrm{~Hz} \text { and down to } \\
2400 \mathrm{~Hz} \\
2: 2500 \mathrm{~Hz}\end{array}$ & $2500 \mathrm{~Hz}$ \\
\hline & F4 & $\begin{array}{l}\text { 1: down from } 3500 \text { to } 3250 \mathrm{~Hz} \\
2: 3300-3400 \mathrm{~Hz}\end{array}$ & $3200-3500 \mathrm{~Hz}$ \\
\hline \multirow{4}{*}{ [i] } & F1 & $\begin{array}{l}1: 250 \mathrm{~Hz} \\
2: \mathrm{NA} \\
\end{array}$ & $\begin{array}{l}1: 201 \mathrm{~Hz} \\
2: \mathrm{NA}\end{array}$ \\
\hline & F2 & $\begin{array}{l}\text { 1: } 1700 \mathrm{~Hz} \\
2: \mathrm{NA}\end{array}$ & $\begin{array}{l}1205 \mathrm{~Hz} \text { (sublaminal formant) } \\
1800-1900 \mathrm{~Hz} \\
2: \mathrm{NA}\end{array}$ \\
\hline & $F_{3}$ & $\begin{array}{l}1: 2200-2300 \mathrm{~Hz} \\
2: \mathrm{NA}\end{array}$ & $\begin{array}{l}\text { 1: } 2217 \mathrm{~Hz} \\
2: \mathrm{NA} \\
\end{array}$ \\
\hline & F4 & $\begin{array}{l}1: 3300 \mathrm{~Hz} \\
2: \mathrm{NA}\end{array}$ & $\begin{array}{l}1: 3302 \mathrm{~Hz} \\
\text { 2: } \mathrm{NA}\end{array}$ \\
\hline
\end{tabular}

Table 53 Summary of the observation of the formant structures of carefully sited boshiboshi and moshimoshis. 
In order to present general formant patterns for these two words, the observed formant values are plotted in Figure 5.5.
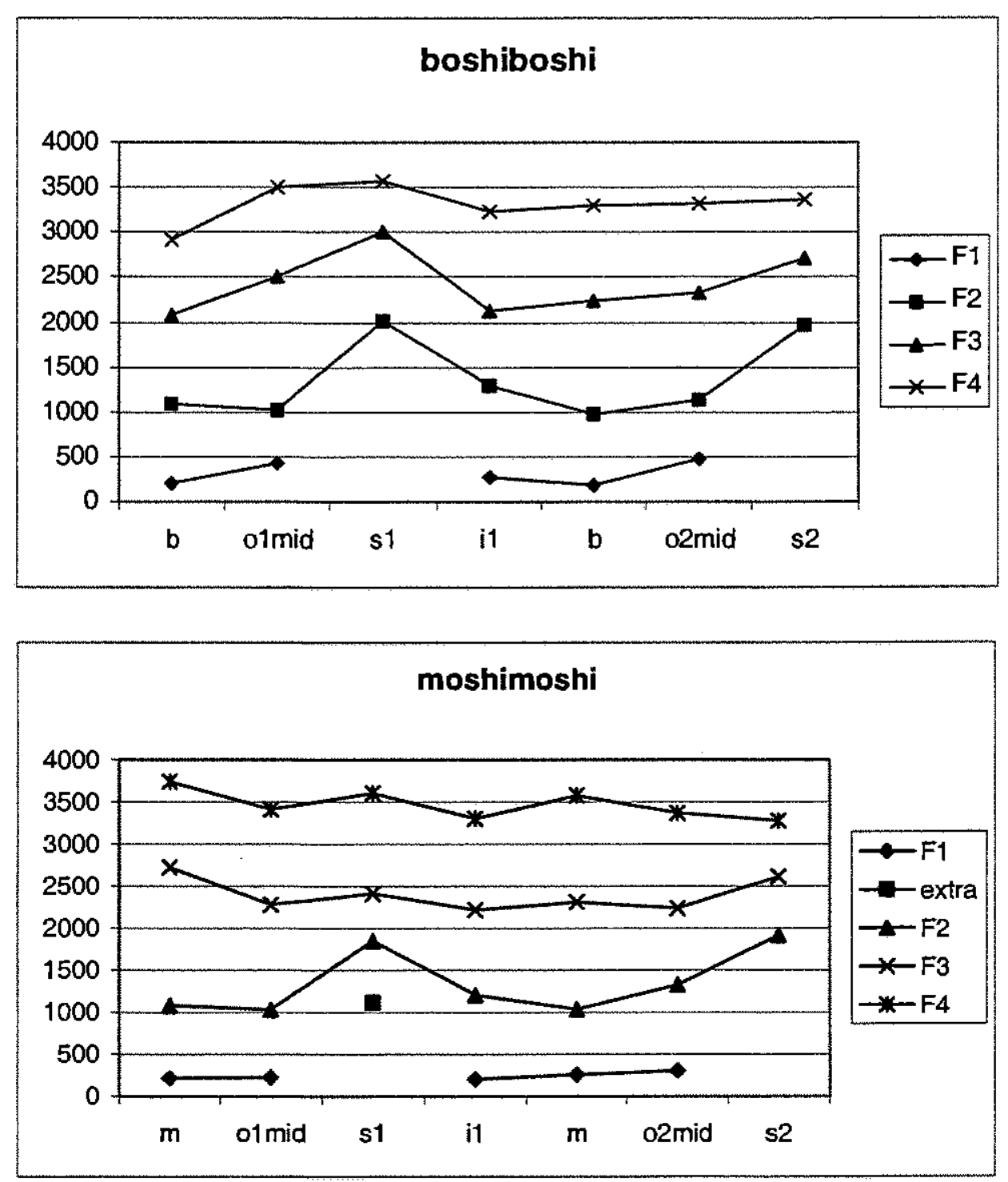

Figure 5.5 Mean F-patterns for boshiboshi and moshimoshi compared.

Figure 5.5 shows that the formant patterns of these two words are generally very similar, with the exception of word initial $/ \mathrm{b} /$ and $/ \mathrm{m} /$. The F-pattens of second halves of these two words are almost 
indistinguishable except that the $\mathrm{F} 1$ of $/ \mathrm{o} /$ is lower for moshimoshi presumably due to the nasalisation. The F-patterns of the first halves, however, differ slightly. The F3 of /o/ and /s/ in boshiboshi are clearly higher than those in moshimoshi.

Generally speaking, however, the identification of formants was much more difficult with moshimoshi than with boshiboshi, due to the nasality of $/ \mathrm{m} /$. The nasality of $/ \mathrm{m} /$ was found to affect the formant structures of the following segments profoundly, especially the lower resonances. The nasality of $/ \mathrm{m} /$ introduces nasal poles to the F-patterns, and the LPC formant extraction function did not perform well in the extraction of lower formants in moshimoshi. LPC sometimes extracted F1 correctly. LPC often ran between a nasal pole and F1, however, interpreting the F1 and nasal pole as a single broad banded spectral peak. The fact that LPC does not pick up F1 correctly will not be a problem, as long as its performance is consistent, for instance, consistently recognising a nasal pole as F1. However, the observations made in the previous sections have shown that, this is not the case. The difficulty in precise measurements possibly introduces extra within-speaker variation, consequently affecting the accuracy of speaker discrimination. These lower formants of nasalised segments are thus not very good parameters for forensic speaker identification.

\subsection{NATURALLY UTTERED MOSHIMOSHI}

Having compared some carefully uttered moshimoshis, this section investigates the naturally uttered moshimoshis spoken by 13 male native Japanese speakers. Most of the speakers were also involved in the research on vowels embedded in different contexts which was described in Chapter 
4. Three more speakers were added for the study in this section. One speaker from the original group (Speaker $\mathrm{KH}$ ), however, had to be removed, as this speaker did not produce sufficient data.

\subsubsection{AUDITORY ANALYSIS}

Through auditory analyses, it was found that the word moshimoshi could contain much withinspeaker variation in its intonation and duration. As described at the beginning of this chapter, the word moshimoshi is the Japanese equivalent of the English word "hello", used exclusively in telephone conversations, especially at the opening of the conversation. In order to elicit spontaneous utterances of moshimoshi, the informants in this study were asked how they would make phone calls to certain individuals, as described in Chapter 3. These hypothetical interlocutors were chosen to vary in their closeness to the informants (e.g. family / stranger) and in the associated socially expected formality (e.g. friends / university lecturers back in Japan). As a result, the utterances in which moshimoshi were embedded differed considerably, although the informants spontaneously used moshimoshi as a part of their response to the question in most situations.

The variations in the interlocutors affected both lexical and phonetic features of the speakers' utterances, making within-speaker variation of the recordings more forensically realistic. The recordings were made not on actual phone calls, but by asking informants how they would make a phone call to certain people. Therefore the influence of the selection of interlocutor was by no means reflected accurately in their speech. Some speakers, however, made an effort to reproduce the situation as realistically as possible by acting, whereas others simply sounded as if they were quoting the words which they would use under the given circumstances. Although the primary purpose of this task design was to elicit naturally and spontaneously occurring moshimoshi rather than recording the variation in phonetic quality caused by the difference in the formality between 
utterances, some of the characteristics associated with differences in speech style will be described in this section.

The hypothetical interlocutors used in the recordings are summarized in Table 5.4. The interlocutors are roughly classified according to formality and the most likely types of interaction.

\begin{tabular}{|c|l|}
\hline Interaction & \multicolumn{1}{|c|}{ Interlocutor } \\
\hline Casual, relaxed & $\begin{array}{l}\text { parents, } \\
\text { friends on their mobile phone, } \\
\text { close friends who live by themselves }\end{array}$ \\
$\downarrow$ & $\begin{array}{l}\text { grandparents, } \\
\text { close friends who live with their parents }\end{array}$ \\
\cline { 2 - 2 } $\begin{array}{c}\text { Formal, possibly a little } \\
\text { nervous }\end{array}$ & $\begin{array}{l}\text { university lecturers in their Japanese university, } \\
\text { total strangers (supposing that they are responding to an advertisement in } \\
\text { newspapers) }\end{array}$ \\
\hline
\end{tabular}

Tahle 5.4 A broad classification of the interlocutors and informants' likely attitudes towards those interlocutors.

An example of one of the most common opening utterances for a telephone conversation is given below. It should be noted, however, that this may vary depending on whether or not the interlocutor identifies whose house he/she is in when the person answers a phone. The following example is a typical utterance where the interlocutor doesn't identify himself $/$ herself when picking up a phone.

Utterance: "( $a$, moshimoshi $X$ desu ga $Y$-san no otaku desu $k a$ " Literal translation: Ah, hello $\mathrm{X}$ is [subject] $\mathrm{Y}^{\prime} \mathrm{s}$ home is [question]

("Hello, this is $\mathrm{X}$ calling. Is that $\mathrm{Y}$ 's place?")

*The glosses showa in [] are particles.

In very casual relaxed situations, the words following moshimoshi are often omitted. It is common to just use "moshimoshi", or "moshimoshi, $\mathrm{Y}$ (the name of the interlocutor)?" expecting the other party to know who is calling by voice. "Moshimoshi, $\mathrm{X}$ (the name of speaker, or words such as ore 
or boku which mean ' $\mathrm{T}$ in Japanese)" is also a common utterance, especially when the other party identifies his / her name first when they answer a phone.

Some speakers varied their utterance-final intonation largely depending upon whom they were supposed to be talking to. In normal utterances, most speakers adopted "moshimoshi t their name" sequence. In casual speech, however, moshimoshi often appeared alone, accompanied by a rising or falling intonation at the end of the word.

Those utterances made by speakers who tried to reproduce the situation realistically could be categorised roughly into two types: formal and casual, according to their auditory impressions as well as lexicon and syntax. The casual speech was further classified into two subjective types, happy / relaxed and blunt speech, mainly on the basis of their auditory impression.

In formal speech, speaker IN replaced moshimoshi with yabun sumimasen (literally, "I'm sorry for calling at night."). Other speakers did not use these words in this interview, as the timing of the call was not specified. More frequent use of this word would be expected if the time of day were specified as nighttime. It is also likely that some speakers will use both moshimoshi and yabun sumimasen as in "(a) moshimoshi yabun sumimasen, $\mathrm{X}$ desuga...". The phonetic characteristics of formal speech were not as conspicuous as casual speech. If anything, formal speech utilises a more precise phonological articulation of the word than casual speech. For instance, vowel prolongation will be avoided in formal speech. When the speakers intend to be more formal or polite, the final ij seems to be often followed by a glottal stop.

Phonetically, the casualness of speech is realised in various ways. The first type of casual speech, happy / relaxed utterance, is realised as prolongation of vowels, as in [mocimo:ci] or [m॰cimscii] 
(e.g. Speakers HA, KF and MO). These are very informal speech styles and the use of this style in the formal situation is regarded as inappropriate.

The utterance made in the other type of casual speech style, namely blunt utterance, stood out from the rest of the data in a few aspects. This style is presumed to be based on the speaker's assumption of closeness, such as "I don't have to worry about what you think about me, because we are so close." In this study, only one token of Speaker MM's casual speech, in a situation where he is calling his good friend on the friend's mobile phone, was classified as a blunt speech. This style is analysed in detail as an example of the effect of paralinguistic information which natural speech inevitably comprises. In this style, Speaker MM reduced the pitch range, with the whole word sounding more monotonous than any other utterance. The differences in pitch range between his normal and blunt utterances are presented in Figure 5.6. The figure also shows the rising intonation at the end of the blunt speech.

[Speaker MM's blunt speech]

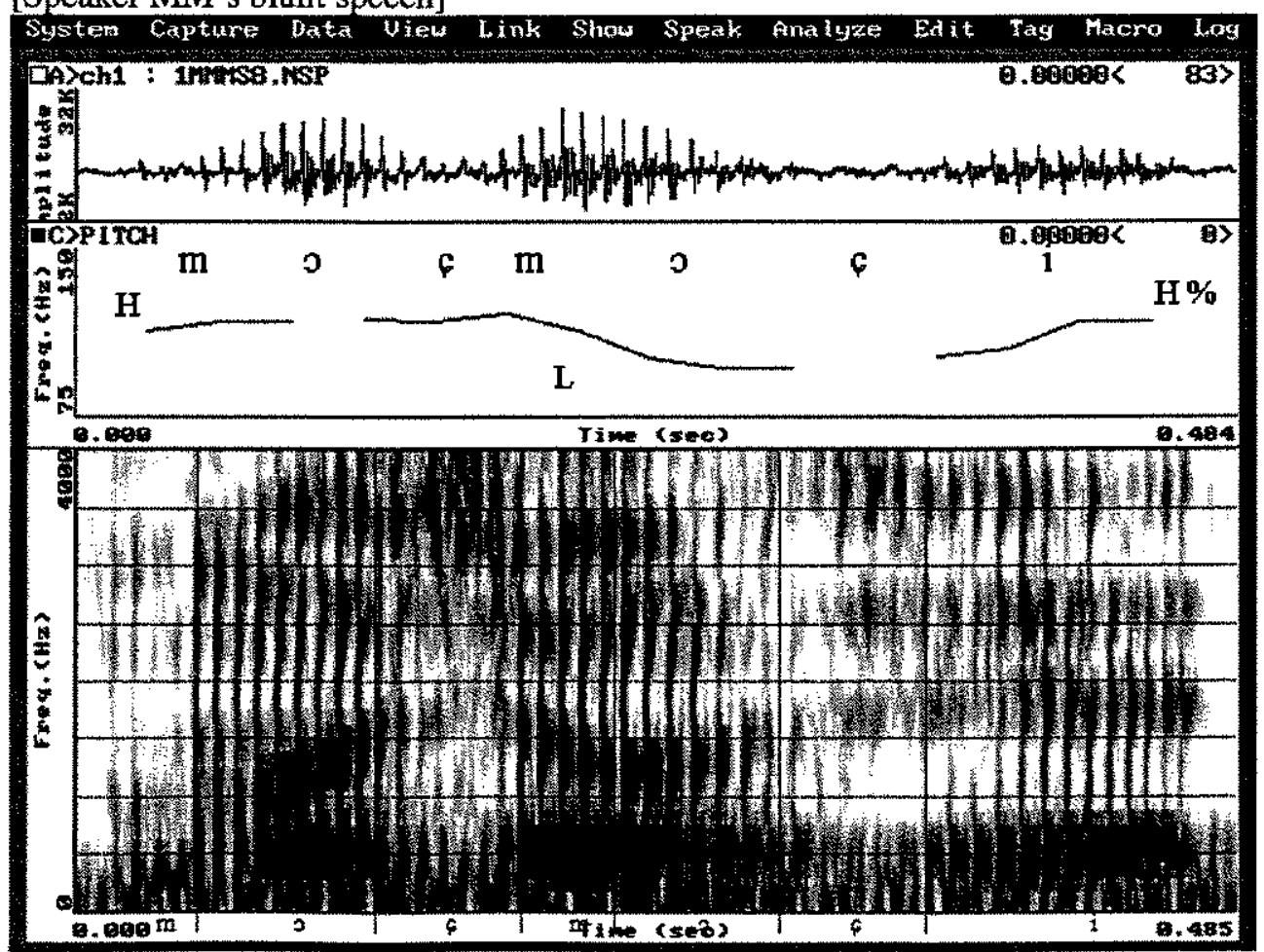




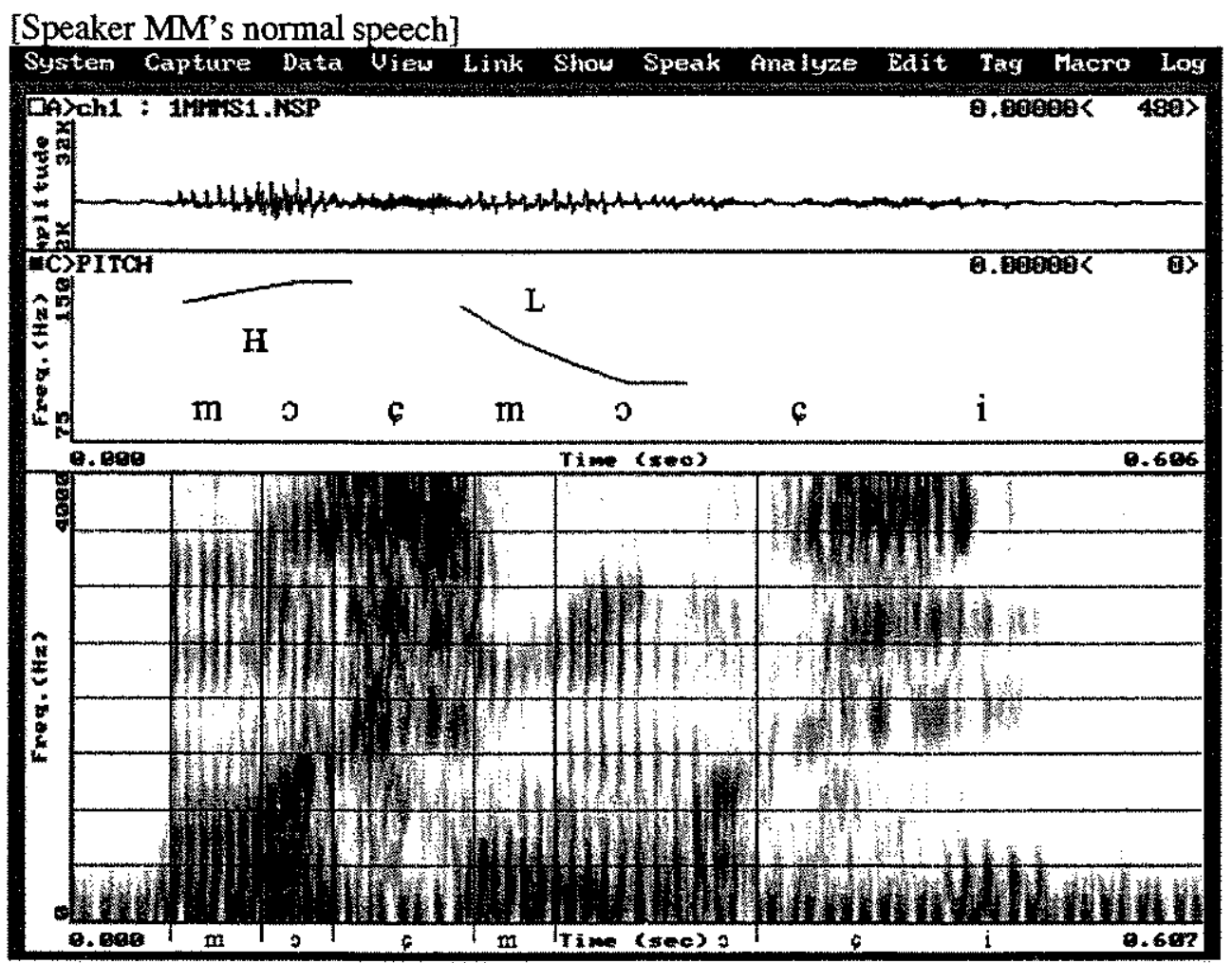

Figure 5.6 Example of the F0 range differences between normal speech and blunt speech of Speaker MM.

It can be seen that the blunt speech has a rising F0 on the final [ci], where the sentence final boundary tone $\mathrm{H} \%$ is used to mark the question. The speaker's blunt speech (top figure) has a clearly narrower and lower F0 range than his normal speech (bottom figure). Table 5.5 summarises the duration and mean F0 of Speaker MM's two moshimoshis presented above.

\begin{tabular}{|c|r|r|r|r|r|}
\hline & Duration & \multicolumn{1}{|c|}{ Range } & \multicolumn{1}{|c|}{ Mean } & \multicolumn{1}{|c|}{ Sd } & \multicolumn{1}{|c|}{ Median } \\
\hline Blunt & 0.456 & $92-133$ & 113 & 10.5 & 118 \\
\hline Normal & 0.467 & $105-151$ & 130.1 & 17.9 & 131.5 \\
\hline
\end{tabular}

Table 5.5 Summary of the duration and F0 of speaker MM's moshimoshi from the blunt and normal tokens.

The relationship between the various acoustic qualities (such as F0, duration, vowel formant structure, and voice quality) and the paralinguistic information speakers intend to convey has been reported by Maekawa (1998). In Maekawa's experiments, no equivalent paralinguistic information type to bluntness was included (admiration, disappointment, suspicion, indifference, focused, and 
neutral were identified information types in his experiments). Maekawa's experiment reports a compression of the pitch range with the expression of disappointment, however, which is a similar result to the data on the blunt utterance in this study. Clearly disappointment and bluntness differ in their communicative intent, making a direct comparison of his result to this study inappropriate. Maekawa's results, however, seem to agree with the current study in identifying that the paralinguistic information can have a significant impact on the pitch range of utterances.

The blunt utterance also sounded less clearly articulated. This impression was possibly caused by the articulation of the first $/ \mathrm{s} /$. The comparison of the audio waveforms in Figure 5.6 demonstrates that the first /s/ blunt utterance (top figure) is voiced. The figure shows that this $/ \mathrm{s} /$ has a periodic wave instead of the non-periodic high frequency wave at the point where the first /s/ is supposed to be located. The duration is also much shorter than the first $/ \mathrm{s} /$ in the example of his normal utterance (bottom figure).

Further, the articulation of the $/ \mathrm{o} /$ vowel for the blunt utterance was examined to determine whether or not the auditory impression of unclear pronunciation was reflected in its acoustics. Since F1 and F2 are known to be correlated with height and frontness respectively, F1 and F2 of the /o/ vowel were examined. Figure 5.7 are the F1/F2 scatterplots of Speaker MM's first and the second /o/ vowels sampled at 3 different parts in their duration. Each scatterplot contains all 8 tokens from the first recording session of this speaker. The location of token 8 (the blunt utterance) is indicated by a square. The top three figures show the onset, middle and offset of the first $/ 0 /$, and the bottom three figures show those of the second $/ \mathrm{o}$. 

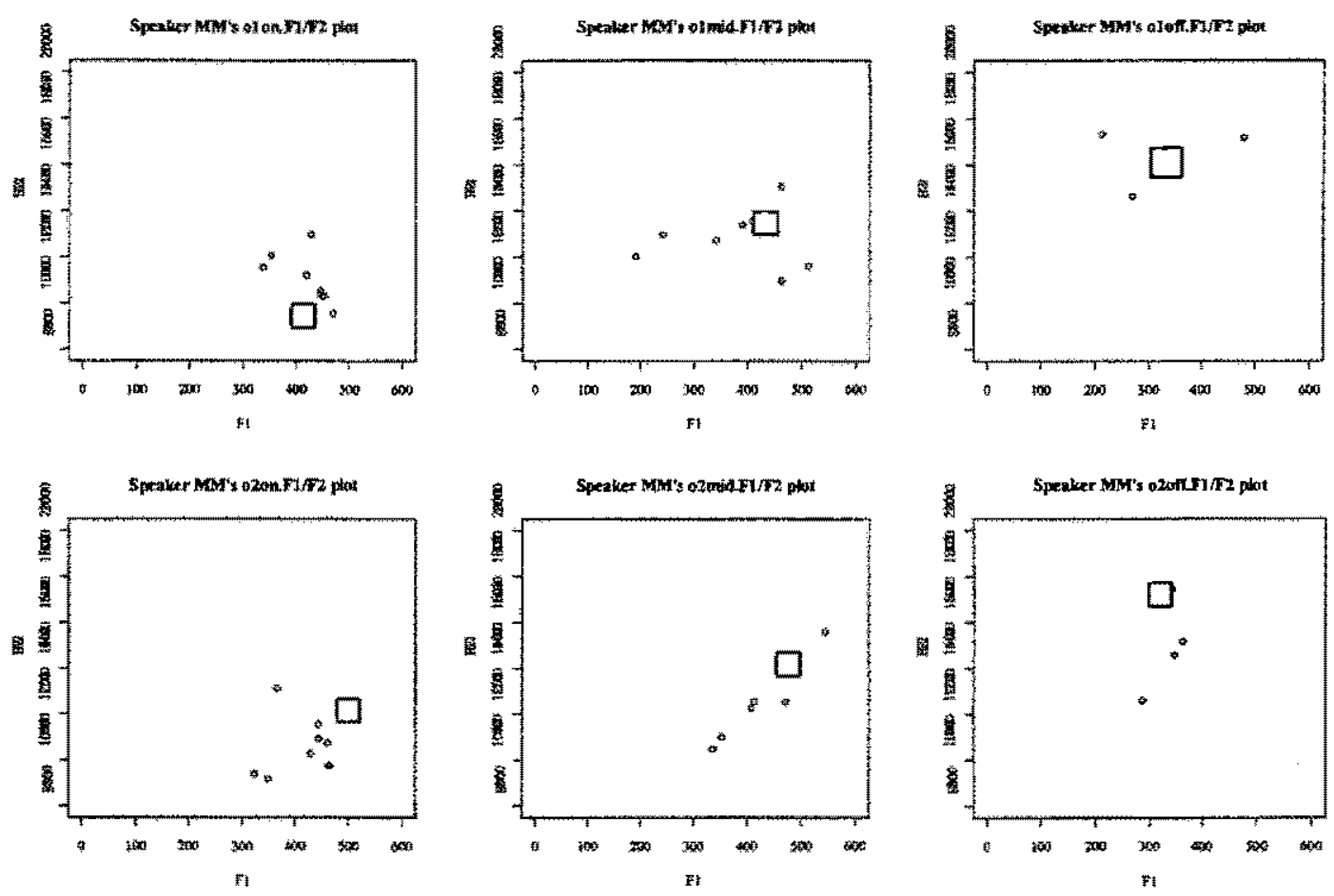

Figure 5.7 Plotted F1 and F2 for the first and the second /o/ of Speaker MM's blunt utterance. The location of blunt speech is indicated by the square.

Figure 5.7 shows that the lack of the clarity in the auditory impression was not reflected in the F1 and F2 placement. As /o/ is a low back vowel, when it is less clearly articulated (i.e. centralised), F1 were expected to be lower and F2 were expected to be higher than the others, as F1 and F2 correlate with height and frontness. This is not observed, however, in Figure 5.7 above. The fact that the paralinguistic factor seems not to have affected the formant structures of this data suggests that formant pattern is not as susceptible as F0 to paralinguistic differences, at least for this token. This makes the formant patterns an even more attractive parameter in forensic phonetics. 


\subsubsection{MEASUREMENTS}

Now we come to the central part of this chapter. The naturally uttered moshimoshis were processed using CSL as described in Chapter 3. In the acoustic measurements of moshimoshi, F1 to F4 were identified on the basis of continuity with the formants of adjacent segments; the observation of the carefully uttered moshimoshi and boshiboshi made in their previous section; and the vowel formants' values measured from Chapter 4. As was anticipated, the formant measurement of moshimoshis was not an easy task. Some speakers had some extra formants, some had missing formants, whilst others had both. As was described in Chapter 3, the middle point of the each segment of moshimoshi was measured in this study. Additionally, for both the first and the second [o]s, both onset and offset were added to the measuring points in order to capture the perturbations at the onset and offset of the vowel. Figure 5.8 shows an example of where measurements were taken. The white circles in the spectrogram indicate the measuring points. 


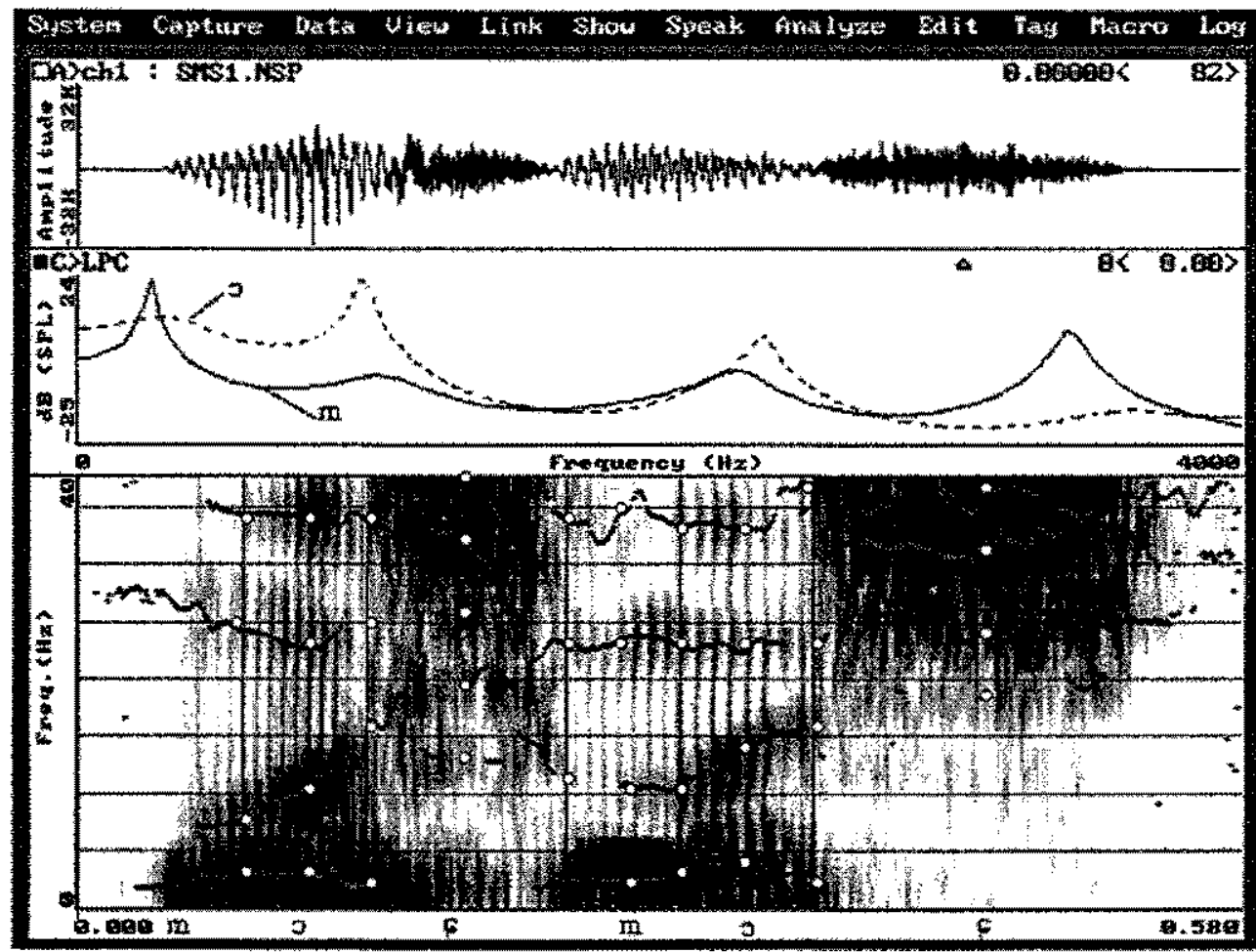

Figure 5.8 Example of the measurement points in a moshimoshi token.

The numbers of moshimoshis measured in this study vary from speaker to speaker, since the speakers sometimes did not respond to the question using this word. The total numbers of moshimoshi recorded for each speaker are listed in Table 5.6.

\begin{tabular}{|l|c|c|c|c|c|c|c|c|c|c|c|c|c|}
\hline Speaker & AA & HA & JN & KA & KF & KO & MM & MN & MO & TN & TS & TY & YH \\
\hline Samples & 12 & 14 & 11 & 8 & 18 & 6 & 17 & 15 & 13 & 13 & 14 & 14 & 12 \\
\hline
\end{tabular}

Table 5.6 Numbers of moshimosini recorded for each speaker.

There are quite a lot of moshimoshis per speaker. It has to be pointed out that in reality we cannot expect to have 6 moshimoshis each from both suspect speech and incriminating speech in a single call. Since it is the opening phrase of a telephone conversation, we cannot expect to have more than one per telephone call, although it is common to have cases where multiple phone calls are intercepted and presented as evidence. 
Having described how F-pattern measurements for moshimoshi were taken, now the results of the measurements are discussed. Firstly the realisation of vowel devoicing is discussed in the next section, and the general formant structures will be analysed subsequently.

\subsubsection{1 [i] vowel}

First of all, it must be noted that the first $/ \mathrm{i} /$ was frequently devoiced by all speakers. The term 'devoiced' is used here according to the traditional Japanese phonological description. The phonetic reality of this $\sqrt{ } /$ vowel is, however, more like omission, rather than devoicing. In Chapter 4 , it was shown that the $/ \mathrm{w}$ / vowels were indeed devoiced, in the sense that they were identifiable in the spectrogram. Their presence could be recognised from their noise-excited formants, although voice excited energy was not observed. The /i/ vowels in moshimoshi, on the other hand, could not be identified in any form, however, suggesting that it is a different type of phonetic phenomenon from the devoicing of the $/ w /$ vowels observed in Chapter 4. The absence of the $/ \mathrm{i} /$ vowels, however, will still be addressed as 'vowel devoicing' in this chapter for convenience.

Although some speakers produced the first /i/fully voiced, this did not occur as often as the devoiced vowel (except for speaker MN, who pronounced the vowel fully voiced with half of his utterances). According to the traditional description on high vowel devoicing (Shibatani 1990), see also Chapters 2 and 4), the first /i/ in moshimosh $i$ is not in a condition where devoicing is preferred, since the vowel is followed by the nasal consonant $/ \mathrm{m} /$. Another observation is that the word final /i/ was more often voiced than the first /i/. This was unexpected, since the vowel is in a condition where devoicing is preferred, conversely to the first $/ \mathrm{i} /$. The numbers of fully voiced $/ \mathrm{i} /$ vowels out of the total numbers of the tokens are summarized in Table 5.7. The table presents the numbers for the two recording sessions and the first and the second / $/$ separately. 


\begin{tabular}{|c|c|c|c|c|}
\hline & & Session 1 & Session 2 & TOTAL \\
\hline AA & $\begin{array}{l}1 \mathrm{st} / \mathrm{i} / \\
2 \mathrm{nd} / \mathrm{j} /\end{array}$ & $\begin{array}{c}0 / 5(0 \%) \\
5 / 5(100 \%) \\
\end{array}$ & $\begin{array}{c}0 / 7(0 \%) \\
6 / 7(85.7 \%) \\
\end{array}$ & $\begin{array}{c}0 / 12(0 \%) \\
11 / 12(91.7 \%)\end{array}$ \\
\hline HA & $\begin{array}{l}1 \mathrm{st} / \mathrm{i} / \\
2 \mathrm{nd} / \mathrm{i} /\end{array}$ & $\begin{array}{c}0 / 6(0 \%) \\
5 / 6(83.3 \%) \\
\end{array}$ & $\begin{array}{c}0 / 8(0 \%) \\
7 / 8(87.5 \%)\end{array}$ & $\begin{array}{c}0 / 14(0 \%) \\
12 / 14(85.7 \%)\end{array}$ \\
\hline $\mathrm{JN}$ & $\begin{array}{l}1 \text { st /i/ } \\
2 \text { nd /i/ }\end{array}$ & $\begin{array}{l}1 / 5(20 \%) \\
1 / 5(20 \%) \\
\end{array}$ & $\begin{array}{l}1 / 6(16.7 \%) \\
1 / 6(16.7 \%)\end{array}$ & $\begin{array}{l}2 / 11(18.2 \%) \\
2 / 11 \quad(18.2 \%) \\
\end{array}$ \\
\hline $\mathrm{KA}$ & $\begin{array}{l}1 \mathrm{st} / \mathrm{i} / \\
2 \mathrm{nd} / \mathrm{i} /\end{array}$ & $\begin{array}{c}0 / 4(0 \%) \\
1 / 4(25 \%)\end{array}$ & $\begin{array}{l}1 / 4(25 \%) \\
3 / 4(75 \%) \\
\end{array}$ & $\begin{array}{c}1 / 8(12.5 \%) \\
4 / 8(0 \%)\end{array}$ \\
\hline $\mathrm{KF}$ & $\begin{array}{l}1 \mathrm{st} / \mathrm{i} / \\
2 \mathrm{nd} / \mathrm{i} /\end{array}$ & $\begin{array}{l}1 / 7(14.3 \%) \\
7 / 7(100 \%)\end{array}$ & $\begin{array}{c}3 / 11(27.3 \%) \\
10 / 11(90.9 \%)\end{array}$ & $\begin{array}{c}4 / 18(22.2 \%) \\
17 / 18(94.4 \%)\end{array}$ \\
\hline KO & $\begin{array}{l}\text { 1st } / \mathrm{i} / \\
2 \mathrm{nd} / \mathrm{i} /\end{array}$ & & & \\
\hline MM & $\begin{array}{l}1 \mathrm{st} / \mathrm{i} / \\
2 \mathrm{nd} / \mathrm{N} /\end{array}$ & $\begin{array}{ll}1 / 9 & (11.1 \%) \\
3 / 9 & (33.3 \%)\end{array}$ & $\begin{array}{c}1 / 8(12.5 \%) \\
6 / 8(75 \%)\end{array}$ & $\begin{array}{l}2 / 17(11.8 \%) \\
9 / 17 \quad(52.9 \%)\end{array}$ \\
\hline MN & $\begin{array}{l}\text { 1st } / \mathrm{i} / \\
\text { 2nd /i/ }\end{array}$ & $\begin{array}{c}1 / 7(14.3 \%) \\
0 / 7(0 \%)\end{array}$ & $\begin{array}{c}7 / 8(87.5 \%) \\
0 / 8(0 \%) \\
\end{array}$ & $\begin{array}{c}8 / 15(53.3 \%) \\
0 / 15(0 \%)\end{array}$ \\
\hline $\mathrm{MO}$ & $\begin{array}{l}\text { 1st } / \mathrm{i} / \\
\text { 2nd } / \mathrm{i} /\end{array}$ & $\begin{array}{c}3 / 6(50 \%) \\
5 / 6(83.3 \%)\end{array}$ & $\begin{array}{l}2 / 7(28.6 \%) \\
3 / 7(42.9 \%)\end{array}$ & $\begin{array}{l}5 / 13(38.5 \%) \\
8 / 13(61.5 \%)\end{array}$ \\
\hline TN & $\begin{array}{l}\text { 1st /i/ } \\
\text { 2nd /i/ }\end{array}$ & $\begin{array}{c}0 / 6(0 \%) \\
4 / 6(22.2 \%) \\
\end{array}$ & $\begin{array}{c}0 / 7(0 \%) \\
4 / 7(57.1 \%) \\
\end{array}$ & $\begin{array}{c}0 / 13(0 \%) \\
8 / 13(61.5 \%)\end{array}$ \\
\hline TS & $\begin{array}{l}1 \mathrm{st} / \mathrm{i} / \\
\text { 2nd / / } /\end{array}$ & $\begin{array}{c}2 / 8(25 \%) \\
5 / 8(62.5 \%) \\
\end{array}$ & $\begin{array}{c}0 / 6(0 \%) \\
1 / 6(16.7 \%)\end{array}$ & $\begin{array}{l}2 / 14(14.3 \%) \\
6 / 14(42.9 \%)\end{array}$ \\
\hline TY & $\begin{array}{l}1 \mathrm{st} / \mathrm{i} / \\
2 \mathrm{nd} / \mathrm{i} /\end{array}$ & $\begin{array}{c}0 / 6(0 \%) \\
2 / 6(33.3 \%)\end{array}$ & $\begin{array}{c}0 / 8(0 \%) \\
5 / 8(62.5 \%)\end{array}$ & $\begin{array}{c}0 / 14(0 \%) \\
7 / 14(50 \%)\end{array}$ \\
\hline YH & $\begin{array}{l}\text { 1st } / \mathrm{i} / \\
\text { 2nd /i/ }\end{array}$ & $\begin{array}{l}1 / 6(16.7 \%) \\
2 / 6(33.3 \%)\end{array}$ & $\begin{array}{l}1 / 6(16.7 \%) \\
2 / 6(33.3 \%)\end{array}$ & $\begin{array}{l}2 / 12(16.7 \%) \\
4 / 12(33.3 \%)\end{array}$ \\
\hline
\end{tabular}

Table 5.7 Incidence of /i/ devoicing in moshimoshi as function of speaker, recording session, and position in word. Numbers of the first and the second $/ \mathrm{i} /$ observed as a fully voiced vowel out of the total numbers of samples.

Referring back to the results of the devoicing of the /u/ vowel discussed in Chapter 4 and also to the descriptions of the carefully uttered boshiboshi and moshimoshi presented earlier in this chapter, the data of 13 speakers' naturally uttered moshimoshis demonstrated that the traditional phonological description of vowel devoicing does not reflect the phonetic reality of natural speech. Firstly, it is noticed that the second /i/ is less often devoiced. The reason for this may be attributed, at least partially, to speech styles. Some speakers uttered this with a prolonged word final syllable, as in /moshimoshi:\% This is not an uncommon speech style in relaxed and casual situations, especially among the younger people. 
The inconsistent nature of devoicing within a speaker is even more obvious, when we look at the realisation of devoicing across the recording sessions. With the exception of Speaker KO, there was no speaker with a constant presence or absence of this /i/ vowel in observation. It is also worth nothing that even the two speakers who showed very constant results in Chapter 4 (Speaker HA had a 100 percent rate of devoicing and Speaker $\mathrm{MN}$ did not devoice the $/ \mathrm{w}$ vowels at all) did not show the same consistency here. In the data of Speaker MN, for instance, only one out of seven first $/ \mathrm{i} / \mathrm{s}$ was observed in the first recording session (14.3\%), whereas seven out of eight first $/ \mathrm{i} / \mathrm{s}$ were observed $(87.5 \%)$, although the result for the second $/ \mathrm{i} /$ was consistent $(0 \%)$.

What are, then, the consequences of this finding for forensic phonetics? Regarding the rates of the incidence of vowel devoicing, it is difficult to judge from this data. As the numbers of tokens vary from speaker to speaker as well as session to session in this dataset (all speakers had four tokens per session in the data presented in Chapter 4), exact matches of the rates are less likely to occur. Thus, with this dataset, it is necessary to determine the threshold for the similarity in order to discuss whether the rates presented here are consistent across two recording sessions. At a quick glance, the rates appear reasonably consistent within a speaker, although there are some exceptions, such as Speaker MO's second $/$ i/ $(83.3 \%$ in the first recording session, but only $42.9 \%$ in the second recording session). The similarity of the rates of devoicing incidence is not researched further in this study.

\subsubsection{Results of measurements}

Table 5.8 gives the means and standard deviations of each speaker for all twelve measuring points in moshimoshi. The data in Table 5.8 are presented according to formants. "ml", "olon", 
"olmid", "o1off", "s1", "i1", "m2", "o2on", "o2mid", and "o2off" all indicate the measuring points which have been shown in Figure 5.8 presented earlier. So, for example, Speaker AA's mean F1 in the middle of the first $/ \mathrm{m} /$ in moshimoshi was $286.9 \mathrm{~Hz}$, with a standard deviation of 107.6. (For individual speaker's measurements, see Appendix 5.1.)

[F1]

\begin{tabular}{|c|c|c|c|c|c|c|c|c|c|c|c|c|}
\hline Speaker & $\mathrm{ml}$ & olon & olmid & oloff & s1 & il & $\mathrm{m} 2$ & o2on & $02 \mathrm{mid}$ & o2off & s2 & $i 2$ \\
\hline $\mathrm{AA}$ & $\begin{array}{l}286.9 \\
107.6\end{array}$ & $\begin{array}{r}407.7 \\
116\end{array}$ & $\begin{array}{l}540.3 \\
101.9\end{array}$ & $\begin{array}{l}410.3 \\
220.4\end{array}$ & & 401 & $\begin{array}{l}353.5 \\
73.43 \\
\end{array}$ & $\begin{array}{l}475.9 \\
156.4\end{array}$ & $\begin{array}{l}410.6 \\
172.5\end{array}$ & $\begin{array}{l}367.8 \\
164.3\end{array}$ & & $\begin{array}{l}326.6 \\
80.97\end{array}$ \\
\hline HA & $\begin{array}{r}513.4 \\
53.7\end{array}$ & $\begin{array}{l}536.5 \\
37.82\end{array}$ & $\begin{array}{l}530.5 \\
106.8\end{array}$ & $\begin{array}{l}515.1 \\
197.9\end{array}$ & 786 & & $\begin{array}{l}428.3 \\
48.43\end{array}$ & $\begin{array}{l}506.3 \\
58.59\end{array}$ & \begin{tabular}{r|}
541 \\
51.4
\end{tabular} & $\begin{array}{l}560.5 \\
190.6\end{array}$ & & $\begin{array}{l}344.6 \\
31.37\end{array}$ \\
\hline $\mathrm{JN}$ & $\begin{array}{l}248.5 \\
37.05\end{array}$ & $\begin{array}{l}328.8 \\
64.22\end{array}$ & $\begin{array}{l}329.5 \\
106.8 \\
\end{array}$ & $\begin{array}{l}178.4 \\
61.91\end{array}$ & $\begin{array}{l}201.7 \\
89.61\end{array}$ & $\begin{array}{r}368 \\
96.17\end{array}$ & $\begin{array}{l}274.7 \\
55.98\end{array}$ & $\begin{array}{l}325.8 \\
48.57\end{array}$ & $\begin{array}{l}360.7 \\
95.74\end{array}$ & $\begin{array}{l}243.9 \\
93.56\end{array}$ & $\begin{array}{r}264 \\
4\end{array}$ & $\begin{array}{r}256 \\
66.93 \\
\end{array}$ \\
\hline $\mathrm{KA}$ & $\begin{array}{l}423.8 \\
102.4\end{array}$ & \begin{tabular}{r|}
509.8 \\
21.7 \\
\end{tabular} & $\begin{array}{r}494.9 \\
24.1\end{array}$ & $\begin{array}{r}363.3 \\
63.7 \\
\end{array}$ & 774 & 471 & \begin{tabular}{r|}
281 \\
37.78 \\
\end{tabular} & $\begin{array}{l}461.4 \\
41.11\end{array}$ & $\begin{array}{r}517.6 \\
113\end{array}$ & $\begin{array}{l}392.6 \\
146.9\end{array}$ & & $\begin{array}{l}330.8 \\
17.65 \\
\end{array}$ \\
\hline $\mathrm{KF}$ & $\begin{array}{l}332.9 \\
81.86\end{array}$ & $\begin{array}{l}394.5 \\
58.03\end{array}$ & $\begin{array}{r}339.4 \\
102\end{array}$ & $\begin{array}{l}355.9 \\
145.3\end{array}$ & $\begin{array}{l}438.8 \\
180.1\end{array}$ & $\begin{array}{l}351.5 \\
43.13\end{array}$ & $\begin{array}{l}320.9 \\
78.93\end{array}$ & $\begin{array}{l}396.8 \\
46.34\end{array}$ & $\begin{array}{r}738.1 \\
1297\end{array}$ & \begin{tabular}{l|}
317.8 \\
81.95
\end{tabular} & & $\begin{array}{l}293.2 \\
43.68\end{array}$ \\
\hline KO & $\begin{array}{r}391.7 \\
93.6\end{array}$ & $\begin{array}{l}354.3 \\
75.66\end{array}$ & $\begin{array}{l}361.8 \\
93.09\end{array}$ & $\begin{array}{l}534.2 \\
291.1\end{array}$ & $\begin{array}{r}398 \\
258.8\end{array}$ & & $\begin{array}{l}413.7 \\
25.48\end{array}$ & $\begin{array}{l}450.4 \\
81.28\end{array}$ & $\begin{array}{l}441.6 \\
67.78\end{array}$ & $\begin{array}{r}461.4 \\
234\end{array}$ & $\begin{array}{r}492 \\
122.7\end{array}$ & \\
\hline $\mathrm{MM}$ & $\begin{array}{l}362.4 \\
72.86\end{array}$ & $\begin{array}{l}423.3 \\
39.11\end{array}$ & $\begin{array}{l}381.1 \\
91.24\end{array}$ & $\begin{array}{r}338.7 \\
94.2\end{array}$ & $\begin{array}{l}442 \\
256\end{array}$ & $\begin{array}{r}386 \\
15.56\end{array}$ & $\begin{array}{l}386.8 \\
46.73\end{array}$ & $\begin{array}{l}423.7 \\
51.82\end{array}$ & $\begin{array}{l}431.5 \\
57.25\end{array}$ & $\begin{array}{r}335 \\
28.36 \\
\end{array}$ & 488 & $\begin{array}{l}347.3 \\
51.21\end{array}$ \\
\hline $\mathrm{MN}$ & $\begin{array}{l}565.1 \\
97.45\end{array}$ & $\begin{array}{l}607.9 \\
81.63\end{array}$ & $\begin{array}{r}524.2 \\
146\end{array}$ & $\begin{array}{l}433.1 \\
173.8\end{array}$ & 886 & $\begin{array}{l}461.7 \\
70.35\end{array}$ & $\begin{array}{r}447 \\
63.64\end{array}$ & $\begin{array}{r}561 \\
45.81\end{array}$ & \begin{tabular}{l|}
569.5 \\
44.85
\end{tabular} & \begin{tabular}{l|}
384.1 \\
183.6
\end{tabular} & & \\
\hline $\mathrm{MO}$ & $\begin{array}{l}226.9 \\
59.44\end{array}$ & $\begin{array}{r}398.4 \\
127\end{array}$ & $\begin{array}{l}380.8 \\
161.2\end{array}$ & 214 & $\begin{array}{l}359.5 \\
54.45\end{array}$ & $\begin{array}{l}299.2 \\
84.11\end{array}$ & $\begin{array}{l}284.9 \\
77.08\end{array}$ & $\begin{array}{l}370.7 \\
103.7\end{array}$ & $\begin{array}{l}354.9 \\
139.1\end{array}$ & $\begin{array}{r}235 \\
50.74\end{array}$ & 289 & $\begin{array}{l}302.4 \\
38.26\end{array}$ \\
\hline $\mathrm{TN}$ & $\begin{array}{l}177.5 \\
25.23\end{array}$ & $\begin{array}{l}205.3 \\
103.8\end{array}$ & $\begin{array}{l}230.7 \\
92.81\end{array}$ & $\begin{array}{r}354 \\
149.9\end{array}$ & & & $\begin{array}{l}204.7 \\
23.52\end{array}$ & $\begin{array}{l}238.7 \\
100.6\end{array}$ & $\begin{array}{l}239.6 \\
141.4\end{array}$ & $\begin{array}{l}685.5 \\
269.6\end{array}$ & $\begin{array}{r}813 \\
9.90\end{array}$ & $\begin{array}{l}299.6 \\
28.29\end{array}$ \\
\hline TS & $\begin{array}{l}320.6 \\
69.63\end{array}$ & $\begin{array}{l}382.9 \\
74.77\end{array}$ & $\begin{array}{l}356.6 \\
120.5\end{array}$ & $\begin{array}{l}173.9 \\
38.48\end{array}$ & $\begin{array}{r}662 \\
229.1\end{array}$ & $\begin{array}{r}369 \\
1.414\end{array}$ & $\begin{array}{l}327.9 \\
77.96\end{array}$ & $\begin{array}{r}374 \\
107.5\end{array}$ & $\begin{array}{l}503.4 \\
105.2\end{array}$ & \begin{tabular}{l|}
370.2 \\
148.3
\end{tabular} & 198 & $\begin{array}{r}312 \\
56.59\end{array}$ \\
\hline TY & $\begin{array}{r}259 \\
56.85\end{array}$ & $\begin{array}{l}404.7 \\
125.6\end{array}$ & $\begin{array}{l}433.1 \\
44.21\end{array}$ & $\begin{array}{l}245.8 \\
88.57\end{array}$ & & & $\begin{array}{r}350.8 \\
95.6\end{array}$ & $\begin{array}{l}454.7 \\
87.05\end{array}$ & $\begin{array}{l}417.9 \\
91.64\end{array}$ & $\begin{array}{r}258.6 \\
81.2\end{array}$ & & $\begin{array}{l}321.2 \\
23.34\end{array}$ \\
\hline $\mathrm{YH}$ & $\begin{array}{l}239.5 \\
119.8\end{array}$ & $\begin{array}{r}32.6 \\
153 \\
\end{array}$ & $\begin{array}{l}247.8 \\
134.2\end{array}$ & $\begin{array}{l}140.5 \\
34.68\end{array}$ & & $\begin{array}{r}358 \\
60.81\end{array}$ & $\begin{array}{l}182.8 \\
59.57\end{array}$ & $\begin{array}{l}289.1 \\
147.8\end{array}$ & $\begin{array}{r}244 \\
139.2\end{array}$ & $\begin{array}{r}252 \\
110.6\end{array}$ & & $\begin{array}{l}276.8 \\
55.14\end{array}$ \\
\hline
\end{tabular}


[F2]

\begin{tabular}{|c|c|c|c|c|c|c|c|c|c|c|c|c|}
\hline eaker & 11 & olon & olmid & oloff & s1 & i1 & $\mathrm{m} 2$ & o2on & $02 \mathrm{mid}$ & o2off & $s 2$ & i2 \\
\hline \multirow[t]{2}{*}{$\mathrm{AA}$} & 795.4 & 702.4 & 1053 & 1418 & 1755 & 1427 & 1100 & 1006 & 1090 & 1602 & 1719 & 184 \\
\hline & & & 195.1 & 194.9 & 228.5 & & 196.4 & 266.7 & 211.8 & 107.3 & 230.5 & 174.1 \\
\hline \multirow[t]{2}{*}{$\mathrm{HA}$} & 101 & 1260 & 1482 & 1762 & 1829 & & 1041 & 1094 & 1437 & 1784 & 1968 & \\
\hline & & & 167.8 & 88.19 & 76.34 & & & 129.9 & & & & \\
\hline \multirow[t]{2}{*}{$\mathbf{J N}$} & 942.2 & 973.4 & 1215 & 1460 & 1551 & 1363 & 1037 & 1344 & 1479 & 1618 & 1750 & 174 \\
\hline & 164 & & & & 109.1 & & & 207.8 & & & 319.6 & 263 \\
\hline & 964.7 & 942.9 & 1185 & 1328 & 1660 & 1201 & 1063 & 1019 & 1314 & 1592 & 1850 & 20 \\
\hline & 156 & & 170 & & 123 & & & 98.77 & & & 118.3 & 66. \\
\hline \multirow[t]{2}{*}{$\mathrm{KF}$} & 975.4 & 984.4 & 1511 & 1965 & 1978 & 2266 & 965.3 & 1034 & 1423 & 1916 & 2089 & 222 \\
\hline & 94.54 & 101.9 & 219.7 & 162.4 & 278.5 & 123 & & 202.5 & 295.1 & 187.8 & 282.6 & 274 \\
\hline \multirow[t]{2}{*}{ KO } & 1225 & 1131 & 1302 & 1474 & 1726 & & 1224 & 1103 & 1346 & 1601 & 1799 & \\
\hline & 139.7 & 131.1 & & 117.2 & 67.39 & & & 182.1 & & 155.9 & 45.19 & \\
\hline \multirow[t]{2}{*}{ MM } & 1290 & 1073 & 1274 & 1569 & 1742 & 1401 & 11 & 1069 & 1288 & 1563 & 1768 & 18 \\
\hline & 148.2 & 102.9 & 116 & & 156.5 & 25. & 136.7 & 120.1 & 137.5 & 123.1 & 86.78 & 166 \\
\hline \multirow[t]{2}{*}{ MN } & 1206 & 1329 & 1520 & 1742 & 2061 & 1669 & 1160 & 1144 & 1305 & 1845 & 2194 & \\
\hline & & & & & & & & & & 85.76 & 97.38 & \\
\hline \multirow[t]{2}{*}{ MO } & 999.3 & 10 & 1516 & 1734 & 173 & 15 & 1108 & 1213 & 1509 & 1685 & 1673 & 20 \\
\hline & 103.4 & 167.2 & & & & & & 165.1 & & 96.87 & 233.4 & \\
\hline \multirow[t]{2}{*}{$\mathrm{TN}$} & 1052 & 1074 & 1492 & 1799 & 1905 & & 1227 & 1395 & 1670 & 1816 & 2109 & \\
\hline & & 124.9 & & 170 & & & & & & 191.1 & 181.6 & \\
\hline \multirow[t]{2}{*}{ TS } & 1014 & 1006 & 1401 & 1748 & 1810 & 1310 & 1102 & 1046 & 1341 & 1791 & 2138 & 20 \\
\hline & 211.7 & 164.9 & 156.9 & 179.3 & 210.5 & & & & 196.3 & & & 158 \\
\hline \multirow[t]{2}{*}{$\mathrm{TY}$} & & & 12 & & 1659 & & & 101 & 1386 & & 157 & 18 \\
\hline & 111.2 & 107.3 & 69.3 & 111.9 & & & 92.18 & 10 & 131 & 68. & 250 & 115 \\
\hline \multirow[t]{2}{*}{$\mathrm{YH}$} & 929.8 & & 143 & & & & & & & & 167 & \\
\hline & & 111.5 & 156.3 & 97.6 & 119. & 14.8 & 114.5 & 113.8 & 82.9 & 150.8 & 80.36 & 274 \\
\hline
\end{tabular}

[F3]

\begin{tabular}{|c|c|c|c|c|c|c|c|c|c|c|c|c|}
\hline eaker & $\mathrm{m} 1$ & olon & 1 mid & oloff & s1 & i1 & $\mathrm{m} 2$ & o2on & $02 \mathrm{mid}$ & 02off & s2 & i2 \\
\hline \multirow[t]{2}{*}{$\mathbf{A A}$} & 2183 & 2236 & 2267 & 2371 & 2578 & 1980 & 2191 & 2248 & 2269 & 2419 & 2509 & 2635 \\
\hline & 106.6 & 58.71 & 84.16 & 91.23 & 132.3 & & 104.7 & 49.62 & 57.8 & 191.1 & 83.83 & 258.2 \\
\hline \multirow[t]{2}{*}{$\mathrm{HA}$} & 2442 & 2442 & 2751 & 2979 & 2963 & & 2470 & 2492 & 2616 & 2876 & & \\
\hline & 160 & 99.6 & 149.7 & 295.4 & 167.8 & & 93.59 & 181 & 284.6 & 190.8 & 157.2 & 27 \\
\hline \multirow[t]{2}{*}{ JN } & 2249 & 2241 & 2178 & 2556 & 2348 & 2415 & 1968 & 2080 & 2109 & 2386 & 2557 & 283 \\
\hline & 1.7 & 150 & & 277.4 & 36.9 & 2.11 & 219 & 159.2 & 191.9 & 92.7 & 80.8 & \\
\hline \multirow[t]{2}{*}{$\mathrm{KA}$} & 2775 & 2909 & 2812 & 2855 & 2586 & 2623 & 2673 & 2325 & 2595 & 2735 & 2796 & 28 \\
\hline & 205.6 & 125.5 & 118.2 & 79.37 & 203.6 & & 135.7 & 169.6 & 210.2 & 145.9 & 95.64 & 65.3 \\
\hline \multirow[t]{2}{*}{$\mathrm{KF}$} & 2348 & 2359 & 2581 & 2898 & 2935 & 3134 & 2480 & 2431 & 2558 & 2732 & 2901 & 291 \\
\hline & 150.2 & 46.33 & & & 215.1 & 687.3 & 115.3 & 230.8 & 207.3 & 266.9 & 145.7 & 145 \\
\hline \multirow[t]{2}{*}{ KO } & 2790 & 2612 & 2585 & 2512 & 2597 & & 2608 & 2563 & 2608 & 2593 & 2635 & \\
\hline & 170.9 & 89.99 & 79.17 & & 113. & & 148.3 & 186.8 & 125,1 & 100.8 & 73.7 & \\
\hline \multirow[t]{2}{*}{$\mathrm{MM}$} & 2381 & 25 & & & 268 & 2305 & 2447 & 2491 & 2517 & 2533 & 269 & 256 \\
\hline & 130.4 & & & 176.3 & 21 & 124.5 & 85.51 & 182.4 & 135 & 102.9 & 142.2 & 146 \\
\hline \multirow[t]{2}{*}{ MN } & 2417 & 2407 & 2283 & 260 & 3050 & 2786 & 2447 & 2419 & 2730 & 2997 & 302 & \\
\hline & 35.28 & 125 & 154.4 & 25.3 & 171.2 & 274.6 & 51.57 & 54.81 & 173.5 & 113.7 & 98.2 & \\
\hline \multirow[t]{2}{*}{ MO } & 2821 & 2807 & 2724 & 2717 & 2738 & 2328 & 2461 & 2426 & 2620 & 2671 & 271 & 28 \\
\hline & 123 & 204.4 & 64.85 & 112.6 & 69.16 & 119.4 & 122.1 & 236.1 & 74.55 & 78.51 & 94.01 & 103 \\
\hline \multirow[t]{2}{*}{ TN } & 2659 & 2556 & 2601 & 2573 & 2693 & & 2481 & 2460 & 2508 & 2626 & 2683 & 271 \\
\hline & 151.3 & & & & 223.5 & & 146.1 & 166.1 & & 01.3 & 85.8 & 79.3 \\
\hline \multirow[t]{2}{*}{ TS } & 2210 & 2205 & 2838 & & 26 & 2012 & 221 & 2177 & 2556 & 281 & 2973 & 288 \\
\hline & 332 & 262.6 & 98.3 & 174.5 & 196.7 & 67.88 & 118.5 & 193.3 & 336.8 & 277.5 & 280 & 341 \\
\hline \multirow[t]{2}{*}{ TY } & 2409 & 2336 & 2379 & 2693 & 2608 & & 2482 & 2423 & 2420 & 284 & 2523 & 270 \\
\hline & 64.85 & 141.6 & 106.3 & 170.7 & 186.7 & & 98.95 & 179 & 211.6 & 113.2 & 172.7 & \\
\hline \multirow[t]{2}{*}{$\overline{Y H}$} & 2124 & & 2325 & & 2440 & & & & 2228 & 231 & 2363 & \\
\hline & & & & & & & & & & & 20.3 & \\
\hline
\end{tabular}


[F4]

\begin{tabular}{|c|c|c|c|c|c|c|c|c|c|c|c|c|}
\hline Speaker & $\mathrm{ml}$ & olon & olmid & oloff & s1 & il & $\mathrm{m} 2$ & $020 n$ & $02 \mathrm{mid}$ & $020 \mathrm{ff}$ & $\mathrm{s} 2$ & $\mathrm{iz}$ \\
\hline \multirow[t]{2}{*}{$\mathrm{AA}$} & 3517 & 3641 & 3817 & 3424 & 3526 & 3047 & 3367 & 3385 & 3425 & 3449 & 3562 & 3481 \\
\hline & 210.8 & 186.2 & 138.5 & 124,1 & 116.5 & & 198.5 & 184.6 & 115.8 & 164.2 & 97.72 & 289.1 \\
\hline \multirow[t]{2}{*}{$\mathrm{HA}$} & 3585 & 3628 & 3638 & 3834 & 3731 & & 3484 & 3580 & 3543 & 3696 & 3684 & 3801 \\
\hline & 223.3 & 104.5 & 238 & 106.2 & 113.6 & & 174.8 & 201.6 & 185.2 & 170.2 & 151.4 & 165.2 \\
\hline \multirow[t]{2}{*}{$\mathrm{IN}$} & 3446 & 3313 & 3492 & 3594 & 3410 & 3560 & 3195 & 3386 & 3216 & 3484 & 3407 & 3629 \\
\hline & 237.5 & 154.3 & 191.6 & 309.9 & 245 & 345.1 & 222.1 & 250.3 & 196.5 & 255.2 & 137.8 & 266.4 \\
\hline \multirow[t]{2}{*}{$\mathrm{KA}$} & 3632 & 3521 & 3586 & 3731 & 3305 & 3520 & 3603 & 3326 & 3397 & 3604 & 3738 & 3754 \\
\hline & 194.4 & 88.06 & 193.8 & 113.9 & 146.2 & & 94.93 & 149.9 & 233.3 & 214 & 177.7 & 127.7 \\
\hline \multirow[t]{2}{*}{$\mathrm{KF}$} & 3962 & 3786 & 3826 & 3918 & 3758 & 4122 & 3841 & 3884 & 3840 & 3784 & 3894 & 3811 \\
\hline & 135.6 & 204.5 & 183.2 & 231.2 & 152.3 & 294.2 & 218.8 & 167 & 141.9 & 215.4 & 169.4 & 272 \\
\hline \multirow[t]{2}{*}{$\mathrm{KO}$} & 3517 & 3529 & 3613 & 3462 & 3363 & & 3208 & 3295 & 3395 & 3544 & 3409 & \\
\hline & 301.5 & 87.1 & 115.3 & 179.8 & 96.76 & & 103.3 & 41.14 & 118.9 & 131.4 & 108.9 & \\
\hline \multirow[t]{2}{*}{$\mathrm{MM}$} & 3022 & 3390 & 3484 & 3573 & 3538 & 2997 & 2987 & 3432 & 3425 & 3561 & 3616 & 3469 \\
\hline & 109,4 & 139.6 & 144,6 & 101 & 103.8 & 116.7 & 249.6 & 212.4 & 108.7 & 117.6 & 124.5 & 180.8 \\
\hline \multirow[t]{2}{*}{$\mathrm{MN}$} & 3053 & 3018 & 3020 & 3406 & 3737 & 3301 & 3455 & 3625 & 3729 & 3720 & 3839 & \\
\hline & 173.4 & 171.1 & 89.33 & 311.4 & 153.7 & 181.9 & 131.6 & 59.71 & 113.4 & 152 & 81.12 & \\
\hline \multirow[t]{2}{*}{ MO } & 3776 & 3637 & 3784 & 3721 & 3692 & 3348 & 3594 & 3577 & 3687 & 3786 & 3896 & 3780 \\
\hline & 272 & 106.2 & 113.8 & 72.97 & 141,2 & 99.57 & 139.9 & 89.59 & 86.35 & 159.7 & 136 & 157.1 \\
\hline \multirow[t]{2}{*}{ TN } & 3762 & 3535 & 3660 & 3833 & 3793 & & 3595 & 3583 & 3687 & 3734 & 3761 & 3707 \\
\hline & 100.3 & 124.6 & 129.8 & 128.1 & 118.4 & & 203 & 178.5 & 200.2 & 139.5 & 119.8 & 169 \\
\hline \multirow[t]{2}{*}{$\mathrm{TS}$} & 3290 & 3422 & 3559 & 3564 & 3459 & 2695 & 3268 & 3312 & 3460 & 3626 & 3743 & 3814 \\
\hline & 337 & 121.1 & 145 & 165.2 & 147.7 & 599.6 & 124.6 & 155.6 & 248.1 & 185.4 & 177.9 & 358 \\
\hline \multirow[t]{2}{*}{ TY } & 3389 & 3433 & 3299 & 3394 & 3317 & & 3696 & 3317 & 3367 & 3495 & 3448 & 3392 \\
\hline & 256.4 & 165.7 & 161.4 & 132.4 & 91.92 & & 282.8 & 105.5 & 198.6 & 191.4 & 194.2 & 214.3 \\
\hline \multirow[t]{2}{*}{ YH } & 3312 & 3519 & 3653 & 3609 & 3474 & 3196 & 3257 & 3303 & 3356 & 3369 & 3340 & 3464 \\
\hline & 230.7 & 233.2 & 187.5 & 181.3 & 194.5 & 113.8 & 198.7 & 146.5 & 143 & 131.8 & 151.9 & 96.27 \\
\hline
\end{tabular}

Table 5.8 Individual speaker' means and standard deviations for F-pattern in moshimoshi.

Overall means and standard deviations for Fl to F4 were also calculated across 13 speakers' means. The results are presented in Table 5.9.

\begin{tabular}{|c|c|c|c|c|c|c|c|c|c|c|c|c|c|}
\hline Speaker & & $\mathrm{ml}$ & olon & olmid & oloff & s1 & il & $\mathrm{m} 2$ & d2on & o2mid & $020 \mathrm{ff}$ & s2 & $i 2$ \\
\hline \multirow[t]{2}{*}{$\mathrm{Fl}$} & $\mathrm{m}$ & 332.7 & 4052 & 394.3 & 322.5 & 551.5 & 383 & 323.9 & 412 & 421.79 & 373.3 & 424 & 311.8 \\
\hline & sd & 111.1 & 99.1 & 100.1 & 120 & 221 & 83.3 & 75.5 & 87.1 & 101.51 & 129.4 & 206.1 & 29.05 \\
\hline \multirow[t]{2}{*}{$\mathrm{F} 2$} & m & 1055.2 & 1032.5 & 1366.8 & 1631 & 1771.4 & 1564 & 1113.4 & 1132 & 1384.8 & 1693.9 & 1865.1 & 1975 \\
\hline & sd & 143.3 & 160.3 & 153.6 & 176.3 & 139.9 & 278 & 81.7 & 124.2 & 131 & 120.5 & 186.4 & 214 \\
\hline \multirow[t]{2}{*}{ F3 } & $\bar{m}$ & 2329.4 & 2332.6 & 2534.1 & 2667.7 & 2683.2 & 2476 & 2385.4 & 2364.2 & 2484.9 & 2653.7 & 2719.9 & 2763 \\
\hline & $5 d$ & 165.7 & 191.9 & 211.7 & 180.8 & 188 & 414 & 189.9 & 147 & 173.5 & 192.5 & 201.2 & 171.33 \\
\hline \multirow[t]{2}{*}{ F4 } & $\mathrm{m}$ & 3477.9 & 3501.1 & 3631.7 & 3615,1 & 3545.9 & 3315 & 3355.1 & 3436.5 & 3486.8 & 3594.4 & 3631.3 & 3635.8 \\
\hline & sd & 261.8 & 1562 & 159 & 155.2 & 170.8 & 390 & 221.9 & 173.5 & 165.1 & 118.6 & 178.3 & 152.9 \\
\hline
\end{tabular}

Table 5.9 Mean F-pattem in moshimoshi across all 13 speakers for each measuring point.

Figure 5.9 below is a line chart based on mean values across speakers. The figure shows a rough approximation of the average formant pattern configuration of the word moshimoshi. The first /if 
is omitted in this figure: as many speakers devoiced this vowel, it is assumed that a better approximation is made without its inclusion. As well as presenting the general F-patterns of moshimoshi, three measuring points are marked by larger black triangles in Figure 5.9. They are the points that will turn out to be the parameters which will be incorporated for the discrimination test in the following chapter, Chapter 6 . As can be seen they are all F3, the first $/ \mathrm{o}$, the second $/ \mathrm{m} /$ and the second $/ \mathrm{s} /$.

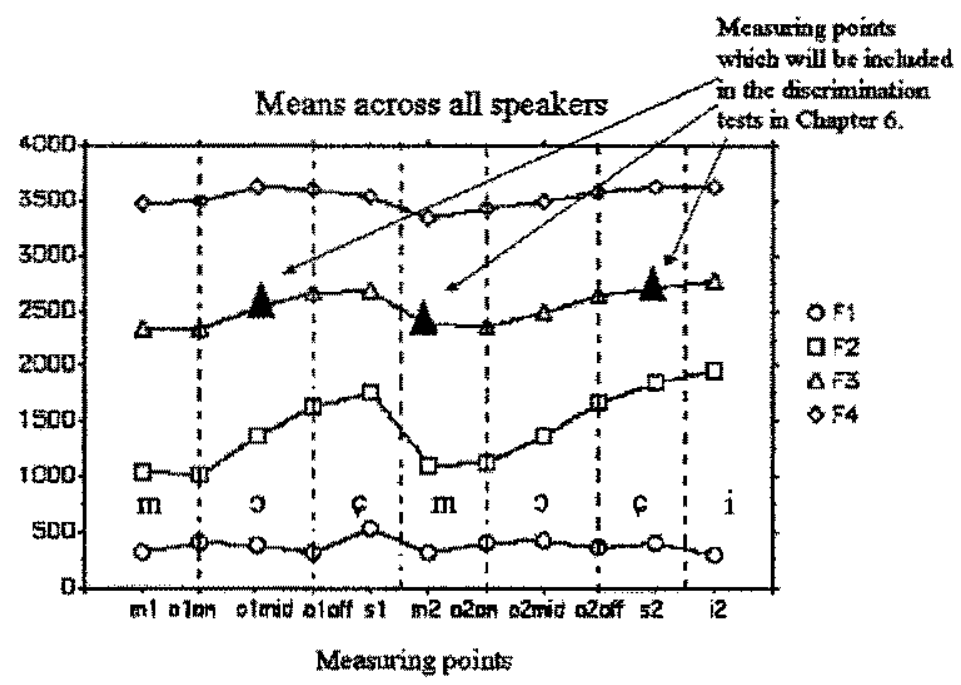

Figure 5.9 Mean F-partern for moshimoshi as function of measurement points.

\subsubsection{CONTEMPORANEOUS / NON-CONTEMPORANEOUS VARIATION}

First of all, the effects of the differences in the recording sessions are examined. Chapter 4 has shown that the difference in the four recording occasions was found to not affect the acoustic output significantly in most measuring points. This effect of the recording occasions is, however, examined again in this section, as comparison of the same words may be more effective to 
illuminate the possible time effect than the comparisons of segments embedded in different contexts.

As described in Chapter 3, there are two sets of data to be compared (ie. moshimoshi from session 1 and that from session 2) for each measuring point of each speaker, since recording sessions were held twice for each speaker. For the statistical analysis, in Rose's (1999b) research on the long* term and short-term speaker variation in hello, three recording sessions are compared by repeatedly testing for each measuring point using one-way ANOVA. Since there are only two sets of data to compare (ie. the data from the first recording session and those from the second recording session) in this study, t-test was used for analysis.

In this section, two types of t-test are presented. One is the repeating t-test for each speaker's sampling point, which is the essentially the same approach as Rose's research except for the comparisons are made between two datasets instead of three. The other is a t-test which tests all speakers at once for each sampling point. The twalue is further examined for significant difference using qq plot. Although a discussion of the appropriate statistical measure is not the aim of this chapter, this demonstrates a problem in the approach which is often practiced.

Firstly, the within-speaker variation of each speaker's measuring point is evaluated with t-test. The results of $t$-test are presented in Table 5.10. The values are probability values (p-value) of the $t$ test, and the top row indicates the measuring points. The results are summarised and then presented for each speaker. The probability values which reached the $95 \%$ confidence level are marked by shading. So, for instance, Speaker AA's F1 of $\mathrm{ml}$ had p-value 0.54 , which did not reach the level of significance. $\mathrm{Fl}$ of $\mathrm{ml}$ for this speaker did not show a significant difference between two recording sessions. F4 of the same segment by this speaker, on the other hand, had the p-value 0.009 , which is marked with shading, as there is significant within-speaker non- 
contemporaneous variation is observed. The cells for the measuring points for which there were no samples to obtain statistics have been left empty.

Speaker AA

\begin{tabular}{|c|c|c|c|c|c|c|c|c|c|c|c|c|}
\hline & m1 & olon & olmid & oloff & s1 & i1 & \multicolumn{1}{c|}{ m2 } & o2on & o2mid & 02off & s2 & i2 \\
\hline F1 & 0.54 & 0.626 & 0.756 & 0.038 & & & 0.313 & 0.196 & 0.911 & 0.829 & 0.996 & 0.955 \\
\hline F2 & 0.178 & 0.71 & 0.111 & 0.002 & 0.4 & & 0.289 & 0.095 & 0.246 & 0.17 & 0.648 & 0.495 \\
\hline F3 & 0.939 & 0.63 & 0.177 & 0.822 & 0.457 & & 0.061 & 0.024 & 0.087 & 0.857 & 0.31 & 0.617 \\
\hline F4 & 0.009 & 0.043 & 0.051 & 0.133 & 0.585 & & 0.933 & 0.088 & 0.263 & 0.743 & 0.056 & \\
\hline
\end{tabular}

Speaker HA

\begin{tabular}{|c|c|r|r|r|c|c|r|r|r|r|c|c|}
\hline & ml & olon & olmid & oloff & s1 & il & \multicolumn{1}{c|}{ m2 } & o2on & o2mid & o2off & s2 & i2 \\
\hline F1 & 0.376 & 0.983 & 0.229 & 0.087 & & & 0.417 & 0.07 & 0.795 & 0.697 & & 0.108 \\
\hline F2 & 0.489 & 0.75 & 0.23 & 0.237 & 0.322 & & 0.487 & 0.647 & 0.521 & 0.485 & 0.463 & 0.098 \\
\hline F3 & 0.961 & 0.315 & 0.3 & 0.65 & 0.159 & & 0.19 & 0.273 & 0.134 & 0.637 & 0.715 & 0.365 \\
\hline F4 & 0.804 & 0.274 & 0.702 & 0.863 & 0.872 & & 0.803 & 0.566 & 0.339 & 0.836 & 0.811 & 0.621 \\
\hline
\end{tabular}

Speaker JN

\begin{tabular}{|c|c|c|c|c|c|c|c|c|c|c|c|r|}
\hline & ml & olon & olmid & oloff & s1 & il & m2 & o2on & o2mid & o2off & s2 & \multicolumn{1}{c|}{ i2 } \\
\hline F1 & 0.908 & 0.054 & 0.515 & 0.188 & 0.727 & & 0.771 & 0.356 & 0.102 & 0.382 & & 0.1 \\
\hline F2 & 0.85 & 0.235 & 0.896 & 0.986 & 0.661 & & 0.053 & 0.252 & 0.617 & 0.266 & 0.055 & 0.192 \\
\hline F3 & 0.455 & 0.063 & 0.639 & 0.133 & 0.751 & & 0.735 & 0.886 & 0.279 & 0.24 & 0.251 & 0.365 \\
\hline F4 & 0.023 & 0.064 & 0.055 & 0.229 & 0.073 & & 0.457 & 0.496 & 0.394 & 0.762 & 0.921 & 0.333 \\
\hline
\end{tabular}

Speaker KA

\begin{tabular}{|c|c|c|c|c|c|c|c|c|c|c|c|c|}
\hline & $\mathrm{ml}$ & olon & olmid & oloff & $\mathrm{s} 1$ & $\mathrm{i} 1$ & $\mathrm{~m} 2$ & o2on & 02mid & o2off & $\mathrm{s} 2$ & $\mathrm{i} 2$ \\
\hline F1 & 0.536 & 0.127 & 0.646 & 0.047 & & & 0.233 & 0.527 & 0.124 & 0.764 & & \\
\hline F2 & 0.287 & 0.758 & 0.214 & 0.487 & 0.273 & & 0.42 & 0.046 & 0.447 & 0.231 & 0.662 & \\
\hline F3 & 0.75 & 0.571 & 0.748 & 0.356 & 0.822 & & 0.806 & 0.212 & 0.201 & 0.12 & 0.655 & \\
\hline F4 & 0.85 & 0.578 & 0.22 & 0.393 & 0.776 & & 0.502 & 0.398 & 0.044 & 0.16 & 0.076 & \\
\hline
\end{tabular}

Speaker KF

\begin{tabular}{|c|c|c|c|c|c|c|c|c|c|c|c|c|}
\hline & m1 & olon & olmid & oloff & s1 & il & m2 & o2on & o2mid & o2off & s2 & i2 \\
\hline F1 & 0.02 & 0.000 & 0.794 & 0.003 & 0.602 & & 0.039 & 0.449 & 0.365 & 0.08 & & 0.619 \\
\hline F2 & 0.075 & 0.408 & 0.652 & 0.158 & 0.208 & & 0.127 & 0.254 & 0.018 & 0.494 & 0.234 & 0.305 \\
\hline F3 & 0.516 & 0.501 & 0.058 & 0.737 & 0.492 & & 0.045 & 0.038 & 0.023 & 0.05 & 0.302 & 0.769 \\
\hline F4 & 0.79 & 0.141 & 0.207 & 0.744 & 0.332 & & 0.144 & 0.427 & 0.024 & 0.392 & 0.716 & 0.766 \\
\hline
\end{tabular}

Speaker KO

\begin{tabular}{|c|c|r|r|r|r|r|r|r|r|r|c|c|}
\hline & ml & olon & olmid & oloff & s1 & i1 & \multicolumn{1}{c|}{ 22 } & o2on & o2mid & o2off & s2 & i2 \\
\hline F1 & 0.478 & 0.11 & 0.098 & 0.45 & & & 0.473 & 0.532 & 0.712 & 0.999 & 0.507 & \\
\hline F2 & 0.353 & 0.465 & 0.203 & 0.3 & 0.476 & & 0.758 & 0.629 & 0.436 & 0.46 & 0.786 & \\
\hline F3 & 0.874 & 0.06 & 0.779 & 0.338 & 0.452 & & 0.413 & 0.094 & 0.322 & 0.61 & 0.335 & \\
\hline F4 & 0.665 & 0.042 & 0.327 & 0.612 & 0.088 & & 0.91 & 0.089 & 0.221 & 0.285 & 0.895 & \\
\hline
\end{tabular}


Speaker MM

\begin{tabular}{|c|c|c|c|c|c|c|c|c|c|c|c|r|}
\hline & $\mathrm{m} 1$ & olon & olmid & oloff & $\mathrm{s} 1$ & $\mathrm{i} 1$ & $\mathrm{~m} 2$ & o2on & o2mid & o2off & $\mathrm{s} 2$ & i2 \\
\hline F1 & 0.271 & 0.935 & 0.794 & 0.175 & & & 0.013 & 0.377 & 0.491 & 0.144 & & 0.55 \\
\hline F2 & 0.511 & 0.248 & 0.632 & 0.402 & 0.529 & & 0.044 & 0.063 & 0.536 & 0.763 & 0.102 & 0.102 \\
\hline F3 & 0.361 & 0.131 & 0.01 & 0.266 & 0.917 & & 0.027 & 0.136 & 0.869 & 0.397 & 0.24 & 0.18 \\
\hline F4 & 0.865 & 0.444 & 0.591 & 0.453 & 0.663 & & 0.067 & 0.189 & 0.171 & 0.114 & 0.343 & 0.029 \\
\hline
\end{tabular}

Speaker MN

\begin{tabular}{|c|r|r|r|r|c|c|c|c|c|c|c|c|}
\hline & $\mathrm{m} 1$ & olon & olmid & oloff & $\mathrm{s} 1$ & $\mathrm{i} 1$ & $\mathrm{~m} 2$ & o2on & 02mid & o2off & $\mathrm{s} 2$ & i2 \\
\hline F1 & 0.5 & 0.904 & 0.581 & 0.798 & & & 0.611 & 0.259 & 0.338 & 0.089 & & \\
\hline F2 & 0.039 & 0.474 & 0.259 & 0.837 & 0.178 & & 0.539 & 0.239 & 0.841 & 0.12 & 0.492 & \\
\hline F3 & 0.56 & 0.971 & 0.278 & 0.166 & 0.579 & & 0.214 & 0.033 & 0.014 & 0.273 & 0.15 & \\
\hline F4 & 0.82 & 0.12 & 0.254 & 0.493 & 0.433 & & 0.335 & 0.408 & 0.021 & 0.931 & 0.732 & \\
\hline
\end{tabular}

Speaker MO

\begin{tabular}{|c|c|c|c|c|c|c|c|c|c|c|c|c|}
\hline & m1 & olon & olmid & oloff & s1 & i1 & \multicolumn{1}{c|}{ 22 } & o2on & o2mid & o2off & s2 & i2 \\
\hline F1 & 0.38 & 0.074 & 0.031 & & 0.513 & 0.142 & 0.76 & 0.959 & 0.004 & & & 0.554 \\
\hline F2 & 0.725 & 0.632 & 0.829 & 0.504 & 0.114 & 0.339 & 0.749 & 0.11 & 0.89 & 0.038 & 0.051 & 0.251 \\
\hline F3 & 0.319 & 0.52 & 0.701 & 0.039 & 0.574 & 0.873 & 0.105 & 0.551 & 0.876 & 0.46 & 0.088 & 0.923 \\
\hline F4 & 0.025 & 0.661 & 0.269 & 0.471 & 0.403 & $3 \mathrm{E}-04$ & 0.004 & 0.004 & 0.209 & 0.086 & 0.736 & 0.589 \\
\hline
\end{tabular}

Speaker TN

\begin{tabular}{|c|r|r|r|c|c|c|c|c|c|c|c|c|}
\hline & $\mathrm{m} 1$ & olon & olmid & oloff & $\mathrm{s} 1$ & $\mathrm{i} 1$ & $\mathrm{~m} 2$ & o2on & 02mid & o2off & $\mathrm{s} 2$ & $\mathrm{i} 2$ \\
\hline F1 & 0.265 & 0.891 & 0.271 & & & & 0.034 & 0.359 & 0.032 & & & 0.028 \\
\hline F2 & 0.87 & 0.01 & 0.013 & 0.898 & 0.838 & & 0.467 & 0.181 & 0.589 & 0.12 & 0.029 & 0.182 \\
\hline F3 & 0.545 & 0.266 & 0.093 & 0.197 & 0.984 & & 0.243 & 0.773 & 0.151 & 0.772 & 0.782 & 0.984 \\
\hline F4 & 0.83 & 0.306 & 0.024 & 0.247 & 0.406 & & 0.202 & 0.008 & 0.054 & 0.025 & 0.055 & 0.018 \\
\hline
\end{tabular}

Speaker TS

\begin{tabular}{|c|r|r|r|c|c|c|c|c|c|c|c|c|}
\hline & m1 & olon & olmid & oloff & s1 & i1 & m2 & o2on & 22mid & o2off & s2 & i2 \\
\hline F1 & 0.062 & 0.012 & $4 \mathrm{E}-05$ & 0.148 & & & 0.016 & 0.03 & 0.727 & & & \\
\hline F2 & 0.04 & 0.119 & 0.561 & 0.629 & 0.47 & & 0.936 & 0.392 & 0.209 & 0.552 & 0.268 & \\
\hline F3 & 0.035 & 0.03 & 0.392 & 0.896 & 0.323 & & 0.005 & 0.096 & 0.738 & 0.31 & 0.129 & \\
\hline F4 & 0.09 & 0.635 & 0.06 & 0.135 & 0.546 & & 0.578 & 0.639 & 0.375 & 0.301 & 0.133 & \\
\hline
\end{tabular}

Speaker TY

\begin{tabular}{|c|r|r|r|r|c|c|c|c|c|c|c|c|}
\hline & \multicolumn{1}{|c|}{ m1 } & olon & olmid & oloff & s1 & il & \multicolumn{1}{c|}{ m2 } & o2on & o2mid & o2off & s2 & i2 \\
\hline F1 & 0.272 & 0.052 & 0.806 & 0.703 & & & 0.864 & 0.594 & 0.683 & 0.586 & & 0.253 \\
\hline F2 & 0.62 & 0.638 & 0.74 & 0.386 & 0.931 & & 0.986 & 0.314 & 0.581 & 0.58 & 0.183 & 0.331 \\
\hline F3 & 0.884 & 0.113 & 0.911 & 0.564 & 0.808 & & 0.005 & 0.17 & 0.072 & 0.371 & 0.062 & 0.148 \\
\hline F4 & 0.303 & 0.014 & 0.022 & 0.35 & 0.009 & & 0.184 & 0.305 & 0.988 & 0.078 & 0.012 & \\
\hline
\end{tabular}


Speaker YH

\begin{tabular}{|c|c|c|c|c|c|c|c|c|c|c|c|c|}
\hline & $\mathrm{m} 1$ & olon & olmid & oloff & s1 & i1 & m2 & o2on & o2mid & o2off & s2 & i2 \\
\hline F1 & 0.028 & 0.528 & 0.718 & 0.003 & & & 0.059 & 0.616 & 0.697 & & & 0.807 \\
\hline F2 & 0.223 & 0.652 & 0.832 & 0.082 & 0.221 & & 0.183 & 0.032 & 0.336 & 0.096 & 0.135 & 0.654 \\
\hline F3 & 0.974 & 0.295 & 0.221 & 0.274 & 0.108 & & 0.087 & 0.18 & 0.356 & 0.501 & 0.625 & 0.877 \\
\hline F4 & 0.634 & 0.776 & 0.347 & 0.145 & 0.022 & & 0.666 & 0.233 & 0.597 & 0.499 & 0.176 & 0.154 \\
\hline
\end{tabular}

Table 5.10 Results of a t-test on F-pattem in non-contemporaneous moshimoshis. The probability values which reached the $95 \%$ confidence level are marked by shading.

At a first glance, it seems that there is not much within-speaker variation between the noncontemporaneous datasets. There are only 59 probability values that reached the $95 \%$ level of significance, of $624 \mathrm{t}$-tests in total. This is about $9.4 \%$ of the total. It should be noted here that data size for t-tests were rather small (see Appendix 5.2). The probability values presented here should therefore be considered as a guideline for interpretation, rather than results which we can rely on.

The small number of data is not the only problem which the statistical procedure presented above suffers from. The level of confidence was, as mentioned earlier, set at $95 \%$. This implies that 5 wrong evaluations per 100 tests can be expected to occur. With this method, t-test was performed many times, 624 times in total. Therefore around 31 tests could have produced random results. Above it was shown that 59 tests out of 624 tests produced the significant results. This is, however, a rather meaningless statement, given that about 31 random results could be included in this number. In the discussion of the statistical approach used to evaluate between-speaker variation effect in Chapter 4 , this random significance was not the problem as the interest there was the size of F-ratio. In the comparison presented here, however, the interest is the examination of whether or not there is a significant difference between the two recordings. Thus this effect of the random significance cannot be ignored. 
The alternative measure to this is performing t-tests combining all speakers, instead of testing each speaker separately. In order to examine the systematic session effect across the speakers, firstly each speaker's means for two recording sessions were calculated, as speakers had different numbers of tokens. Speaker KF had 18 tokens and Speaker KO had 6, for example. Thus without adjusting this difference, statistics will be biased. T-test was then carried out, comparing the difference between two sessions to 0 . If there is little difference between the two sessions, the value should not differ from 0 significantly. The results are summarised in Table 5.11. The columns headed 'df' and 'p value' show degree of freedom and probability value, respectively. Values which reached the level of significance are indicated by shading.

\begin{tabular}{|c|r|r|r|r|r|r|r|r|r|}
\hline & & $\mathrm{t}$ value & \multicolumn{1}{c|}{ df } & $\mathrm{p}$ value & & $\mathrm{t}$ value & df & $\mathrm{p}$ value \\
\hline $\mathrm{m} 1$ & $\mathrm{~F} 1$ & 0.81 & 12 & 0.43 & $\mathrm{~m} 2$ & $\mathrm{~F} 1$ & 0.34 & 12 & 0.74 \\
& $\mathrm{~F} 2$ & -0.03 & 12 & 0.98 & & $\mathrm{~F} 2$ & -0.17 & 12 & 0.87 \\
& $\mathrm{~F} 3$ & -0.40 & 12 & 0.69 & & $\mathrm{~F} 3$ & -0.31 & 12 & 0.76 \\
& $\mathrm{~F} 4$ & -0.05 & 12 & 0.96 & & $\mathrm{~F} 4$ & 1.19 & 12 & 0.26 \\
\hline o1 on & $\mathrm{F} 1$ & 0.09 & 12 & 0.93 & 02 on & $\mathrm{F} 1$ & -0.40 & 12 & 0.70 \\
& $\mathrm{~F} 2$ & -0.31 & 12 & 0.76 & & $\mathrm{~F} 2$ & -0.63 & 12 & 0.54 \\
& $\mathrm{~F} 3$ & -0.78 & 12 & 0.45 & & $\mathrm{~F} 3$ & -1.25 & 12 & 0.24 \\
& $\mathrm{~F} 4$ & 1.78 & 12 & 0.10 & & $\mathrm{~F} 4$ & 1.02 & 12 & 0.33 \\
\hline o1 mid & $\mathrm{F} 1$ & -0.41 & 12 & 0.69 & $02 \mathrm{mid}$ & $\mathrm{F} 1$ & -1.39 & 12 & 0.19 \\
& $\mathrm{~F} 2$ & -0.62 & 12 & 0.55 & & $\mathrm{~F} 2$ & -1.09 & 12 & 0.30 \\
& $\mathrm{~F} 3$ & -0.19 & 12 & 0.85 & & $\mathrm{~F} 3$ & -0.62 & 12 & 0.55 \\
& $\mathrm{~F} 4$ & 0.10 & 12 & 0.92 & & $\mathrm{~F} 4$ & 0.90 & 12 & 0.39 \\
\hline o1 off & $\mathrm{F} 1$ & -0.48 & 11 & 0.64 & 02 off & $\mathrm{F} 1$ & -0.71 & 10 & 0.49 \\
& $\mathrm{~F} 2$ & -0.33 & 12 & 0.74 & & $\mathrm{~F} 2$ & 0.01 & 12 & 0.99 \\
& $\mathrm{~F} 3$ & 1.07 & 12 & 0.31 & & $\mathrm{~F} 3$ & 0.18 & 12 & 0.86 \\
& $\mathrm{~F} 4$ & 0.66 & 12 & 0.52 & & $\mathrm{~F} 4$ & 0.95 & 12 & 0.36 \\
\hline $\mathrm{s} 1$ & $\mathrm{~F} 1$ & -1.37 & 4 & 0.24 & $\mathrm{~s} 2$ & $\mathrm{~F} 1$ & & & \\
& $\mathrm{~F} 2$ & 0.60 & 12 & 0.56 & & $\mathrm{~F} 2$ & -0.33 & 12 & 0.75 \\
& $\mathrm{~F} 3$ & -0.01 & 12 & 0.99 & & $\mathrm{~F} 3$ & -1.65 & 12 & 0.13 \\
& $\mathrm{~F} 4$ & -0.41 & 12 & 0.69 & & $\mathrm{~F} 4$ & -1.17 & 12 & 0.26 \\
\hline i1 & $\mathrm{F} 1$ & 0.40 & 5 & 0.71 & $\mathrm{i} 2$ & $\mathrm{~F} 1$ & 0.63 & 10 & 0.55 \\
& $\mathrm{~F} 2$ & 0.81 & 5 & 0.46 & & $\mathrm{~F} 2$ & 3.61 & 10 & 0.00 \\
& $\mathrm{~F} 3$ & -0.75 & 5 & 0.49 & & $\mathrm{~F} 3$ & 1.22 & 10 & 0.25 \\
& $\mathrm{~F} 4$ & -1.82 & 5 & 0.13 & & $\mathrm{~F} 4$ & 0.87 & 10 & 0.4045 \\
\hline
\end{tabular}

Table 5.11 Results of the t-test, all speakers forming a single data set. $t$-values, differences of freedom, and probability values are presented. The significant result is indicated by shading. 
The results of this t-test show that only $F 2$ of the second /i/ showed a significant difference between the two recording sessions. This is $2 \%$ ( 1 out of 48 tests) and much less than $9.4 \%$ shown in the first set of the t-tests. This indicates that repeating the t-test many times indeed can produce unreliable results.

This approach, however, also repeated the t-test for each measuring point as many as 48 times, and we cannot ignore the possibility of the random errors in this result as well. The results are thus further examined using a qq plot. A qq plot here consists where a plot of the ordered values of tvalues presented above in Table 5.11 (y-axis) versus corresponding quantiles of a t-distribution with mean zero and variance one ( $\mathrm{x}$-axis) are plotted. Linear qq plot indicates that the data is normally distributed (MathSoft Inc, Data Analysis Products division 1999:57). If the results in Table 5.11 are reliable without including random erroneous results, all tokens but iF2 should come out fairly linear. Figure 5.10 below presents 6 qq plots for the t-test presented above, produced by using 6 different random selections of samples from t-distribution. Degree of freedom was set at 12 , since this is the appropriate value for most of the tests. 

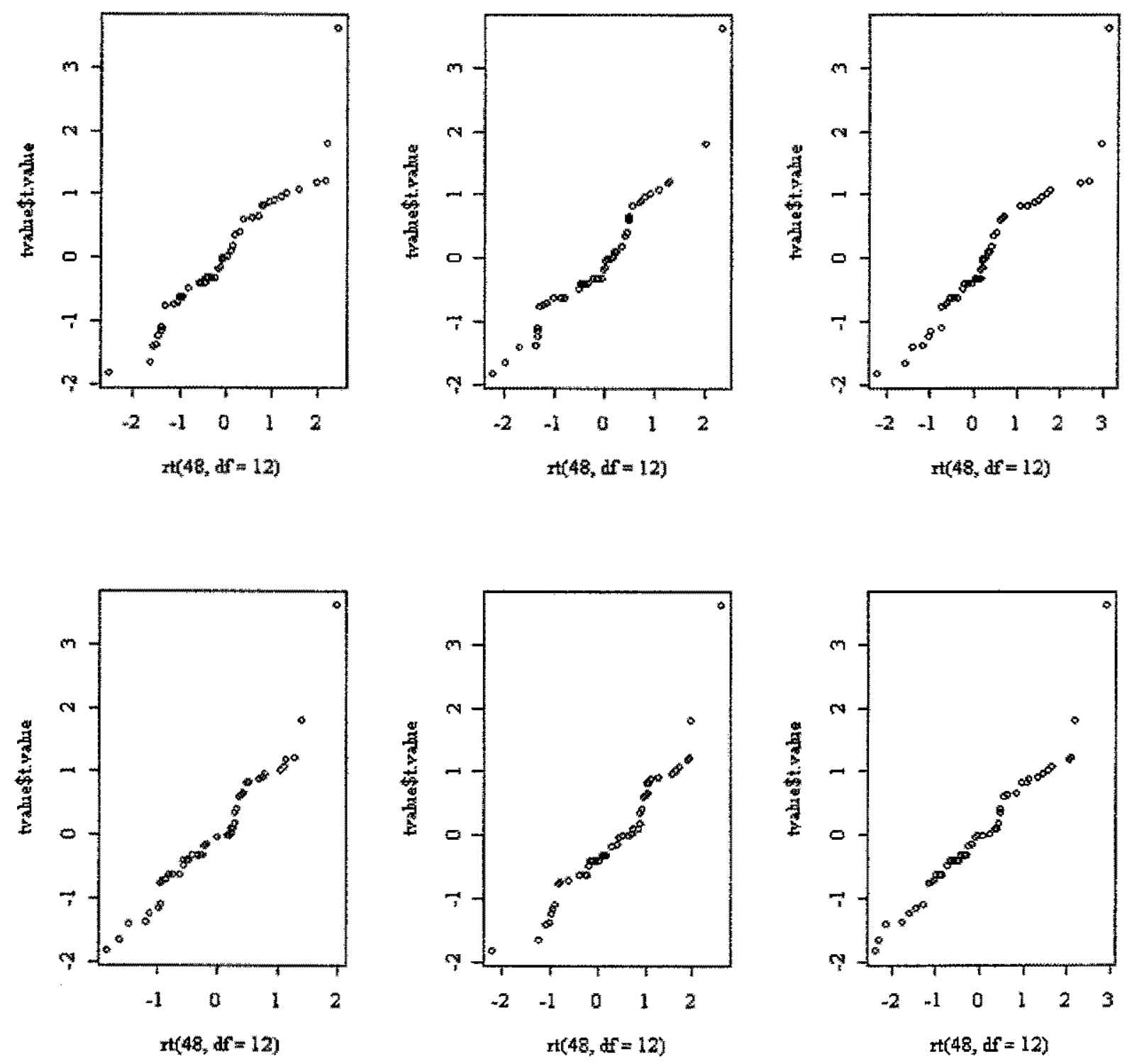

Figure 5.10 qq plots of the t-values.

All six qq plots presented above show that all but one of the $t$-values are distributed normally. The outlier identified from the qq plots is the $F 2$ of the second $/ \mathrm{i} /$ which was evaluated to have a significant difference between the two recording sessions. This indicates that there was no randomly produced error in the results presented in Table 5.11 . 
In sum, it has been shown that there is hardly any systematic session effect across speakers for the acoustic values of moshimoshi. Only F2 of the second /i/ was found to be affected by the session difference. Why only this measuring point showed large within-speaker variation remains unclear. It may be, however, attributed to the difference in the boundary tone.

This section also demonstrated a problem in the commonly practiced statistical method of repeating t-tests (or ANOVA for the same reason). How many significant differences will be found can vary considerably depending on the ways of applying the given statistics. In order to obtain a picture of systematic effect of the session difference, the second approach shown in this section should be followed.

\subsubsection{BETWEEN-SPEAKER VARIATION}

The results of the previous section have shown that the differences between the recording sessions do not significantly affect between-speaker variation in the formant patterns (F-patterns) of moshimoshi. The two datasets from both recording sessions were thus combined below for an examination of between-speaker variation. Following the same procedures as Chapter 4, one-way ANOVA was performed repeatedly for all measuring points taking each speaker as a separate group. F-ratios which were over 20 were marked by shading. Table 5.12 presents F-ratios and the total numbers of measurements made at individual measuring points. The total number (when all tokens of all speakers were measured) is 145 . 


\begin{tabular}{|c|c|c|c|c|c|}
\hline measuring $\mathrm{pt}$. & & 51 & F2 & $\mathrm{F3}$ & F4 \\
\hline \multirow[t]{2}{*}{$\mathrm{ml}$} & F-ratio & 210 & 11.3 & 20,5 & 15.0 \\
\hline & no. & 130 & 132 & 127 & 122 \\
\hline \multirow[t]{2}{*}{ ol on } & F-ratio & 15.1 & 17.2 & 249 & 16.1 \\
\hline & no. & 134 & 143 & 138 & 137 \\
\hline \multirow[t]{2}{*}{ ol mid } & F-ratio & 14.1 & 10.8 & 275 & 220 \\
\hline & no. & 137 & 143 & 142 & 140 \\
\hline \multirow[t]{2}{*}{01 off } & F-ratio & 7.5 & 18.0 & 9.3 & 11.5 \\
\hline & no. & 103 & 143 & 139 & 143 \\
\hline \multirow[t]{2}{*}{ s1 } & F-ratio & 3.5 & 8.3 & 13.5 & 15.7 \\
\hline & no. & 22 & 142 & 140 & 144 \\
\hline \multirow[t]{2}{*}{ il } & F-ratio & 2.5 & 8.4 & 12.8 & 4.7 \\
\hline & no. & 23 & 23 & 23 & 23 \\
\hline \multirow[t]{2}{*}{$\mathrm{m}^{2}$} & F-ratio & 207 & 29 & 272 & 15.1 \\
\hline & no. & 141 & 139 & 142 & 128 \\
\hline \multirow[t]{2}{*}{02 on } & F-ratio & 12.4 & 6.1 & 8.4 & 13.9 \\
\hline & no. & 134 & 139 & 141 & 135 \\
\hline \multirow[t]{2}{*}{$02 \mathrm{mid}$} & F-ratio & 9.0 & 8.8 & 10.0 & 13.8 \\
\hline & no. & 129 & 144 & 143 & 139 \\
\hline \multirow[t]{2}{*}{02 off } & F-ratio & 6.3 & 9.1 & 13.8 & 6.8 \\
\hline & no. & 95 & 142 & 143 & 142 \\
\hline \multirow[t]{2}{*}{$\$ 2$} & F-ratio & 11.8 & 11.8 & 266 & 223 \\
\hline & no. & 12 & 141 & 141 & 143 \\
\hline \multirow[t]{2}{*}{12} & F-ratio & 3.2 & 10.8 & 6.4 & 4.4 \\
\hline & no. & 83 & 84 & 85 & 81 \\
\hline
\end{tabular}

Table 5.12 F-ratios for between-speaker variation and the number of measurements made for each segment. Larger F-ratios (over 20) are marked by shading.

Table 5.12 thus shows that the promising segment / formant combinations in moshimoshi are:

F1 (21.4) and F3 (20.5) at the middle point of the first $/ \mathrm{m} /$

F3 (24.9) at the onset of the first $/ o /$,

$\mathrm{F} 3(27.5)$ and F4 (22.0) at the middle point of the first $/ \mathrm{o} /$,

$\mathrm{F} 1$ (20.7) and F3 (27.2) at the middle point of the second $/ \mathrm{m} /$,

F3 (26.6) and F4 (22.3) at the middle point of the second/s/ (phonetically [c]) 
The word moshimoshi is a repeat of the morpheme moshi, so that each segment appears twice in this word. The results of the first half and the latter half of this word are therefore expected to be similar. Despite this expectation, Table 5.12 showed that the size of F-ratio between the first and the second halves of moshimoshi was not constant. For instance, in the first 'moshi', F3 of the onset and middle of /o/ and F4 of the middle of /o/ were found to have higher F-ratios (24.9, 27.5, and 22.0 respectively), but these segment / formant combinations did not show higher F-ratios for the second 'moshi' (8.4, 10.0, and 13.8 respectively). For the F3 and F4 of /s/ conversely, the higher F-ratios were found in the second 'moshi' (26.6 and 22.3), but not in the first (13.5 and 15.7).

The explanation for this inconsistency may lie in pitch accent. The first $/ 0 /$ which had high Fratios, carries an accent. Ingram et al. (1996:138) have reported an experiment on connected speech revealed that the segments containing a stress accent distinguish between speakers more successfully. Ingram et al.'s report agrees with the results in this study where the first $/ 0 /$ had higher F-ratios than the second $/ \alpha$, despite the fact that English and Japanese have a different accent marking system. This is a clear indication of the sensitivity of the segmental variation to prosodic structure.

The first $/ \mathrm{m} /$, which is also a part of the accented syllable, however, did not show higher F-ratios than the second $/ \mathrm{m} /$. In fact, the F-ratio of F3 of the first $/ \mathrm{m} /(20.5)$ was noticeably lower than that of the second $/ \mathrm{m} /(27.2)$. This is probably attributed to the fact that the first $/ \mathrm{m} / \mathrm{s}$ were considerably shorter and weaker in intensity, compared to the second / $\mathrm{m} /$ for many speakers and, consequently, the higher formants of the first $/ \mathrm{m} /$ could not be measured in some cases. The smaller sampling size may have affected the size of the F-ratio of the first $/ \mathrm{m} /$. 
Although the first $/ \mathrm{s} /$ is not a part of accented syllable, as the intensity of it was clearly affected by the preceding accented syllable, it may be predicted that the first / $/$ / would produce higher F-ratio than the second $/ \mathrm{S} /$. F-ratios of $/ \mathrm{S} /$ showed, however, a contradicting result. The durational difference between the first and the second $/ S /$ probably affected the difference in their F-ratios. Many speakers had a longer duration for the second $/ \mathrm{s} /$ than for the first $/ \mathrm{s} /$. The durational difference found in two $/ \mathrm{s} / \mathrm{s}$ was even larger than that of the two $/ \mathrm{m} / \mathrm{s}$ which was mentioned earlier. The second $/ \mathrm{s} /$ probably reached the stable stage of configuration at the middle of its duration, but the first /s/ may not have had long enough duration to achieve a stable state.

\subsection{COMPARISON WITH THE RESULTS OF THE PREVIOUS CHAPTER}

Now the results for measurements are compared to those of the previous chapter. In Chapter 4 of this thesis, vowels embedded in different contexts were also examined with one-way ANOVA. Since the two sets of the data have a different structure, direct comparison of the F-ratio cannot be made. It is, however, possible to compare the relationship between two vowels $/ \mathrm{f}$ and $/ 0 /$, which were included in both experiments. In the study of moshimoshi, it was found that $f o /$ vowel produces one of the highest $F$-ratios. F-ratios of the two /o/ vowels were generally larger than $F$ ratios of $/ \mathrm{i}$. In Chapter 4 , on the other hand, i// was found to be the powerful parameter, but not fol. /o/ embedded in the different context did not produce any high F-ratio. This discrepancy between the experiments can be interpreted in two ways: the $/ \mathrm{i} /$ vowel was performed more poorly in the study of moshimoshi than it should, and the /o/ vowel performed more poorly in the study in Chapter 4 than it should. 
One possible explanation for the poor performance of the /i/ vowels in moshimosh $i$ is the small sampling numbers for this vowel. As mentioned earlier, many speakers devoiced this vowel, especially the first $/ \mathrm{i} /$. The sample numbers of this vowel were therefore much smaller than that of any other segments. For instance, $23 \mathrm{first} / \mathrm{i} / \mathrm{s}$ and $84 \mathrm{~second} / \mathrm{i} / \mathrm{s}$ were analysed with respect to their F2. F2 of the /o/ were measured on average 142.3 times, however. The smaller F-ratio of $/ \mathrm{i} /$ compared to $/ \mathrm{o} /$ in moshimoshi thus may be explained by the small sampling number for the $/ \mathrm{i} /$ vowels.

It is, however, noticed that the second / $\mathrm{i} /$ in moshimoshi produced lower F-ratios than the first $/ \mathrm{i} /$, despite its sampling number being larger than of the first /i/. Thus there must be other factors affecting the F-ratio of the second $/ \mathrm{i}$. Intonation may be nominated as the explanation for the low F-ratio of the second /i/ vowel. As mentioned earlier, some speakers varied their utterance final intonation largely according to their hypothetical interlocutor. In casual speech, moshimoshi was often accompanied by a sentence final rising or falling intonation. This may have amplified the within-speaker variation of the second $/ \mathrm{l}$, resulting in smaller F-ratios.

An interpretation of the discrepancy between the experiments in the poor performance of $/ 0 /$ when it is embedded in the different words, is discussed next. This interpretation suggests that $/ 0 /$ vowel is more susceptible to coarticulation with other segments. The fact that the $/ \mathrm{d} /$ in measurements produced an F-ratio as high as that of $/ \mathrm{m} /$ and $/ \mathrm{s} /([\mathrm{c}]$, phonetically) is noteworthy. These segments are expected to show much more speaker specific characteristics. The fact that $/ 0 /$ vowel (which was not found to be a particularly promising vowel in the study of the five Japanese vowels) is actually capable of producing F-ratios as high as F-ratios of those supposedly powerful consonants is fairly significant from the forensic phonetics standpoint. That is, even vowels other than /i/ and /e/ which produced high F-ratio in Chapter 4 can be very useful as speaker 
identification parameters, as long as they are embedded in the same word. This finding will allow us a wider selection of the target for the measurements in forensic speaker identification.

\subsection{EXTRA FORMANTS}

The differences in the centre frequencies of F1, F2, F3, and F4 of the segments have been examined so far. Differences between speech samples are not only reflected in the frequencies of those formants, however. The tokens collected for this study involved many formants as well as some missing values. The term "extra formants" is defined here as those formants which could not be classified as F1, F2, F3 or F4, because they are not in continuity with the equivalent formants of their adjacent segments and/or there are other formants with better continuity than the formants of the adjacent segments in that region.

Table 5.13 presents the summary of the extra formants observed in moshimoshi for all speakers. The means and standard deviations and numbers of instances observed are shown there. The top row of the table shows the measuring points for those formants, and the second row indicates where the formants were located relative to the other formants at the measuring points. "F1-2," for instance, means that the formants were found in between F1 and F2. "F4t" means that the formants are above $\mathrm{F} 4$, and below $4000 \mathrm{~Hz}$ (the measurement of this study was limited to values below $4000 \mathrm{~Hz}$, as mentioned in Chapter 3). Those formants shown in the columns headed "F4+" may or may not be F5, but they were not examined in detail in this study, as they have less relevance to forensic phonetic application because high frequencies are often not picked up in telephone signals used as incriminating evidence. The rows headed "no." present how many times each formant was observed. As mentioned in the earlier section of this chapter, speakers do not 
have the same numbers of samples (for details, see Table 5.6 in p.208). The extra formants which appeared relatively regularly (for more than $75 \%$ of total sample number) are marked by shading.

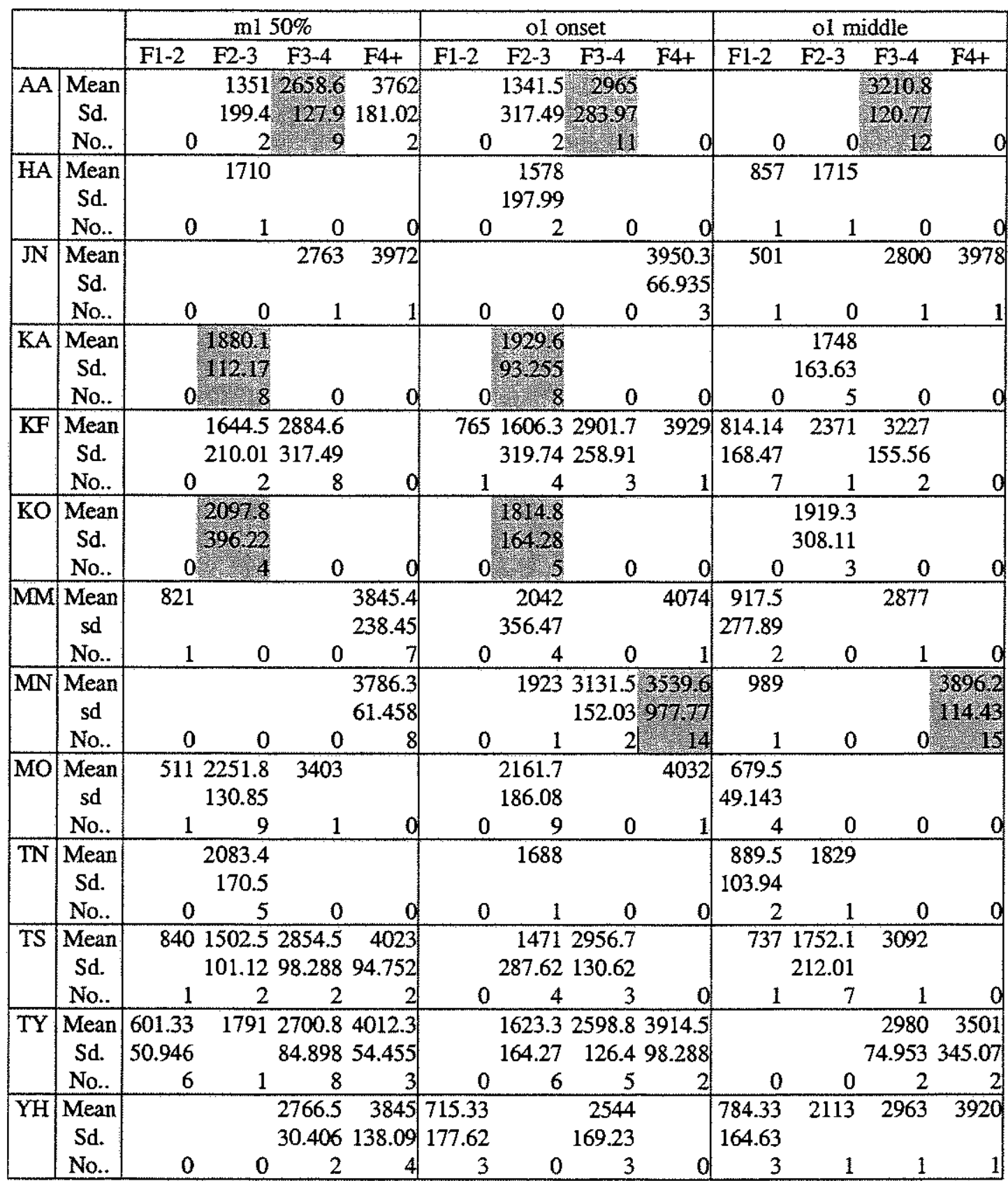




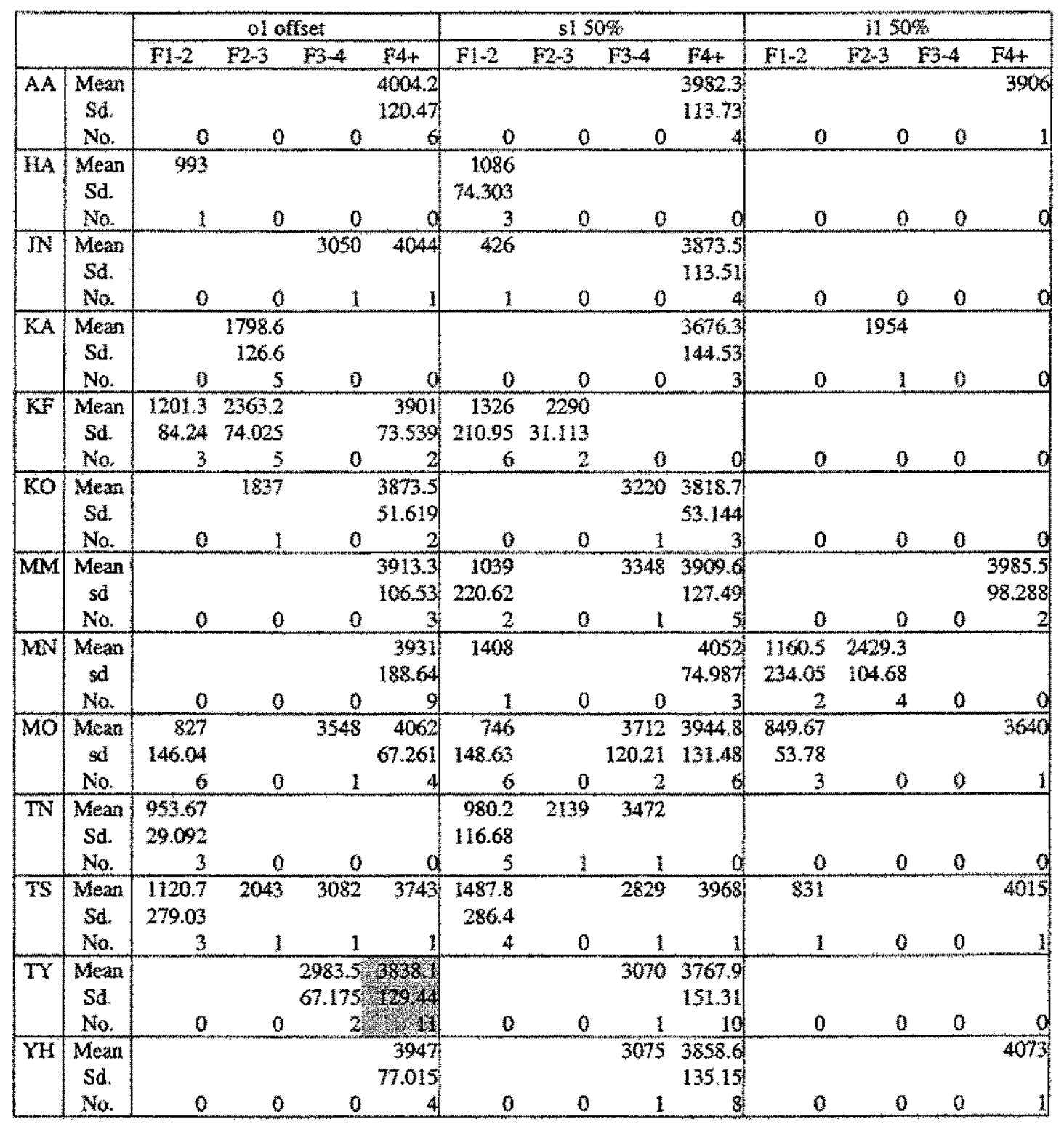




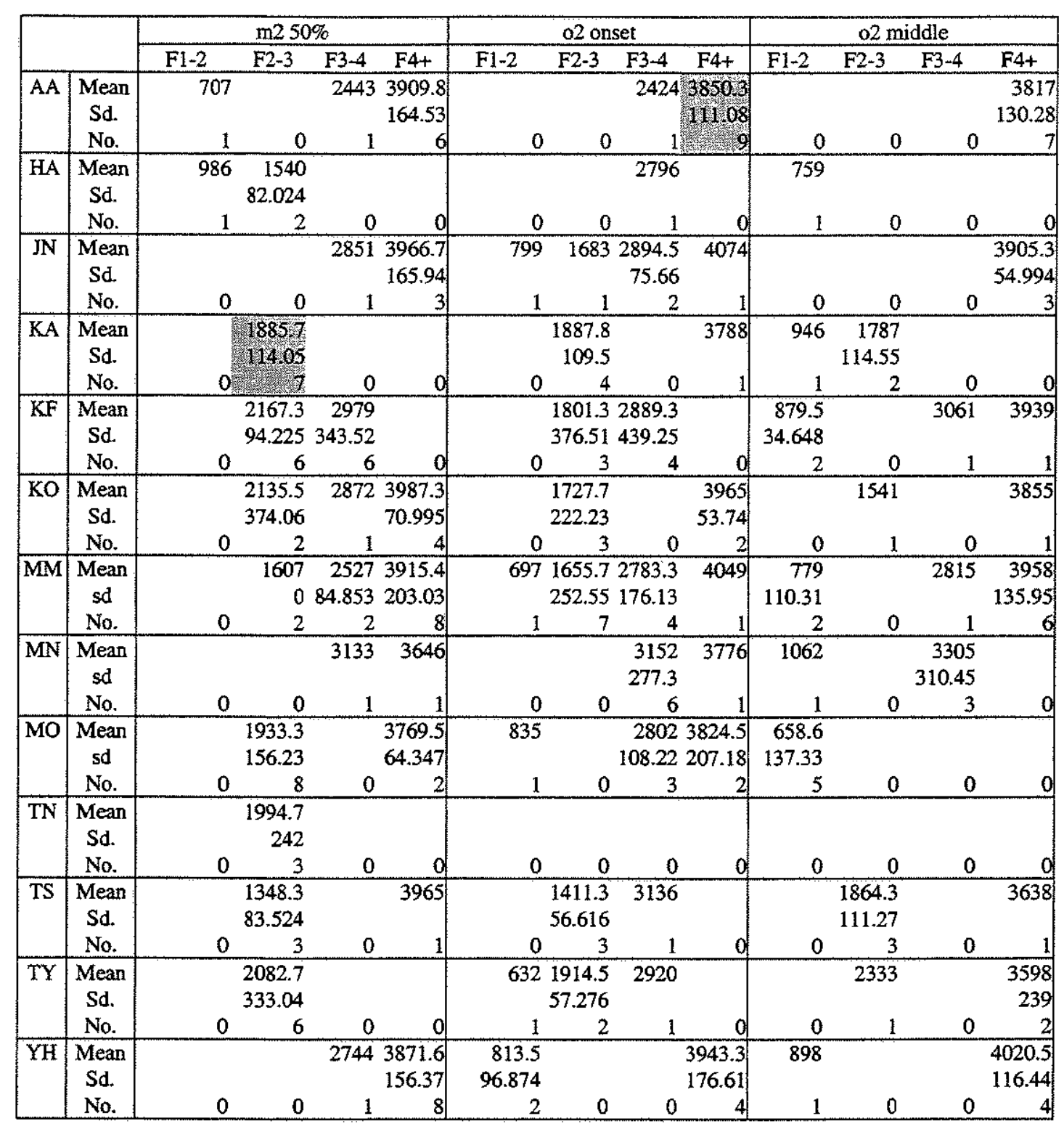




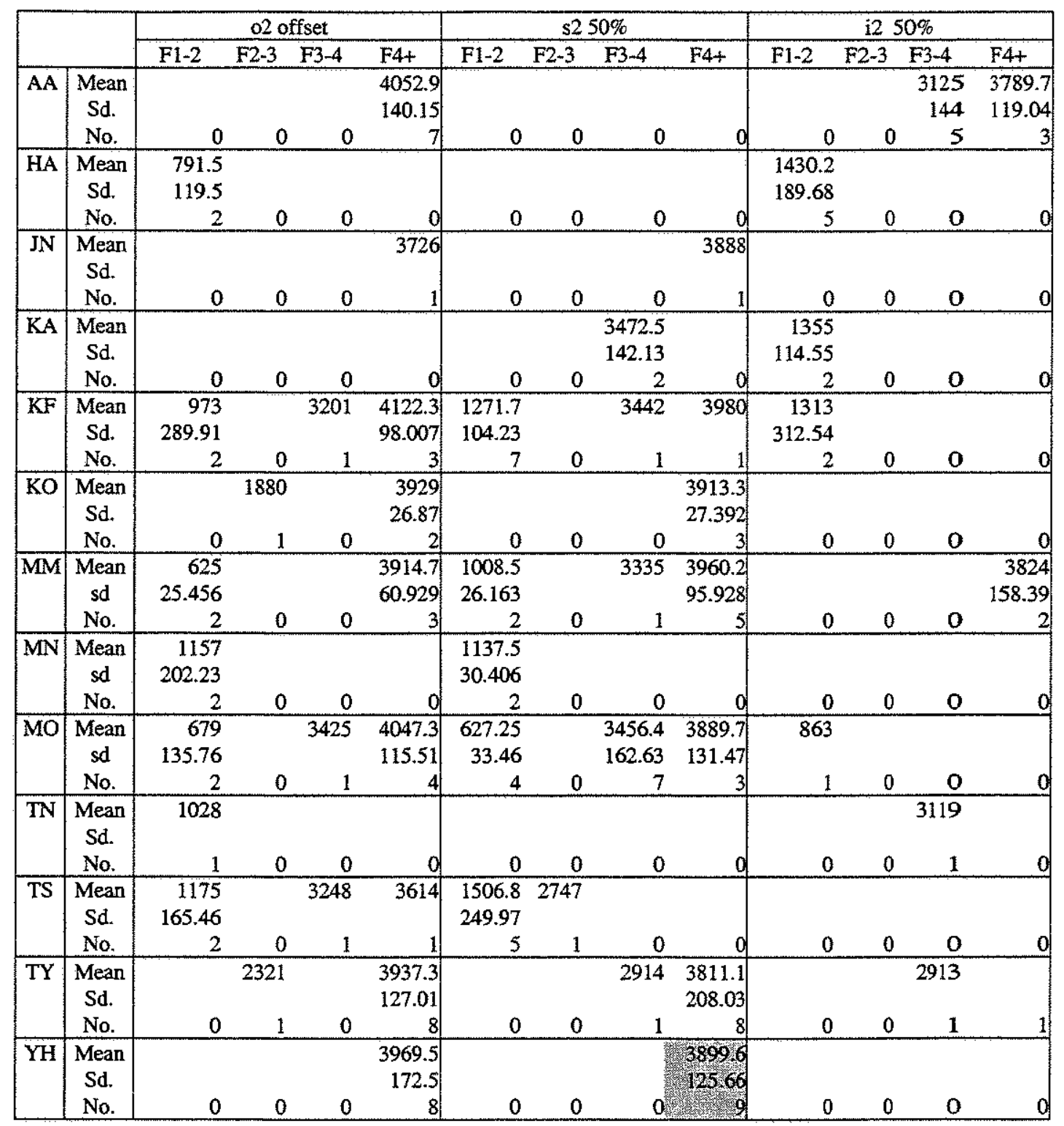

Table 5.13 Extra formants observed in moshimoshi for all speakers.

Table 5.13 above shows that the number of extra formants varies from speaker to speaker and also from segment to segment, to a great extent. It appears that the first component of moshimoshi had more of the extra formants. The extra formants which appeared relatively regularly are summarised in Table 5.14. The measuring points listed here are those observed in more than $75 \%$ of all samples. 'Mean' and 'Sd.' represent the mean and standard deviation of the extra formants. The column headed 'No.' shows how many times the extra formants were observed and the total 
number of observations, and the columns headed '\%' show the rate of the appearance of the extra formants in percentage form.

\begin{tabular}{|c|l|r|r|r|r|}
\hline Speaker & Measuring points & \multicolumn{1}{|c|}{ Mean } & \multicolumn{1}{|c|}{ Sd } & \multicolumn{1}{|c|}{ No. } & \multicolumn{1}{c|}{$\%$} \\
\hline & -m1 F3-4 & 2658.6 & 127.9 & $9 / 12$ & $75 \%$ \\
AA & -01 onset F3-4 & $\mathbf{2 9 6 5}$ & $\mathbf{2 8 3 . 9 7}$ & $\mathbf{1 1 / 1 2}$ & $\mathbf{9 1 . 7 \%}$ \\
& -01 middle F3-4 & $\mathbf{3 2 1 0 . 8}$ & $\mathbf{1 2 0 . 7 7}$ & $\mathbf{1 2 / 1 2}$ & $\mathbf{1 0 0 \%}$ \\
& -o2 onset F4+ & 3850.3 & 151.31 & $10 / 12$ & $83.3 \%$ \\
\hline & -m1 F2-3 & $\mathbf{1 8 8 0 . 1}$ & $\mathbf{1 1 2 . 1 7}$ & $\mathbf{8 / 8}$ & $\mathbf{1 0 0 \%}$ \\
KA & -o1 onset F2-3 & $\mathbf{1 9 2 9 . 6}$ & $\mathbf{9 3 . 2 5 5}$ & $\mathbf{8 / 8}$ & $\mathbf{1 0 0 \%}$ \\
& -m2 F2-3 & 1885.7 & 114.05 & $7 / 8$ & $87.5 \%$ \\
\hline KO & -o1 onset F2-3 & 1814.8 & 164.28 & $5 / 6$ & $83.3 \%$ \\
\hline MN & -m1 F4+ & $\mathbf{3 5 3 9 . 6}$ & $\mathbf{9 7 7 . 7 7}$ & $\mathbf{1 4 / 1 5}$ & $\mathbf{9 3 . 3 \%}$ \\
& -o1 onset F4+ & $\mathbf{3 8 9 6 . 2}$ & $\mathbf{1 1 4 . 4 3}$ & $\mathbf{1 5 / 1 5}$ & $\mathbf{1 0 0 \%}$ \\
\hline TY & -01 offset F4+ & $\mathbf{3 8 3 8 . 1}$ & $\mathbf{1 2 9 . 4 4}$ & $\mathbf{1 1 / 1 4}$ & $\mathbf{7 8 . 6 \%}$ \\
\hline YH & -s2 F4+ & 3899.6 & 125.66 & $9 / 12$ & $75 \%$ \\
\hline
\end{tabular}

Table 5.14 Summary of the higher rates ( $75 \%$ and above) of the incidence of extra formants.

Table 5.14 above suggests a few things. Firstly, the extra formants are found mostly in the first component of moshimoshi, especially in the first syllable /mo/. This is the syllable which carries a pitch accent, so it may be the case that the extra formants are more commonly found in accented syllables. Furthermore, Table 5.14 shows that the location of the extra formants depends on the individual. For instance, Speaker AA's extra formants were found very regularly at the onset and the middle of the [0] vowel, in between the F3 and F4 (91.7\% and $100 \%$, respectively). Speaker KA's extra formants were, on the other hand, found at the measuring points at $\mathrm{m} 1$ and the onset of $\mathrm{o} 1$ in between the F2 and F3 in all utterances. In addition, Table 5.13 and Table 5.14 show that extra formants are generally found above F2. No extra formants which are between F1 and F2 reached $75 \%$ consistency in their appearance.

The possible use of extra formants as forensic speaker identification parameters has been studied also by Rose (1999a) in his research on hello uttered by 6 Australian speakers. Rose examined between-speaker variability of those formants in terms of their incidence, frequencies and bandwidths. He reports that although the formant frequency of the extra formants is not so 
powerful and bandwidth is useless, the incidence of the extra formants can be a fairly powerful diagnostic.

The extra formants in the current study differ considerably from Rose's research, however, with respect to the consistency of their incidence of across speakers. Rose's study shows that all speakers had the formant which Rose called trachea F3 in his study. There are also two speakers who had a so-called singer's formant, and one of these two speakers also produced another extra formant around $1500-2000 \mathrm{~Hz}$ region. The variability in terms of where the extra formants were found is, however, not large. In the current study, on the other hand, the extra formants are found to vary greatly from one speaker to another. It is impossible to compare the frequencies of extra formants across speakers and use them as additional speaker identification parameters.

The incidence of extra formants can, however, add some more information about a speaker's identity. Table 5.14 above has shown that some of the parameters occur very consistently within a speaker, and where the consistent extra formants are found varies from speaker to speaker. For instance, Speaker AA had an extra formant at the middle of the first $/ \mathrm{o}$ in all tokens, but none of the other speakers had this formant consistently. The examination of Table 5.13 shows that all other speakers had very few incidences of this extra formant. Speakers KF and TY had two occurrences of this formant out of 18 and 14 tokens respectively, and the rest had either one or zero occurrence. Thus this extra formant of Speaker AA can be a diagnostic to discriminate Speaker AA from other speakers.

The situation where the extra formants can be useful is, however, strictly limited to the cases where those extra formants are observed very consistently in one set of recordings but not in another, or where both have a very consistent appearance of an extra formant, and also where many closely comparable words appear in both incriminating evidence and suspect's recordings. These extra 
formants have to be found at the same measuring point of the same segment, as speakers were shown to articulate the extra formants at different locations. The condition regarding the pitch accent also has to be the same, since Table 5.13 showed that the extra formants are observed much more consistently in the syllable which carries pitch accent than in the syllables which do not. Given the fact that the word moshimoshi is the repetition of exactly the same string of segments 'moshi', the reason for the more frequent appearance of the extra formants in the first $/ \mathrm{m} /$ and $/ \mathrm{o} /$ rather than in the second $/ \mathrm{m} /$ and $/ \mathrm{o} /$ is solely attributed to the difference in the pitch accent.

\subsection{SUMMARY}

This chapter set out to determine which segment / formant combinations in a Japanese word moshimoshi carry more speaker specific information, with additional research on the effect of the difference in recording occasions and incidence of extra formants. The findings, discussions, and analyses presented in this chapter are now summarised.

\subsubsection{MAIN FINDINGS}

This chapter studied which segment / formant combinations in moshimoshi are more suitable for speaker identification parameters, using one-way ANOVA. Higher F-ratios were found with following measuring points: 
F1 (21.4) and F3 (20.5) at the middle point of the first $/ \mathrm{m} /$

F3 (24.9) at the onset of the first /o/,

F3 (27.5) and F4 (22.0) at the middle point of the first /o/,

F1 (20.7) and F3 (27.2) at the middle point of the second $/ \mathrm{m} /$,

F3 (26.6) and F4 (22.3) at the middle point of the second /s/ (phonetically [6])

The observations of the F-ratios showed that the accented syllable is associated with higher Fratios. This is in agreement with a previous study (Ingram et al. 1996).

\subsubsection{ADDITIONAL FINDINGS}

This chapter firstly described and compared the F-patterns of carefully uttered moshimoshi and the nonsense word boshiboshi. This process was considered to be useful to obtain a general picture of the formant structure of the word moshimoshi, as it was anticipated that nasality of $/ \mathrm{m} /$ would cause difficulties in measurements of this word.

The observation of the carefully uttered words moshimoshi and boshiboshi revealed some noteworthy findings. Firstly, it should be noted that CSL presented difficulties in tracing lower resonances when the nasal formants were present. Nasal poles in adjacent vowels were introduced by $/ \mathrm{m} /$ and, as a result, the lower frequency region (below $1000 \mathrm{~Hz}$ ) came to have a higher density of resonances. CSL formant tracing could not separate out those resonances located close to each 
other. CSL sometimes picked up only one of the two adjacent formants or traced in the middle of the two formants. This indicates that accurate and consistent measurements for the lower frequencies of nasalised segments (including nasalised vowels as well as nasal consonants) are very difficult and, because of that, lower resonances of nasalised segments are not a practical choice as a forensic speaker identification parameter.

For the naturally uttered moshimoshi, the importance of the auditory analysis prior to the acoustic analysis became evident. For instance, Speaker MM exhibited an example of a very distinctive speech style. One of his utterances was labelled as blunt speech in this study, because of the auditory impression the sample gave. This particular sample showed a distinctly narrow pitch range with a rise at the end of the word. Although this study could not investigate long tem Fo, it is known to be one of the robust speaker recognition parameters (see Sambur 1975). There is no doubt that long-term fundamental frequency has the potential to be a powerful indicator of the speaker's identity, it is also known to be susceptible to paralinguistic factors (Maekawa 1998; Stevens 1971). The observation of Speaker MM's blunt utterance confirmed that the use of F0 can be a dangerous practice, unless the comparability of the data was established first by careful auditory analysis.

Furthermore, the analysis of blunt speech showed that formant patterns were not largely affected by the change of speech style. This suggests that formant patterns are more resistant against the differences in paralinguistic content of speech, favouring the use of formant patterns as parameters for forensic speaker identification.

Part of the attraction of the word moshimoshi is that this word consists of segments, $/ \mathrm{m} /, / \mathrm{s} /$, and $/ \mathrm{i} /$, which supposedly contain large amounts of speaker specific information. However, this study showed that, it is often the case that we cannot use as a parameter $/ 1 /$ in moshimoshi because of 
vowel devoicing. Being followed by a nasal consonant $/ \mathrm{m} /$ the first $/ \mathrm{J} /$ vowel should not have been devoiced, according to the traditional description of vowel devoicing. This study showed that, on the contrary, the first $/ \mathrm{j} /$ was more often devoiced than the second $/ V$. (see Table 5.7). The discrepancy between the traditional phonological descriptions and the phonetic reality was again revealed in this chapter.

It was also shown that whether or not a vowel is devoiced was not consistent within a speaker. There were very few speakers who showed constant devoicing or voicing across all moshimoshis they uttered. This observation indicates that whether or not vowel devoicing occurs is probably not a very useful parameter for forensic speaker identification, if it was analysed with respect to whether or not the speaker devoices certain vowels. The possibility of using the rate of the incidence of devoicing, however, remains.

Similarly to the result described in the previous chapter, the difference in F-patterns between the two recording sessions was found to be very small for this word as well. It seems that, for this single word, the two weeks' time difference between the recording sessions did not affect the size of the within-speaker variation greatly. No particular formant or measuring point seems to feature a larger non-contemporaneous within-speaker yariation.

Finally, this study also investigated the extra formants. Extra formants were found to have a potential as speaker identification parameter, since the measuring points and location of extra formants appears varied from speaker to speaker. As suggested in Chapter 4 in the discussion of devoicing, this categorical parameter also can be expressed in Bayesian terms, presenting likelihood ratios. It is, however, difficult to quantify the probability on this parameter, as the amount of data will be fairly limited. 


\section{Chapter 6}

\section{STATISTICAL EVALUATION}

\subsection{OBJECTIVES}

As has been stated at the beginning of this thesis, this study has two main questions. They are:

1) "Can formants discriminate speakers?"

and, if they do,

2) "How well do they perform?"

This chapter provides the answers to these questions.

In the process of the quest, this chapter also discusses what is the best and realistic statistical procedure available today to perform forensic speaker identification. Three different statistical approaches are going to be examined in this chapter. Statistical evaluation of data is now an indispensable part of the procedure of any credible acoustic phonetic analysis. Forensic phonetics, however, requires slightly different types of statistics from what is used for standard acoustic analyses, because of its highly specialized objectives. The statistical approaches proposed in this chapter are extensively discussed in terms of their strengths and weaknesses, as well as examined with respect to their performance in the discrimination of speakers. The selection of parameters 
which are going to be used in this examination was made on the basis of the results of the experiments in the previous two chapters (Chapters 4 and 5).

\subsection{DISCRIMINATION OR IDENTIFICATION?}

In real life forensic phonetic investigations, forensic phoneticians are typically given two (or more) speech samples; one is an unknown speaker's incriminating speech, and the other is a recording of the suspect's speech. The task which experts face here is to provide an evaluation of the given speech samples, which is to assist the court to decide whether or not the suspect and criminal are the same individual. In other words, the court ultimately wants a yes / no answer to the question of whether the suspect is indeed the criminal, but not to identify a certain speaker from a various multiple candidates.

The nature of the task involved bere is therefore discriminating the same-speaker pair from different-speaker pair, rather than identifying a speaker from multiple suspects. The statistical approach involved in forensic speaker identification therefore must be in such a form that the outcome will assist the juries to make the necessary judgement.

A speaker verification test can produce four types of judgements: identifying a speaker correctly, discriminating speakers correctly, identifying a speaker wrongfully, and discriminating a speaker wrongfully. Those four possible judgements are from now on referred to, respectively, as valid positive discrimination, valid negative discrimination, false positive discrimination, and false negative discrimination in this chapter. Table 6.1 below summarises the four possible judgements made in the speaker discrimination tests. 


\begin{tabular}{|c|c|c|}
\hline & Discrimination & Non-discrimination (identification) \\
\hline Correct & valid posifive discrimination & valid luegative discrimination \\
\hline Wrong & false positive discrimination & false negative discrimination \\
\hline
\end{tabular}

Table 6.1 Summary of the four possible judgements made in a speaker discrimination test. The correct judgments were indicated by shading.

Furthermore, in the discussion of the rates of the successful judgments, the term successful discrimination rate is used in this thesis. This refers to both types of correct judgement, ie. valid positive discriminations and valid negative discriminations, which were marked with the shading in Table 6.1 above.

\subsection{STATISTICAL APPROACHES TESTED IN THIS STUDY}

As has been mentioned, three different statistical procedures are discussed in this chapter. All three approaches incorporate multiple variables. This is one of the desiderata of the statistical procedure for speaker identification. As no acoustic parameter is powerful enough to profile a speaker by itself, it is absolutely essential for speaker identification to use multiple parameters. Comparing the performance of multiple values as they are is, however, far more complex than comparing the performance of single values, and analyses of such data are not an easy task. The preferred statistical approach in forensic phonetics is one which allows us to incorporate multiple acoustic parameters and derives from them a smaller set of values or, better still, a single figure.

The three approaches discussed in this chapter are firstly categorised into two types. These are named identification-discrimination and forensic discrimination in this study. The difference between these two types lies in the tasks they perform. The first type, identification-discrimination, identifies a speaker from the multiple candidates available on the basis of the given information. The latter type, forensic discrimination, tests whether or not two given samples are from the same 
individual. It is the latter which is thus more closely analogous to tasks in the realistic forensic situation.

The latter type, forensic discrimination, can be categorised further into two. Both of them use the distant-based measures, in the sense that they both analyse the difference between two given samples and make judgements on the speaker's identity referring to a threshold which classifies the given difference into either within-speaker variation or between-speaker variation. However, the distances used were derived from different statistical measures. The second analysis discussed in this chapter is a discriminant score-based distant measure, and the final analysis is likelihood ratiobased distance measure. For the summary of the three statistical measures, see Figure 6.1.

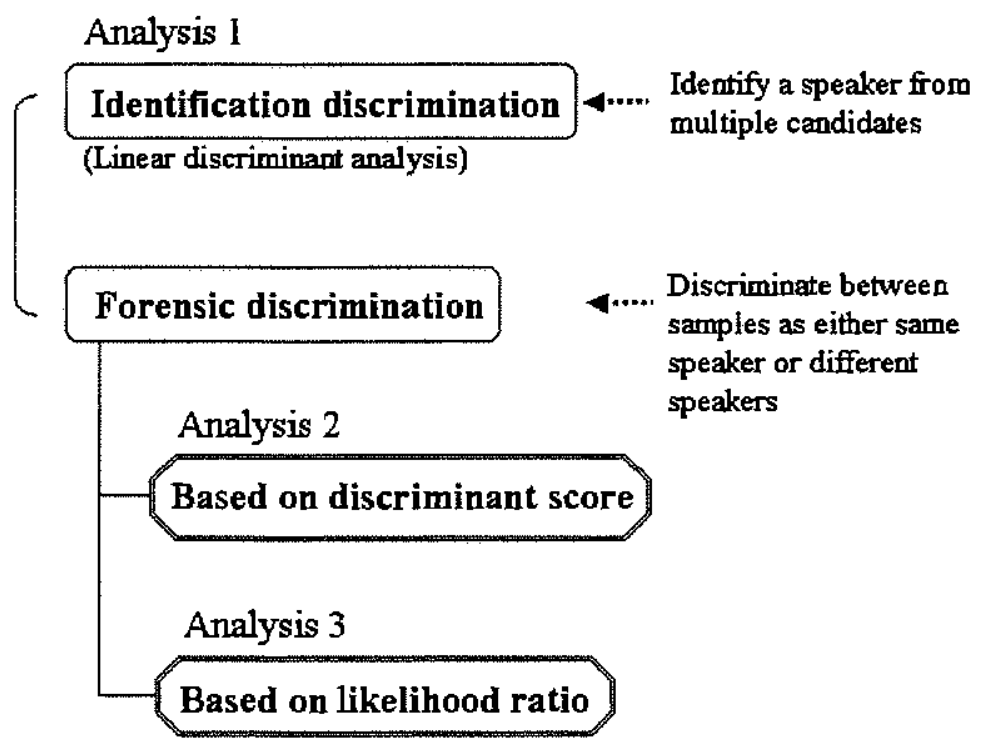

Figure 6.1 Summary of the three statistical measures tested in this chapter.

In the first set of analysis, "identification-discrimination" is performed. This is a statistical approach which forensic phonetic experiments often use (see Ingram et al. 1996; Jassem 1995). In this study, the estimation of correct discrimination is made using cross validation, which is a 
statistical technique used to obtain realistic estimates under the circumstance where the dataset is too small to have a test set separated from the training set (Ripley 1996: 69). (details of the cross validation approach will be discussed in the section 6.4.3).

In the second set of analysis, "forensic discrimination based on linear discriminant scores", linear discriminant scores generated in the process of discriminant analysis are used as a measure of dimension reduction. In this analysis, however, those scores are used for distance measurements, not for finding out the successful identification rates. The magnitudes of the differences between linear discriminant scores are compared for both within- and between-speaker variation. This method is derived from the idea that between-speaker variation has to be larger than within-speaker variation in order to discriminate speakers on the basis of their voices, and the larger the difference is, the more likely the two samples under comparison are from different speakers.

Unlike the first two analyses, the final method, "forensic discrimination based on likelihood ratio" does not use the discriminant scores to reduce dimensions of the data. Instead, the third approach in this study uses the Bayesian likelihood ratio.

A Bayesian approach is the most commonly used method in actual forensic cases (but not necessarily in forensic phonetics). The likelihood ratio here is, however, not used in the way that it is originally intended. The likelihood ratio is developed to express the probability of a certain event to occur under a given condition. In forensic situation, likelihood ratio is usually used to quantify how strongly the evidence supports the prosecution (or defence) hypothesis. In this study, however, the likelihood ratio is used as a new set of values on which the distance measure is performed, although there are a few reasons why I hesitate to use the likelihood ratio in forensic speaker identification in the usual method (detailed discussion on this matter is in the later sections of this chapter, 6.6.1 and 6.6.1.2). 
In this final analysis, likelihood ratios for the parameters which are selected on the basis of the results of Chapters 4 and 5 are first calculated. Those likelihood ratios are then combined into a single figure using the Bayesian approach. In the calculation of the likelihood ratio as well, cross validation estimates were employed.

\subsection{IDENTIFICATION-DISCRIMINATION}

This section examines how well the selected parameters discriminate speakers using discriminant analysis. Discriminant analysis can be described as a technique with two functions. One is to confirm that apparent clusters are real, and the other is to classify any newly added data into the right cluster (Woods et al. 1986). So, when viewed in the forensic speaker identification context, discriminant analysis examines if the given speech samples form clusters according to the speakers' identity, and also estimates how well any newly added speech samples can be classified into the correct speaker groups. For instance, suppose that an incriminating telephone call was intercepted. Also suppose that there are only three people on earth who could possibly have made the phone call, as the record showed that the incriminating phone call was made from a certain house and there were only three people at home at the time of the crime. In a closed set case like that, discriminant analysis will be an ideal statistic, as the task here is to find the closest match to the incriminating recordings from the three samples.

As has been discussed in sections 6.2 and 6.3 above, however, this is often not what needs to be achieved in most forensic speaker identification cases. In most cases, what needs to be done is an evaluation of how well two samples are discriminated as same speaker or different speaker. Nevertheless the approach based on the discriminant analysis is presented here as the first set of 
analysis, in order to demonstrate the difference between identification-discrimination and forensic discrimination.

Discriminant analysis has certain advantages from which any speaker identification benefits greatly. First of all, discriminant analysis reduces the dimensions of the given data and produces smaller sets of figures which separate the groups of values maximally. Also, the discriminant function takes the correlation between variables into account. As acoustic parameters produced from a single vocal mechanism are quite possibly correlated, how this correlation should be dealt with is a serious question.

\subsubsection{DISCRIMINANT FUNCTION AND SCORE}

Discriminant analysis is going to be performed on the data of the vowel formants in this first set of tests.

Discriminant analysis is a statistical approach which creates functions to separate groups optimally, incorporating multiple variables. In discriminant analysis, datasets are discriminated based on the linear or non-linear functions. Once discriminant functions are calculated on the basis of the datasets, the raw values from the datasets are apphed to the function. The values obtained as the result are discriminant scores. This study consists of 11 groups to be discriminated, as 11 speakers are included in this study. When there are more than two groups to be discriminated, like in this study, more than one line is needed to separate the groups. In such cases, the number of discriminant functions will be "number of groups - 1" (Everitt and Dunn 1991:228), and the number of the discriminant scores produced varies according to the number of the functions. Whether or not the function classifies the test samples into known categories correctly is then 
evaluated referring to the discriminant scores, and an estimation of how well the different sets of data are discriminated is made (Ishimura 1992:129; Woods et al. 1986: 266).

\subsubsection{CALCULATION IN THIS STUDY}

The results of the experiment in Chapter 4 showed that, because they had the highest F-ratios, F2 of Ii/ and F2 and F3 of /e/ are promising candidates for speaker identification parameters, and they are the parameters incorporated in the discriminant analysis performed in this section. The parameters from moshimoshi in Chapter 5 are not going to be included, as the nature of the data from Chapter 4 and Chapter 5 are inherently different. Although discriminant analysis allows us to incorporate multiple parameters, combining datasets with different structures severely complicates the analysis.

The difference between datasets from Chapters 4 and 5 lies in the phonological environments in which the target segments were embedded. The formant measurements of vowels in Chapter 4 were made from 5 different words. The measurements on Chapter 5 , on the other hand, were made constantly from the same word moshimoshi. They are, as has been shown, different in terms of the effects which phonological environments potentially imposed upon the target segments. Combining these datasets without necessary adjustments in respect to the specific effects of phonological environments can distort the statistics. In order to make this adjustment, however, the estimate for this effect produced on the basis of the large population is necessary, and this is not really a practical solution here or in real forensic cases. In this section, therefore, the methodology is demonstrated only using parameters selected in Chapter 4, and without those in Chapter 5.

As mentioned above, the experiment in Chapter 4 revealed that iF2, eF2, and eF3 were the three most promising vowel / formant combinations, and they thus were selected as the parameters for 
discriminant analysis. In the data collection for these formants, five words per vowel were elicited for the measurements of the vowel formants, as described in Chapter 3 in detail. Speakers uttered each of those five words four times altogether, as they were recorded at two recording sessions and the tasks were repeated at each recording session. Prior to discriminant analysis, each speaker's means for parameters $\mathrm{iF} 2$, eF2, and eF3 were calculated across the four different occasions of recording, and separately for each of the five words. The five mean values for each speaker were treated as a set of data, and discriminant analysis was performed on these data sets.

Means were calculated in the manner described above, since the investigation on the within-speaker variation revealed that non-contemporaneity of datasets did not show any large effects in the analysis presented in Chapter 4, but some of the phonological environments in which the target vowels were embedded were found to affect the formant values more largely (Kinoshita and Maindonald 2000). It was hence considered that the data is represented more appropriately in this mean calculation rather than calculation in which the means were produced across words, but separately for the four occasions of recordings.

The data analysis in this section includes 11 speakers, and therefore there were 11 groups to be separated. Discriminant analysis produced 10 (11 groups -1) discriminant functions. All discriminant scores do not, however, have equal importance in the discrimination of groups. The software used in this analysis, $\mathrm{R}$, produces values called proportion of trace, which indicate the proportion of the information each function carries. In this particular data, the proportions of trace were found to be: LD1 $0.93, \mathrm{LD} 20.0551, \mathrm{LD} 30.0152$. These values are interpreted as the following: the first discriminant function carries $93 \%$, the second one carries $5.5 \%$, the third one carries $1.1 \%$ of information to discriminate speakers, and the fourth to tenth functions carry the rest, ie. $0.4 \%$ of the information. By far the most important information for speaker discrimination is, thus, contained in the first discriminant function, some in the second discriminant functions, and 
from the third to the tenth discriminant functions hardly carry any effective information. In this study, thus, 5 first and second discriminant scores are calculated by applying the 5 means to the first and the second discriminant functions. Table 6.2 presents the discriminant scores. LD1 and LD2 indicate the discriminant scores produced by the first and the second discriminant functions, respectively, and tokens 1- 5 represent the five different words.

\begin{tabular}{|c|c|c|c|}
\hline Speaker & Word & LDI & LD2 \\
\hline \multirow{5}{*}{$\mathrm{AA}$} & 1 & 2.044 & 0.0145 \\
\hline & 2 & 2.573 & 0.6826 \\
\hline & 3 & 2.255 & -0.5995 \\
\hline & 4 & 1.636 & -0.7963 \\
\hline & 5 & 0.875 & -0.1538 \\
\hline \multirow{5}{*}{$\mathrm{HA}$} & 1 & -5.166 & 1.4141 \\
\hline & 2 & -4.471 & 0.2971 \\
\hline & 3 & -6.058 & 0.7712 \\
\hline & 4 & -5.565 & 0.8018 \\
\hline & 5 & -7.99 & -0.5984 \\
\hline \multirow{5}{*}{$\mathrm{JN}$} & 1 & 3.93 & 0.9245 \\
\hline & 2 & 4.3 & 1.7019 \\
\hline & 3 & 4.378 & 0.4597 \\
\hline & 4 & 5.572 & -0.4864 \\
\hline & 5 & 4.361 & 0.3447 \\
\hline \multirow{5}{*}{$\mathrm{KA}$} & 1 & 1.572 & 0.6625 \\
\hline & 2 & -0.177 & 0.7541 \\
\hline & 3 & -0.485 & -0.8756 \\
\hline & 4 & 1.899 & -2.1896 \\
\hline & 5 & -1.454 & -1.0775 \\
\hline \multirow{5}{*}{$\mathbf{K F}$} & 1 & -3.32 & 0.1247 \\
\hline & 2 & -2.054 & -0.656 \\
\hline & 3 & -1.167 & -0.9887 \\
\hline & 4 & -0.506 & -1.0775 \\
\hline & 5 & -3.796 & -1.2982 \\
\hline \multirow{5}{*}{$\mathrm{KH}$} & 1 & 1.318 & 1.2058 \\
\hline & 2 & 0.415 & 0.9763 \\
\hline & 3 & 1.614 & -0.4081 \\
\hline & 4 & 2.207 & -0.2029 \\
\hline & 5 & 0.279 & -1.0936 \\
\hline
\end{tabular}

\begin{tabular}{|c|c|c|c|}
\hline Speaker & Word & LD1 & $\mathrm{LD2}$ \\
\hline \multirow{5}{*}{$\mathrm{KO}$} & 1 & 0.827 & 0.6557 \\
\hline & 2 & 1.476 & 1.5869 \\
\hline & 3 & 2.162 & 0.2544 \\
\hline & 4 & 1.524 & 0.7657 \\
\hline & 5 & -0.709 & 0.4676 \\
\hline \multirow{5}{*}{$\mathrm{MN}$} & 1 & -3.611 & 1.9639 \\
\hline & 2 & -3.482 & 1.1069 \\
\hline & 3 & -4.226 & -0.5155 \\
\hline & 4 & -4.326 & -0.9173 \\
\hline & 5 & -5.093 & -1.1625 \\
\hline \multirow{5}{*}{$\mathrm{TN}$} & 1 & -1.223 & 0.2097 \\
\hline & 2 & -0.253 & -0.1464 \\
\hline & 3 & -0.265 & -1.6902 \\
\hline & 4 & 0.541 & -2.334 \\
\hline & 5 & -1.544 & -2.6369 \\
\hline \multirow{5}{*}{ TS } & 1 & -0.894 & 2.4511 \\
\hline & 2 & -0.534 & 3.4352 \\
\hline & 3 & 0.131 & 0.5544 \\
\hline & 4 & 1.027 & 0.2573 \\
\hline & 5 & 0.462 & -0.3506 \\
\hline \multirow{5}{*}{ TY } & 1 & 3.625 & -0.0896 \\
\hline & 2 & 4.856 & 0.17 \\
\hline & 3 & 4.435 & -1.1322 \\
\hline & 4 & 3.683 & -0.6108 \\
\hline & 5 & 2.391 & +0.9912 \\
\hline
\end{tabular}

Table 6.2 Discriminant scores calculated for each word for each speaker. 


\subsubsection{CROSS VALIDATION}

Before proceeding to the discussion of the results of the discrimination test, the cross validation approach needs to be discussed. Cross-validation is a statistical technique used to obtain realistic estimates under the circumstance where the dataset is too small to have a test set separated from the training set (Ripley 1996: 69). If the test sample is actually a part of the reference samples, the results of statistics will be optimistic, because whatever formula is used for the discrimination is modelled to discriminate the given data optimally. If the model is tested with a new set of data which is not included in the process of modelling the formula, it is most likely that the speakers will not be as well discriminated. This is particularly so in the case where the datasets are small like in this study. Statistical analyses using a test set which is a part of the reference samples are thus misleading, and the results produced by such procedures are not reliable, and it is not appropriate to refer to those results in the practice of actual forensic speaker identification.

Furthermore, using the same test set and reference set is unrealistic in terms of real forensic phonetics. In forensic speaker identification, the test sample is always independent from the reference sample. The successful identification rate is thus not appropriately referred to in actual forensic cases, unless the rate was derived from the discrimination test where the test sample and the reference sample are independent.

In the basic cross-validation approach, the training dataset is randomly divided into, say, $\mathrm{N}$ pieces. One of these $\mathrm{N}$ sets of data is removed from the training set and is used as a test sample, so that the test samples are independent from the training sets. This process is repeated $\mathrm{N}$ times, using each of the $\mathrm{N}$ pieces of data as a test sample. As a result, $\mathrm{N}$ estimates are obtained and they are averaged to finally produce overall estimates. 
This method can be taken further: the leave-one-out method. Instead of dividing the whole data into $\mathrm{N}$ pieces, one is taken out as the test sample, and the rest becomes a reference sample. This is again repeated by taking one dataset after another out of the population. In this way, a test sample and a reference population can be independent. Also repeating the tests with many different test sets reduces the chance of being strongly biased by a peculiar piece of data which happened to be the test sample. This statistical technique is thus very valuable in research where the sample size is small. In this study, datasets from a pair of speakers were considered as one test set, and all possible speaker combinations were taken out from the reference population in turn and tested.

\subsubsection{RESULTS OF DISCRIMINANT ANALYSIS}

The discriminant analysis was carried out using the three vowel formants, F2 of /i/ and F2 and F3 of /e/, as they were revealed to have large F-ratios in Chapter 4. Each speaker's discriminant scores for words 1 to 5 presented in Table 6.2 in the earlier section (6.4.1) were tested as to whether or not those scores were classified into the correct speaker group, in addition to counting how many times the speaker was identified correctly. All speaker combinations were thus tested five times each as each speaker's dataset includes five mean values which were made for five different words.

Table 6.3 presents the results of the discriminant analysis. Each column indicates a speaker group for test samples to be classified into, and the identities of the speakers who uttered the test sample are shown by rows. As each speaker has five linear discriminant scores to be classified, the total for each row should be 5 . If all five test samples for each speaker are classified into the correct speaker group, we should see 5 in the shaded cells. For instance, with Speaker AA's row, a total of four is in the shaded cell, meaning that four out of five words of Speaker AA were identified correctly; one of the words was, however, misidentified as speaker $\mathrm{KH}$. For an example of poor performance, see 
Speaker $\mathrm{KH}$ instead. Speaker KH's five words were not classified correctly - not even once. They were classified as Speaker AA twice, and as Speakers KO, KA, and TS once each.

\begin{tabular}{|c|c|c|c|c|c|c|c|c|c|c|c|}
\hline & AA & HA & $\mathrm{JN}$ & $\mathrm{KA}$ & $\mathrm{KF}$ & $\mathrm{KH}$ & $\mathrm{KO}$ & $\mathrm{MN}$ & TN & $\mathrm{TS}$ & $T Y$ \\
\hline $\mathrm{AA}$ & $4 x^{2}$ & 0 & 0 & 0 & 0 & 1 & 0 & 0 & 0 & 0 & 0 \\
\hline $\mathrm{HA}$ & 0 & 44 & 0 & 0 & 0 & 0 & 0 & 1 & 0 & 0 & 0 \\
\hline $\mathrm{JN}$ & 0 & 0 & 4 . & 0 & 0 & 0 & 0 & 0 & 0 & 0 & 1 \\
\hline $\mathrm{KA}$ & 1 & 0 & 0 & ank & 1 & 1 & 0 & 0 & 1 & 1 & 0 \\
\hline $\mathrm{KF}$ & 0 & 0 & 0 & 0 & 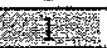 & 0 & 0 & 2 & 2 & 0 & 0 \\
\hline$\overline{\mathrm{KH}}$ & 2 & 0 & 0 & 1 & 0 & 0 & 1 & 0 & 0 & 1 & 0 \\
\hline $\mathrm{KO}$ & 0 & 0 & 0 & 0 & 0 & 0 & S & 0 & 0 & 2 & 0 \\
\hline $\mathrm{MN}$ & 0 & 2 & 0 & 0 & 0 & 0 & 0 & 3.5 & 0 & 0 & 0 \\
\hline $\mathrm{TN}$ & 0 & 0 & 0 & 2 & 2 & 0 & 0 & 0 & 1 1. & 0 & 0 \\
\hline TS & 0 & 0 & 0 & 1 & 0 & 2 & 0 & 0 & 0 & 2 & 0 \\
\hline TY & 1 & 0 & 1 & 0 & 0 & 0 & 0 & 0 & 0 & 0 & W \\
\hline
\end{tabular}

Table 6.3 Results of the discriminant analysis. See text for explanation.

The data for 11 speakers were used in this discriminant analysis, and there were five discriminant scores to be classified for each speaker, producing 55 scores to be tested altogether. The number of the scores which were classified correctly was 25 . The correct classifications were thus made $25 / 55$ (which is $45.45 \%$ ) of time, and the error rate is $30 / 55(54.55 \%)$. The successful classification rate by chance is $1 / 11$ as there are 11 speakers to be classified into. The correct classification rate $45.45 \%$ may seem low, but this is in tact five times better than chance. This then shows that speakers can be identified by discriminant analysis on the basis of formants to a small extent.

\subsubsection{PROBLEMS WITH THE DISCRIMINANT ANALYSIS}

Although it has its own advantages as described, there are also some problems in the use of discriminant analysis in forensic speaker identification. First of all, incorporating datasets with different structure is difficult, as described earlier in the section 6.4.2. This imposes a severe restriction on the application of discriminant analysis to actual forensic speaker identification. Given that we have absolutely no control over the contents or quality of forensic recordings, what is 
available for analysis in both incriminating recording and suspect's recording may not always be similar. We may have the /i/ vowels which are prosodically comparable but in different phonological contexts, ịma 'now' and sashimi_ 'sashimi,' one in incriminating recording and the other in recordings of suspect's speech. We may also have the same word eki 'station' to compare in both recordings. Since it is obvious that the comparison of the same word is more powerful, we cannot exclude this word from the data just because it has a different nature from the other part of the data. We also cannot afford to discard the $/ 1 /$ vowels in different contexts either, as a single word $e k i$ 'station' probably would not provide us with enough infornation to make a reliable evaluation.

It is thus quite possible that we have great difficulties in applying discriminant analysis to real life forensic investigations. If the datasets in both incriminating and suspect's speech are large, enabling one to discard some of the measurements to make the structure of data consistent, it may not be a problem. This is, however, a very unlikely luxury in most forensic situations.

A much more serious problem with discriminant analysis is the incompatibility of its judgement making style to the tasks in actual forensic phonetic investigations. As has been described, discriminant analysis creates discriminant functions which optimally classify samples into correct groups. In most forensic investigations, however, this is not what is needed. The task in forensic speaker identification is to answer the question of whether or not the given datasets are from the same individual, rather than identifying one speaker from several suspects. 


\subsection{FORENSIC DISCRIMINATION: DISCRIMINANT SCORE- BASED DISTANCE APPROACH}

\subsubsection{METHODOLOGY AND RESULT}

This section discusses the method which is based on the distances between tokens. Admittedly, this method is statistically very crude. However, it directly reflects the basic idea of speaker identification, and it is therefore easy to comprehend. The linear discriminant scores calculated in the previous section for discriminant analysis and shown in Table 6.2 were used as data and plotted as a two dimensional representation of the differences between tokens of all speakers in Figure 6.2. The initials in the figure indicate individual speakers.

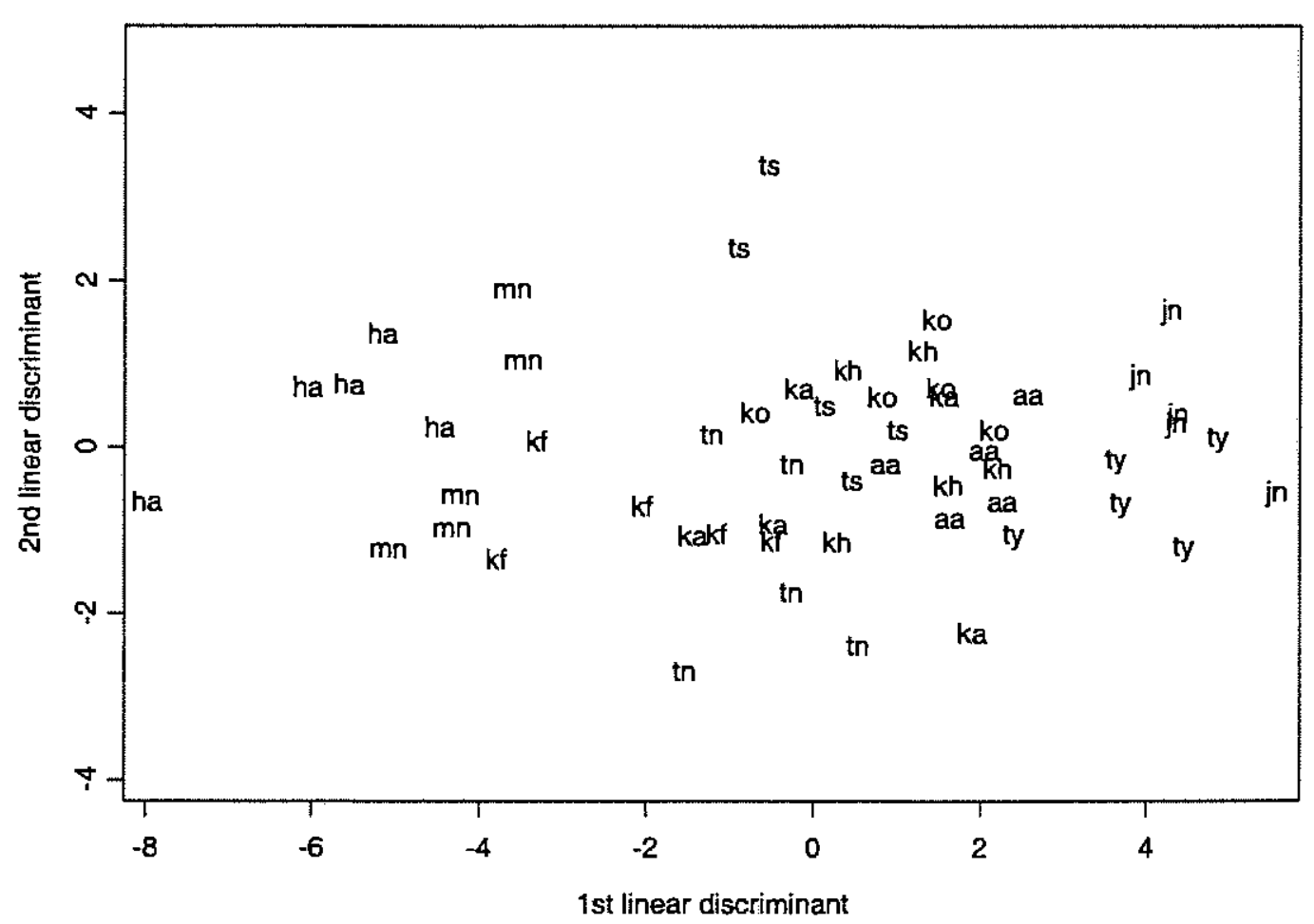

Figure 6.2 Scatterplots of the five linear discriminant scores for each of the 11 speakers. 
Each speaker"s five words, which are plotted based on the first and the second discriminant scores, appear to form a cluster. Some speaker combinations, such as Speakers HA, KF, TS, and JN, did not overlap with each other at all, but there were also some speakers (eg. Speakers $\mathrm{KO}, \mathrm{KH}$, and AA) who were very similarly distributed and thus overlapped heavily.

For the application of this result to real life forensic cases, we shall examine here how successfully two sets of data can be discriminated both positively and negatively. As repeatedly pointed out, speakers can be discriminated from each other only when within-speaker variation is overall smaller than between-speaker variation. If the difference between two comparable samples is large, it is more likely to be between-speaker variation (in other words, the two samples come from two different individuals). If the difference is small, on the other hand, it is more likely to be withinspeaker variation (ie. the recordings were made by the same speaker). The question which arises here now is how small (or large) the distance between tokens should be in order to classify them as within- (or between-) speaker variation.

The size difference between these two types of variation is expected to be a continuum, and they even partly overlap. Therefore to classify an observed difference into between-speaker or within* speaker variation, an appropriate threshold must be set. Once the threshold between within- and between-speaker variation is set, whether or not the two given samples are indeed from the same speaker can be determined sirmply by referring to the threshold. For instance, when the threshold is set at $X$, if the size of variation between two samples is larger than $X$, the two samples are assumed to be from two different individuals. Conversely, when the threshold is smaller than $\mathrm{X}$, it is assumed to be from the same speaker. After the discrimination tests, the error rate produced by the discrimination tests using the threshold also needs to be estimated. The threshold is then manipulated until an equal error rate is achieved. 
In this section, the Euclidian distance between two plotted linear discriminant scores was calculated to represent the difference between samples. As those scores were produced to discriminate speakers optimally by incorporating the three most promising vowel formants, the differences between speakers are assumed to be reflected more clearly in discriminant scores than in raw formant values. In the calculation of Euclidean distance, the distance between two vectors $\left(\mathrm{x}_{\mathrm{l}}, \mathrm{y}_{\mathrm{l}}\right)$ and $\left(x_{2}, y_{2}\right)$ these two tokens is represented as $\sqrt{(x 1-x 2)^{2}+(y 1-y 2)^{2}}$. Examples of calculations are given below using the three sets of discriminant scores extracted from Table 6.2 (p.248). The first and second discriminant scores of Speaker AA's words 1 and 2 and Speaker KF's word 1 are:

$\begin{array}{lcc} & 1^{\text {a }} \text { discriminant score } & 2^{\text {od }} \text { discriminant scores } \\ \text { Speaker AA's word 1 } & 2.044 & 0.0145 \\ \text { Speaker AA's word 2 } & 2.573 & 0.6826 \\ \text { Speaker KF's word 1 } & 0.827 & 0.6557\end{array}$

The distances between Speaker AA's words 1 and 2 representing within-speaker variation and that between Speaker AA's words 1 and Speaker KF's words 1, which shows the size of betweenspeaker variation, are calculated as:

Speaker AA's words $1 / 2$

$$
\sqrt{(2.044-2.573)^{2}+(0.0145-0.6826)^{2}}=0.853273
$$

Speaker AA's words 1 / Speaker KF's words 1

$$
\sqrt{(2.044-0.827)^{2}+(0.0145-0.6557)^{2}}=1.217297
$$

This calculation was made for all possible combinations of discriminant scores. For within-speaker comparison, ten distances were measured for each of the 11 speakers, since five sets of linear 
discriminant scores were compared to each other as in word 1-2, word 1-3, word $1-4, \ldots$ and so on, to word 4-5. 110 (11 speakers * ten comparisons) within-speaker distances were thus calculated. Of course between-speaker comparisons produced many more distances values. Five sets of discriminant scores per speaker were compared to the five of the other speakers for each speaker combination, producing 25 between-speaker distance values. Furthermore, 11 speakers produce 55 possible speaker combinations, (the number of possible pairs of $\mathrm{N}$ speakers is as $\mathrm{N}^{*}(\mathrm{~N}-1) / 2$ ), so 1375 (55 speaker combinations * 25 comparisons) distances were calculated altogether. Table 6.4 and Table 6.5 below summarise the results of the distance calculations. The column "speakers" in Table 6.5 shows the pairs of speakers compared. The means of overall within-speaker distance and between-speaker distance were also calculated and presented with Table 6.4 and Table 6.5.

[Within-speaker distance]

\begin{tabular}{|c|c|c|c|}
\hline Speaker & Distance & Speaker & Distance \\
\hline $\mathrm{AA}$ & 1.163 & $\mathrm{KO}$ & 1.551 \\
\hline $\mathrm{HA}$ & 1.877 & $\mathrm{MN}$ & 1.891 \\
\hline $\mathrm{JN}$ & 1.264 & $\mathrm{TN}$ & 2.053 \\
\hline $\mathrm{KA}$ & 2.534 & $\mathrm{TS}$ & 1.968 \\
\hline $\mathrm{KF}$ & 1.949 & $\mathrm{TY}$ & 2.053 \\
\hline $\mathrm{KH}$ & 1.706 & & \\
\hline
\end{tabular}

\begin{tabular}{|c|c|}
\hline Mean & 1.82 \\
\hline Sd. & 0.39 \\
\hline
\end{tabular}

Table 6.4 Mean within-speaker distances calculated by comparing five tokens of each speaker. The columns "speaker" indicate each speaker $(n=10)$.

[Between-speaker distance]

\begin{tabular}{|l|c|l|l|l|l|l|l|}
\hline Speakers & Distance & Speakers & Distance & Speakers & Distance & Speakers & Distance \\
\hline AA - HA & 7.81 & $\mathrm{HA}-\mathrm{KO}$ & 6.952 & $\mathrm{KA}-\mathrm{KH}$ & 2.122 & $\mathrm{KH}-\mathrm{TN}$ & 2.658 \\
\hline $\mathrm{AA}-\mathrm{JN}$ & 2.886 & $\mathrm{HA}-\mathrm{MN}$ & 2.384 & $\mathrm{KA}-\mathrm{KO}$ & 2.242 & $\mathrm{KH}-\mathrm{TS}$ & 2.579 \\
\hline $\mathrm{AA}-\mathrm{KA}$ & 2.31 & $\mathrm{HA}-\mathrm{TN}$ & 5.796 & $\mathrm{KA}-\mathrm{MN}$ & 4.809 & $\mathrm{KH}-\mathrm{TY}$ & 3.184 \\
\hline $\mathrm{AA}-\mathrm{KF}$ & 4.158 & $\mathrm{HA}-\mathrm{TS}$ & 5.935 & $\mathrm{KA}-\mathrm{TN}$ & 2.228 & $\mathrm{KO}-\mathrm{MN}$ & 5.436 \\
\hline $\mathrm{AA}-\mathrm{KH}$ & 1.412 & $\mathrm{HA}-\mathrm{TY}$ & 9.134 & $\mathrm{KA}-\mathrm{TS}$ & 2.294 & $\mathrm{KO}-\mathrm{TN}$ & 2.948 \\
\hline $\mathrm{AA}-\mathrm{KO}$ & 1.649 & $\mathrm{JN}-\mathrm{KA}$ & 4.628 & $\mathrm{KA}-\mathrm{TY}$ & 3.982 & $\mathrm{KO}-\mathrm{TS}$ & 2.237 \\
\hline $\mathrm{AA}-\mathrm{MN}$ & 6.221 & $\mathrm{JN}-\mathrm{KF}$ & 6.887 & $\mathrm{KF}-\mathrm{KH}$ & 3.632 & $\mathrm{KO}-\mathrm{TY}$ & 3.26 \\
\hline $\mathrm{AA}-\mathrm{TN}$ & 2.969 & $\mathrm{JN}-\mathrm{KH}$ & 3.507 & $\mathrm{KF}-\mathrm{KO}$ & 3.731 & $\mathrm{MN}-\mathrm{TN}$ & 4.266 \\
\hline $\mathrm{AA}-\mathrm{TS}$ & 3.056 & $\mathrm{JN}-\mathrm{KO}$ & 3.559 & $\mathrm{KF}-\mathrm{MN}$ & 2.689 & $\mathrm{MN}-\mathrm{TS}$ & 4.622 \\
\hline $\mathrm{AA}-\mathrm{TY}$ & 2.704 & $\mathrm{JN}-\mathrm{MN}$ & 8.822 & $\mathrm{KF}-\mathrm{TN}$ & 2.33 & $\mathrm{MN}-\mathrm{TY}$ & 7.595 \\
\hline $\mathrm{HA}-\mathrm{JN}$ & 10.403 & $\mathrm{JN}-\mathrm{TN}$ & 5.585 & $\mathrm{KF}-\mathrm{TS}$ & 3.661 & $\mathrm{TN}-\mathrm{TS}$ & 3.44 \\
\hline $\mathrm{HA}-\mathrm{KA}$ & 6.354 & $\mathrm{JN}-\mathrm{TS}$ & 5.059 & $\mathrm{KF}-\mathrm{TY}$ & 5.801 & $\mathrm{TN}-\mathrm{TY}$ & 4.728 \\
\hline $\mathrm{HA}-\mathrm{KF}$ & 4.067 & $\mathrm{JN}-\mathrm{TY}$ & 2.318 & $\mathrm{KH}-\mathrm{KO}$ & 1.531 & $\mathrm{TS}-\mathrm{TY}$ & 4.266 \\
\hline $\mathrm{HA}-\mathrm{KH}$ & 7.121 & $\mathrm{KA}-\mathrm{KF}$ & 2.838 & $\mathrm{KH}-\mathrm{MN}$ & 5.568 & & \\
\hline
\end{tabular}

Table 6.5 Mean distances of linear discriminant functions between speakers. All the possible combinations of speakers were calculated. The columns "speakers" indicate combinations of speakers $(n=25)$. 
The mean of overall within-speaker distances was 1.819, and that of between-speaker distances was 4.261. As expected, mean between-speaker distances turned out to be generally larger than mean within-speaker distances.

Next, the threshold which optimally partitions within-speaker from between-speaker variation has to be determined. In this research, the threshold was decided to be at the point where the withinand between-speaker variations produce an equal error rate. It should be noted, however, that the threshold does not always have to yield an equal error rate. For instance, one might wish to be conservative with identifying a speaker in order to avoid false negative discrimination, ie. misidentifying someone innocent as a criminal. The threshold can be lowered in such a situation, as lowered thresholds will result in lower false negative discrimination (less chance of wrongfully identifying an innocent individual as a criminal), but higher error rates for false positive discrimination (more chance of letting the actual perpetrator go). Figure 6.3 below presents hypothetical distributions of within-speaker and between-speaker distances and the possible locations for the threshold, assuming that they have normal distribution. 


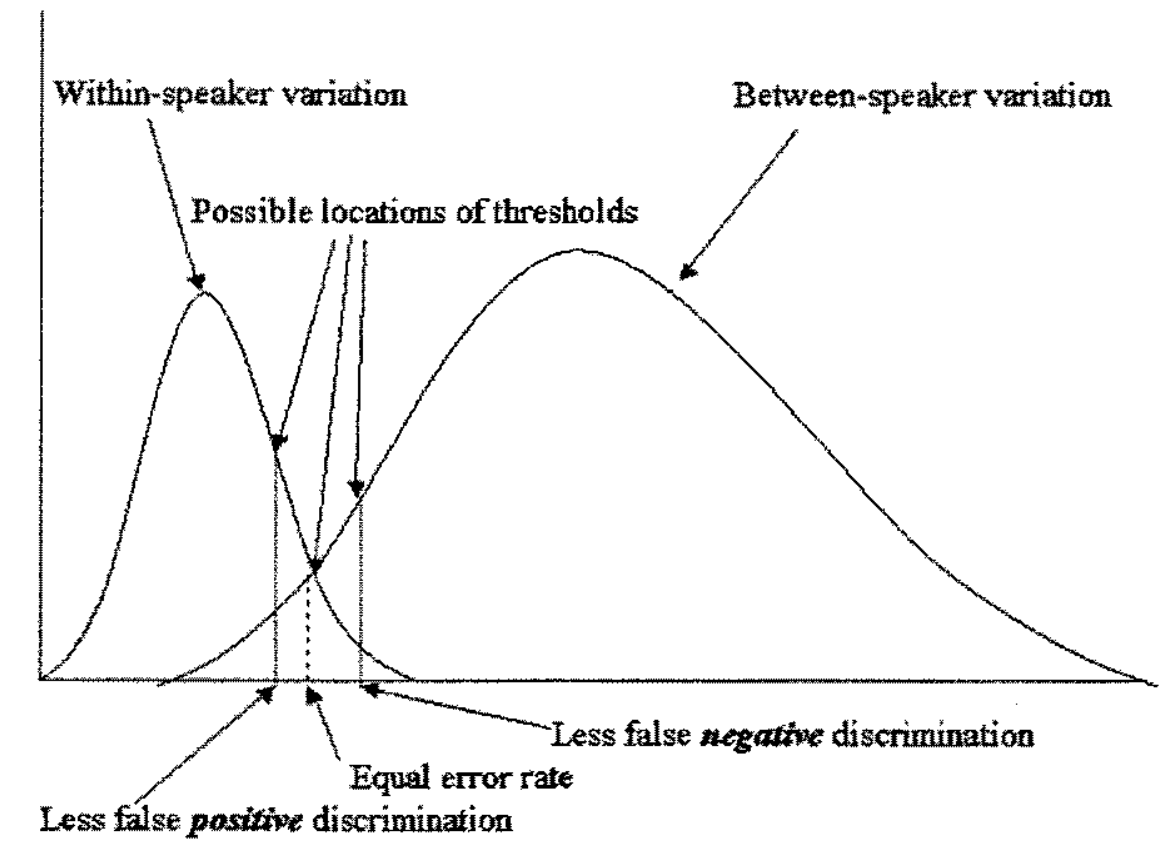

Figure 6.3 Model of the locations of thresholds.

For the selection of the threshold in this study, the error rate for both within-speaker and betweenspeaker variation was firstly calculated, applying a tentatively approximated threshold. This tentative threshold was selected, referring to the mean of within-speaker distance, 1.819 , and that of between-speaker distance, 4.261 . Although the value between these two means is 3.04 , a smaller value, 2.5 , was chosen as the first approximate threshold, since the within-speaker variation has a much narrower distribution than the between-speaker variation, as is seen in the standard deviations ( 0.39 and 2.07 for within- and between- speaker variation, respectively). After this first calculation of error rate, the tentative threshold was adjusted according to the obtained exror rates. If the error rates were larger for within-speaker variation, the threshold was raised, whereas it was lowered when the error rates were larger for between-speaker variation. This process was repeated until an equal error rate between within-speaker and between-speaker variation was achieved. It should be noted here that this trial and enror approach for the search of equal error ratio would not have been 
necessary, if those two curves were normal curves. The threshold for equal error ratio could be determined then by simply finding the location of the intersection of the two normal curves. It was not found to be the case in this study, however.

As mentioned in 6.4.1 above, discriminant scores used here were calculated for five means derived separately for each of the five words, so that there were five vectors to be compared for each speaker. In the within-speaker variation table, the five vectors were compared to each other, as in token 1 vs. 2 , token 1 vs. 3 , token 1 vs. $4, \ldots$ and so on, making ten within- speaker comparisons per speaker altogether. As for between-speaker variation, 25 comparisons were made for each combination of speakers, since five tokens of one speaker were compared to five tokens of the other speaker. The number of comparisons per speaker or speaker combination are summarised below.

\author{
[Within-speaker comparison per speaker] \\ five words compared to each other $\Rightarrow$ ten word combinations \\ [Between-speaker comparison per speaker combination] \\ five words from two speakers compared to each other $\Rightarrow 25$ word combinations
}

The results of the calculations are presented in Table 6.6 in terms of the number of correct classifications. The far left columns of the tables show speakers for within-speaker comparison. For between-speaker comparison on the right, pairs of speakers were compared. The values in the top row, namely $2.5,2.53$, and 2.55 , indicate the thresholds at which were tested. The values in the bottom three rows of each table show the overall means of the valid positive and negative discrimination rates and error rates. 
[Within-speaker comparison]

\begin{tabular}{|c|c|c|c|}
\hline & 2.5 & 2.53 & 2.55 \\
\hline AA & 10 & 10 & 10 \\
\hline HA & 7 & 7 & 7 \\
\hline JN & 9 & 9 & 10 \\
\hline KA & 4 & 4 & 4 \\
\hline KF & 7 & 7 & 7 \\
\hline KH & 9 & 10 & 10 \\
\hline KO & 9 & 9 & 9 \\
\hline MN & 6 & 6 & 6 \\
\hline TN & 7 & 7 & 7 \\
\hline TS & 6 & 6 & 6 \\
\hline TY & 6 & 6 & 6 \\
\hline Mean & 7.273 & 7.364 & 7.455 \\
\hline Suc.\% & 72.73 & 73.64 & 74.55 \\
\hline Err.\% & 27.27 & 2636 & 25.45 \\
\hline
\end{tabular}

[Between-speaker comparison]

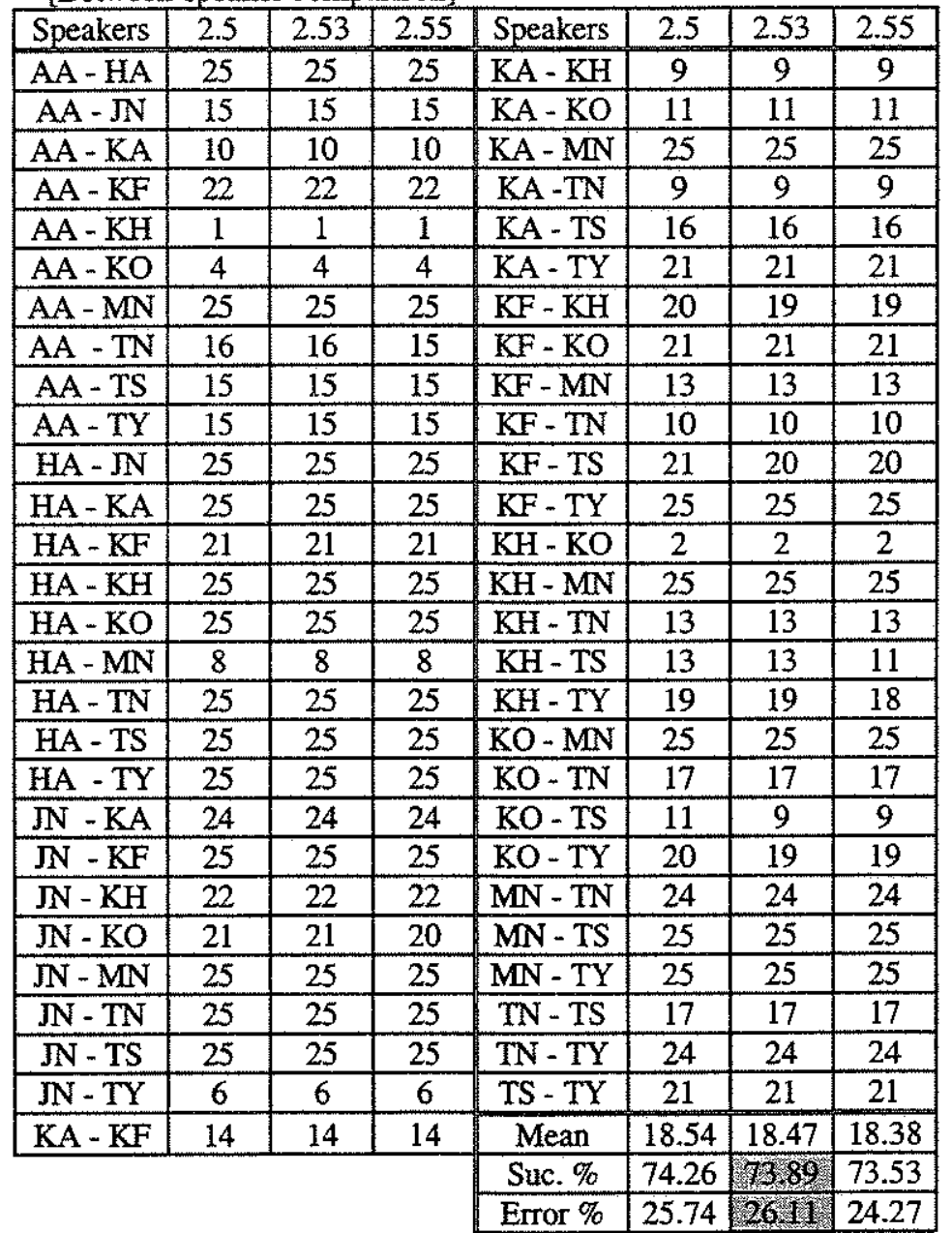

Table 6.6 Summary of the results of the classification tests with the thresholds $2.5,2.53$, and 2.5. The figures presented in the tables show how many valid positive and negative discriminations were made with the given threshold in the top row. There were ten comparisons to be identified for within-speaker distance table, and 25 to be discriminated for between-speaker distance. The values labelled "Suc. \%" and "Enor \%" at the bottom of both tables show successful discrimination rates and error rates, respectively.

Table 6.6 shows, for instance, that Speaker JN was correctly identified nine times out of ten with thresholds 2.5 and 2.53 , and when the threshold was set at 2.55 , all ten comparisons made for this speaker were correctly classified. The between-speaker comparison table shows that differences between the tokens of Speakers $\mathrm{AA}$ and $\mathrm{HA}$ were all correctly discriminated with all three thresholds tested here, whereas the differences between Speakers AA and KH were correctly discriminated only once out of 25 with all three threshold tested. 
Although 2.5 was chosen for the first approximation for the threshold, this threshold yielded larger error rates for within-speaker comparison than for between-speaker comparison. Threshold 2.5 yielded the successful discrimination rate $72.73 \%$ for within-speaker comparison, and $74.26 \%$ for between-speaker comparison. Next, 2.55 was tested as a threshold, but this threshold made the successful discrimination rate of within-speaker comparison too small ( $74.55 \%$ for within-speaker comparison, and $75.53 \%$ for between-speaker comparison). Eventually 2.53 was found to produce a more or less equal error rate between within-speaker and between-speaker comparison. When the threshold was set at 2.53 for testing, the successful discrimination rate for within-speaker comparison and for between-speaker comparison become fairly close: $73.64 \%$ and $73.89 \%$, respectively. The threshold which produced the equal error rate is, therefore, 2.53 .

In sum, using the discriminant score-based distance method, approximately $74 \%$ of the successful discrimination rate was achieved. That is, 81 out of 110 pairs for within-speaker comparison were correctly identified as the same speaker, and 1016 out of 1375 pairs for between-speaker comparison were correctly discriminated from each other. Given that the results presented here were just based on three vowel formants, the correct classification with just below $74 \%$ accuracy seems a good result. This is, however, an overestimation of the reality. In the next section, two fundamental problems with this method are discussed.

\subsubsection{PROBLEMS WITH DISCRIMINANT SCORE-BASED DISTANCE METHOD}

First of all, the discrimination tests and the estimation of the threshold were carried out with the same dataset (ie. reference and test samples are the same dataset) in this analysis. It was expected that the discrimination rate would be deteriorated considerably if the threshold was tested with a 
different data set, as the threshold 2.53 was a tailored threshold for the optimal classification of the current datasets. It is most likely that this threshold does not perform as well with different sets of data as it did with the current dataset.

Secondly, but more crucially, the small size of the dataset needs to be pointed out. As only 11 speakers were involved in this study, being plotted according to their linear discriminant scores, the speakers are scattered much more sparsely than what the population in reality would be. Adding more speakers to the dataset, the space between speakers would be filled up, and it is predicted that the discrimination of speakers based purely on the distance between speakers would become considerably more difficult.

Although this approach reflects the fundamental notion of speaker identification very directly, and is also conceptually very easy to comprehend, this method is thus regarded as inadequate for practical application.

\subsection{FORENSIC DISCRIMINATION: LIKELIHOOD RATIO- BASED DISTANCE APPROACH}

Finally, the distance approach based on Bayesian likelihood ratios is discussed in this section. The Bayesian likelihood ratio in this study is not used in the way in which it was originally developed. The Bayesian likelihood ratio is properly used to quantify the strength of evidence. This study uses the likelihood ratio as a scale which measures the distance between the two samples. 


\subsubsection{LIKELIHOOD RATIO AND BAYESIAN APPROACH}

\subsubsection{What do likelihood ratio and Bayesian approach evaluate?}

In this section, I first discuss what is the Bayesian likelihood ratio in its original sense. Robertson and Vignaux (1995) claim that "...expert evidence should be restricted to the likelihood ratio given by the test or observation (or its components)" (p. 21). Their claim is based on the role of experts in court. Experts in a court are not in the position to pass judgment as to whether or not the suspect is guilty. It is the juries, or judges in the countries which do not have juries, who make this final judgment. Thus, for providing those who are in charge of making a judgment with objective evaluations of evidence, the results of comparisons have to be described and presented on the basis of the likelihood ratio.

Presenting the evaluation of evidence in the form of a likelihood ratio is crucial in forensic speaker identification, also because it is technically very difficult or almost impossible to reach conclusions as to a speaker's identity just based on speech recordings. As discussed in Chapter 1 extensively, the human speech mechanism is very complex and is also highly flexible. A comparison of speech samples is thus nothing like a comparison of fingerprints in terms of its complexity. Speech samples made by a single speaker can vary from occasion to occasion considerably, depending on emotional state, health condition, or who the interlocutor is. Furthermore, the same phonetic target can be achieved by different anatomy, using different articulators. For instance, low formants can be produced by an anatomically long vocal tract or by lowering the larynx with a speaker's conscious effort. Moreover, it is often the case that the size and the recording quality of the speech samples being compared are far from ideal. This makes comparing speech samples and reaching firm conclusions even more difficult. There may be a fortuitous case, such as a situation where there are only two possible perpetrators and only one of them has very heavy speech impediments, which are extremely similar to the incriminating speech. In such cases, it is then surely possible to 
come to a very firm conclusion on the basis of speech evidence. It is, however, not the kind of situation which we can expect to happen often. Statements, such as "the suspect IS (or IS NOT) the same individual as the criminal" are therefore not only legally inappropriate, but also technically impossible in most cases, if the evaluation is made simply on the basis of speech samples.

The Bayesian likelihood ratio measures the strength of evidence, taking both prosecution and defense hypotheses into account. Where LR is likelihood ratio, $\mathrm{P}$ means probability, E means evidence, $H$ and $\overline{\mathrm{H}}$ show hypothesis and null hypothesis respectively, the formula for likelihood ratio is shown as:

$$
L R=\frac{P(E \mid H)}{P(E \mid \bar{H})}
$$

In the context of forensic speaker identification, the likelihood ratio can be paraphrased as the ratio of "the probability of finding the observed difference between the recordings when incriminating speech and suspect's speech are from the same speaker" to "the probability of obtaining that difference when those samples are not from the same individuals." A likelihood ratio of 3 , thus, means that it is three times more likely to observe the evidence, if the given samples have been uttered by the same speaker than by different speakers. A likelihood ratio of 1 means that the evidence is just as probable if it is the same or different speakers; the evidence hence provides no information as to the identity of the speaker. A very small likelihood ratio or a very large likelihood ratio in relation to 1 , on the other hand, suggests that the piece of information makes a useful contribution to the decision making process.

A very simple demonstration of the calculation of the likelihood ratio should be given here using the categorical data obtained from the analysis of the vowel devoicing incidence rates in Chapter 4. 
The tables presented in Chapter 4 are reproduced here again in order to show the detail of the rates of the illegal vowel devoicing incidence.

[Within-speaker comparison]
\begin{tabular}{|c|c|c|}
\hline & Session 1 & Session 2 \\
\hline AA & $25 \%$ & $25 \%$ \\
\hline HA & $100 \%$ & $100 \%$ \\
\hline JN & $100 \%$ & $75 \%$ \\
\hline KA & $100 \%$ & $75 \%$ \\
\hline KF & $50 \%$ & $75 \%$ \\
\hline KH & $0 \%$ & $0 \%$ \\
\hline KO & $50 \%$ & $50 \%$ \\
\hline MN & $0 \%$ & $0 \%$ \\
\hline TN & $75 \%$ & $75 \%$ \\
\hline TS & $100 \%$ & $75 \%$ \\
\hline TY & $75 \%$ & $75 \%$ \\
\hline
\end{tabular}

[Between-speaker comparison]

\begin{tabular}{|c|c|c|c|}
\hline Comparison & Same rates & Total no. & $\%$ \\
\hline Session1/1 & 8 & 55 & $15 \%$ \\
\hline Session1/2 & 4 & 55 & $7 \%$ \\
\hline Session2/1 & 9 & 55 & $16 \%$ \\
\hline Session2/2 & 13 & 55 & $24 \%$ \\
\hline TOTAL & 34 & 220 & $15 \%$ \\
\hline
\end{tabular}

Table 6.7 Rates of the illegal vowel devoicing incidence (originally presented in Chapter 4).

The analysis of the consistency of the rate of vowel devoicing incidence across different speech samples within a speaker showed that the probability that the vowel devoicing incidence rates match when they are produced by the same speaker was $7 / 11(=63.6 \%)$. The probability that two rates match when they are produced by different speakers was found to be between $7 \%$ and $24 \%$, and the mean was $15 \%$. If we use the mean for the calculation, the likelihood ratio of this particular evidence will be:

$$
L R=64.6 \% / 15 \%=4.307
$$

This means that if the vowel devoicing incidence rates match across two samples, it is 4.307 times more likely for the samples to be uttered by the same speaker than not. 
As described in Chapter 1 as well, the Bayesian approach tells us how to incorporate the information of newly added evidence into an existing probability. This process is performed by multiplying the prior odds by the likelihood ratio of the new piece of evidence. The result of this multiplication, presented as posterior odds, is the likelihood ratio after incorporating the new piece of evidence. The likelihood ratio for the incidence of illegal devoicing presented above thus can be combined with the likelihood ratio produced on the basis of formants, which is going to be discussed in the following sections by simple multiplication, although it was not incorporated in the production of final posterior odds in this study.

The advantage of the Bayesian approach over the discriminant analysis lies in the fact that incorporating a new piece of information into the existing results is extremely simple providing these pieces of evidence are not correlated. There is no need to worry about the difference of the structures between these parameters. Given that we do not have any control over the sampling size in real-life forensic situation, this is a significant advantage.

\subsubsection{Likelihood ratio calculation}

Aitkin (1995: 181) proposes a formula for the likelihood ratio calculation of continuous data, using the reflective indices of glass fragments as an example of the evidence. Aitkin's formula for the likelihood ratio for continuous data is as follows:

$$
V \cong \frac{\tau}{a \sigma} \times \exp \left\{-\frac{(\bar{x}-\bar{y})^{2}}{2 a^{2} \sigma^{2}}\right\} \times \exp \left\{-\frac{(w-\mu)^{2}}{2 \tau^{2}}+\frac{(z-\mu)^{2}}{\tau^{2}}\right\}
$$

(Aitkin 1995: 181) 
In Aitkin's formula, the calculation of likelihood ratio takes seven values into account. The seven values are:

1) Number of measurements of criminal samples $(m)$

2) Mean of criminal samples $(\bar{x})$

3) Number of measurements of suspect samples $(n)$

4) Mean of suspect samples $(\bar{y})$

5) Variance of criminal and suspects samples $\left(\sigma^{2}\right)$

6) Overall mean of population $(\mu)$

7) Overall variance of population $\left(\tau^{2}\right)$

Furthermore, $z, w$, and $a^{2}$ are derived using the values above as follows:

$$
\begin{aligned}
& z=(\bar{x}+\bar{y}) / 2 \\
& w=(m \bar{x}+n \bar{y}) /(m+n) \\
& a^{2}=1 / m+1 / n
\end{aligned}
$$

Aitkin composed this formula in such a way that it reflects two important characteristics of the samples: the similarity between samples which are in comparison, and the typicality of those samples. Strong similarity between samples and low typicality both contribute to higher likelihood ratios. Similarity and typicality are expressed in Aitkin's formula as indicated below.

$$
V \equiv \frac{\tau}{a \sigma} \times \exp \left\{-\frac{(\bar{x}-\bar{y})^{2}}{2 a^{2} \sigma^{2}}\right\} \times \exp \left\{-\frac{(w-\mu)^{2}}{2 \tau^{2}}+\frac{(z-\mu)^{2}}{\tau^{2}}\right\}
$$

Similarity measures are obviously how similar the two samples are. The similarity is evaluated in terms of how close those samples are located in the distribution of the reference population. The 
other characteristic, typicality, evaluates how usual or unusual the given samples are. The samples which are close to the population mean are interpreted as samples with ordinary voice quality. This suggests that many speakers could produce those similar values by coincidence, so that similarity between samples near the population mean does not tell us much about whether the samples are from two different speakers or not. Unusual voices located on the skirts of the distribution curve, on the other hand, provide us with more information as to the speaker's identity. Clearly it is far less likely to obtain two unusual and also very similar voices from two different samples just by coincidence. Those two samples are more likely to be produced by the same speaker. Similarly, if one sample is located at one end of the distribution and the other sample at the opposite end, those two voices are both very unusual and also very different from each other, so that those samples are very unlikely to have been produced by the same speaker.

Aitkin's formula presented above is, however, not totally appropriate for the calculation of the likelihood ratio of speech data for a few reasons. The next section points out the concern about the use of Aitkin's formula in forensic speaker identification, and the likelihood ratio-based distance approach as an alternative measure is discussed.

\subsubsection{Problems in Aitkin fs $f$ or ml a and andternati ve approacht of his st ud}

The formula presented in the previous section is designed to deal with the transfer of evidence like reflective indices of glass, which do not change their value depending on the occasions of measurements. The glass fragments which a criminal carries on his clothing without knowing will not change their reflective indices whether they are discovered and measured on the next day or three months later. The human voice is, on the other hand, very flexible and it is known that the time factor can affect it greatly, as has been repeatedly pointed out. Thus the application of Aitkin's 
formula inherently has a problem of not taking the plasticity of voice production into account, although the experiment of this study has shown that two weeks time difference between recording sessions does not affect the size of within-speaker variation.

The other problem is the correlation between parameters. The Bayesian approach enables us to combine multiple parameters in a very simple and yet principled way. However, the simple multiplication is appropriate only when the parameters incorporated are not correlated. When there are some correlations between parameters, it is necessary to take the relationship between those parameters into consideration.

Suppose there are two forensic phonetic parameters, $\mathrm{E}_{1}$ and $\mathrm{E}_{2}$, and the value of $\mathrm{E}_{2}$ is dependent on $\mathrm{E}_{1}$. As the probability of $\mathrm{E}_{2}$ is determined by not only hypothesis, but also the first parameter, the likelihood ratios for each parameter are expressed as:

$$
\begin{aligned}
& \text { For } \mathrm{E}_{1}, \quad L R=\frac{p\left(E_{1} \mid H\right)}{p\left(E_{1} \mid \bar{H}\right)} \\
& \text { For } \mathrm{E}_{2}, \quad L R=\frac{p\left(E_{2} \mid E_{1} \text { and } H\right)}{p\left(E_{2} \mid E_{1} \text { and } \bar{H}\right)}
\end{aligned}
$$

Then suppose two parameters, $E_{1}$ and $E_{2}$, were $F 2$ and $F 3$ of the same vowel. They are expected to be correlated to some extent, given that they are produced by a single vocal cord for the production of the same vowel in a single occasion. It is not possible with these parameters to tell which parameter is dependent on which, and what the degree of dependency is. Furthermore, for forensic speaker identification, we would like to take as many parameters as possible into account for the accuracy of performance. The expression of correlation under these circumstances becomes even more complex. The calculation of the posterior odds incorporating the comelation is therefore 
practically impossible, as we do not have enough knowledge on the dependency of parameters to each other to perform this calculation. Although the degree of the correlation between them in this study is going to be discussed in the following section and it is shown that it is not significant, this is a point we have to bear in our mind.

Furthermore, Aitkin's formula assumes that the samples are normally distributed. This may not be the case in human speech. In fact, some of the parameters which were sampled in this study show abnormal distribution (the distributions of parameters are presented in the later section, 6.6.4.2).

These problems suggest the possibility that the posterior odds produced by the likelihood calculation and the Bayesian approach may not provide us with accurate estimates of the strength of the speech evidence, although Aitkin's formula is still the closest approximation available currently to the author's knowledge. This thesis, therefore, proposes a different use of the Bayesian likelihood ratio. That is the use of posterior odds as the scores for a discrimination test, rather than the scale which evaluates the strength of the evidence. The Bayesian likelihood ratio-based distance approach discussed in the following sections classifies the posterior odds into withinspeaker variation or between-speaker variation, depending on whether or not they are over or below 1 , and the successful discrimination rates are calculated. The threshold was set at 1 , as the likelihood ratio above 1 favours the hypothesis that samples came from the same speaker and below 1 favours the hypothesis that they did not. 


\subsubsection{CORRELATION AND PARAMETER SELECTION}

\subsubsection{Selection of parameters}

In Chapters 4 and 5, the parameters which produce high F-ratios were revealed. The study of vowel formants embedded in different contexts in Chapter 4 showed that the F-ratio of F2 of /i/, F2 and F3 of /e/, and effective F2 of /e/ had high F-ratios (26, 31, 27, and 43.3 respectively). In Chapter 5, the analysis of the phrase moshimoshi, revealed that more measuring points produced high F-ratios. The parameters which produced F-ratios over 20 were:

$\begin{array}{ll}\text { The first } / \mathrm{m} / & \text { F1 (21.4), F3 (20.5) } \\ \text { The first } / \mathrm{o} / \text { onset } & \text { F3 (24.9) } \\ \text { The first } / \mathrm{o} / \text { middle } & \text { F3 (27.5), F4 (22.0) } \\ \text { The second /m/ } & \text { F1 (20.7), F3 (27.2) } \\ \text { The second /s/ } & \text { F3 (26.6), F4 (22.3) }\end{array}$

As has been discussed, forensic cases often involve recordings of telephone conversations as incriminating speech samples. It is particularly so with this phrase, as moshimoshi is almost exclusively used in telephone conversation. When samples are recorded from telephone lines, the formants located outside of the telephone bandwidth (usually approximately $300-3000 \mathrm{~Hz}$ ) are lost or attenuated. This means that in many circumstances, F1 and F4 are not available. Since there is no F2 which had a high F-ratio, the parameters to be used in calculations are limited to F3 in this study.

Furthermore, for the selection of the parameters for speaker discrimination, correlation between parameters also had to be taken into consideration. Given that all sampling points were extracted from a single word, there is an obvious possibility of a strong correlation between parameters. If 
two selected parameters reflect similar information on an individual's voice, incorporating both of those parameters will not increase the useful information for profiling the individuals' characteristics, but on the contrary it will distort the posterior odds severely.

The points discussed above led me to select $\mathrm{F} 3$ of the first $/ \mathrm{O} / \mathrm{F} 3$ of the second $/ \mathrm{m} /$, and $\mathrm{F} 3$ of the second /s/ for this study. Those three segments had higher F-ratios, and were also considered to be less directly correlated to each other, as they are not adjacent to each other. The continuity between these selected segments is thus expected to be relatively low, although it will be still inevitable that these words will be correlated to some extent, as they constitute the same F3 produced by a single vocal tract in the the production of a single word.

In summary, the parameters chosen for use in this approach ane thus,

F2 of / / $/$

F2 and F3of /e/

F3 of the mid point of the first $/ 0 /$

F 3 of the second $/ \mathrm{m} /$

F3 of the second $/ \mathrm{s} /$

In addition, effective $\mathrm{F} 2$ is also examined. These parameters are labelled $\mathrm{iF2}, \mathrm{eF} 2, \mathrm{eF} 3, \mathrm{eF}^{\prime} 2$, olmid, $\mathrm{m} 2$, and $\mathrm{s} 2$ henceforth for convenience in the discussion.

The treatment of effective F2 in the application of the Bayesian approach should also be noted here. The effective $F 2$ is, as discussed, the parameter which incorporates $F 1, F 2$ and F3 into a single figure. The F-ratio of effective F2 of the /e/ vowel was found to be much higher than any of the other F-ratios, and its potential as a speaker identification parameter was pointed out in earlier 
chapters. In the Bayesian approach, however, the likelihood ratios calculated for each parameter are combined into a single figure by multiplying. In the Bayesian approach, the advantages of the use of effective $\mathrm{F} 2$ instead of the conventional $\mathrm{F} 2$ and $\mathrm{F} 3$ become unclear. The posterior odds using conventional F2 and F3 and that of using effective F2 instead of F2 and F3 may not yield different results. Posterior odd calculations using the Bayesian approach in this chapter, therefore, test both calculations to see whether effective F2 does in fact have an advantage over separate F2 and F3.

\subsubsection{Effect of between-parameter correlation on statistics}

As briefly discussed in Chapter 3, most acoustic parameters produced by a single speaker are potentially correlated, because they were produced using the same vocal cords, the same oral and nasal cavities and so forth. It seems very difficult to find acoustic parameters with no correlation except for some temporal factors.

As has been described, the Bayesian approach can properly combine different pieces of information by multiplying the likelihood ratios only when the parameters are independent. Although there is a possibility of correlation between parameters, this study does not take the correlation between parameters into account, as this complicates the calculation too much. In this way, if the parameters are strongly correlated, the multiplication of the likelihood ratios becomes a multiplication of very similar values, and this will distort the results severely by disproportionately amplifying the common features across those acoustic parameters.

If the correlation is expected to cause problems, then, the extent to which those parameters are indeed correlated should be statistically examined. Thus an examination was carried out separately for each speaker to see the correlation between the parameters. This is because if correlation is 
examined across speakers and if speakers have different patterns of between-parameter correlation, the correlation estimated across speakers will obscure the underlying speaker-specific betweenparameter correlation. The results of Pearson's $r$ correlation test are presented in the form of a correlation matrix in Table 6.8. Numbers of comparisons for each parameter pair are shown as well to the right of the $\mathrm{r}$ value (degree of freedom is $n-2$ ). The values which reached the level of significance ( $95 \%$ level of confidence) are indicated by shading. The parameters are divided by a dotted line, according to whether the segments were embedded in the different words, or the segments were elicited from a single phrase, moshimoshi.

\begin{tabular}{|c|c|c|c|c|c|c|c|c|c|c|c|c|c|}
\hline & iF 2 & & $e^{F} 2$ & & $e F 3$ & & $e F 2$ & & olmid & & $m 2$ & & $s 2$ \\
\hline iF 2 & 1 & & & & & & & & & & & & \\
\hline $\mathrm{eF} 2$ & 0.25 & 19 & 1 & & & & & & & & & & \\
\hline $\mathrm{eF3}$ & 0.29 & $19 \%$ & 0.4 & 20 & 1 & & & & & & & & \\
\hline $\mathrm{eF} 2$ & 0.29 & 19 & 028 & 20 & 06 & 20 & 1 & & & & & & \\
\hline olmid & 0.47 & 11 . & 0,5 & 12 & 0.32 & 12 & (1) & 12 & 1 & & & & \\
\hline $\mathrm{m} 2$ & 0.24 & 11 & 0.27 & 12 & 0.37 & 12 & 0.33 & 12 & 0.48 & 12 & 1 & & \\
\hline $\mathrm{s} 2$ & -0.02 & 11 & 0.31 & 12 & 0.16 & 12 & 0.34 & 12 & -0.03 & 12 & 0.13 & 12 & 1 \\
\hline
\end{tabular}

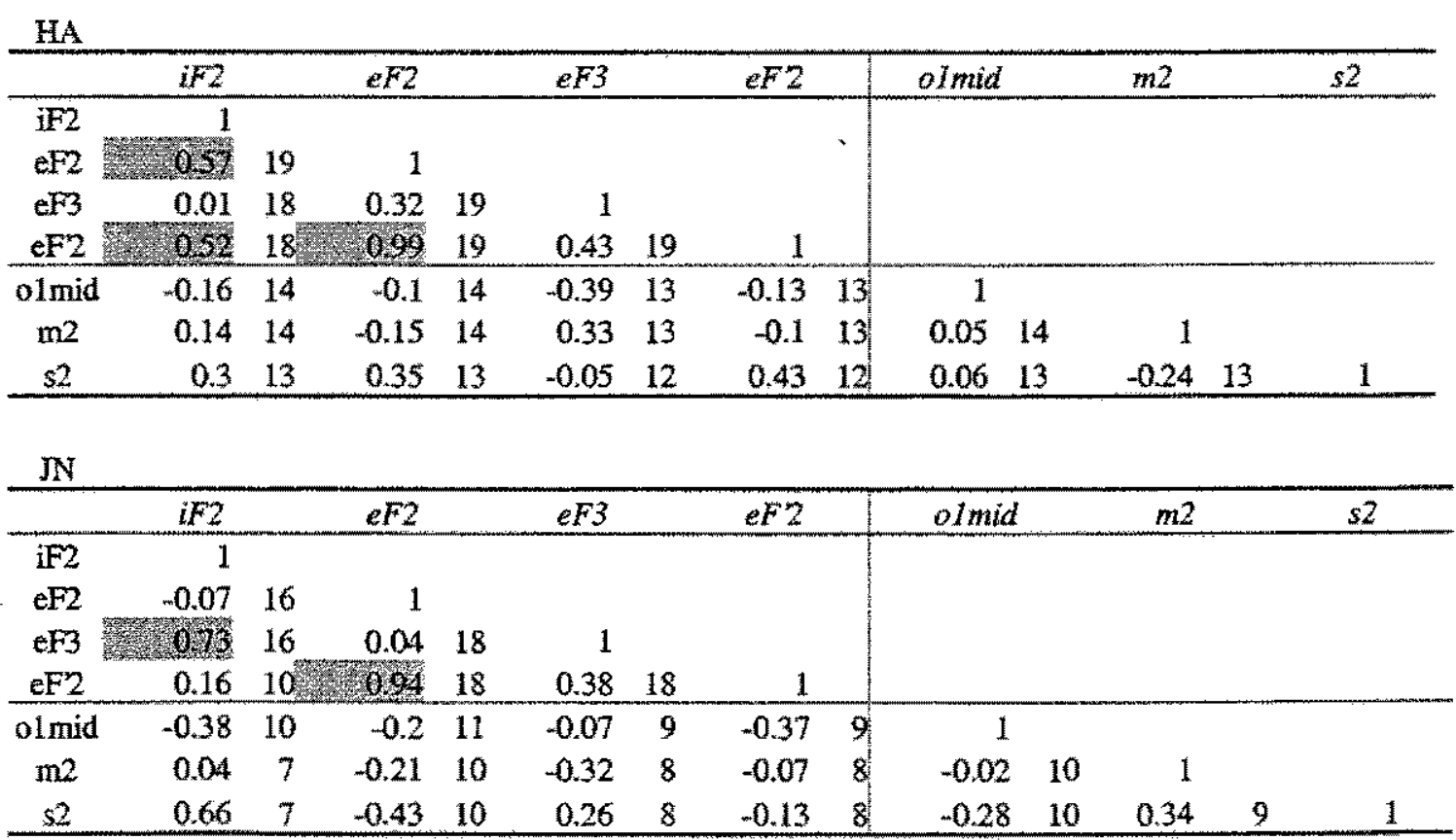




\begin{tabular}{|c|c|c|c|c|c|c|c|c|c|c|c|c|c|}
\hline & $i F 2$ & & $e F 2$ & & $e F 3$ & & $e F 2$ & & olmid & & $m 2$ & & $s 2$ \\
\hline $\mathrm{iF} 2$ & 1 & & & & & & & & & & & & \\
\hline eF2 & -0.4 & 19 & 1 & & & & & & & & & & \\
\hline $\mathrm{eF} 3$ & -0.08 & 19 & 0.41 & 19 & 1 & & & & & & & & \\
\hline $\mathrm{eF} 2$ & -0.36 & 19 & 0.92 & 19 & 0.62 & 19 & 1 & & & & & & \\
\hline olmid & -0.13 & 8 & 0.46 & 8 & 0.46 & 8 & 0.54 & 8 & 1 & & & & \\
\hline $\mathrm{m} 2$ & 078 & 7 & -0.11 & 7 & 0.16 & 7 & -0.04 & 7 & 0.2 & 7 & 1 & & \\
\hline$s 2$ & -0.41 & 8 & -0.1 & 8 & 0.71 & 8 & 0.18 & 8 & 0.07 & 8 & -0.32 & 7 & 1 \\
\hline \multicolumn{14}{|l|}{$\mathrm{KF}$} \\
\hline & $i F 2$ & & $e F 2$ & & $e F 3$ & & $e F 2$ & & olmid & & $m^{2}$ & & $s 2$ \\
\hline $\mathrm{iF} 2$ & 1 & & & & & & & & & & & & \\
\hline $\mathrm{eF} 2$ & 0.09 & 19 & 1 & & & & & & & & & & \\
\hline $\mathrm{eF3}$ & 0.11 & 19 & 0.64 & 20 & 1 & & & & & & & & \\
\hline $\mathrm{eF}^{2}$ & 0.1 & 19 & 0.98 & $20^{\circ}$ & 0.79 & 20 & 1 & & & & & & \\
\hline olmid & 0.42 & 15 & 0.14 & 16 & 0.31 & 16 & 0.2 & 16 & 1 & & & & \\
\hline $\mathrm{m} 2$ & 0.12 & 16 & 0.08 & 17 & 0.19 & 17 & 0.12 & 17 & 0.46 & 16 & 1 & & \\
\hline $\mathrm{s} 2$ & 0.16 & 16 & -0.26 & 17 & -0.01 & 17 & -0.2 & 17 & 0.32 & 17 & 0.37 & 17 & 1 \\
\hline \multicolumn{14}{|l|}{$\mathrm{KO}$} \\
\hline & $i F 2$ & & $e F 2$ & & $e F 3$ & & $e F 2$ & & olmid & & $m 2$ & & $s 2$ \\
\hline $\mathrm{iF2}$ & 1 & & & & & & & & & & & & \\
\hline $\mathrm{eF} 2$ & 0.13 & 14 & 1 & & & & & & & & & & \\
\hline $\mathrm{eF3}$ & -0.29 & 14 & -0.07 & 15 & 1 & & & & & & & & \\
\hline $\mathrm{eF}^{\prime 2}$ & 0.06 & 14. & 098 & 15 & 0.13 & 15 & 1 & & & & & & \\
\hline olmid & -0.21 & 6 & -0.62 & 6 & 0.3 & 6 & -0.6 & 6 & 1 & & & & \\
\hline $\mathrm{m} 2$ & 0.45 & 6 & 0.22 & 6 & -0.12 & 6 & 0.2 & 6 & -0.26 & 6 & 1 & & \\
\hline $\mathrm{s} 2$ & 0.93 & 6 & 0.51 & 6 & 0,94 & 6 & 0.35 & 6 & -0.27 & 6 & 0.18 & 6 & 1 \\
\hline \multicolumn{14}{|l|}{$\mathrm{MN}$} \\
\hline & $i F 2$ & & $e F 2$ & & $e F 3$ & & $e F 2$ & & olmid & & $m 2$ & & $s 2$ \\
\hline $\mathrm{iF} 2$ & 1 & & & & & & & & & & & & \\
\hline $\mathrm{eF} 2$ & 0.18 & 20 & 1 & & & & & & & & & & \\
\hline $\mathrm{eF} 3$ & -0.08 & 20 & 0.14 & 20 & 1 & & & & & & & & \\
\hline $\mathrm{eF} 2$ & 0.13 & $20^{\circ}$ & 0.98 & 20 & 0.3 & 20 & 1 & & & & & & \\
\hline olmid & -0.36 & 11 & -0.25 & 11 & 0.38 & 11 & -0.17 & 11 & 1 & & & & \\
\hline $\mathrm{m} 2$ & -0.28 & 15 & 0.27 & 15 & -0.09 & 15 & 0.23 & 15 & -0.24 & 11 & 1 & & \\
\hline $\mathrm{s} 2$ & -0.1 & 15 & -0.26 & 15 & -0.13 & 15 & -0.29 & 15 & 0.02 & 15 & 0.02 & 15 & 1 \\
\hline
\end{tabular}




\begin{tabular}{|c|c|c|c|c|c|c|c|c|c|c|c|c|c|}
\hline & $i F 2$ & & $e F 2$ & & $e F 3$ & & $e F 2$ & & olmid & & $m 2$ & & $s 2$ \\
\hline $\mathrm{iF} 2$ & 1 & & & & & & & & & & & & \\
\hline $\mathrm{eF} 2$ & 0.02 & 20 & 1 & & & & & & & & & & \\
\hline $\mathrm{eF} 3$ & -0.1 & 20 & -0.36 & 20 & 1 & & & & & & & & \\
\hline eF2 & -0.01 & 20 & 0.95 & 20 & -0.07 & 20 & 1 & & & & & & \\
\hline olmid & -0.18 & 12 & 0.4 & 12 & -0.25 & 12 & 0.4 & 12 & 1 & & & & \\
\hline m2 & -0.24 & 12 & -0.17 & 12 & 0.28 & 12 & -0.14 & 12 & 0.16 & 12 & 1 & & \\
\hline s2 & 0.03 & 11 & -0.26 & 11 & 0.19 & 11 & -0.29 & 11 & 0.02 & 11 & 0.75 & 11 & 1 \\
\hline \multicolumn{14}{|l|}{$\mathrm{TS}$} \\
\hline & $i F 2$ & & $e F 2$ & & eF3 & & $e F 2$ & & olmid & & $m 2$ & & $s 2$ \\
\hline $\mathrm{iF} 2$ & 1 & & & & & & & & & & & & \\
\hline $\mathrm{eF} 2$ & 0.4 & 20 & 1 & & & & & & & & & & \\
\hline $\mathrm{eF} 3$ & 062 & 20 & -0.3 & 20 & 1 & & & & & & & & \\
\hline $\mathrm{eF2}$ & 0.15 & 20 & 0,9 & 20 & 0.14 & 20 & 1 & & & & & & \\
\hline olmid & -0.13 & 14 & 0.12 & 14 & 0.06 & 14 & 0.11 & 14 & 1 & & & & \\
\hline $\mathrm{m} 2$ & -0.18 & 14 & -0.35 & 14 & 0.13 & 14 & -0.24 & 14 & 0.06 & 14 & 1 & & \\
\hline $\mathrm{s} 2$ & -0.27 & 14 & -0.17 & 14 & 0.36 & 14 & 0.06 & 14 & 0.38 & 14 & 0.61 & 14 & 1 \\
\hline \multicolumn{14}{|l|}{ TY } \\
\hline & $i F 2$ & & $e F 2$ & & $e F 3$ & & $e F^{2}$ & & olmid & & $m 2$ & & $s 2$ \\
\hline $\mathrm{iF} 2$ & 1 & & & & & & & & & & & & \\
\hline $\mathrm{eF} 2$ & 0.01 & 20 & 1 & & & & & & & & & & \\
\hline $\mathrm{eF3}$ & 0.18 & 20 & 0.15 & 20 & 1 & & & & & & & & \\
\hline $\mathrm{eF} 2$ & 0.05 & 20 & 0.97 & 20 & 0.39 & 20 & 1 & & & & & & \\
\hline olmid & -0.24 & 14 & 0.36 & 14 & -0.28 & 14 & 0.28 & 14 & 1 & & & & \\
\hline $\mathrm{m} 2$ & 0.01 & 14 & -0.06 & 14 & 0.05 & 14 & -0.03 & 14 & -0.05 & 14 & 1 & & \\
\hline$s 2$ & -0.16 & 13 & -0.38 & 13 & 0.35 & 13 & -0.26 & 13 & 0.11 & 13 & 0.46 & 13 & 1 \\
\hline
\end{tabular}

Table 6.8 Correlation between parameters and the numbers of comparisons made for each parameter combination.

Table 6.8 shows that, despite the expectation, there is not much correlation between the parameters.

Seven parameters make 21 parameter combinations. Speaker AA had the largest number of correlated parameter combinations, which was five. Speakers MN, TN, and TY had only one parameter combination which correlated significantly. The parameter combination found to be correlated for these speakers was F2 of /e/ and effective F2 (shown as eF'2), and these two parameters were revealed to be strongly correlated in all speakers' tables. This is, however, hardly surprising since effective $F 2$ was calculated based chiefly on F2, taking F1 and F3 into account (see Chapter 4). It has to be noted that, except for this F2 of /e/ and effective F2 combination, none of 
the parameter combinations seem to be correlated consistently across speakers. For instance, it would seem quite obvious that F2 and F3, which were measured from the same vowel at the same measuring point (the middle point of the vowel duration), are closely correlated. Table 6.8 shows, however, that is not the case. Only two out of ten speakers' F2 of /e/ was correlated to F3 of the same vowel.

It should also be noted that the correlation between the formants of the vowels embedded in the same words showed no stronger correlation than the formants of segments which were measured from the different words. The dotted lines in the table separate the parameters according to the elicitation style. The bottom right quarter presents the correlation between the segments which were measured from a single utterance, moshimoshi, in the top left quarter comparison between iF2 and $\mathrm{eF} 2$, $\mathrm{eF} 3$, or effective $\mathrm{F} 2$ shows the correlation among the parameters which were measured from different words. This seems to suggest that it is valid to incorporate parameters which were extracted from a single word, as long as the sampling point was selected sensibly, perhaps by sampling no adjacent phonetic targets for incorporation. The reason why not much correlation between words was found is maybe attributed to the small sampling size of the data and also to the small magnitude of the within-parameter variation.

\subsubsection{SPEAKER POOL FOR THIS TEST}

Now, the number of speakers involved in this test needs to be described. As described in Chapter 3 , 11 speakers were included in the speaker pool for the vowel formant data. Three more speakers were recorded later and added to the measurements of moshimoshi. One speaker (Speaker $\mathrm{KH}$ ), however, had to be removed, as this speaker did not produce a sufficient number of moshimoshi tokens to perform statistics. Thirteen speakers are thus included in the speaker pool of the 
moshimoshi data. Although 11 speakers for the parameters from Chapter 4 , and 13 speakers for the parameters from Chapter 5 were included in the speaker pool, only the speakers who were measured on all parameters were included for the discrimination test. This number tumed out to be ten.

The means, standard deviations and sampling numbers which were used for the likelihood calculation are presented in Table 6.9. Speakers marked by shading are those who were included in the speaker pool but not used for the discrimination test based on the posterior odds, as they lacked the data either from the vowel formants or moshimoshi. "Ses 1 " and "Ses 2 " indicate the reconding sessions 1 and 2 , and the columns headed "no." show the number of samples measured for the particular speaker / parameter combination. These numbers vary from speaker to speaker especially with parameters extracted from moshimoshi (namely olmid, $\mathrm{m} 2$, and $\mathrm{s} 2$ ).

\begin{tabular}{|c|c|c|c|c|c|c|}
\hline \multicolumn{7}{|c|}{ Ses 1} \\
\hline & mean & sd & no. & meann & sd & no. \\
\hline AA & 2040.3 & 92.23 & 10 & 2085.4 & 107.89 & 9 \\
\hline $\mathrm{HA}$ & 2498.3 & 184.31 & 10 & 2482 & 184.99 & 9 \\
\hline $\mathrm{JN}$ & 19949 & 204.37 & 8 & 1784.9 & 79.45 & 8 \\
\hline $\mathrm{KA}$ & 2075.9 & 206.77 & 10 & 2202.7 & 148.17 & 9 \\
\hline $\mathrm{KF}$ & 2242 & 168.43 & 9 & 2264.8 & 100.48 & 10 \\
\hline 8 & 20522 & 67.6 & 5 & 21646 & 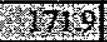 & 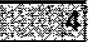 \\
\hline $\mathrm{KO}$ & 2021.6 & 138.84 & 10 & 1955 & 226.37 & 10 \\
\hline $\mathrm{MN}$ & 2466 & 127.69 & 10 & 2341.7 & 167.05 & 10 \\
\hline $\mathrm{TN}$ & 2188.2 & 128.02 & 10 & 2203.5 & 92.51 & 10 \\
\hline $\mathrm{TS}$ & 2215.4 & 94.75 & 10 & 2174.6 & 79.66 & 10 \\
\hline$T Y$ & 19706 & 158.83 & 10 & 1982.5 & 83.76 & 10 \\
\hline
\end{tabular}

$[\mathrm{e} / \mathrm{F} 2]$

\begin{tabular}{|c|c|c|c|c|c|c|}
\hline & \multicolumn{3}{|c|}{ Ses 1} & \multicolumn{3}{|c|}{ Ses 2} \\
\hline & mean & sd & no. & mean & sd & no. \\
\hline$\overline{\mathrm{AA}}$ & 1866.9 & 92.09 & 10 & 1951.9 & 63.13 & 10 \\
\hline $\mathrm{HA}$ & 2223.9 & 136.0 & 10 & 2164.4 & 72.92 & 10 \\
\hline IN & 1744.5 & 85.66 & 10 & 1749.5 & 108.23 & 10 \\
\hline$\overline{K A}$ & 2019.9 & 128.23 & 10 & 1999.5 & 169.86 & 10 \\
\hline$\overline{K F}$ & 21729 & 84.83 & 10 & 2070.9 & 94.5 & 10 \\
\hline Ka & 18788 & 80182 & 8) & 19604 & 8650 & 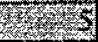 \\
\hline $\mathrm{KO}$ & 1942.6 & 71.95 & 5 & 1912.8 & 89.31 & 10 \\
\hline $\mathrm{MN}$ & 2222.2 & 149.99 & 10 & 2081.5 & 98.3 & 10 \\
\hline TN & 2133,8 & 110.81 & 10 & 2053.9 & 77.72 & 10 \\
\hline TS & 1897.5 & $86 \mathrm{A1}$ & 10 & 1863.5 & 75.71 & 10 \\
\hline$T Y$ & 18227 & 79.52 & 10 & 1861.8 & 78.6 & 10 \\
\hline
\end{tabular}

[/e/ F3]

\begin{tabular}{|c|c|c|c|c|c|c|}
\hline & \multicolumn{3}{|c|}{ Ses 1} & \multicolumn{3}{|c|}{$\operatorname{Ses} 2$} \\
\hline & mean & sd & no. & mean & $\mathrm{sd}$ & no. \\
\hline $\mathrm{AA}$ & 2527.4 & 84.31 & 10 & 2626.1 & 79.76 & 10 \\
\hline $\mathrm{HA}$ & 2959.2 & 53.6 & 9 & 2915.1 & 45.37 & 10 \\
\hline $\mathrm{JN}$ & 2597.5 & 197.63 & 8 & 2440.5 & 42.21 & 10 \\
\hline $\mathrm{KA}$ & 2622.5 & 183.39 & 10 & 2639.7 & 93.61 & 10 \\
\hline $\mathrm{KF}$ & 2750.2 & 109.06 & 10 & 2673.7 & 77.47 & 10 \\
\hline 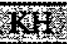 & 326176 & 7.77.6 & 8 & $641 / 8$ & 896 & 5 \\
\hline $\mathrm{KO}$ & 26322 & 35.25 & 5 & 2695.8 & 86.76 & 10 \\
\hline $\mathrm{MN}$ & 29005 & 82.8 & 10 & 2782.5 & 86.78 & 10 \\
\hline $\mathrm{TN}$ & 2638 & 54.35 & 10 & 2605.7 & 100.56 & 10 \\
\hline TS & 26724 & 210.96 & 10 & 2760.4 & 77.2 & 10 \\
\hline$T Y$ & 2457.6 & 85.86 & 10 & 2484.8 & 77.6 & 10 \\
\hline
\end{tabular}

[/e/ F f 2

\begin{tabular}{|c|c|c|c|c|c|c|}
\hline & \multicolumn{3}{|c|}{ Ses 1} & \multicolumn{3}{|c|}{$\operatorname{Ses} 2$} \\
\hline & mexn & $\mathrm{sd}$ & no. & mean & so & no. \\
\hline AA & 2089.5 & 87.3 & 10 & 2182.9 & 61.8 & 10 \\
\hline $\mathrm{HA}$ & 2477.6 & 127.6 & 9 & 2423.6 & 51.119 & 10 \\
\hline $\mathrm{JN}$ & 2004.3 & 87.4 & 8 & 1976.5 & 86.6 & 10 \\
\hline $\mathrm{KA}$ & 22342 & 131.2 & 10 & 2222.4 & 153,4 & 10 \\
\hline $\mathrm{KF}$ & 2390.7 & 87.7 & 10 & 2292.1 & 83.9 & 10 \\
\hline 59 & 21162 & 605 & 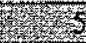 & 8184 & 10 & $7 x$ \\
\hline $\mathrm{KO}$ & 2190.2 & 54.7 & 5 & 2165.8 & 81.0 & 10 \\
\hline $\mathrm{MN}$ & 2457.1 & 118.1 & 10 & 2319.5 & 86.6 & 10 \\
\hline $\mathrm{TN}$ & 2324.1 & 69.5 & 10 & 2258.5 & 55.5 & 10 \\
\hline TS & 2144.3 & 81.8 & 10 & 2135.8 & 60.5 & 10 \\
\hline $\mathrm{TY}$ & 2038.2 & 60.0 & 10 & 2079.7 & 78.9 & 10 \\
\hline
\end{tabular}


$[/ / 1$ middle point $\mathrm{F} 3]$

\begin{tabular}{|c|c|c|c|c|c|c|}
\hline & \multicolumn{3}{|c|}{ Ses 1} & \multicolumn{3}{|c|}{ Ses 2} \\
\hline & mean & $5 d$ & no. & mean & sol & no. \\
\hline $\mathrm{AA}$ & 2218.8 & 112.3 & 5 & 2301.7 & 35.2 & 7 \\
\hline HA & 2724.2 & 119.9 & 6 & 2776.8 & 1825 & 6 \\
\hline $\mathrm{JN}$ & 2114,3 & 199.1 & 5 & 2140 & 106.2 & 6 \\
\hline $\mathrm{KA}$ & 27965 & 169.3 & 4 & 2827.5 & 57.3 & 4 \\
\hline $\mathrm{KF}$ & 2664.6 & 175.5 & 5 & 2464.4 & 214.6 & 6 \\
\hline $\mathrm{KO}$ & 2574.3 & 103.6 & 3 & 2596 & 67.7 & 3 \\
\hline 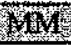 & 253198 & 2807 & 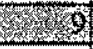 & 276 & 1028 & 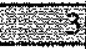 \\
\hline $\mathrm{MN}$ & 2310 & 144.6 & 7 & 2220.3 & 189.8 & 5 \\
\hline 10 & 778 & or 3 & (2) & $2 r 85$ & 718 & 6 \\
\hline TN & 2550.5 & 83.9 & 6 & 2661.4 & 134.2 & 6 \\
\hline $\mathrm{TS}$ & 2848.1 & 99.6 & 8 & 2817.3 & 106.8 & 4 \\
\hline TY & 2411.3 & 118.8 & 6 & 2345.8 & 90.4 & 6 \\
\hline (1) & 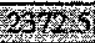 & 11102 & 58 & 2783 & 1367 & 6 \\
\hline
\end{tabular}

$[/ \mathrm{s} / 2 \mathrm{~F} 3]$

\begin{tabular}{|c|c|c|c|c|c|c|}
\hline & \multicolumn{3}{|c|}{ Ses 1} & \multicolumn{3}{|c|}{$\operatorname{Ses} 2$} \\
\hline & mean & sd & no. & mean & sd & no. \\
\hline AA & 2523.6 & 93.5 & 5 & 2498.9 & 82.2 & 7 \\
\hline $\mathrm{HA}$ & 3035 & 151.0 & 6 & 3012.7 & 162.7 & \\
\hline $\mathrm{IN}$ & 2494.8 & 210.5 & 5 & 2618.8 & 140.1 & 6 \\
\hline $\mathrm{KA}$ & 2779.5 & 110.5 & 4 & 2813.3 & 91.6 & \\
\hline $\mathrm{KF}$ & 2914.4 & 150.4 & 5 & 2882.4 & 154.0 & \\
\hline $\mathrm{KO}$ & 2667.7 & 59.5 & 3 & 26023 & 82.9 & 3 \\
\hline Mu & 2688 & 122.9 & 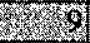 & 1800 & 170,8 & \\
\hline $\mathrm{MN}$ & 2990.6 & 97.8 & 7 & 3055.5 & 77.4 & 5 \\
\hline NO & 26067 & 589 & 6 & 2722,4 & 84 & 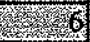 \\
\hline $\mathrm{TN}$ & 2657 & 46.7 & 6 & 27043 & 112.5 & 6 \\
\hline TS & 2865,3 & 171.3 & 8 & 3113.4 & 354.4 & 4 \\
\hline $\mathrm{TY}$ & 2440.4 & 101.6 & 6 & 2562 & 197.0 & \\
\hline$x^{2}$ & 23808 & $1 / 86$ & 9 & 23603 & 130 & \\
\hline
\end{tabular}

Table 6.9 Means and standard deviations used for the likelihood calculations.

In the calculation of likelihood ratios, a cross validation approach was once again used in order to avoid overestimation of the discrimination power of the parameters. Two speakers were firstly removed from the speaker pool and the mean and the standard deviation of the speaker pool without those two speakers were calculated. These two speakers who were removed from the speaker pool were used as a hypothetical suspect and criminal. Thus, in each case of within-speaker and between-speaker comparison, the evaluation was made with respect to a speaker pool that did not contain test samples. 
Ten speakers produced 45 possible speaker combinations (i.e. AA/HA, AAJN, AA/KA ... and so on), so that the whole process of the likelihood ratio calculation was repeated 45 times. Each speaker's dataset consisted of two parts, the data from the first recording session and the data from the second recording session. Each speaker combination, therefore, produced 6 different comparisons: two comparisons to be identified (non ${ }^{-}$contemporaneous comparisons between session 1 and 2 within a single speaker, and two speakers were in each speaker combination), and four comparisons to be discriminated (Speaker A's session 1 vs. Speaker B's session 1, Speaker A's session 2 vs. Speaker B's session 2, Speaker A's session 1 vs. Speaker B's session 2, Speaker A's session 2 vs. Speaker B's session 1). Figure 6.4 summarises the comparisons involved in each test. The vertical arrows indicate the comparisons made within a single speaker (comparisons which should be identified as the same speaker), and the horizontal and diagonal anows indicate the between-speaker comparisons (which should be discriminated as two different individuals).

to be discriminated

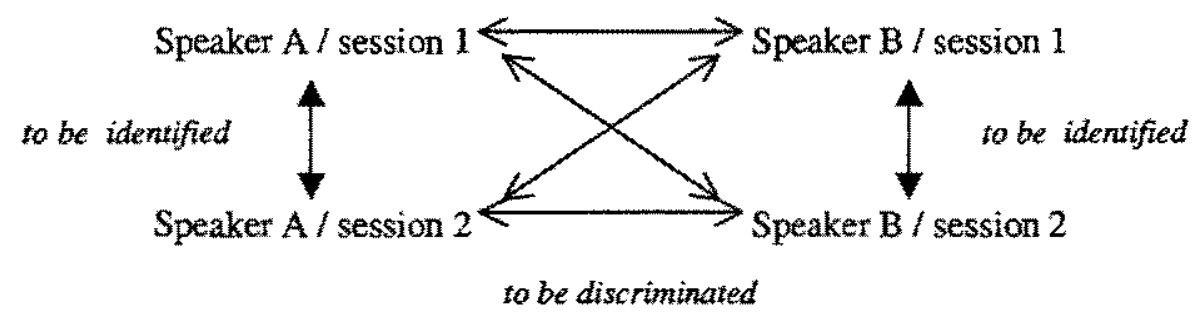

Figure 6.4 Summary of the comparison which each speaker combination comprised. The vertical arrows indicate comparisons made within a single speaker, and the horizontal and diagonal arrows indicate the between speaker-comparisons.

Since the test was repeated 45 times using different speaker combinations as test samples, the whole process produced the following numbers of likelihood ratios. 
Within-speaker non-contemporaneous comparisons

90 (45 speaker combinations * two comparisons for each test)

Between-speaker comparisons

180 (45 speaker combinations * four comparisons for each test)

It has to be emphasised here again that, in each case of 90 within-speaker comparisons and 180 between-speaker comparisons, the evaluations were made with respect to reference data that did not contain their tokens, as a cross validation approach was used.

\subsubsection{PERFORMANCE WITH WITHIN-SPEAKER COMPARISONS}

\subsubsection{Results}

This section discusses the results of the within-speaker comparisons. Each speaker was tested nine times as to whether or not they were identified correctly on the basis of their posterior odds. This is because all speakers had to be taken out from the speaker pool nine times to be combined with the nine other speakers in order to be tested against all other speakers. As each speaker has different acoustic values, naturally the means and standard deviations of the speaker pool vary depending on which two speakers were taken out of the pool. The likelihood ratio calculation therefore had to be performed using nine different means and standard deviations of the speaker pool for each speaker combination, although speakers had only one type of within-speaker comparison (the first recording session against the second one).

The likelihood ratios obtained for each parameter and posterior odds are presented in Table 6.10. The parameters are headed accordingly and columns headed "posterior" on the far right present the posterior odds, ie. the results of the multiplication of the likelihood ratios for all parameters. 
Separate tables are presented for each speaker. The first ten tables show the results of the posterior odds calculations using conventional $F_{2}$ and $F 3$, and the next ten tables present the results of the posterior odds calculated replacing F2 and F3 with effective F2. For instance, Speaker AA's first posterior odd 1.55 was produced by multiplying six likelihood ratios for parameters $\mathrm{iF} 2, \mathrm{eF} 2, \mathrm{eF}$, olmidF3, m2F3, and $\mathrm{s} 2 \mathrm{~F} 3$.

As these are the comparisons between the two recording sessions of single speakers, the likelihood ratio has to be over 1 for the results of the test to be correct. Those which were incorrectly evaluated (ie. a posterior ratio under 1) are marked by shading in the tables.

[Calculation using $\mathrm{F}_{2}$ and $\mathrm{F} 3$ ]

\begin{tabular}{|c|r|r|r|r|r|r|r|}
\hline Speaker & \multicolumn{1}{|c|}{$\mathrm{iF2}$} & \multicolumn{1}{c|}{$\mathrm{eF} 2$} & \multicolumn{1}{c|}{$\mathrm{eF}$} & \multicolumn{1}{c|}{ olmid F3 } & \multicolumn{1}{c|}{ m2 F3 } & s2 F3 & posterior \\
\hline AA & 2.4368 & 0.298084 & 0.138495 & 2.3903 & 1.1902 & 5.4049 & 1.55 \\
AA & 3.148592 & 0.360425 & 0.1968 & 4.2075 & 2.3526 & 6.638782 & 14.68 \\
AA & 2.8419 & 0.32361 & 0.1708 & 2.3320 & 1.1237 & 5.6236 & 2.31 \\
AA & 2.939237 & 0.323988 & 0.1819 & 2.5808 & 1.1911 & 6.3739 & 3.39 \\
AA & 2836045 & 0.3236 & 0.1708 & 2.3326 & 2.3326 & 4.0668 & 3.47 \\
AA & 2.695491 & 0.312211 & 0.1614 & 3.2209 & 1.2014 & 5.4075 & 2.84 \\
AA & 3.083955 & 0.332765 & 0.1904 & 2.5266 & 1.1814 & 6.2458 & 3.64 \\
AA & 3.083658 & 0.361306 & 0.1812 & 2.3473 & 1.3553 & 5.4878 & 3.52 \\
AA & 3.180707 & 0.361383 & 0.2029 & 3.0104 & 1.4825 & 8.0044 & 8.33 \\
\hline
\end{tabular}

\begin{tabular}{|c|r|r|r|r|r|r|r|}
\hline Speaker & \multicolumn{1}{|c|}{ iF2 } & \multicolumn{1}{|c|}{ eF2 } & \multicolumn{1}{c|}{ eF3 } & olmid F3 & \multicolumn{1}{c|}{ m2 F3 } & \multicolumn{1}{|c|}{ s2 F3 } & posterior \\
\hline HA & 17.8209 & 5.353916 & 22.2372 & 3.1796 & 0.6830 & 6.1893 & 28516.13 \\
HA & 26.7364 & 5.791804 & 29.7320 & 3.2092 & 0.5578 & 5.9615 & 49135.06 \\
HA & 19.77392 & 6.060932 & 21.5675 & 4.0264 & 0.7226 & 7.1832 & 54018.37 \\
HA & 33.9108 & 9.102164 & 31.3420 & 3.5707 & 0.7387 & 8.1877 & 208937.59 \\
HA & 18.30841 & 5.389542 & 26.2986 & 3.5983 & 0.7314 & 6.4169 & 43821.03 \\
HA & 110.6788 & 5.463967 & 11.4797 & 3.1863 & 0.7404 & 11.0067 & 180272.16 \\
HA & 21.70245 & 7.858922 & 21.3319 & 3.6273 & 0.7370 & 6.5672 & 63872.05 \\
HA & 21.71592 & 5.224247 & 32.7942 & 4.0905 & 0.7005 & 10.3857 & 110724.91 \\
HA & 18.39671 & 5.197255 & 36.2748 & 3.3352 & 0.7392 & 6.2136 & 53127.55 \\
\hline
\end{tabular}

\begin{tabular}{|c|c|c|c|c|c|c|c|}
\hline Speaker & if 2 & $\mathrm{eF2}$ & eF3 & olmid F3 & $\mathrm{m} 2 \mathrm{~F} 3$ & $52 F_{3}$ & posterior \\
\hline IN & 0.414833 & 23.20343 & 0.2993 & 29.1043 & 92.1886 & 1.6637 & 12861.37 \\
\hline $\mathrm{JN}$ & 0.409376 & 19.473 & 0.2227 & 17.1633 & 26.4314 & 1.3666 & 0.58 \\
\hline $\mathrm{N}$ & & & & & & & \\
\hline $\mathrm{N}$ & 0.340672 & 17.1 & 0.25 & & 26.8209 & 1.4602 & 891.06 \\
\hline $\mathbb{N}$ & 0.558085 & 21.33008 & 0.2645 & & 30.8375 & 008 & 2228.18 \\
\hline $\mathrm{IN}$ & & & & & & & \\
\hline $\mathrm{N} N$ & 0.361008 & 16.80515 & 0.2821 & 14.2728 & 27.4791 & 1.5796 & 1060.22 \\
\hline $\mathbb{N} \mathbf{N}$ & 0.361705 & 26.08205 & 0.2587 & 14.9163 & 58.3798 & 1.3855 & 2944.89 \\
\hline & & & & & & & \\
\hline
\end{tabular}




\begin{tabular}{|c|r|r|r|r|r|r|r|}
\hline Speaker & \multicolumn{1}{|c|}{ iF2 } & eF2 & eF3 & olmid F3 & m2 F3 & s2 F3 & posterior \\
\hline KA & 0.7549 & 1.987961 & 2.0102 & 4.4948 & 4.2684 & 2.5446 & 147.28 \\
KA & 0.679396 & 2.035999 & 1.6686 & 6.0372 & 5.6127 & 2.6847 & 209.97 \\
KA & 0.740795 & 1.843491 & 2.1391 & 4.5667 & 5.6952 & 2.6394 & 200.53 \\
KA & 0.802852 & 2.133924 & 2.1906 & 5.2228 & 5.6206 & 2.7598 & 304.05 \\
KA & 0.802579 & 2.174674 & 2.2151 & 5.2553 & 7.0920 & 2.7151 & 391.23 \\
KA & 0.744913 & 2.084257 & 1.9672 & 4.6250 & 5.4554 & 5.4554 & 420.41 \\
KA & 0.828836 & 2.165925 & 2.2319 & 5.3354 & 5.7948 & 2.7442 & 339.94 \\
KA & 0.828526 & 2.124831 & 2.1841 & 6.7770 & 4.8734 & 2.6790 & 340.22 \\
KA & 0.920677 & 2.066393 & 2.1065 & 4.6979 & 4.8330 & 2.5724 & 234.06 \\
\hline
\end{tabular}

\begin{tabular}{|c|c|c|c|c|c|c|c|}
\hline Speaker & iF2 & $\mathrm{eF} 2$ & eF3 & olmid F3 & $\mathrm{m} 2 \mathrm{F3}$ & s2 F3 & posterior \\
\hline $\mathrm{KF}$ & 3.179184 & 0.315832 & 0.7836 & 0.5761 & 0.4279 & 2.6046 & . 0.51 \\
\hline $\mathrm{KF}$ & 3.431943 & 0.401306 & 0.7460 & 0.6077 & 0.4644 & 3.3988 & 0.99 \\
\hline KF & 2.7414 & 0.2773 & 0.7371 & 0.5263 & 0.3492 & 2.8557 & 0.29 \\
\hline $\mathrm{KF}$ & 3.264357 & 0.34073 & 0.8113 & 0.5964 & 0.4554 & 3.1140 & 0.76 \\
\hline $\mathrm{KF}$ & 3.040159 & 0.320161 & 0.8233 & 0.6219 & 0.4602 & 2.9506 & 0.68 \\
\hline $\mathrm{KF}$ & 3.371122 & 0.407551 & 0.7913 & 0.5775 & 0.4648 & 3.3978 & 0.99 \\
\hline $\mathrm{KF}$ & 3.325575 & 0.370038 & 0.8079 & 0.6201 & 0.4629 & 2.9998 & 0.86 \\
\hline $\mathrm{KF}$ & 3.323809 & 0.309089 & 0.8211 & 0.5917 & 0.4391 & 3.3413 & 013 \\
\hline $\mathrm{KF}$ & 3.018448 & 0.300069 & 0.7053 & 0.6045 & 0.4642 & 2.7824 & 0.50 \\
\hline
\end{tabular}

\begin{tabular}{|c|r|r|r|r|r|r|r|}
\hline Speaker & \multicolumn{1}{c|}{$\mathrm{iF}$} & \multicolumn{1}{c|}{ eF2 } & eF3 & olmid F3 & m2 F3 & s2 F3 & posterior \\
\hline KO & 2.411645 & 2.5758 & 3.0430 & 2.6727 & 2.6727 & 2.1626 & 292.02 \\
KO & 2.149946 & 2.418841 & 2.8134 & 2.8614 & 1.9892 & 2.0747 & 172.78 \\
KO & 3.416213 & 2.702425 & 3.0821 & 2.4595 & 1.6350 & 2.3515 & 269.06 \\
KO & 2.692051 & 2.824164 & 3.3552 & 2.8148 & 2.4173 & 2.3087 & 400.71 \\
KO & 2.465623 & 2.623644 & 3.3632 & 2.8945 & 1.9890 & 2.2293 & 279.23 \\
KO & 2.296626 & 2.528862 & 3.1429 & 2.6982 & 1.9616 & 2.0742 & 200.39 \\
KO & 2.612107 & 2.692025 & 3.3443 & 2.9087 & 2.0171 & 2.3544 & 324.85 \\
KO & 2.61314 & 2.850947 & 3.3571 & 2.7954 & 1.7844 & 2.0953 & 261.40 \\
KO & 3.002709 & 2.824379 & 2.9665 & 2.8263 & 2.0045 & 2.3383 & 333.28 \\
\hline
\end{tabular}

\begin{tabular}{|c|r|r|r|r|r|r|r|}
\hline Speaker & \multicolumn{1}{c|}{ iF2 } & \multicolumn{1}{c|}{ eF2 } & eF3 & olmid F3 & m2 F3 & s2 F3 & posterior \\
\hline MN & 1.495061 & 0.332835 & 0.1089 & 4.1459 & 5.5486 & 5.9894 & 7.47 \\
MN & 5.5995 & 0.362629 & 0.1224 & 3.0948 & 5.9485 & 10.7921 & 49.36 \\
MN & 1.494947 & 0.310154 & 0.1073 & 5.3120 & 4.5432 & 6.0628 & 7.28 \\
MN & 1.591255 & 0.365699 & 0.1132 & 3.0409 & 5.7098 & 5.7098 & 6.53 \\
MN & 1.980107 & 0.453549 & 0.1263 & 3.3308 & 5.9406 & 7.9602 & 17.87 \\
MN & 1.449551 & 0.336858 & 0.1213 & 3.2877 & 5.8220 & 6.2041 & 7.03 \\
MN & 1.674099 & 0.42121 & 0.1123 & 3.2627 & 5.9182 & 6.3501 & 9.71 \\
MN & 1.673685 & 0.325192 & 0.1275 & 3.0256 & 5.6807 & 10.1742 & 12.14 \\
MN & 1.442425 & 0.317787 & 0.1050 & 3.6928 & 5.9399 & 6.0175 & 6.35 \\
\hline
\end{tabular}

\begin{tabular}{|c|r|r|r|r|r|r|r|}
\hline Speaker & \multicolumn{1}{c|}{$\mathrm{iF} 2$} & \multicolumn{1}{c|}{$\mathrm{eF} 2$} & $\mathrm{eF}$ & $\mathrm{olmid} F 3$ & $\mathrm{~m} 2 \mathrm{F3}$ & $\mathrm{s} 2 \mathrm{F3}$ & posterior \\
\hline TN & 3.748943 & 0.943316 & 2.9329 & 1.0207 & 0.2739 & 2.9550 & 8.57 \\
TN & 3.393235 & 1.148053 & 2.1871 & 1.1021 & 0.2994 & 2.7183 & 7.64 \\
TN & 3.270289 & 0.80536 & 2.8607 & 0.9302 & 0.2239 & 2.9790 & 4.67 \\
TN & 3.80485 & 1.003565 & 2.9566 & 1.0885 & 0.2979 & 2.9925 & 10.95 \\
TN & 3.783078 & 1.06895 & 2.8897 & 1.1060 & 0.2990 & 2.9060 & 11.23 \\
TN & 3.612033 & 0.956633 & 2.9244 & 1.1144 & 0.2991 & 3.0188 & 10.17 \\
TN & 3.600034 & 1.095698 & 2.5850 & 1.0232 & 0.2992 & 2.7172 & 8.48 \\
TN & 3.839179 & 0.924924 & 2.8807 & 1.0827 & 0.2813 & 2.7381 & 8.53 \\
TN & 3.590808 & 0.895575 & 2.8331 & 1.0742 & 0.2994 & 2.9372 & 8.61 \\
\hline
\end{tabular}




\begin{tabular}{|c|r|r|r|r|r|r|r|}
\hline Speaker & \multicolumn{1}{|c|}{ iF2 } & \multicolumn{1}{|c|}{ eF2 } & \multicolumn{1}{c|}{ eF3 } & olmid F3 & \multicolumn{1}{c|}{ 2 F3 } & \multicolumn{1}{|c|}{ 2 F3 } & posterior \\
\hline TS & 3.056988 & 3.66617 & 1.0265 & 7.3395 & 0.0144 & 1.2202 & 1.49 \\
TS & 2.761733 & 2.980196 & 0.9980 & 9.8259 & 0.0138 & 2.1303 & 2.37 \\
TS & 2.667144 & 4.042266 & 0.9650 & 7.3960 & 0.0148 & 1.2364 & 1.41 \\
TS & 3.102118 & 3.50789 & 1.0638 & 10.8337 & 0.0123 & 1.4157 & 2.18 \\
TS & 3.083041 & 3.223198 & 1.0799 & 8.3077 & 0.0138 & 1.6067 & 1.97 \\
TS & 2.945647 & 3.64484 & 1.0809 & 8.3542 & 0.0138 & 1.2670 & 1.69 \\
TS & 2.93224 & 3.11506 & 1.0462 & 7.3460 & 0.0139 & 2.1313 & 2.08 \\
TS & 3.131234 & 3.308312 & 1.0591 & 8.5030 & 0.0136 & 1.2966 & 1.65 \\
TS & 2.928389 & 3.772926 & 0.9620 & 7.5442 & 0.2886 & 1.2247 & 28.35 \\
\hline
\end{tabular}

\begin{tabular}{|c|r|r|r|r|r|r|r|}
\hline Speaker & \multicolumn{1}{|c|}{ iF2 } & \multicolumn{1}{|c|}{ eF2 } & \multicolumn{1}{c|}{ eF3 } & olmid F3 & \multicolumn{1}{c|}{ 22 F3 } & s2 F3 & posterior \\
\hline TY & 5.831337 & 4.196108 & 12.7717 & 3.0293 & 0.2484 & 2.5664 & 603.60 \\
TY & 4.483337 & 3.349946 & 11.8707 & 2.6729 & 0.3062 & 1.6889 & 246.40 \\
TY & 7.406008 & 5.631912 & 17.5371 & 3.0753 & 0.2298 & 2.0919 & 1081.59 \\
TY & 4.643106 & 3.871741 & 10.8326 & 2.5962 & 0.2671 & 1.8524 & 250.12 \\
TY & 5.057153 & 3.55754 & 9.6326 & 2.8415 & 0.3058 & 1.7766 & 267.51 \\
TY & 6.229303 & 4.125193 & 9.8254 & 2.8385 & 0.3046 & 1.9945 & 435.46 \\
TY & 4.737437 & 3.47057 & 9.4268 & 2.9956 & 0.3062 & 1.6896 & 240.21 \\
TY & 5.362002 & 3.637166 & 11.0684 & 2.8180 & 0.3053 & 1.9492 & 362.00 \\
TY & 5.364687 & 4.314152 & 8.1870 & 2.5603 & 0.2886 & 1.7153 & 240.18 \\
\hline
\end{tabular}

[Calculation using effective F2]

\begin{tabular}{|c|r|r|r|r|r|r|}
\hline Speaker & \multicolumn{1}{|c|}{$\mathrm{i} F 2$} & \multicolumn{1}{|c|}{$\mathrm{F}^{2} 2$} & olmid F3 & \multicolumn{1}{c|}{$\mathrm{m}^{2} \mathrm{~F} 3$} & \multicolumn{1}{c|}{$\mathrm{s} 2 \mathrm{~F} 3$} & posterior \\
\hline $\mathrm{AA}$ & 2.4368 & 0.1321 & 2.3903 & 1.1902 & 5.4049 & 4.95 \\
$\mathrm{AA}$ & 3.148592 & 0.1709 & 4.2075 & 2.3526 & 6.638782 & 35.37 \\
$\mathrm{AA}$ & 2.8419 & 0.1502 & 2.3320 & 1.1237 & 5.6236 & 6.29 \\
$\mathrm{AA}$ & 2.939237 & 0.1513685 & 2.5808 & 1.1911 & 6.3739 & 8.72 \\
$\mathrm{AA}$ & 2.836045 & 0.1502 & 2.3326 & 2.3326 & 4.0668 & 9.43 \\
$\mathrm{AA}$ & 2.695491 & 0.1433161 & 3.2209 & 1.2014 & 5.4075 & 8.08 \\
$\mathrm{AA}$ & 3.083955 & 0.1578281 & 2.5266 & 1.1814 & 6.2458 & 9.07 \\
$\mathrm{AA}$ & 3.083658 & 0.1677877 & 2.3473 & 1.3553 & 5.4878 & 9.03 \\
$\mathrm{AA}$ & 3.180707 & 0.1692443 & 3.0104 & 1.4825 & 8.0044 & 19.23 \\
\hline
\end{tabular}

\begin{tabular}{|c|r|r|r|r|r|r|}
\hline Speaker & \multicolumn{1}{|c|}{$\mathrm{iF2}$} & \multicolumn{1}{|c|}{$\mathrm{eF}^{\prime} 2$} & olmid F3 & \multicolumn{1}{c|}{$\mathrm{m}^{2} \mathrm{~F} 3$} & \multicolumn{1}{|c|}{ 2 F3 } & posterior \\
\hline $\mathrm{HA}$ & 17.8209 & 9.6267 & 3.1796 & 0.6830 & 6.1893 & 2305.77 \\
$\mathrm{HA}$ & 26.7364 & 5.7918045 & 17.1632 & 0.5578 & 5.9615 & 1652.60 \\
$\mathrm{HA}$ & 19.77392 & 10.809569 & 4.0264 & 0.7226 & 7.1832 & 4466.94 \\
$\mathrm{HA}$ & 33.9108 & 9.1021642 & 3.5707 & 0.7387 & 8.1877 & 6666.38 \\
$\mathrm{HA}$ & 18.30841 & 9.8233295 & 3.5983 & 0.7314 & 6.4169 & 3037.08 \\
$\mathrm{HA}$ & 110.6788 & 33.456791 & 3.1863 & 0.7404 & 11.0067 & 96155.25 \\
$\mathrm{HA}$ & 21.70245 & 7.8589217 & 3.6273 & 0.7370 & 6.5672 & 2994.21 \\
$\mathrm{HA}$ & 21.71592 & 9.4568255 & 4.0905 & 0.7005 & 10.3857 & 6111.81 \\
$\mathrm{HA}$ & 18.39671 & 9.7972094 & 3.3352 & 0.7392 & 6.2136 & 2760.85 \\
\hline
\end{tabular}

\begin{tabular}{|c|r|r|r|r|r|r|}
\hline Speaker & \multicolumn{1}{|c|}{$\mathrm{iF} 2$} & \multicolumn{1}{|c|}{$\mathrm{eF}^{2} 2$} & \multicolumn{1}{|c|}{ olmid F3 } & \multicolumn{1}{c|}{$\mathrm{m} 2 \mathrm{~F} 3$} & \multicolumn{1}{c|}{$\mathrm{s} 2 \mathrm{~F} 3$} & posterior \\
\hline $\mathbb{N}$ & 0.414833 & 17.0136 & 29.1043 & 92.1886 & 1.663651 & 31504.07 \\
$\mathbb{N}$ & 0.409376 & 19.473809 & 3.2092 & 26.4314 & 1.3666 & 4942.54 \\
$\mathbb{N}$ & 0.390974 & 13.1063 & 14.5803 & 39.2012 & 1.5214 & 4455.86 \\
$\mathbb{N}$ & 0.340672 & 12.320467 & 14.9813 & 26.8209 & 1.4602 & 2462.57 \\
$\mathbb{N}$ & 0.558085 & 14.581295 & 14.3346 & 30.8375 & 1.6008 & 5758.50 \\
$\mathbb{N}$ & 0.353871 & 13.108891 & 28.4799 & 26.2818 & 1.3667 & 4745.47 \\
$\mathbb{N}$ & 0.361008 & 12.309078 & 14.2728 & 27.4791 & 1.5796 & 2752.98 \\
$\mathbb{N}$ & 0.361705 & 16.409936 & 14.9163 & 58.3798 & 1.3855 & 7161.06 \\
$\mathbb{N}$ & 0.5819 & 26.13475 & 19.6927 & 26.5890 & 1.6691 & 13290.86 \\
\hline
\end{tabular}




\begin{tabular}{|c|r|r|r|r|r|r|}
\hline Speaker & \multicolumn{1}{c|}{ iF2 } & \multicolumn{1}{c|}{ eF'2 } & o1mid F3 & m2 F3 & s2 F3 & posterior \\
\hline KA & 0.7549 & 2.0911 & 4.4948 & 4.2684 & 2.5446 & 77.07 \\
KA & 0.679396 & 2.0337457 & 6.0372 & 5.6127 & 2.6847 & 125.70 \\
KA & 0.740795 & 1.9925 & 4.5667 & 5.6952 & 2.6394 & 101.32 \\
KA & 0.802852 & 2.2387 & 5.2228 & 5.6206 & 2.7598 & 145.62 \\
KA & 0.802579 & 2.3095272 & 5.2553 & 7.0920 & 2.7151 & 187.57 \\
KA & 0.744913 & 2.1519429 & 4.6250 & 5.4554 & 5.4554 & 220.65 \\
KA & 0.828836 & 2.2956681 & 5.3354 & 5.7948 & 2.7442 & 161.43 \\
KA & 0.828526 & 2.2815716 & 6.7770 & 4.8734 & 2.6790 & 167.26 \\
KA & 0.920677 & 2.1607999 & 4.6979 & 4.8330 & 2.5724 & 116.19 \\
\hline
\end{tabular}

\begin{tabular}{|c|r|r|r|r|r|r|}
\hline Speaker & \multicolumn{1}{|c|}{$\mathrm{iF2}$} & \multicolumn{1}{|c|}{$\mathrm{eF}^{\prime} 2$} & $\mathrm{olmid} F 3$ & $\mathrm{~m} 2 \mathrm{F3}$ & $\mathrm{s} 2 \mathrm{F3}$ & posterior \\
\hline $\mathrm{KF}$ & 3.179184 & 0.2797928 & 0.5761 & 0.4279 & 2.6046 & 0.57 \\
$\mathrm{KF}$ & 3.431943 & 0.448085 & 0.6077 & 0.4644 & 3.3988 & 1.48 \\
$\mathrm{KF}$ & 1.8357 & 0.2454508 & 0.5263 & 0.3492 & 2.8557 & 0.24 \\
$\mathrm{KF}$ & 2.176013 & 0.2988457 & 0.5964 & 0.4554 & 3.1140 & 0.55 \\
$\mathrm{KF}$ & 2.007697 & 0.2887 & 0.6219 & 0.4602 & 2.9506 & 0.49 \\
$\mathrm{KF}$ & 2.453293 & 0.3495785 & 0.5775 & 0.4648 & 3.3978 & 0.78 \\
$\mathrm{KF}$ & 2.232104 & 0.3128503 & 0.6201 & 0.4629 & 2.9998 & 0.60 \\
$\mathrm{KF}$ & 3.323809 & 0.2809253 & 0.5917 & 0.4391 & 3.3413 & 081 \\
$\mathrm{KF}$ & 3.018448 & 0.2628376 & 0.6045 & 0.4642 & 2.7824 & 0.62 \\
\hline
\end{tabular}

\begin{tabular}{|c|r|r|r|r|r|r|}
\hline Speaker & \multicolumn{1}{|c|}{ iF2 } & \multicolumn{1}{c|}{ eF'2 } & olmid F3 & \multicolumn{1}{c|}{ 2 F3 } & \multicolumn{1}{c|}{ 2 F3 } & posterior \\
\hline KO & 2.411645 & 2.8820 & 2.6727 & 2.6727 & 2.1626 & 107.37 \\
KO & 2.149946 & 2.630292 & 2.8614 & 1.9892 & 2.0747 & 66.78 \\
KO & 3.416213 & 2.9405007 & 2.4595 & 1.6350 & 2.3515 & 94.99 \\
KO & 2.692051 & 3.1859589 & 2.8148 & 2.4173 & 2.3087 & 134.73 \\
KO & 2.465623 & 2.9965218 & 2.8945 & 1.9890 & 2.2293 & 94.82 \\
KO & 2.296626 & 2.844128 & 2.6982 & 1.9616 & 2.0742 & 71.71 \\
KO & 2.612107 & 3.107389 & 2.9087 & 2.0171 & 2.3544 & 112.12 \\
KO & 2.61314 & 3.189282 & 2.7954 & 1.7844 & 2.0953 & 87.10 \\
KO & 3.002709 & 3.0923265 & 2.8263 & 2.0045 & 2.3383 & 123.00 \\
\hline
\end{tabular}

\begin{tabular}{|c|r|c|r|r|r|r|}
\hline Speaker & \multicolumn{1}{|c|}{ iF2 } & eF2 & olmid F3 & \multicolumn{1}{c|}{ 2 F3 } & \multicolumn{1}{c|}{ 2 F3 } & posterior \\
\hline MN & 1.495061 & 0.1279353 & 4.1459 & 5.5486 & 5.9894 & 26.35 \\
MN & 5.5995 & 0.3115334 & 3.0948 & 5.9485 & 10.7921 & 346.58 \\
MN & 1.494947 & 0.1214375 & 5.3120 & 4.5432 & 6.0628 & 26.56 \\
MN & 1.591255 & 0.1392233 & 3.0409 & 5.7098 & 5.7098 & 21.96 \\
MN & 1.980107 & 0.1735882 & 3.3308 & 5.9406 & 7.9602 & 54.14 \\
MN & 1.449551 & 0.1319339 & 3.2877 & 5.8220 & 6.2041 & 22.71 \\
MN & 1.674099 & 0.1536644 & 3.2627 & 5.9182 & 6.3501 & 31.54 \\
MN & 1.673685 & 0.1277276 & 3.0256 & 5.6807 & 10.1742 & 37.38 \\
MN & 1.442425 & 0.121997 & 3.6928 & 5.9399 & 6.0175 & 23.23 \\
\hline
\end{tabular}

\begin{tabular}{|c|r|r|r|r|r|r|}
\hline Speaker & \multicolumn{1}{|c|}{$\mathrm{iF} 2$} & \multicolumn{1}{c|}{$\mathrm{eF}^{\prime} 2$} & olmid F3 & \multicolumn{1}{c|}{$\mathrm{m} 2 \mathrm{~F} 3$} & \multicolumn{1}{c|}{ 2 F3 } & posterior \\
\hline TN & 3.748943 & 0.503779 & 1.0207 & 0.2739 & 2.9550 & 1.56 \\
TN & 3.393235 & 1.1480529 & 1.1021 & 0.2994 & 2.7183 & 3.49 \\
TN & 3.270289 & 0.4322745 & 0.9302 & 0.2239 & 2.9790 & 0.88 \\
TN & 3.80485 & 0.5286889 & 1.0885 & 0.2979 & 2.9925 & 1.95 \\
TN & 3.783078 & 0.5374905 & 1.1060 & 0.2990 & 2.9060 & 1.95 \\
TN & 3.612033 & 0.5179674 & 1.1144 & 0.2991 & 3.0188 & 1.88 \\
TN & 3.600034 & 0.5373412 & 1.0232 & 0.2992 & 2.7172 & 1.61 \\
TN & 3.839179 & 0.5071102 & 1.0827 & 0.2813 & 2.7381 & 1.62 \\
TN & 3.590808 & 0.4732524 & 1.0742 & 0.2994 & 2.9372 & 1.61 \\
\hline
\end{tabular}




\begin{tabular}{|c|r|c|r|r|r|r|}
\hline Speaker & \multicolumn{1}{|c|}{ iF2 } & eF2 & olmid F3 & m2 F3 & s2 F3 & posterior \\
\hline TS & 3.056988 & 5.2026464 & 7.3395 & 0.0144 & 1.2202 & 2.06 \\
TS & 2.761733 & 4.1276359 & 9.8259 & 0.0138 & 2.1303 & 3.29 \\
TS & 2.667144 & 5.2528627 & 7.3960 & 0.0148 & 1.2364 & 1.90 \\
TS & 3.102118 & 5.0999547 & 10.8337 & 0.0123 & 1.4157 & 2.98 \\
TS & 3.083041 & 4.7284102 & 8.3077 & 0.0138 & 1.6067 & 2.68 \\
TS & 2.945647 & 5.1804114 & 8.3542 & 0.0138 & 1.2670 & 2.22 \\
TS & 2.93224 & 4.4781824 & 7.3460 & 0.0139 & 2.1313 & 2.86 \\
TS & 3.131234 & 4.9272563 & 8.5030 & 0.0136 & 1.2966 & 2.32 \\
TS & 2.928389 & 5.2398713 & 7.5442 & 0.2886 & 1.2247 & 40.92 \\
\hline
\end{tabular}

\begin{tabular}{|c|c|r|r|r|r|r|}
\hline Speaker & \multicolumn{1}{|c|}{ iF } & eF2 & olmid F3 & m2 F3 & s2 F3 & posterior \\
\hline TY & 5.831337 & 4.4098593 & 3.0293 & 0.2484 & 2.5664 & 49.67 \\
TY & 4.483337 & 3.43402 & 2.6729 & 0.3062 & 1.6889 & 21.28 \\
TY & 7.406008 & 6.4791761 & 3.0753 & 0.2298 & 2.0919 & 70.95 \\
TY & 4.643106 & 3.9831428 & 2.5962 & 0.2671 & 1.8524 & 23.75 \\
TY & 5.057153 & 3.6565426 & 2.8415 & 0.3058 & 1.7766 & 28.54 \\
TY & 6.229303 & 4.1811698 & 2.8385 & 0.3046 & 1.9945 & 44.92 \\
TY & 4.737437 & 3.5545949 & 2.9956 & 0.3062 & 1.6896 & 26.10 \\
TY & 5.362002 & 3.7878565 & 2.8180 & 0.3053 & 1.9492 & 34.06 \\
TY & 5.364687 & 4.3709986 & 2.5603 & 0.2886 & 1.7153 & 29.72 \\
\hline
\end{tabular}

Table 6.10 Summary of each speaker's likelihood ratios and posterior odds. The first ten tables show calculations using natural $F_{2}$ and $F 3$, and the latter ten tables show calculations replacing F2 and F3 with effective $\mathrm{F} 2$,

The distributions of the posterior odds presented in Table 6.10 are shown as below. The first figure shows the distribution of the posterior odds calculated using natural F2 and F3, and the second figure shows the distribution of those calculated using effective F2 instead of natural F2 and F3.

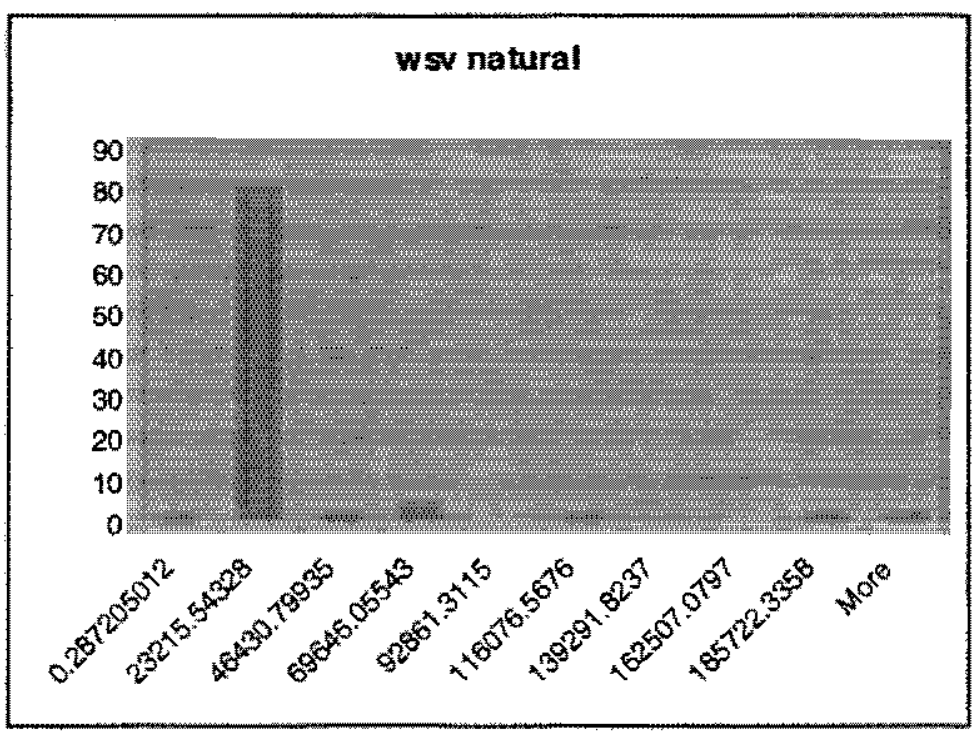




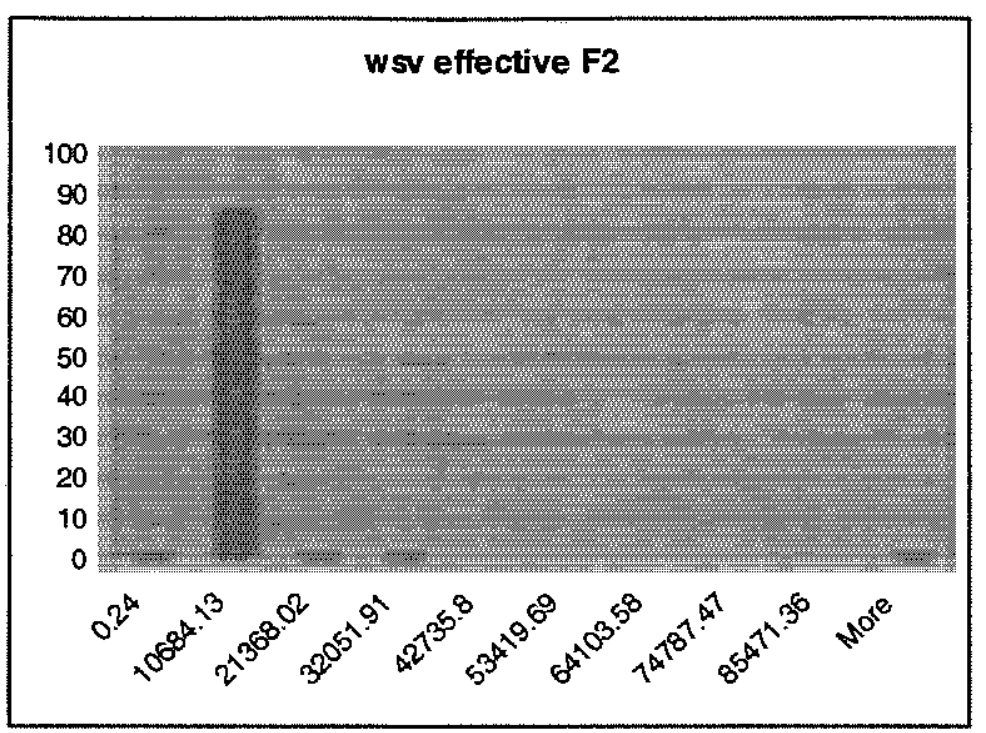

Figure 6.5 Distribution of the posterior odds presented in Table 6.10.

All the tables in Table 6.10 present good identification rates. Although Speaker KF could not be identified correctly in both types of calculations, all the other speakers were successfully identified in the calculation with natural F2 and F3. In the calculation with effective F2, Speaker TN was not identified once. This produces an approximately $90 \%$ valid negative discrimination rate. It is also noticed that the results are very consistent across the nine tests conducted for each speaker. The tables for the calculations with natural F2 and F3 showed that all posterior odds in Speaker KF's table were below 1 , but all the posterior odds for the other speakers were above 1 . The sets of tables with the effective F2 also showed similar results. In this set of results, one of Speaker KF's posterior odds was over 1 (ie. correctly identified), one of Speaker TN's posterior odds was found to be below 1 (ie. not identified), and rest of the speakers had posterior odds over one in all nine tests.

Now, the characteristics which speakers presented will be examined. The means across 9 posterior odds were calculated as a rough indicator to find out which speakers produced particularly high or low posterior odds. The means of the posterior odds for each speaker are presented in Table 6.11. 
The shaded row indicates the speaker whom the likelihood ratio-based distance approach failed to identify (ie. produced posterior odds below 1).

\begin{tabular}{|c|r|r|r|r|}
\hline \multirow{2}{*}{ Speaker } & \multicolumn{2}{|c|}{ Using F2\&3 } & \multicolumn{2}{c|}{ Using effective F2 } \\
\cline { 2 - 5 } & \multicolumn{1}{|c|}{ Mean } & \multicolumn{1}{c|}{ Sd } & Mean & \multicolumn{1}{c|}{ Sd } \\
\hline AA & 4.86 & 4.14 & 12.24 & 9.55 \\
\hline HA & 83487.04 & 64817.09 & 13863.39 & 30848.85 \\
\hline JN & 3423.12 & 3939.43 & 8563.77 & 9178.76 \\
\hline KA & 287.52 & 93.76 & 144.76 & 44.76 \\
\hline TF & 0.70 & 0.23 & 968 & 034 \\
\hline KO & 281.52 & 68.78 & 99.18 & 22.51 \\
\hline MN & 13.75 & 13.86 & 65.61 & 105.85 \\
\hline TN & 8.76 & 1.97 & 1.84 & 0.70 \\
\hline TS & 4.80 & 8.84 & 6.80 & 12.80 \\
\hline TY & 414.12 & 278.75 & 36.56 & 16.00 \\
\hline
\end{tabular}

Table 6.11 Means of the posterior likelihood ratios.

It was noticed that there was a wide range among the mean values of the posterior odds. For the calculation with natural F2 and F3, the means range from 0.70 to 83487 , and for the calculation with effective $\mathrm{F} 2$, from 0.68 to 13863.39 . Speaker $\mathrm{KF}$ produced likelihood ratios below 1 , whereas Speakers HA and JN's likelihood ratios were remarkably high. For Speakers HA and JN, the mean posterior odds were 83487.0 and 3423.12 using F2 and F3 for calculation, and 13863.4 and 8563.77 with effective F2, respectively. It means that, for example, we can say that, having Speaker HA's data, it is 83487 times more likely that the two samples are from the same speaker than they are not, whereas we cannot say much when we have Speaker KF's data. Without the calculation of means, the difference would be even larger.

For a discussion of the causes of these extreme posterior likelihood ratios, the parameters which contributed to those very high or low posterior likelihood ratios should be looked at. The examination of Table 6.10 shows that the constant low posterior likelihood ratios for Speaker $\mathrm{KF}$ were caused by several parameters, namely eF2, eF3, eF' 2 , o1mid, and $\mathrm{m} 2$. His likelihood ratios for those parameters were constantly below 1 . 
It should be noted that Speakers HA and JN, who produced extremely high posterior odds, still had some parameters which actually produced likelihood ratios below 1 . The parameter $\mathrm{m} 2$ for Speaker HA, and the parameters iF2 and eF3 for speaker JN produced likelihood ratios below 1. The parameters $\mathrm{iF} 2$ and eF3 for Speaker HA and the parameters eF2, o1mid, m2 for Speaker JN, however, produced very high likelihood ratios (quite a few of them reached 30 ), the posterior odds for those speakers thus become extremely large. The observations discussed above are summarised in Table 6.12. The columns headed "Low LR parameter" and "High LR parameter" present the parameters which produced the Table 6.12 likelihood ratios below 1 and the parameters which produced particularly high likelihood ratios, respectively. "Low posterior odds" and "High posterior odds" in the far left column indicate whether the speakers shown in the next columns had posterior odds below 1 or extremely high posterior odds.

\begin{tabular}{|l|l|l|l|}
\hline & Speaker & \multicolumn{1}{|c|}{ Low LR parameter } & \multicolumn{1}{|c|}{ High LR parameter } \\
\hline Low posterior odds & $\mathrm{KF}$ & $\mathrm{eF} 2, \mathrm{eF} 3, \mathrm{eF} 2$, olmid, $\mathrm{m} 2$ & $\mathrm{~N} / \mathrm{A}$ \\
\hline \multirow{2}{*}{ High posterior odds } & $\mathrm{HA}$ & $\mathrm{m} 2$ & $\mathrm{iF} 2, \mathrm{eF} 3$ \\
\cline { 2 - 4 } & $\mathrm{JN}$ & $\mathrm{iF} 2, \mathrm{eF} 3$ & $\mathrm{eF} 2, \mathrm{olmid}, \mathrm{m} 2$ \\
\hline
\end{tabular}

Table 6.12 Summary of the observation of the likelihood ratios produced by speaker with extreme posterior odds. The parameters which produced particularly high / low likelihood ratios are presented.

The fact that even the speakers who produced very high posterior odds had some likelihood ratios below 1 with some parameters demonstrates the importance of the use of multiple parameters. If the speaker HA was evaluated only on the basis of parameter $m 2$, for instance, two samples from this speaker could not be correctly evaluated, despite the potential of other parameters to evaluate the sample with great confidence.

In addition, Table 6.10 and Table 6.12 above show that there was no parameter which worked consistently well across speakers. Speaker KF's eF2 wrongfully discriminated, whereas, for Speaker $\mathrm{JN}$, this parameter constantly produced very high likelihood ratios ranging from 16.8 to 
33.38. All other parameters which produced high likelihood ratios in Speaker JN's data (namely, $\mathrm{iF} 2, \mathrm{eF} 3$, olmid, and $\mathrm{m} 2$ ) also produced a likelihood ratio below 1 in other speakers' data.

\subsubsection{Causes of high / low posterior likelihood ratios}

This section discusses what might have caused the unusually high or low likelihood ratios in the evaluation of the same-speaker pairs observed in previous section. In the description of likelihood ratio in the earlier section (6.6.1.2), it was noted that similarity between samples and typicality of these samples are the two major factors which affect the size of likelihood ratios. To what extent similarity and typicality of the data can be attributed to the extreme likelihood ratios is examined in this section.

For the discussion of similarity between samples, the means and standard deviations of those parameter / speaker combinations which produced unusually high or low likelihood ratios are summarised, together with the means and standard deviations of the whole speaker pool in Table 6.13. The speakers who had high posterior odds (namely Speakers HA and JN) are distinguished from those who had low posterior odds by shading. The means and standard deviations of the speaker pool were calculated across all speakers without taking any pairs of speakers out from the presentation in this table. Standard deviations are presented in parentheses beneath the means. 


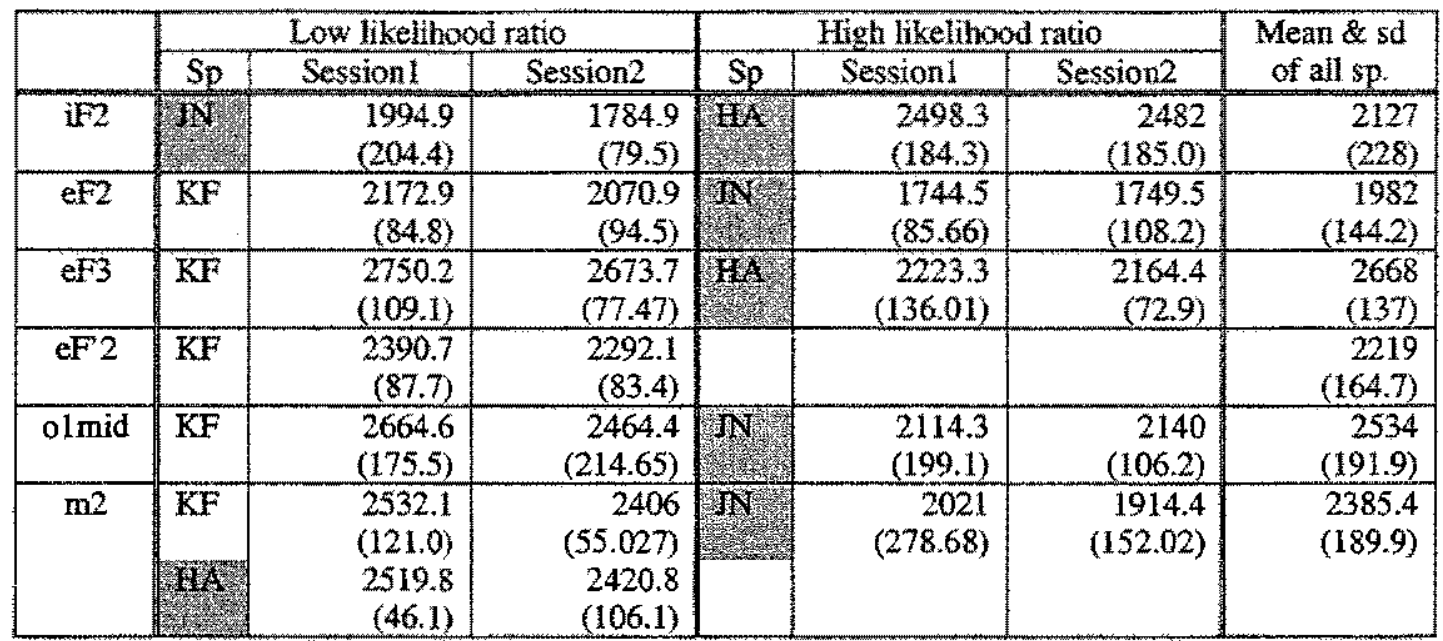

Table 6.13 Means and standard deviations for the parameters which produced particularly high or low likelihood ratios. The speakers who had high posterior odds (speakers HA and JN) are indicated by shading.

An examination of Table 6.13 suggests that the unusually high or low likelihood ratios were caused by both similarity and typically in measured values. The similarity of two samples can be illustrated more clearly in terms of the mean differences between the first and the second sessions. Table 6.14 summarises the difference between two recording sessions in their means for the speaker I parameter combinations in question. The values presented here are the absolute values of the mean difference between two recording sessions. Here again, the speakers who had high posterior odds (namely Speakers HA and $\mathrm{JN}_{\mathrm{N}}$ ) are distinguished from those who had low posterior odds by shading.

\begin{tabular}{|c|c|c|c|c|}
\hline & \multicolumn{2}{|c|}{ Low likelihood ratio } & \multicolumn{2}{|c|}{ High likelihood ratio } \\
\hline & $\$ p$ & Difference & $\mathrm{Sp}$ & Difference \\
\hline $\mathrm{iF2}$ & 3) & $210 \mathrm{~Hz}$ & Her & $16.3 \mathrm{~Hz}$ \\
\hline $\mathrm{eF} 2$ & $\overline{K F}$ & $102 \mathrm{~Hz}$ & TN & $5 \mathrm{Fz}$ \\
\hline $\mathrm{eF} 3$ & KF & $76.5 \mathrm{~Hz}$ & WII & $58.9 \mathrm{~Hz}$ \\
\hline $\mathrm{aF}^{2}$ & $\mathrm{KF}$ & $98.6 \mathrm{~Hz}$ & & \\
\hline olmid & $\mathrm{KF}$ & $200.2 \mathrm{~Hz}$ & $n+$ & $25.7 \mathrm{~Hz}$ \\
\hline $\mathrm{m} 2$ & KF & $\begin{array}{r}126.1 \mathrm{~Hz} \\
99 \mathrm{~Hz}\end{array}$ & NI & $106.6 \mathrm{~Hz}$ \\
\hline
\end{tabular}

Table 6.14 Difference between two recording sessions within a speaker in the means for the speaker $f$ parameter combinations in question. Speakers who had high posterior odds are indicated by shading. 
Table 6.14 above shows that the parameter / speaker combinations which produced high likelihood ratios (on the right in the table) had very small between-session difference, with the exception of Speaker IN's m2. This is more evident when those values are compared to those of the speaker / parameter combination which yielded low likelihood ratios, on the left in the table.

There are, however, a few speaker / parameter combinations which cannot be explained by the mere similarity between two means. For instance, the size of the difference between the two recording sessions of Speaker KF's eF3 $(76.5 \mathrm{~Hz})$, eF'2 $(98.6 \mathrm{~Hz})$, and Speaker HA's m2 $(99 \mathrm{~Hz})$ and Speaker JN"s m2 $(106.6 \mathrm{~Hz})$ do not differ by much. JN"s m2 produced high likelihood ratios, however, the others produced likelihood ratios below 1 . The similarity by itself thus fails to provide a satisfactory explanation for all the unusual likelihood ratios of those speaker / parameter combinations.

Now, the other factor, typicality, should be examined. The typicality of each speaker is probably best shown by the location of the speaker's mean in relation to the distribution of the speaker pool. Figure 6.6 below presents the distributions of the whole speaker pool for different parameters, and the means of the speakers who had particularly low or high likelihood ratios with the particular parameters are also shown in the figure. Two lines pointing from each speaker in the figure indicate the location of the mean of both sessions 1 and 2 in the distribution for the given parameter. 

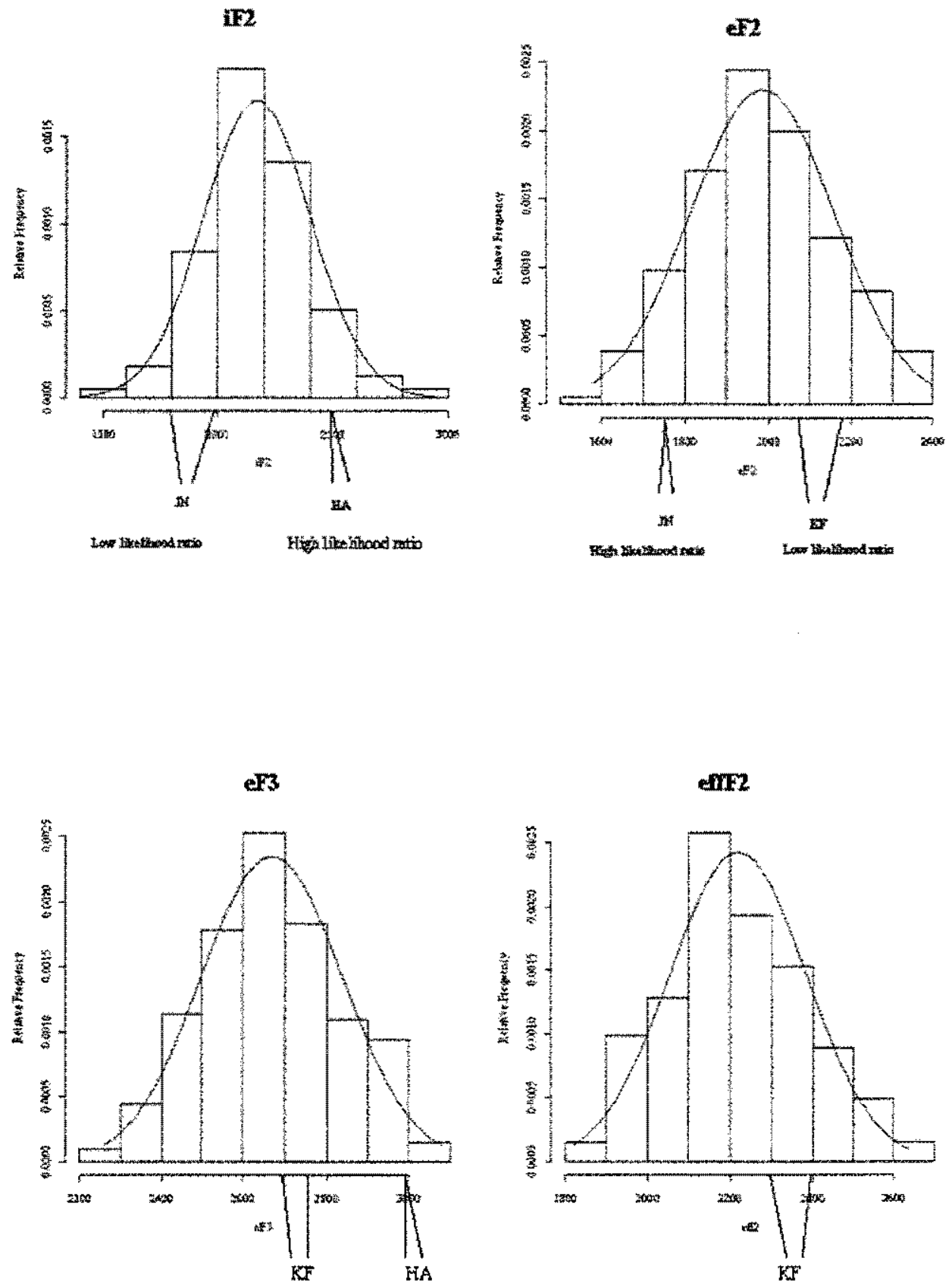

Law likelihood ratio

Fitgits liketihood ratio

Low lakelikood ratio 

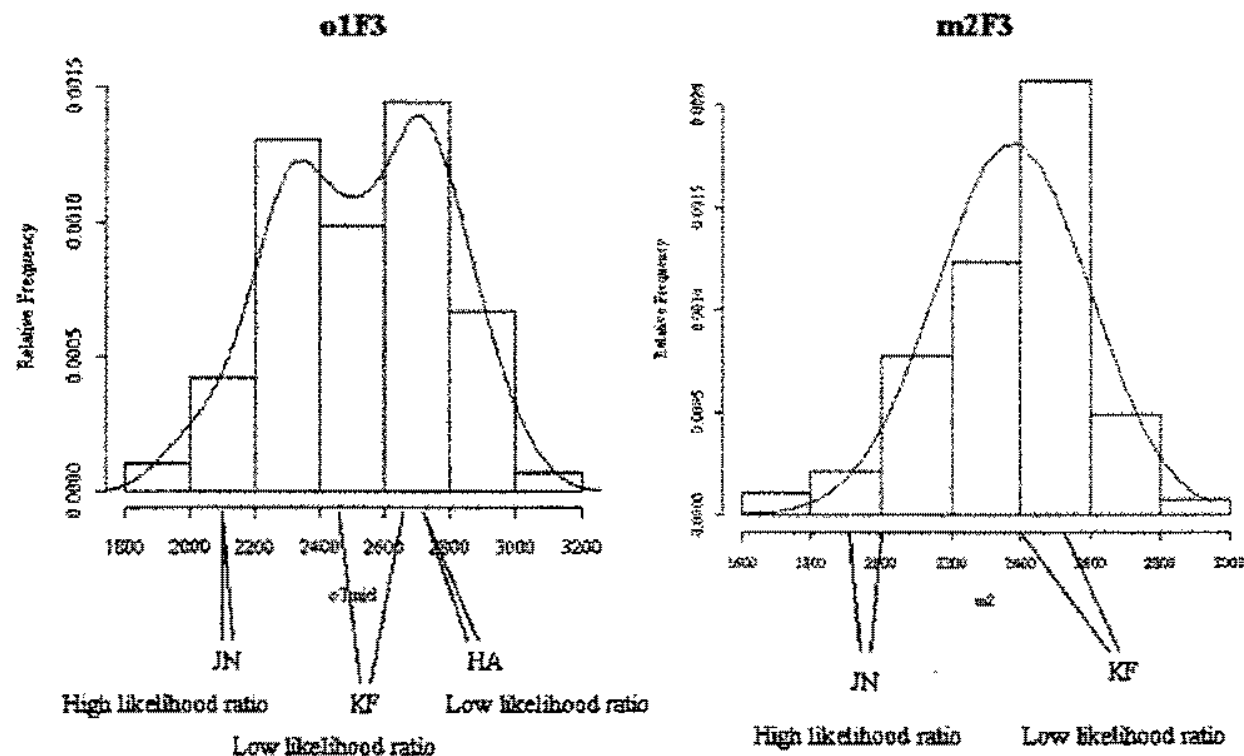

High litedihood ratio Low bikelisood ratio

*2173

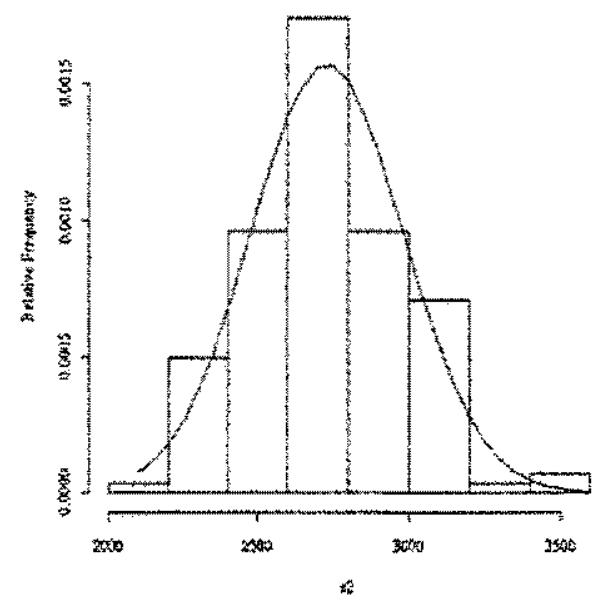

Figure 6.6 Frequency distribution of each parameter. Means of the speakers who had particularly low or high likelihood ratios with the parameters are shown.

Figure 6.6 above shows that all speaker / parameter combinations with high likelihood ratios are located in the ontskirts of the distribution, which means that those speakers who were identified with strong confidence level had relatively unusual formant values for the particular parameter. 
The likelihood ratio of Speaker IN's m2 could not be explained by the lack of similarity between samples, but both means of this speaker are at the edge of the distribution. It is thus assumed that the between-session difference of $99 \mathrm{~Hz}$ was offset by the low typicality of those means in speaker pool. Speaker KF's eF3, and eF'2, and Speaker HA's m2, on the other hand, were both located near the population mean.

Thus, the unusually high or low likelihood ratios seem to be explained by the expected causes.

\subsubsection{Distribution of formants}

The histograms presented in the previous section (Figure 6.6) showed that most formants have a reasonably normal distribution. The distribution of $\mathrm{m}^{2}$ was, however, very skewed, and the distribution of olmid even appears to be bimodal. This is not a desirable result, since Aitkin's formula for the likelihood ratio calculation is used assuming that the data distribute normally.

Although the distribution of olmid appears to be bimodal, actually it cannot be easily judged whether or not this vowel truly has a bimodal distribution from this figure. Since the bin at the dipping part of distribution is still reasonably high, the manipulation of the bin size possibly alters the shape of distribution. To see whether or not this vowel has bimodal distribution, the histograms were produced with three different bin sizes. See Figure 6.7 below. 


\section{Distribution of olmid with different bin size}

$\operatorname{Bin}=10$

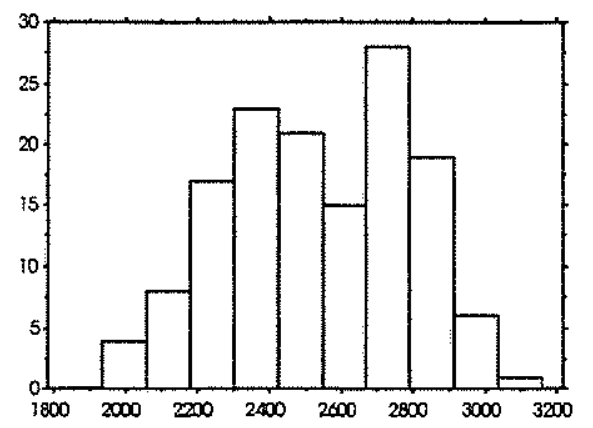

$\operatorname{Bin}=20$

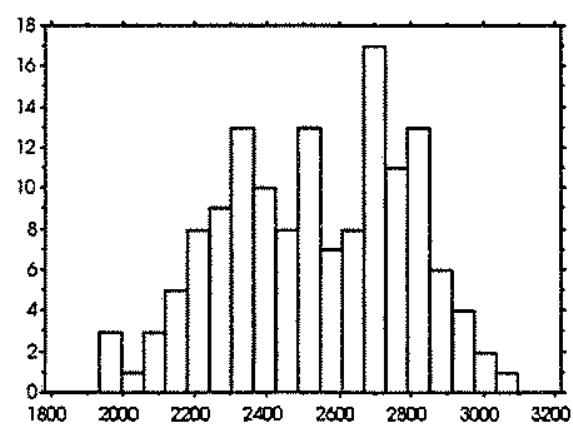

$\operatorname{Bin}=15$

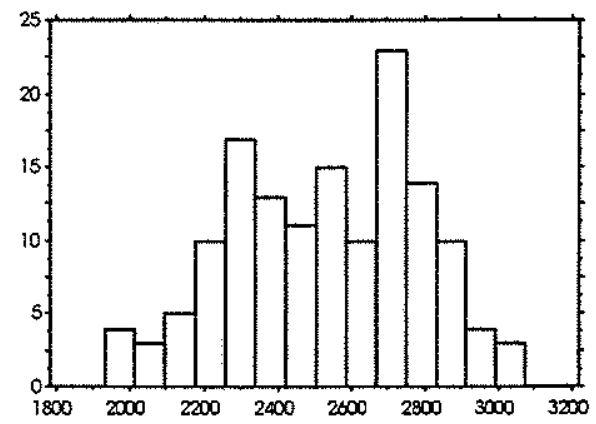

Figure 6.7 Distribution of olmid with three different bin sizes, 10,15, and 20 .

Even after the alteration of the bin size, olmid still appears to have bimodal distribution. If this vowel formant does indeed have a bimodal distribution, then, what would be the cause? There are a couple of possible causes for this bimodal distribution.

As mentioned in the discussion of the flexibility of human speech in Chapter 1 , it is possible to achieve the same phonetic target with different area functions, known as the Stevens and House modelling (Stevens and House 1955). It is not possible, however, to achieve different phonetic targets with the same area function. Thus the fact that two different modes were found in the distribution probably means that they were produced by different articulations. It is known that lip 
rounding lowers F2 and F3 for back vowels (Stevens 1998). Thus maybe some speakers produced the /o/ vowel with more lip rounding than others do.

The other possible interpretation is the effect of the nasal consonant preceding this /o/ vowel. Since this vowel is extracted from the phrase moshimoshi, this vowel is likely to be nasalised. As CSL had problems with extracting formant frequencies with this word, it is possible that two modes were caused by the measurement of two different resonances, nasal pole and $\mathrm{F} 3$ of the /o/ vowel.

The possible causes discussed above are nothing more than hypothetical suppositions, and the conclusive reason remains unclear. It should be noted here, however, that this study has shown that the distribution of acoustic values of forensic phonetic parameters can be non-unimodal. Since the formula presented in 6.6.1.2 is based on the idea that the datasets are normally distributed, this can be a problem in terms of its accuracy of the likelihood ratio. Although the discrimination test performed in the subsequent sections achieved high success rates, it seems certain that the formula still has room for improvement.

\subsubsection{PERFORMANCE WITH BETWEEN-SPEAKER COMPARISONS}

\subsubsection{Results}

Next, the results of the between-speaker comparisons are discussed. As mentioned in the earlier section, each test set produced four different between-speaker comparisons, since the data for a single speaker consisted of the data from recording session 1 and that from session 2 , and two sets of data for two speakers result in four different comparisons. In this section, as all comparisons were between-speaker comparison, the likelihood ratio has to be below 1 if Bayesian likelihood 
ratio discriminates the two speakers correctly. The results of likelihood ratio calculations and posterior odds for between-speaker comparisons are presented in Table 6.15. The likelihood ratios obtained for each parameter are presented in the columns labelled accordingly. The columns headed "posterior" show the posterior odds. The first table shows the results using F2 and F3, and the second one shows the result with effective F2. Comparisons which did not discriminate speakers correctly (ie. produced a likelihood ratio larger than 1) are marked with shading.

[Calculation using F2 and F3]

\begin{tabular}{|c|c|c|c|c|c|c|c|c|}
\hline Speaker & Speaker 2 & $\mathrm{iF} 2$ & $\mathrm{eF} 2$ & $\mathrm{eF} 3$ & olmid & $\mathrm{m} 2 \mathrm{~F} 3$ & s2 F3 & posterior \\
\hline \multirow[t]{4}{*}{$\mathrm{HA}$} & $\mathrm{AA}$ & $7.6 \mathrm{E}-10$ & $1.1 \mathrm{E}-12$ & $3.9 \mathrm{E}-32$ & $1.1 \mathrm{E}-06$ & $7.8 \mathrm{E}-05$ & $1.163 \mathrm{E}-06$ & $3.30 \mathrm{E}-69$ \\
\hline & & $3.7 \mathrm{E}-06$ & $1.5 \mathrm{E}-06$ & $1.7 \mathrm{E}-21$ & $1.4 \mathrm{E}-14$ & $2.3 \mathrm{E}-07$ & $2.669 \mathrm{E}-12$ & $8.37 \mathrm{E}-65$ \\
\hline & & $7.1 \mathrm{E}-09$ & 1.7E-09 & $1.2 \mathrm{E}-33$ & $2.4 \mathrm{E}-09$ & $2 \mathrm{E}-19$ & $4.605 \mathrm{E}-08$ & $3.16 \mathrm{E}-85$ \\
\hline & & $6.5 \mathrm{E}-07$ & $1.8 \mathrm{E}-09$ & $2.7 \mathrm{E}-19$ & $1.3 \mathrm{E}-08$ & 0.04691 & $3.676 \mathrm{E}-10$ & $7.16 \mathrm{E}-53$ \\
\hline \multirow[t]{4}{*}{$\mathrm{N}$} & $\mathrm{AA}$ & $6.6 \mathrm{E}-07$ & 0.44957 & 0.32086 & 12.6415 & 2.61392 & 4.6597643 & $1.47 \mathrm{E}-05$ \\
\hline & & 1.78413 & 3.1E-07 & 2.07918 & 1.4887 & 3.04633 & 1.9774722 & $1.05 \mathrm{E}-05$ \\
\hline & & 3.33715 & 0.15919 & 1.82762 & 11.3424 & 15.2756 & 3.5177509 & $592 \mathrm{I}+02$ \\
\hline & & $1.1 \mathrm{E}-07$ & $5.5 \mathrm{E}-05$ & $5 E-08$ & 0.05902 & 0.00735 & 1.009889 & $1.38 \mathrm{E}-22$ \\
\hline \multirow[t]{4}{*}{$\mathrm{KA}$} & $\mathrm{AA}$ & 0.07473 & 0.28453 & 0.11056 & $1.2 \mathrm{E}-16$ & $5.7 \mathrm{E}-06$ & 0.0006676 & $1.09 \mathrm{E}-27$ \\
\hline & & 2.48869 & 1.0936 & 2.16373 & $4.2 \mathrm{E}-10$ & $4.6 \mathrm{E}-11$ & 0.0002765 & $3.16 \mathrm{E}-23$ \\
\hline & & 2.56067 & 0.04145 & 0.94524 & $9.8 \mathrm{E}-07$ & $2.1 \mathrm{E}-25$ & 0.0117962 & $2.47 \mathrm{E}-34$ \\
\hline & & 0.56161 & 1.75177 & 3.27575 & $7.3 \mathrm{E}-63$ & 0.0001 & $2.887 \mathrm{E}-06$ & $7.07 \mathrm{E}-72$ \\
\hline \multirow[t]{4}{*}{$\overline{\mathrm{KF}}$} & $\mathrm{AA}$ & $2.2 \mathrm{E}-05$ & 3.08698 & 0.0028 & 0.34983 & $1.3 \mathrm{E}-08$ & 0.0005434 & $4.57 \mathrm{E}-19$ \\
\hline & & 0.24159 & $1.5 \mathrm{E}-08$ & 0.07367 & $2.4 \mathrm{E}-05$ & 0.00046 & 4.44E-07 & $1.27 \mathrm{E}-24$ \\
\hline & & 0.02477 & $7.9 \mathrm{E}-12$ & $2.6 \mathrm{E}-05$ & 0.00027 & $3.6 \mathrm{E}-07$ & 0.0001501 & $7.41 \mathrm{E}-32$ \\
\hline & & 0.00723 & 0.02921 & 1.7606 & 0.64229 & 0.01579 & $3.174 \mathrm{E}-06$ & $1.20 \mathrm{E}-11$ \\
\hline \multirow[t]{4}{*}{$\mathrm{KO}$} & $\overline{\mathrm{AA}}$ & 4.00986 & 2.32677 & 0.20097 & $8.5 \mathrm{E}-05$ & $4.2 \mathrm{E}-05$ & 2.1146342 & $1.41 \mathrm{E}-08$ \\
\hline & & 2.41165 & 3.7116 & 1.28333 & $1.1 \mathrm{E}-06$ & $9.4 \mathrm{E}-05$ & 0.0132819 & $1.52 \mathrm{E}-11$ \\
\hline & & 1.92218 & 1.01862 & 0.00394 & 0.00151 & $2.1 \mathrm{E}-12$ & 0.4104618 & $1.01 \mathrm{E}-17$ \\
\hline & & 2.06192 & 2.35945 & 0.72542 & $5.1 \mathrm{E}-14$ & 0.00076 & 0.4879397 & $6.68 \mathrm{E}-17$ \\
\hline \multirow[t]{4}{*}{$\mathrm{MN}$} & $\overline{A A}$ & $4 \mathrm{E}-05$ & 2.5612 & $6.8 \mathrm{E}-09$ & 7.42316 & $4 \mathrm{E}-09$ & $7.783 \mathrm{E}-17$ & $1.62 \mathrm{E}-36$ \\
\hline & & $1.4 \mathrm{E}-09$ & $1.5 \mathrm{E}-05$ & $3.5 \mathrm{E}-11$ & 7.66785 & 0.00018 & $1.926 \mathrm{E}-19$ & $1.92 \mathrm{E}-46$ \\
\hline & & 2.2E-14 & $3 \mathrm{E}-08$ & 1.2E-19 & 4.17837 & $1.2 \mathrm{E}-14$ & $1.067 \mathrm{E}-12$ & $4.29 \mathrm{E}-66$ \\
\hline & & 0.00288 & 0.01531 & 0.00131 & 4.78935 & 0.0022 & $1.686 \mathrm{E}-25$ & $1.03 E-34$ \\
\hline \multirow[t]{4}{*}{$\mathrm{TN}$} & $\overline{\mathrm{AA}}$ & 0.00426 & 3.97378 & 0.9267 & $2.6 \mathrm{E}-06$ & $8 \mathrm{E}-08$ & 0.1375332 & $4.46 \mathrm{E}-16$ \\
\hline & & 0.73185 & 0.00042 & 4.52412 & 3E-09 & 0.11064 & 0.0044742 & $2.02 \mathrm{E}-15$ \\
\hline & & 0.07664 & $6.3 \mathrm{E}-07$ & 0.02334 & $1.3 \mathrm{E}-05$ & 0.00041 & 0.1297487 & $7.78 \mathrm{E}-19$ \\
\hline & & 0.22062 & 0.04337 & 3.3992 & $1.2 \mathrm{E}-08$ & 4.7E-05 & 0.0104338 & $1.90 \mathrm{E}-16$ \\
\hline \multirow[t]{4}{*}{ TS } & $\overline{\mathrm{AA}}$ & 0.02209 & 0.00021 & $3 \mathrm{E}-08$ & $1.9 \mathrm{E}-11$ & 0.0100 & 0.0093562 & $2.46 \mathrm{E}-28$ \\
\hline & & 0.12581 & 1.5135 & 1.63461 & $1 \mathrm{E}-35$ & 1.7601 & $2.69 \mathrm{E}-05$ & $1.51 \mathrm{E}-40$ \\
\hline & & 0.00176 & 3.76491 & 0.35027 & $1.2 \mathrm{E}-20$ & 10.0717 & 0.0019258 & $5.33 \mathrm{E}-25$ \\
\hline & & 0.6746 & 0.14995 & 0.00552 & $2.2 \mathrm{E}-24$ & 1.0334 & 0.0006114 & $7.59 \mathrm{E}-31$ \\
\hline \multirow[t]{4}{*}{ TY } & $\mathrm{AA}$ & 2.70257 & 0.00023 & 5.66163 & 1.33323 & $6.8 \mathrm{E}-10$ & 3.5926484 & $1.12 \mathrm{E}-11$ \\
\hline & & 0.9177 & 0.00411 & 0.00064 & 0.69543 & 0.00351 & 7.3423298 & $4.32 \mathrm{E}-08$ \\
\hline & & 2.60113 & 3.62304 & 2.60959 & 0.26622 & $1 \mathrm{E}-10$ & 4.154433 & $2.76 \mathrm{E}-09$ \\
\hline & & 0.49458 & 0.15096 & 0.00461 & 5.57865 & $1.8 \mathrm{E}-05$ & 3.7601661 & $1.28 \mathrm{E}-07$ \\
\hline
\end{tabular}




\begin{tabular}{|c|c|c|c|c|c|c|c|c|}
\hline & peaker 2 & $\mathrm{iF} 2$ & $\mathrm{eF} 2$ & eF3 & $1 \mathrm{mid}$ & $\mathrm{m} 2 \mathrm{F3}$ & $\mathrm{s} 2 \mathrm{~F} 3$ & posterior \\
\hline JN & $\mathrm{HA}$ & $\begin{array}{l}1.4 \mathrm{E}-20 \\
1.5 \mathrm{E}-05 \\
2.9 \mathrm{E}-06 \\
3.8 \mathrm{E}-19 \\
\end{array}$ & $\begin{array}{r}5.6 \mathrm{E}-15 \\
1.9 \mathrm{E}-27 \\
9 \mathrm{E}-18 \\
4.9 \mathrm{E}-20 \\
\end{array}$ & \begin{tabular}{r|}
$1 \mathrm{E}-107$ \\
$4.3 \mathrm{E}-05$ \\
$1.2 \mathrm{E}-05$ \\
$1 \mathrm{E}-114$ \\
\end{tabular} & $\begin{array}{l}0.52863 \\
0.02064 \\
2.5 \mathrm{E}-07 \\
1.43304 \\
\end{array}$ & \begin{tabular}{l|}
$8.3 \mathrm{E}-16$ \\
0.03798 \\
0.00109 \\
$4.9 \mathrm{E}-08$ \\
\end{tabular} & $\begin{array}{l}9.785 \mathrm{E}-05 \\
0.0002909 \\
0.0002784 \\
0.0005496\end{array}$ & $\begin{array}{r}7.77 \mathrm{E}-175 \\
3.25 \mathrm{E}-47 \\
2.34 \mathrm{E}-41 \\
1.80 \mathrm{E}-172 \\
\end{array}$ \\
\hline $\mathrm{KA}$ & $\mathrm{HA}$ & $\begin{array}{l}0.00686 \\
0.00034 \\
8.3 \mathrm{E}-05 \\
0.01804 \\
\end{array}$ & $\begin{array}{l}0.03022 \\
0.06134 \\
0.02157 \\
0.09543\end{array}$ & $\begin{array}{l}1.6 \mathrm{E}-15 \\
6.8 \mathrm{E}-05 \\
1.4 \mathrm{E}-05 \\
1.3 \mathrm{E}-13 \\
\end{array}$ & $\begin{array}{r}2.7456 \\
4.35122 \\
3.7933 \\
5.34475 \\
\end{array}$ & \begin{tabular}{r|}
0.99574 \\
0.00445 \\
0.00212 \\
0.161 \\
\end{tabular} & $\begin{array}{l}0.2302715 \\
0.2376085 \\
0.1096654 \\
0.4581828 \\
\end{array}$ & $\begin{array}{l}2.06 \mathrm{E}-19 \\
6.59 \mathrm{E}-12 \\
2.27 \mathrm{E}-14 \\
8.95 \mathrm{E}-17 \\
\end{array}$ \\
\hline $\mathrm{KF}$ & $\mathrm{HA}$ & $\begin{array}{l}0.03975 \\
0.16804 \\
0.08158 \\
0.09548\end{array}$ & $\begin{array}{r}0.18273 \\
16.3298 \\
11.603 \\
0.52421 \\
\end{array}$ & $\begin{array}{r}3.6 \mathrm{E}-16 \\
0.00207 \\
0.0001 \\
7.7 \mathrm{E}-14 \\
\end{array}$ & $\begin{array}{r}0.1449 \\
1.89596 \\
2.74328 \\
0.09962 \\
\end{array}$ & \begin{tabular}{r|}
0.01359 \\
1.0934 \\
4.5568 \\
3.96612 \\
\end{tabular} & \begin{tabular}{|l|}
1.5287617 \\
3.1752596 \\
2.6841359 \\
2.0558221 \\
\end{tabular} & $\begin{array}{l}1.97 \mathrm{E}-19 \\
2.23 \mathrm{E}-01 \\
4.01 \mathrm{E}-02 \\
3.27 \mathrm{E}-14 \\
\end{array}$ \\
\hline KO & $\mathrm{HA}$ & \begin{tabular}{l|}
$1.3 \mathrm{E}-08$ \\
$2.2 \mathrm{E}-06$ \\
$3.2 \mathrm{E}-07$ \\
$1.1 \mathrm{E}-07$
\end{tabular} & $\begin{array}{r}9.7 \mathrm{E}-16 \\
4.6 \mathrm{E}-06 \\
0.0006 \\
2.1 \mathrm{E}-09\end{array}$ & $\begin{array}{l}1.2 \mathrm{E}-12 \\
6.6 \mathrm{E}-19 \\
7.4 \mathrm{E}-20 \\
1.6 \mathrm{E}-09\end{array}$ & $\begin{array}{l}1.05773 \\
0.63351 \\
0.78728 \\
0.81003\end{array}$ & & \begin{tabular}{l|}
0.0004448 \\
0.0142085 \\
0.0034354 \\
0.0024348 \\
\end{tabular} & \\
\hline MN & $\mathrm{HA}$ & $\begin{array}{l}5.09082 \\
92.9683 \\
104.376 \\
7.03626 \\
\end{array}$ & $\begin{array}{l}5.2 \mathrm{E}-07 \\
5.69054 \\
11.7539 \\
0.82855 \\
\end{array}$ & $\begin{array}{l}6.5 \mathrm{E}-05 \\
26.0594 \\
8.90795 \\
0.00331\end{array}$ & $\begin{array}{r}2 \mathrm{E}-05 \\
3.3 \mathrm{E}-05 \\
5.8 \mathrm{E}-06 \\
8.6 \mathrm{E}-05 \\
\end{array}$ & \begin{tabular}{r|}
.59412 \\
4.3798 \\
0.09757 \\
3.16485 \\
\end{tabular} & & $\begin{array}{r}9.02 \mathrm{E}-14 \\
2.12 \mathrm{E}+010 \\
7.08 \mathrm{E}-02 \\
6.75 \mathrm{E}-05 \\
\end{array}$ \\
\hline TN & $\mathrm{HA}$ & & $\begin{array}{l}1.1 \mathrm{E}-08 \\
7.77721 \\
3.32483 \\
0.06367\end{array}$ & $\begin{array}{l}.1 \mathrm{E}-17 \\
1.1 \mathrm{E}-29 \\
3.3 \mathrm{E}-32 \\
2.2 \mathrm{E}-15\end{array}$ & $\begin{array}{l}3.03062 \\
0.14108 \\
0.12823 \\
1.86693\end{array}$ & & & $\begin{array}{l}\mathrm{EE}-30 \\
9 \mathrm{E}-36 \\
0 \mathrm{E}-41 \\
1 \mathrm{E}-21\end{array}$ \\
\hline TS & $\mathrm{HA}$ & $\begin{array}{r}4.9 \mathrm{E}-05 \\
0.0049 \\
0.00153 \\
0.00021\end{array}$ & $\begin{array}{r}9.9 \mathrm{E}-20 \\
5.2 \mathrm{E}-11 \\
3.1 \mathrm{E}-08 \\
4 \mathrm{E}-16\end{array}$ & & & & \begin{tabular}{|l|}
9233 \\
1831 \\
4911 \\
0313
\end{tabular} & $\begin{array}{l}E-32 \\
E-19 \\
E-30\end{array}$ \\
\hline TY & $\mathrm{HA}$ & \begin{tabular}{l|}
$5.9 \mathrm{E}-13$ \\
$1.6 \mathrm{E}-08$ \\
$1.5 \mathrm{E}-09$ \\
$8.3 \mathrm{E}-12$
\end{tabular} & $\begin{array}{l}1.3 \mathrm{E}-19 \\
9.4 \mathrm{E}-20 \\
5.8 \mathrm{E}-13 \\
1.1 \mathrm{E}-15\end{array}$ & $\begin{array}{r}7.8 \mathrm{E}-46 \\
1.4 \mathrm{E}-43 \\
2.3 \mathrm{E}-44 \\
6 \mathrm{E}-45 \\
\end{array}$ & & & & $\begin{array}{l}3.06 \mathrm{E}-86 \\
7.29 \mathrm{E}-83 \\
2.61 \mathrm{E}-81 \\
1.08 \mathrm{E}-78 \\
\end{array}$ \\
\hline $\mathrm{KA}$ & $\mathrm{JN}$ & \begin{tabular}{r|}
0.17764 \\
0.01335 \\
1.8896 \\
$1.1 \mathrm{E}-09$
\end{tabular} & $\begin{array}{r}0.00106 \\
3 \mathrm{E}-05 \\
2.3 \mathrm{E}-06 \\
0.00312\end{array}$ & & & & \begin{tabular}{|r|}
0.0831795 \\
0.55201 \\
0.1774888 \\
0.2318701 \\
\end{tabular} & $\begin{array}{l}4.97 \mathrm{E}-16 \\
1.14 \mathrm{E}-33 \\
5.87 \mathrm{E}-15 \\
1.53 \mathrm{E}-51 \\
\end{array}$ \\
\hline $\mathrm{KF}$ & $\sqrt{\mathrm{N}}$ & $\begin{array}{r}0.00686 \\
1.5 \mathrm{E}-09 \\
0.07506 \\
2 \mathrm{E}-23 \\
\end{array}$ & $\begin{array}{r}6 \mathrm{E}-13 \\
8.6 \mathrm{E}-19 \\
6.9 \mathrm{E}-25 \\
5.5 \mathrm{E}-10 \\
\end{array}$ & $\begin{array}{l}.17347 \\
8.4 \mathrm{E}-14 \\
0.26227 \\
1.5 \mathrm{E}-13 \\
\end{array}$ & & $\begin{array}{l}0.03574 \\
7.4 \mathrm{E}-10 \\
0.00565 \\
6.9 \mathrm{E}-10 \\
\end{array}$ & \begin{tabular}{|r|}
0.011754 \\
0.0227593 \\
0.0089572 \\
0.0438758 \\
\end{tabular} & $\begin{array}{l}2.71 \mathrm{E}-19 \\
1.48 \mathrm{E}-44 \\
1.10 \mathrm{E}-33 \\
1.42 \mathrm{E}-44 \\
\end{array}$ \\
\hline KO & $\sqrt{N}$ & $\begin{array}{l}.81422 \\
2.77509 \\
3.75621 \\
0.00504\end{array}$ & $\begin{array}{r}6 \mathrm{E}-06 \\
0.01292 \\
0.00085 \\
0.01642\end{array}$ & $\begin{array}{l}0.81641 \\
8.6 \mathrm{E}-23 \\
1.01014 \\
1.3 \mathrm{E}-13\end{array}$ & \begin{tabular}{c|}
0.00392 \\
$5.1 \mathrm{E}-06$ \\
0.01064 \\
$5.1 \mathrm{E}-08$
\end{tabular} & $\begin{array}{l}0.00614 \\
4.5 \mathrm{E}-09 \\
0.01539 \\
1.9 \mathrm{E}-07\end{array}$ & \begin{tabular}{|l}
1.638423 \\
2.2643424 \\
0.9259245 \\
2.7092979
\end{tabular} & $\begin{array}{l}7.38 \mathrm{E}-10 \\
1.59 \mathrm{E}-37 \\
4.87 \mathrm{E}-07 \\
2.81 \mathrm{E}-31\end{array}$ \\
\hline MN & JN & $\begin{array}{l}0.00176 \\
8.7 \mathrm{E}-34 \\
3.3 \mathrm{E}-07 \\
9.3 \mathrm{E}-15\end{array}$ & $\begin{array}{l}0.91079 \\
3.4 \mathrm{E}-13 \\
2.4 \mathrm{E}-15 \\
2.5 \mathrm{E}-10 \\
\end{array}$ & $\begin{array}{l}0.07429 \\
5.4 \mathrm{E}-48 \\
0.00037 \\
1.2 \mathrm{E}-24\end{array}$ & $\begin{array}{l}10.3611 \\
1.33875 \\
2.57826 \\
11.6518 \\
\end{array}$ & $\begin{array}{l}0.02122 \\
6.7 \mathrm{E}-14 \\
0.00573 \\
8.5 \mathrm{E}-10\end{array}$ & $\begin{array}{l}7.259 \mathrm{E}-06 \\
5.216 \mathrm{E}-06 \\
8.098 \mathrm{E}-06 \\
4.504 \mathrm{E}-07\end{array}$ & $\begin{array}{r}1.90 \mathrm{E}-10 \\
7.69 \mathrm{E}-112 \\
3.57 \mathrm{E}-32 \\
1.28 \mathrm{E}-62 \\
\end{array}$ \\
\hline TN & IN & $\begin{array}{l}0.07256 \\
1.3 \mathrm{E}-11 \\
0.18279 \\
1.1 \mathrm{E}-19\end{array}$ & $\begin{array}{l}1.31041 \\
2.6 \mathrm{E}-12 \\
2.5 \mathrm{E}-15 \\
2.4 \mathrm{E}-10\end{array}$ & $\begin{array}{l}2.21429 \\
8.7 \mathrm{E}-16 \\
1.94288 \\
0.00026\end{array}$ & $\begin{array}{r}1.8 \mathrm{E}-05 \\
6 \mathrm{E}-10 \\
0.00025 \\
3.1 \mathrm{E}-10\end{array}$ & $\begin{array}{l}0.00093 \\
2.6 \mathrm{E}-07 \\
0.05921 \\
1.5 \mathrm{E}-11\end{array}$ & $\begin{array}{l}0.4224715 \\
3.2458289 \\
0.7866007 \\
1.7160177\end{array}$ & $\begin{array}{l}1.48 \mathrm{E}-09 \\
1.52 \mathrm{E}-53 \\
1.02 \mathrm{E}-20 \\
5.13 \mathrm{E}-53\end{array}$ \\
\hline
\end{tabular}




\begin{tabular}{|c|c|c|c|c|c|c|c|c|}
\hline Speaker 1 & Speaker 2 & $\mathrm{iF} 2$ & $\mathrm{eF} 2$ & eF3 & olmid & $\mathrm{m} 2 \mathrm{~F} 3$ & S2F3 & posterior \\
\hline \multirow[t]{4}{*}{ TS } & \multirow[t]{4}{*}{ JN } & 0.17019 & 0.45363 & 0.15191 & $2.3 \mathrm{E}-07$ & 0.4490 & 0.0185278 & $2.21 \mathrm{E}-11$ \\
\hline & & $3.1 \mathrm{E}-20$ & 0.0519 & 0.01534 & $1.3 \mathrm{E}-30$ & 0.2547 & 0.0654217 & $5.14 \mathrm{E}-55$ \\
\hline & & 0.04841 & 0.00843 & 1.0987 & $1.3 \mathrm{E}-14$ & 10.8073 & 0.0114615 & $7.41 \mathrm{E}-1$ \\
\hline & & $2.6 \mathrm{E}-20$ & 0.40085 & 8.1E-26 & $3.2 \mathrm{E}-17$ & 0.0002 & 0.0396889 & $1.96 \mathrm{E}-67$ \\
\hline \multirow[t]{4}{*}{ TY } & \multirow[t]{4}{*}{ JN } & 5.2383 & 0.44181 & 1.20135 & 0.62052 & 0.00126 & 2.5186146 & $5.47 \mathrm{E}-03$ \\
\hline & & 0.39354 & 4.65597 & 34.9409 & 0.00591 & $6.3 \mathrm{E}-11$ & 0.3441475 & \\
\hline & & 4.46476 & 2.82664 & 0.75431 & 0.1029 & 0.02168 & 4.3263722 & \\
\hline & & 0.00034 & 0.5554 & 11.015 & 0.05167 & $9.5 \mathrm{E}-13$ & 2.3535267 & $2.38 \mathrm{E}-16$ \\
\hline \multirow[t]{4}{*}{$\mathrm{KF}$} & \multirow[t]{4}{*}{$\mathrm{KA}$} & 0.12557 & 2.07483 & 1.7007 & 0.13755 & $8.1 \mathrm{E}-10$ & 1.5355461 & \\
\hline & & 2.39318 & 0.07792 & 0.22394 & 1.18344 & 1.65558 & 34471 & \\
\hline & & 0.42686 & 0.05049 & 0.42642 & 1.79872 & 1.30618 & 1.1184144 & \\
\hline & & 2.1375 & 1.41905 & 2.62349 & 0.03195 & 0.05052 & & \\
\hline \multirow[t]{4}{*}{ KO } & \multirow[t]{4}{*}{$\mathrm{KA}$} & 2.38322 & 0.37057 & 1.61895 & 0.68149 & 39504 & 2764 & $236 \mathrm{E}+0$ \\
\hline & & 0.07213 & 1.50153 & 2.14658 & 0.01025 & 2.09559 & 2422 & \\
\hline & & 1.35459 & 1.21173 & 1.35947 & 0.55481 & 0.2533 & & \\
\hline & & 0.10043 & 1.00659 & 1.4969 & 0.00117 & 4.98763 & 0422 & \\
\hline \multirow[t]{4}{*}{ MN } & \multirow[t]{4}{*}{$\mathrm{KA}$} & 0.02516 & 1.70661 & 0.13228 & 0.00025 & 0.00026 & 0.00 & \\
\hline & & 0.00208 & 0.04284 & $1.5 \mathrm{E}-08$ & 1.2E-08 & & & \\
\hline & & $2.8 \mathrm{E}-05$ & 0.03618 & 0.00052 & $6.5 \mathrm{E}-05$ & $3.4 \mathrm{E}-11$ & 0.04 & \\
\hline & & 0.61211 & 1.18166 & 0.01268 & 7.2E-07 & 0.34314 & & \\
\hline \multirow[t]{4}{*}{ TN } & \multirow[t]{4}{*}{$\mathrm{KA}$} & 0.65419 & 2.45618 & 2.29395 & 1.71106 & 6077 & 8479 & \\
\hline & & 3.04658 & 0.3917 & 4.31372 & & & 2186 & \\
\hline & & 0.97707 & 0.46236 & 2.4112 & 0.09182 & 0.00241 & 0.40 & \\
\hline & & 3.54925 & 1.82298 & 2.6692 & 0.62201 & 2.93463 & 1.11 & $3.53 E+01$ \\
\hline \multirow[t]{4}{*}{ TS } & \multirow[t]{4}{*}{$\mathrm{KA}$} & 1.15122 & 0.03252 & 0.26478 & 6.00179 & 0.0000 & 5995 & \\
\hline & & 3.46573 & 0.69993 & 1.79091 & 13.8262 & 0.0000 & 2.2959 & \\
\hline & & 0.4987 & 0.18386 & 1.41021 & 7.21985 & 0.0000 & 6941 & \\
\hline & & 3.22998 & 0.24292 & 0.04439 & 11.1694 & 0.0435 & 8219 & \\
\hline \multirow[t]{4}{*}{$T Y$} & \multirow[t]{4}{*}{$\mathrm{KA}$} & 1.61408 & 0.03085 & 0.40595 & $1.8 \mathrm{E}-05$ & 1.066 & 7762 & \\
\hline & & 0.02483 & 0.05172 & 0.00055 & $1.6 \mathrm{E}-07$ & 0.06576 & & \\
\hline & & 1.39657 & 0.001 & & 0.002 & & 0.00 & \\
\hline & & 0.00271 & 0.23256 & 0.00361 & $1.2 \mathrm{E}-15$ & & & \\
\hline \multirow[t]{4}{*}{$\mathrm{KO}$} & \multirow[t]{4}{*}{$\mathrm{KF}$} & 0.03297 & $7.9 \mathrm{E}-09$ & 0.54589 & 1.91287 & 03368 & 6841 & \\
\hline & & 0.00211 & & & & & & \\
\hline & & 0.02727 & $2.5 \mathrm{E}$ & 1.30937 & 1.62 & 0.13641 & 408 & \\
\hline & & 0.00039 & & 3.35222 & 1.15691 & 0.06976 & 0.06 & \\
\hline$\overline{\mathrm{MN}}$ & $\mathrm{KF}$ & 1.65554 & 0.82393 & 3.44867 & 0.00556 & & & \\
\hline & & 0.00892 & 0.26002 & & & 2191 & & \\
\hline & & 0.04644 & & 0.03224 & & 787. & 5413 & \\
\hline & & & & 0.08275 & & & & \\
\hline TN & $\mathrm{KF}$ & 2.81591 & 0.05645 & 0.04263 & 2.86894 & 88755 & 0.165716 & $2.33 \mathrm{E}-\mathrm{C}$ \\
\hline & & & & & & 3.95372 & & \\
\hline & & 2.25747 & 5.71378 & 0.08281 & & & & $7.27 \mathrm{E}-\mathrm{C}$ \\
\hline & & 1.98246 & 4.29941 & 0.99485 & 0.49669 & 0.14357 & 0.3237134 & $1.97 \mathrm{E}-\mathrm{C}$ \\
\hline TS & $\mathrm{KF}$ & 1.90203 & $1.5 \mathrm{E}-05$ & 4.04409 & 1.47881 & 0.1612 & 2.4584563 & $8.71 \mathrm{E}-\mathrm{C}$ \\
\hline & & 2.7677 & 0.0009 & 2.00323 & & 0.0000 & & \\
\hline & & 3.10656 & $3.1 \mathrm{E}-10$ & 1.24117 & 0.40533 & 0.0000 & 3.0695541 & $2.31 \mathrm{E}-1$ \\
\hline & & 0.54602 & $6.8 \mathrm{E}-06$ & 0.26212 & 0.05757 & 1.8312 & 1.9082252 & $4.54 \mathrm{E}-\mathrm{C}$ \\
\hline TY & $\overline{\mathrm{KF}}$ & 0.00074 & $1.5 \mathrm{E}-05$ & $7.1 \mathrm{E}-08$ & 0.0059 & 3.77671 & 0.0225059 & $5.42 \mathrm{E}-1$ \\
\hline & & $5.1 \mathrm{E}-05$ & $4.6 \mathrm{E}-08$ & 7.7E-07 & 2.19463 & 5.9939 & $\mid 1.626 \mathrm{E}-06$ & $5.04 \mathrm{E}-1$ \\
\hline & & 0.00721 & $7 \mathrm{E}-18$ & $7.2 \mathrm{E}-09$ & 0.09328 & 0.73924 & $3.731 \mathrm{E}-07$ & $1.30 \mathrm{E}-2$ \\
\hline & & $3.5 \mathrm{E}-09$ & $7.8 \mathrm{E}-06$ & $7.9 \mathrm{E}-06$ & 1.44719 & 0.16208 & 0.0322282 & $2.09 \mathrm{E}-1$ \\
\hline
\end{tabular}




\begin{tabular}{|c|c|c|c|c|c|c|c|c|}
\hline peaker & Speaker 2 & $\mathrm{iF} 2$ & $\mathrm{eF} 2$ & $\mathrm{eF3}$ & olmid & $\mathrm{m}^{2} \mathrm{~F} 3$ & $\mathrm{~s} 2 \mathrm{~F} 3$ & posterior \\
\hline \multirow[t]{4}{*}{$\mathrm{MN}$} & $\mathrm{KO}$ & 0.00039 & 0.08879 & 0.24417 & 0.06748 & 1.04008 & $3.396 \mathrm{E}-09$ & $1.99 \mathrm{E}-15$ \\
\hline & & $4.6 \mathrm{E}-11$ & $2.3 \mathrm{E}-06$ & $1.3 \mathrm{E}-05$ & 0.03236 & 0.17162 & $1.352 \mathrm{E}-06$ & $1.02 \mathrm{E}-29$ \\
\hline & & $6.1 \mathrm{E}-08$ & 0.00224 & $4.2 \mathrm{E}-06$ & 0.08527 & 0.03957 & $3.707 \mathrm{E}-05$ & $7.20 \mathrm{E}-23$ \\
\hline & & 0.00014 & 0.00235 & 0.44597 & 0.03166 & 0.57531 & $3.736 \mathrm{E}-10$ & $1.02 \mathrm{E}-18$ \\
\hline \multirow[t]{4}{*}{ TN } & $\mathrm{KO}$ & 0.02602 & 0.15 & 0.93162 & & 3.34148 & 2.736 & \\
\hline & & 0.10031 & $6.6 \mathrm{E}$ & 1.05824 & 3.18 & & 2.880 & \\
\hline & & 0.06977 & 0.012 & 1.61106 & 3.35324 & 0.54577 & 5.747 & $1.47 \mathrm{E}-02$ \\
\hline & & 0.01768 & 0.006 & 0.43521 & 2.29328 & & 1.438 & $E-04$ \\
\hline \multirow[t]{4}{*}{ TS } & KO & 0.0663 & 0.7906 & 0.539 & 0.12846 & $\overline{0161}$ & & $E-05$ \\
\hline & & 0.00923 & 4.1819 & 1.91296 & 0.00 & & 0.12 & \\
\hline & & 0.0168 & 2.29243 & 1.4802 & 0.00 & 0.0000 & 0.3877409 & $9.28 \mathrm{E}-15$ \\
\hline & & 0.06515 & 2.30203 & 1.0701 & 0.11 & 0.0978 & 0.1146566 & $2.08 \mathrm{E}-04$ \\
\hline \multirow[t]{4}{*}{ TY } & KO & 4.69723 & 1.16046 & 1.1E-05 & 0.03622 & 4.28249 & 1.39 & $1.33 \mathrm{E}-05$ \\
\hline & & 3.67693 & 0.43944 & $1.7 \mathrm{E}-07$ & 0.26 & & 0.46 & \\
\hline & & 4.4641 & 0.123 & $4 \mathrm{E}-06$ & 0.55357 & 0.02348 & 0.0266304 & $7.63 \mathrm{E}-10$ \\
\hline & & 4.62483 & 2.22888 & $1.7 \mathrm{E}-06$ & 0.00343 & 2.18772 & 2.0577187 & 2.72E-07 \\
\hline \multirow[t]{4}{*}{$\mathrm{TN}$} & MN & $2.5 \mathrm{E}-05$ & 0.05 & $4 \mathrm{E}-10$ & 0.00052 & & 0.0 & $2-23$ \\
\hline & & 0.31687 & 3.0 & 0.00056 & 0.008 & & & $8-24$ \\
\hline & & 0.000 & 3.16 & $1.1 \mathrm{~F}$ & 0.01 & & & $E-28$ \\
\hline & & 0.37396 & 3.81 & 0.00112 & 0.00063 & 514 & 3.0 & $3.10 \mathrm{E}-12$ \\
\hline \multirow[t]{4}{*}{ TS } & $\mathrm{MN}$ & $3 \mathrm{E}-07$ & $3.5 \mathrm{E}-09$ & 0.00951 & $5.4 \mathrm{E}-07$ & 0.3 & 7.8 & $\mathrm{E}-23$ \\
\hline & & 0.57509 & 0.00045 & 0.75567 & $1.6 \mathrm{E}-11$ & & & \\
\hline & & $9.5 \mathrm{E}-05$ & $3.9 \mathrm{E}$ & 0.03286 & $1 \mathrm{E}-13$ & 0.0 & & $8-37$ \\
\hline & & 0.09801 & $3.2 \mathrm{E}$ & 4.65043 & $8.5 \mathrm{E}-06$ & 0.4 & 10.7 & E-11 \\
\hline \multirow[t]{4}{*}{ TY } & $\mathrm{MN}$ & $9.7 \mathrm{E}-20$ & $3.8 \mathrm{E}-09$ & $1.9 \mathrm{E}-26$ & 4.70167 & 0.4894 & .0000 & $7.08 \mathrm{E}-58$ \\
\hline & & $2 \mathrm{E}-05$ & $2.2 \mathrm{E}$ & & & & 0.0000 & \\
\hline & & $7.9 \mathrm{E}-12$ & $3.9 \mathrm{E}$ & $3.5 \mathrm{E}$ & 1.91 & 6.7524 & 0.0000 & $3.53 \mathrm{E}-65$ \\
\hline & & $1.7 \mathrm{E}-07$ & $3.7 \mathrm{E}$ & $5.4 \mathrm{E}-13$ & 2.23819 & 1.5169 & 0.0000 & $3.08 \mathrm{E}-29$ \\
\hline \multirow[t]{4}{*}{ TS } & TN & 3.99245 & $4 \mathrm{E}$ & 0.00253 & 0.00221 & 2.0230 & 0.0454127 & $8.22 \mathrm{E}-14$ \\
\hline & & 4.68352 & 0.001 & 1.35966 & 0.13124 & 0.0000 & & \\
\hline & & 3.50659 & $9.9 \mathrm{E}-06$ & 1.85356 & $1.8 \mathrm{E}-06$ & 0.0001 & 0.0774022 & $1.30 \mathrm{E}-15$ \\
\hline & & 3.99959 & & 0.00439 & 1.06009 & 0.0595 & 294 & $6.43 \mathrm{E}-10$ \\
\hline \multirow[t]{4}{*}{ TY } & TN & 0.00125 & $4.8 \mathrm{E}-08$ & $5.4 \mathrm{E}-05$ & 0.00495 & 0.44363 & 1.7203849 & $1.22 \mathrm{E}-17$ \\
\hline & & 0.00258 & $1.5 \mathrm{E}-08$ & 0.02266 & 0.02396 & 0.24554 & 0.0023017 & E-17 \\
\hline & & & & & & & & \\
\hline & & & & & & & & \\
\hline \multirow[t]{4}{*}{ TS } & $\mathrm{TY}$ & $1.2 \mathrm{E}-06$ & 3.6333 & 0.1004 & $1.2 \mathrm{E}-17$ & 0.0000 & 0.0337595 & $3.81 \mathrm{E}-36$ \\
\hline & & & & $1.4 \mathrm{E}-13$ & $1.8 \mathrm{E}-05$ & 1.0040 & 0.0005845 & $6.08 \mathrm{E}-23$ \\
\hline & & 0.00131 & 1.0061 & 0.04478 & $1.6 \mathrm{E}-10$ & 0.0000 & $9.767 \mathrm{E}-06$ & $2.34 \mathrm{E}-28$ \\
\hline & & $2.3 \mathrm{E}-05$ & 6.59634 & $1.6 \mathrm{E}-12$ & $5.9 \mathrm{E}-10$ & 0.0473 & 0.0263733 & $1.83 \mathrm{E}-28$ \\
\hline
\end{tabular}


[Calculation with effective F2]

\begin{tabular}{|c|c|c|c|c|c|c|c|}
\hline Speaker 1 & Speaker 2 & $\mathrm{iF} 2$ & eF2 & olmid & $\mathrm{m} 2 \mathrm{~F} 3$ & $\mathrm{~s} 2 \mathrm{F3}$ & posterior \\
\hline \multirow[t]{4}{*}{$\mathrm{HA}$} & \multirow[t]{4}{*}{$\mathrm{AA}$} & $7.6 \mathrm{E}-10$ & $2.1 E-21$ & 1.1E-06 & $7.8 \mathrm{E}-05$ & $1.163 \mathrm{E}-06$ & $1.57 \mathrm{E}-46$ \\
\hline & & $3.7 \mathrm{E}-06$ & $3 E-08$ & $1.4 \mathrm{E}-14$ & 2.3E-07 & $2.669 \mathrm{E}-12$ & $9.36 \mathrm{E}-46$ \\
\hline & & $7.1 \mathrm{E}-09$ & $4.9 \mathrm{E}-12$ & $2.4 \mathrm{E}-09$ & $2 \mathrm{E}-19$ & $4.605 \mathrm{E}-08$ & $7.48 \mathrm{E}-55$ \\
\hline & & $6.5 \mathrm{E}-07$ & $1.7 \mathrm{E}-17$ & $1.3 \mathrm{E}-08$ & 0.04691 & $3.676 \mathrm{E}-10$ & $2.58 \mathrm{E}-42$ \\
\hline \multirow[t]{4}{*}{$\mathrm{JN}$} & \multirow[t]{4}{*}{$\mathrm{AA}$} & $6.6 \mathrm{E}-07$ & 0.28003 & 12.6415 & 2.61392 & 4.6597643 & $2.85 \mathrm{E}-05$ \\
\hline & & 1.78413 & $8.4 \mathrm{E}-05$ & 1.4887 & 3.04633 & 1.9774722 & $1.34 \mathrm{E}-03$ \\
\hline & & 3.33715 & 1.58265 & 11.3424 & 15.2756 & 3.5177509 & $322 \mathrm{E}+03$ \\
\hline & & $1.1 \mathrm{E}-07$ & $3.8 \mathrm{E}-07$ & 0.05902 & 0.00735 & 1.009889 & $1.91 \mathrm{E}-17$ \\
\hline \multirow[t]{4}{*}{$\mathrm{KA}$} & \multirow[t]{4}{*}{$\mathrm{AA}$} & 0.07473 & 0.20506 & $1.2 \mathrm{E}-16$ & $5.7 \mathrm{E}-06$ & 0.0006676 & $7.08 \mathrm{E}-27$ \\
\hline & & 2.48869 & 1.67647 & $4.2 \mathrm{E}-10$ & $4.6 \mathrm{E}-11$ & 0.0002765 & $2.24 \mathrm{E}-23$ \\
\hline & & 2.56067 & 0.06517 & $9.8 \mathrm{E}-07$ & $2.1 \mathrm{E}-25$ & 0.0117962 & $4.11 \mathrm{E}-34$ \\
\hline & & 0.56161 & 2.0025 & 7.3E-63 & 0.0001 & $2.887 \mathrm{E}-06$ & $2.47 \mathrm{E}-72$ \\
\hline \multirow[t]{4}{*}{ KF } & \multirow[t]{4}{*}{ AA } & $2.2 \mathrm{E}-05$ & 0.15137 & 0.34983 & $1.3 \mathrm{E}-08$ & 0.0005434 & $7.99 \mathrm{E}-18$ \\
\hline & & 0.24159 & 0.27979 & $2.4 \mathrm{E}-05$ & 0.00046 & $4.44 \mathrm{E}-07$ & $3.30 \mathrm{E}-16$ \\
\hline & & 0.02477 & $9.7 \mathrm{E}-12$ & 0.00027 & $3.6 \mathrm{E}-07$ & 0.0001501 & $3.51 \mathrm{E}-27$ \\
\hline & & 0.00723 & 0.03094 & 0.64229 & 0.01579 & $3.174 \mathrm{E}-06$ & $7.20 \mathrm{E}-12$ \\
\hline \multirow[t]{4}{*}{$\mathrm{KO}$} & \multirow[t]{4}{*}{ AA } & 4.00986 & 0.68069 & $8.5 \mathrm{E}-05$ & 4.2E-05 & 2.1146342 & $2.06 \mathrm{E}-08$ \\
\hline & & 2.41165 & 4.04318 & $1.1 \mathrm{E}-06$ & $9.4 \mathrm{E}-05$ & 0.0132819 & $1.29 \mathrm{E}-11$ \\
\hline & & 1.92218 & 0.29817 & 0.00151 & $2.1 \mathrm{E}-12$ & 0.4104618 & $7.50 \mathrm{E}-16$ \\
\hline & & 2.06192 & 3.83849 & $5.1 \mathrm{E}-14$ & 0.00076 & 0.4879397 & $1.50 \mathrm{E}-16$ \\
\hline \multirow[t]{4}{*}{$\mathrm{MN}$} & \multirow[t]{4}{*}{$\mathrm{AA}$} & $4 \mathrm{E}-05$ & $5 \mathrm{E}-07$ & 7.42316 & $4 \mathrm{E}-09$ & $7.783 \mathrm{E}-17$ & $4.61 \mathrm{E}-35$ \\
\hline & & $1.4 \mathrm{E}-09$ & $2.4 \mathrm{E}-08$ & 7.66785 & 0.00018 & $1.926 \mathrm{E}-19$ & $8.78 \mathrm{E}-39$ \\
\hline & & $2.2 \mathrm{E}-14$ & $1.8 \mathrm{E}-12$ & 4.17837 & 1.2E-14 & $1.067 \mathrm{E}-12$ & $2.12 \mathrm{E}-51$ \\
\hline & & 0.00288 & 0.00252 & 4.78935 & 0.0022 & $1.686 \mathrm{E}-25$ & $1.29 \mathrm{E}-32$ \\
\hline \multirow[t]{4}{*}{ TN } & \multirow[t]{4}{*}{$\mathrm{AA}$} & 0.00426 & $2.8 \mathrm{E}-05$ & $2.6 \mathrm{E}-06$ & $8 \mathrm{E}-08$ & 0.1375332 & $3.37 \mathrm{E}-21$ \\
\hline & & 0.73185 & 0.00016 & $3 \mathrm{E}-09$ & 0.11064 & 0.0044742 & $1.70 \mathrm{E}-16$ \\
\hline & & 0.07664 & $9.4 E-09$ & $1.3 \mathrm{E}-05$ & 0.00041 & 0.1297487 & $4.96 \mathrm{E}-19$ \\
\hline & & 0.22062 & 0.13258 & $1.2 \mathrm{E}-08$ & 4.7E-05 & 0.0104338 & $1.71 \mathrm{E}-16$ \\
\hline \multirow[t]{4}{*}{$\mathrm{TS}$} & \multirow[t]{4}{*}{$\mathrm{AA}$} & 0.02209 & 2.47767 & $1.9 \mathrm{E}-11$ & 0.0100 & 0.0093562 & $9.86 \mathrm{E}-17$ \\
\hline & & 0.12581 & 2.59397 & $1 E-35$ & 1.7601 & $2.69 \mathrm{E}-05$ & $1.58 \mathrm{E}-40$ \\
\hline & & 0.00176 & 1.96885 & $1.2 \mathrm{E}-20$ & 10.0717 & 0.0019258 & $7.96 \mathrm{E}-25$ \\
\hline & & 0.6746 & 1.55252 & $2.2 \mathrm{E}-24$ & 1.0334 & 0.0006114 & $1.43 \mathrm{E}-27$ \\
\hline \multirow[t]{4}{*}{ TY } & \multirow[t]{4}{*}{$\mathrm{AA}$} & 2.70257 & 6.40675 & 1.33323 & $6.8 \mathrm{E}-10$ & 3.5926484 & $5.65 \mathrm{E}-08$ \\
\hline & & 0.9177 & $2.3 \mathrm{E}-05$ & 0.69543 & 0.00351 & 7.3423298 & $3.76 \mathrm{E}-07$ \\
\hline & & 2.60113 & 3.02661 & 0.26622 & $1 \mathrm{E}-10$ & 4.154433 & $8.83 \mathrm{E}-10$ \\
\hline & & 0.49458 & 0.04866 & 5.57865 & $1.8 \mathrm{E}-05$ & 3.7601661 & $8.96 \mathrm{E}-06$ \\
\hline \multirow[t]{4}{*}{$\mathrm{JN}$} & $\mathrm{HA}$ & $1.4 \mathrm{E}-20$ & $3.4 \mathrm{E}-20$ & 0.52863 & $8.3 \mathrm{E}-16$ & $9.785 \mathrm{E}-05$ & $4.12 \mathrm{E}-73$ \\
\hline & & $1.5 \mathrm{E}-05$ & 2.3E-31 & 0.02064 & 0.03798 & 0.0002909 & $8.97 \mathrm{E}-47$ \\
\hline & & $2.9 \mathrm{E}-06$ & $2.5 \mathrm{E}-15$ & $2.5 \mathrm{E}-07$ & 0.00109 & 0.0002784 & $5.59 \mathrm{E}-34$ \\
\hline & & $3.8 \mathrm{E}-19$ & $8.2 \mathrm{E}-39$ & 1.43304 & $4.9 \mathrm{E}-08$ & 0.0005496 & $2.47 \mathrm{E}-77$ \\
\hline $\mathrm{KA}$ & $\mathrm{HA}$ & 0.00686 & 0.00456 & 2.7456 & 0.99574 & 0.2302715 & $1.97 \mathrm{E}-05$ \\
\hline & & 0.00034 & 0.00146 & 4.35122 & 0.00445 & 0.2376085 & $2.28 \mathrm{E}-09$ \\
\hline & & $8.3 \mathrm{E}-05$ & 0.00284 & 3.7933 & 0.00212 & 0.1096654 & $2.08 \mathrm{E}-10$ \\
\hline & & 0.01804 & 0.00392 & 5.34475 & 0.161 & 0.4581828 & $2.79 \mathrm{E}-05$ \\
\hline$\overline{\mathrm{KF}}$ & $\mathrm{HA}$ & 0.03975 & 0.0195 & 0.1449 & 0.01359 & 1.528762 & $2.33 \mathrm{E}-06$ \\
\hline & & 0.16804 & 14.4852 & 1.89596 & 1.0934 & 3.175260 & $16015+0$ \\
\hline & & 0.08158 & 6.33212 & 2.74328 & 4.5568 & 2.684136 & 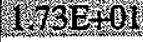 \\
\hline & & 0.09548 & 0.00372 & 0.09962 & 3.96612 & 2.055822 & $2.88 \mathrm{E}-04$ \\
\hline $\mathrm{KO}$ & $\mathrm{HA}$ & $1.3 \mathrm{E}-08$ & $4.3 \mathrm{E}-08$ & 1.05773 & 1.43572 & 0.0004448 & $3.66 \mathrm{E}-19$ \\
\hline & & $2.2 \mathrm{E}-06$ & $1.5 \mathrm{E}-12$ & 0.63351 & 0.78567 & 0.0142085 & $2.44 \mathrm{E}-20$ \\
\hline & & $3.2 \mathrm{E}-07$ & 0.00013 & 0.78728 & 6.15987 & 0.0034354 & $7.01 \mathrm{E}-13$ \\
\hline & & $1.1 \mathrm{E}-07$ & $3.9 \mathrm{E}-14$ & 0.81003 & 0.32757 & 0.0024348 & $2.71 \mathrm{E}-24$ \\
\hline
\end{tabular}




\begin{tabular}{|c|c|c|c|c|c|c|c|}
\hline Speaker 1 & Speaker 2 & iF2 & $\mathrm{eF} 2$ & olmid & $\mathrm{m}^{2} \mathrm{F3}$ & $52 F 3$ & posterior \\
\hline \multirow[t]{4}{*}{$\mathrm{MN}$} & \multirow[t]{4}{*}{$\mathrm{HA}$} & 5.09082 & 0.21545 & $2 \mathrm{E}-05$ & 1.59412 & 16622614 & $5.69 \mathrm{E}-04$ \\
\hline & & 92.9683 & 40.1475 & $3.3 \mathrm{E}-05$ & 4.3798 & 10.71976 & 6331400 \\
\hline & & 104.376 & 70.9204 & $5.8 \mathrm{E}-06$ & 0.09757 & 11.357831 & $4.79 \mathrm{E}-02$ \\
\hline & & 7.03626 & 0.14471 & $8.6 \mathrm{E}-05$ & 3.16485 & 12.845929 & $3.56 \mathrm{E}-03$ \\
\hline \multirow[t]{4}{*}{ TN } & \multirow[t]{4}{*}{$\mathrm{HA}$} & 0.00065 & 0.00016 & 3.03062 & 323143 & 0.0015659 & $1.60 \mathrm{E}-09$ \\
\hline & & 0.00341 & 0.03549 & 0.14108 & 3.10832 & $5.582 \mathrm{E}-05$ & $2.96 \mathrm{E}-09$ \\
\hline & & 0.00103 & 0.09487 & 0.12823 & 0.41342 & $2.352 \mathrm{E} 06$ & $121 E^{-11}$ \\
\hline & & 0.00224 & $5 \mathrm{E}-09$ & 1.86693 & 0.45874 & 0.0073661 & $7.04 \mathrm{E}-14$ \\
\hline \multirow[t]{4}{*}{ TS } & \multirow[t]{4}{*}{$\mathrm{HA}$} & $4.9 \mathrm{E} 05$ & $2.9 \mathrm{E}-11$ & 3.507 & 0.0025 & 9.7539233 & $1.23 \mathrm{E}-16$ \\
\hline & & 0.0049 & $2 E-16$ & 5.42382 & 0.0000 & 1.8101831 & $2.25 \mathrm{E}-22$ \\
\hline & & 0.00153 & $3.1 \mathrm{E} 09$ & 1.25654 & 0.0000 & 1.2994911 & $1.89 \mathrm{E}-28$ \\
\hline & & 0,00021 & $9.6 \mathrm{E}-26$ & 5.17518 & 1.6068 & 8.2000313 & $1.35 \mathrm{E}-27$ \\
\hline \multirow[t]{4}{*}{ TY } & \multirow[t]{4}{*}{$\mathrm{HA}$} & $5.9 \mathrm{E}-13$ & $1.7 \mathrm{E}-13$ & $4.6 \mathrm{E}-07$ & 5.02992 & 0.0002284 & $5.33 \mathrm{E}-35$ \\
\hline & & $1.6 \mathrm{E}-08$ & $8.4 \mathrm{E}-47$ & 0.00226 & 0.30616 & $5.25 \mathrm{E}-10$ & $4.80 \mathrm{E}-67$ \\
\hline & & $1.5 \mathrm{E}-09$ & $9.5 \mathrm{E}-19$ & 0.00061 & 0.03341 & $6.542 \mathrm{E}-12$ & $1.88 \mathrm{E}-43$ \\
\hline & & $8.3 \mathrm{E}-12$ & $3.1 \mathrm{E}-26$ & $3.7 \mathrm{E}-0 \mathrm{~S}$ & 0.70089 & 0.0007997 & $5.29 \varepsilon-45$ \\
\hline \multirow[t]{4}{*}{$\mathrm{KA}$} & \multirow[t]{4}{*}{$\mathbf{N}$} & 0.17764 & 0.01148 & 1.3E-08 & 0.00137 & 0.0831795 & $2.97 \mathrm{E}-15$ \\
\hline & & 0.01335 & $2.3 \mathrm{E}-05$ & $2.7 \mathrm{E}-10$ & $3.1 \mathrm{E}-16$ & 0.55201 & $1.42 \mathrm{E}-32$ \\
\hline & & 1.8896 & 0.00118 & $1.4 \mathrm{E}, 05$ & 0.00033 & 0.1774888 & $1.80 \mathrm{E}-12$ \\
\hline & & $1.1 \mathrm{E}-09$ & 0.0006 & $1.2 \mathrm{E}-24$ & $5.1 \mathrm{E}-09$ & 0.2318701 & $9.31 \mathrm{E}-46$ \\
\hline \multirow[t]{4}{*}{$\mathrm{KF}$} & \multirow[t]{4}{*}{$\mathrm{JN}$} & 0.00686 & $7.1 \mathrm{E}-10$ & 0.15768 & 0.03574 & 0.011754 & $3.24 \mathrm{E}-16$ \\
\hline & & $1.5 \mathrm{E}-09$ & $2.9 \mathrm{E}-22$ & $6.7 \mathrm{E}-07$ & $7,4 \mathrm{E}-10$ & 0.0227593 & $5.04 \mathrm{E}-48$ \\
\hline & & 0.07506 & $6.1 \mathrm{E}-17$ & 0.00042 & 0.00565 & 0.0089572 & $9.59 \mathrm{E}-26$ \\
\hline & & $25-23$ & $1.7 \mathrm{E}-13$ & 0.04225 & $6.9 \mathrm{E}-10$ & 0.0438758 & $4.42 \mathrm{E}-48$ \\
\hline \multirow[t]{4}{*}{$\mathrm{KO}$} & \multirow[t]{4}{*}{$\mathrm{JN}$} & 3.81422 & $8.1 \mathrm{E}-10$ & 0.00392 & 0.00614 & 1.638423 & $1.21 \mathrm{E}-13$ \\
\hline & & 2.77509 & $7.1 \mathrm{E}-05$ & $5.1 F_{m} 06$ & $4.5 \mathrm{E}-09$ & 2.2643424 & $1.01 \mathrm{E}-17$ \\
\hline & & 3.75621 & 0.00193 & 0.01064 & 0.01539 & 0.9259245 & $1.10 \mathrm{E}-06$ \\
\hline & & 0.00504 & $8.1 \mathrm{E} .05$ & $5.1 \mathrm{E}-08$ & $1.9 \mathrm{E}_{0} 07$ & 2.7092979 & $1.11 \mathrm{E}-20$ \\
\hline \multirow[t]{4}{*}{$\mathrm{MN}$} & \multirow[t]{4}{*}{$\mathrm{JN}$} & 0.00176 & $1.7 \mathrm{E}-11$ & 10.3611 & 0.02122 & $7.259 \mathrm{E}-06$ & $492 \mathrm{E}-20$ \\
\hline & & $8.7 \mathrm{E}-34$ & $2.3 E-21$ & 1.33875 & $6.7 \mathrm{E}-14$ & $5.216 \mathrm{E}-06$ & $9.28 \mathrm{E}-73$ \\
\hline & & $3.3 \mathrm{E}-07$ & $3.6 E-16$ & 2.57826 & 0.00573 & $8.098 \mathrm{E}-06$ & $1.43 \mathrm{E}-29$ \\
\hline & & $9.3 \mathrm{E}-15$ & $1.7 \mathrm{E}-15$ & 11.6518 & $8.5 \mathrm{E}-10$ & $4.504 \mathrm{E}-07$ & $6.98 \mathrm{E}-44$ \\
\hline \multirow[t]{4}{*}{$\mathrm{TN}$} & \multirow[t]{4}{*}{$\mathrm{JN}$} & 0.07256 & $7.3 \mathrm{E}-11$ & $1.8 \mathrm{E}-05$ & 0.00093 & 0.4224715 & $3.71 \mathrm{E}-20$ \\
\hline & & $1.3 \mathrm{E}-11$ & $3.1 \div-19$ & $6 \mathrm{E}-10$ & $2.6 \mathrm{E}-07$ & 32458289 & $2.11 \mathrm{E}-45$ \\
\hline & & 0.18279 & $1.5 \mathrm{E}-14$ & 0.00025 & 0.05921 & 0.7866007 & $315 \mathrm{E}-20$ \\
\hline & & $1.1 \mathrm{E}-19$ & $1.2 \mathrm{E}-14$ & $3.1 \mathrm{E}-10$ & $1.5 \mathrm{E}-11$ & 1.7160177 & $9.65 \mathrm{E}-54$ \\
\hline \multirow[t]{4}{*}{ TS } & $\mathrm{NN}$ & 0.17019 & 0.01755 & $2.3 \mathrm{E}-07$ & 0.4490 & 0.0185278 & $5.62 \mathrm{E}-12$ \\
\hline & & $3.1 \mathrm{E}-20$ & 0.00121 & $1.3 \mathrm{E}-30$ & 0.2547 & 0.0654217 & $7.83 \mathrm{E}-55$ \\
\hline & & 0.04841 & 0.03435 & $1.3 \mathrm{E}-14$ & 10.8073 & 0.0114615 & $2.75 \mathrm{E}-18$ \\
\hline & & $2.6 \mathrm{E}-20$ & 0.00039 & $3.2 \mathrm{E}-17$ & 0.0002 & 0,0396889 & $2.35 \mathrm{E}-45$ \\
\hline TY & $\mathrm{JN}$ & 5.2383 & 2.98498 & 0.62052 & 0.00126 & 2.5186146 & $3.08 \mathrm{E}-02$ \\
\hline & & 0.39354 & 6.4408 & 0.00591 & $6.3 \mathrm{E}-11$ & 0.3441475 & $3.25 \mathrm{E}-13$ \\
\hline & & 4.46476 & 15.3036 & 0.1029 & 0.02168 & 4.3263722 & $6.59 \mathrm{E}-01$ \\
\hline & & 0.00034 & 0.58404 & 0.05167 & $9.5 \mathrm{E}-13$ & 2.3535267 & $2.27 \mathrm{E}-17$ \\
\hline $\mathrm{KF}$ & $\mathrm{KA}$ & 0.12557 & 1.70034 & 0.13755 & $8.1 \mathrm{E}-10$ & 1.5355461 & $3.64 \mathrm{E}-11$ \\
\hline & & 2.39318 & 0.05555 & 1.18344 & 1.65558 & 1.7284471 & $4.50 \mathrm{E}-01$ \\
\hline & & 0.42686 & 0.04542 & 1.79872 & 1.30618 & 1.1184144 & $509 \mathrm{E}-02$ \\
\hline & & 2.1375 & 1.35788 & 0.03195 & 0.05052 & 2.2111963 & $1.04 \mathrm{E}-02$ \\
\hline $\mathrm{KO}$ & $\mathrm{KA}$ & 2.38322 & 1.2703 & 0.68149 & 6.39504 & 0.3792764 & $800 \mathrm{~T}+00$ \\
\hline & & 0.07213 & 1.90112 & 0.01025 & 2.09559 & 0.4142422 & $1.22 \mathrm{E}-0 \mathrm{O}$ \\
\hline & & 1.35459 & 1.91594 & 0.55481 & 0.2533 & 1.1354307 & $4.14 \mathrm{E}-01$ \\
\hline & & 0.10043 & 1.70378 & 0.00117 & 4.98763 & 0.0870422 & $8.65 E-05$ \\
\hline
\end{tabular}




\begin{tabular}{|c|c|c|c|c|c|c|c|}
\hline Speaker 1 & Speaker 2 & $\mathrm{iF2}$ & $\mathrm{eF} 2$ & olmid & $\mathrm{m} 2 \mathrm{~F} 3$ & $\mathrm{~s} 2 \mathrm{~F} 3$ & posterior \\
\hline \multirow[t]{4}{*}{$\mathrm{MN}$} & \multirow[t]{4}{*}{$\mathrm{KA}$} & 0.02516 & 0.85768 & 0.00025 & 0.00026 & 0.0026552 & $3.72 \mathrm{E}-12$ \\
\hline & & 0.00208 & 0.005 & $1.2 \mathrm{E}-08$ & 0.07416 & 0.1299974 & $1.24 \mathrm{E}-15$ \\
\hline & & $2.8 \mathrm{E}-05$ & 0.00326 & $6.5 \mathrm{E}-05$ & 3.4E-11 & 0.0485852 & $9.73 \mathrm{E}-24$ \\
\hline & & 0.61211 & 0.71688 & 7.2E-07 & 0.34314 & 0.0045229 & $4.88 \mathrm{E}-10$ \\
\hline \multirow[t]{4}{*}{ TN } & \multirow[t]{4}{*}{$\mathrm{KA}$} & 0.65419 & 2.97001 & 1.71106 & 3.6077 & 1.8948479 & $227 E+01$ \\
\hline & & 3.04658 & 0.59006 & $1.1 \mathrm{E}-05$ & 0.11276 & 0.0312186 & $7.13 \mathrm{E}-08$ \\
\hline & & 0.97707 & 0.67511 & 0.09182 & 0.00241 & 0.4017376 & $5.87 \mathrm{E}-05$ \\
\hline & & 3.54925 & 2.34901 & 0.62201 & 2.93463 & 1.1190417 & $170 \mathrm{E}+01$ \\
\hline \multirow[t]{4}{*}{ TS } & \multirow[t]{4}{*}{$\mathrm{KA}$} & 1.15122 & 0.41175 & 6.00179 & 0.0000 & $\begin{array}{l}0.5995 \\
\end{array}$ & $4.45 \mathrm{E}-07$ \\
\hline & & 3.46573 & 1.11599 & 13.8262 & 0.0000 & 2.2959 & $6.24 \mathrm{E}-06$ \\
\hline & & 0.4987 & 0.67354 & 7.21985 & 0.0000 & 1.6941 & $4.35 \mathrm{E}-22$ \\
\hline & & 3.22998 & 0.84933 & 11.1694 & 0.0435 & 0.8219 & $109 \mathrm{E}+00$ \\
\hline \multirow[t]{4}{*}{$\mathrm{TY}$} & \multirow[t]{4}{*}{$\mathrm{KA}$} & 1.61408 & 0.03344 & $1.8 \mathrm{E}-05$ & 1.066 & 0.371762 & $3.78 \mathrm{E}-07$ \\
\hline & & 0.02483 & 0.01214 & $1.6 \mathrm{E}-07$ & 0.06576 & $2.779 \mathrm{E}-06$ & $8.67 \mathrm{E}-18$ \\
\hline & & 1.39657 & 0.00092 & 0.002 & $1.4 \mathrm{E}-10$ & 0.0001478 & $5.46 \mathrm{E}-20$ \\
\hline & & 0.00271 & 0.13632 & $1.2 \mathrm{E}-15$ & 1.53867 & 0.1937565 & $1.36 \mathrm{E}-19$ \\
\hline \multirow[t]{4}{*}{ KO } & \multirow[t]{4}{*}{$\mathrm{KF}$} & 0.03297 & $5 \mathrm{E}-07$ & 1.91287 & 0.03368 & 0.0336841 & $3.55 \mathrm{E}-11$ \\
\hline & & 0.00211 & 0.24109 & 1.29107 & 0.25878 & 0.2587815 & $4.40 \mathrm{E}-05$ \\
\hline & & 0.02727 & 0.00025 & 1.62811 & 0.13641 & 0.136408 & $2.08 \mathrm{E}-07$ \\
\hline & & 0.00039 & 0.01995 & 1.15691 & 0.06976 & 0.0697568 & $4.40 \mathrm{E}-08$ \\
\hline \multirow[t]{4}{*}{ MN } & \multirow[t]{4}{*}{$\mathrm{KF}$} & 1.65554 & 1.70245 & 0.00556 & 1.99416 & 1.81 & $5.69 \mathrm{E}-02$ \\
\hline & & 0.00892 & 0.02323 & 0.99725 & 3.92191 & 2753 & $1.71 \mathrm{E}-03$ \\
\hline & & 0.04644 & 5.80341 & 0.00524 & 1.07787 & 3.7795413 & $5.75 \mathrm{E}-03$ \\
\hline & & 2.3645 & 4.06636 & 0.53598 & 2.57101 & 0.8219476 & $109 E+01$ \\
\hline \multirow[t]{4}{*}{ TN } & \multirow[t]{4}{*}{$\mathrm{KF}$} & 2.81591 & 0.00432 & 2.86894 & 3.08755 & 0.165716 & $1.79 \mathrm{E}-02$ \\
\hline & & 1.49355 & 3.74576 & 1.72503 & 3.95372 & 3054 & $9.27 \mathrm{E}-01$ \\
\hline & & 2.25747 & 1.5101 & 1.33676 & 0.7778 & 0.0054212 & $1.92 \mathrm{E}-02$ \\
\hline & & 1.98246 & 3.10779 & 0.49669 & 0.14357 & 0.32 & $1.42 \mathrm{E}-01$ \\
\hline \multirow[t]{4}{*}{ TS } & \multirow[t]{4}{*}{$\mathrm{KF}$} & 1.90203 & $3 \mathrm{E}-11$ & 1.47881 & 0.1612 & 34563 & $1.67 \mathrm{E}-11$ \\
\hline & & 2.7677 & 0.00296 & 0.00058 & 0.0000 & 3.2698882 & $1.54 \mathrm{E}-12$ \\
\hline & & 3.10656 & $2.3 \mathrm{E}-08$ & 0.40533 & 0.0000 & 3.0695541 & $1.69 \mathrm{E}-15$ \\
\hline & & 0.54602 & 0.00015 & 0.05757 & 1.8312 & 1.9082252 & $9.97 \mathrm{E}-06$ \\
\hline \multirow[t]{4}{*}{ TY } & \multirow[t]{4}{*}{$\mathrm{KF}$} & 0.00074 & $9.7 \mathrm{E}-14$ & 0.0059 & 3.77671 & 0.0225059 & $3.60 \mathrm{E}-20$ \\
\hline & & 5.1E-05 & $6.2 \mathrm{E}-12$ & 2.19463 & 5.9939 & $1.626 \mathrm{E}-06$ & $6.81 \mathrm{E}-21$ \\
\hline & & 0.00721 & $1.2 \mathrm{E}-21$ & 0.09328 & & 3.73 & $2.30 \mathrm{E}-31$ \\
\hline & & $3.5 \mathrm{E}-09$ & $8.5 \mathrm{E}-07$ & 1.44719 & 0.16208 & 0.0322282 & $2.29 \mathrm{E}-17$ \\
\hline \multirow[t]{4}{*}{ MN } & KO & 0.00039 & 0.05621 & 0.06748 & 1.04008 & $3.396 \mathrm{E}-09$ & $5.16 \mathrm{E}-15$ \\
\hline & & $4.6 \mathrm{E}-11$ & $3.3 \mathrm{E}-08$ & 0.03236 & 0.17162 & $1.352 \mathrm{E}-06$ & $1.15 \mathrm{E}-26$ \\
\hline & & $6.1 \mathrm{E}-08$ & 0.00012 & 0.08527 & 0.03957 & $3.707 \mathrm{E}-05$ & $8.76 \mathrm{E}-19$ \\
\hline & & & & 0.03166 & & $3.736 \mathrm{E}-10$ & $1.91 \mathrm{E}-18$ \\
\hline TN & KO & 0.02602 & 0.52035 & 1.83017 & 3.34148 & 2.7360015 & $2.27 \mathrm{E}-01$ \\
\hline & & 0.10031 & 0.00022 & 3.18646 & 0.24576 & 2.8803704 & $5.06 \mathrm{E}-05$ \\
\hline & & 0.06977 & 0.00863 & 3.35324 & 0.54577 & 5.7473069 & $6.33 \mathrm{E}-03$ \\
\hline & & 0.01768 & 0.08571 & 2.29328 & 2.94964 & 1.4385403 & $1.47 \mathrm{E}-02$ \\
\hline TS & KO & 0.0663 & 1.37341 & 0.12846 & 0.0161 & 0.2097909 & $3.95 \mathrm{E}-05$ \\
\hline & & 0.00923 & 3.84233 & 0.00513 & 0.0000 & 0.1240666 & $2.42 \mathrm{E}-11$ \\
\hline & & 0.0168 & 2.15308 & 0.00491 & 0.0000 & 0.3877409 & $5.89 \mathrm{E}-15$ \\
\hline & & 0.06515 & 3.48889 & 0.11579 & 0.0978 & 0.1146566 & $2.95 \mathrm{E}-04$ \\
\hline $\mathrm{TY}$ & $\mathrm{KO}$ & 4.69723 & 0.1302 & 0.03622 & 4.28249 & 1.3979545 & $1.33 \mathrm{E}-01$ \\
\hline & & 3.67693 & 0.00487 & 0.26071 & 0.16991 & 0.4686764 & $3.72 \mathrm{E}-04$ \\
\hline & & 4.4641 & 0.00028 & 0.55357 & 0.02348 & 0.0266304 & $4.25 \mathrm{E}-07$ \\
\hline & & 4.62483 & 0.38568 & 0.00343 & 2.18772 & 2.0577187 & $2.75 \mathrm{E}-02$ \\
\hline
\end{tabular}




\begin{tabular}{|c|c|r|r|r|r|r|r|}
\hline Speaker 1 & Speaker 2 & \multicolumn{1}{c|}{ iF2 } & \multicolumn{1}{c|}{ eF2 } & \multicolumn{1}{c|}{ olmid } & \multicolumn{1}{c|}{ 2 F3 } & \multicolumn{1}{c|}{ s2 F3 } & posterior \\
\hline TN & MN & $2.5 \mathrm{E}-05$ & 0.0002 & 0.00052 & 0.33401 & 0.0001555 & $1.38 \mathrm{E}-16$ \\
& & 0.31687 & 5.9745 & 0.008 & 2.52825 & $1.504 \mathrm{E}-19$ & $5.76 \mathrm{E}-21$ \\
& & 0.00013 & 0.12886 & 0.01564 & 3.46905 & $9.409 \mathrm{E}-11$ & $8.80 \mathrm{E}-17$ \\
& & 0.37396 & 1.09045 & 0.00063 & 0.99514 & $3.085 \mathrm{E}-06$ & $7.93 \mathrm{E}-10$ \\
\hline TS & MN & $3 \mathrm{E}-07$ & $1.4 \mathrm{E}-11$ & $5.4 \mathrm{E}-07$ & 0.3254 & 7.8351542 & $5.66 \mathrm{E}-24$ \\
& & 0.57509 & 0.00021 & $1.6 \mathrm{E}-11$ & 0.0000 & 0.7973612 & $1.66 \mathrm{E}-23$ \\
& & $9.5 \mathrm{E}-05$ & $2 \mathrm{E}-09$ & $1 \mathrm{E}-13$ & 0.0000 & 1.7405538 & $4.72 \mathrm{E}-38$ \\
& & 0.09801 & $5 \mathrm{E}-06$ & $8.5 \mathrm{E}-06$ & 0.4849 & 10.740366 & $2.18 \mathrm{E}-11$ \\
\hline TY & MN & $9.7 \mathrm{E}-20$ & $4.9 \mathrm{E}-14$ & 4.70167 & 0.4894 & 0.0000 & $4.65 \mathrm{E}-37$ \\
& & $2 \mathrm{E}-05$ & $4.5 \mathrm{E}-14$ & 0.76815 & 3.9867 & 0.0000 & $1.10 \mathrm{E}-39$ \\
& & $7.9 \mathrm{E}-12$ & $8.8 \mathrm{E}-20$ & 1.91519 & 6.7524 & 0.0000 & $2.28 \mathrm{E}-47$ \\
& & $1.7 \mathrm{E}-07$ & $2.2 \mathrm{E}-08$ & 2.23819 & 1.5169 & 0.0000 & $3.45 \mathrm{E}-19$ \\
\hline TS & TN & 3.99245 & $3.5 \mathrm{E}-08$ & 0.00221 & 2.0230 & 0.0454127 & $2.79 \mathrm{E}-11$ \\
& & 4.68352 & 0.01147 & 0.13124 & 0.0000 & 0.4715698 & $3.10 \mathrm{E}-12$ \\
& & 3.50659 & $1.4 \mathrm{E}-05$ & $1.8 \mathrm{E}-06$ & 0.0001 & 0.0774022 & $1.00 \mathrm{E}-15$ \\
& & 3.99959 & 0.00024 & 1.06009 & 0.0595 & 0.1433294 & $8.82 \mathrm{E}-06$ \\
\hline TY & TN & 0.00125 & $1.2 \mathrm{E}-10$ & 0.00495 & 0.44363 & 1.7203849 & $5.51 \mathrm{E}-16$ \\
& & 0.00258 & $3.8 \mathrm{E}-14$ & 0.02396 & 0.24554 & 0.0023017 & $1.32 \mathrm{E}-21$ \\
& & 0.01941 & $5.4 \mathrm{E}-19$ & 0.39888 & 3.85667 & 0.000562 & $9.04 \mathrm{E}-24$ \\
& & $3.8 \mathrm{E}-06$ & $9.5 \mathrm{E}-07$ & 0.00026 & 3.70232 & 0.9374535 & $3.23 \mathrm{E}-15$ \\
\hline TS & TY & $1.2 \mathrm{E}-06$ & 1.3193 & $1.2 \mathrm{E}-17$ & 0.0000 & 0.0337595 & $1.38 \mathrm{E}-35$ \\
& & 0.01007 & 0.0243 & $1.8 \mathrm{E}-05$ & 1.0040 & 0.0005845 & $2.53 \mathrm{E}-12$ \\
& & 0.00131 & 0.0526 & $1.6 \mathrm{E}-10$ & 0.0000 & $9.767 \mathrm{E}-06$ & $2.73 \mathrm{E}-28$ \\
& & $2.3 \mathrm{E}-05$ & 1.5832 & $5.9 \mathrm{E}-10$ & 0.0473 & 0.0263733 & $2.68 \mathrm{E}-17$ \\
\hline
\end{tabular}

Table 6.15 Results of the likelihood ratio calculations. First table presents the results of the calculations using the conventional F2 and F3. The latter one presents the results of the calculation which uses effective $F 2$, instead of $F 2$ and F3. The likelihood ratios over 1 are marked by shading.

The distributions of the posterior odds presented in Table 6.15 are shown as below. Since the posterior odds are very small, the distributions were presented on the basis of their log. The top figure shows the distribution of the posterior odds calculated using natural F2 and F3, and the bottom figure shows the distribution of those calculated using effective F2 instead of natural F2 and F3. 

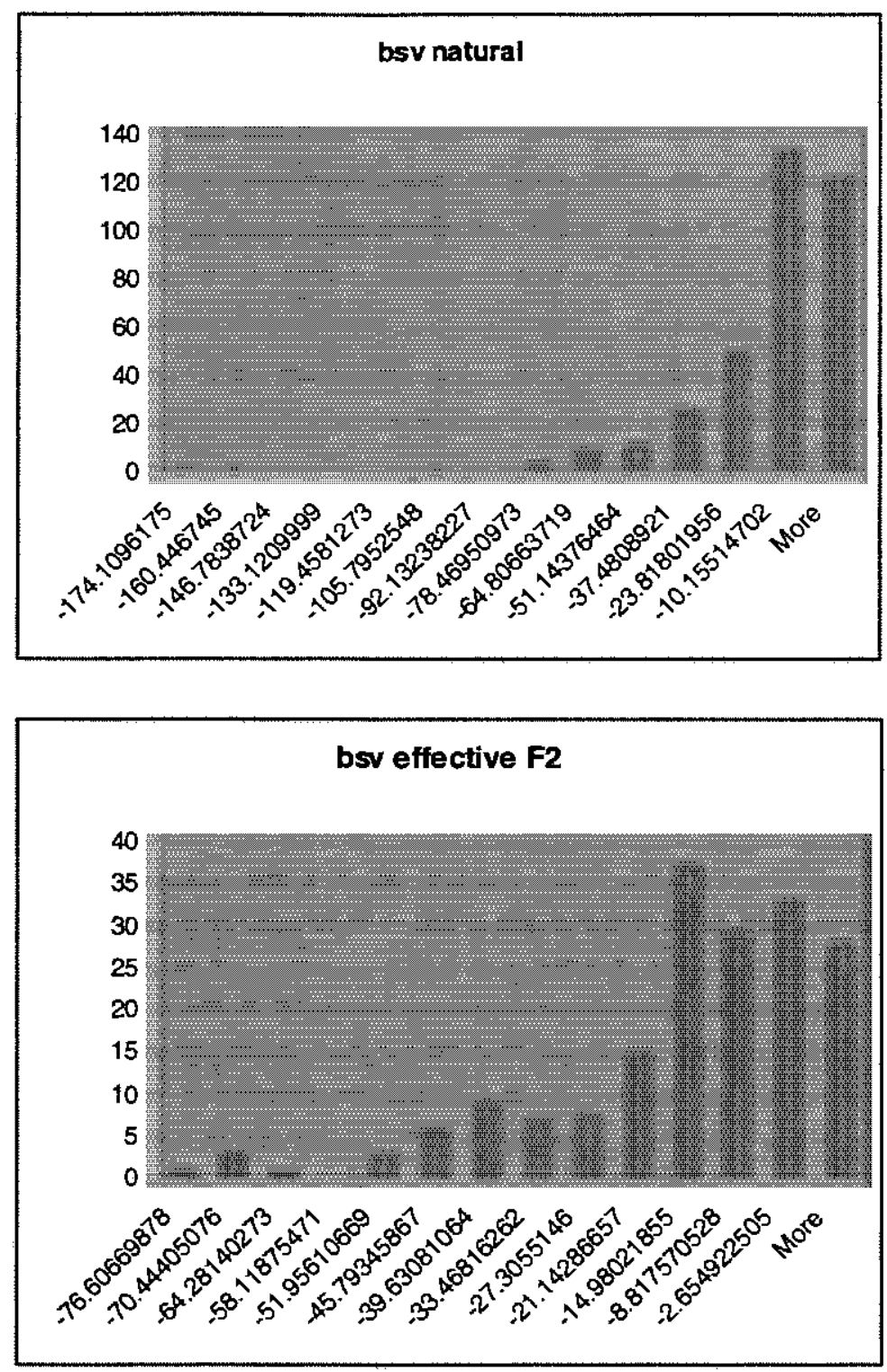

Figure 6.8 Distribution of the log of the posterior odds presented in Table 6.15.

The results in Table 6.15 above show that the Bayesian likelihood ratio approach works even better for between-speaker discrimination. In the table with F2 and F3, there were 6 occurrences where the test did not discriminate two speakers successfully and 9 occasions in the table with effective F2. This means the success rates for the discrimination with each type of calculation are approximately $96.67 \%$ (with F2 and F3) and $95 \%$ (with effective F2), as 180 tests (45 speaker 
combinations * four ways of comparing the pair of speakers) for discrimination were performed altogether.

These are almost surprisingly high success rates, but since each pair of speakers consists of four comparisons, some may argue that counting all four comparisons for the same speaker combination as separate tests is misleading. Another way of looking at these data is to consider the four comparisons for each speaker pair as a set of results. This latter approach produces 45 sets of results. In the table 5 pairs of speakers were misidentified once or twice out of four occasions. If those sets of comparisons were counted as cases of unsuccessful discrimination, the successful discrimination rate becomes $88.8 \%$.

There seems no clear answer to the question of which way of looking at the data is more appropriate. As mentioned above, taking four comparisons from the same speaker pairs as four independent tests may overestimate the results. Those four combinations are, however, four different comparisons, not duplicates of the same comparisons, unlike the within-speaker comparison presented earlier where the same data were compared nine times per speaker using different speaker pools. In the second calculation of successful discrimination rates, if one false discrimination makes us discard three successful discriminations, this would seem to result in an underestimation of the success rate. What can be said here is, even if the calculation was made in a very conservative way, the likelihood ratio-based distance approach seems to produce still high success rates.

Also, with between-speaker comparison as well, a large range in the posterior odds was found. For instance, the first comparison between Speakers JN and HA was found to be $7.77^{*} 10^{175}$ and the third comparison between Speakers JN and AA was 591.7. This is a very large difference. In fact, the incredibly small figure for the posterior odds $7.77^{*} 10^{\text {ths }}$ suggests the possibility of an underflow 
in the calculation software (Excel has been used in this study). Whether or not there was an underflow effect was examined by producing logs of individual likelihood ratios and adding them. In fact, some claim this additive method, with a logged likelihood ratio, instead of the multiplication of the individual likelihood ratios, is a more appropriate way to evaluate the weight of evidence (Good 1991). The result of the log-additive method for this particular combination came out very close to $7.77 * 10^{-175}$, as -174.1096 , and is therefore probably not the result of underflow. Moreover, this value could, of course, differ from machine to machine depending on the maximum bit representation implemented in the hardware. This will not really effect its interpretation for the court, since any values for the log LR bigger than 4 would count as 'very strong' evidence (Champod and Evett 2000: 240).

\subsubsection{Details of unsuccessful discrimination}

The details of the unsuccessful discriminations are examined next. Four comparisons made for each speaker combination are numbered one to four for convenience. These comparisons are listed as follows:

Comparison 1: Speaker A's session 1 vs. Speaker B's session 2

Comparison 2: Speaker A's session 2 vs. Speaker B's session 1

Comparison 3: Speaker A's session 1 vs. Speaker B's session 1

Comparison 4: Speaker A's session 2 vs. Speaker B's session 2

The comparisons, which produced posterior odds over 1 , are summarised in Table 6.16. The numbers shown in parentheses next to the speaker combinations are the comparison numbers as listed above. Shaded columns show the speaker combinations which produced inconsistent 
likelihood ratios across two types of calculations ie. with $F 2$ and $F 3$, and with effective $F 2$. The posterior odds of correctly discriminated speaker combinations in one calculation but not in the other, are shown in bold case.

\begin{tabular}{|c|c|c|}
\hline \multirow{2}{*}{$\begin{array}{c}\text { Speaker } \\
\text { Combination }\end{array}$} & $F_{2}$ and $F_{3}$ & Effective $\mathrm{F} 2$ \\
\hline & Posterior odds & Posterior odds \\
\hline $\mathbb{N}-A A(3)$ & 591.74 & 3219.03 \\
\hline $\mathrm{KO}-\mathrm{KA}(1)$ & 2.36 & 5.00 \\
\hline $\mathrm{MN}-\mathrm{HA}(2)$ & 21.16 & 5.72 \\
\hline $\mathrm{MN}-\mathrm{KF} \quad(2)$ & 12.22 & 10.89 \\
\hline $\mathrm{TN}-\mathrm{KA} \quad(1)$ & 43.11 & 22.73 \\
\hline (4) & 35.28 & 17.03 \\
\hline KF H. (2) & 0.22 & 16.02 \\
\hline$=(3)$ & 0.04 & 17.33 \\
\hline
\end{tabular}

Table 6.16 Summary of the between-speaker comparisons which produced posterior adds over 1 (false negative discrinination).

It is noticed that the combination of Speakers IN and AA had a very high posterior likelihood ratio in both types of calculations ( 591.74 for the calculation with natural F2 and F3 and 3219.03 for the calculation with effective F2). The combination of Speakers $\mathrm{NN}$ and AA produced very high posterior odds of 591.74 (using natural F2 and F3) and 3219.03 (using effective F2). This suggests that, in a particular case, extremely high likelihood ratios do not necessarily implicate the same individual, as the conventional use of likelihood suggests. In other words, even if an extremely high posterior likelihood is obtained from speech evidence, there is still a possibility of false negative discrimination, although the probability is low. This demonstrates the danger of the conventional use of likelihood ratio in forensic speaker identification. This will not be a problem, however, if the evidence is evaluated on the basis of the likelihood ratio for the accuracy of the discrimination test itself. The likelihood ratio which evaluates the strength of the evidence produced using this approach is discussed later (6.6.7).

Next, the results of the two posterior odds calculation, ie. one with conventional F2 and F3 and the other with effective $\mathrm{F} 2$, are compared. Generally, the results seem reasonably consistent across the two types of calculations. There were two occasions (Speakers KF and HA's second and third 
comparisons), where the calculation with $\mathrm{F} 2$ and F3 discriminated the speakers correctly, although the calculation with effective $\mathrm{F} 2$ failed to do so. The posterior odds for these comparisons were 0.22 and 0.04 for the calculation with $\mathrm{F} 2$ and F3, and 16.02 and 17.33 for the calculation with effective F2. This may suggest that, when the Bayesian approach is employed as the method for evaluation, the effective $F 2$ performs less effectively than incorporating $F 2$ and $F 3$ separately. Only one example of discrepancy between the two calculations was observed in the current data and no conclusive remark can be made, it seems, however, that the posterior odds produced using effective F2 does not work as well as that using natural F2 and F3. The results of within-speaker comparisons (see in 6.6.4.1) also showed more consistent and accurate results for the calculation with natural $F 2$ and $F 3$ instead of effective $F 2$.

Hypothesizing that the natural F2 and F3 performed better, the reason that they perform better than effective F2 may lead us to a concern about the correlation between parameters which were incorporated in this study. If there is a strong correlation between F2 and F3 of the same vowel, multiplying these strongly correlated variables may have resulted in disproportional emphasis on their discriminant power and thus may have distorted the statistics. In this case, those variables were selected on the basis of their high speaker discriminating power. The results of multiplication yielded larger likelihood ratios than they actually have. It is quite likely that the calculation of $F 2$ and $F 3$ produces lower correct discrimination rates than the calculation with effective $F 2$ if a vowel with a weak discriminant power was incorporated into the Bayesian calculation, as the weakness of both $\mathrm{F} 2$ and $\mathrm{F} 3$ are disproportionally amplified by the multiplication.

It has been shown in discussion of the between-parameter correlation (see section 6.6.2.2), however, that the correlation between $\mathrm{F} 2$ and $\mathrm{F} 3$ is not necessarily strong. Thus the reason for the better performance by the posterior odds with F2 and F3 must be sought elsewhere. One of the possible reasons lies in the formula of effective F2. Since effective F2 is based more heavily on F2 than F3, 
the speaker specific information F3 contains is not fully reflected in effective F2 and, as a result, calculation with conventional F2 and F3 adds more speaker specific information than calculation with effective $\mathrm{F} 2$ alone.

There is also a possibility that the reason why effective F2 performed slightly more poorly is because that effective $\mathrm{F} 2$ incorporates $F 1$ as well as F2 and F3 and it is F1 which is degrading the results.

Although the observation that effective F2 performs less effectively is not yet conclusive, the tendency seems to be there. In this study, therefore, the rest of the discussion will concentrate on the posterior odds calculated with natural F2 and F3. The discussion and the results for effective F2 are relegated to the appendices.

\subsubsection{Causes for unsuccessful discrimination}

To see what caused the unsuccessful discriminations, the likelihood ratio for each parameter and the posterior odds of speaker combinations which could not be discriminated correctly are summarised and presented in Table 6.17. The likelihood ratios which are under 1, ie. correctly discriminated the speakers, are marked by shading. (The results for the calculation of effective $F 2$ are presented in Appendix 6.1.) 


\begin{tabular}{|c|c|c|c|c|c|c|c|}
\hline Speakers & $\mathrm{iF} 2$ & $\mathrm{eF} 2$ & $\mathrm{eF} 3$ & olmid F3 & $\mathrm{m} 2 \mathrm{~F}_{3}$ & $82 \mathrm{~F} 3$ & Posterior \\
\hline $\bar{N}-A A(3)$ & 3.33715 & 0.15919 & 1.82762 & 11.3424 & 15.2756 & 3.51775 & 591.74 \\
\hline $\mathrm{KO}-\mathrm{KA}(1)$ & 2.38322 & 0.37057 & 1.61895 & 0.6836 & 6.3950 & 037927 & 2.36 \\
\hline $\mathrm{MN}-\mathrm{HA}(2)$ & 92.9683 & 5.69054 & 26.0594 & 3.8505 & 4.3798 & 10.7198 & 21.16 \\
\hline$\overline{T N}-\mathrm{KA}(1)$ & 065419 & 2.45618 & 2.29395 & 1.71106 & 3.6077 & 1.89485 & 43.11 \\
\hline (4) & 3.54925 & 1.82298 & 2.6692 & 0.62201 & 293463 & 1.11904 & 35.28 \\
\hline
\end{tabular}

Table 6.17 Likelihood ratios and the posterior odds for the between-speaker combinations which were not discriminated correctly. The likelihood ratios below 1 (valid negative discrimination) are marked by shading.

Table 6.17 shows that these speaker combinations produced likelihood ratios which were over 1 (ie. false negative discrimination) with many different parameters. The cause of this unsuccessful discrimination therefore cannot be attributed to any particular parameter.

Although the characteristics of the wrongfully identified pairs of speakers will be analysed in this section, the examination will focus on the parameters which discriminated the speakers correctly, despite the fact that the posterior odds for the particular comparison were over 1 (ie. false negative discrimination).

\subsection{Similarity}

Table 6.18 presents the mean values of the datasets which were used in the between-speaker comparisons which the Bayesian approach failed to discriminate. For easier analysis of the tables, the speaker pool mean for each parameter is also presented here. The mean values of the parameters which produced likelihood ratios below 1 (valid discrimination) in Table 6.17 are indicated by shading. (The results for the calculation with effective F2 are presented in Appendix 6.2.) 


\begin{tabular}{|c|c|c|c|c|c|c|c|}
\hline Sp. combinatio & Dataset & iF2 & $\mathrm{eF} 2$ & $\mathrm{eF} 3$ & olmid & $\mathrm{m} 2$ & $s 2$ \\
\hline \multirow{2}{*}{$\mathbb{N}-A A(3)$} & JN 1 & 1994.9 & 17145 & 2597.45 & 2114.3 & 2021 & 2494.8 \\
\hline & $A A 1$ & 2040.3 & 18669 & 2527.4 & 2218.8 & 2127.4 & 2523.6 \\
\hline \multirow[t]{2}{*}{$\mathrm{KO}-\mathrm{KA}(1)$} & $\mathrm{KO} 2$ & 1955 & 19128 & 2695.8 & 2596 & 2668.3 & $2608-5$ \\
\hline & $\mathrm{KAI}$ & 2075.9 & 20199 & 2622.5 & 27965 & 2657 & 27705 \\
\hline \multirow[t]{2}{*}{$\mathrm{MN}-\mathrm{HA}(2)$} & $\mathrm{MN1}$ & 2466 & 2222.2 & 2900.5 & 2310 & 2439.7 & 2990.6 \\
\hline & HA 2 & 2482 & 2164.4 & 2915.1 & 27768 & 2420.8 & 3012.7 \\
\hline \multirow[t]{4}{*}{$T N-K A(1)$} & $\operatorname{TN2} 2$ & 22635 & 2053.9 & 2605.7 & 2661.4 & 2574 & 2704.3 \\
\hline & $\mathrm{KAl}$ & 20759 & 2019.9 & 2622.5 & 2796.5 & 2657 & 2779.5 \\
\hline & TN2 & 2203.5 & 2053.9 & 2605.7 & 5661.4 & 2574 & 2704.3 \\
\hline & $\mathrm{KA} 2$ & 2202.7 & 1999.5 & 2639.7 & 2827.5 & 2693.7 & 2813.3 \\
\hline
\end{tabular}

[Population means and standard deviations]

\begin{tabular}{|c|c|c|c|c|c|c|c|}
\hline Parameters & if 2 & eF2 & $\mathrm{eF3}$ & $\mathrm{eF}^{\prime 2}$ & olmid & $\mathrm{m} 2$ & $\$ 2$ \\
\hline Mean & 2169 & 1982 & 2668 & 2218.8 & 2534.1 & 2385.4 & 27152 \\
\hline $\mathrm{Sd}$ & 228 & 144.2 & 137 & 164.65 & 191.9 & 189.9 & 201.2 \\
\hline
\end{tabular}

Table 6.18 Means of the datasets which the Bayesian approach failed to discriminate. The means of the parameters which produced likelihood ratios below 1 are indicated by shading. Means of reference population and standard deviations are also presented here for the discussion.

For the examination of the similarity between datasets, the means presented in Table 6.18 were subtracted. Table 6.19 presents the absolute values of the subtraction of one dataset from the other. For instance, the value 45.4 for iF2 of the comparison between Speakers JN and AA was obtained by |Speaker AA's iF2 (2040.3 Hz) - Speaker JN's (1994.9 Hz)|. The larger the value presented in Table 6.19 is, the less similar the two sets of data are. Those values for the parameters which discriminated the speakers correctly are indicated by shading. (The results for the calculation with effective $F 2$ are presented in Appendix 6.3.)

\begin{tabular}{|c|c|c|c|c|c|c|}
\hline Sp. combination & if 2 & $\mathrm{eF} 2$ & eF3 & olmidF & $\mathrm{m}_{2} \mathrm{~F}_{3}$ & $\$ 2 F 3$ \\
\hline W? AA (3) & 45.4 & 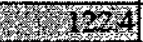 & 70.05 & 104.5 & 106.4 & 28.8 \\
\hline $\mathrm{KO}$ ? KA (1) & 120.9 & 35 & 73.3 & 2000 & 11.3 & 列 1779 \\
\hline $\mathrm{MN} ? \mathrm{HA}(2)$ & 16 & 57.8 & 14.6 & 5660 & 18.9 & 22.1 \\
\hline $\mathrm{TN}$ ? KA (1) & 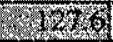 & 34 & 16.8 & 135.1 & 83 & 75.2 \\
\hline (4) & 0.8 & 54.4 & 34 & 166) & 119.7 & 109 \\
\hline
\end{tabular}

Table 6.19 Absolute values of the subtraction of one dataset from the other. The parameters which discriminated the speakers correctly (see Table 6.17) are indicated by shading.

It seems that, generally, the parameters which discriminated speakers correctly had a larger difference between the two data sets. The mean and standard deviation, and the minimum and maximum values for the difference are summarised in Table 6.20 below. Those differences in 
means for the parameters which failed to discriminate speakers are shown in the left part of the table, and those of the parameters which correctly discriminated speakers are shown in the right part of the table. (The results for the calculation with effective $F 2$ are presented in Appendix 6.3.)

\begin{tabular}{|c|c|c|c|c|c|c|c|}
\hline \multicolumn{3}{|c|}{ Failed to discriminate speakers } & \multicolumn{3}{c|}{ Correctly discriminated speakers } \\
\hline mean & 58.78 & $\min$ & 0.8 & mean & 195.39 & $\min$ & 107.1 \\
\hline sd & 41.50 & $\max$ & 135.1 & sd & 124.23 & $\max$ & 466.8 \\
\hline
\end{tabular}

Table 6.20 Means, standard deviations, minimum and maximum values of the difference between 2 speakers. Those parameters which failed to discriminate speakers are shown on the left, and the parameters which discriminated speakers correctly are shown on right.

All of the means, standard deviations, maximum values, and minimum values are clearly smaller in the left part of Table 6.20 , ie. the comparisons which could not be discriminated, than for the comparisons discriminated correctly. Table 6.20 thus seems to support the assumption that the similarity between two speakers contributes to the confusion in the speaker discrimination.

There are, however, some parameters which could not discriminate the speakers despite the relatively large between-speaker differences in their mean. Table 6.19 shows that olmid did not discriminate Speakers $\mathrm{TN}$ and $\mathrm{KA}$ in the first comparison, despite the fact that the difference between these two speakers $(135.1 \mathrm{~Hz})$ was larger than some of the differences which successfully discriminated two speakers. For example, the difference between Speakers TN and KA's first comparison was $127.6 \mathrm{~Hz}$ and the difference between Speakers $\mathrm{IN}$ and AA's third comparison was $122.4 \mathrm{~Hz}$, and yet those comparisons were correctly discriminated.

\subsection{Typicality}

What cannot be explained by the similarity between speakers may be explained by their typicality. As mentioned in an earlier section (6.6.1.2), when the typicality of the two samples is low, the 
significance of the similarity between the data is greater, as there are less people who could produce such formant values. Conversely, what the size of the difference implies is less significant, if the two sets of data are located near the mode of the population. So those pairs of speakers who had been confused despite a large difference between their means, may have been within a range of common voice quality. A histogram is presented in Figure 6.9 below for the examination of the typicality of the speakers in the parameters for which similarity failed to provide a satisfactory explanation (olmid for Speakers TN and KA's first comparison).

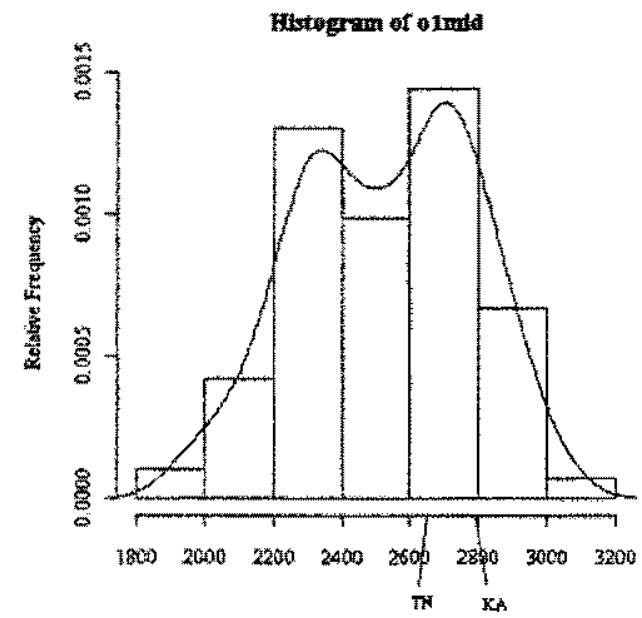

Figure 6.9 Distribution of the population and the locations of Speakers TN and KA, who could not be distinguished despite the large difference between them.

Figure 6.9 shows that Speakers TN and KA's means were located on each side of one of the peaks of the distribution. The area where those two speakers means were located are high-density areas, so the dissimilarity between those two speakers may have been offset by this.

As an alternative explanation, the standard deviation can be put forth. Although the mean of these two pairs of datasets are reasonably separate, it is possible that those datasets included large variation within the dataset, so that those sets of data actually overlap heavily. This clearly prevents discrimination between these two speakers. Figure 6.10 presents the distribution of those datasets. 


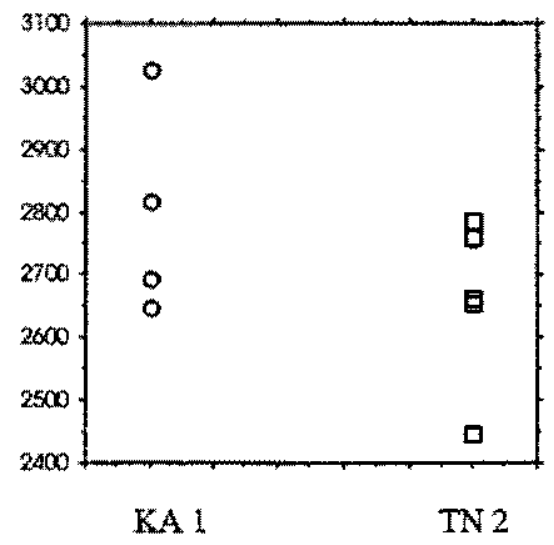

Figure 6.10 Distribution of olmid of the data sets, $\mathrm{KA} 1$ and TN2.

Figure 6.10 shows that 4 out of 5 samples by Speaker TN overlap with Speaker KA's. This naturally will contribute to discrimination between these two speakers. This suggests that likelihood ratio may not discriminate speech samples well, when samples to be compared consist of a large within-speaker variation.

\subsubsection{NUMBERS OF PARAMETERS AND DISCRIMINATION}

Given that the likelihood ratio based distance approach is the result of combining several quasiindependent parameters, it seems sensible to look at how the successful positive and negative discrimination rate changes by increasing the number of parameters incorporated in the discrimination. It has been demonstrated that, by incorporating multiple parameters, formant frequencies can discriminate speakers with a high confidence level. Then, the question that arises here is "how many parameters are many enough?" Ideally as many parameters as possible should be incorporated for reliable speaker discrimination. Practically, however, the numbers of the parameters extracted can vary greatly from case to case, and it is quite possible that there are some 
cases in which only a small set of parameters can be extracted. It thus seems useful to know what, if any, are the absolute minimum numbers for reliable speaker discrimination.

Another reason for looking at this is that once a sufficient number of parameters is incorporated, it is possible that the successful discrimination rate will reach an asymptotic state. Since the results are expected to vary according to many factors, there will not be a simple answer to the questions: what the minimum number of parameters to obtain reliable results is, or what the effect of the numbers of parameter on successful discrimination rate is like. We may, however, be able to find out at which points the successful discrimination rate changes critically or reaches an asymptotic state, or if there are any points like that at all.

In order to investigate the relationship between the number of parameters incorporated and the successful discrimination rate, the successful discrimination rates were calculated in case those one to five parameters are incorporated, instead of all six parameters. Depending on how many parameters were incorporated, the numbers of possible patterns of combinations vary. For instance, when only one parameter is incorporated, the possible combination patterns are six. When two parameters were incorporated, the six parameters produce 15 possible combination patterns of parameters, as in $\mathrm{iF} 2 / \mathrm{eF} 2, \mathrm{iF} 2 / \mathrm{eF} 3, \mathrm{iF} / \mathrm{olmid}$, and so on. When three parameters were combined, even more combination patterns, namely 20 , are possible. The successful discrimination rates were calculated for each combination, and then means across the possible combinations were also calculated. Table 6.21 presents the results of the calculations. The columns headed "Total" show the total numbers of valid positive and negative discriminations out of 90 and 180 comparisons, respectively, and the columns headed "\%" present the successful discrimination rates. Sums, means, and standard deviations are also presented at the bottom section of each table. So for example, when only one parameter is taken, there are six possible outcomes because there are six different parameters. Table 6.21 shows that if the single parameter is $\mathrm{iF} 2$, there are 72190 within-speaker 
comparisons $(=80 \%)$ correct, and $132 / 180(=73 \%)$ between speaker comparisons correct. If only one parameter is taken, the average correct rate for within-speaker comparisons is $73 \%$ and for between-speaker comparisons it is $72 \%$.

\begin{tabular}{|c|c|c|c|c|}
\hline \multirow{2}{*}{$\begin{array}{c}\text { Possible } \\
\text { parameter combinations }\end{array}$} & \multicolumn{2}{|c|}{ wsv } & \multicolumn{2}{|c|}{ bsv } \\
\hline & & & & \\
\hline & & & & \\
\hline $\mathrm{iF} 2$ & 72 & 80 & 132 & 73.33 \\
\hline $\mathrm{eF} 2$ & 58 & 64.44 & 133 & 73.89 \\
\hline $\mathrm{eF} 3$ & 51 & 56.67 & 129 & 71.67 \\
\hline olmid F3 & 80 & 88.89 & 129 & 71.67 \\
\hline $\mathrm{m} 2 \mathrm{~F} 3$ & 45 & 50 & 129 & 71.67 \\
\hline s2F3 & 90 & 100 & 125 & 69.44 \\
\hline Sum & 396 & & 777 & \\
\hline Mean & 66 & 73.33 & 129.5 & 71.94 \\
\hline Standard deviation & 17.54 & & 2.81 & \\
\hline
\end{tabular}

\begin{tabular}{|c|c|c|c|c|}
\hline \multirow{2}{*}{$\begin{array}{c}\text { Possible } \\
\text { parameter combinations }\end{array}$} & \multicolumn{2}{|c|}{ wsv } & \multicolumn{2}{|c|}{ bsv } \\
\hline & Total $/ 90$ & $\%$ & Total $/ 180$ & $\%$ \\
\hline $\mathrm{iF} 2 * \mathrm{eF} 2$ & 74 & 82.22 & 152 & 84.44 \\
\hline $\mathrm{iF} 2 * \mathrm{eF} 3$ & 63 & 70.00 & 146 & 81.11 \\
\hline $\mathrm{iF} 2 *^{\circ}$ olmid & 90 & 100.00 & 159 & 88.33 \\
\hline $\mathrm{iF} 2{ }^{*} \mathrm{~m} 2$ & 79 & 87.78 & 161 & 89.44 \\
\hline $\mathrm{iF} 2 * \mathrm{~s} 2$ & 81 & 90.00 & 145 & 80.56 \\
\hline $\mathrm{eF} 2 * \mathrm{eF} 3$ & 63 & 70.00 & 150 & 83.33 \\
\hline $\mathrm{eF} 2 *$ olmid & 71 & 78.89 & 163 & 90.56 \\
\hline $\mathrm{eF} 2 * \mathrm{~m} 2$ & 55 & 61.11 & 151 & 83.89 \\
\hline $\mathrm{eF} 2 * \mathrm{~s} 2$ & 86 & 95.56 & 154 & 85.56 \\
\hline $\mathrm{eF} 3 *^{*}$ olmid & 63 & 70.00 & 160 & 88.89 \\
\hline $\mathrm{eF} 3 * \mathrm{~m} 2$ & 45 & 50.00 & 164 & 91.11 \\
\hline $\mathrm{eF}^{*} \mathrm{~s} 2$ & 70 & 77.78 & 152 & 84.44 \\
\hline $01 \mathrm{mid}^{*} \mathrm{~m} 2$ & 55 & 61.11 & 151 & 83.89 \\
\hline olmid*s2 & 90 & 100.00 & 150 & 83.33 \\
\hline $\mathrm{m} 2 * \mathrm{~s} 2$ & 62 & 68.89 & 151 & 83.89 \\
\hline Sum & 1047 & & 2309 & \\
\hline Mean & 69.8 & 77.56 & 153.93 & 85.52 \\
\hline Standard deviation & 13.52 & & 5.99 & \\
\hline
\end{tabular}




\begin{tabular}{|c|c|c|c|c|}
\hline \multirow{2}{*}{$\begin{array}{c}\text { Possible } \\
\text { parameter combinations }\end{array}$} & \multicolumn{2}{|c|}{ wsv } & \multicolumn{2}{|c|}{ bsv } \\
\hline & Total $/ 90$ & $\%$ & Total $/ 180$ & $\%$ \\
\hline $\mathrm{iF} 2 * \mathrm{eF} 2 * \mathrm{eF} 3$ & 65 & 72.22 & 157 & 87.22 \\
\hline $\mathrm{iF} 2 * \mathrm{eF} 2 * \mathrm{o} 1 \mathrm{mid}$ & 81 & 90.00 & 166 & 92.22 \\
\hline $\mathrm{iF} 2 * \mathrm{eF} 2 * \mathrm{~m} 2$ & 68 & 75.56 & 162 & 90.00 \\
\hline $\mathrm{iF} 2 * \mathrm{eF} 2 * \mathrm{~s} 2$ & 90 & 100.00 & 158 & 87.78 \\
\hline $\mathrm{iF} 2{ }^{*} \mathrm{eF} 3{ }^{*}$ olmid & 81 & 90.00 & 164 & 91.11 \\
\hline $\mathrm{iF} 2 * \mathrm{eF} 3 * \mathrm{~m} 2$ & 69 & 76.67 & 169 & 93.89 \\
\hline $\mathrm{iF} 2 * \mathrm{eF} 3 * \mathrm{~s} 2$ & 78 & 86.67 & 155 & 86.11 \\
\hline $\mathrm{iF} 2 *_{\mathrm{o}} 1 \mathrm{mid}^{*} \mathrm{~m} 2$ & 72 & 80.00 & 167 & 92.78 \\
\hline $\mathrm{iF} 2 *$ olmid*s 2 & 90 & 100.00 & 159 & 88.33 \\
\hline $\mathrm{iF} 2 * \mathrm{~m} 2 * \mathrm{~s} 2$ & 82 & 91.11 & 164 & 91.11 \\
\hline $\mathrm{eF} 2 * \mathrm{eF} 3 *$ olmid & 63 & 70.00 & 171 & 95.00 \\
\hline $\mathrm{eF} 2 * \mathrm{eF} 3 * \mathrm{~m} 2$ & 46 & 51.11 & 168 & 93.33 \\
\hline $\mathrm{eF} 2 * \mathrm{eF} 3{ }^{*} \mathrm{~s} 2$ & 65 & 72.22 & 160 & 88.89 \\
\hline $\mathrm{eF} 2{ }^{*} \mathrm{o} 1 \mathrm{mid}^{*} \mathrm{~m} 2$ & 60 & 66.67 & 168 & 93.33 \\
\hline $\mathrm{eF} 2 *_{\mathrm{o}} 1 \mathrm{mid} *_{\mathrm{s}} 2$ & 81 & 90.00 & 167 & 92.78 \\
\hline $\mathrm{eF} 2 * \mathrm{~m} 2 * \mathrm{~s} 2$ & 64 & 71.11 & 162 & 90.00 \\
\hline eF3*olmid*m2 & 56 & 62.22 & 168 & 93.33 \\
\hline $\mathrm{eF3}$ *o1mid*s2 & 90 & 100.00 & 162 & 90.00 \\
\hline $\mathrm{eF} 3 *^{\mathrm{m}} 2 * \mathrm{~s} 2$ & 77 & 85.56 & 165 & 91.67 \\
\hline $\mathrm{olmid} * \mathrm{~m} 2 * s 2$ & 65 & 72.22 & 162 & 90.00 \\
\hline Sum & 1443 & & 3274 & \\
\hline Mean & 72.15 & 80.17 & 163.70 & 90.94 \\
\hline Standard deviation & 12.07 & & 4.39 & \\
\hline
\end{tabular}

4 parameters (15 patterns)
\begin{tabular}{|c|cc|cc|}
\hline Possible & \multicolumn{2}{|c|}{ wsv } & \multicolumn{2}{|c|}{ bsv } \\
\cline { 2 - 5 } parameter combinations & Total /90 & $\%$ & Total /180 & $\%$ \\
\hline eF3*olmid*m2*s2 & 73 & 81.11 & 168 & 93.33 \\
eF2*olmid*m2*s2 & 66 & 73.33 & 171 & 95.00 \\
eF2*eF3*m2*s2 & 64 & 71.11 & 169 & 93.89 \\
eF2*eF3*olmid*s2 & 69 & 76.67 & 172 & 95.56 \\
eF2*eF3*oimid*m2 & 48 & 53.33 & 174 & 96.67 \\
iF2*olmid*ms*s2 & 82 & 91.11 & 166 & 92.22 \\
iF2*eF2*eF3*m*s2 & 81 & 90.00 & 169 & 93.89 \\
iF2*eF3*olmid*s2 & 82 & 91.11 & 166 & 92.22 \\
iF2*eF3*o1mid*m2 & 72 & 80.00 & 171 & 95.00 \\
iF2*eF2*m2*s2 & 81 & 90.00 & 165 & 91.67 \\
iF2*eF2*olmid*s2 & 90 & 100.00 & 165 & 91.67 \\
iF2*eF2*o1mid*m2 & 78 & 86.67 & 170 & 94.44 \\
iF2*eF2*eF3*s2 & 78 & 86.67 & 164 & 91.11 \\
iF2*eF2*eF3*m2 & 56 & 62.22 & 170 & 94.44 \\
iF2*eF2*eF3*olmid & 63 & 70.00 & 170 & 94.44 \\
\hline Sum & 1083 & & 2530 & \\
Mean & 72.20 & 80.22 & 168.67 & 93.70 \\
\hline Standard deviation & 11.28 & & 2.92 & \\
\hline
\end{tabular}




\begin{tabular}{|c|c|c|c|c|}
\hline \multirow{2}{*}{$\begin{array}{l}\text { Possible } \\
\text { parameter combinations }\end{array}$} & \multicolumn{2}{|c|}{ wsv } & \multicolumn{2}{|c|}{ bsv } \\
\hline & Total 190 & $\%$ & Total $/ 180$ & 曾 \\
\hline $\mathrm{iF} 2 * \mathrm{~F} 2 * \mathrm{eF} 3{ }^{*}$ olmid*m2 & 73 & 81.11 & 172 & 95.56 \\
\hline $\mathrm{iF} 2 * \mathrm{eF}^{2} \mathrm{eF}{ }^{*}$ olmid*s2 & 89 & 98,89 & 169 & 93.89 \\
\hline $\mathrm{iF} 2 * \mathrm{eF}^{2}{ }^{*} \mathrm{eF} 3{ }^{*} \mathrm{~m} 2{ }^{*} \mathrm{~s} 2$ & 76 & 84.44 & 172 & 95.56 \\
\hline $\mathrm{iF} 2 * \mathrm{eF} 2 * 01 \mathrm{mid} * \mathrm{~m} 2 * 52$ & 84 & 93.33 & 172 & 95.56 \\
\hline $\mathrm{iF} 2 * \mathrm{eF} 3 * \mathrm{olmid} * \mathrm{~m} 2 * \mathrm{~s} 2$ & 82 & 91,11 & 171 & 95.00 \\
\hline $\mathrm{eF} 2 * \mathrm{eF}^{2}{ }^{*}$ olmid*m2 ${ }^{*} \mathrm{~s} 2$ & 71 & 78.89 & 175 & 97.22 \\
\hline Sum & 475 & & 1031 & \\
\hline Mean & 79.17 & 87.96 & 171.83 & 95.46 \\
\hline Standard deviation & 6.97 & & 1.94 & \\
\hline
\end{tabular}

6 parameters

\begin{tabular}{|c|c|c|c|c|}
\hline & ws & & bs & \\
\hline All & 81 & 90 & 174 & 96.67 \\
\hline
\end{tabular}

Table 6.21 Total numbers, means, standard deviations, and percentages of successful discrimination for each number of parameters incorporated in discrimination tests.

Table 6.21 shows that the overall discrimination power seems to improve as the number of parameters incorporated becomes larger. It can be seen, however, that the parameter combinations vary greatly in their discrimination power. There are large differences depending on which parameter is incorporated even when the same number of parameters was used for discrimination tests. This is particularly so when the discrimination tests were carried out on 1 parameter or two parameters. The worst parameter combinations for within-speaker comparison produced successful discrimination rates of merely $50 \%$, when only one or two parameters were included, whereas the best combination achieved $100 \%$ successful discrimination.

Table 6.21 also shows that the within-speaker comparisons have larger standard deviations of successful discrimination rate than the between-speaker comparisons do. Standard deviations of successful discrimination rates for within-speaker comparisons range from 6.97 to 17.54 (the mean of 5 standard deviations is 12.28), whereas standard deviations for between-speaker comparisons range from 1.94 to 5.99 (the mean of 5 standard deviations is 3.61 ). This seems to suggest that the within-speaker comparisons are more susceptible to which parameters are included in the discrimination tests. All parameters used in the discrimination tests were selected on the basis of 
their large F-ratios, that is, on the assumption that they are associated with small within-speaker variation and large between-speaker variation. The parameter to parameter (or combination to combination) variation in the successful discrimination rates here is therefore supposed to be relatively small, compared to the variations which randomly selected parameters (or parameter combinations) would produce. This large combination-dependent variance in successful discrimination rates therefore highlights once again the importance of the incorporation of multiple parameters.

Table 6.22 summarises the parameter combinations which produce the worst and the best successful discrimination rates. The values shown in parentheses are the successful discrimination rates for given parameter combinations.

\begin{tabular}{|c|c|c|c|}
\hline & $\begin{array}{l}\text { No. of } \\
\text { parameters }\end{array}$ & Within-speaker comparison & Between-speaker comparison \\
\hline \multirow{5}{*}{$\begin{array}{c}\text { Worst } \\
\%\end{array}$} & 1 parameter & $\mathrm{m} 2(50 \%)$ & $\$ 2(69.44 \%)$ \\
\hline & 2 parameters & $\mathrm{eF} 3 * \mathrm{~m} 2(50 \%)$ & if $2 * s 2(80.56 \%)$ \\
\hline & 3 parameters & $\mathrm{eF} 2 * \mathrm{eF} 3 * \mathrm{~m} 2(51.11 \%)$ & $\mathrm{iF} 2 * \mathrm{eF} 3 * \mathrm{~s} 2(86.11 \%)$ \\
\hline & 4 parameters & $\mathrm{eF} 2 * \mathrm{eF}^{*}$ olmid*m2 $(53.33 \%)$ & $\mathrm{iF} 2 * \mathrm{eF} 2 * \mathrm{eF} 3 * \mathrm{~s} 2(91.11 \%)$ \\
\hline & 5 parameters & $\mathrm{eF}^{*} \mathrm{eF} 3^{*} \mathrm{olmid} \mathrm{m}^{*} \mathrm{~s} 2(78.89 \%)$ & 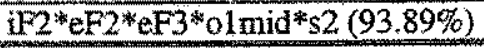 \\
\hline \multirow{5}{*}{$\begin{array}{c}\text { Best } \\
\%\end{array}$} & 1 parameter & $\mathrm{s} 2(100 \%)$ & $\mathrm{eF} 2(73.89 \%)$ \\
\hline & 2 parameters & $\begin{array}{l}\text { iF2*olmid }(100 \%) \\
\text { olmid*s }(100 \%)\end{array}$ & $\mathrm{eF} 3 * \mathrm{~m} 2(91.11 \%)$ \\
\hline & 3 parameters & $\begin{array}{l}\mathrm{iF} 2 * 01 \mathrm{mid} * \mathrm{*} 2(100 \%) \\
\mathrm{eF} 3 * \text { olmid*s } 2(100 \%)\end{array}$ & eF2*eF3;olmid (95\%) \\
\hline & 4 parameters & $\mathrm{iF} 2 * \mathrm{~F} 2 * 01 \mathrm{mid} * \mathrm{~s} 2(100 \%)$ & $\mathrm{eF} 2 * \mathrm{eF} 3$ olmid*m2 $(96.67 \%)$ \\
\hline & 5 parameters & iF ${ }^{*} \mathrm{eF} 2 * \mathrm{eF}{ }^{*}$ olmid*s $2(98.89 \%)$ & eF2*eF3*olmid*m2*s2 $(97.72 \%)$ \\
\hline
\end{tabular}

Table 6.22 Parameter combinations which produced best and worst successful discrimination rates.

The importance of the incorporation of multiple parameters discussed above is even clearer in this table. Table 6.22 shows that when the number of parameters used for the tests were 1 to 4 , the lowest successful discrimination rates for within-speaker comparisons are as low as about $\mathbf{5 0}$ to $53.33 \%$, which is effectively just chance. This means that unless more than five parameters are included, the effects of the selection of parameters are too large to perform reliable negative discrimination, especially with within-speaker comparisons. When less than 4 parameters are 
included, the possibility of false negative discrimination (ie. identifying an innocent person as a criminal) becomes alarmingly high, depending on which parameters are incorporated.

Figure 6.11 presents the data of Table 6.22 in graphic form. It shows how the discrimination rates improve when the number of the parameters is increased. The figure contains 3 lines each for within-speaker and between-speaker comparisons. This is so as to present the change in both best and worst of the successful discrimination rates among the all possible parameter combinations as well as the mean discrimination rates. The mean of the discrimination rates for both within-speaker and between-speaker comparisons are shown by thicker lines.

Number of incorporated parameters and improvement in successful discrimination rates

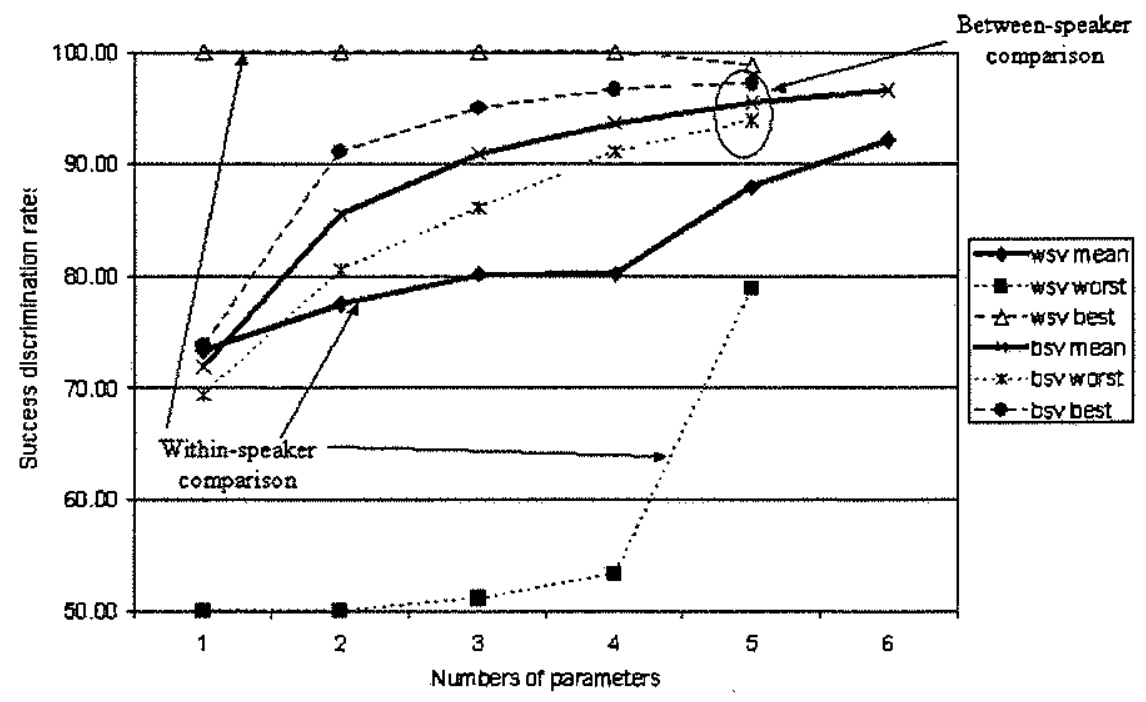

Figure 6.11 Numbers of incorporated parameters in discrimination tests and improvement in successful discrimination rates for the worst and best parameter combinations and means.

Apart from the change in the best parameter combination for within-speaker comparison, it is evident that the addition of parameters improves the successful discrimination rates. It can be seen that the mean between-speaker comparison curve increases steadily with number of parameters incorporated, from about $72 \%$ for one parameter to $97 \%$ for all six parameters. The irregular 
move of the best discrimination rates for within-speaker comparisons are presumably caused by its relatively small data size. The total number of comparisons made for within-speaker comparisons were 90 , which is the half of the number of between-speaker comparisons made in this study. Furthermore this figure 90 is, in fact, ten comparisons * nine repetitions as described earlier.

With the mean of the within-speaker comparisons, it can be seen that there was not much improvement when the parameter number was increased from three to four and then the successful discrimination rate jumped up when another parameter was added. This is an intriguing result and the cause is not clear.

Is it possible to say from this which parameters are more powerful than the others? It seems, for example, that $\mathrm{m} 2$ and $\mathrm{s} 2$ are the least effective parameters for both valid negative discrimination (ie. to identify two samples as a single individual) and valid positive discrimination (to discriminate two samples as different individuals). On the contrary, it seems that speakers were more accurately discriminated when $s 2$ for within-speaker comparisons and $\mathrm{eF} 2$ or $\mathrm{eF} 3$ for the between-speaker comparisons were included in the parameters. This is, however, a simplification of the matter. As pointed out in the discussion of the details of unsuccessful discrimination for the within-speaker comparison in 6.6.5.2, there was no parameter which constantly performed well across all speakers. Examination of each speaker's mean likelihood ratios for the six parameters demonstrates this clearly. This is shown in Table 6.23 , which summarises the likelihood ratios produced by withinspeaker comparisons. Since these likelihood ratios are produced as results of within-speaker comparisons, if the likelihood ratios evaluate speakers' identity correctly, the likelihood ratio should be more than 1 . The largest likehihood ratios (ie. the most powerful likelihood ratios) for each speaker are indicated with shading. 


\begin{tabular}{|c|c|c|c|c|c|c|c|}
\hline & & iF 2 & $\mathrm{eF} 2$ & $\mathrm{eF} 3$ & olmid F3 & $\mathrm{m} 2 \mathrm{F3}$ & s2 F3 \\
\hline \multirow[t]{2}{*}{$\mathrm{AA}$} & Mean & 2.92 & 0.33 & 0.18 & 2.77 & 1.49 & 592 \\
\hline & St & 0.24 & 0.02 & 0.02 & 0.63 & 0.50 & 1.08 \\
\hline \multirow[t]{2}{*}{$\mathrm{HA}$} & Mean & $=32.12$ & 6.16 & 25.90 & 3.54 & 0.71 & 7.57 \\
\hline & St & 29.91 & 1.38 & 7.59 & 0.35 & 0.06 & 1.90 \\
\hline \multirow[t]{2}{*}{ JN } & Mean & 0.42 & 21.48 & 0.28 & 18.61 & 39.36 & 1.51 \\
\hline & St & 0.09 & 5.43 & 0.05 & 6.03 & 22.43 & 0.12 \\
\hline \multirow[t]{2}{*}{ KA } & Mean & 0.79 & 2.07 & 2.08 & 5.22 & 5.4 & 2.98 \\
\hline & St & 0.07 & 0.10 & 0.18 & 0.77 & 0.79 & 0.93 \\
\hline \multirow[t]{2}{*}{$\mathrm{KF}$} & Mean & 3.12 & 0.34 & 0.78 & 0.59 & 0.44 & 3.05 \\
\hline & $\mathrm{St}$ & 0.22 & 0.05 & 0.04 & 0.03 & 0.04 & 0.29 \\
\hline \multirow[t]{2}{*}{ KO } & Mean & 2.63 & 2.67 & 316 & 2.77 & 2.05 & 2.22 \\
\hline & St & 0.38 & 0.15 & 0.20 & 0.14 & 0.31 & 0.12 \\
\hline \multirow[t]{2}{*}{ MN } & Mean & 2.04 & 0.36 & 0.12 & 3.58 & 5.67 & 725 \\
\hline & St & 1.34 & 0.05 & 0.01 & 0.74 & 0.45 & 1.95 \\
\hline \multirow[t]{2}{*}{ TN } & Mean & 3.63 & 0.98 & 2.78 & 1.06 & 0.29 & 2.88 \\
\hline & St & 0.19 & 0.11 & 0.25 & 0.06 & 0.03 & 0.12 \\
\hline \multirow[t]{2}{*}{ TS } & Mean & 2.96 & 3.47 & 1.03 & 838 & 0.04 & 1.50 \\
\hline & $\mathrm{St}$ & 0.16 & 0.34 & 0.05 & 1.22 & 0.09 & 0.38 \\
\hline \multirow[t]{2}{*}{$T Y$} & Mean & 5.46 & 4.02 & 1124 & 2.83 & 0.28 & 1.93 \\
\hline & $\mathrm{St}$ & 0.92 & 0.69 & 2.73 & 0.19 & 0.03 & 0.28 \\
\hline
\end{tabular}

Table 6.23 Each speaker's mean likelihood ratios and standard deviations for each parameter. The largest likelihood ratios (ie. the most powerful likelihood ratio for negative discrimination) are indicated with shading.

Table 6.23 thus demonstrates clearly that the most powerful parameter for discrimination varies from speaker to speaker and, generalizations about the most powerful parameter cannot be made.

\subsubsection{EQUAL ERROR RATE AND PRODUCTION OF LIKELIHOOD RATIO FOR THE TEST}

Finally, this section discusses the production of a new likelihood ratio, based on the results of the discrimination tests demonstrated so far. It has been shown that the likelihood ratio-based distance approach can discriminate speakers rather well. Then, the question arises: how strong the evidence will be, when the likelihood ratio-based distance approach indicates that the two samples were from 
the same individual? This question can be answered by the estimating equal error ratio and then forming a new likelihood ratio for the strength of the evidence produced by this test.

The sections so far estimated the successful positive and negative discrimination rates using the likelihood ratio as the score for the testing and 1 as the threshold. The successful discrimination was calculated in two ways in section 6.6.5.1. One is simply to count the number of correct discriminations and divide them with the total number of tests, ie. 90 for within-speaker comparisons and 180 for between speaker comparisons. With this type of calculation, the discrimination rates were found to be $90 \%$ successful for within-speaker comparisons and $96.7 \%$ successful for between-speaker comparisons. The other way of calculation takes each speaker (for within-speaker comparisons) or speaker combination (for between-speaker comparisons) as a set of results and considers the discrimination was successful only when all tests for the particular speaker or combination of speakers were discriminated correctly. This method produces lower successful discrimination rates, which were $90 \%$ for within-speaker comparisons and $88.9 \%$ for between speaker comparisons.

An equal error rate means that the error ratio for within-speaker and between-speaker comparisons are equal. The threshold which produces the equal enror rate thus allows us to discriminate speakers with the same accuracy in both positive and negative discrimination. As can be seen in the successful discrimination rates presented above, however, neither of the two types of calculation produced equal error rates, when the threshold for the classification was set at 1 . In this section, the threshold which produces the equal error rate, and what the equal error rate is with this likelihood ratio-based distance approach, are determined.

The threshold for the classification was manipulated to find out the equal error ratio for both types of discrimination rates calculation in the same way as described in section 6.5 .1 for the second test 
(discriminant score-based distance method). Table 6.24 presents results of the threshold manipulations. The row headed as "No. of wrong" lists the number of false discriminations in the top table. In the second table, "\%" shows the successful discrimination rates for the number of speaker or speaker combinations which contained at least one false discrimination. The discrimination rates which are more or less equal for both within- and between-speaker comparisons are indicated by shading.

[Calculation counting each successful discrimination]

\begin{tabular}{|r|l|r|r|r|r|r|r|r|r|r|}
\hline & & 0.2 & 0.3 & 0.4 & 0.5 & 0.6 & 0.7 & 0.8 & 0.9 & \multicolumn{1}{|c|}{1} \\
\hline \multirow{2}{*}{ wsv } & No. of wrong & 0 & 1 & 1 & 2 & & 4 & 6 & 7 & 9 \\
\cline { 2 - 10 } & Successful \% & 100 & 98.889 & 98.89 & 97.78 & 96.67 & 95.56 & 93.33 & 92.22 & 90 \\
\hline \multirow{2}{*}{ bsv } & No. of wrong & 11 & 9 & 8 & 8 & & 6 & 6 & 6 & 6 \\
\cline { 2 - 10 } & Successful \% & 93.9 & 95 & 95.6 & 95.6 & 96.7 & 96.7 & 96.7 & 96.7 & 96.7 \\
\hline
\end{tabular}

[Calculation counting a set of tests for each speaker or speaker combination as 1 result]

\begin{tabular}{|c|c|c|c|c|c|c|c|c|c|c|}
\hline & & 0.4 & 0.5 & 0.6 & 0.7 & 0.8 & 0.9 & 1 & 1.1 & 1.2 \\
\hline wsv & $\begin{array}{l}\text { No. of wrong } \\
\text { Successful \% }\end{array}$ & $\begin{array}{r}1 \\
90\end{array}$ & $\begin{array}{r}1 \\
90\end{array}$ & 90 & & & & & & 90 \\
\hline bsv & $\begin{array}{l}\text { No. of wrong } \\
\text { Successful \% }\end{array}$ & $\begin{array}{r}7 \\
84.4\end{array}$ & $\begin{array}{r}7 \\
84.4 \\
\end{array}$ & $\begin{array}{r}6 \\
86.7\end{array}$ & 88. & 88 & 88 & & 88 & 889 \\
\hline
\end{tabular}

Table 6.24 Summary of the successful discrimination rates for different thresholds. Top row shows withinspeaker comparisons, and bottom row shows between-speaker variation. Discrimination rates which are more or less equal for both within-speaker and between-speaker comparisons are indicated by shading.

It seems that equal error rate calculation does not work as well in the bottom table as in the top table. This is presumably because the numbers of results for equal enor rate calculation are reduced greatly by taking the tests for each speaker and speaker combination as one set of results. This way of calculation reduces the number of results from 90 to ten for within-speaker comparisons and from 180 to 45 for between speaker comparisons, as nine tests and four tests respectively were treated as one set of results. The calculation for the within-speaker variation in the top table is admittedly not appropriate, as the supposedly 90 comparisons are in fact the repeat of ten comparisons with nine different population means and standard deviations. However, as this 
research does not have a large population, the figures in the top table will be used to demonstrate the derivation of a new likelihood ratio, this time for the discrimination test itself.

The top panel of Table 6.24 shows that an equal error ratio just above $96 \%$ can be achieved, using 0.6 as the threshold instead of 1 . This means that the likelihood ratio associated with the test is:

$$
\mathrm{LR}=\frac{\mathrm{P}(\mathrm{E} \mid \mathrm{H})}{\mathrm{P}(\mathrm{E} \mid \overline{\mathrm{H}})}=\frac{0.96}{(1-0.96)}=\frac{0.96}{0.04}=24
$$

Thus, assuming that the calculation of the successful discrimination rates are appropriate in the first place, when the posterior odds for the comparison of two speech samples turns out to be over 0.6 , an expert may say "It is 24 times more likely for the given two speech samples to come from the same speaker than they did not."

A likelihood ratio of 24 is nowhere near as big as those that can be found with DNA evidence (Robertson and Vignaux 1995:22). However, according to the verbal scales suggested by Champod and Evett 2000: 240), it still constitutes 'moderate evidence' in favour of the prosecution case. The forensic phonetic evidence can therefore still be a useful piece of information in situations where not much other evidence is available. Furthermore, given the results from the previous section, we can expect to improve the equal error rate by increasing the number of the parameters to be used, which results in higher likelihood ratio for this test. Thus the use of likelihood ratio proposed in this chapter seems promising for the application in actual forensic speaker identification. 


\subsubsection{PROBLEMS WITH LIKELIHOOD RATIO-BASED DISTANCE APPROACH}

It seems that the likelihood ratio-based distance approach works very well in speaker discrimination. The successful discrimination rates for within-speaker comparison were $90 \%$ and for between-speaker comparison, were $96.7 \%$ (with a relatively optimistic calculation) or, being conservatively estimated, were $88.9 \%$. There are, however, aspects of this approach which do not give us complete confidence in the results, as noted in the course of the discussion so far. It is appropriate here to discuss and summarise the limitations of the approach.

The main problem concerning the likelihood ratio-based distance approach presented in this study is the small sample size. The sample of this study is 11 speakers for the vowels in different words and 13 for moshimoshi. It has been discussed in Chapter 3 that the ways for speakers to vary from each other are not infinite, and also variation annong a homogeneous population is assumed to be narrower than general population. If the subject group itself is a smaller part of the population with a reasonably homogeneous speech style, it may be just enough to produce a general picture of this particular population. However, although the crucial part of the information (whether or not the likelihood ratio is over 1) stayed very stable, the actual value of the likelihood ratios varied depending on who were extracted from the population as test samples (see Table 6.10 for the identification test, and Table 6.15 for the discrimination test). This indicates that each speaker had a fairly strong influence on the mean of the speaker pool. In other words, that is, the sample size was too small.

Correlation between parameters is another concern. Although the correlation between parameters was shown to be very small in the earlier section, it nonetheless still exists. The problem here is that it is not predictable which parameters are going to be more correlated than others (with the 
exception of F2 and effective F2, which was chiefly derived from F2). There is speaker-to-speaker difference regarding which parameters correlate more, and to what extent these parameters correlate. Although the effect of correlation seems marginal, we still need to be aware that there is possible distortion of statistics from this feature.

Furthermore, Aitkin's formula for likelihood ratio is not optimal for speech data for two reasons. Firstly, it does not take occasion-to-accasion difference into account. Secondly, Aitkin's formula is composed assuming that the data has a normal distribution. Speech data are, however, not always normally distributed. This has been shown in Figure $6.9 \mathrm{in}$ 6.6.4.2 and discussed in 6.6.4.3.

Finally, although it was not discussed in the main body of the study, the effect of rounding off numbers should be mentioned. Aitkin's likelihood ratio calculation formula usually produces a likelihood ratio with very many decimal points. As usual practice for dealing with numeral data, we often round the numbers up to figures which are considered to have significance in the discussion of the data. Two decimal points are probably a common choice in many acoustic studies. In the production of the posterior odds from multiplying the individual likelihood ratios, however, it was found that rounding off the individual likelihood ratios can affect the posterior odd considerably. Table 6.25 demonstrates the effect of rounding decimal points. The table presents individual likelihood ratio for one of speaker HA's within-speaker cornparisons. This is the comparison which produced the largest posterior odds of all. The row shown as "0" presents the calculation without any rounding for individual likelihood ratio. The rows " 2 " and " 1 " show the calculations when the individual likelihood ratio was rounded up to 2 and 1 decimal points, respectively. 


\begin{tabular}{|c|c|c|c|c|c|c|c|}
\hline & iF2 & $\mathrm{eF} 2$ & $\mathrm{eF} 3$ & olmid $\mathrm{F}_{3}$ & $\mathrm{~m}^{2} \mathrm{~F} 3$ & $\$ 2 F 3$ & posterior \\
\hline 0 & 33.9107597902 & 9.1021641986 & 31.3419815946 & 3.5707200239 & 0.7387353624 & 8.187725228 & 1208937.59 \\
\hline 2 & 33.91 & 9.1 & $\begin{array}{r}31.34 \\
\end{array}$ & 357 & 0.74 & 8.188 & 8209192.42 \\
\hline 1 & 33.9 & 9.1 & 31.3 & 3.6 & 0.7 & & 199526.15 \\
\hline
\end{tabular}

Table 6.25 Example of effects of rounding on calculation of posterior odds.

It can be seen that the difference between posterior odds are over 10000 , depending on the rounding. This is a very large difference, although there hardly will be a tangible difference for a jury between 209000 times more likely and 199500 times more likely. Although this round-off effect will be proportional to the size of original posterior odds, and probably would not alter the original posterior odds 1 to 1000 , it is clear that rounding-off can distort the data. Thus care should be taken not to round off the likelihood ratios prior to the production of posterior odds.

\subsection{SUMMARY}

This chapter set out to find out the answers to the two main questions in this thesis: Q1) "Can formants discriminate speakers?" and, if so, Q2) "How well do they perform?" The answers to these questions found in this chapter were:

Al) Yes, they can.

A2) They do very well.

It was demonstrated that formant frequencies indeed discriminate speakers rather well with forensically realistic datasets, ie. non-contemporaneous natural speech from a homogeneous speaker pool. 
This chapter has examined how well formants, which were selected on the basis of the investigations in Chapters 4 and 5, perform in the discrimination of speakers, using three different methods; discriminant analysis, discriminant score-based distance method, and likelihood ratiobased distance method. The correct classification rates turned out to be approximately $45.5 \%$ for discriminant analysis, $74 \%$ for the discriminant score-based distance method, and about $90 \%$ for within-speaker comparisons for the likelihood ratio-based distance method, and $96.7 \%$ (or, a conservatively estimated, $88.9 \%$ ) for between-speaker comparisons when F2 and F3 were used in the calculation.

All approaches presented in this chapter have their strengths and weaknesses. Extensive discussion of those has shown that the likelihood ratio-based distance approach is the most practical method among those three. Although there are some concerns, the Bayesian approach is superior to the other commonly used method, discriminant analysis, in certain important aspects.

Firstly, using discriminant analysis, it is difficult to incorporate datasets which have a different structure of variables. For instance, the formants of vowels elicited from different phonological contexts and the formants of segments elicited from identical words differ in their structure qua variable, as those two types of formant measurements differ in the effects they have from their phonological environment. This can cause serious problems in actual forensic phonetic investigations, since what is available for measurements can be very limited, and usually we cannot afford to discard any of the available parameters just because they have different structures.

Secondly and more importantly, what discriminant analysis evaluates can rarely be helpful in actual forensic speaker identification. Discriminant analysis classifies a newly added unknown speaker into one of the known speakers. Except for fairly special cases, this is not the task forensic phoneticians are expected to perform. In most situations, what needs to be found out is the degree 
of support for the hypothesis whether or not given samples came from the same individual, rather than to identify the owner of incriminating speech from multiple suspects. Despite the fact that discriminant analysis is a commonly used statistic in the research on speaker identification, it is not clear how to apply it in most actual forensic cases.

Next, the discriminant score based distance approach was used. Although it is an approach which represents the concept of within-speaker variation and between-speaker variation, this is statistically a very crude procedure and it was shown that it is not appropriate for practical use.

Finally, the likelihood ratio-based distance approach was discussed. In this study, the likelihood ratio was used as scores for the discrimination test, so the usage in this thesis was very different from what it is originally developed for. The likelihood ratios were classified into within-speaker variation or between-speaker variation according to whether or not the likelihood ratio is over 1 . Within-speaker comparison was correctly classified with a $90 \%$ successful discrimination rate, and between-speaker variation was correctly classified with a $96.7 \%$, (or a very conservative estimate $88.9 \%$ ) success rate.

After the estimation of the successful discrimination rates, as there was a difference between within-speaker and between-speaker comparisons in their rates, an equal error rate was estimated by manipulating the threshold. The calculation was not entirely appropriate, as the data size for the within-speaker comparison was too small to estimate the equal error rate. Nevertheless the process for the production of a new likelihood ratio, which evaluates the strength of the test using the likelihood ratio-based distance approach, was demonstrated. An equal enror rate was achieved at a threshold 0.6, and that was $96 \%$. This equal enror rate enabled us to calculate the likelihood ratio, which evaluated the strength of evidence based on this test. The likelihood ratio was found to be 
24. It was pointed out that more within-speaker comparison data is required, however, to produce a reliable equal error rate.

Concerning this likelihood ratio-based distance approach, the relationship between the successful discrimination rate and the number of parameters included in the discrimination test was also investigated. There were two main findings: firstly, the successful discrimination rate improved when the number of parameters was increased and, secondly, the successful discrimination rates varied greatly depending on which parameters were included in the test. The successful discrimination rates for within-speaker comparisons were found to be more susceptible to which parameters were used than those of between-speaker comparisons, varying the successful discrimination rates from $50 \%$ to $100 \%$, when only one or two parameters were used. The results seem to suggest that it is ideal to incorporate as many parameters as possible for reliable discrimination, and five parameters is probably the smallest acceptable number.

It was further pointed out that the likelihood ratio presented in this chapter is still not a perfect way of evaluating speech samples forensically, since this calculation was developed for objects like the refractive indices of glass fragments, but not for human speech. The formula does not take into account the possible variation within a sample, depending on the occasions of measurements. The development of an optimal evaluation method is clearly beyond most phoneticians' expertise. Further discussion and developments in this matter should thus be left to collaboration with statisticians. 


\section{Chapter 7}

\section{CONCLUSION}

This thesis set out to investigate the potential of using formants as forensic phonetic speaker discrimination parameters, extracted from forensically realistic data, namely: non-contemporaneous natural speech from a homogeneous speaker pool. The research was conducted in two stages: a search for segment / formant combinations with high F-ratios; and an evaluation of the performances of those selected parameters using various realistic statistical approaches. This chapter summarises results, and also canvasses future tasks.

\subsection{ANSWERS TO THE RESEARCH QUESTIONS OF THIS THESIS}

At the beginning of this thesis, two main research questions and three sub-questions were asked. These were:

Main questions: 1) Do formants discriminate speakers?

2) If they do, how well do they perform? 


$$
\begin{aligned}
& \text { Sub-questions: 1) What are the optimal parameters? } \\
& \text { 2) What is the optimal statistical approach available now? } \\
& \text { 3) Are there any language specific differences in forensic speaker } \\
& \text { identification? }
\end{aligned}
$$

The answers to these questions were investigated and discussed in Chapters 4 to 6 of this thesis, and the results are summarised in this section.

\subsubsection{ANSWERS TO THE MAIN QUESTIONS}

The answers to the two main questions, do formants discriminate speakers? and if they do, how well do they perform?, were determined in Chapter 6. This thesis used two types of realistic data: vowels in different phonological contexts and vowels and consonant segments in the word moshimoshi, and Chapters 4 and 5 are devoted to their examination. It was found that selected formants did indeed discriminate same-speaker and different-speaker pairs and, what is more, they did it rather well. The perfornance of formants was tested using three different statistical methods. The best successful discrimination rates produced in this study were $90 \%$ for within-speaker comparison and $97 \%$ for between-speaker comparison. This is not a bad result, given that the data in this study is forensically realistic. The speaker discrimination was performed based on natural and non-contemporaneous speech with a homogeneous speaker pool, all these facts make the discrimination supposedly more difficult, since between-speaker variation is assumed to be smaller in a bomogeneous speaker pool. This thesis thus concludes that forensic speaker identification using formants, as currently practiced, is possible. 


\subsubsection{ANSWERS TO THE SUB-QUESTIONS}

\subsubsection{What are the optimal parameters?}

Chapters 4 and 5 provided the answer to the sub-question number one: "what are the optimal parameters?" In these chapters, ANOVA was carried out to find out what is the best formant / segment combination to discriminate speakers. As F-ratio is the ratio of between-group variation to within-group variation, a larger F-ratio was assumed to be an indirect indicator of the stronger discrimination potential. The potential candidates for the speaker identification parameters were therefore selected according to the size of F-ratio which the parameters produced. The potential candidates were found to be:

For the vowels which are in different phonological contexts:

F2 of the /i/ vowel,

F2, F3 and effective F2 of the /e/ vowel,

For the moshimoshi segments :

$\mathrm{F} 1$ and $\mathrm{F} 3$ of the first $/ \mathrm{m} /$

$\mathrm{F} 3$ and $\mathrm{F} 4$ of the middle of the first /o/

$\mathrm{Fl}$ and $\mathrm{F} 3$ of the second $/ \mathrm{m} /$

$\mathrm{F} 3$ and $\mathrm{F} 4$ of the second $/ \mathrm{s} /$

\subsubsection{What is the optimal statistical approach available?}

The second sub-question, "What is the optimal statistical approach available now?" was studied in Chapter 6. As well as the conventional discriminant analysis, this study proposed two approaches 
basing on the notion of between-sample distances: a discriminant score-based distance approach and likelihood ratio-based distance approach. The successful discrimination rates using these three approaches were $45.45 \%, 74 \%$, and $90 \%$ (within-speaker comparisons) / 96.7\% (for betweenspeaker comparisons), respectively, and this study must therefore conclude that a likelihood ratiobased distance approach was the most effective and practical method currently available.

The high successful discrimination rate is not the only reason why the likelihood ratio-based approach is preferable. The first two approaches were found to have several crucial shortcomings.

Conventional discriminant analysis, although commonly used in forensic speaker identification research, does not provide the information which most forensic cases demand. Discriminant analysis classifies a newly added unknown speech sample into one of a known speaker group. The most commonly asked question in forensic speaker identification is, however, whether or not the given two speech samples are uttered by the same speaker, but not which one among several suspects matches the incriminating speech most closely.

The discriminant score-based distance approach is simply statistically very crude, and the results presented in this study for this approach were heavily influenced by a small number of reference population and also by the fact that the test sample and the reference sample were not independent. This means that if the number of speakers to be tested is increased or the same threshold is tested with different datasets, the discrimination will deteriorate. Although this approach reflects the idea of between-speaker distance very directly, it is not suitable for any practical application.

In addition, the first two approaches (i.e. discriminant analysis and discriminant score-based distance approach) incorporated only three parameters, namely F2 of $/ i /$ and F2 and F3 of /el. This is because there was a structural difference between the parameters selected in Chapters 4 and 5 . The differences in formants obtained from the same segments embedded in different phonological 
contexts (samples in Chapter 4) are not equivalent to the differences between the formants measured off the same segments elicited from the same word (samples in Chapter 5), in terms of the effect which phonological environments impose upon the formants. Combining different types of data by calculating discriminant scores without adjustments will distort the statistics severely, and sufficient data to make the adjustments with respect to the effects of the phonological environment is not available. This inflexibility in the incorporation of parameters can be a serious problem in real life forensic cases. In an actual forensic situation, it is not likely that we have the luxury of discarding any parameters, as the amount of the information tends to be very limited in the first place.

The likelihood ratio-based distance approach is a very different method from the conventional use of the Bayesian likelihood ratio, where it is used as the scale to measure the strength of the evidence for the given hypothesis. In this study, a likelihood ratio was used as the score for the discrimination test. Likelihood ratios were calculated for 7 parameters, F2 of $/ \mathrm{i} /$ and F2, F3 and effective F2 of /e/ (those parameters were selected from Chapter 4), F3 of the first $/ \mathrm{O} /, \mathrm{F} 3$ of the second $/ \mathrm{m} /$ and F3 of the second $/ \mathrm{s} /$ ([c], phonetically, and those parameters were from Chapter 5). They were then combined by multiplying with each other, according to Bayes' rule. Two types of posterior odds calculations were made. One includes 6 parameters and the other includes 5 , depending on whether the calculation includes F2 and F3 of /e/ or effective F2 instead.

The posterior odds were classified into two groups: within-speaker variation and between-speaker variation, according to whether or not the posterior odds are larger than a given threshold. The successful discrimination rate was then calculated on the basis of the numbers of valid discrimination, ie. successful discrimination between same-speaker and different-speaker pairs. The posterior odds produced by the combination including natural F2 and F3, rather than effective F2, generally discriminated speakers better. 
Ignoring for the moment the inappropriateness of using each within-speaker comparison as independent comparisons, an equal error rate of $96 \%$ was determined by the manipulation of the threshold which was originally set at 1 . This was further converted into a likelihood ratio for the test itself which evaluates results produced by this approach with respect to its reliability. The likelihood ratio was found to be 24 . Supposing that this test was used for an actual forensic speaker identification and the posterior odds for the given two samples were over 0.6 , which is the threshold for the equal error rate, experts then can say "The difference between the incriminating speech and suspect's speech is 24 times more likely to be produced by a single speaker than they were produced by two different individuals." Likelihood ratio of 24 is not very large compared to the likelihood ratios obtained from other types of evidence, such as DNA. It is, however, undoubtedly useful information under circumstances where a limited amount of information is available from other evidence.

\subsubsection{Are there any language specific differences??}

The third sub-question in this thesis was "Are there any language specific differences in forensic speaker identification?" The answer to this question is "quite possibly."

The investigation on vowels in Chapter 4 showed that F2 of $/ \mathrm{i} /$ and $\mathrm{F} 2$ and $\mathrm{F} 3$ of /e/ have high Fratios. A comparison with previous studies on Australian English vowels or on American English vowels revealed, however, that the results of this study have some peculiarity. Both Mokhtari and Clermont (1996) and Sambur (1975) report that speaker specific information is reflected more strongly in the F2 of front vowels and F3 of back vowels in English. In this study, however, F3 of back vowels were not found to have a high F-ratio. Instead F3 of a high vowel /e/ was found to produce a high F-ratio. The causes for this discrepancy remain inconclusive. The difference between Japanese and American or Australian English in phonological structure, such as vowel 
space, should also be considered as one of the possible causes for this difference. It must be noted that there is a possibility of language-to-language differences in what are the most effective speaker identification parameters, due to the differences in the phonological structure of the languages.

Speaker identification parameters can be not only acoustic parameters, but also linguistic parameters. In addition to the acoustic parameters discussed above, therefore, there is a possibility that different languages offer different linguistic parameters, which can be useful in forensic speaker identification. In this study, vowel devoicing was considered to be a candidate for a language specific linguistic parameter in Japanese. Although it was revealed that the withinspeaker variation in the realisation of vowel devoicing is too large to provide any reliable information on individuality in most cases, if many tokens of this parameter are available in both incriminating evidence and suspect's speech samples, and also the realisation of the devoicing (whether the realisation is present or absent) is very consistent in each of those samples, vowel devoicing can be a parameter for speaker discrimination.

\subsection{ADDITIONAL FINDINGS}

The calculation of the likelihood ratios for each parameter and examination of these revealed not surprisingly that there is no parameter which works particularly well for all speakers. Parameters which discriminate one speaker or speaker combination very effectively do not necessarily discriminate other speakers or speaker combinations successfully.

One of the advantages of the likelihood ratio-based distance approach is its simplicity in the incorporation of multiple parameters. The significance of this advantage cannot be emphasised 
enough, as it is absolutely necessary to incorporate many more than a single parameter in order to perform reliable speaker discrimination.

The question which then arises is how many parameters are sufficient for reliable speaker discrimination. In the course of discussion of the likelihood ratio-based distance approach, the relationship between the successful discrimination rates and the number of parameters incorporated for the discrimination test was also investigated. The results showed two very important aspects. Firstly, the successful discrimination rates vary greatly depending on which parameters were combined for the discrimination test. The other point is that the difference between parameter combinations was larger with within-speaker comparisons, indicating that the within-speaker comparison is more susceptible to differences in parameter combinations than between-speaker comparisons. The successful discrimination rates for within-speaker comparisons range from about $50 \%$ to $100 \%$ when one to four parameters are combined. This strongly suggests that at least 5 parameters are probably necessary in order to have reliable results in speaker discrimination.

Finally, the effect of the speech analysis software should be noted. A comparison of moshimoshi and boshiboshi showed that the formant tracing of CSL was not very accurate when the lower resonances associated with nasalised segments were measured. In acoustic analysis, the data we deal with is not a direct record of human voice, but an artefact of the transformation through the equipment we use. Although this study has achieved an over $90 \%$ successful discrimination rate, it is thus very important for the experts to be aware of the potential error in measurements which the speech analysis package may impose. Furthermore, the use of speech analysis software should be consistent across the speech samples to be compared. 


\subsection{FUTURE TASKS}

Although this study found that the likelihood ratio-based distance approach is an effective and practical approach for forensic speaker identification, many aspects of this approach remain for further research and testing.

First of all, the formula used in the likelihood ratio calculation in this study was not entirely appropriate to be applied to speech data. The formula of the likelihood ratio used was developed for data where the difference in the occasions of the measurements has no effect on their values, such as reflective indices of glass, and the flexible human voice is clearly not one of those data Furthermore, Aitkin's formula assumes that the data in question are normally distributed. It has been demonstrated, however, that some of the parameters, namely olmid and $\mathrm{m} 2$, do not have normal distribution. This formula thus needs some modification with respect to these shortcomings for the use of speech data. This, however, suggests that there is a possibility of further improvement in the accuracy of likelihood ratio-based forensic speaker identification, which is an exciting thought. As for the actual modification of the formula, however, this is clearly beyond a phonetician's expertise. Collaboration with statisticians is thus essential to improve the likelihood ratio.

The small size of the speaker pool in this study also leads to a further research task. It is not yet established that this result is applicable to the speaker groups with different backgrounds in terms of their socio-economic and / or age groups. One way to estimate the applicability of this result to the general population is in the comparison of the mean and standard deviation of the speaker pool in this study to those of a larger datasets. If the mean and standard deviation of this study are found to be similar to those of the larger datasets, we can assume that this approach will work with a larger and more general population. 


\section{APPENDICES}

\section{PROCEDURE}

Appendix 3.1 Map and information sheet for task
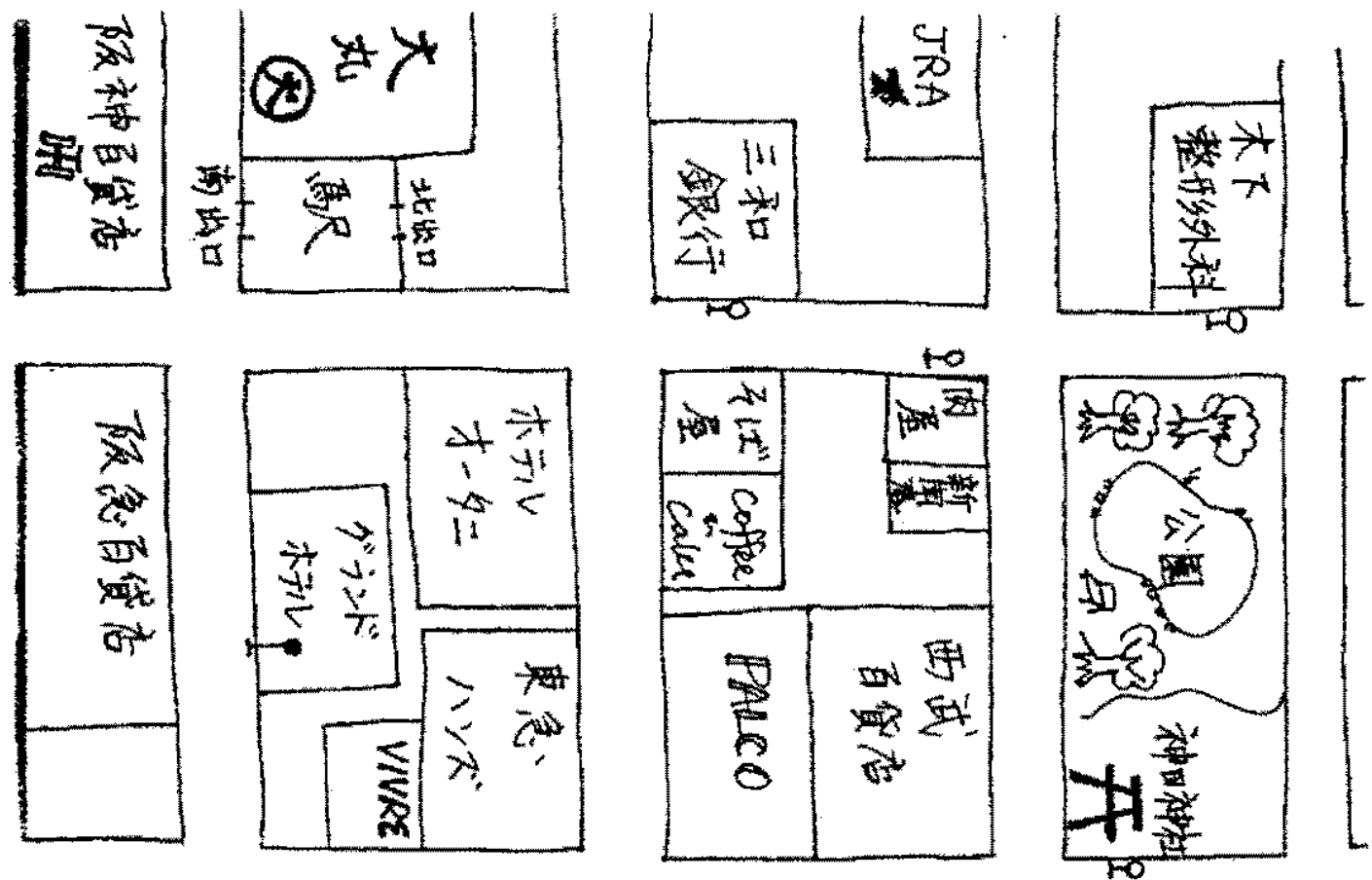

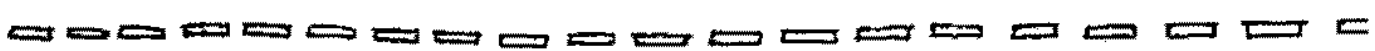
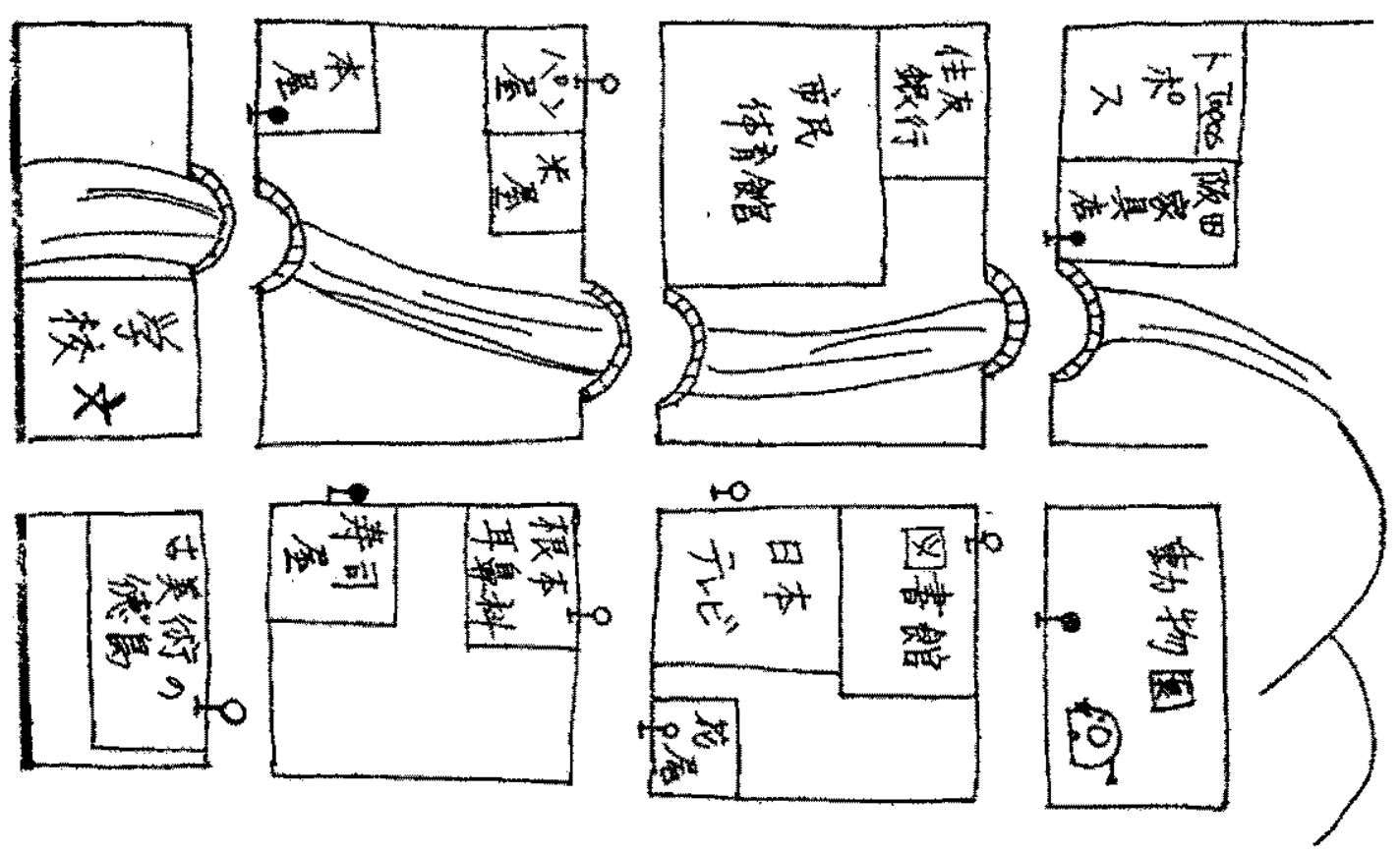
N

II

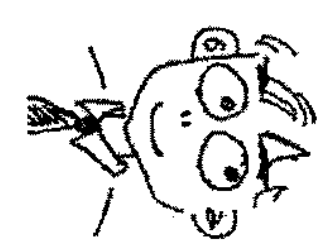

하

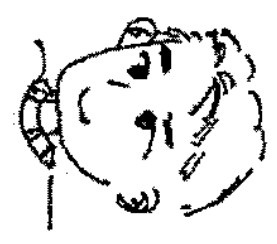

田 神
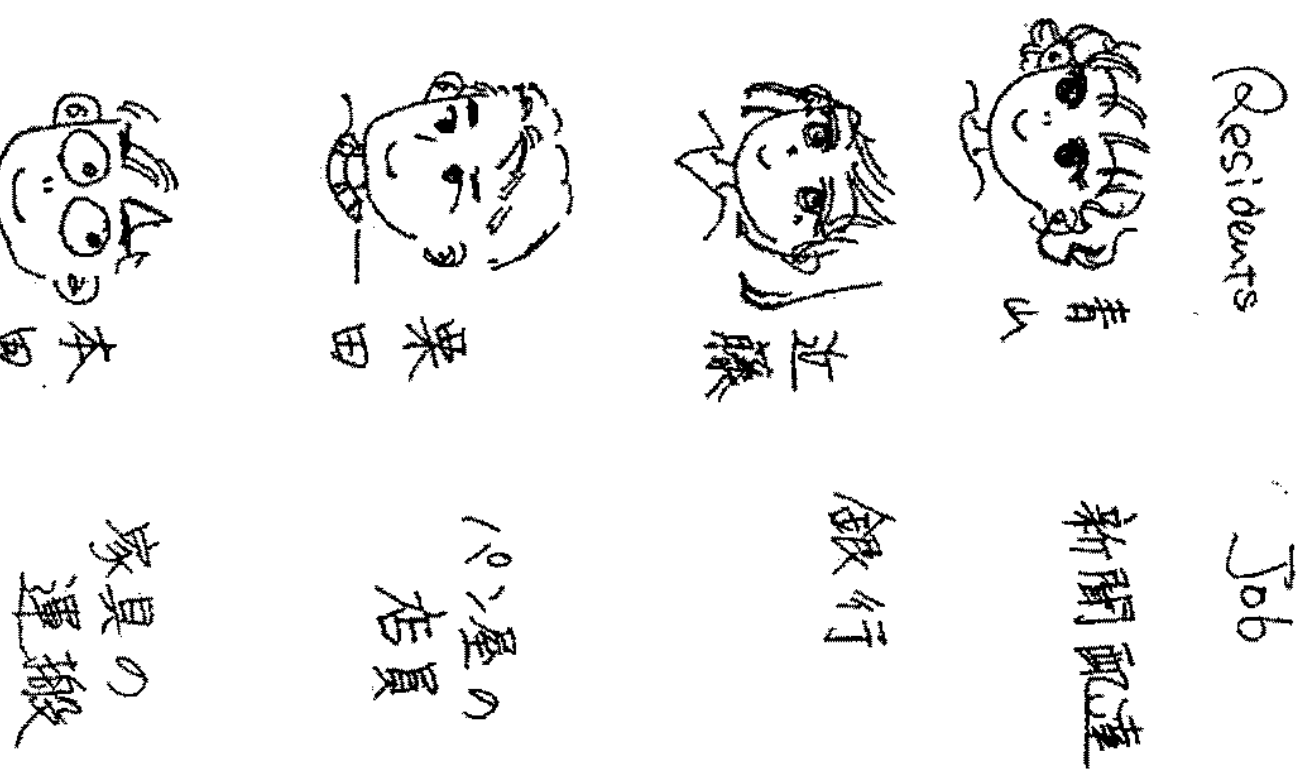

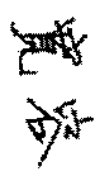

त्य
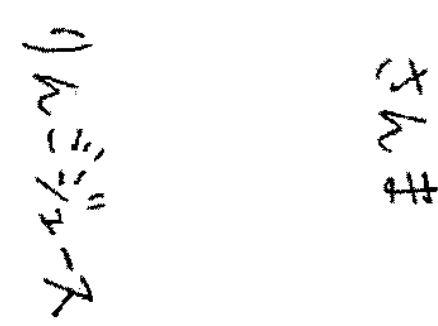

4
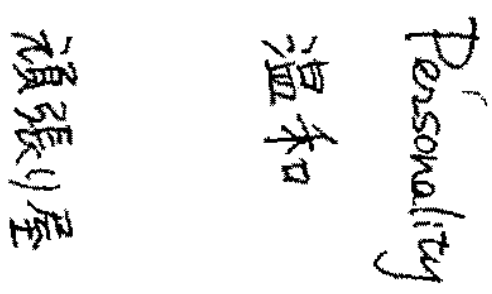

$\sum_{i=\frac{8}{8}}^{\frac{g}{g}}$ 


\section{VOWEL FORMANTS}

Appendix 4.1 Between-speaker comparisons of the rate of illegal devoicing incidence. 11 speakers makes 55 possible combinations, and 2 recording sessions make 4 different comparisons per speaker combination. The combinations which showed no between-speaker variation in devoicing rates are indicated by shading.

[Session 1 vs. Session 1]

\begin{tabular}{|c|c|c|c|}
\hline Speaker & Session 1 & Speaker & Session 1 \\
\hline $\mathrm{AA}$ & 25 & $\mathrm{HA}$ & 100 \\
\hline $\mathrm{AA}$ & 25 & $\mathrm{JN}$ & 100 \\
\hline $\mathrm{AA}$ & 25 & KA & 100 \\
\hline $\mathrm{AA}$ & 25 & $\mathrm{KF}$ & 50 \\
\hline $\mathrm{AA}$ & 25 & $\mathrm{KH}$ & 0 \\
\hline $\mathrm{AA}$ & 25 & $\mathrm{KO}$ & 50 \\
\hline $\mathrm{AA}$ & 25 & $\mathrm{MN}$ & 0 \\
\hline $\mathrm{AA}$ & 25 & TN & 75 \\
\hline $\mathrm{AA}$ & 25 & TS & 100 \\
\hline $\mathrm{AA}$ & 25 & TY & 75 \\
\hline $\mathrm{HA}$ & 25 & $\mathrm{IN}$ & 100 \\
\hline $\mathrm{NIA}$ & 100 & KaA & 100 \\
\hline $\mathrm{HA}$ & 100 & $\mathrm{KF}$ & 50 \\
\hline $\mathrm{HA}$ & 100 & $\mathrm{KH}$ & 0 \\
\hline $\mathrm{HA}$ & 100 & $\mathrm{KO}$ & 50 \\
\hline $\mathrm{HA}$ & 100 & $\mathrm{MN}$ & 0 \\
\hline $\mathrm{HA}$ & 100 & TN & 75 \\
\hline IX & 100 & WTS & 100 \\
\hline $\mathrm{HA}$ & 100 & TY & 75 \\
\hline IN & P 100 & $\mathrm{KA}$ & 2100 \\
\hline $\mathrm{JN}$ & 100 & $\mathrm{KF}$ & 50 \\
\hline $\mathrm{JN}$ & 100 & $\mathrm{KH}$ & 0 \\
\hline $\mathrm{JN}$ & 100 & $\mathrm{KO}$ & 50 \\
\hline $\mathrm{JN}$ & 100 & $\mathrm{MN}$ & 0 \\
\hline $\mathrm{JN}$ & 100 & $\mathrm{TN}$ & 75 \\
\hline$=2 \mathrm{~T}$ & 100 & Th & 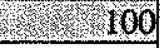 \\
\hline $\mathrm{N}$ & 100 & TY & 75 \\
\hline
\end{tabular}

\begin{tabular}{|c|c|c|c|}
\hline Speaker & Session 1 & Speaker & Session1 \\
\hline $\mathrm{KA}$ & 100 & $\mathrm{KF}$ & 50 \\
\hline $\mathrm{KA}$ & 100 & $\mathrm{KH}$ & 0 \\
\hline $\mathrm{KA}$ & 100 & $\mathrm{KO}$ & 50 \\
\hline $\mathbf{K A}$ & 100 & MN & 0 \\
\hline $\mathrm{KA}$ & 100 & TN & 75 \\
\hline KA & 100 & ThS & 100 \\
\hline $\mathrm{KA}$ & 100 & TY & 75 \\
\hline $\mathrm{KF}$ & 50 & $\mathrm{KH}$ & 0 \\
\hline KF & r & $\mathrm{KO}$ & 50 \\
\hline $\mathrm{KF}$ & 50 & MN & 0 \\
\hline $\mathrm{KF}$ & 50 & $\mathrm{TN}$ & 75 \\
\hline $\mathbf{K F}$ & 50 & TS & 100 \\
\hline $\mathrm{KF}$ & 50 & $\mathrm{TY}$ & 75 \\
\hline $\mathbf{K H}$ & 0 & KO & 50 \\
\hline $\mathbf{K H}$ & 0 & MN & 0 \\
\hline $\mathrm{KH}$ & 0 & TN & 75 \\
\hline $\mathrm{KH}$ & 0 & TS & 100 \\
\hline $\mathrm{KH}$ & 0 & TY & 75 \\
\hline KO & 50 & $\mathrm{MN}$ & 0 \\
\hline KO & 50 & TN & 75 \\
\hline KO & 50 & TS & 100 \\
\hline KO & 50 & TY & 75 \\
\hline $\mathrm{MN}$ & 0 & TN & 75 \\
\hline MN & 0 & TS & 100 \\
\hline MN & 0 & TY & 75 \\
\hline TN & 75 & TS & 100 \\
\hline TN & ris & TY & 95 \\
\hline TS & 100 & TY & 75 \\
\hline
\end{tabular}


[Session 1 vs. Session 2]

\begin{tabular}{|c|c|c|c|c|c|c|c|}
\hline Speaker & Session 1 & Speaker & Session 2 & Speaker & Session 1 & Speaker & Session 2 \\
\hline $\mathrm{AA}$ & 25 & $\mathrm{HA}$ & 100 & $\mathrm{KA}$ & 100 & $\mathrm{KF}$ & 75 \\
\hline $\mathrm{AA}$ & 25 & $\mathrm{JN}$ & 75 & $\mathrm{KA}$ & 100 & $\mathrm{KH}$ & 0 \\
\hline $\mathrm{AA}$ & 25 & $\mathrm{KA}$ & 75 & $\mathrm{KA}$ & 100 & $\mathrm{KO}$ & 50 \\
\hline $\mathrm{AA}$ & 25 & $\mathrm{KF}$ & 75 & KA & 100 & MN & 0 \\
\hline AA & 25 & $\mathrm{KH}$ & 0 & $\mathrm{KA}$ & 100 & TN & 75 \\
\hline $\mathrm{AA}$ & 25 & $\mathrm{KO}$ & 50 & $\mathrm{KA}$ & 100 & TS & 75 \\
\hline $\mathrm{AA}$ & 25 & MN & 0 & $\mathrm{KA}$ & 100 & TY & 75 \\
\hline $\mathrm{AA}$ & 25 & $\mathrm{TN}$ & 75 & $\mathrm{KF}$ & 50 & $\mathrm{KH}$ & 0 \\
\hline $\mathrm{AA}$ & 25 & TS & 75 & KF & $5 \quad 50$ & $\mathrm{KO}$ & W \\
\hline $\mathrm{AA}$ & 25 & $\mathrm{TY}$ & 75 & $\mathrm{KF}$ & 50 & MN & 0 \\
\hline $\mathrm{HA}$ & 25 & $\mathrm{JN}$ & 75 & $\mathrm{KF}$ & 50 & TN & 75 \\
\hline HA & 100 & KA & 75 & $\mathrm{KF}$ & 50 & TS & 75 \\
\hline $\mathrm{HA}$ & 100 & $\mathrm{KF}$ & 75 & $\mathrm{KF}$ & 50 & TY & 75 \\
\hline $\mathrm{HA}$ & 100 & $\mathrm{KH}$ & 0 & $\mathrm{KH}$ & 0 & $\mathrm{KO}$ & 50 \\
\hline $\mathrm{HA}$ & 100 & $\mathrm{KO}$ & 50 & $\mathrm{KH}$ & 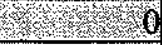 & $\mathrm{MN}$ & 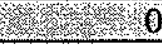 \\
\hline $\mathrm{HA}$ & 100 & $\mathrm{MN}$ & 0 & $\mathrm{KH}$ & 0 & TN & 75 \\
\hline $\mathrm{HA}$ & 100 & TN & 75 & $\mathrm{KH}$ & 0 & TS & 75 \\
\hline $\mathrm{HA}$ & 100 & TS & 75 & $\mathrm{KH}$ & 0 & TY & 75 \\
\hline $\mathrm{HA}$ & 100 & TY & 75 & $\mathrm{KO}$ & 50 & $\mathrm{MN}$ & 0 \\
\hline $\mathrm{JN}$ & 100 & $\mathrm{KA}$ & 75 & $\mathrm{KO}$ & 50 & TN & 75 \\
\hline JN & 100 & $\mathrm{KF}$ & 75 & $\mathrm{KO}$ & 50 & TS & 75 \\
\hline $\mathrm{JN}$ & 100 & $\mathrm{KH}$ & 0 & $\mathrm{KO}$ & 50 & $\mathrm{TY}$ & 75 \\
\hline $\mathrm{JN}$ & 100 & $\mathrm{KO}$ & 50 & $\mathrm{MN}$ & 0 & $\mathrm{TN}$ & 75 \\
\hline JN & 100 & $\mathrm{MN}$ & 0 & MN & 0 & TS & 75 \\
\hline $\mathrm{JN}$ & 100 & TN & 75 & MN & 0 & TY & 75 \\
\hline JN & 100 & TS & 75 & 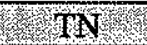 & 25) & TS & 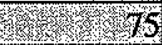 \\
\hline JN & 100 & TY & 75 & TiN & 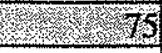 & XI & $\begin{array}{r}75 \\
\end{array}$ \\
\hline & & & & TS & 100 & TY & 75 \\
\hline
\end{tabular}


[Session 2 vs. Session 1]

\begin{tabular}{|c|c|c|c|}
\hline Speaker & Session 2 & Speaker & Session1 \\
\hline $\mathrm{AA}$ & 25 & HA & 100 \\
\hline $\mathrm{AA}$ & 25 & $\mathrm{JN}$ & 100 \\
\hline$\overline{\mathrm{AA}}$ & 25 & $\overline{\mathrm{KA}}$ & 100 \\
\hline $\mathrm{AA}$ & 25 & $\mathrm{KF}$ & 50 \\
\hline $\mathrm{AA}$ & 25 & $\mathrm{KH}$ & 0 \\
\hline $\mathrm{AA}$ & 25 & $\mathrm{KO}$ & 50 \\
\hline AA & 25 & MN & 0 \\
\hline $\mathrm{AA}$ & 25 & TN & 75 \\
\hline $\mathrm{AA}$ & 25 & TS & 100 \\
\hline $\mathbf{A A}$ & 25 & TY & 75 \\
\hline $\mathrm{HA}$ & 25 & $\mathrm{JN}$ & 100 \\
\hline $\mathrm{HA}$ & 100 & XA & 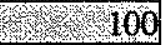 \\
\hline $\mathrm{HA}$ & 100 & $\mathrm{KF}$ & 50 \\
\hline $\mathrm{HA}$ & 100 & $\mathrm{KH}$ & 0 \\
\hline $\mathrm{HA}$ & 100 & $\mathrm{KO}$ & 50 \\
\hline $\mathrm{HA}$ & 100 & MN & 0 \\
\hline $\mathrm{HA}$ & 100 & $\mathrm{TN}$ & 75 \\
\hline $\mathrm{HA}$ & 100 & TS & 100 \\
\hline $\mathrm{HA}$ & 100 & TY & 75 \\
\hline $\mathrm{JN}$ & 75 & $\mathrm{KA}$ & 100 \\
\hline JN & 75 & $\mathrm{KF}$ & 50 \\
\hline JN & 75 & $\mathrm{KH}$ & 0 \\
\hline $\mathrm{JN}$ & 75 & KO & 50 \\
\hline $\mathrm{JN}$ & 75 & MN & 0 \\
\hline $\mathbb{N} N$ & 75 & $\mathbb{1 N}$ & 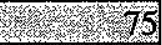 \\
\hline $\mathrm{JN}$ & 75 & TS & 100 \\
\hline $\mathbf{N}$ & 75 & TX & 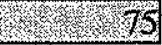 \\
\hline
\end{tabular}

\begin{tabular}{|c|c|c|c|}
\hline Speaker & Session 2 & Speaker & Session1 \\
\hline $\mathrm{KA}$ & 75 & $\mathrm{KF}$ & 50 \\
\hline $\mathrm{KA}$ & 75 & $\mathrm{KH}$ & 0 \\
\hline $\mathrm{KA}$ & 75 & KO & 50 \\
\hline $\mathrm{KA}$ & 75 & $\mathrm{MN}$ & 0 \\
\hline $\mathrm{KA}$ & W & $\mathrm{nN}$ & 75 \\
\hline $\mathrm{KA}$ & 75 & TS & 100 \\
\hline KA & 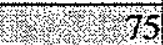 & TYY & 75 \\
\hline $\mathrm{KF}$ & 25 & $\mathrm{KH}$ & 0 \\
\hline $\mathrm{KF}$ & 25 & $\mathrm{KO}$ & 50 \\
\hline $\mathrm{KF}$ & 25 & $\mathrm{MN}$ & 0 \\
\hline $\mathrm{KF}$ & 25 & TN & 75 \\
\hline $\mathrm{KF}$ & 25 & TS & 100 \\
\hline $\mathrm{KF}$ & 25 & TY & 75 \\
\hline $\mathrm{KH}$ & 0 & $\mathrm{KO}$ & 50 \\
\hline Ka & 0 & $\mathrm{MN}$ & 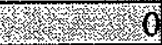 \\
\hline $\mathrm{KH}$ & 0 & $\mathrm{TN}$ & 75 \\
\hline $\mathrm{KH}$ & 0 & TS & 100 \\
\hline $\mathrm{KH}$ & 0 & TY & 75 \\
\hline $\mathrm{KO}$ & 50 & $\mathrm{MN}$ & 0 \\
\hline KO & 50 & TN & 75 \\
\hline $\mathrm{KO}$ & 50 & TS & 100 \\
\hline KO & 50 & TY & 7 \\
\hline MN & 0 & TN & 7. \\
\hline $\mathrm{MN}$ & 0 & TS & 100 \\
\hline MN & 0 & $\mathrm{TY}$ & 7 \\
\hline $\mathrm{TN}$ & 75 & TS & 100 \\
\hline TN & 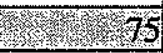 & $T$ & $\sqrt{75}$ \\
\hline TS & 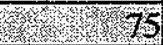 & TY & \\
\hline
\end{tabular}


[Session 2 vs. Session 2]

\begin{tabular}{|c|c|c|c|}
\hline Speaker & Session 1 & Speaker & Session1 \\
\hline AA & 25 & $\mathrm{HA}$ & 100 \\
\hline AA & 25 & $\mathrm{JN}$ & 75 \\
\hline $\mathrm{AA}$ & 25 & $\mathrm{KA}$ & 75 \\
\hline $\mathrm{AA}$ & 25 & $\mathrm{KF}$ & 75 \\
\hline $\mathrm{AA}$ & 25 & $\mathrm{KH}$ & 0 \\
\hline $\mathrm{AA}$ & 25 & $\mathrm{KO}$ & 50 \\
\hline AA & 25 & $\mathrm{MN}$ & 0 \\
\hline $\mathrm{AA}$ & 25 & TN & 75 \\
\hline A.A & 25 & rs & 75 \\
\hline $\mathrm{AA}$ & 25 & TY & 75 \\
\hline $\mathrm{HA}$ & 25 & $\mathrm{JN}$ & 75 \\
\hline $\mathrm{HA}$ & 100 & KA & 75 \\
\hline HA & 100 & $\mathrm{KF}$ & 75 \\
\hline $\mathrm{HA}$ & 100 & $\mathrm{KH}$ & 0 \\
\hline $\mathrm{HA}$ & 100 & $\mathrm{KO}$ & 50 \\
\hline $\mathrm{HA}$ & 100 & MN & 0 \\
\hline $\mathrm{HA}$ & 100 & TN & 75 \\
\hline $\mathrm{HA}$ & 100 & TS & 75 \\
\hline $\mathrm{HA}$ & 100 & TY & 75 \\
\hline 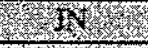 & 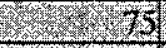 & $\mathrm{K}_{\mathrm{K}}$ & $4 x$ \\
\hline $2 x$ & $y-2 x=75$ & KF & 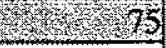 \\
\hline $\mathrm{JN}$ & 75 & $\mathrm{KH}$ & 0 \\
\hline IN & 75 & $\mathrm{KO}$ & 50 \\
\hline JN & 75 & $\mathrm{MN}$ & 0 \\
\hline N N & 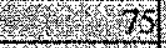 & N T & 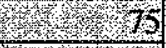 \\
\hline (1) & 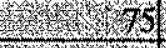 & S & - \\
\hline 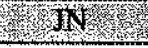 & 7ty & ST & 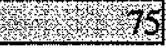 \\
\hline
\end{tabular}

\begin{tabular}{|c|c|c|c|}
\hline Speaker & Session 1 & Speaker & Session1 \\
\hline $\mathrm{K}$ & -5 & S & 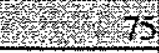 \\
\hline $\mathrm{KA}$ & 75 & $\mathrm{KH}$ & 0 \\
\hline $\mathrm{KA}$ & 75 & KO & 50 \\
\hline $\mathrm{KA}$ & 75 & MN & 0 \\
\hline Kt & S & STN & 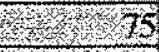 \\
\hline $8 \%$ & 4 & $4 \mathrm{TS}$ & Herar \\
\hline $\mathrm{Kat}$ & 35 & IV & 45 \\
\hline $\mathrm{KF}$ & 25 & $\mathrm{KH}$ & 0 \\
\hline $\mathrm{KF}$ & 25 & KO & 50 \\
\hline $\mathrm{KF}$ & 25 & $\mathrm{MN}$ & 0 \\
\hline $\mathrm{KF}$ & 25 & TN & 75 \\
\hline $\mathrm{KF}$ & 25 & TS & 75 \\
\hline $\mathrm{KF}$ & 25 & $\mathrm{TY}$ & 75 \\
\hline $\mathrm{KH}$ & 0 & KO & 50 \\
\hline $\mathrm{KH}$ & 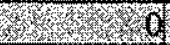 & $\mathrm{NN}$ & 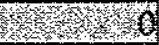 \\
\hline $\mathrm{KH}$ & 0 & $\mathrm{TN}$ & 75 \\
\hline $\mathrm{KH}$ & 0 & TS & 75 \\
\hline $\mathrm{KH}$ & 0 & TY & 75 \\
\hline Ko & 50 & $\mathrm{MN}$ & 0 \\
\hline KO & 50 & $\mathrm{TN}$ & 75 \\
\hline Ko & 50 & TS & 75 \\
\hline Ko & 50 & $\mathrm{TY}$ & 75 \\
\hline $\mathrm{MN}$ & 0 & $\mathrm{TN}$ & 75 \\
\hline $\mathrm{MN}$ & 0 & TS & 75 \\
\hline $\mathrm{MN}$ & 0 & $\mathrm{TY}$ & 75 \\
\hline TN & 等 & TS & 75 \\
\hline 鵄 & 浱 & WIY & 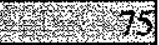 \\
\hline TS & Yer & Urix & 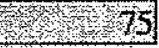 \\
\hline
\end{tabular}


Appendix 4.2 Each speaker's raw measurements for vowel formants.

[Speaker AA]

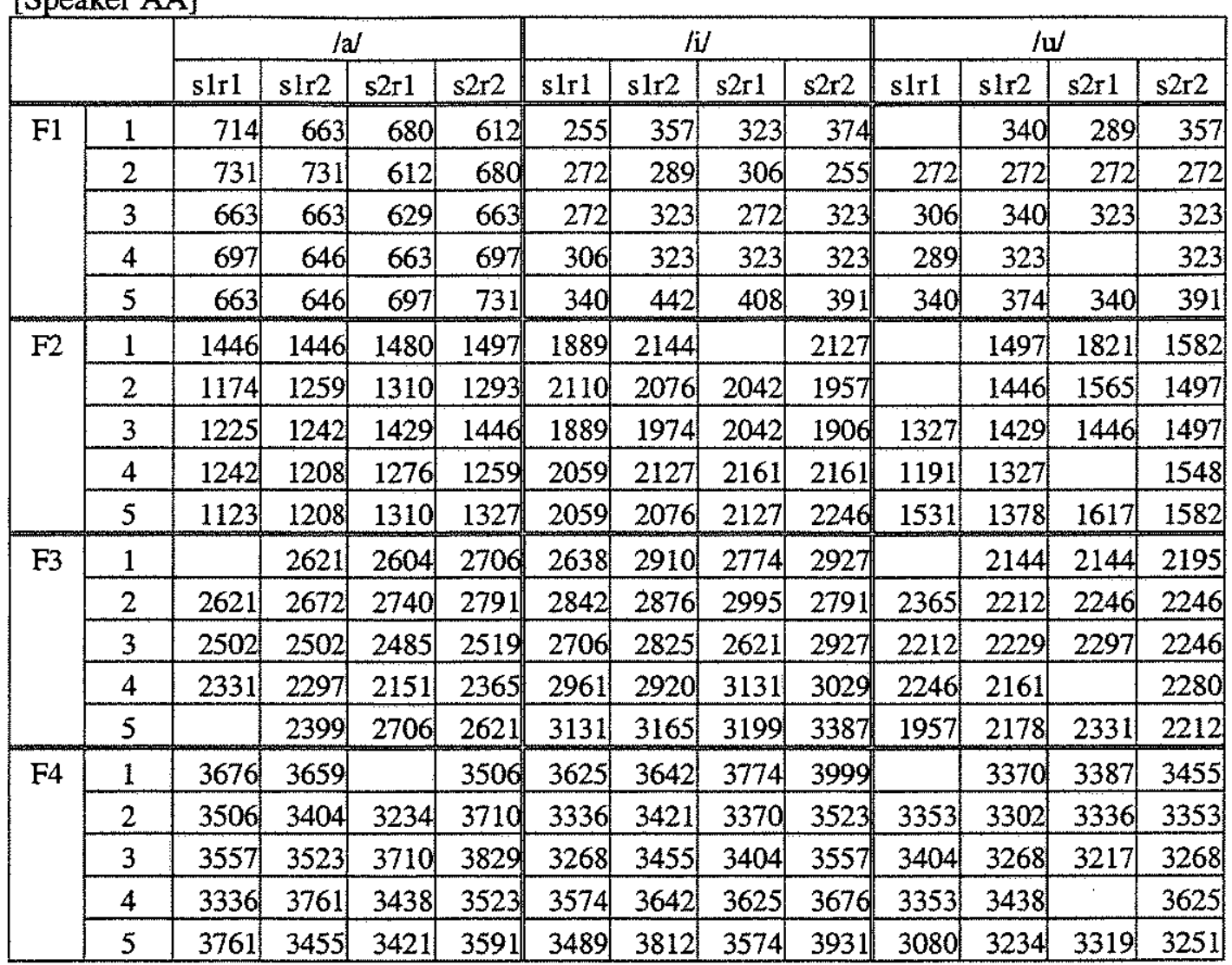

\begin{tabular}{|c|c|c|c|c|c|c|c|c|c|}
\hline & \multicolumn{4}{|c|}{ lel } & \multicolumn{4}{|c|}{$10 /$} \\
\hline & & sirl & $\sin 2$ & $\mathrm{~s} 2 \mathrm{r} 1$ & $s 2 \times 2$ & slr1 & slr1 & s1r2 & $\mathrm{s} 2 \mathrm{r} 1$ \\
\hline \multirow[t]{5}{*}{$\mathrm{F} 1$} & 1 & 544 & 442 & 544 & 476 & 493 & 527 & 476 & 578 \\
\hline & 2 & 510 & 493 & 476 & 476 & 459 & 459 & 493 & 476 \\
\hline & 3 & 425 & 459 & 476 & 425 & 408 & 442 & 459 & 459 \\
\hline & 4 & 459 & 442 & 459 & 476 & 527 & 731 & 578 & 595 \\
\hline & 5 & 391 & 374 & 357 & 425 & 459 & 442 & 425 & 544 \\
\hline \multirow[t]{5}{*}{$F 2$} & 1 & 1753 & 1940 & 1872 & 1991 & 919 & 1821 & 953 & 1038 \\
\hline & 2 & 1668 & 1838 & 1821 & 1923 & 1004 & 1140 & 1089 & 1157 \\
\hline & 3 & 1889 & 1889 & 2025 & 2008 & 1089 & 1225 & 1072 & 1208 \\
\hline & 4 & 1872 & 1957 & 1974 & 1974 & 731 & & 851 & \\
\hline & 5 & 1940 & 1923 & 1957 & 1974 & 982 & 919 & 885 & 1004 \\
\hline \multirow[t]{5}{*}{$\mathrm{F} 3$} & 1 & 2434 & 2604 & 2621 & 2655 & 2348 & 2451 & 2365 & 2212 \\
\hline & 2 & 2536 & 2570 & 2485 & 2740 & 2127 & 2178 & 2280 & 2246 \\
\hline & 3 & 2468 & 2553 & 2689 & 2553 & 2417 & 2587 & 2570 & 2417 \\
\hline & 4 & 2399 & 2502 & 2689 & 2570 & 2042 & 2621 & 2331 & 2161 \\
\hline & 5 & 2519 & 2689 & 2689 & 2570 & 2399 & 2365 & 2246 & 2417 \\
\hline \multirow[t]{5}{*}{ F4 } & 1 & 3404 & 3625 & 3642 & 3744 & 3114 & 3234 & 3421 & 2672 \\
\hline & 2 & 3693 & 3727 & 3625 & 3778 & 3097 & 3217 & 3114 & 3131 \\
\hline & 3 & 3438 & 3489 & 3914 & 3608 & 3251 & 3421 & 3251 & 3319 \\
\hline & 4 & 3302 & 3404 & 3642 & 3642 & 3165 & 3302 & 3302 & 3302 \\
\hline & 5 & 3285 & 3421 & 3489 & 3608 & 3029 & 3165 & 3182 & 3234 \\
\hline
\end{tabular}


[Speaker HA]

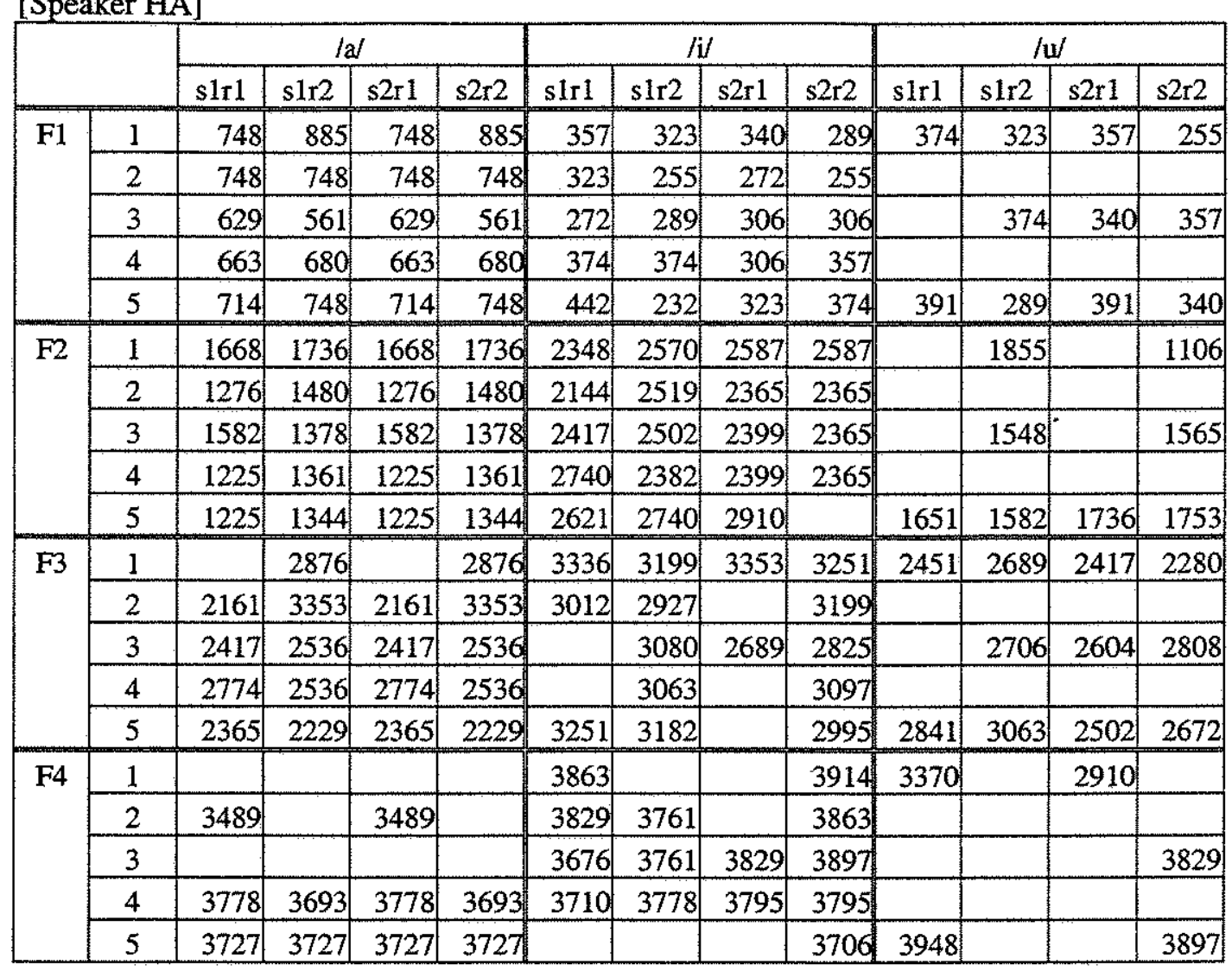

\begin{tabular}{|c|c|c|c|c|c|c|c|c|c|}
\hline & \multicolumn{4}{|c|}{ le/ } & \multicolumn{4}{|c|}{$10 /$} \\
\hline & & slr1 & $\operatorname{sir2}$ & $\mathrm{s} 2 \mathrm{r} 1$ & $s 2 r 2$ & s1r1 & $\operatorname{slr} 2$ & $\mathrm{~s} 2 \mathrm{r} 1$ & $\mathrm{~s} 2 \mathrm{r} 2$ \\
\hline \multirow[t]{5}{*}{ F1 } & 1 & 595 & 493 & 510 & 493 & 585 & 595 & 510 & 561 \\
\hline & 2 & 408 & 459 & 425 & 459 & 493 & 459 & 442 & 459 \\
\hline & 3 & 408 & 408 & 442 & 425 & & 476 & 425 & 391 \\
\hline & 4 & 408 & 442 & 442 & 442 & 753 & 510 & 544 & 527 \\
\hline & 5 & 442 & 408 & 408 & 408 & 357 & 459 & 527 & 442 \\
\hline \multirow[t]{5}{*}{$\mathrm{F} 2$} & 1 & 2008 & 2059 & 2110 & 2161 & & 1174 & 1157 & 1174 \\
\hline & 2 & 2059 & 2263 & 2212 & 2178 & 1242 & 1089 & 1038 & 1140 \\
\hline & 3 & 2365 & 2246 & 2178 & 2093 & 906 & 1242 & 1089 & 1174 \\
\hline & 4 & 2280 & 2229 & 2076 & 2093 & & 919 & 1038 & 1038 \\
\hline & 5 & 2348 & 2382 & 2297 & 2246 & 1089 & 953 & 834 & 902 \\
\hline \multirow[t]{5}{*}{ F3 } & 1 & 2944 & 2910 & 2893 & 2978 & 3080 & 2399 & 2468 & 2331 \\
\hline & 2 & 2893 & & 2876 & 2893 & & 2502 & 2076 & 2263 \\
\hline & 3 & 3064 & 2995 & 2893 & 2978 & 3178 & 2672 & 2587 & 2944 \\
\hline & 4 & 2961 & 2910 & 2961 & 2927 & 2980 & 2910 & 2553 & 2485 \\
\hline & 5 & 2961 & 2995 & 2842 & 2910 & 2961 & 2825 & 2553 & 2723 \\
\hline \multirow[t]{5}{*}{ F4 } & 1 & & 3829 & 3829 & 4000 & & 3438 & 3489 & 3438 \\
\hline & 2 & & 3961 & 3961 & 3982 & & 3353 & & 3302 \\
\hline & 3 & & & & & & 3999 & 3591 & \\
\hline & 4 & & 3914 & 3914 & & & 3812 & 3812 & 3693 \\
\hline & 5 & & 3982 & 3982 & 3795 & 3880 & 3829 & 3659 & 3659 \\
\hline
\end{tabular}


[Speaker JN]

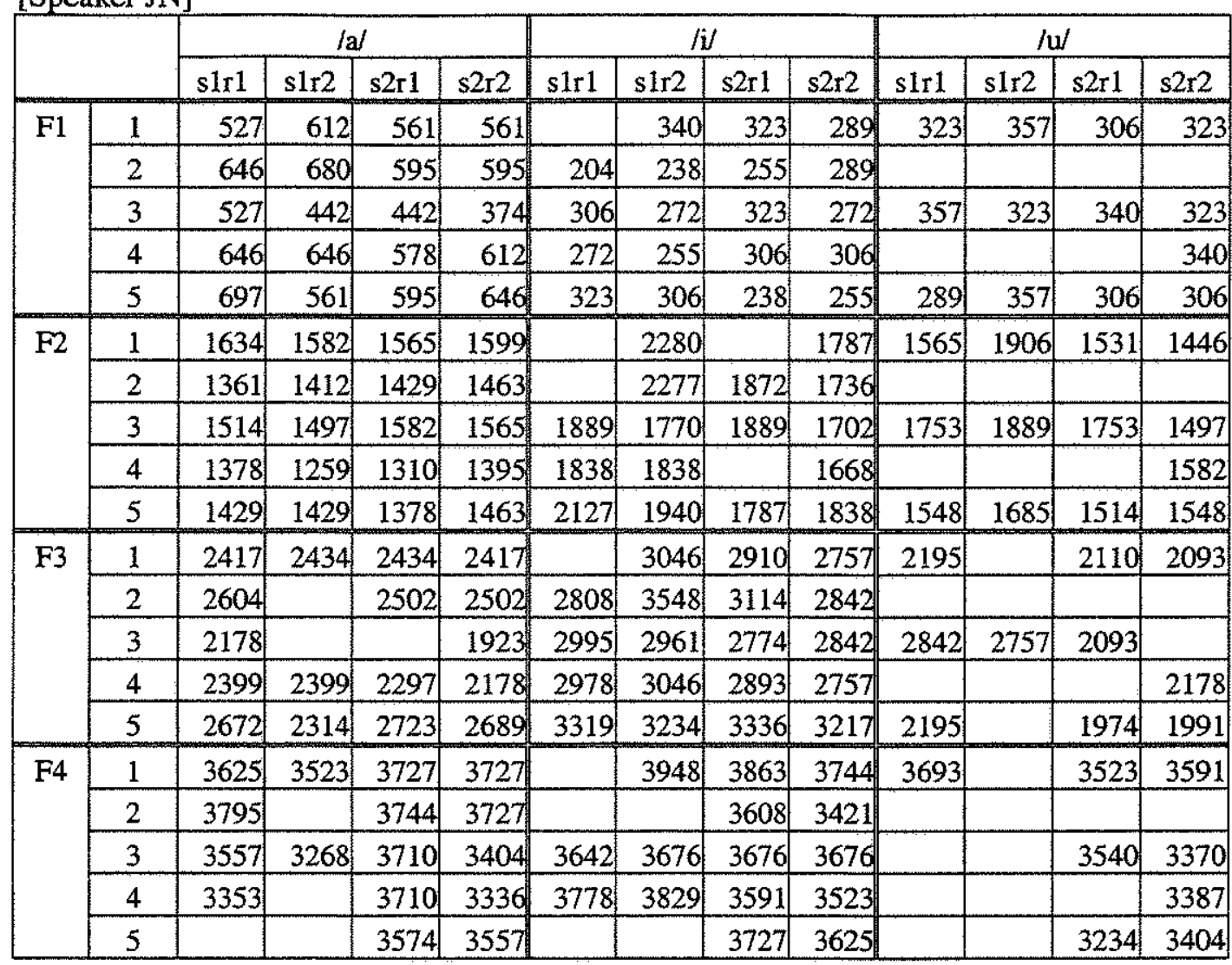

\begin{tabular}{|c|c|c|c|c|c|c|c|c|c|}
\hline & \multicolumn{4}{|c|}{$/ \mathrm{e} /$} & \multicolumn{4}{|c|}{101} \\
\hline & & slr1 & $\sin 2$ & s2r1 & $\mathrm{s} 2 \mathrm{r} 2$ & slr1 & $\mathrm{s} 1 \mathrm{r} 2$ & $s 2 \mathrm{r} 1$ & $\mathrm{~s} 2 \mathrm{r} 2$ \\
\hline \multirow[t]{5}{*}{ F1 } & 1 & 476 & 425 & 459 & 391 & 408 & 391 & 340 & 374 \\
\hline & 2 & 374 & 408 & 391 & 374 & 255 & 340 & 374 & 306 \\
\hline & 3 & 425 & 391 & 374 & 357 & 289 & 476 & 374 & 357 \\
\hline & 4 & 391 & 391 & 357 & 323 & 306 & 340 & 323 & 357 \\
\hline & 5 & 306 & 374 & 374 & 306 & 391 & 425 & 391 & 374 \\
\hline \multirow[t]{5}{*}{$\mathrm{F} 2$} & 1 & 1651 & 1753 & 1787 & 1668 & 1259 & 1463 & 1310 & 1446 \\
\hline & 2 & 1651 & 1685 & 1736 & 1582 & 1106 & 1089 & 1293 & 1514 \\
\hline & 3 & 1838 & 1787 & 1753 & 1804 & 1208 & 1208 & 1344 & 1225 \\
\hline & 4 & 1821 & 1634 & 1770 & 1957 & 1004 & 902 & 970 & 902 \\
\hline & 5 & 1872 & 1753 & 1821 & 1617 & 1021 & 1072 & 1072 & 1072 \\
\hline \multirow[t]{5}{*}{ F3 } & 1 & 2280 & 2825 & 2502 & 2468 & 2042 & & & 2246 \\
\hline & 2 & 2502 & 2825 & 2451 & 2451 & & 2468 & 2127 & \\
\hline & 3 & & 2791 & 2451 & 2365 & & 2757 & 2314 & 2195 \\
\hline & 4 & 2485 & & 2399 & 2399 & 2451 & 2161 & 2127 & 2280 \\
\hline & 5 & 2570 & 2502 & 2485 & 2434 & 2536 & & 2314 & 2127 \\
\hline \multirow[t]{5}{*}{ F4 } & 1 & 3557 & 3676 & 3540 & 3710 & 3557 & & 3744 & 3744 \\
\hline & 2 & 3591 & & 3523 & 3472 & 3370 & 3370 & 3438 & \\
\hline & 3 & 3574 & 3642 & 3591 & 3591 & 3557 & & 3438 & 3387 \\
\hline & 4 & 3540 & 3506 & 3591 & 3727 & 3642 & 3165 & & \\
\hline & 5 & 3421 & 3591 & 3523 & 3404 & 3506 & 3455 & 3812 & 3268 \\
\hline
\end{tabular}


[Speaker KA]

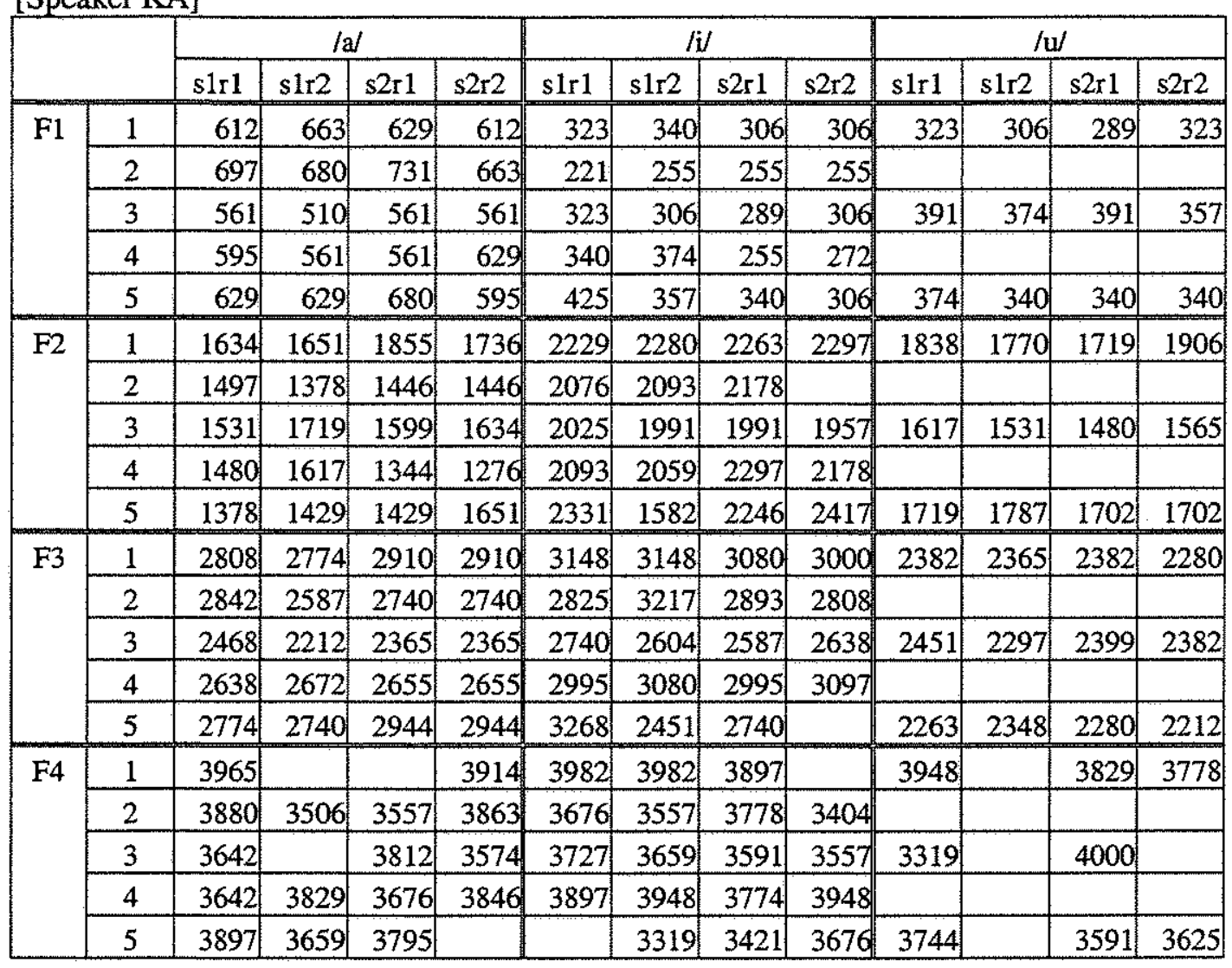

\begin{tabular}{|c|c|c|c|c|c|c|c|c|c|}
\hline & \multicolumn{4}{|c|}{ lel } & \multicolumn{4}{|c|}{$10 /$} \\
\hline & & slr1 & $\sin 2$ & $\mathrm{~s} 2 \mathrm{r} 1$ & $\mathrm{~s} 2 \mathrm{r} 2$ & slrl & s1r2 & $s 2 \mathrm{r} 1$ & $\mathrm{~s} 2 \mathrm{r} 2$ \\
\hline \multirow[t]{5}{*}{ F1 } & 1 & 442 & 493 & 510 & 510 & 459 & 510 & 476 & 510 \\
\hline & 2 & 408 & 476 & 459 & 408 & 459 & 442 & 493 & 476 \\
\hline & 3 & 374 & 391 & 408 & 323 & 425 & 408 & 425 & 442 \\
\hline & 4 & 357 & 357 & 357 & 391 & 476 & 391 & 527 & 476 \\
\hline & 5 & 391 & 374 & 357 & 391 & 408 & 425 & 476 & 476 \\
\hline \multirow[t]{5}{*}{ F2 } & 1 & 1957 & 1770 & 1753 & 1719 & 1259 & 1225 & 1463 & 1327 \\
\hline & 2 & 2008 & 2008 & 1940 & 1872 & 1004 & 987 & 1106 & 1123 \\
\hline & 3 & 2161 & 2093 & 2195 & 2076 & 1123 & 1191 & 1140 & 1174 \\
\hline & 4 & 2008 & 1889 & 2093 & 2059 & 936 & 902 & 987 & 1004 \\
\hline & 5 & 2110 & 2195 & 2178 & 2110 & 1565 & 765 & 919 & 970 \\
\hline \multirow[t]{5}{*}{ F3 } & 1 & 2638 & 2604 & 2468 & 2587 & 2944 & 2757 & 2587 & 2348 \\
\hline & 2 & 2791 & 2740 & 2638 & 2740 & 2399 & 2417 & 2382 & 2178 \\
\hline & 3 & 2638 & 2774 & 2791 & 2604 & 2774 & 2706 & 2740 & 2604 \\
\hline & 4 & 2331 & 2263 & 2553 & 2638 & 2893 & & 2791 & 2161 \\
\hline & 5 & 2757 & 2689 & 2689 & 2689 & 2910 & 2842 & 2808 & 2689 \\
\hline \multirow[t]{5}{*}{ F4 } & 1 & 3319 & 3863 & & 3778 & 3608 & 3557 & 3472 & 3574 \\
\hline & 2 & 3982 & 3948 & & 3880 & 3557 & 3472 & 3404 & 3387 \\
\hline & 3 & 3880 & 3864 & & 3846 & 3744 & 3676 & & 3574 \\
\hline & 4 & 3812 & & & 3761 & 3744 & 2944 & & 3012 \\
\hline & 5 & 3880 & 3761 & 3982 & 3693 & 3489 & & 3557 & 3506 \\
\hline
\end{tabular}


[Speaker KF]

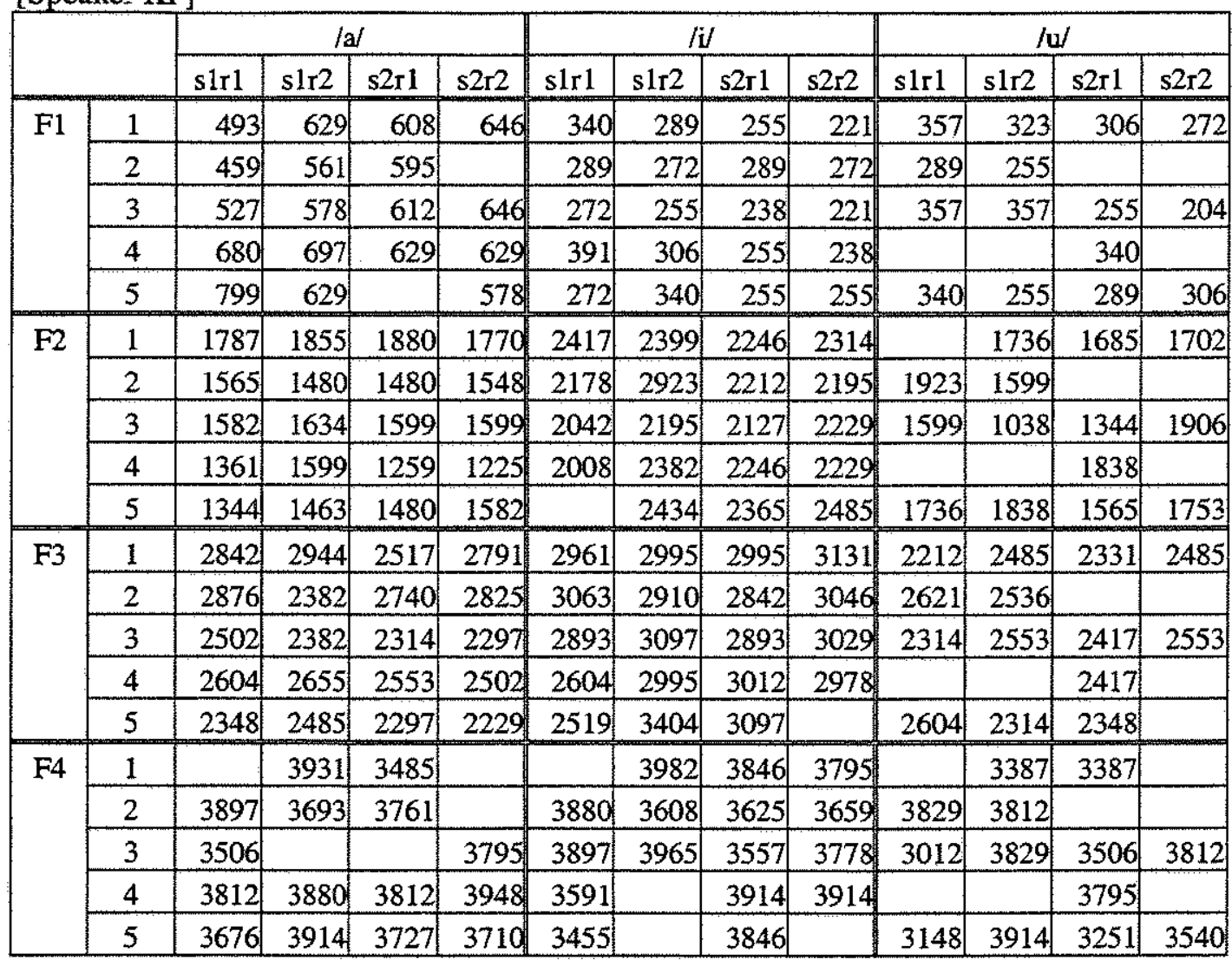

\begin{tabular}{|c|c|c|c|c|c|c|c|c|c|}
\hline & \multicolumn{4}{|c|}{ lel } & \multicolumn{4}{|c|}{$10 /$} \\
\hline & & slr1 & s1r2 & $s 2 \pi 1$ & $\mathrm{~s} 2 \mathrm{r} 2$ & s1r1 & s1r2 & $\mathrm{s} 2 \mathrm{r} 1$ & $\mathrm{~s} 2 \mathrm{r} 2$ \\
\hline \multirow[t]{5}{*}{$\mathrm{F} 1$} & 1 & 306 & 408 & 408 & 289 & 357 & 408 & 391 & 408 \\
\hline & 2 & 374 & 408 & 408 & 340 & 374 & 374 & 357 & 374 \\
\hline & 3 & 374 & 391 & 340 & 357 & 408 & 391 & 340 & 357 \\
\hline & 4 & 357 & 374 & 408 & 357 & 391 & 357 & 357 & 323 \\
\hline & 5 & 357 & 374 & 374 & 289 & 391 & 357 & 221 & 272 \\
\hline \multirow[t]{5}{*}{$\mathrm{F} 2$} & 1 & 2178 & 2178 & 2025 & 2093 & 1327 & 1378 & 1327 & 1327 \\
\hline & 2 & 2195 & 2042 & 1957 & 2127 & 1208 & 1174 & 1259 & 1344 \\
\hline & 3 & 2127 & 2280 & 1906 & 2195 & 1089 & 1157 & 1174 & 1361 \\
\hline & 4 & 2093 & 2093 & 2025 & 2059 & 1174 & 953 & 1038 & 1106 \\
\hline & 5 & 2297 & 2246 & 2144 & 2178 & 868 & 1106 & 1242 & 987 \\
\hline \multirow[t]{5}{*}{ F3 } & 1 & 2825 & 2893 & 2791 & 2740 & 2791 & 2417 & 2382 & 2331 \\
\hline & 2 & 2774 & 2655 & 2621 & 2706 & 2791 & & 2110 & 2297 \\
\hline & 3 & 2604 & 2791 & 2621 & 2740 & 2382 & 2553 & 2519 & 2399 \\
\hline & 4 & 2672 & 2655 & 2519 & 2655 & 2604 & 2570 & 2331 & 2502 \\
\hline & 5 & 2927 & 2706 & 2689 & 2655 & 2655 & 2791 & 2502 & 2365 \\
\hline \multirow[t]{5}{*}{ F4 } & 1 & & & 3540 & 3625 & 3761 & & & 3982 \\
\hline & 2 & & 3795 & 3710 & 3761 & & & 3421 & 3523 \\
\hline & 3 & & & 3710 & 3965 & 3812 & & 3336 & 3727 \\
\hline & 4 & & 3591 & 3659 & 3812 & & & 3676 & 3863 \\
\hline & 5 & & & 3594 & & 4000 & 3948 & 3114 & 3523 \\
\hline
\end{tabular}


[Speaker KH]

\begin{tabular}{|c|c|c|c|c|c|c|c|c|c|c|c|}
\hline & \multicolumn{2}{|c|}{$\mid \mathrm{a} /$} & \multicolumn{2}{|c|}{ /i } & \multicolumn{2}{|c|}{$/ \mathrm{w}$} & \multicolumn{2}{|c|}{ lel } & \multicolumn{2}{|c|}{101} \\
\hline & & $s 1$ & s2 & s1 & s2 & s1 & s2 & s1 & s2 & s1 & $s 2$ \\
\hline \multirow[t]{5}{*}{ F1 } & 1 & 663 & 748 & 238 & 340 & 323 & 340 & 255 & 612 & 268 & 204 \\
\hline & 2 & 680 & 799 & 255 & 289 & 306 & 323 & 442 & 459 & 391 & 459 \\
\hline & 3 & 646 & 561 & 238 & 289 & 289 & 357 & 374 & 408 & 442 & 442 \\
\hline & 4 & 646 & 612 & 289 & 306 & 323 & & 340 & 374 & 221 & 289 \\
\hline & 5 & 748 & 731 & 255 & 306 & 408 & 340 & 323 & 391 & 272 & 476 \\
\hline \multirow[t]{5}{*}{ F2 } & 1 & 1668 & 1719 & 2042 & 2008 & 1582 & 1889 & 1787 & 1940 & 1174 & 1531 \\
\hline & 2 & 1344 & 1565 & 2110 & 2161 & 1531 & 1702 & 1804 & 1991 & 970 & 1208 \\
\hline & 3 & 1446 & 1480 & 2076 & 2059 & 1429 & 1208 & 1940 & 1940 & 1208 & 1140 \\
\hline & 4 & 1327 & 1344 & 1940 & 2144 & 1719 & & 1872 & 1923 & 851 & 885 \\
\hline & 5 & 1344 & 1310 & 2093 & 2451 & 1685 & 1582 & 1991 & 2008 & 953 & 1055 \\
\hline \multirow[t]{5}{*}{ F3 } & 1 & 2740 & 2723 & 2825 & 2995 & 2382 & 2348 & 2655 & 2723 & 2655 & 2246 \\
\hline & 2 & 2297 & 2995 & 2638 & 2893 & 2587 & 2536 & 2638 & 2757 & 2178 & 2348 \\
\hline & 3 & 2280 & 2212 & 2791 & 2740 & 2485 & 2536 & 2604 & 2553 & 2587 & 2536 \\
\hline & 4 & 2434 & 2604 & 2757 & 2978 & 2485 & & 2536 & 2587 & 2331 & 2246 \\
\hline & 5 & 2587 & 2519 & 2808 & 3131 & 2706 & 2451 & 2519 & 2604 & & 2570 \\
\hline \multirow[t]{5}{*}{ F4 } & 1 & 3812 & 3999 & 3812 & 3812 & 3557 & & 3540 & 3982 & 3234 & 3438 \\
\hline & 2 & 2941 & 3829 & 3472 & 3795 & 3438 & 3557 & 3421 & 3914 & 3131 & 3285 \\
\hline & 3 & 3506 & 3863 & 3472 & 3812 & 3523 & 3710 & 3897 & 3965 & 3540 & 3659 \\
\hline & 4 & 3519 & 3812 & 3591 & 3846 & 3506 & & 3863 & 3931 & 3217 & \\
\hline & 5 & 3438 & 3625 & 3976 & 3982 & 3506 & 3574 & 3523 & 3659 & & 3693 \\
\hline
\end{tabular}

[Speaker KO]

\begin{tabular}{|c|c|c|c|c|c|c|c|c|c|c|c|c|c|c|c|c|}
\hline & \multicolumn{3}{|c|}{ la/ } & \multicolumn{3}{|c|}{ ii/ } & \multicolumn{3}{|c|}{$/ w^{\prime}$} & \multicolumn{3}{|c|}{ lel } & \multicolumn{3}{|c|}{$\mathrm{lo} /$} \\
\hline & & s1 & $\mathrm{s} 2 \mathrm{r} 1$ & $\mathrm{~s} 2 \mathrm{r} 1$ & sl & $s 2 \times 1$ & $\mathrm{~s} 2 \mathrm{r} 1$ & s1 & $\mathrm{s} 2 \mathrm{rl}$ & $\mathrm{s} 2 \mathrm{rl}$ & sI & $s 2 \mathrm{r} 1$ & $\mathrm{~s} 2 \mathrm{r} 1$ & s1 & $\mathrm{s} 2 \mathrm{r} 1$ & $\mathrm{~s} 2 \mathrm{r} 1$ \\
\hline \multirow[t]{5}{*}{$\mathrm{F} 1$} & 1 & 731 & 527 & 748 & 323 & 306 & 374 & 374 & & 306 & 272 & 408 & 493 & 493 & 374 & 476 \\
\hline & 2 & 731 & 868 & 527 & 272 & 306 & 323 & & 340 & & 408 & 425 & 476 & 442 & 476 & 442 \\
\hline & 3 & 578 & 493 & 561 & 340 & 306 & 306 & 340 & & 255 & 442 & 408 & 425 & 374 & 442 & 459 \\
\hline & 4 & 646 & 663 & 561 & 374 & 340 & 357 & 306 & & & 442 & 459 & 425 & 799 & 544 & 476 \\
\hline & 5 & 851 & 987 & 799 & 306 & 272 & 374 & 357 & 340 & 323 & 476 & 459 & 408 & 408 & 425 & 408 \\
\hline \multirow[t]{5}{*}{$\mathrm{F} 2$} & 1 & 1651 & 1634 & 1617 & 2059 & 1429 & 2093 & 1855 & & 1906 & 2055 & 1923 & 1991 & 1225 & 1395 & 1276 \\
\hline & 2 & 1361 & 1327 & 1446 & 2127 & 2093 & 2008 & & 1855 & & 1906 & 1804 & 1719 & 1174 & 1106 & 1344 \\
\hline & 3 & 1463 & 1599 & 1514 & 1804 & 1957 & 1855 & 1259 & & 1753 & 1889 & 1957 & 1940 & 1174 & 1123 & 1089 \\
\hline & 4 & 1344 & 1242 & 1259 & 1974 & 1906 & 2025 & 1838 & & & 1889 & 1923 & 1889 & 1361 & 919 & 936 \\
\hline & 5 & 1412 & 1310 & 1293 & 2144 & & 2229 & 1753 & 1804 & 1821 & 1974 & 1974 & 2008 & 817 & 970 & 970 \\
\hline \multirow[t]{5}{*}{ F3 } & 1 & 2195 & 2706 & 2927 & 2791 & 2706 & 2859 & 2553 & & 2212 & 2638 & 2774 & 2808 & 2468 & 2468 & 2995 \\
\hline & 2 & 2944 & 2417 & 2399 & 2655 & 2944 & 2961 & & 2195 & & 2655 & 2655 & 2740 & 2451 & 2331 & 2434 \\
\hline & 3 & 2774 & 2536 & 2485 & 2553 & 2451 & 2672 & 2417 & & 2348 & 2706 & 2553 & 2655 & 2842 & 2859 & 2808 \\
\hline & 4 & 2502 & 2502 & 2468 & 2791 & 2774 & 2842 & 2842 & & & 2723 & 2638 & 2655 & 3012 & 2382 & 2451 \\
\hline & 5 & 2314 & 2434 & 2263 & 2995 & 2750 & 2944 & 2178 & 2178 & 2212 & 2689 & 2825 & 2655 & 2621 & 2723 & 2672 \\
\hline \multirow[t]{5}{*}{ F4 } & 1 & 3948 & 3778 & 3778 & 3812 & 3914 & 3829 & 3557 & & 3131 & 3778 & 3914 & 3863 & 3489 & 3540 & 3608 \\
\hline & 2 & 3625 & 3506 & 3693 & 3642 & 3608 & 3591 & & 3165 & & 3727 & 3744 & 3744 & 3336 & 3302 & 3148 \\
\hline & 3 & 3761 & 3829 & 3795 & 3523 & 3353 & 3608 & 3744 & & 3234 & 3693 & 3591 & 3608 & 3523 & 3489 & 3642 \\
\hline & 4 & 3472 & 3574 & 3523 & 3659 & 3642 & 3693 & 3557 & & & 3608 & 3489 & 3625 & 3608 & 3472 & 3097 \\
\hline & 5 & 3591 & 3795 & 3676 & 3727 & 3731 & 3659 & 3523 & 3199 & 3199 & 3421 & 3591 & 3438 & 3472 & 3506 & 3523 \\
\hline
\end{tabular}


[Speaker MN]

\begin{tabular}{|c|c|c|c|c|c|c|c|c|c|c|c|c|c|}
\hline & \multicolumn{4}{|c|}{$\mid \mathrm{a} /$} & \multicolumn{4}{|c|}{ iv } & \multicolumn{4}{|c|}{$\mathrm{lu}$} \\
\hline & & slr1 & slr2 & $\mathrm{s} 2 \mathrm{r} 1$ & $\mathrm{~s} 2 \mathrm{r} 2$ & s1rl & slr2 & $\mathrm{s} 2 \mathrm{r} 1$ & $s 2+2$ & slr1 & slr2 & s2r1 & $s 2 \times 2$ \\
\hline \multirow[t]{5}{*}{ F1 } & 1 & & 680 & 748 & 799 & 306 & 357 & 374 & 493 & 391 & 374 & 357 & 374 \\
\hline & 2 & 765 & 782 & 799 & 868 & 323 & 340 & 340 & 391 & 391 & 425 & 391 & 408 \\
\hline & 3 & 765 & 663 & 714 & 799 & 357 & 340 & 323 & 340 & 408 & 391 & 408 & 442 \\
\hline & 4 & 714 & 697 & 748 & 748 & 306 & 340 & 340 & 340 & 425 & 459 & 476 & 493 \\
\hline & 5 & 834 & 714 & 834 & 799 & 357 & 374 & 459 & 422 & 408 & 408 & 357 & 391 \\
\hline \multirow[t]{5}{*}{$F 2$} & 1 & 1411 & 1702 & 1753 & 1753 & 2434 & 2485 & 2434 & 2451 & 1514 & 1463 & 1548 & 1634 \\
\hline & 2 & 1480 & 1395 & 1378 & 1361 & 2356 & 2468 & 2382 & 2076 & 1378 & 1429 & 1259 & 1344 \\
\hline & 3 & 1446 & 1497 & 1412 & 1361 & 2297 & 2341 & 2161 & 2127 & 1140 & 1327 & 1293 & 1191 \\
\hline & 4 & 1259 & 1259 & 1429 & 1310 & 2570 & 2399 & 2297 & 2434 & 1327 & 1651 & 2025 & 1906 \\
\hline & 5 & 1327 & 1327 & 1276 & 1293 & 2689 & 2621 & 2519 & 2536 & 1497 & 1531 & 1497 & 1412 \\
\hline \multirow[t]{5}{*}{ F3 } & 1 & 2961 & 2944 & 2808 & 2961 & 3199 & 3285 & 3268 & 3251 & 2356 & 2655 & 2570 & 2536 \\
\hline & 2 & 2757 & 3097 & 2859 & & 2825 & 3063 & 2910 & 3165 & 2740 & 2842 & 2672 & 2706 \\
\hline & 3 & 2893 & 2621 & 2689 & 2655 & 3012 & 2978 & 2842 & 3064 & 2944 & 2791 & 2706 & 2672 \\
\hline & 4 & 2672 & 2604 & 2672 & 2621 & 3336 & 3268 & 2825 & 3217 & 2740 & 2876 & 2740 & 2672 \\
\hline & 5 & 3080 & 3421 & 2910 & 2365 & 3268 & 3182 & 3251 & 3029 & 2417 & 2468 & 2706 & 2587 \\
\hline \multirow[t]{5}{*}{ F4 } & 1 & & & & & 3897 & 3795 & & & 3523 & 3574 & 3421 & 3438 \\
\hline & 2 & 3778 & 3812 & 3710 & 3846 & 3540 & 3523 & 3421 & & 3965 & 3591 & 3336 & 3472 \\
\hline & 3 & 3693 & 3761 & 3931 & 3846 & & 3421 & 3608 & 3574 & 3761 & 3574 & 3591 & 3982 \\
\hline & 4 & 3812 & 3948 & 3795 & 3897 & 3897 & & 3404 & & 3778 & & 3727 & 3523 \\
\hline & 5 & 3812 & 3812 & 3693 & 3897 & 3591 & 3557 & 3506 & 3421 & 3234 & 3438 & 3676 & 3251 \\
\hline
\end{tabular}

\begin{tabular}{|c|c|c|c|c|c|c|c|c|c|}
\hline & \multicolumn{4}{|c|}{ /el } & \multicolumn{4}{|c|}{ lod } \\
\hline & & slr1 & $\mathrm{s} 1 \mathrm{r} 2$ & $s 2 r 1$ & $s 2 \times 2$ & slr1 & $\sin 2$ & $\mathrm{~s} 2 \mathrm{r} 1$ & $\mathrm{~s} 2 \mathrm{r} 2$ \\
\hline \multirow[t]{5}{*}{$\mathrm{F} 1$} & 1 & 680 & 646 & 612 & 663 & 646 & 646 & 612 & 663 \\
\hline & 2 & 517 & 544 & 527 & 561 & 527 & 561 & 527 & 595 \\
\hline & 3 & 425 & 493 & 476 & 476 & 510 & 510 & 493 & 510 \\
\hline & 4 & 442 & 425 & 476 & 459 & 578 & 629 & 578 & 578 \\
\hline & 5 & 462 & 425 & 476 & 476 & 476 & 442 & 425 & 493 \\
\hline \multirow[t]{5}{*}{$F 2$} & 1 & 2042 & 2008 & 1906 & 1940 & 1344 & 1344 & 1395 & 1259 \\
\hline & 2 & 2069 & 2093 & 2042 & 2076 & 1106 & 1208 & 1208 & 1276 \\
\hline & 3 & 2348 & 2348 & 2178 & 2161 & 1191 & 1191 & 1089 & 1242 \\
\hline & 4 & 2399 & 2314 & 2076 & 2110 & 1106 & 987 & 1123 & 987 \\
\hline & 5 & 2287 & 2314 & 2212 & 2114 & 1123 & 1106 & 1123 & 987 \\
\hline \multirow[t]{5}{*}{ F3 } & 1 & 3046 & 2927 & 2706 & $287 \epsilon$ & 2961 & 2859 & 2655 & 2485 \\
\hline & 2 & 2968 & 2842 & 2808 & 2893 & 2553 & 2553 & 2604 & 2587 \\
\hline & 3 & 2910 & 2842 & 2825 & 2893 & 2808 & 2144 & 2740 & 2706 \\
\hline & 4 & 2995 & 2825 & 2689 & 2672 & 2348 & 2229 & 2468 & 2485 \\
\hline & 5 & 2859 & 2791 & 2723 & 2740 & 2655 & 2774 & 2740 & 2740 \\
\hline \multirow[t]{5}{*}{ F4 } & 1 & 3812 & 3897 & 3829 & & 3659 & 3761 & 3404 & 3455 \\
\hline & 2 & 3649 & 3608 & 3744 & & 3165 & 3114 & 3114 & 3131 \\
\hline & 3 & 3914 & 3659 & 3676 & & 3727 & 2923 & 3965 & \\
\hline & 4 & 3982 & 3625 & 3523 & & 3693 & 3795 & 3761 & 3982 \\
\hline & & 3921 & 3540 & 3574 & 3727 & 3608 & 3829 & & 3778 \\
\hline
\end{tabular}


[Speaker TN]

\begin{tabular}{|c|c|c|c|c|c|c|c|c|c|c|c|c|c|}
\hline & \multicolumn{4}{|c|}{$\mid \mathrm{a} /$} & \multicolumn{4}{|c|}{ ii } & \multicolumn{4}{|c|}{$h w^{\prime}$} \\
\hline & & slr1 & $\operatorname{sir2}$ & $\mathrm{s} 2 \mathrm{r} 1$ & $\mathrm{~s} 2 \mathrm{r} 2$ & s1r1 & $\operatorname{sir} 2$ & $\mathrm{~s} 2 \mathrm{rl}$ & $\mathrm{s} 2 \mathrm{r} 2$ & s1r1 & $\sin 2$ & $\mathrm{~s} 2 \mathrm{r} 1$ & $\mathrm{~s} 2 \mathrm{r} 2$ \\
\hline \multirow[t]{5}{*}{$\mathrm{F} 1$} & 1 & 765 & 680 & 663 & 697 & 238 & 221 & 221 & 221 & & & 238 & \\
\hline & 2 & 561 & 680 & 629 & 697 & 221 & 272 & 238 & 204 & 204 & 187 & 204 & 221 \\
\hline & 3 & 561 & 612 & 510 & 578 & 357 & 459 & 289 & 340 & & 459 & & \\
\hline & 4 & 663 & 663 & 680 & 799 & 204 & 255 & 306 & 221 & 187 & 221 & 255 & 289 \\
\hline & 5 & 1889 & 1787 & 1770 & 1736 & 2297 & 2229 & 2127 & 2212 & 2076 & 1821 & 2076 & 1719 \\
\hline \multirow[t]{5}{*}{$\mathrm{F} 2$} & 1 & 1531 & 1480 & 1565 & 1429 & 2127 & 2246 & 2178 & 2280 & & & 1140 & \\
\hline & 2 & 1378 & 1514 & 1480 & 1361 & 2042 & 2195 & 2076 & 2127 & 1208 & 902 & 1004 & 1463 \\
\hline & 3 & 1497 & 1582 & 1463 & 1446 & 1957 & 2110 & 2229 & 2161 & & 1685 & & \\
\hline & 4 & 1668 & 1548 & 1565 & 1463 & 2331 & 2348 & 2399 & 2246 & 1685 & 1395 & 1548 & 1497 \\
\hline & 5 & 2961 & 2723 & 2723 & 2825 & 3046 & 2646 & 2944 & 2944 & 2348 & 2468 & 2672 & 2399 \\
\hline \multirow[t]{5}{*}{ F3 } & 1 & 2842 & 2621 & 2689 & 2706 & 2655 & 2655 & 2553 & 3063 & & & 2451 & \\
\hline & 2 & 2365 & 2434 & 2502 & 2451 & 2757 & 2808 & 2638 & 2723 & 2417 & & 2485 & 2451 \\
\hline & 3 & 2451 & 2519 & 2553 & 2434 & 2502 & 2502 & 2825 & 2757 & & 2808 & & \\
\hline & 4 & 2604 & 2604 & 2774 & 2740 & 3131 & 3182 & 2961 & 3302 & 2519 & 2127 & 2246 & 2348 \\
\hline & 5 & & 3914 & 3897 & 3880 & 3982 & 3029 & 3812 & 3931 & 3608 & 3234 & 3608 & 3625 \\
\hline \multirow[t]{5}{*}{ F4 } & 1 & 3659 & 3863 & 3727 & 3659 & 3778 & 3353 & 3710 & 3710 & & & 3642 & \\
\hline & 2 & 3676 & 3472 & 3693 & 3523 & 3710 & 3659 & 3523 & 3455 & 3472 & & 3778 & 3710 \\
\hline & 3 & 3353 & 3489 & 3693 & 3455 & 3795 & 3251 & 3931 & 3523 & & 3676 & & \\
\hline & 4 & 3880 & 3863 & 3778 & 3676 & 3761 & 3846 & 3472 & 3778 & 3540 & 3012 & 3863 & 3234 \\
\hline & 5 & & & & & & & & & & & & \\
\hline
\end{tabular}

\begin{tabular}{|c|c|c|c|c|c|c|c|c|c|}
\hline & \multicolumn{4}{|c|}{$/ \mathrm{e} /$} & \multicolumn{4}{|c|}{$10 /$} \\
\hline & & slr1 & $\operatorname{sir} 2$ & $\mathrm{~s} 2 \mathrm{r} 1$ & $\$ 2 \pi 2$ & s1r1 & s1r2 & $\mathrm{s} 2 \mathrm{r} 1$ & $s 2 \mathrm{r} 2$ \\
\hline \multirow[t]{5}{*}{$\mathrm{F} 1$} & 1 & 374 & 476 & 408 & 306 & 476 & 663 & 306 & 357 \\
\hline & 2 & 374 & 476 & 357 & 357 & 391 & 612 & 289 & 323 \\
\hline & 3 & 306 & 510 & 374 & 357 & 357 & 544 & 238 & 255 \\
\hline & 4 & 340 & 442 & 306 & 357 & 391 & 629 & 357 & 340 \\
\hline & 5 & 2042 & 1974 & 2076 & 2008 & 1344 & 1344 & 1395 & 1259 \\
\hline \multirow[t]{5}{*}{$F 2$} & 1 & 2042 & 2042 & 1923 & 1957 & 1106 & 1208 & 1208 & 1276 \\
\hline & 2 & 2059 & 2246 & 2093 & 2127 & 1191 & 1191 & 1089 & 1242 \\
\hline & 3 & 2212 & 2212 & 2008 & 2059 & 1106 & 987 & 1123 & 987 \\
\hline & 4 & 2246 & 2263 & 2144 & 2144 & 1123 & 1106 & 1123 & 987 \\
\hline & 5 & 2723 & 2706 & 2774 & 2723 & 2961 & 2859 & 2655 & 2485 \\
\hline \multirow[t]{5}{*}{ F3 } & 1 & 2672 & 2655 & 2689 & 2621 & 2553 & 2553 & 2604 & 2587 \\
\hline & 2 & 2655 & 2604 & 2621 & 2570 & 2808 & 2144 & 2740 & 2706 \\
\hline & 3 & 2570 & 2570 & 2485 & 2502 & 2348 & 2229 & 2468 & 2485 \\
\hline & 4 & 2638 & 2587 & 2485 & 2587 & 2655 & 2774 & 2740 & 2740 \\
\hline & 5 & 3863 & 3897 & 3710 & 3812 & 3659 & 3761 & 3404 & 3455 \\
\hline \multirow[t]{5}{*}{ F4 } & 1 & 3812 & 3931 & 3812 & 3727 & 3165 & 3114 & 3114 & 3131 \\
\hline & 2 & 3931 & 4000 & 3846 & 3829 & 3727 & 2923 & 3965 & \\
\hline & 3 & 3846 & 3965 & 3931 & 3829 & 3693 & 3795 & 3761 & 3982 \\
\hline & 4 & 3812 & 3914 & 3812 & 3795 & 3608 & 3829 & & 3778 \\
\hline & 5 & 765 & 680 & 663 & 697 & 238 & 221 & 221 & 221 \\
\hline
\end{tabular}


[Speaker TS]

\begin{tabular}{|c|c|c|c|c|c|c|c|c|c|c|c|c|c|}
\hline & \multicolumn{4}{|c|}{$\mathrm{a} /$} & \multicolumn{4}{|c|}{ /i } & \multicolumn{4}{|c|}{$\mathrm{w}$} \\
\hline & & slr1 & $\operatorname{sir} 2$ & $\mathrm{~s} 2 \mathrm{r} 1$ & $\mathrm{~s} 2 \mathrm{r} 2$ & s1r1 & $\mathrm{s} 1 \mathrm{r} 2$ & $\mathrm{~s} 2 \mathrm{r} 1$ & $\mathrm{~s} 2 \mathrm{r} 2$ & s1r1 & $\operatorname{sir} 2$ & $\mathrm{~s} 2 \mathrm{r} 1$ & $\mathrm{~s} 2 \mathrm{r} 2$ \\
\hline \multirow[t]{5}{*}{ F1 } & 1 & 629 & 714 & 561 & 612 & 289 & 357 & 255 & 289 & 357 & 340 & 301 & 323 \\
\hline & 2 & 595 & 697 & & 663 & 340 & 289 & 204 & 204 & & & 238 & \\
\hline & 3 & 612 & 527 & 527 & & 289 & 306 & 255 & 289 & 323 & 340 & 272 & 306 \\
\hline & 4 & 612 & 629 & 595 & 595 & 340 & 340 & 289 & 272 & & & & \\
\hline & 5 & 629 & 595 & 578 & 527 & 357 & 391 & 255 & 340 & 357 & 340 & 306 & 238 \\
\hline \multirow[t]{5}{*}{ F2 } & 1 & 1497 & 1565 & 1736 & 1685 & 2144 & 2280 & 2178 & 2076 & 1531 & 1480 & 1719 & 1753 \\
\hline & 2 & 1174 & 1191 & 1157 & 1293 & 2059 & 2178 & 2212 & 2076 & & & 1838 & \\
\hline & 3 & 1327 & 1327 & 1327 & 1361 & 2127 & 2161 & 2144 & 2110 & 1293 & 1651 & 1497 & 1446 \\
\hline & 4 & 1140 & 1174 & 1208 & 1157 & 2297 & 2246 & 2246 & 2212 & & & & \\
\hline & 5 & 1123 & 1123 & 1106 & 1089 & 2314 & 2348 & 2161 & 2331 & 1480 & 1617 & 1719 & 1716 \\
\hline \multirow[t]{5}{*}{ F3 } & 1 & 2706 & 2638 & 2689 & 2604 & 2995 & 2927 & 3012 & 2995 & 2451 & 2485 & 2331 & \\
\hline & 2 & 2672 & 2570 & 2706 & 2297 & 2706 & 2825 & 2876 & 2842 & & & 2859 & \\
\hline & 3 & 2774 & 2621 & 2638 & 2553 & 3268 & 3029 & 2672 & 2740 & 2348 & 2399 & 2502 & 2757 \\
\hline & 4 & 2451 & 2485 & 2485 & 2621 & 3097 & 2825 & 2995 & 3029 & & & & \\
\hline & 5 & 2757 & 2859 & 2859 & 2757 & 3131 & 3268 & 2961 & 2825 & 2723 & 2468 & 2314 & 2638 \\
\hline \multirow[t]{5}{*}{ F4 } & 1 & & & & & 3591 & & & 3948 & & 3829 & 3761 & 3812 \\
\hline & 2 & 3744 & 3812 & 3812 & & & 3659 & 3914 & & & & 3625 & \\
\hline & 3 & 3863 & 4000 & 3948 & 3965 & 3880 & 3795 & 3931 & 3744 & & 3727 & 3625 & 3642 \\
\hline & 4 & & 3370 & 3897 & 3523 & 3846 & 3795 & 3829 & 3931 & & & & \\
\hline & 5 & 3642 & 3795 & 3812 & 3778 & 3897 & 3846 & 3591 & 3829 & & 3557 & 3693 & \\
\hline
\end{tabular}

\begin{tabular}{|c|c|c|c|c|c|c|c|c|c|}
\hline & \multicolumn{4}{|c|}{ lel } & \multicolumn{4}{|c|}{$\mathrm{Iol}$} \\
\hline & & s1r1 & $\mathrm{s} 1 \mathrm{r} 2$ & $\mathrm{~s} 2 \mathrm{r} 1$ & $s 2 \times 2$ & slr1 & s1r2 & $\mathrm{s} 2 \mathrm{rl}$ & $\mathrm{s} 2 \mathrm{r} 2$ \\
\hline \multirow[t]{5}{*}{ FI } & 1 & 476 & 442 & 425 & 459 & 476 & 476 & 425 & 391 \\
\hline & 2 & 459 & 459 & 459 & 476 & 425 & 459 & 357 & 408 \\
\hline & 3 & 425 & 442 & 476 & 425 & 425 & 442 & 374 & 357 \\
\hline & 4 & 442 & 442 & 374 & 425 & 662 & 459 & 408 & 561 \\
\hline & 5 & 425 & 425 & 408 & 442 & 459 & 442 & 340 & 272 \\
\hline \multirow[t]{5}{*}{$\mathrm{F} 2$} & 1 & 1804 & 1974 & 1889 & 1804 & 1446 & 1429 & 1293 & 1242 \\
\hline & 2 & 1838 & 1821 & 1821 & 1685 & 1055 & 1038 & 1004 & 1174 \\
\hline & 3 & 1991 & 1974 & 1940 & 1872 & 1038 & 1123 & 1055 & 1072 \\
\hline & 4 & 1906 & 1753 & 1906 & 1906 & 1293 & 987 & 834 & 1310 \\
\hline & 5 & 1974 & 1940 & 1923 & 1889 & 1634 & & 868 & 987 \\
\hline \multirow[t]{5}{*}{ F3 } & 1 & 2791 & 2944 & 2774 & 2842 & 2502 & 2791 & 2246 & 2348 \\
\hline & 2 & 3063 & 2744 & 2791 & 2910 & 2331 & 2485 & & 2519 \\
\hline & 3 & 2672 & 2570 & 2757 & 2774 & 2621 & 2842 & 2689 & 2808 \\
\hline & 4 & 2502 & 2434 & 2723 & 2706 & 2195 & 2212 & & \\
\hline & 5 & 2485 & 2519 & 2655 & 2672 & 2995 & & 2927 & 2655 \\
\hline \multirow[t]{5}{*}{$\mathrm{F} 4$} & 1 & & & 3761 & 3880 & 3421 & 3761 & 3302 & 3625 \\
\hline & 2 & & & & & 3506 & 3642 & 3574 & 3455 \\
\hline & 3 & & 3131 & 3727 & 3880 & 3676 & 3744 & 3591 & 3795 \\
\hline & 4 & 3931 & & & 3829 & & 3489 & & 3438 \\
\hline & 5 & & & 3795 & 3812 & 3744 & & & 3846 \\
\hline
\end{tabular}


[Speaker TY]

\begin{tabular}{|c|c|c|c|c|c|c|c|c|c|c|c|c|c|}
\hline & \multicolumn{4}{|c|}{$|a|$} & \multicolumn{4}{|c|}{ /i/ } & \multicolumn{4}{|c|}{$\mathrm{lu} /$} \\
\hline & & sir1 & $\operatorname{sir2}$ & $\mathrm{s} 2 \mathrm{r} 1$ & $\mathrm{~s} 2 \mathrm{r} 2$ & slrl & $\operatorname{sir} 2$ & $\mathrm{~s} 2 \mathrm{r} 1$ & $\mathrm{~s} 2 \mathrm{r} 2$ & slrl & s1r2 & $\mathrm{s} 2 \mathrm{r} 1$ & $\mathrm{~s} 2 \mathrm{r} 2$ \\
\hline \multirow[t]{5}{*}{ F1 } & 1 & 442 & 765 & 680 & 629 & 323 & 289 & 272 & 289 & 391 & 374 & 391 & 374 \\
\hline & 2 & 799 & 748 & 680 & 714 & 289 & 289 & 289 & 272 & & & & 306 \\
\hline & 3 & 561 & 595 & 595 & 595 & 306 & 272 & 289 & 272 & 340 & 323 & 340 & 391 \\
\hline & 4 & 697 & 646 & 646 & 561 & 323 & 323 & 306 & 374 & 340 & & & \\
\hline & 5 & 782 & 799 & 748 & 646 & 289 & 306 & 340 & 306 & 357 & 391 & 374 & 357 \\
\hline \multirow[t]{5}{*}{ F2 } & 1 & 1395 & 1548 & 1412 & 1565 & 2076 & 2127 & 1974 & 2093 & 1327 & 1412 & 1106 & 1582 \\
\hline & 2 & 1344 & 1293 & 1293 & 1259 & 1838 & 1991 & 1991 & 1991 & & & & 1395 \\
\hline & 3 & 1038 & 1242 & 1208 & 1242 & 1872 & 1940 & 1804 & 2008 & 1361 & 1174 & 1140 & 1259 \\
\hline & 4 & 1293 & 1191 & 1259 & 1174 & 1702 & 1838 & 1991 & 1889 & 1259 & & & \\
\hline & 5 & 1276 & 1327 & 1293 & 1208 & 2178 & 2144 & 2008 & 2076 & 1599 & 1429 & 1497 & 1514 \\
\hline \multirow[t]{5}{*}{ F3 } & 1 & 2331 & 2348 & 2417 & 2417 & 2570 & 2638 & 2757 & 2774 & 2008 & 2195 & 2144 & 2195 \\
\hline & 2 & 2178 & 2246 & 2314 & 2178 & 2451 & 2672 & 2638 & 2706 & & & & 2395 \\
\hline & 3 & 2331 & 1957 & 2502 & 1940 & 2263 & 2604 & 2178 & 2587 & 2127 & 2348 & 2434 & 2399 \\
\hline & 4 & 2280 & 2229 & 2246 & 2212 & 2451 & 2604 & 2655 & 2502 & 2348 & & & \\
\hline & 5 & 2042 & 2246 & 2382 & 2399 & & & 2791 & 3012 & 2025 & 2178 & 2127 & 2229 \\
\hline \multirow[t]{5}{*}{$\mathrm{F} 4$} & 1 & 3591 & 3523 & 3897 & 3608 & 3336 & 3438 & 3472 & 3727 & 3336 & 3319 & 3795 & 3608 \\
\hline & 2 & 3659 & 3472 & 3608 & 3489 & 3472 & 3336 & 3302 & 3336 & & & & \\
\hline & 3 & 3659 & 3268 & 3727 & & 3574 & 3370 & 3268 & 3523 & & 3948 & & \\
\hline & 4 & 3574 & 3438 & 3914 & 3897 & 3285 & 3353 & 3642 & 3625 & 3761 & & & \\
\hline & 5 & 3557 & 3438 & 3574 & 3557 & 3199 & 3319 & 3489 & 3676 & 3659 & & 3353 & 3472 \\
\hline
\end{tabular}

\begin{tabular}{|c|c|c|c|c|c|c|c|c|c|}
\hline & \multicolumn{4}{|c|}{$\mathrm{le} /$} & \multicolumn{4}{|c|}{$10 /$} \\
\hline & & s1r1 & $\sin 2$ & $\mathrm{~s} 2 \mathrm{r} 1$ & $\mathrm{~s} 2 \mathrm{r} 2$ & $s \operatorname{lr} 1$ & slr 2 & $\mathrm{~s} 2 \mathrm{r} 1$ & $s 2 \mathrm{r} 2$ \\
\hline \multirow[t]{5}{*}{$\mathrm{F} 1$} & 1 & 476 & 493 & 391 & 442 & 459 & 476 & 442 & 408 \\
\hline & 2 & 391 & 425 & 442 & 425 & 425 & 442 & 442 & 459 \\
\hline & 3 & 374 & 391 & 357 & 357 & 374 & 425 & 408 & 391 \\
\hline & 4 & 408 & 425 & 391 & 357 & 459 & 408 & 357 & 442 \\
\hline & 5 & 408 & 374 & 374 & 374 & 374 & 391 & 408 & 425 \\
\hline \multirow[t]{5}{*}{ F2 } & 1 & 1753 & 1804 & 1770 & 1838 & 1055 & 1055 & 1191 & 1106 \\
\hline & 2 & 1719 & 1702 & 1753 & 1770 & 1055 & 1055 & 1055 & 1106 \\
\hline & 3 & 1855 & 1838 & 1872 & 1906 & 1089 & 1089 & 1157 & 1106 \\
\hline & 4 & 1906 & 1821 & 1872 & 1991 & 765 & 765 & 782 & 765 \\
\hline & 5 & 1889 & 1940 & 1923 & 1923 & & & 697 & 748 \\
\hline \multirow[t]{5}{*}{ F3 } & 1 & 2655 & 2365 & 2399 & 2485 & 2212 & 2297 & 2365 & 2331 \\
\hline & 2 & 2417 & 2519 & 2434 & 2434 & 2161 & 2025 & 2178 & 1940 \\
\hline & 3 & 2451 & 2382 & 2348 & 2519 & 2417 & 2348 & 2485 & 2468 \\
\hline & 4 & 2417 & 2519 & 2536 & 2570 & 2434 & 2399 & 2502 & 2468 \\
\hline & 5 & 2417 & 2434 & 2570 & 2553 & 2468 & & 2587 & 2604 \\
\hline \multirow[t]{5}{*}{ F4 } & 1 & 3948 & 3710 & 3948 & 3863 & 3438 & 3659 & & 3472 \\
\hline & 2 & 3387 & 3455 & 3710 & 3540 & 3080 & 3251 & 3591 & 3336 \\
\hline & 3 & 3489 & 3438 & 3387 & 3880 & 3812 & 3455 & 3812 & 3829 \\
\hline & 4 & 3982 & 3795 & & 3319 & 3625 & 3608 & 3914 & 3608 \\
\hline & 5 & & 3540 & 3965 & 3965 & 3795 & 3846 & 3829 & 3829 \\
\hline
\end{tabular}




\section{MOSHIMOSHI}

Appendix 5.1 F-pattern measurements for each speaker.

\begin{tabular}{|c|c|c|c|c|c|c|c|c|c|c|c|c|c|}
\hline \multirow[t]{2}{*}{ AA } & & \multicolumn{5}{|c|}{1} & \multicolumn{7}{|c|}{2} \\
\hline & & 1 & 2 & 3 & 4 & 5 & 1 & 2 & 3 & 4 & 5 & 6 & 7 \\
\hline \multirow{4}{*}{ ml $50 \%$} & $\mathrm{~F} 1$ & 419 & 167 & 208 & & 488 & 257 & & 248 & 294 & 291 & 148 & 349 \\
\hline & $\mathrm{F} 2$ & & 608 & 843 & 692 & 790 & 875 & 742 & 1007 & 711 & 900 & 786 & \\
\hline & F3 & 2121 & & 2042 & 2294 & 2259 & 2144 & 2282 & 1992 & 2263 & 2249 & 2100 & 2263 \\
\hline & F4 & 3432 & 3240 & 3462 & 3080 & 3448 & 3662 & 3710 & 3733 & 3774 & 3571 & 3671 & 3425 \\
\hline \multirow{4}{*}{ ol on } & $\mathrm{F} 1$ & 477 & 403 & 353 & & 499 & 491 & & & 173 & & 458 & \\
\hline & F2 & 633 & 711 & 765 & 614 & 838 & 631 & 758 & 667 & 729 & 723 & & 657 \\
\hline & F3 & 2182 & 2209 & 2339 & 2311 & 2193 & 2146 & 2304 & 2209 & 2252 & 2192 & 2259 & 2232 \\
\hline & F4 & 3562 & 3256 & 3436 & 3673 & 3626 & 3670 & 3793 & 3593 & 3740 & 3583 & 3759 & 4002 \\
\hline \multirow{4}{*}{ ol mid } & $\mathrm{F} 1$ & 443 & 606 & 634 & 433 & 530 & 459 & 536 & 694 & 640 & 587 & 573 & 349 \\
\hline & $\mathrm{F} 2$ & 993 & 1130 & 1137 & 1404 & 1115 & 834 & 904 & 1045 & 1329 & 1057 & 701 & 983 \\
\hline & F3 & 2058 & 2198 & 2282 & 2359 & 2197 & 2306 & 2333 & 2297 & 2324 & 2305 & 2320 & 2227 \\
\hline & $\mathrm{F} 4$ & 3877 & 3738 & 3558 & 3841 & 3652 & 3826 & 3870 & & 4033 & 3824 & 3952 & \\
\hline \multirow{4}{*}{ ol off } & $\mathrm{F} 1$ & 356 & 174 & 107 & & 373 & 686 & 474 & & 216 & 668 & 639 & \\
\hline & $\mathrm{F} 2$ & 1611 & 1420 & 1757 & 1596 & 1593 & 1305 & 1181 & 1253 & 1499 & 1383 & 1277 & 1136 \\
\hline & F3 & 2295 & 2297 & 2494 & 2390 & 2342 & 2300 & 2297 & 2352 & 2323 & 2336 & 2582 & 2442 \\
\hline & F4 & 3369 & 3502 & 3541 & 3549 & 3457 & 3232 & 3205 & 3422 & 3461 & 3284 & 3511 & 3553 \\
\hline \multirow{4}{*}{ s1 $50 \%$} & Fl & & & & & & & & & & & & \\
\hline & $\mathbf{F} 2$ & 1696 & 1960 & 2067 & 1581 & 1803 & 1814 & 2140 & 1614 & 1329 & 1705 & 1551 & 1799 \\
\hline & F3 & 2583 & 2588 & 2543 & 2486 & 2534 & 2561 & 2981 & 2547 & 2525 & 2479 & 2595 & 2512 \\
\hline & $\mathrm{F} 4$ & 3448 & 3678 & 3455 & 3602 & 3326 & 3601 & 3678 & 3357 & 3475 & 3552 & 3619 & 3526 \\
\hline \multirow{4}{*}{ il $50 \%$} & $\overline{F l}$ & 401 & & & & & & & & & & & \\
\hline & F2 & 1427 & & & & & & & & & & & \\
\hline & F3 & 1980 & & & & & & & & & & & \\
\hline & F4 & 3047 & & & & & & & & & & & \\
\hline \multirow{4}{*}{$\mathrm{m} 250 \%$} & FI & 436 & 332 & 425 & 328 & 370 & 440 & 328 & 403 & & 318 & 187 & 321 \\
\hline & $\mathrm{F} 2$ & 935 & 804 & 1179 & 1148 & & 1502 & 1084 & 1273 & & 1053 & 928 & 1094 \\
\hline & F3 & 2023 & 2170 & 2069 & 2198 & 2177 & 2127 & 2152 & 2433 & 2222 & 2226 & 2292 & 2202 \\
\hline & F4 & 3492 & & 3219 & & & 3184 & 3587 & 3278 & 3682 & 3117 & 3488 & 3260 \\
\hline \multirow{4}{*}{02 onset } & $\mathrm{FI}$ & 413 & 594 & 280 & 288 & & 429 & 402 & 359 & 651 & 673 & 670 & \\
\hline & $\mathrm{F} 2$ & 827 & & 982 & 905 & 685 & & 1291 & 786 & 1560 & 1192 & 972 & 857 \\
\hline & F3 & 2162 & 2232 & 2257 & 2187 & 2223 & 2250 & 2286 & 2349 & 2262 & 2282 & 2276 & 2213 \\
\hline & $\mathrm{F} 4$ & 3424 & 3533 & 3451 & 3553 & & 3604 & 3177 & 3277 & 3578 & 3151 & 3402 & 3086 \\
\hline \multirow{4}{*}{02 middle } & $\mathrm{F} 1$ & 159 & 548 & 565 & 283 & 533 & 157 & 442 & 213 & 585 & & 475 & 557 \\
\hline & $\mathbf{F} 2$ & 746 & 951 & 949 & 1015 & 1343 & 1009 & 782 & 1210 & 1209 & 1387 & 1279 & 1202 \\
\hline & F3 & 2276 & 2239 & 2281 & 2206 & 2178 & 2269 & 2326 & 2348 & 2327 & 2337 & 2237 & 2199 \\
\hline & $\mathrm{F} 4$ & & 3310 & 3541 & 3232 & 3373 & 3482 & 3454 & 3387 & 3655 & 3457 & 3337 & 3444 \\
\hline \multirow{4}{*}{02 offset } & $\mathrm{Fl}$ & 402 & & 247 & 500 & 267 & 237 & 624 & 316 & 567 & & & 150 \\
\hline & $F 2$ & 1449 & 1669 & 1686 & 1730 & 1756 & 1585 & 1568 & 1535 & 1510 & 1689 & 1613 & 1430 \\
\hline & F3 & 2391 & 2854 & 2286 & 2349 & 2283 & 2530 & 2263 & 2327 & 2415 & 2168 & 2651 & 2511 \\
\hline & F4 & 3436 & & 3481 & 3502 & 3447 & 3694 & 3259 & 3360 & 3330 & 3614 & 3649 & 3166 \\
\hline \multirow{4}{*}{ s2 $50 \%$} & F1 & & & & & & & & & & & & \\
\hline & $\mathbf{F} 2$ & 1771 & 1223 & 1835 & 1793 & 1969 & 1679 & 1658 & 1930 & 1458 & 1490 & 1832 & 1986 \\
\hline & F3 & 2446 & 2474 & 2518 & 2496 & 2684 & 2547 & 2525 & 2602 & 2391 & 2557 & 2392 & 2478 \\
\hline & F4 & 3439 & 3569 & 3508 & 3516 & 3612 & 3428 & 3560 & 3471 & 3562 & 3690 & 3747 & 3636 \\
\hline \multirow{4}{*}{ i2 $50 \%$} & F1 & 324 & 319 & 331 & 419 & 508 & 267 & 359 & 276 & & 253 & 220 & 317 \\
\hline & $\mathbf{F} 2$ & 1758 & 1964 & 2078 & 1674 & 1776 & 1720 & 1777 & 1998 & & 1827 & 1598 & 2142 \\
\hline & F3 & 2641 & 2768 & 2864 & 2294 & 2295 & 2536 & 2432 & 2977 & & 2884 & 2386 & 2905 \\
\hline & F4 & 3498 & 3306 & 3740 & 3270 & 3348 & 3166 & 3021 & 3589 & & 4002 & 3627 & 3727 \\
\hline
\end{tabular}




\begin{tabular}{|c|c|c|c|c|c|c|c|c|c|c|c|c|}
\hline \multirow[t]{2}{*}{$\mathrm{HA}$} & & \multicolumn{5}{|c|}{1} & \multicolumn{6}{|c|}{2} \\
\hline & & 1 & 2 & 3 & 4 & 5 & 6 & 1 & 2 & 3 & 4 & $\$$ \\
\hline \multirow{4}{*}{$\mathrm{m} 150 \%$} & $F 1$ & 476 & 493 & 544 & 425 & & 539 & 598 & 545 & 562 & 525 & 430 \\
\hline & $F_{2}$ & 987 & 1191 & 1344 & 1004 & 834 & 1087 & 1130 & 1129 & 1186 & 1032 & 1128 \\
\hline & 53 & 2382 & 2348 & 2200 & 2451 & 2685 & 2442 & & 2275 & 2723 & 2486 & 2527 \\
\hline & 54 & 3625 & 3761 & 3591 & 3880 & 3243 & 3307 & & 3442 & 3621 & 3708 & 3898 \\
\hline \multirow{4}{*}{ of on } & FI & 493 & 544 & 493 & 578 & 509 & 544 & 562 & 603 & 562 & 551 & 518 \\
\hline & $\mathrm{F} 2$ & 1038 & 1327 & 1634 & 1157 & 1137 & 1236 & 1298 & 1235 & 1301 & 1055 & 1442 \\
\hline & F3 & 2417 & 2399 & 2706 & 2365 & 2380 & 2384 & 2436 & 2371 & 2505 & 2371 & 2431 \\
\hline & F4 & 3609 & 3693 & 3744 & 3863 & 3484 & 3532 & 3598 & 3598 & 3543 & 3576 & 3594 \\
\hline \multirow{4}{*}{ ol mid } & F1 & 510 & 510 & 459 & 561 & 447 & 402 & 507 & 748 & 732 & 542 & 482 \\
\hline & $\mathrm{F} 2$ & 1310 & 1514 & 1651 & 1599 & 1091 & & 1488 & 1409 & 1494 & 1464 & 1630 \\
\hline & E3 & 2791 & 2502 & 2740 & 2689 & 2786 & 2837 & 2736 & 2645 & 3052 & 2563 & 2733 \\
\hline & $\mathrm{F} 4$ & 3846 & 3574 & 3625 & 3710 & 3626 & 3678 & 3853 & 3438 & 3434 & 3093 & 3981 \\
\hline \multirow{4}{*}{01 off } & $\mathrm{FI}$ & 476 & 272 & 527 & 709 & 804 & 770 & 452 & 172 & 662 & 534 & 487 \\
\hline & $\mathrm{F} 2$ & 1634 & 1719 & 1736 & 1916 & 1806 & 1815 & 1664 & 1841 & 1782 & 1753 & 1639 \\
\hline & F3 & 2859 & 2706 & 3723 & 3077 & 2844 & 2988 & 2839 & 2929 & 3199 & 3089 & 2524 \\
\hline & F4 & 4000 & 3778 & 3829 & 3840 & 3743 & 3712 & 3921 & 3739 & 3973 & 3975 & 3771 \\
\hline \multirow{4}{*}{ si 50 \% } & $\mathrm{Fl}$ & & & & & & & & & 786 & & \\
\hline & $\mathrm{F} 2$ & 1770 & 1821 & 1804 & 1889 & 1923 & 1823 & 1696 & 1875 & 1509 & 1914 & 1805 \\
\hline & $F$ & 2927 & 2961 & 2859 & 2996 & 3139 & 3307 & 2836 & 2996 & 2801 & 3110 & 2677 \\
\hline & 174 & 3778 & 3880 & 3557 & 3662 & 3682 & 3731 & 3566 & 3900 & 3859 & 3775 & 3700 \\
\hline i1 $50 \%$ & $\begin{array}{l}\mathrm{F} 1 \\
\mathrm{~F} 2 \\
\mathrm{F3} \\
\mathrm{F4}\end{array}$ & & & & & & & & & & & \\
\hline \multirow{4}{*}{$\mathrm{m}^{2} 50 \%$} & $F 1$ & 425 & 442 & 408 & 368 & 456 & 397 & 436 & 343 & 445 & 537 & 430 \\
\hline & $F 2$ & 1072 & 1157 & 1072 & 1005 & 1116 & 1032 & 1031 & 912 & 709 & 1143 & 1182 \\
\hline & $\mathrm{F3}$ & 2553 & 2434 & 2536 & 2517 & 2516 & 2563 & 2469 & 2487 & 2469 & 2435 & 2458 \\
\hline & F4 & 3591 & & & 3735 & 3590 & 3152 & 3526 & 3629 & 3479 & 3506 & 3333 \\
\hline \multirow{4}{*}{02 onset } & FI & 340 & 493 & 493 & 520 & 464 & 544 & 537 & 557 & 540 & 532 & 535 \\
\hline & $\mathrm{F} 2$ & 102 & 1089 & 1259 & 1100 & 1085 & 958 & 1195 & 1003 & 1239 & 826 & 1246 \\
\hline & $\mathrm{F3}$ & 2570 & 2502 & 2417 & 2220 & 2436 & 2583 & 2388 & 2755 & 2849 & 2275 & 2401 \\
\hline & $\mathrm{Fu}$ & 3574 & & 3319 & 3563 & 3509 & 3740 & 3455 & 3862 & 3960 & 3334 & 3569 \\
\hline \multirow{4}{*}{02 middle } & FI & 510 & 510 & 510 & 517 & 653 & 610 & 570 & 568 & 500 & 513 & 553 \\
\hline & $\mathrm{Fz}$ & 1344 & $137 \mathrm{~B}$ & 1651 & 1535 & 1357 & 1016 & 1428 & 1442 & 1382 & 1801 & 1568 \\
\hline & $\mathrm{F3}$ & 2434 & 2451 & 2791 & 2481 & 2243 & 2617 & 2341 & 2901 & 2420 & 2982 & 2562 \\
\hline & $\mathrm{F} 4$ & & 3710 & 3455 & 3431 & 3586 & 3859 & 3228 & 3670 & 3442 & 3710 & 3537 \\
\hline \multirow{4}{*}{02 offset } & $\mathrm{Fl}$ & & 527 & 510 & 455 & 504 & 881 & 779 & 141 & 626 & 506 & 668 \\
\hline & F2 & 1838 & 1617 & 1787 & 1939 & 1803 & 1796 & 1814 & 1747 & 1741 & 1980 & 1708 \\
\hline & F3 & 3165 & 2502 & 2876 & 2992 & 3065 & 2858 & 2837 & 2966 & 2753 & 3083 & 2746 \\
\hline & $\mathrm{E} 4$ & 3829 & 3812 & 3523 & 3381 & 3783 & 3647 & 3648 & 3642 & 3828 & 4035 & 3620 \\
\hline \multirow{4}{*}{$\mathrm{s} 250 \%$} & $\mathrm{FI}$ & & & & & & & & & & & \\
\hline & F2 & 1889 & 2042 & 2059 & 1991 & 1737 & 1873 & 1853 & 1880 & 2002 & 1594 & 2375 \\
\hline & F 3 & 2859 & 2995 & 3046 & 3040 & 3310 & 2960 & 2761 & 2817 & 3084 & 3135 & $317 \%$ \\
\hline & F4 & 3591 & 3897 & 3625 & 3738 & 3725 & 3544 & 3387 & 3846 & 3522 & 3731 & 3774 \\
\hline \multirow{4}{*}{$1250 \%$} & F1 & 306 & 357 & 374 & 321 & 279 & & 352 & 382 & 337 & 355 & 357 \\
\hline & $\mathrm{F2}$ & 2519 & 2348 & 2536 & 2370 & 2700 & & 2460 & 2345 & 2150 & 2131 & 2291 \\
\hline & F3 & 2995 & 3131 & 3080 & 3138 & 3317 & & 3216 & 3253 & 2972 & 2532 & 2881 \\
\hline & F4 & & 3774 & 3965 & 3717 & 3747 & & 407 & 3875 & 3866 & 3526 & 3662 \\
\hline
\end{tabular}




\begin{tabular}{|c|c|c|c|c|c|c|c|c|c|c|c|c|}
\hline \multirow[t]{2}{*}{$\mathrm{JN}$} & & \multicolumn{5}{|c|}{1} & \multicolumn{6}{|c|}{2} \\
\hline & & 1 & 2 & 3 & 4 & 5 & 1 & 2 & 3 & 4 & 5 & 6 \\
\hline \multirow{4}{*}{$\mathrm{ml} 50 \%$} & F1 & 289 & 228 & 255 & 215 & 207 & 276 & 329 & 221 & 221 & 243 & 249 \\
\hline & F2 & 707 & 907 & 1242 & 847 & 948 & 779 & 1072 & 1122 & 869 & 1062 & 809 \\
\hline & F3 & 2059 & 2357 & 2611 & 2249 & 1937 & 2002 & 2361 & 2362 & 2240 & 2320 & 2243 \\
\hline & $\mathrm{F} 4$ & 3784 & 3622 & 3427 & 3787 & 3026 & 3606 & 3208 & 3333 & 3313 & 3463 & 3332 \\
\hline \multirow{4}{*}{ ol on } & F1 & 397 & 400 & 402 & 305 & 369 & 313 & 334 & 286 & 358 & 233 & 220 \\
\hline & $\mathbf{F} 2$ & 952 & 1024 & 918 & 726 & 1073 & 997 & 1012 & 798 & 956 & 999 & 1252 \\
\hline & F3 & 2439 & 2458 & 2272 & 2230 & 2173 & 1910 & 2333 & 2168 & 2245 & 2273 & 2148 \\
\hline & F4 & 3139 & 3292 & & 3070 & 3542 & 3476 & 3295 & 3416 & 3150 & 3380 & 3373 \\
\hline \multirow{4}{*}{ ol mid } & F1 & 150 & 410 & 448 & 459 & 408 & 166 & 384 & 265 & 308 & 271 & 356 \\
\hline & F2 & 1087 & 1383 & 1254 & 1166 & 1334 & 1239 & 1316 & 1054 & 1313 & 929 & 1291 \\
\hline & F3 & 1961 & & 2334 & 2231 & 1931 & 1962 & 2245 & 2136 & 2080 & 2227 & 2190 \\
\hline & F4 & 3775 & 3765 & 3518 & 3485 & 3098 & & 3525 & 3433 & 3461 & 3372 & 3486 \\
\hline \multirow{4}{*}{01 off } & F1 & 142 & 243 & 337 & 183 & 161 & 172 & 156 & 110 & 157 & 143 & 158 \\
\hline & $\mathrm{F} 2$ & 1324 & 1604 & 1442 & 1474 & 1425 & 1432 & 1504 & 1416 & 1525 & 1430 & 1487 \\
\hline & F3 & 2084 & 2375 & 2545 & & 2950 & 2401 & 2736 & 2415 & 2374 & 2764 & 2915 \\
\hline & $\mathrm{F} 4$ & 3653 & 3594 & 3646 & 3989 & 4217 & 3173 & 3293 & 3668 & 3359 & 3632 & 3311 \\
\hline \multirow{4}{*}{ sl $50 \%$} & $\mathrm{~F} 1$ & & 170 & & 208 & 152 & & & 107 & 205 & & 368 \\
\hline & $\mathrm{F} 2$ & 1663 & 1555 & 1511 & 1541 & 1448 & 1660 & 1535 & 1633 & 1561 & 1657 & 1298 \\
\hline & F3 & 2039 & 2465 & 2298 & 2739 & 1962 & 2459 & 2361 & 2480 & 2103 & 2588 & 2339 \\
\hline & $\mathrm{F} 4$ & 3711 & 3567 & 3465 & 3463 & 3136 & 3162 & 3177 & 3496 & 3109 & 3378 & 3845 \\
\hline \multirow{4}{*}{ i1 $50 \%$} & $\mathrm{~F} 1$ & & & & 436 & & 300 & & & & & \\
\hline & F2 & & & & 1380 & & 1346 & & & & & \\
\hline & F3 & & & & 2116 & & 2713 & & & & & \\
\hline & F4 & & & & 3316 & & 3804 & & & & & \\
\hline \multirow{4}{*}{$\mathrm{m} 250 \%$} & Fl & 131 & 287 & 342 & 301 & 263 & 256 & & 299 & 300 & 296 & 272 \\
\hline & F2 & 951 & 1003 & 986 & 764 & 1351 & 1135 & & 1002 & 1185 & 911 & 1083 \\
\hline & F3 & 2025 & 2194 & 1639 & 1885 & 2362 & 1722 & & 1943 & 2067 & 2045 & 1795 \\
\hline & $\mathrm{F} 4$ & 3730 & 3246 & 3105 & 3044 & 3269 & 2880 & & 3222 & 3211 & 3183 & 3059 \\
\hline \multirow{4}{*}{02 onset } & F1 & 359 & 415 & 342 & 275 & 356 & 279 & 375 & 321 & 260 & 318 & -284 \\
\hline & F2 & & 1420 & 1614 & 1317 & 1434 & 1255 & 1470 & 1265 & 1591 & 935 & 1137 \\
\hline & F3 & 2203 & 2125 & & 1943 & 1949 & 2385 & & 1988 & & 2114 & 1933 \\
\hline & $\mathrm{F} 4$ & 3297 & 3954 & 3452 & 3186 & 3563 & 3117 & 3166 & 3446 & & 3218 & 3457 \\
\hline \multirow{4}{*}{ o2 middle } & Fl & 378 & 439 & 439 & 381 & 346 & 343 & 346 & 297 & 115 & 430 & 454 \\
\hline & $\mathbf{F} 2$ & 1195 & 1661 & 1380 & 1525 & 1417 & 1498 & 1626 & 1496 & 1618 & 1273 & 1582 \\
\hline & F3 & 2118 & 2616 & 1982 & 2182 & 2023 & & 2110 & 2023 & 1930 & 2046 & 2063 \\
\hline & $\mathrm{F} 4$ & 3210 & 3618 & 3125 & 3225 & 3152 & 3024 & 3257 & 3323 & 3043 & 2945 & 3458 \\
\hline \multirow{4}{*}{02 offset } & F1 & 105 & 268 & 335 & 122 & 305 & 153 & 308 & 348 & & 251 & \\
\hline & F2 & 1369 & & 1614 & 1590 & 1896 & 1556 & 1414 & 1702 & 1974 & 1509 & 1557 \\
\hline & F3 & 2273 & 2009 & 2598 & 2105 & 2376 & 2764 & 2291 & 2875 & 2659 & 2155 & 2143 \\
\hline & F4 & 3579 & 3105 & 3700 & 3694 & 3615 & 3874 & 3411 & 3652 & 3247 & 3138 & 3314 \\
\hline \multirow{4}{*}{ s2 50\% } & Fl & & 260 & & & 268 & & 264 & & & & \\
\hline & $\mathrm{F} 2$ & 1457 & 1816 & 1172 & 1535 & 1952 & 2263 & 1698 & 1693 & 2069 & 2056 & 1536 \\
\hline & F3 & 2603 & 2685 & 2268 & 2265 & 2653 & 2857 & 2568 & 2492 & 2614 & & 2563 \\
\hline & F4 & 3401 & 3420 & 3595 & 3238 & 3616 & 3459 & 3521 & 3188 & 3355 & 3282 & 3407 \\
\hline \multirow{4}{*}{ i2 $50 \%$} & $\mathrm{Fl}$ & 245 & & 139 & 209 & 268 & 208 & 217 & 284 & 301 & 375 & 314 \\
\hline & $\mathrm{F} 2$ & 1837 & & 1873 & & 1972 & 2179 & 1348 & 1438 & 1803 & 1643 & 1588 \\
\hline & F3 & 3548 & & 2954 & 2663 & 3025 & 2915 & 2293 & 2802 & 2672 & 2728 & 2745 \\
\hline & $\mathrm{F} 4$ & 3416 & & 3769 & 3293 & 4063 & 3793 & 3450 & 3398 & 3752 & 3941 & 3410 \\
\hline
\end{tabular}




\begin{tabular}{|c|c|c|c|c|c|c|c|c|c|}
\hline \multicolumn{2}{|l|}{$\mathrm{KA}$} & \multicolumn{3}{|c|}{1} & \multirow[b]{2}{*}{4} & \multicolumn{3}{|c|}{2} & \multirow[b]{2}{*}{4} \\
\hline & & 1 & 2 & 3 & & 1 & 2 & 3 & \\
\hline \multirow{4}{*}{ ml $50 \%$} & F1 & 510 & 325 & 351 & 409 & 451 & 269 & 530 & 545 \\
\hline & $\mathrm{F} 2$ & 763 & 886 & 1044 & 904 & 903 & 998 & 1255 & \\
\hline & F3 & 2636 & 2976 & 2879 & 2712 & 2946 & 2867 & 2830 & 2350 \\
\hline & F4 & 3644 & 3276 & 3799 & 3749 & 3658 & 3712 & 3817 & 3398 \\
\hline \multirow{4}{*}{ ol on } & $\mathrm{F1}$ & 504 & 508 & 505 & 474 & 541 & 491 & 528 & 527 \\
\hline & $\mathrm{F} 2$ & 924 & 887 & 977 & 946 & 881 & 921 & 1110 & 897 \\
\hline & $\mathbf{F 3}$ & 2954 & & 2944 & & 2817 & & 2756 & 3076 \\
\hline & F4 & 3390 & 3517 & 3644 & 3612 & 3439 & 3524 & 3581 & 3463 \\
\hline \multirow{4}{*}{$01 \mathrm{mid}$} & F1 & 478 & 507 & 527 & 485 & 468 & 464 & 518 & 512 \\
\hline & $\mathrm{F} 2$ & 1007 & 1165 & 1138 & 1097 & 1280 & 1126 & 1561 & 1105 \\
\hline & F3 & 2693 & 2817 & 3027 & 2649 & 2887 & 2828 & 2750 & 2845 \\
\hline & $F_{4}$ & 3413 & 3366 & 3649 & 3556 & 3593 & 3702 & 3968 & 3444 \\
\hline \multirow{4}{*}{ ol off } & $\mathrm{F} 1$ & 320 & 347 & 235 & 370 & 380 & 424 & 400 & 430 \\
\hline & $\mathrm{F} 2$ & 1251 & 1499 & 1186 & 1189 & 1390 & 1311 & 1643 & 1156 \\
\hline & F3 & 2739 & 2873 & 2936 & 2760 & 2886 & 2957 & 2806 & 2886 \\
\hline & F4 & 3749 & 3580 & 3867 & 3879 & 3598 & 3705 & 3801 & 3667 \\
\hline \multirow{4}{*}{ si $50 \%$} & $\mathrm{Fl}$ & & & & 774 & & & & \\
\hline & $\mathrm{F} 2$ & 1494 & 1610 & 1631 & 1697 & 1813 & 1582 & 1859 & 1593 \\
\hline & F3 & 2482 & 2416 & 2920 & 2465 & 2757 & 2476 & & \\
\hline & $\mathrm{F} 4$ & 3507 & 3156 & 3222 & 3402 & 3435 & 3110 & 3393 & 3217 \\
\hline \multirow{4}{*}{ i1 $50 \%$} & F1 & & & & & & & 471 & \\
\hline & $\mathrm{F} 2$ & & & & & & & 1201 & \\
\hline & F3 & & & & & & & 2623 & \\
\hline & F4 & & & & & & & 3520 & \\
\hline \multirow{4}{*}{$\mathrm{m} 250 \%$} & $\mathrm{~F} 1$ & 250 & 240 & 335 & 240 & 310 & 300 & & 292 \\
\hline & $\mathrm{F} 2$ & 1121 & 937 & 1024 & 1093 & 1144 & 1034 & & 1090 \\
\hline & F3 & 2647 & 2717 & 2655 & 2609 & 2898 & 2732 & & 2451 \\
\hline & F4 & 3625 & 3538 & 3475 & 3673 & 3753 & 3623 & & 3531 \\
\hline \multirow{4}{*}{02 onset } & $\mathrm{Fl}$ & 373 & 461 & 499 & 471 & 452 & 484 & 503 & 448 \\
\hline & $\mathrm{F} 2$ & 892 & 913 & 1030 & 974 & 976 & 1088 & 1100 & 1179 \\
\hline & $\mathrm{F3}$ & 2475 & 2097 & 2366 & 2024 & 2437 & 2391 & 2453 & 2360 \\
\hline & F4 & 3536 & 3069 & 3297 & 3198 & 3457 & 3344 & 3426 & 3277 \\
\hline \multirow{4}{*}{02 middle } & $\mathrm{F} 1$ & 679 & 701 & 464 & 488 & 393 & 505 & 491 & 420 \\
\hline & $\mathrm{F} 2$ & 1497 & 1166 & 1239 & 1190 & 1202 & 1295 & 1478 & 1442 \\
\hline & F3 & 2347 & 2479 & 2860 & 2264 & 2691 & 2690 & 2776 & 2655 \\
\hline & $\mathrm{F} 4$ & 3362 & 3268 & 3374 & 2946 & 3567 & 3454 & 3748 & 3460 \\
\hline \multirow{4}{*}{02 offset } & $\mathrm{Fl}$ & 663 & 180 & 469 & 330 & 343 & 283 & 388 & 485 \\
\hline & $F 2$ & 1677 & 1469 & 1540 & 1492 & 1645 & 1548 & 1788 & 1576 \\
\hline & F3 & 2657 & 2418 & 2802 & 2713 & 2848 & 2816 & 2760 & 2867 \\
\hline & $\mathrm{F} 4$ & 3554 & 3144 & 3621 & 3647 & 3678 & 3729 & 3890 & 3568 \\
\hline \multirow{4}{*}{ s2 $50 \%$} & $\mathrm{~F} 1$ & & & & & & & & \\
\hline & $\mathrm{F} 2$ & 1993 & 1902 & 1819 & 1601 & 1881 & 1804 & 1944 & 1854 \\
\hline & F3 & 2783 & 2923 & 2757 & 2655 & 2695 & 2789 & 2899 & 2870 \\
\hline & F4 & 3663 & 3561 & 3457 & 3831 & 3843 & 3994 & 3869 & 3686 \\
\hline \multirow{4}{*}{ i2 $50 \%$} & $\mathrm{Fl}$ & & & 316 & & 329 & & 356 & 322 \\
\hline & $\mathrm{F} 2$ & & & 2011 & & 2120 & & 1982 & 1980 \\
\hline & $\mathrm{F3}$ & & & 2808 & & 2891 & & 2929 & 2959 \\
\hline & $\mathrm{F} 4$ & & & 3586 & & 3852 & & 3855 & 3724 \\
\hline
\end{tabular}




\begin{tabular}{|c|c|c|c|c|c|c|c|c|c|c|c|c|c|c|c|c|c|c|}
\hline \multirow[t]{2}{*}{$\mathrm{KF}$} & & \multicolumn{7}{|c|}{1} & \multicolumn{10}{|c|}{2} \\
\hline & & 1 & 2 & 3 & 4 & 5 & 6 & 7 & 1 & 2 & 3 & 4 & 6 & 7 & 8 & 9 & 10 & 11 \\
\hline \multirow{4}{*}{$\mathrm{ml} 50 \%$} & $\mathrm{Fl}$ & 380 & 357 & $\overline{323}$ & 310 & 308 & 388 & 539 & 268 & 236 & 282 & 247 & 357 & 325 & 373 & 294 & 221 & 206 \\
\hline & $\mathrm{F} 2$ & 1052 & 964 & 876 & 1059 & 986 & 987 & 1166 & 1043 & 865 & 918 & 948 & 841 & 914 & 900 & 971 & 1011 & 930 \\
\hline & $\mathrm{F} 3$ & 2135 & 2353 & 2344 & 2649 & 2335 & 2593 & 2240 & 2225 & 2363 & 2279 & 2229 & 2425 & 2378 & 2413 & 2440 & 2195 & 2328 \\
\hline & F4 & 3832 & 4110 & 4219 & 3975 & 3858 & & 3883 & 3977 & 3943 & 4124 & 3850 & 3812 & 4106 & 4137 & 3820 & 3921 & 3900 \\
\hline \multirow{4}{*}{ ol on } & $\mathrm{Fl}$ & 416 & 468 & 469 & 381 & 362 & 431 & 477 & 400 & 353 & 325 & 338 & 314 & 313 & 415 & 334 & 317 & 316 \\
\hline & $\mid F 2$ & 1017 & 970 & 825 & 983 & 956 & 1080 & 1211 & 992 & 1011 & 881 & 1019 & 868 & 857 & 939 & 877 & 1133 & 1024 \\
\hline & F3 & 2430 & 2317 & 2420 & 2335 & 2369 & 2405 & 2320 & 2316 & 2309 & 2310 & 2397 & 2383 & 2382 & 2254 & 2483 & 2428 & 2235 \\
\hline & F4 & & 3599 & 3520 & 3514 & 3906 & 3944 & 3718 & 3956 & 3607 & 3793 & 4034 & 4054 & 4011 & 3731 & 3572 & 3866 & 3891 \\
\hline \multirow{4}{*}{ ol mid } & $F 1$ & 248 & 442 & 400 & 284 & & 462 & 245 & 505 & 396 & 242 & 259 & 250 & 243 & 476 & 387 & 259 & 313 \\
\hline & $\mathrm{F} 2$ & 1653 & 1418 & 1140 & 1660 & 1225 & 1595 & 1962 & 1380 & 1636 & 1410 & 1471 & 1586 & 1799 & & & 1882 & 1475 \\
\hline & F3 & 2701 & 2518 & 2449 & 2680 & 2593 & 2992 & 2719 & 2220 & 2551 & 2786 & 2393 & 2372 & 2506 & & 2360 & 2649 & 2508 \\
\hline & F4 & 3930 & 3932 & 3523 & 3883 & 3750 & 4226 & 3919 & 3885 & 3769 & 3813 & 3649 & 3627 & 3550 & 3779 & 3993 & 3909 & 3571 \\
\hline \multirow{4}{*}{ o1 off } & F1 & 326 & 430 & 338 & 555 & 552 & 567 & & 235 & 228 & 204 & 259 & 221 & 209 & 310 & 171 & 242 & 205 \\
\hline & $\mid F 2$ & 1903 & 1977 & & 1770 & 2085 & 2008 & 1771 & 1957 & 2264 & 2026 & 2114 & 1736 & 1829 & 2090 & 2150 & 2349 & 1880 \\
\hline & F3 & 2776 & 2863 & 2966 & 3122 & 2865 & 3210 & 2471 & 2640 & 3220 & 2634 & 2953 & 3054 & 2799 & 3010 & 2573 & 2980 & 2705 \\
\hline & F4 & 3612 & 3998 & 3913 & 4120 & 3745 & 4208 & 3666 & 3788 & 3926 & 4373 & 3708 & 3957 & 3819 & 3998 & 3524 & 3954 & 3507 \\
\hline \multirow{4}{*}{ s1 50\% } & F1 & & 288 & & & & 728 & & 499 & 335 & & 344 & & & & 149 & 274 & 501 \\
\hline & F2 & 2063 & 1809 & 1974 & 2192 & 2170 & 1929 & 2313 & 1522 & & 2388 & 1622 & 1778 & 1830 & 2216 & 1947 & 1943 & 2003 \\
\hline & F3 & 2752 & 2707 & 3191 & 2875 & 2890 & 2970 & 3093 & 2720 & 3343 & 3085 & 2634 & 2956 & 2774 & 3053 & 2544 & 2684 & 2740 \\
\hline & F4 & 3692 & 3591 & 3831 & 3866 & 3703 & 3685 & 3680 & 3854 & 3696 & 3989 & 3502 & 4002 & 3685 & 4235 & 3442 & 3914 & 3744 \\
\hline \multirow{4}{*}{ il $50 \%$} & $\mathrm{FI}$ & & & 382 & & & & & & 321 & & & & & 309 & & 282 & \\
\hline & $\mathrm{F} 2$ & & & 2353 & & & & & & 2179 & & & & & 2107 & & 1208 & \\
\hline & $\mid \mathrm{F} 3$ & & & 2648 & & & & & & 3620 & & & & & 2403 & & 2371 & \\
\hline & F4 & & & 3914 & & & & & & 4330 & & & & & 3721 & & 3372 & \\
\hline \multirow{4}{*}{$\mathrm{m} 250 \%$} & $\mid \mathrm{F} 1$ & 385 & 402 & 282 & 461 & 332 & 285 & 318 & 275 & 231 & 416 & 243 & 221 & 249 & 252 & 349 & 272 & 288 \\
\hline & $\mid F 2$ & 955 & 952 & 964 & 1093 & 969 & 933 & 953 & 981 & 794 & 1117 & 912 & 961 & 860 & 859 & 933 & 890 & 876 \\
\hline & $\mid \mathrm{F} 3$ & 2600 & 2269 & 2601 & 2621 & 2562 & 2522 & 2550 & 2379 & 2375 & 2440 & 2351 & 2485 & 2415 & 2309 & 2389 & 2604 & 2361 \\
\hline & F4 & 4132 & 3853 & 3870 & 3970 & 3808 & 3976 & 3624 & 4015 & 3456 & 3526 & 4021 & & 3626 & 3605 & 3418 & 3829 & 4061 \\
\hline \multirow{4}{*}{02 onset } & $\mathrm{Fl}$ & 445 & 444 & 442 & 391 & 345 & 323 & 401 & 442 & 419 & 422 & 351 & 337 & 360 & 390 & 380 & 357 & 357 \\
\hline & $F 2$ & 1061 & 1031 & 920 & 1647 & 955 & 918 & 1034 & 1036 & 859 & 1002 & 935 & 1010 & 976 & 822 & 1003 & 839 & 1074 \\
\hline & F3 & 2443 & 2288 & 2407 & 2770 & 2624 & 2611 & 2798 & 2129 & 2432 & 2139 & 2180 & 2353 & 2579 & 2402 & 2336 & 2632 & 2344 \\
\hline & F4 & 3782 & 3815 & 3730 & 4055 & 3855 & 3771 & 3722 & 3970 & 4283 & 3813 & 3790 & 4019 & 4283 & 3530 & 3659 & 3877 & 3702 \\
\hline \multirow{4}{*}{02 middle } & $\mathrm{Fl}$ & 453 & 475 & 435 & 261 & 321 & 323 & 413 & 4848 & 371 & 395 & 340 & 222 & 249 & 418 & 453 & 421 & 407 \\
\hline & $\mathrm{F2}$ & 1456 & 1462 & 1273 & 1927 & 1745 & 1628 & 1520 & 1388 & 903 & 1289 & 952 & 1535 & 1476 & 995 & 1385 & 1275 & 1547 \\
\hline & F3 & 2242 & 2635 & 2567 & 2768 & 2685 & 2692 & 2787 & 2276 & 2388 & 2390 & 2842 & 2420 & 2586 & 2043 & 2179 & 2211 & 2380 \\
\hline & F4 & 3670 & 3935 & 3934 & 3962 & 3837 & 3844 & 4018 & 3678 & 3621 & 3720 & 3821 & 4038 & 3518 & 3262 & 3573 & 3825 & 3794 \\
\hline \multirow{4}{*}{02 offset } & $F 1$ & 412 & 380 & 361 & & & 292 & 267 & 429 & 217 & 298 & & 204 & 170 & 277 & & 324 & 186 \\
\hline & $\mathrm{F} 2$ & 1691 & 1911 & 1817 & 2142 & 2252 & 1987 & 2040 & 1620 & 1931 & 2016 & 1875 & 1707 & 2022 & 2100 & 1846 & 2084 & 1951 \\
\hline & F3 & 2500 & 2876 & 2890 & 2865 & 2970 & 2737 & 2963 & 2640 & 3021 & 2813 & 2231 & 2277 & 2585 & 2670 & 2467 & 2892 & 2537 \\
\hline & F4 & 3442 & 3681 & 4026 & 3797 & 3835 & 3663 & 4106 & 3620 & 4053 & 3845 & 3552 & & 4132 & 3337 & 3418 & 3568 & 3620 \\
\hline \multirow[t]{3}{*}{ s2 $50 \%$} & $\begin{array}{l}\mathrm{F} 1 \\
\mathrm{~F} 2\end{array}$ & 1995 & 1723 & 2392 & 2286 & 2410 & 2245 & 2220 & & 2356 & 1723 & 1917 & 1707 & 2128 & 2310 & 1679 & 2255 & 2113 \\
\hline & F3 & 2707 & 2724 & 3016 & 2859 & 3048 & 2997 & 3050 & 2684 & 3070 & 2778 & 2903 & 2977 & 2892 & 2790 & 2505 & 2883 & 2852 \\
\hline & F4 & 3627 & 3966 & 4024 & 3742 & 4016 & 3831 & 3791 & 3918 & 4038 & 3863 & 3693 & 4221 & 4126 & 3970 & 3568 & 3764 & 3727 \\
\hline \multirow{4}{*}{ i2 $50 \%$} & $\mathrm{Fl}$ & 334 & 367 & 249 & 287 & 313 & 306 & 298 & & 278 & & 208 & 292 & 278 & 265 & 442 & 273 & 313 \\
\hline & $\mathrm{F} 2$ & 2054 & 2088 & 1795 & 2501 & 2382 & 2796 & 2402 & 2113 & 2049 & & 2081 & 2251 & 2268 & 2239 & 1931 & 2183 & 2176 \\
\hline & F3 & 2823 & 2794 & 2615 & 2976 & 3074 & 3086 & 2976 & 2912 & 3080 & & 2822 & 2955 & 2924 & 2968 & 2634 & 2945 & 2701 \\
\hline & F 4 & 3769 & 3577 & 3231 & 4075 & 3621 & 4209 & 4044 & 3834 & 3837 & & 3756 & 3967 & 3757 & 3840 & 3454 & 3717 & 3555 \\
\hline
\end{tabular}




\begin{tabular}{|c|c|c|c|c|c|c|c|}
\hline \multicolumn{2}{|l|}{$\mathrm{JN}$} & \multicolumn{3}{|c|}{1} & \multicolumn{3}{|c|}{2} \\
\hline & & 1 & 2 & 3 & 1 & 2 & 3. \\
\hline \multirow{4}{*}{$\mathrm{ml} \mathrm{50 \%}$} & Fl & 295 & 482 & 492 & 274 & 436 & 371 \\
\hline & F2 & 1078 & 1397 & 1389 & 1219 & 1097 & 1169 \\
\hline & F3 & 2583 & 2899 & 2926 & 2916 & 2853 & 2560 \\
\hline & F4 & 3269 & 4030 & & 3387 & 3531 & 3369 \\
\hline \multirow{4}{*}{ ol on } & F1 & 304 & 245 & 366 & 441 & 433 & 337 \\
\hline & $\mathrm{F} 2$ & 1111 & 1069 & 1068 & 1055 & 1089 & 1396 \\
\hline & F3 & 2570 & 2475 & 2595 & 2655 & 2744 & 2632 \\
\hline & F4 & 3454 & 3505 & 3427 & 3656 & 3533 & 3601 \\
\hline \multirow{4}{*}{ ol mid } & $\mathrm{F} 1$ & 284 & 245 & 369 & 477 & 460 & 336 \\
\hline & F2 & 1162 & 1362 & 1221 & 1315 & 1352 & 1401 \\
\hline & F3 & 2502 & 2693 & 2528 & 2630 & 2518 & 2640 \\
\hline & F4 & 3473 & 3723 & 3481 & 3742 & 3640 & 3616 \\
\hline \multirow{4}{*}{01 off } & F1 & 861 & 279 & 144 & 662 & 449 & 810 \\
\hline & F2 & 1276 & 1504 & 1476 & 1473 & 1643 & 1470 \\
\hline & F3 & 2568 & 2568 & 2517 & 2546 & 2524 & 2347 \\
\hline & F4 & 3247 & 3445 & 3561 & 3738 & 3485 & 3293 \\
\hline \multirow{4}{*}{ s1 $50 \%$} & F1 & 581 & 215 & & & & \\
\hline & F2 & 1734 & 1752 & 1625 & 1829 & 1692 & 1725 \\
\hline & F3 & 2639 & 2781 & 2494 & 2630 & 2471 & 2569 \\
\hline & F4 & 3377 & 3513 & 3397 & 3363 & 3223 & 3305 \\
\hline \multirow{3}{*}{ il 50\% } & $\begin{array}{l}\mathrm{F} 1 \\
\mathrm{~F} 2\end{array}$ & & & & & & \\
\hline & F3 & & & & & & \\
\hline & F4 & & & & & & \\
\hline \multirow{4}{*}{$\mathrm{m} 250 \%$} & FI & 381 & 417 & 417 & 457 & 413 & 397 \\
\hline & F2 & 1322 & 1374 & 1039 & 1073 & 1227 & 1310 \\
\hline & F3 & 2531 & 2596 & 2513 & 2551 & 2905 & 2549 \\
\hline & F4 & 3325 & 3144 & 3138 & 3317 & & 3118 \\
\hline \multirow{4}{*}{02 onset } & $\mathrm{Fl}$ & 553 & 413 & 465 & 485 & & 336 \\
\hline & F2 & 1007 & 957 & 1216 & 1116 & 920 & 1401 \\
\hline & F3 & 2575 & 2264 & 2468 & 2607 & 2824 & 2640 \\
\hline & F4 & 3288 & 3239 & 3284 & 3350 & & 3316 \\
\hline \multirow{4}{*}{02 middle } & $\mathrm{F} 1$ & 402 & 476 & 400 & 545 & & 385 \\
\hline & F2 & 1261 & 1191 & 1450 & 1414 & 1260 & 1499 \\
\hline & E3 & 2796 & 2721 & 2487 & 2544 & 2595 & 2502 \\
\hline & F4 & 3563 & 3362 & 3450 & 3383 & 3409 & 3200 \\
\hline \multirow{4}{*}{02 offset } & $\mathrm{Fl}$ & 743 & & 179 & 651 & 423 & 311 \\
\hline & $F 2$ & 1669 & 1347 & 1626 & 1659 & 1507 & 1800 \\
\hline & F3 & 2668 & & 2577 & 2685 & 2431 & 2602 \\
\hline & F4 & 3567 & 3667 & 3600 & 3649 & 3324 & 3454 \\
\hline \multirow{4}{*}{$\$ 250 \%$} & F1 & 672 & 429 & & 402 & 465 & \\
\hline & $\mathrm{F} 2$ & 1820 & 1778 & 1817 & 1782 & 1864 & 1732 \\
\hline & F3 & 2718 & 2683 & 2602 & 2643 & 2657 & 2507 \\
\hline & F4 & 3378 & 3459 & 3413 & 3553 & 3430 & 3223 \\
\hline \multirow{4}{*}{ i2 $50 \%$} & $\mathrm{Fl}$ & & & & & & \\
\hline & F2 & 295 & 482 & 492 & 274 & 436 & 371 \\
\hline & F3 & 1078 & 1397 & 1389 & 1219 & 1097 & 1169 \\
\hline & F4 & 2583 & 2899 & 2926 & 2916 & 2853 & 2560 \\
\hline
\end{tabular}




\begin{tabular}{|c|c|c|c|c|c|c|c|c|c|c|c|c|c|c|c|c|c|}
\hline \multirow{2}{*}{\multicolumn{2}{|c|}{ MM }} & \multicolumn{9}{|c|}{2} & \multicolumn{7}{|c|}{2} \\
\hline & & 1 & 2 & 3 & 4 & 5 & 6 & 7 & 8 & 9 & 1 & 2 & 3 & 4 & 5 & 6 & \\
\hline \multirow{4}{*}{$\mathrm{m} 150 \%$} & F1 & 367 & 170 & 362 & 343 & 414 & 472 & 417 & 348 & 393 & 318 & 374 & 371 & 298 & 302 & 379 & \\
\hline & $F 2$ & & 1048 & 1470 & 1047 & 1324 & 1417 & 1312 & 1176 & 1485 & 1301 & 1293 & 1319 & 1338 & 1335 & 1402 & 132 \\
\hline & F3 & 2420 & 2251 & 2434 & 2075 & 2429 & 2373 & 2439 & 2454 & 2620 & 2404 & 2337 & 2341 & 2260 & 2332 & 2370 & 229 \\
\hline & F4 & 3124 & & 3197 & 2905 & 2963 & & 2841 & 3007 & & 3079 & 3057 & 3023 & 2972 & 3043 & 3042 & 280 \\
\hline \multirow{4}{*}{01 on } & F1 & 429 & 453 & 470 & 427 & 419 & 355 & 337 & 445 & 446 & 432 & 446 & 420 & 411 & 422 & 383 & 41 \\
\hline & $F 2$ & 957 & 1028 & 955 & 1295 & 1117 & 1206 & 1154 & 1036 & 1051 & 1018 & 991 & 1070 & 1130 & 997 & 978 & 111 \\
\hline & F3 & 2671 & 2554 & 2388 & 2498 & 2395 & 2607 & 2491 & 2602 & 2685 & 2885 & 2930 & 2399 & 2639 & 2864 & 2846 & 264 \\
\hline & F4 & 3401 & 3583 & 3484 & 3056 & 3322 & 3544 & 3322 & 3442 & 3345 & 3484 & 3401 & 3290 & 3274 & 3391 & 3414 & 3305 \\
\hline \multirow{4}{*}{ ol mid } & F1 & 193 & 463 & 244 & 513 & 391 & 409 & 342 & 426 & 462 & 393 & 399 & 338 & 334 & 411 & 397 & 37 \\
\hline & F2 & 1202 & 1502 & 1295 & 1158 & 1338 & 1355 & 1271 & 1347 & 1096 & 1115 & 1271 & 1337 & 1361 & 1288 & 1338 & 145 \\
\hline & F3 & 2511 & 2672 & 2814 & 2129 & 2742 & 2527 & 2353 & 2470 & 2564 & 2742 & 2679 & 2880 & 2740 & 2781 & 2844 & 267 \\
\hline & F4 & 3551 & 3767 & 3510 & 3671 & 3410 & 3340 & 3387 & 3343 & 3324 & 3571 & 3367 & 3570 & 3380 & 3495 & 3553 & 335 \\
\hline \multirow{4}{*}{ ol off } & $\mathrm{F} 1$ & & 214 & & & 479 & & 361 & 311 & 271 & & 396 & & 492 & & & \\
\hline & F2 & 1641 & 1730 & 1388 & 1628 & 1713 & 1270 & 1562 & 1596 & 1458 & 1650 & 1574 & 1618 & 1610 & 1747 & 438 & 160 \\
\hline & F3 & 2704 & 2704 & 2305 & 2615 & 2827 & 2938 & 2472 & 2710 & 2633 & 2910 & 2644 & 2767 & 2692 & 2773 & 2601 & \\
\hline & F4 & 3595 & 3548 & 3448 & 3655 & 3522 & 3806 & 3477 & 3441 & 3586 & 3587 & 669 & 3643 & 3496 & 3571 & 608 & 362 \\
\hline \multirow{4}{*}{ s1 50\% } & $\mathrm{Fl}$ & & & & & & & & 261 & & 623 & & & & 637 & & \\
\hline & $F$ & 1963 & 1936 & 1848 & 1800 & 1727 & 1621 & 1765 & 1367 & 1723 & 1728 & 1786 & 1643 & 1752 & 1743 & 1749 & 167 \\
\hline & F3 & 2844 & 2674 & 3059 & 2333 & 2895 & 2810 & 2589 & 2334 & 2663 & 2650 & 601 & 2732 & 2634 & 2641 & 3063 & 268 \\
\hline & $\mathrm{F} 4$ & 3582 & 3592 & 3653 & 3513 & 3395 & 3295 & 3489 & 3541 & 3562 & 3603 & 3641 & 3588 & 3509 & 3481 & 3546 & 357 \\
\hline \multirow{4}{*}{ i1 $50 \%$} & $F 1$ & & & & & 375 & & & & & 397 & & & & & & \\
\hline & $F_{2}$ & & & & & 1419 & & & & & 1383 & & & & & & \\
\hline & F3 & & & & & 2393 & & & & & 2217 & & & & & & \\
\hline & F4 & & & & & 2914 & & & & & 3079 & & & & & & \\
\hline \multirow{4}{*}{$\mathrm{m} 250 \%$} & $F 1$ & 284 & 428 & 414 & 414 & 385 & 418 & 407 & 452 & 330 & 351 & 376 & 383 & 302 & 336 & 298 & 31 \\
\hline & $F$ & 1348 & 1042 & 1327 & 1101 & 949 & 1210 & 1026 & 1263 & & 1235 & 1280 & 1302 & 1314 & 1191 & 1330 & \\
\hline & F3 & 2375 & 2408 & 2436 & 2531 & 2461 & 2438 & 2535 & 2318 & 2634 & 2390 & 2455 & 2387 & 2284 & 2355 & 2270 & 2187 \\
\hline & $\mathrm{F} 4$ & 2812 & 3412 & 2740 & 3098 & & & 3116 & 3175 & & 2819 & & 2720 & 2736 & 2833 & 2834 & \\
\hline \multirow{4}{*}{02 onset } & F1 & 366 & 445 & 463 & 460 & 442 & 428 & 349 & 493 & 325 & 430 & 412 & 471 & 352 & 407 & & 39 \\
\hline & $F 2$ & 1311 & 1089 & 974 & 1070 & 1153 & 1024 & 914 & 1175 & 939 & & & 1043 & 1041 & 933 & 944 & 973 \\
\hline & F3 & 2391 & 2618 & 2709 & 2569 & 2640 & 2364 & 2809 & 2358 & 2552 & 2357 & 2295 & 2228 & 2747 & 2641 & 2288 & 2299 \\
\hline & F4 & 3267 & 3504 & 3476 & 3201 & & & 591 & 3249 & 3865 & 3466 & & 3271 & 3677 & 3279 & 3128 & 2759 \\
\hline \multirow{4}{*}{$02 \mathrm{mid}$} & $\mathrm{Fl}$ & 338 & 544 & 354 & 471 & 415 & 469 & & 469 & 407 & 428 & 430 & 421 & & 348 & 875 & 45 \\
\hline & F2 & 1048 & 1555 & 1099 & 1250 & 1252 & 1380 & 1406 & 1383 & 1223 & 1230 & 317 & 1318 & 1299 & 1263 & 1465 & 1289 \\
\hline & F3 & 2733 & 2574 & 2450 & 2425 & 2443 & 2439 & 2827 & 2403 & 2438 & 2531 & 415 & 2520 & 2587 & 2633 & 2517 & 2593 \\
\hline & F4 & & 3612 & 3386 & 3345 & 3486 & & 3552 & 3451 & 3381 & 3399 & 3409 & 3225 & 3413 & 3468 & & 350 \\
\hline \multirow{4}{*}{02 offset } & $F 1$ & 289 & 342 & 347 & & & & & 332 & 365 & & & & 164 & & & 239 \\
\hline & $\mathrm{F} 2$ & 1261 & 1742 & 1453 & 1659 & 1568 & 1563 & 1610 & 1678 & 1514 & 1622 & 1539 & 1550 & 1586 & 1597 & 1602 & 1498 \\
\hline & $F 3$ & 2590 & 2713 & 2512 & 2554 & 2551 & 2325 & 2652 & 2560 & 2401 & 2544 & 2473 & 2517 & 2681 & 2628 & 2635 & 2503 \\
\hline & F4 & 3579 & 3811 & 3479 & 3735 & 3538 & 3566 & 3507 & 3599 & 3355 & 3529 & 3526 & 3509 & 3499 & 3493 & 3501 & 3378 \\
\hline \multirow{4}{*}{ s2 50\% } & F1 & & & & & & & & 488 & & & & & & & & \\
\hline & $\mathrm{F} 2$ & 1851 & 1747 & 1912 & 1649 & 1702 & 1920 & 1802 & 1741 & 1722 & 1738 & 1750 & 1679 & 1665 & 1777 & 1734 & 165 \\
\hline & F3 & 2703 & 2619 & 2787 & 2566 & 2550 & 2901 & 2614 & 2525 & 2663 & 2986 & 2765 & 2651 & 2721 & 2714 & 2733 & 2732 \\
\hline & $\mathrm{F} 4$ & 3663 & 3814 & 3730 & 3635 & 3287 & 3599 & 3647 & 3614 & 3619 & 3647 & 3584 & 3551 & 3598 & 3558 & 3498 & 3501 \\
\hline \multirow{4}{*}{ i2 $50 \%$} & $\mathrm{~F} 1$ & & 340 & 326 & & & & 358 & & & 423 & 268 & 369 & 330 & 335 & & \\
\hline & F2 & & 1972 & 1941 & & & & 1900 & & & 1955 & 1531 & 1856 & 1942 & 1398 & & \\
\hline & F3 & & 2720 & 2688 & & & & 2603 & & & 2515 & 2313 & 2544 & 2786 & 2322 & & \\
\hline & F4 & & 3601 & 3696 & & & & 3522 & & & 3210 & 3312 & 3471 & 3340 & 3217 & & \\
\hline
\end{tabular}




\begin{tabular}{|c|c|c|c|c|c|c|c|c|c|c|c|c|c|c|c|c|}
\hline \multirow{2}{*}{\multicolumn{2}{|c|}{ MN }} & \multicolumn{7}{|c|}{1} & \multicolumn{8}{|c|}{2} \\
\hline & & 1 & 2 & 3 & 4 & 5 & 6 & 7 & 1 & 2 & 3 & 4 & 5 & 6 & 7 & 8 \\
\hline \multirow{4}{*}{$\mathrm{ml} 50 \%$} & F1 & 340 & 630 & & 602 & & & & 616 & 616 & 538 & 629 & 550 & & 650 & \\
\hline & F2 & 1030 & 1187 & & 1008 & & & & 1262 & 1422 & 1431 & 1270 & 1036 & & 1316 & \\
\hline & F3 & 2449 & 2421 & & 2388 & & & & 2416 & 2355 & & 2445 & 2448 & & 2346 & \\
\hline & $\mathrm{F} 4$ & 3244 & 3056 & & 2915 & & & & 2866 & 3328 & 2938 & & 3021 & & & \\
\hline \multirow{4}{*}{ ol on } & $\overline{F 1}$ & 623 & 620 & 640 & 620 & 764 & 656 & 417 & 606 & 566 & 546 & 581 & 656 & 648 & 611 & 703 \\
\hline & $F 2$ & 1067 & 1354 & 1276 & 1025 & 1309 & 1314 & 1773 & 1378 & 1566 & 1464 & 1382 & 1039 & 1254 & 1620 & 1384 \\
\hline & F3 & 2443 & 2446 & 2374 & 2237 & 2379 & 2400 & 2670 & 2362 & 2503 & & 2208 & 2458 & 2310 & 2706 & \\
\hline & F4 & 2817 & 3215 & 3096 & 2792 & & & & 2870 & 3114 & 2944 & 3046 & 3264 & 3245 & 3828 & 3787 \\
\hline \multirow{4}{*}{ ol mid } & $\mathrm{Fl}$ & 642 & 537 & 546 & 613 & 605 & 534 & 116 & 506 & 487 & 442 & 570 & 692 & 651 & 487 & 616 \\
\hline & F2 & 1131 & 1723 & 1551 & 1351 & 1507 & 1497 & 1623 & 1580 & 1683 & 1692 & 1547 & 1354 & 1496 & 1718 & 1607 \\
\hline & F3 & 2395 & 2488 & 2433 & 2309 & 2121 & 2121 & 2303 & & & 2006 & 2367 & 2288 & 2103 & & \\
\hline & F4 & 3209 & 2988 & 3017 & 3111 & 3008 & 2908 & & 2887 & 3006 & 3056 & 2976 & 3051 & 3011 & 2925 & 2934 \\
\hline \multirow{4}{*}{ ol off } & F1 & 603 & 437 & 508 & 572 & 158 & 336 & 402 & 452 & 120 & 499 & 677 & & 539 & 263 & 297 \\
\hline & F2 & 1553 & 1867 & 1704 & 1771 & 2016 & 1504 & 1732 & 1743 & 1679 & 1798 & 1896 & 1635 & 1608 & 1821 & 1830 \\
\hline & F3 & 2449 & 2758 & 2586 & & & 2294 & 2580 & 2819 & 2261 & 2881 & 2861 & 2530 & 2619 & 2802 & 2786 \\
\hline & F4 & 3216 & 3796 & 2767 & 3419 & 3308 & 3270 & 3624 & 3483 & 3036 & 3629 & 3850 & 3473 & 3157 & 3397 & 3591 \\
\hline \multirow{4}{*}{ s1 $50 \%$} & $\mathrm{Fl}$ & 886 & & & & & & & & & & & & & & \\
\hline & F2 & 2017 & 2182 & 2106 & 2009 & 2187 & 2294 & 1954 & 2051 & 2055 & 2152 & 1840 & 1882 & 2007 & 2182 & 1977 \\
\hline & F3 & 2922 & 3016 & 3047 & 3094 & 3222 & 3270 & 2614 & 3102 & 3080 & 3215 & 2992 & 3025 & 3090 & 3051 & 3054 \\
\hline & F4 & 3526 & 3866 & 3537 & 3807 & 3833 & 3922 & 3571 & 3668 & 3911 & 3729 & 3581 & 3890 & 3945 & 3654 & 3948 \\
\hline \multirow{4}{*}{ i1 $50 \%$} & $\mathrm{Fl}$ & & & 365 & & & & & 554 & 397 & 508 & 461 & 485 & 455 & 402 & \\
\hline & $F 2$ & & & 1667 & & & & & 1891 & 1672 & 1601 & 1775 & 1407 & 1355 & 1633 & \\
\hline & F3 & & & 2357 & & & & & 3114 & 2596 & 2802 & 2998 & 2849 & 2887 & 2798 & \\
\hline & F4 & & & 3148 & & & & & 3622 & 3240 & 3308 & 3124 & 3364 & 3426 & 3101 & \\
\hline \multirow{4}{*}{$\mathrm{m} 250 \%$} & $\mathrm{Fl}$ & 440 & 408 & 353 & 473 & 485 & 364 & 518 & 434 & 364 & 506 & 480 & 539 & 380 & 418 & 489 \\
\hline & F2 & 1161 & 1185 & 1113 & 1196 & 1202 & 1106 & 1149 & 1198 & 1164 & 1131 & 1153 & 1156 & 1113 & 1118 & 1149 \\
\hline & F3 & 2470 & 2490 & 2452 & 2423 & 2441 & 2444 & 2358 & 2474 & 2520 & 2501 & 2436 & 2351 & 2530 & 2493 & 2482 \\
\hline & F4 & 3572 & 3513 & 3573 & 3452 & 3209 & 3553 & 3539 & 3415 & 3393 & 3352 & 3260 & 3623 & 3495 & 3447 & 3417 \\
\hline \multirow{4}{*}{02 onset } & $\overline{F l}$ & 533 & 595 & 493 & 592 & 485 & 534 & 624 & 608 & 523 & 591 & 580 & 574 & 558 & 605 & 585 \\
\hline & F2 & 1096 & 1137 & 1125 & 1167 & 1202 & 1172 & 1170 & 1115 & 1137 & 1146 & 1182 & 1082 & 1120 & 1125 & 1147 \\
\hline & F3 & 2432 & 2431 & 2450 & 2355 & 2441 & 2322 & 2357 & 2500 & 2480 & 2442 & 2369 & 2444 & 2453 & 2471 & 2511 \\
\hline & F4 & 3653 & 3604 & 3627 & 3691 & 3582 & 3658 & 3548 & 3666 & 3634 & 3662 & 3489 & 3681 & 3585 & 3511 & 3534 \\
\hline \multirow{4}{*}{$02 \mathrm{mid}$} & $\mathrm{Fl}$ & 561 & 634 & 460 & 559 & 610 & 544 & 607 & 604 & 544 & 573 & 580 & 558 & 633 & 631 & 621 \\
\hline & $\mathrm{F} 2$ & 1387 & 1179 & 1283 & 1317 & 1239 & 1344 & 1371 & 1244 & 1275 & 1238 & 1479 & 1301 & 1351 & 1152 & 1463 \\
\hline & F3 & 2828 & 2384 & 2745 & 2649 & 2495 & 2735 & 2649 & 2727 & 2803 & 2865 & 3045 & 2832 & 2776 & 2917 & 2773 \\
\hline & F4 & 3736 & 3603 & 3635 & 3827 & 3728 & 3618 & 3540 & 3769 & 3837 & 3718 & 3923 & 3819 & 3745 & 3730 & 3773 \\
\hline \multirow{4}{*}{02 offset } & F1 & & & 221 & 484 & 480 & 263 & 209 & 341 & 304 & 829 & 309 & 401 & 631 & 603 & 609 \\
\hline & F2 & 1820 & 1768 & 2000 & 1886 & 1892 & 1907 & 1876 & 1784 & 1805 & 1901 & 1838 & 1663 & 1873 & 1774 & 1877 \\
\hline & F3 & 2960 & 2918 & 3050 & 3011 & 3084 & 2925 & 3118 & 3228 & 2859 & 2868 & 2887 & 3052 & 2883 & 2891 & 2907 \\
\hline & F4 & 3843 & 3585 & 3624 & 3793 & 3497 & 3637 & 3815 & 3779 & 3720 & 3585 & 3698 & 4064 & 3708 & 3481 & 3502 \\
\hline \multirow{4}{*}{ s2 $50 \%$} & $\mathrm{~F} 1$ & & & & & & & & & & & & & & & \\
\hline & $F 2$ & 2314 & 2073 & 2193 & 2216 & 2074 & 2267 & 2250 & 2123 & 2094 & 2139 & 2377 & 2213 & 2158 & 2125 & 2080 \\
\hline & F3 & 3180 & 2893 & 3014 & 3034 & 2930 & 2964 & 2919 & 2962 & 3062 & 3098 & 3165 & 3127 & 3039 & 3052 & 2972 \\
\hline & F4 & 3830 & 3817 & 3831 & 3743 & 3898 & 3826 & 3788 & 3777 & 3759 & 3973 & 4006 & 3825 & 3828 & 3801 & 3699 \\
\hline i2 $50 \%$ & $\begin{array}{l}F 1 \\
F 2 \\
F 3 \\
F 4\end{array}$ & & & & & & & & & & & & & & & \\
\hline
\end{tabular}




\begin{tabular}{|c|c|c|c|c|c|c|c|c|c|c|c|c|c|c|}
\hline \multirow{2}{*}{\multicolumn{2}{|c|}{ MO }} & \multicolumn{6}{|c|}{1} & \multicolumn{7}{|c|}{2} \\
\hline & & 181 & 227 & 184 & 252 & 218 & 218 & 383 & 151 & & 218 & 245 & 219 & 252 \\
\hline \multirow{4}{*}{$\mathrm{ml} \mathrm{50 \%}$} & FI & 954 & 932 & 961 & 971 & 1044 & 1222 & 1115 & 1050 & 961 & 1051 & 841 & 890 & 1043 \\
\hline & F2 & 2865 & 2770 & 3076 & 2756 & 2700 & & & 2805 & & 2776 & & & 2407 \\
\hline & F3 & 4075 & 3831 & 4179 & 3810 & 3840 & & & & 3569 & 3873 & 3367 & 3441 & 3780 \\
\hline & F4 & 454 & 441 & 301 & 163 & 465 & 155 & 373 & 461 & 535 & 444 & 485 & 504 & 447 \\
\hline \multirow{4}{*}{ ol on } & F1 & 1089 & 990 & 1086 & 903 & 1137 & 1551 & 920 & 1012 & 1135 & 1040 & 1119 & 1180 & 1132 \\
\hline & F2 & 2763 & 2548 & 3028 & 2812 & 2819 & 2626 & 3188 & 2591 & 2748 & 2881 & 2607 & 3068 & \\
\hline & F3 & 3697 & 3507 & 3632 & 3670 & 3612 & 3713 & 3906 & 3575 & 3649 & 3565 & 3525 & 3593 & 3460 \\
\hline & F4 & 93 & 322 & 332 & 460 & 480 & 120 & 496 & 452 & 515 & & & 538 & 490 \\
\hline \multirow{4}{*}{ ol mid } & F1 & 1564 & 1495 & 1354 & 1360 & 1416 & 1849 & 1347 & 1537 & 1518 & 1546 & 1542 & 1669 & 1517 \\
\hline & $F 2$ & 2695 & 2640 & 2770 & 2692 & 2817 & 2699 & 2733 & 2668 & 2675 & 2803 & 2665 & 2827 & 2536 \\
\hline & F3 & 3653 & 3693 & 3882 & 3896 & 3734 & 4022 & 3669 & 3779 & 3714 & 3745 & 3722 & 3895 & 3610 \\
\hline & F4 & & & & & & & & 214 & & & & & \\
\hline \multirow{4}{*}{ ol off } & F1 & 1655 & 1668 & 2076 & 1501 & 1699 & 1642 & 1747 & 1730 & 1803 & 1707 & 1776 & 1809 & 177 \\
\hline & $F 2$ & 2722 & 2691 & & 2604 & 2532 & 2662 & 2794 & 2704 & 2715 & 2978 & 2758 & 2731 & 266 \\
\hline & F3 & 3623 & 3827 & 3645 & 3712 & 3715 & 3663 & 3741 & 3860 & 3684 & 3733 & 3785 & 3665 & 363 \\
\hline & F4 & 321 & & & & & & & & 398 & & & & \\
\hline \multirow{4}{*}{ sl $50 \%$} & F1 & 1613 & 1314 & 2224 & 1832 & 1490 & 1651 & 1827 & 1936 & 1770 & 1557 & 1746 & 1865 & 177 \\
\hline & F2 & 2742 & 2652 & 2764 & 2754 & 2654 & 2633 & 2840 & 2674 & 2780 & 2772 & 2764 & 2828 & 2666 \\
\hline & F3 & 3736 & 3427 & 3807 & 3967 & 3752 & 3653 & 3759 & 3592 & 3782 & 3529 & 3659 & 3637 & 377 \\
\hline & F4 & 212 & 357 & & 231 & & & 285 & & 411 & & & & \\
\hline \multirow{4}{*}{ i1 $50 \%$} & $\mathrm{~F} 1$ & 1546 & 1323 & & 1522 & & & 1655 & & 1592 & & & & \\
\hline & $\mathrm{F}$ & 2318 & 2151 & & 2367 & & & 2323 & & 2483 & & & & \\
\hline & F3 & 3290 & 3239 & & 3497 & & & 3389 & & 3325 & & & & \\
\hline & F4 & 233 & 274 & 184 & 229 & 226 & 196 & 296 & 369 & 309 & 405 & 288 & 410 & 37 \\
\hline \multirow{4}{*}{$\mathrm{m} 250 \%$} & $F$ & 1020 & 998 & 1022 & 1066 & 1055 & 1581 & 1042 & 1042 & 1132 & 1156 & 1023 & 1155 & 1103 \\
\hline & F2 & 2472 & 2511 & 2382 & 2495 & 2286 & 2390 & 2457 & 2376 & 2379 & 2702 & 2410 & 2670 & 2149 \\
\hline & F3 & 3773 & 3885 & 3580 & 3620 & 3565 & 3492 & 3685 & 3434 & 3441 & 3562 & 3649 & 3436 & 3441 \\
\hline & F4 & 338 & 296 & 339 & 256 & 168 & & 495 & 431 & 492 & 368 & 440 & 455 & 415 \\
\hline \multirow{4}{*}{02 onset } & F1 & 1209 & 1049 & 1090 & 1028 & 1395 & 1489 & 1188 & 1044 & 1200 & 1214 & 1149 & 1497 & 1143 \\
\hline & F2 & 2037 & 2430 & 2255 & 2230 & 2508 & 2332 & 2077 & 2581 & 2702 & 2664 & 2684 & 2609 & 220 \\
\hline & F3 & 3611 & 3527 & 3682 & 3648 & 3632 & 3414 & 3699 & 3428 & 3593 & 3537 & 3571 & 3576 & 3470 \\
\hline & F4 & 148 & & & 291 & 203 & & & & 492 & 444 & 446 & 460 & 639 \\
\hline \multirow{4}{*}{$02 \mathrm{mid}$} & F1 & 1543 & 198 & 1429 & 1520 & 1513 & 1503 & 382 & 1541 & 1422 & 1585 & 1510 & 1659 & 1448 \\
\hline & F2 & 2695 & 2491 & 2681 & 2681 & 2573 & 2521 & 2597 & 2562 & 2634 & 2716 & 2598 & 2695 & 2499 \\
\hline & F3 & 3744 & 3594 & 3769 & 3897 & 3642 & 3638 & 3688 & 3592 & 3674 & 3655 & 3629 & 3723 & 3545 \\
\hline & F4 & & & & & 225 & & & 290 & & 190 & & & 14 \\
\hline \multirow{3}{*}{02 offset } & $\mathrm{F}$ & & & 1715 & 1651 & 1502 & 1615 & 836 & 1743 & 1724 & 1682 & 1660 & 1811 & 1681 \\
\hline & F2 & 747 & & 2626 & 2791 & 575 & 2561 & 678 & 2691 & 2700 & 2723 & 684 & 2730 & 2542 \\
\hline & F3 & 3920 & 3628 & 3944 & 4103 & 3651 & 3904 & 3768 & 3815 & 3734 & $\begin{array}{r}3779 \\
280\end{array}$ & 3559 & 3629 & \\
\hline \multirow{4}{*}{ s2 50\% } & $\mathrm{Fl}$ & 59 & & 114 & 770 & 1805 & 1629 & 1803 & 1734 & 1848 & 1739 & 1892 & 1875 & 1714 \\
\hline & $F$ & 2682 & & 2621 & 2711 & 2708 & 2564 & 2858 & 2616 & 2765 & 2764 & 2812 & 2818 & 2563 \\
\hline & $F$ & 3939 & 3918 & 3912 & 4085 & 3692 & 3692 & 3897 & 3816 & 3998 & 4120 & 3912 & 3775 & 3313 \\
\hline & F & 289 & 30 & 240 & 353 & 293 & & 330 & 343 & 269 & & & & \\
\hline \multirow{4}{*}{ i2 $50 \%$} & F & 2374 & 1960 & 2006 & 1946 & 2004 & & 1985 & 1966 & 1892 & & & & \\
\hline & $F$ & & & 2843 & 2831 & 2916 & & 2982 & 2713 & 2860 & & & & \\
\hline & $F$ & 3941 & 35 & 3933 & 3733 & 3871 & & 3832 & 3603 & & & & & \\
\hline & F4 & 181 & 227 & 184 & 252 & 218 & 218 & 383 & 151 & & 218 & 245 & 219 & 252 \\
\hline
\end{tabular}




\begin{tabular}{|c|c|c|c|c|c|c|c|c|c|c|c|c|c|c|}
\hline \multirow{2}{*}{\multicolumn{2}{|c|}{ TN }} & \multicolumn{6}{|c|}{1} & \multicolumn{7}{|c|}{2} \\
\hline & & 1 & 2 & 3 & 4 & 5 & 6 & 1 & 2 & 3 & 4 & 5 & 6 & 7 \\
\hline \multirow{4}{*}{$\mathrm{m} 150 \%$} & $\mathrm{~F} 1$ & 125 & 210 & 161 & 189 & 161 & & 204 & 173 & 194 & & 168 & 190 & 203 \\
\hline & F2 & 1001 & 1128 & 856 & 1227 & & & 1014 & 1060 & & & 1144 & 983 & 989 \\
\hline & F3 & 2389 & 2687 & 2751 & & & & 2580 & 2669 & 2880 & & & 2654 & \\
\hline & F4 & 3637 & 3879 & 3727 & 3902 & 3686 & & 3804 & 3761 & 3747 & & 3861 & 3611 & 3903 \\
\hline \multirow{4}{*}{ ol on } & $\mathrm{F} 1$ & 152 & 458 & 145 & 106 & & & 221 & 241 & 169 & & 207 & 149 & 231 \\
\hline & F2 & 1165 & 1162 & 1139 & 1026 & 1332 & 1089 & 1007 & 972 & 856 & & 1059 & 1011 & 1007 \\
\hline & F3 & 2542 & 2472 & 2508 & 2807 & 2606 & & 2622 & 2488 & 2362 & & 2409 & 2746 & 2167 \\
\hline & F4 & 3541 & 3715 & 3614 & 3577 & 3569 & & 3674 & 3341 & 3476 & & 3499 & 3344 & 3771 \\
\hline \multirow{4}{*}{ ol mid } & F1 & 115 & 225 & 179 & & & 218 & 393 & 335 & 130 & & 190 & 291 & 134 \\
\hline & $\mathrm{F} 2$ & 1536 & 1650 & 1688 & 1446 & 1599 & 1543 & 1340 & 1574 & 1239 & & 1400 & 1398 & 1451 \\
\hline & F3 & 2685 & 2495 & 2554 & 2593 & 2535 & 2441] & 2445 & 2758 & 2787 & & 2663 & 2654 & 2670 \\
\hline & F4 & 3809 & 3602 & 3834 & 3774 & 3848 & 3531 & 3510 & 3640 & 3567 & & 3575 & 3566 & 3508 \\
\hline \multirow{4}{*}{ ol off } & F1 & & & & & & 248 & & 460 & & & & & \\
\hline & F2 & 1868 & 1798 & 2128 & 1676 & 1503 & 1852 & 1964 & 1738 & 1905 & & 1702 & 1656 & 1782 \\
\hline & F3 & 2532 & 2518 & 2842 & 2299 & 2065 & 2708 & 2472 & 2716 & 2875 & & 2557 & 2721 & 2710 \\
\hline & F4 & 3986 & 3836 & 3923 & 3886 & 3813 & 3822 & 3710 & 3710 & 3704 & & 4084 & 3694 & 3843 \\
\hline \multirow{3}{*}{ s1 50\% } & $\begin{array}{l}\text { F1 } \\
\text { F2 }\end{array}$ & 811 & 2015 & 1848 & 1828 & 1718 & 2118 & 1945 & 1874 & 1840 & & 2056 & 1902 & 1810 \\
\hline & F3 & 2855 & 2816 & 2661 & & 2331 & 2826 & 2998 & 2738 & 2386 & & 2476 & 2842 & 2730 \\
\hline & F4 & 3948 & 3683 & 3758 & 3903 & 3673 & 3893 & 3929 & 3821 & 3672 & & 3825 & 3616 & 3620 \\
\hline \multirow[b]{2}{*}{ i1 $50 \%$} & $\begin{array}{l}\mathrm{F} 1 \\
\mathrm{~F} 2\end{array}$ & & & & & & & & & & & & & \\
\hline & $\begin{array}{l}\mathrm{F} \\
\mathrm{F} 4\end{array}$ & & & & & & & & & & & & & \\
\hline \multirow{4}{*}{$\mathrm{m} 250 \%$} & $\mathrm{Fl}$ & 154 & 200 & 203 & 182 & 204 & 215 & 219 & 224 & 206 & & 199 & 246 & 268 \\
\hline & F2 & 1212 & 1171 & 1318 & 1263 & 1377 & 1272 & 1167 & 1293 & 993 & & 984 & 1443 & 1348 \\
\hline & F3 & 2433 & 2501 & 2381 & 2516 & 2213 & 2380 & 2580 & 2389 & 2622 & & 2760 & 2519 & 2225 \\
\hline & F4 & 3512 & 3837 & 3348 & 3661 & 3789 & 3739 & 3423 & 3311 & 3785 & & 3764 & 3376 & 3196 \\
\hline \multirow{4}{*}{02 onset } & $\mathrm{Fl}$ & 114 & & & 127 & 259 & 350 & 187 & 202 & 383 & & 179 & 347 & 396 \\
\hline & F2 & 1190 & 1349 & 1468 & 1214 & 1509 & 1313 & 1567 & 1568 & 1239 & & 1281 & 1649 & 1488 \\
\hline & F3 & 2474 & 2448 & 2441 & 2489 & 2512 & 2316 & 2590 & 2749 & 2085 & & 2411 & 2547 & 2471 \\
\hline & F4 & 3469 & 3674 & 3869 & 3693 & 3851 & 3599 & 3534 & 3568 & 3298 & & 3410 & 3451 & 3178 \\
\hline \multirow{4}{*}{$02 \mathrm{mid}$} & F1 & & 95 & 108 & & 154 & 131 & 313 & 507 & 383 & & 283 & 182 & 98 \\
\hline & F2 & 1607 & 1886 & 1651 & 1426 & 1723 & 1600 & 1882 & 1743 & 1399 & & 1576 & 1878 & 1745 \\
\hline & F3 & 2657 & 2343 & 2523 & 2544 & 2391 & 2347 & 2595 & 2580 & 2464 & & 2450 & 2689 & 2634 \\
\hline & F4 & 3844 & 3858 & 3988 & 3686 & 3458 & 3827 & 3702 & 3639 & 3297 & & 3522 & 3735 & 3430 \\
\hline \multirow{4}{*}{02 offset } & $\mathrm{F} 1$ & & 288 & & & & & 884 & 764 & & & 806 & & \\
\hline & $F 2$ & 1939 & 1950 & 1834 & 1697 & 1460 & 1513 & 2024 & 1838 & 1768 & & 2000 & 1954 & 1826 \\
\hline & F3 & 2633 & 2604 & 2673 & 2680 & 2148 & 2894 & 2728 & 2471 & 2647 & & 2541 & 2865 & 2588 \\
\hline & F4 & 3835 & 3791 & 3792 & 3759 & 3814 & 3899 & 3809 & 3600 & 3418 & & 3763 & 3595 & 3648 \\
\hline \multirow{4}{*}{ s2 50\% } & $F 1$ & & & & & & & 820 & & & & 806 & & \\
\hline & F2 & 2062 & 2033 & 2060 & 2458 & 2344 & 2249 & 2107 & 1790 & 2079 & & 2034 & 1988 & 1757 \\
\hline & F3 & 2651 & 2649 & 2590 & 2718 & & 2677 & 2671 & 2536 & 2778 & & 2738 & 2826 & 2494 \\
\hline & $F 4$ & 3639 & 3830 & 3929 & 3836 & 3819 & 3830 & 3686 & 3506 & 3848 & & 3739 & 3704 & 3571 \\
\hline \multirow{4}{*}{ i2 $50 \%$} & $\mathrm{Fl}$ & 308 & 323 & 332 & & & 321 & 300 & & 256 & & 297 & 260 & \\
\hline & F2 & 2176 & 2436 & 2203 & & & 2221 & 2168 & & 2172 & & 2168 & 1706 & \\
\hline & F3 & 2771 & 2638 & 2705 & & & 2758 & 2753 & & 2783 & & 2778 & 2563 & \\
\hline & F4 & 3704 & 3961 & 3856 & & & 3813 & 3583 & & 3663 & & 3658 & 3420 & \\
\hline
\end{tabular}




\begin{tabular}{|c|c|c|c|c|c|c|c|c|c|c|c|c|c|c|c|}
\hline \multirow{2}{*}{\multicolumn{2}{|c|}{ TS }} & \multicolumn{8}{|c|}{1} & \multicolumn{6}{|c|}{2} \\
\hline & & 1 & 2 & 3 & 4 & 5 & 6 & 7 & 8 & 1 & 2 & 3 & 4 & 5 & \\
\hline \multirow{4}{*}{$\mathrm{ml} \mathrm{50 \%}$} & Fl & 278 & 379 & 286 & 282 & 371 & 346 & 470 & 325 & 357 & 201 & 288 & 264 & 262 & 296 \\
\hline & F2 & 1198 & 1295 & 964 & 930 & 719 & 1331 & 992 & 1226 & 1012 & 884 & 948 & 673 & 749 & \\
\hline & F3 & 2088 & 1727 & 2145 & 2051 & 2061 & 2035 & 1982 & 2299 & & 2728 & 2866 & 2324 & 2012 & 2763 \\
\hline & $\mathrm{F4}$ & 2727 & 2907 & 3098 & 3382 & 3682 & 3454 & 3223 & & 3028 & 3572 & 3823 & 3393 & 3894 & 3542 \\
\hline \multirow{4}{*}{01 on } & F1 & 442 & 411 & 426 & 426 & 388 & 396 & 482 & 380 & 373 & 339 & 350 & 182 & 327 & 205 \\
\hline & F2 & 917 & 1174 & 832 & 832 & 706 & 929 & 1201 & 975 & 1134 & 1044 & 1181 & 1144 & 1000 & 911 \\
\hline & F3 & 2172 & 1895 & 2430 & 1838 & 2234 & 2005 & 1977 & & 2301 & 2228 & 2671 & 2501 & 2202 & 2230 \\
\hline & F4 & 3466 & 3431 & 3392 & 3392 & 3445 & 3342 & & 3618 & 3515 & 3458 & 3457 & 3128 & 3546 & 3329 \\
\hline \multirow{4}{*}{01 mid } & $\mathrm{F} 1$ & 480 & 391 & 433 & 433 & 490 & 453 & 321 & 439 & 269 & 175 & 249 & 146 & & 205 \\
\hline & F2 & 1285 & 1402 & 1292 & 1337 & 1258 & 1352 & 1682 & 1403 & 1438 & 1174 & 1509 & 1675 & 1505 & 1274 \\
\hline & F3 & 2905 & 2800 & 2882 & 2881 & 2955 & 2929 & 2649 & 2784 & 2758 & 2697 & 2898 & 2916 & 2956 & 2185 \\
\hline & F4 & 3574 & 3475 & 3569 & 3584 & 3578 & 3523 & 3976 & 3553 & 3387 & 3528 & 3434 & 3522 & 3522 & 3057 \\
\hline \multirow{4}{*}{01 off } & F1 & 203 & 233 & & 109 & 162 & 162 & & 179 & 169 & & & & 116 & 109 \\
\hline & F2 & 1812 & 1724 & 1824 & 1729 & 1819 & 2038 & 1796 & 1616 & 1472 & 2023 & 1484 & 1637 & 2207 & 1536 \\
\hline & F 3 & 3022 & 2863 & 2919 & 2811 & 2922 & 2932 & 2670 & 2627 & 2433 & 2819 & 2816 & 3044 & 3196 & 2861 \\
\hline & F4 & 3523 & 3664 & 3575 & 3626 & 3777 & 3620 & 3790 & 3340 & 3297 & 3378 & 3471 & 3706 & 3585 & 3492 \\
\hline \multirow{4}{*}{ s1 $50 \%$} & F1 & 500 & & & & & & & & & & 824 & & & \\
\hline & F2 & 1731 & 1900 & 2151 & 1659 & 2175 & 1840 & 1587 & 1516 & 1849 & 1578 & 1900 & 1832 & 2293 & \\
\hline & F3 & 2685 & 2834 & 2771 & 2451 & 2764 & 2733 & 2418 & 2508 & 2928 & 2358 & 2940 & 2642 & 3078 & 2693 \\
\hline & F4 & 3572 & 3299 & 3359 & 3701 & 3660 & 3395 & 3381 & 3334 & 3587 & 3431 & 3536 & 3250 & & 3217 \\
\hline \multirow{4}{*}{ il $50 \%$} & F1 & & 370 & & & 368 & & & & & & & & & \\
\hline & $\mathrm{F}$ & & 1379 & & & 1240 & & & & & & & & & \\
\hline & F3 & & 1964 & & & 2060 & & & & & & & & & \\
\hline & F4 & & 3119 & & & 2271 & & & & & & & & & \\
\hline \multirow{4}{*}{$\mathrm{m} 250 \%$} & F1 & 400 & 412 & 394 & 348 & 364 & 356 & 327 & 334 & 322 & 131 & 312 & 235 & 327 & 203 \\
\hline & F2 & 1428 & 682 & 1386 & 956 & 828 & 832 & 1323 & 1284 & 1289 & 1096 & 1027 & 1091 & 1070 & 1022 \\
\hline & F3 & 2186 & 2245 & 2140 & 1961 & 2108 & 2176 & 2182 & 2189 & 2374 & 2334 & 2365 & 2292 & 2323 & 2146 \\
\hline & F4 & 3166 & 3120 & 3268 & 3248 & 3345 & 3292 & 3369 & 3408 & 3504 & 3250 & 3085 & 3157 & 3342 & 3052 \\
\hline \multirow{4}{*}{02 onset } & $\mathrm{F} 1$ & 463 & 470 & 447 & 393 & 403 & 449 & 382 & 403 & & 211 & 361 & 132 & 282 & \\
\hline & F2 & 1627 & 731 & 901 & 698 & 870 & 996 & 1036 & 1058 & 1007 & 1499 & 1145 & 979 & 844 & 1200 \\
\hline & F3 & 2234 & 2503 & 1966 & 1942 & 2181 & 2049 & 2024 & 2079 & 2122 & 2544 & 2298 & 2178 & 2395 & \\
\hline & F4 & 3279 & 3163 & 3143 & 3513 & 3319 & 3239 & 3543 & 3170 & 3468 & 3509 & 3252 & 3143 & 3205 & 2883 \\
\hline \multirow{4}{*}{$\begin{array}{c}02 \\
\text { mid }\end{array}$} & $\mathrm{F} 1$ & 479 & 461 & 470 & 512 & 470 & 480 & 437 & 506 & 793 & & 426 & & 371 & \\
\hline & F2 & 1193 & 1292 & 1220 & 1185 & 1276 & 1307 & 1214 & 1334 & 1465 & 1904 & 1408 & 1295 & 976 & 1552 \\
\hline & $\mathrm{F3}$ & 2105 & 2815 & 2310 & 2958 & 2835 & 2735 & 1822 & 2836 & 2708 & 2503 & 2536 & 2514 & 2211 & \\
\hline & F4 & 3361 & 3692 & 3074 & 3982 & 3670 & 3444 & 3209 & 3239 & 3453 & 3598 & 3331 & 3472 & 3247 & 2756 \\
\hline \multirow{4}{*}{02 offset } & F1 & & 334 & 157 & 397 & 393 & 570 & & & & & & & & \\
\hline & F2 & 2118 & 1839 & 1593 & 1841 & 1841 & 1836 & 1794 & 1722 & 1617 & 2063 & 1678 & 1548 & 1872 & \\
\hline & F3 & 3213 & 2772 & 2919 & 3208 & 2717 & 2765 & 2804 & 2492 & 2547 & 3124 & 2917 & 2338 & 2873 & 2331 \\
\hline & $\mathrm{F} 4$ & 3812 & 3700 & 3655 & 3903 & 3499 & 3497 & 3451 & 3734 & 3532 & 3832 & 3638 & 3260 & 3712 & 3141 \\
\hline \multirow{4}{*}{$\$ 250 \%$} & F1 & & & & & & & & & & 198 & & & & \\
\hline & F2 & 2332 & 1898 & 1985 & 2182 & 2284 & 2080 & 2550 & 1994 & 1733 & 2035 & 2482 & 2104 & 1956 & 1656 \\
\hline & F3 & 3061 & 2767 & 2860 & 2713 & 2613 & 3044 & 3054 & 2810 & 2842 & 2936 & 3566 & 3413 & 3505 & 2676 \\
\hline & F4 & 3836 & 3753 & 3837 & 3634 & 3631 & 3638 & 3608 & 3427 & 3689 & 3886 & 4119 & 3854 & 3959 & 3511 \\
\hline \multirow{4}{*}{ i2 $50 \%$} & $\mathrm{~F} 1$ & 334 & & & 333 & 341 & & 341 & & 211 & & & & & \\
\hline & $F 2$ & 2071 & & 1985 & 2192 & 2089 & & 2053 & & 1727 & & & & & \\
\hline & F3 & 2934 & & 2860 & 3128 & 3343 & & 2638 & & 2386 & & & & & \\
\hline & F4 & 3900 & & 3837 & 4188 & 4161 & & 3520 & & 3279 & & & & & \\
\hline
\end{tabular}




\begin{tabular}{|c|c|c|c|c|c|c|c|c|c|c|c|c|c|c|c|}
\hline \multirow[t]{2}{*}{$\mathrm{TY}$} & & \multicolumn{6}{|c|}{1} & \multicolumn{8}{|c|}{2} \\
\hline & & 1 & 2 & 3 & 4 & 5 & 6 & 1 & 2 & 3 & 4 & 5 & 6 & 7 & \\
\hline \multirow{4}{*}{$\mathrm{ml} 50 \%$} & F1 & & & 181 & 264 & 247 & & & 262 & 383 & 258 & 247 & 230 & & \\
\hline & F2 & 1084 & 1437 & 1206 & 1245 & 1242 & & 1001 & 1187 & 1258 & 1220 & 1258 & 1289 & 1028 & 1399 \\
\hline & F3 & 2474 & 2324 & 2389 & 2403 & 2394 & 2420 & 2315 & 2417 & 2375 & 2507 & 2522 & 2369 & 2370 & 2369 \\
\hline & F4 & 3681 & 3421 & 2961 & & & 3229 & 3158 & 3412 & 3627 & & & 3623 & 3472 & 3886 \\
\hline \multirow{4}{*}{ ol on } & $\mathrm{Fl}$ & 45 & 318 & & 250 & 250 & 420 & & 567 & 318 & 409 & 626 & 430 & 383 & 665 \\
\hline & $F_{2}$ & 778 & 915 & 625 & 741 & 720 & 926 & 692 & 751 & 680 & 878 & 954 & 799 & 646 & 1179 \\
\hline & F3 & 2447 & 2319 & 2404 & 2509 & 2506 & 2417 & 2230 & 2267 & 2196 & 2126 & 2137 & 2472 & 2616 & 2453 \\
\hline & F4 & 3324 & 3311 & 3443 & 3314 & 3269 & 3285 & 3333 & 3718 & 3751 & 3454 & 3421 & 3572 & 3358 & 3461 \\
\hline \multirow{4}{*}{$01 \mathrm{mid}$} & $\mathrm{Fl}$ & 461 & 414 & 34 & 446 & 443 & & 476 & 457 & 369 & 470 & 452 & 442 & 420 & 330 \\
\hline & $F_{2}$ & 1187 & 1291 & 1276 & 1224 & 1216 & 1434 & 1198 & 1263 & 1252 & 1219 & 1309 & 1333 & 1219 & 1268 \\
\hline & F3 & 2365 & 2265 & 2292 & 2525 & 2521 & 2500 & 2333 & 2359 & 2463 & 2276 & 2220 & 2424 & 2847 & 2445 \\
\hline & F4 & 3218 & 3303 & 2981 & 3246 & 3228 & 3229 & 3106 & 3441 & 3485 & 3478 & 3395 & 3480 & 3354 & 3256 \\
\hline \multirow{4}{*}{ ol off } & $F 1$ & & 197 & 241 & & & 405 & 266 & 314 & 190 & 108 & 245 & & 325 & 313 \\
\hline & $F 2$ & 1349 & 1462 & 1593 & 1616 & 1609 & 1687 & 1524 & 1778 & 1585 & 1606 & 1545 & 1694 & 1599 & 1524 \\
\hline & F3 & 3025 & 2539 & 2654 & 2714 & 2695 & & 2854 & 2615 & 2587 & 2675 & 2861 & 2404 & 2646 & 2696 \\
\hline & F4 & 3540 & 3410 & 3294 & 3375 & 3367 & 3235 & 3486 & 3466 & 3379 & 3628 & 3411 & 3137 & 3487 & 3476 \\
\hline \multirow{3}{*}{ s1 $50 \%$} & $\begin{array}{l}\text { F1 } \\
F 2\end{array}$ & 1661 & 574 & 1551 & 1649 & 1645 & 1855 & 1660 & 1688 & 1723 & 1608 & & 1634 & 1656 & 1651 \\
\hline & F3 & 2661 & 2374 & 2447 & 2850 & 2825 & & 2903 & 2647 & 2532 & 2493 & 2384 & 2567 & 2661 & 2640 \\
\hline & F4 & 3268 & 3144 & 3245 & 3296 & 3293 & 3322 & 3486 & 3458 & 3311 & 3386 & 3283 & 3316 & 3392 & 3366 \\
\hline i1 $50 \%$ & $\begin{array}{l}F 1 \\
F 2 \\
F 3 \\
F 4\end{array}$ & & & & & & & & & & & & & & \\
\hline \multirow{4}{*}{$\mathrm{m} 250 \%$} & Fl & & & 279 & 455 & 456 & 270 & 270 & 292 & 450 & 259 & 430 & 496 & 284 & 275 \\
\hline & F2 & 062 & 1191 & 1181 & 1219 & 1219 & 1108 & 1204 & 1298 & 998 & 1185 & 1075 & 1019 & 1196 & 1339 \\
\hline & F3 & 2411 & 2378 & 2511 & 2418 & 2426 & 2389 & 2720 & 2538 & 2422 & 2525 & 2580 & 2465 & 2579 & 2617 \\
\hline & $F 4$ & 3819 & 3714 & 3720 & & 3567 & 3151 & 3589 & 3985 & 3575 & 4144 & & & & 3857 \\
\hline \multirow{4}{*}{02 onset } & F1 & 549 & 398 & 371 & 519 & 466 & 485 & 606 & 451 & 489 & 339 & 474 & 309 & 530 & 784 \\
\hline & F2 & 1012 & 948 & 874 & 1097 & 1192 & 1020 & 1082 & 956 & 1041 & 1003 & 1138 & 854 & 1405 & 1443 \\
\hline & F3 & 2365 & 2369 & 2244 & 2417 & 2445 & 2388 & 2078 & 2443 & 2418 & 2560 & 2829 & 2520 & 2634 & 2454 \\
\hline & F4 & 3316 & 3209 & 3542 & 3246 & 3259 & 3176 & 3263 & 3414 & 3309 & 3404 & 3346 & & & 3406 \\
\hline \multirow{4}{*}{$02 \mathrm{mid}$} & F1 & 490 & 536 & 486 & 436 & 356 & 378 & 556 & 485 & 322 & 312 & 290 & 368 & 525 & 552 \\
\hline & $\mathrm{F} 2$ & 1318 & 1324 & 1156 & 1498 & 1565 & 1241 & 1429 & 1230 & 1531 & 1454 & 1485 & 1396 & 1243 & 1373 \\
\hline & F3 & 2373 & 2030 & 2295 & 2443 & 2530 & 2325 & 2173 & 2383 & 2469 & 2822 & 2672 & 2525 & 2937 & 2447 \\
\hline & F4 & 3107 & 3371 & 3253 & 3592 & 3600 & 3216 & 3020 & 3366 & 3379 & 3545 & 3634 & 3320 & 3217 & 3358 \\
\hline \multirow{4}{*}{02 offset } & F1 & 411 & 219 & & 327 & 305 & 285 & 188 & 253 & 290 & 198 & 104 & 265 & 261 & 631 \\
\hline & $\mathrm{F} 2$ & 1618 & 1623 & & 1732 & 1673 & 1585 & 1633 & 1575 & 1739 & 1516 & 1571 & 1654 & 1606 & 1708 \\
\hline & F3 & 2852 & 2880 & 2977 & 2585 & 2763 & 2899 & 2957 & 2931 & 2787 & 2921 & 2860 & 2720 & 2819 & 3159 \\
\hline & F4 & 3137 & 3569 & 3692 & 3381 & 3410 & 3300 & 3661 & 3294 & 3492 & 3582 & 3757 & 3663 & 3713 & 3684 \\
\hline \multirow{3}{*}{ s2 50\% } & $\begin{array}{l}F 1 \\
F 2\end{array}$ & & & & & & & & & & & & & & \\
\hline & $F_{3}$ & 19 & 2404 & 2327 & & 2572 & 2380 & 2793 & 2457 & 2740 & 2275 & 2706 & 2583 & 2531 & 2708 \\
\hline & F & 3295 & 3232 & 3239 & & 3380 & 3358 & 3816 & 3339 & 3674 & 3423 & 3500 & 3669 & 3458 & 3290 \\
\hline \multirow{4}{*}{ i2 $50 \%$} & $\mathbf{F}$ & & 344 & 331 & & & & & 330 & & 278 & 313 & 331 & & 348 \\
\hline & $F_{2}$ & & 1822 & 1818 & & & & & 2062 & & 1756 & 1863 & 1745 & & 2063 \\
\hline & F3 & & 2551 & 2660 & & & & & 2774 & & 2936 & 2783 & 2514 & & 2798 \\
\hline & F4 & & & 3064 & & & & & 3388 & & 3607 & 3558 & 3343 & & 3351 \\
\hline
\end{tabular}




\begin{tabular}{|c|c|c|c|c|c|c|c|c|c|c|c|c|c|}
\hline \multirow{2}{*}{\multicolumn{2}{|c|}{ YH }} & \multicolumn{5}{|c|}{1} & & \multicolumn{6}{|c|}{2} \\
\hline & & 1 & 2 & 3 & 4 & 5 & 6 & 1 & 2 & 3 & 4 & 5 & 6 \\
\hline \multirow{4}{*}{$\mathrm{ml} 50 \%$} & F1 & 136 & 221 & 369 & 385 & 452 & 278 & 225 & 121 & 120 & 227 & & 101 \\
\hline & F2 & 963 & 855 & 990 & 1015 & 1145 & 837 & 881 & 908 & 753 & 1010 & 869 & 932 \\
\hline & F3 & 2375 & 2188 & 2025 & 2140 & 1894 & 2131 & 2327 & 2055 & 2091 & 2218 & 1908 & 2136 \\
\hline & F4 & 3346 & 3673 & 3107 & 3078 & & 3547 & & 3265 & 3083 & 3591 & 3067 & 3367 \\
\hline \multirow{4}{*}{ ol on } & $\mathrm{Fl}$ & 87 & 379 & 380 & 491 & 508 & 262 & & 133 & 317 & 409 & 465 & 118 \\
\hline & F2 & 1022 & 908 & 1073 & 1227 & 1219 & 900 & 1014 & 1197 & 1177 & 1072 & 1036 & 1041 \\
\hline & F3 & 2378 & 2212 & 1986 & 2137 & 2135 & 1843 & 2346 & 2391 & 2175 & 1984 & 2161 & 2275 \\
\hline & $\mathrm{F} 4$ & 3432 & 3782 & 3043 & 3335 & 3725 & 3675 & 3351 & 3605 & 3328 & 3841 & 3645 & 3469 \\
\hline \multirow{4}{*}{ ol mid } & $\mathrm{F} 1$ & 157 & 220 & 122 & 161 & 402 & 509 & 158 & 152 & 140 & 402 & & 303 \\
\hline & $\mathrm{F} 2$ & 1362 & 1237 & 1226 & 1493 & 1531 & 1793 & 1518 & 1435 & 1483 & 1328 & 1297 & 1456 \\
\hline & F3 & 2367 & 2341 & 2581 & 2377 & 2313 & 2256 & 2346 & 2435 & 2379 & 2169 & 2268 & 2073 \\
\hline & F4 & 3370 & 3467 & 3933 & 3513 & 3721 & 3592 & 3673 & 3543 & 3693 & 4040 & 3675 & 3614 \\
\hline \multirow{4}{*}{ ol off } & F1 & 142 & 161 & 158 & 175 & 178 & 182 & 109 & 91 & 130 & & 138 & 82 \\
\hline & F2 & 1712 & 1392 & 1641 & 1557 & 1545 & 1508 & 1699 & 1680 & 1557 & 1681 & 1666 & 1675 \\
\hline & F3 & 2899 & 2463 & 2431 & 2466 & 2321 & 2438 & 2385 & 2328 & 2290 & 2492 & 2412 & 2478 \\
\hline & F4 & & 3471 & 3451 & 3479 & 3440 & 3771 & 3624 & 3585 & 3557 & 4008 & 3809 & 3507 \\
\hline \multirow{3}{*}{ sl $50 \%$} & $\begin{array}{l}F 1 \\
F 2\end{array}$ & 1495 & 1767 & 1761 & 1726 & 1652 & 1559 & 1686 & 1498 & 1651 & 1573 & 1654 & 1376 \\
\hline & F3 & 2475 & 2564 & 2722 & 2427 & 2352 & 2460 & 2454 & 2338 & 2347 & 2209 & 2509 & 2420 \\
\hline & F4 & 3304 & 3391 & 3424 & 3265 & 3254 & 3443 & 3563 & 3433 & 3454 & 3724 & 3934 & 3501 \\
\hline \multirow{4}{*}{ i1 $50 \%$} & F1 & & & & & & 401 & & & & 315 & & \\
\hline & F2 & & & & & & 1496 & & & & 1517 & & \\
\hline & F3 & & & & & & 2361 & & & & 1895 & & \\
\hline & F4 & & & & & & 3115 & & & & 3276 & & \\
\hline \multirow{4}{*}{$\mathrm{m} 250 \%$} & $\overline{F I}$ & 183 & 214 & 308 & 164 & 181 & 238 & 152 & 116 & 130 & 251 & 120 & 136 \\
\hline & $F 2$ & 1027 & 1041 & 1122 & 1193 & 1323 & 1060 & 1024 & 1102 & 1152 & 1109 & 962 & 874 \\
\hline & F3 & 2288 & 2129 & 2220 & 1994 & 2635 & 2233 & 2169 & 1902 & 1965 & 2083 & 2158 & 2063 \\
\hline & $F 4$ & 3129 & 3096 & 3086 & 3050 & 3546 & 3478 & 3263 & 3565 & 3168 & 3175 & 3069 & 3463 \\
\hline \multirow{4}{*}{02 onset } & F1 & 466 & & 242 & & 264 & & 520 & 124 & 375 & 347 & 130 & 134 \\
\hline & F2 & 1225 & 1457 & 1260 & 1222 & 1239 & 1382 & 1264 & 1120 & 1268 & 1163 & 1045 & 1115 \\
\hline & F3 & 2249 & 2835 & 2105 & 2099 & 1975 & 2167 & 2122 & 2060 & 1945 & 1956 & 1975 & 2180 \\
\hline & F4 & 3080 & 3280 & 3354 & 3372 & 3133 & & 3406 & 3278 & 3629 & 3201 & 3307 & 3294 \\
\hline \multirow{4}{*}{$\begin{array}{c}02 \\
\text { middle }\end{array}$} & F1 & 405 & 113 & 189 & 342 & 354 & 168 & 126 & 66 & & 433 & & \\
\hline & & 1504 & 1445 & 1455 & 1481 & 1462 & 1529 & 1482 & 1311 & 1546 & 1267 & 1483 & 1487 \\
\hline & F3 & 2546 & 2235 & 2320 & 2178 & 2162 & 2196 & 2287 & 2185 & 2271 & 1923 & 2038 & 2394 \\
\hline & $\mathrm{F} 4$ & 3412 & 3269 & 3448 & 3285 & 3337 & 3239 & & 3372 & 3734 & 3297 & 3298 & 3228 \\
\hline \multirow{4}{*}{02 offset } & $\overline{F l}$ & 439 & 315 & 150 & 247 & 152 & 209 & & & & & & \\
\hline & $\mathrm{F} 2$ & 1561 & 1562 & 1621 & 1808 & 1535 & 1659 & 1400 & 1551 & 1615 & 1188 & 1489 & 1618 \\
\hline & F3 & 2494 & 2586 & 2258 & 2227 & 2259 & 2254 & 2024 & 2353 & 2339 & 2165 & 2270 & 2531 \\
\hline & F4 & 3510 & 3490 & 3263 & 3244 & 3337 & 3208 & 3470 & 3337 & 3651 & 3304 & 3326 & 3292 \\
\hline \multirow{3}{*}{ s2 $50 \%$} & $\mathrm{FI}$ & 1709 & 1600 & 1770 & 1642 & 18 & 1696 & 1672 & 1631 & 1726 & 1547 & 1565 & 1675 \\
\hline & F3 & 2463 & 2583 & 2322 & 2334 & 2268 & 2315 & 2134 & 2434 & 2332 & 2279 & 2379 & 2509 \\
\hline & $F 4$ & 3377 & 3397 & 3182 & 3180 & 3261 & 3268 & 3555 & 3280 & 3586 & 3179 & 3260 & 3551 \\
\hline \multirow{4}{*}{ i2 $50 \%$} & F1 & 328 & 206 & & & & & 312 & 261 & & & & \\
\hline & F2 & 1916 & 1500 & & & & & 1306 & 1780 & & & & \\
\hline & F3 & 2648 & 2237 & & & & & 2206 & 2581 & & & & \\
\hline & F4 & 3579 & 3489 & & & & & 3349 & 3437 & & & & \\
\hline
\end{tabular}


Appendix 5.2 Summary of the numbers of tokens measured for each sampling point.

\begin{tabular}{|c|c|c|c|c|c|c|c|c|c|c|c|c|c|c|c|c|c|c|c|c|c|c|c|c|c|c|c|}
\hline & \multicolumn{2}{|c|}{$\mathrm{AA}$} & \multicolumn{2}{|c|}{$\mathrm{HA}$} & \multicolumn{2}{|c|}{$\mathrm{JN}$} & \multicolumn{2}{|c|}{$\mathrm{KA}$} & \multicolumn{2}{|c|}{$\mathrm{KF}$} & \multicolumn{2}{|c|}{$\mathrm{KO}$} & \multicolumn{2}{|c|}{$\mathrm{MM}$} & \multicolumn{2}{|c|}{$\mathrm{MN}$} & \multicolumn{2}{|c|}{$\mathrm{MO}$} & \multicolumn{2}{|c|}{$\mathrm{TN}$} & $T$ & & $\mathrm{~T}$ & & YH & \\
\hline & & 1 & 2 & 1 & 2 & 1 & 2 & 1 & 2 & 1 & 2 & 1 & 2 & 1 & & 1 & 2 & 1 & 2 & & & & 2 & & & & 2 \\
\hline & $\mathrm{F} 1$ & 4 & 6 & 5 & 8 & 5 & 6 & 4 & 4 & 7 & 10 & 3 & 3 & 9 & 8 & 3 & 6 & 6 & 6 & 5 & 6 & 7 & 6 & 3 & 4 & 6 & 6 \\
\hline & $\mathrm{F} 2$ & 4 & 6 & 6 & 8 & 5 & 6 & 4 & 3 & 7 & 10 & 3 & 3 & 8 & 8 & 3 & 6 & 6 & 7 & 4 & 5 & 7 & 5 & 6 & 6 & 6 & 6 \\
\hline $\mathrm{ml}$ & F3 & 4 & 7 & 6 & 7 & 5 & 6 & 4 & 4 & 7 & 10 & 3 & 3 & 9 & 8 & 3 & 5 & 5 & 3 & 3 & 4 & 7 & 5 & 7 & 6 & 6 & 6 \\
\hline & $\mathrm{F} 4$ & 5 & 7 & 6 & 7 & 5 & 6 & 4 & 4 & 6 & 10 & 2 & 3 & 6 & 8 & 3 & 4 & 5 & 5 & 5 & 6 & 7 & 6 & 5 & 4 & 5 & \\
\hline & $F 1$ & 4 & 3 & 6 & 8 & 5 & 6 & 4 & 4 & 7 & 10 & 3 & 3 & $\overline{9}$ & 8 & 7 & 8 & 6 & 7 & 4 & 6 & 7 & 6 & 5 & 6 & 6 & 5 \\
\hline ol & $\mathrm{F}$ & 5 & 6 & 6 & 8 & 5 & 6 & 4 & 4 & 7 & 10 & 3 & 3 & 9 & 8 & 7 & 8 & 6 & 7 & 6 & 6 & 7 & 6 & 7 & 6 & 6 & 6 \\
\hline onse & F3 & 5 & 7 & 6 & 8 & 5 & 6 & 2 & 3 & 7 & 10 & 3 & 3 & 9 & 8 & 7 & 6 & 6 & 6 & 5 & 6 & 7 & 6 & 7 & 6 & 6 & 6 \\
\hline & F4 & 5 & 7 & 6 & 8 & 4 & 6 & 4 & 4 & 6 & 10 & 3 & 3 & 9 & 8 & 4 & 8 & 6 & 7 & 5 & 6 & 6 & 6 & 7 & 6 & 6 & 6 \\
\hline & F1 & 5 & 7 & 6 & 8 & 5 & 6 & 4 & 4 & 6 & 10 & 3 & 3 & 9 & 8 & 7 & 8 & 6 & 5 & 4 & 6 & 7 & 5 & 6 & 6 & 6 & 6 \\
\hline 01 & F & 5 & 7 & 5 & 8 & 5 & 6 & 4 & 4 & 7 & & 3 & 3 & 9 & 8 & 7 & 8 & 6 & 7. & 6 & 6 & 7 & 6 & 7 & 6 & 6 & 6 \\
\hline mid & F3 & 5 & 7 & 6 & 8 & 4 & 6 & 4 & 4 & 7 & & 3 & 3 & 9 & 8 & 7 & 4 & 6 & 7 & 6 & 6 & 7 & 6 & 7 & 6 & 6 & 6 \\
\hline & F4 & 5 & 5 & 6 & 8 & 5 & 5 & 4 & 4 & 7 & 10 & 3 & 3 & 9 & 8 & 6 & 8 & 6 & 7 & 6 & 6 & 7 & 6 & 7 & 6 & 6 & 6 \\
\hline & $\mathrm{F} 1$ & 4 & 5 & 6 & 8 & 5 & 6 & 4 & 4 & 6 & 10 & 3 & 3 & 5 & 2 & 7 & 7 & 0 & 1 & 1 & 1 & 5 & 3 & 4 & 5 & 6 & 6 \\
\hline ol & F2 & 5 & 7 & 6 & 8] & 5 & 6 & 4 & 4 & 6 & 10 & 3 & 3 & 9 & 8 & 7 & 8 & 6 & 7 & 6 & 6 & 7 & 6 & 7 & 6 & 6 & 6 \\
\hline off & F3 & 5 & 7 & 6 & 8 & 4 & 6 & 4 & 4 & 7 & 10 & 3 & 3 & 9 & 8 & 5 & 8 & 5 & 7 & 6 & 6 & 7 & 6 & 6 & 6 & 6 & 6 \\
\hline & F4 & 5 & 7 & 6 & 8 & 5 & 6 & 4 & 4 & 7 & 10 & 3 & 3 & 9 & 8 & 7 & 8 & 6 & 7 & 6 & 6 & 7 & 6 & 7 & 6 & 5 & 6. \\
\hline & $\mathbf{F}$ & 0 & 0 & 0 & 1 & 3 & 3 & 1 & 0 & 2 & & 2 & 0 & 1 & 2 & 1 & 0 & 1 & 1 & 0 & 0 & 1 & 1 & 0 & 0 & 0 & 0 \\
\hline & $F$ & 5 & 7 & 6 & 8 & 5 & 6 & 4 & 4 & 7 & 9 & 3 & 3 & 9 & 8 & 7 & 8 & 6 & 7 & 6 & 6 & 7 & 6 & 7 & 5 & 6 & 6 \\
\hline sl & F3 & 5 & 7 & 6 & 8 & 5 & 6 & 4 & 2 & 7 & 10 & 3 & 3 & 9 & 8 & 7 & 8 & 6 & 7 & 5 & 6 & 7 & 6 & 6 & 6 & 6 & 6 \\
\hline & F4 & 5 & 7 & 6 & 8 & 5 & 6. & 4 & 4 & 7 & 10 & 3 & 3 & 9 & 8 & 7 & 8 & 6 & 7 & 6 & 6 & 7 & 5 & 7 & 6 & 6 & 6 \\
\hline & F1 & 1 & 0 & 0 & 0 & 1 & $\mathrm{I}$ & 0 & 1 & 1 & & 0 & 0 & 1 & 1 & 1 & 7 & 3 & 2 & 0 & 0 & 2 & 9 & 0 & 0 & 1 & 1 \\
\hline & F & 1 & 0 & 0 & o & 1 & 1 & 0 & 1 & 1 & & 0 & 0 & 1 & 1 & 1 & 7 & 3 & 2 & 0 & 0 & 2 & 0 & 0 & 0 & 1 & \\
\hline in & $\mathrm{F}$ & 1 & 0 & 0 & o & 1 & 1 & 0 & 1 & 1 & 3 & 0 & 0 & 1 & 1 & 1 & 7 & 3 & 2 & 0 & 0 & 2 & 0 & 0 & 0 & 1 & \\
\hline & F4 & 1 & 0 & 0 & 0 & 1 & 1 & 0 & 1 & 1 & 3 & 0 & 0 & 1 & 1 & 1 & 7 & 3 & 2 & 0 & 0 & 2 & 0 & 0 & 0 & 1 & 1 \\
\hline & FI & 5 & 6 & 6 & 8 & 5 & 5 & 4 & 3 & 7 & 10 & 3 & 3 & 9 & 8 & 7 & 8 & 6 & 7 & 6 & 6 & 7 & 6 & 7 & 6 & 6 & 6 \\
\hline & $F_{2}$ & 4 & 6 & 6 & 8 & 5 & 5 & 4 & 3 & 7 & 10 & 3 & 3 & 8 & 7 & 7 & 8 & 6 & 7 & 6 & 6 & 7 & 6 & 7 & 6 & 6 & 6 \\
\hline $\mathrm{m} 2$ & F3 & 5 & 7 & 6 & 8 & 5 & 5 & 4 & 3 & 7 & 10 & 3 & 3 & 9 & 8 & 7 & 8 & 6 & 7 & 6 & 6 & 7 & 6 & 7 & 6 & 6 & 6 \\
\hline & $\mathrm{F} 4$ & 2 & 7 & 4 & 8 & 5 & 5 & 4 & 3 & 7 & 9 & 3 & 2 & 6 & 6 & 7 & 8 & 6 & 7 & 6 & 6 & 7 & 6 & 6 & 3 & 6 & 6 \\
\hline & $\mathbf{F}$ & 4 & 6 & 6 & 8 & 5 & 6 & 4 & 4 & 7 & 10 & 3 & 2 & 9 & 7 & 7 & 8 & 5 & 7 & 4 & 6 & 7 & 4 & 7 & 6 & 3 & 3 \\
\hline 02 & $\mathrm{~F} 2$ & 4 & 6 & 6 & 8 & 4 & 6 & 4 & 4 & 7 & 10 & 3 & 3 & 9 & 6 & 7 & 8 & 6 & 7 & 6 & 6 & 7 & 6 & 7 & 6 & 6 & 6 \\
\hline onset & F3 & 5 & 7 & 6 & 8 & 4 & 4 & 4 & 4 & 7 & 10 & 3 & 3 & 9 & 7 & 7 & 8 & 6 & 7 & 6 & 6 & 7 & 5 & 7 & 6 & 6 & 6 \\
\hline & $F_{4}$ & 4 & 7 & 5 & 8 & 5 & 5 & 4 & 4 & 7 & 10 & 3 & 2 & 7 & 7 & 7 & 8 & 6 & 7 & 6 & 6 & 7 & 6 & 7 & 4 & 5 & \\
\hline & $F$ & 5 & 6 & 6 & 8 & 5 & 6 & 4 & 4 & 7 & 10 & 3 & 2 & 8 & 6 & 7 & 8 & 3 & & 4 & 6 & 7 & 3 & 7 & 6 & 6 & 6 \\
\hline 0 & $F_{2}$ & 5 & 7 & 6 & 8 & 5 & 6 & 4 & 4 & 7 & 10 & 3 & 3 & 9 & 8 & 7 & 8 & 6 & & 6 & 6 & 7 & 6 & 7 & 6 & 6 & 6 \\
\hline middle & $F_{3}$ & 5 & 7 & 6 & 8 & 5 & 5 & 4 & 4 & 7 & 10 & 3 & 3 & 9 & 8 & 7 & 8 & 6 & 7 & 6 & 6 & 7 & 5) & 7 & 6 & 6 & 6 \\
\hline & $F_{4}^{4}$ & 4 & 7 & 5 & 8 & 5 & 6 & 4 & 4 & 7 & 10 & 3 & 3 & 7 & 7 & 7 & 8 & 6 & 7 & 6 & 6 & 7 & 6 & 7 & 6 & 6 & 5 \\
\hline & $F$ & 4 & 5 & 5 & 8 & 5 & 4 & 4 & 4 & 5 & 8 & 2 & 3 & 5 & 2 & 5 & 8 & 1 & 3 & 1 & 3 & 5 & 0 & 6 & 6 & 6 & 5 \\
\hline 02 & $F_{2}$ & 5 & 7 & 6 & 8 & 4 & 6] & 4 & 4 & 7 & 10 & 3 & 3 & 9 & 8 & 7 & 8 & 6 & 7 & 6 & 6 & 7 & 5 & 6 & 6 & 6 & 6 \\
\hline offset & $F_{3}$ & 5 & 7 & 6 & 8 & 5 & 6 & 4 & 4 & 7 & 10 & 2 & 3 & 9 & 8 & 7 & 8 & 6 & $7]$ & 6 & 6 & 7 & 6 & 7 & 6 & 6 & 6 \\
\hline & F4 & 4 & 7 & 6 & 8 & 5 & 6 & 4 & 4 & 7 & 9 & 3 & 3 & 9 & 8 & 7 & 8 & 6 & 7 & 6 & 6 & 7 & 6 & 7 & 6 & 6 & 6 \\
\hline & F1 & 0 & 0 & 0 & 0 & 2 & 1) & 0 & 0 & 0 & 0 & 2 & 2 & 1 & 0 & 0 & 0 & 0 & 1 & 0 & 2 & 0 & 1 & 0 & 0 & 0 & 0 \\
\hline s2 & $F_{2}$ & 5 & 7 & 6 & 7 & 5 & 6 & 4 & 4 & 7 & 9 & 3 & 3 & 9 & 8 & 7 & 8 & 6 & 7 & 6 & 6 & 7 & 6 & 5 & 6 & 6 & 6 \\
\hline & $\mathrm{F}$ & 5 & 7 & 6 & 7 & 5 & 5 & 4 & 4 & 7 & 10 & 3 & 3 & 9 & 8 & 7 & 8 & 6 & 7 & 5 & 6 & 7 & 6 & 6 & 6 & 6 & 6 \\
\hline & F4 & 5 & 7 & 6 & 7 & 5 & 6 & 4 & 4 & 7 & 10 & 3 & 3 & 9 & 8 & 7 & 8 & 6 & 7 & 6 & 6 & 7 & 6 & 6 & 6 & 6 & 6 \\
\hline & F1 & 5 & 6 & 5 & 7 & 4 & 6 & 1 & 3 & 7 & 8 & 0 & 0 & 3 & 6 & 0 & 0 & 5 & 3 & 4 & 4 & 4 & 1 & 2 & 4 & 2 & 2 \\
\hline i2 & $F_{2}$ & 5 & 6 & 5 & 7 & 3 & 6 & 1 & 3 & 7 & 9 & 0 & 0 & 3 & 6 & 0 & 0 & 5 & 3 & 4 & 4 & 5 & 1 & 2 & 4 & 2 & 2 \\
\hline & $F_{3}$ & 5 & 6 & 5 & 6 & 4 & 6 & 1 & 3 & 7 & 9 & 0 & 0 & 3 & 6 & 0 & 요 & 5 & 3 & 4 & 4 & 5 & 1 & 2 & 4 & 2 & 2 \\
\hline & F4 & 5 & 6 & 4 & 6 & 4 & 6 & 1 & 3 & 7 & 9 & 0 & 0 & 3 & 6 & 0 & 0 & 5 & 4 & 4 & 4 & 5 & 1) & 1 & 4 & 2 & 21 \\
\hline
\end{tabular}




\section{STATISTICAL EVALUATION}

Appendix 6.1 Likelihood ratios for each parameter and the posterior odds for the speaker combinations which were not discriminated correctly for the calculation using effective F2. The likelihood ratios below 1 are marked by shading.

\begin{tabular}{|c|c|c|r|r|r|r|}
\hline Speakers & iF2 & eF'2 & olmid F3 & m2 F3 & s2 F3 & Posterior \\
\hline NN - AA (3) & 3.33715 & 1.58265 & 11.3424 & 15.2756 & 3.51775 & 3219.03 \\
\hline KO - KA (1) & 2.38322 & 1.2703 & 0.68149 & 6.39504 & 033728 & 5.00 \\
\hline $\mathrm{MN}-\mathrm{HA}(2)$ & 92.9683 & 40.1475 & $3.3 \mathrm{E}-05$ & 4.3798 & 10.7198 & 5.73 \\
\hline $\mathrm{TN}-\mathrm{KA}(1)$ & 0.65419 & 2.97001 & 1.71106 & 3.6077 & 1.89485 & 16.02 \\
$(4)$ & 3.54925 & 2.34901 & 0.62201 & 2.93463 & 1.11904 & 17.33 \\
\hline $\mathrm{KF}-\mathrm{HA}(2)$ & 0.16804 & 14.4852 & 1.89596 & 1.0934 & 3.17526 & 345.14 \\
\hline
\end{tabular}

Appendix 6.2 The mean values of the datasets for the calculation using effective $F 2$ which the Bayesian approach failed to discriminate. The mean values of the parameters which produced likelihood ratios below 1 (correct discrimination) were indicated by shading.

\begin{tabular}{|r|l|r|r|r|r|r|}
\hline Sp. combination & Dataset & \multicolumn{1}{|c|}{ IF2 } & \multicolumn{1}{c|}{ eF'2 $^{\prime}$} & \multicolumn{1}{c|}{ olmid } & \multicolumn{1}{c|}{ 2 } & \multicolumn{1}{c|}{ s2 } \\
\hline JN - AA (3) & JN 1 & 1994.9 & 2004.3 & 2114.3 & 2021 & 2494.8 \\
& AA 1 & 2040.3 & 2089.5 & 2218.8 & 2127.4 & 2523.6 \\
\hline KO-KA (1) & KO 2 & 1955 & 2165.8 & 2596 & 2668.3 & 2602.3 \\
& KA 1 & 2075.9 & 2234.2 & 2796.5 & 2657 & 2779.5 \\
\hline MN - HA (2) & MN 1 & 2466 & 2457.1 & 2310 & 2439.7 & 2990.6 \\
& HA 2 & 2482 & 2477.6 & 2776.8 & 2420.8 & 3012.7 \\
\hline TN - KA (1) & TN 2 & 2203.5 & 2258.5 & 2661.4 & 2574 & 2704.3 \\
& KA 1 & 2075.9 & 2234.2 & 2796.5 & 2657 & 2779.5 \\
\cline { 2 - 7 } & TN 2 & 2203.5 & 2258.5 & 2661.4 & 2574 & 2704.3 \\
& KA 2 & 2202.7 & 2222.4 & 2827.5 & 2693.7 & 2813.3 \\
\hline KF-HA (2) & KF 1 & 2242 & 2390.7 & 2664.6 & 2532.1 & 2914.4 \\
& HA 2 & 2482 & 2423.6 & 2776.8 & 2420.8 & 3012.7 \\
\cline { 2 - 7 } (3) & KF 1 & 2242 & 2390.7 & 2664.6 & 2532.1 & 2914.4 \\
& HA 1 & 2498.3 & 2477.6 & 2724.2 & 2519.8 & 3035 \\
\hline
\end{tabular}

Appendix 6.3 Absolute values of the subtraction of one dataset from the other for the calculation using effective F2. Those values for the parameters which discriminated the speakers correctly are indicated by shading.

\begin{tabular}{|c|c|c|c|c|c|}
\hline Sp. combination & $\mathrm{iF} 2$ & eF f & olmid F3 & $\mathrm{m} 2 \mathrm{~F} 3$ & s2 F3 \\
\hline JN ? AA (3) & 45.4 & 85.2 & 104.5 & 106.4 & 28.8 \\
\hline $\mathrm{KO} ? \mathrm{KA}(1)$ & 120.9 & 68.4 & 2005 & 11.3 & 172 \\
\hline $\mathrm{MN}$ ? HA (2) & 16 & 20.5 & 4668 & 18.9 & 22.1 \\
\hline TN? KA (1) & 127.6 & 24.3 & 135.1 & 83 & 75.2 \\
\hline (4) & 0.8 & 36.1 & 166.1 & 119.7 & 109 \\
\hline $\mathrm{KF}$ ? HA (2) & 240 & 32.9 & 112.2 & 111.3 & 98.3 \\
\hline (3) & 256.3 & 86.9 & 59.6 & 12.3 & 120.6 \\
\hline
\end{tabular}


Appendix 6.4 The mean, standard deviation, minimum and maximum values of the difference between 2 speakers for the calculation using effective F2. Those parameters which failed to discriminate speakers are shown in the left part of the table, and those of the parameters which correctly discriminated speakers are shown in the right part of the table.

\begin{tabular}{|c|r|c|r|c|c|c|c|}
\hline \multicolumn{3}{|c|}{ Failed to discriminate speakers } & \multicolumn{4}{c|}{ Correctly discriminated speakers } \\
\hline mean & 66.63 & $\min$ & 0.8 & mean & 232.5 & $\min$ & 127.6 \\
\hline sd & 42.79 & $\max$ & 135.1 & sd & 111.81 & $\max$ & 466.8 \\
\hline
\end{tabular}




\section{BIBLIOGRAPHY}

Aitkin, C. G. C., and D. A. Stoney. 1991. "Editors' introduction to chapter 3." in C. G. C. Aitkin and D. A. Stoney (eds.) The use of statistics in forensic science. Chichester: Ellis Horwood series in forensic science. Pp. 83-84

Aitkin, C. G. C. 1995. Statistics and the Evaluation of Evidence for Forensic Scientists: Wilely. Akamatsu, T. 2000. Japanese Phonology - Functional Approach. Muenchen: Lincom Europa.

Atal, B. S. 1972. "Automatic Speaker Recognition Based on Pitch Contour." Journal of Acoustical Society of America 52: 1687-1697.

- 1976. "Automatic recognition of speakers from their voices." Proceedings of the IEEE 64: $460-475$.

Backen, R. J. 1996. Clinical Measurement of Speech and Voice. San Diego: Singular Publishing Group.

Beck, J. M. 1997. "Organic variation of the vocal apparatus." in W. Hardcastle and J. M. D. Laver (eds.) The Handbook Of Phonetic Sciences. Oxford: Blackwell. Pp.256-297

Beckman, M. 1982. "Segment duration and the 'mora' in Japanese." Phonetica 39: 113-135.

Bö, L.-J. 2000. "Forensic voice identification in France." Speech Communication 31: 205-224.

Boss, D. 1996. "The problem of F0 and real-life speaker identification: case study." Forensic Linguistics 3: 155-159.

Bricker, P. D., and S. Pruzansky. 1976. "Speaker recognition." in N. J. Lass (eds.) Contemporary Issues In Experimental Phonetics. New York: Academic Press. Pp. 295326

Broad, D. J. 1976. "Toward defining acoustic phonetic equivalence for vowels." Phonetica 33: 401-424.

Broeders, A.P.A. 1999 "Some observations on the use of probability scales in forensic identification", Forensic Linguistics 6/2: 228-229.

Champod, C. and Evett, I. 2000. "Commentary on Broeders (1999)", Forensic Linguistics 7/2: 238-243.

Champod, C., and D. Meuwley 2000. "The inference of identity in forensic speaker recognition." Speech Communication 31:193-203

Clermont, F., and S. Itahashi. 2000. "Static and dynamic vowels in a "cepstro-phonetic" subspace." Journal of Acoustical Society of Japan 21: 221-223.

Crystal, D. 1991. A Dictionary of Linguistics and Phonetics. Oxford: Blackwell.

Elliott, J. 2000. "Comparing the acoustic properties of normal and shouted speech: a study in forensic phonetics." in M. Barlow (eds.) The Eighth Australian International Conference on Speech Science ad Technology. Canberra: Australian Speech Science and Technology Association. Pp. 154-159

Everitt, B. S., and G. Dunn. 1991. Applied Multivariate Data Analysis. London: Amold.

Evett, I. W. 1991. "Interpretation: a personal odyssey." in C. G. C. Aitkin and D. A. Stoney (eds.) The use of statistics in forensic science. Chichester: Ellis Horwood series in forensic science. Pp. 9-22

Fant, G. 1973. Speech Sound and Features. Cambridge: The MIT Press.

Farnetani, E. 1997. "Coarticulation and connected speech processes." in W. Hardcastle and J. M. D. Laver (eds.) The Handbook Of Phonetic Sciences. Oxford: Blackwell. Pp. 371404

French, P. 1994. "An overview of forensic phonetics with particular reference to speaker identification." Forensic Linguistics 1: 169-181.

Fromkin, V., R. Rodman, P. Collins, and D. Blair. 1994. An Introduction to Language. Sydney: Holt, Linehart, and Winston.

Fujimura, O. 1962. "Analysis of nasal consonants." Journal of Acoustical Society of America 34: $1865-1875$. 
Fujimura, O., and D. Erickson. 1997. "Acoustic Phonetics." in W. Hardcastle and J. M. D. Laver (eds.) The Handbook Of Phonetic Sciences. Oxford: Blackwell. Pp. 65-115

Garvin, P. L., and P. Ladefoged. 1963. "Speaker identification and message identification in speech recognition." Phonetica 9: 193-199.

Glenn, J. W., and N. Kleiner. 1968. "Speaker identification based on Nasal Phonation." Journal of Acoustical Society of America 43: 368-372.

Greisbach, R., O. Esser, and C. Weinstock. 1995. "Speaker identification by formant contours." Studies in Forensic Phonetics BEIPHOL 64: 49-55.

Goggin, J. P., C. P. Thompson, G. Strube, and L. R. Simental. 1991. "The role of language familiarity in voice identification." Memory and Cognition 19: 448-58.

Goldstein, A. G., P. Knight, K. Bailis, and J. Conover. 1981. "Recognition memory for accented and unaccented voices." Bulletin of Psychonomic Society 17: 217-220.

Good, I. J. 1991. "Weight of evidence and the Bayesian likelihood rato." in C. G. C. Aitkin and D. A. Stoney (eds.) The use of statistics in forensic science. Chichester: Ellis Horwood series in forensic science. Pp. 85-106

Guessenhoven, C., and H. Jacobs. 1998. Understanding Phonology. London: Arnold.

Hattori, S., K. Yamamoto, and F. O. 1958. "Nasalisation of vowels in relation to nasals." Joumal of Acoustical Society of America 30: 267-274.

Hatch, E., and A. Lazaraton. 1991. The Research Manual Design and Statistics for Applied Linguistics. Boston: Heinle \& Heinle.

Hazan, B. 1973. "Effects of differing phonetic contexts on spectrographic speaker identification." Joumal of Acoustical Society of America 54: 650-659.

Hirson, A., P. French, and D. Howard. 1995. "Speech fundamental frequency over the telephone and face-to-face: some implications for forensic phonetics." in Studies in General and English Phonetics: Routledge.

Hollien, H., R. Huntley, H. J. Künzel, and P. A. Hollien. 1995. "Criteria for earwitness lineups." Forensic Linguistics 2: 143-153.

Hollien, H., and C. A. Martin. 1996. "Conducting research on the effects of intoxication on speech." Forensic Linguistics 3: 107-128.

Huntley Bahr, R., and K. J. Pass. 1996. "The influence of style-shifting on voice identification." Forensic Linguistic 3: 24-38.

Hymann, L. M. 1977. "On the nature of linguistic stress." in L. M. Hyman (eds.) Studies in Stress and Accent. Southerm California University. Pp. 37-82

Imai, K. 1980. "Onsee gakuteki hikaku" [Phonetic comparison] in T. Kunihiro (eds.) Onsee to Keitai, Tokyo: Taishuukan Publisher. Pp. 7 - 68

Imaishi, M., and J. Mitsuwa. 1989. "Boin no Onkyoo teki tokuchoo - hoogen ni yoru sai." [Acoustic characteristics of vowels in Japanese dialects] in M. Sugitoo (eds.) Nihongo no Onsee, Onin. Tokyo: Meiji Shoin. Pp. 85-108

Ingram, J. C. L., R. Prandolini, and S. Ong. 1996. "Formant trajectories as indices of phonetic variation for speaker identification." Forensic Linguistics 3: 129-145.

Ishimura, S. 1992. Sugu Wakaru Tahenryoo Kaiseki. [Quick understanding for multivariate analysis] Tokyo: Tokyo Tosho.

Jassem, W. 1995. "Discriminant analysis and its application in voice recognition." Studies in Forensic Phonetics BEIPHOL 64: P.132-145.

Jessen, M. 1997. "Speaker-specific information in voice quality parameters." Forensic Linguistics 4: 84-103.

Jiang, M. 1996. "Fundamental frequency vector for a speaker identification system." Forensic Linguistics 3: 95-106.

Johnson, K. 1997. Acoustic and Auditory Phonetics. Oxford: Blackwell.

Kawakami, S. 1977. Nihongo Onsee Gaisetsu. [Introduction to Japanese phonetics] Tokyo: Oohuusha.

Kersta, L. G. 1962. "Voiceprint identification." Nature 196: 1253-1257. 
Kindaichi, H. 1967. "'Satooya" to "sato:ya": hikionsetsu no teisho:" ["Satooya" and "Sato:ya": proposition on interpretation of Japanese long vowels] in Kokugo on in-ron. Tokyo: Tokyodo.

Kinoshita, Y. 1998. "Japanese forensic phonetics: Non-contemporaneous within-speaker variation in natural and read-out speech." in H. M. Mannell and J. R. -R. Robert (eds.) The 5th International Conference on Spoken Language Processing. Sydney: Australian Speech Science and Technology Association. (CD ROM publication).

Kinoshita, Y., and J. Maindonald. 2000. "Statistical quantification of differential vowel comparability in forensic phonetic samples." in M. Barlow (eds.) The Eighth Australian Intemational Conference on Speech Science ad Technology. Canberra: Australian Speech Science and Technology Association. Pp. 166-171

Koenig, B. E. 1986. "Spectrographic voice identification: A forensic survey." Journal of Acoustical Society of America 79: 2088-2090.

Köster, O., M. M. Hess, O. Schiller, and H. J. Künzel. 1998. "The correlation between auditory speech sensitivity and speaker recognition ability." Forensic Linguistics 5: 22-32.

Köster, O., and N. O. Schiller. 1996. "Evaluation of a foreign speaker in forensic phonetics: a report." Forensic Linguistics 3: 176-185.

-. 1997. "Different influences of the native language of a listener on speaker recognition." Forensic Linguistics 4: 18-27.

- 1998. "The ability of expert witnesses to identify voices: comparison between trained and untrained listeners." Forensic Linguistics 5: 1-9.

Kubozono, H., and S. Ota. 1998. Onin Koozoo to Akusento. [Phonological structure and word accent] Tokyo: Kenkyuusha Shuppan.

Künzel, H. 1994. "On problem of speaker identification by victims and witnesses." Forensic Linguistics 1: 45-57.

- 1995. "Field procedures in forensic speaker recognition." in J. W. Lewis (eds.) Studies in General and English Phonetics. Essays in Honour of J. D. O'Connor. London: Routledge. Pp. 68-84

Ladd, D. R. 1996. Intonational Phonology. Cambridge: Cambridge University Press.

Ladefoged, P. 1978. "Expectation affects identification by listening." UCLA Working Paper in Phonetics 41. Pp.41-42

Ladefoged, P. 1993. A Course In Phonetics. Fort Worth: Harcourt Brace \& Company.

Ladefoged, P., and J. Ladefoged. 1980. "The ability of listeners to identify voices." UCLA Working paper in Phonetics 49.

Ladefoged, P., and I. Maddieson. 1996. The Sounds Of the World's Languages. Oxford: Blackwell.

Lancker, D. V., J. Kreiman, and K. Emmorey. 1985. "Familiar voice recognition: Patterns and parameters, Part 1: recognition of rate-aitered voices." Journal of Phonetics 13: 19-38.

Laubstein, A. S. 1997. "Problems of voice line-ups." Forensic Linguistics 4: 262-279.

Lass, R. 1984. Phonology. Cambridge: Cambridge University Press.

Maddieson, I. 1985. "Phonetic Cues to Syllabification." in V. A. Fromkin (eds.) Phonetic Linguistics: Essays in Honor of Peter Ladefoged. Orland: Academic Press. Pp. 203-221

Maekawa, K. 1989. "Boin no museeka" [Vowel devoicing] in M. Sugitoo (eds.) Nihongo no Onsee, Onin. Tokyo: Meiji Shoin. Pp.135-153

- 1997a. "Nikkan Taishoo Onseegaku Kakken" [Phonetic Comparison of Korean and Japnaese] in Kokuritu Kokugogaku Kenkyuusho (eds.) Nihongo to Choosengo. Tokyo: Kuroshio. Pp. 173-191

-. 1997b. "Onseegaku" [Phonetics] in Y. Otsu, T. Gunji, Y. Takubo, M. Nagao, K. Masuda, T. Masuoka and H. Matsumoto (eds.) Onsei, edited by . Tokyo: Iwanami Shoten. Pp. 1-52

- 1998. "Phonetic and phonological characteristics of paralinguistic information in spoken Japanese." in H. M. Mannell and J. R. -R. Robert (eds.) The 5th International Conference on Spoken Language Processing. Sydney: Australian Speech Science and Technology Association. (CD ROM publication). 
Majewski, W., and C. Basztura. 1996. "Integrated approach to speaker recognition in forensic applications." Forensic Linguistics 3: 50-64.

Markham, D. 1999. "Listeners and disguised voices: the imitation and perception of dialectal accent." Forensic Linguistics 6: 289-299.

MathSoft Inc (Data Analysis Products division), M. I. D. A. P. 1999. S-plus 2000 guide to Statistics. Seattle: (Manual for software, S-Plus 2000).

McGhee, F. 1937. "The reliability of the identification of the human voice." Joumal of General Psychology 17: 249-271.

- 1944. "An experimental study in voice recognition." Joumal of General Psychology 31: 53 65.

McCawley, J. D. 1977. "Accent in Japanese." Studies in stress and accent: Southern Califomia occasional papers in linguistics 4:261-302.

Mokhtari, P., and F. Clermont. 1996. "A methodology for investigating vowel-speaker interactions in the acoustic-phonetic domain." in P. McCormack and A. Russell (eds.) The sixth Australian International Conference on Speech Science and Technology. Adelaide: Australian Speech Science and Technology Association. Pp. 127-132

Monastersky, R. 1998. "Courting Reliable Science." Science News 18/4/1998: 249-250.

Noda, H. 1992. "Washaninshiki no hookagakuteki jitsuyoo nikansuru kenkyuu." [The application of speaker identification to the forensic investigation] Unpublished $\mathrm{PhD}$ thesis submitted to Engineering Department, Kyuushuu kogyoo daigaku.

Noda, H., M. Tanimoto, and S. Kijima. 1981. "Individuality in the static and dynamic feature of speech." Reports of National Institution Research of Police Science 34: 19-22.

Noda, H., M. Tanimoto, T. Suzuki, S. Kijima, and I. Hayama. 1982. "Speaker verification by the word-independent threshold." Reports of National Research Institution of Police Science 35: 30-34.

Nolan, F. 1983. The Phonetic Bases of Speaker Recognition. Cambridge: Cambridge University Press.

- 1990. "The limitations of auditory-phonetic speaker identification." in H. Kniffka (eds.) Texte zur Theorie and Praxis forensischer Linguistik. Tubingen: Max Niemayer Verlag. Pp. $457-479$

-. 1996. "Forensic Phonetics." in Australian Linguistics Institute. ANU: unpublished course material.

- 1997. "Speaker recognition and forensic phonetics." in W. Hardcastle and J. M. D. Laver (eds.) The Handbook of Phonetic Sciences. Oxford: Blackwell. Pp. 744-7670xford: Blackwell.

Osanai, T., and K. Ozeki. 1995. "Speaker verification using short sentences based on Hidden Markov Model of Isolated vowel utterances." Reports of National Institution Research of Police Science 48: 13-20.

Osanai, T., M. Tanimoto, H. Kido, and T. Suzuki. 1995. "Text dependent speaker verification using isolated word utterances based on dynamic programming." Reports of National Research Institution of Police Science 48: 15-19.

Pahn, J. and Pahn, E. 1991. "Formblatt, Eigenschafeten, Ablauf und Bedeutung des Tests der Sensibilitat formaler sprachlicher Elemente im Finblick auf Perzeption und Produktion." Sprache-Stimme-Gehör, 15: 19-23

Pierrehumbert, J., and M. Beckman. 1988. Japanese Tone Structure. Cambridge Massachusetts: The MIT Press.

Reich, A. R., K. L. Moll, and J. F. Curtis. 1976. "Effects of selected vocal disguises upon spectrographic speaker identification." Journal of Acoustical Society of America 60: 919-925.

Ripley, B. D. 1996. Pattem Recognition and Neural Network. Cambridge: Cambridge University Press.

Robertson, B., and G. A. Vignaux. 1995. Interpreting Evidence. Chichester: Wiley.

Rose, P. J. 1996. "Between- and within-speaker variation in the fundamental frequency of Cantonese Tones." in P. J. Davis and N. H. Fletcher (eds.) Vocal Fold Phvsiology. San Diego/London: Singular Publishing Group, Inc. Pp.307-324 
- 1998. "Difference and discriminability in the acoustic characteristics of words in voices of similar-sounding speakers - a forensic phonetic investigation." in ICSLP 98. Sydney.

-1999a. "Differences and distinguishability in the acoustic characteristics of Hello in voices of similar-sounding speakers." Australian Review of Applied Linguistics 21: 1-42.

- 1999b. "Long-and short term within-speaker differences in the formants of hello." Journal of International Phonetic Association 29: 1-30.

Rose, P. J., and F. Clermont. 2000. "Comparative performance of cepstrum- and formant-based analysis on similar-sounding speakers for forensic speaker identification." in M. Barlow (eds.) The Eighth Australian International Conference on Speech Science ad Technology. Canberra: Australian Speech Science and Technology Association. Pp. 172-177

Rose P. and Clermont, F. 2001. "A Comparison of Two Acoustic Methods for Forensic Discrimination", Acoustics Australia 29/1: 31-35.

Rose, P. J., and S. Duncan. 1995. "Naive auditory identification and discrimination of similar voices by familiar listeners." Forensic Linguistics 2: 1-17.

Rose, P. J., and A. Simmons. 1996. "F-pattem variability in disguise and over the telephone comparisons for forensic speaker identification." in P. McCormack and A. Russell (eds.) The sixth Australian International Conference on Speech Science and Technology. Adelaide: Australian Speech Science and Technology Association. Pp. 121-126.

Sambur, M. R. 1975. "Selection of acoustic features for speaker identification." IEEE Transactions on Acoustics, Speech, and Signal Processing ASSP-23: 178-182.

Sato, Y. 1993. "The durations of syllable-final nasals an $d$ the mora hypothesis in Japanese." Phonetica 50: 44-67.

Schiller, N. O., O. Köster, and M. Duckworth. 1997. "The effect of removing linguistic information upon identifying speakers of a foreign language." Forensic Linguistics 4: 117.

Schmidt-Nielsen, A., and K. R. Stern. 1985. "Identification of known voices as a function of familiarity and narrow-band coding." Journal of Acoustical Society of America 77: 658663.

Shibata, T. 1955/1980. "Nihongo no akusento taikei." [Accentuation system in Japnaese] in T. Sibata, H. Kitamura and H. Kindaichi (eds.) Nihongo no Gengogaku: Onin. Tokyo: Taishuukan Publishing Company. Pp. 405-442.

Shibatani, M. 1990. The Language of Japan. Cambridge: Cambridge University Press.

Shibatani, M., T. Kageyama, and I. Tamori. 1981. Gengo no koozoo-Riron to bunseki-: Onsee, Onin. [Structure of language: theory and analysis in phonetics and phonology] Tokyo: Kuroshio Shuppan.

Simmons, A. 1997. "Difference and distinguishability: a Bayesian approach to forensic speaker identification." unpublished Honours thesis submitted to The Australian National University.

Stevens, K. N. 1971. "Sources of inter- and intra-speaker variability in the acoustic properties of speech sounds." in 7th international Congress of Phonetic Sciences. Montreal: Mouton and Company. Pp. 206-232

- 1997. "Articulatory-acoustic-auditory relationships." in W. Hardcastle and J. M. D. Laver (eds.) The Handbook Of Phonetic Sciences. Oxford: Blackwell. Pp. 462-506.

-. 1998. Acoustic Phonetics. Cambridge: MIT Press.

Stevens, K. N., and A. S. House. 1955. "Development of a quantative description of vowel articulation." Journal of Acoustical Society of America 27: 484-93.

Sugito, M. 1989. "Onsetsu ka haku ka -Chooon, hatsuon, sokuon-" [Syllable or Mora? long vowels, moraic nasals, and geminate consonants] in M. Sugitoo (eds.) Nihongo no Onsee, Onin. Tokyo: Meiji Shoin. Pp. 154-177.

- 1996a. "Boin no museeka -Tokyo to Osaka no baai-" [Vowel devoicing in Tokyo and Osaka] Pp. $28-42$ in M. Sugito (eds.) Nihongo no on. Tokyo: izumishoin.

- 1996b. "Nihon no 8 toshi ni okeru boin no museeka" [Vowel devoicing in 8 cities in Japan] in M. Sugito (eds.) Nihongo no on. Tokyo: Izumishoin. Pp. 43-54 
-. 1996c. Nihongo no On. [Sound of Japanese] Tokyo: Izumi Shoin.

Suzuki, T. 1973a. "Analysis of female voice by sound-spectrograph with $500 \mathrm{~Hz}$ band-pass." Reports of National Research Institution of Police Science 26: 49-54.

- 1973b. "Variation of voice-print with changing vocal pitch." Reports of National Research Institution of Police Science 26: 7-23.

- 1978. "Voice at the time of adolescent voice change." Reports of National Research Institution of Police Science 31: 120-129.

Suzuki, T., and H. Inomata. 1975. "Individuality of pitch occurrence distribution in speech." Reports of National Research Institution of Police Science 28: 40-50.

Suzuki, T., and K. Odagawa. 1976. "Disguised voice spectrograms: the voice spectrograms of the phonation in closed nostrils." Reports of National Research Institution of Police Science 29: 39-51.

Suzuki, T., T. Kasai, and Y. Takahashi. 1978. "Spectrograms of whispered voices in Japanese 5 vowels." Reports of National Research Institution of Police Science 31: 31-38.

Suzuki, T., M. Tanimoto, T. Osanai, and H. Kido. 1994. "Speaker verification rates of five vowels uttered isolatedly by large number of adult male speakers." Reports of National Research Institution of Police Science 47: 1-6.

Tosi, O., H. Oyer, W. Lashbrook, C. Pedrey, J. Nicol, and E. Nash. 1972. "Experiment on voice identification." Journal of Acoustical Society of America 5: 2030-2043.

Tsukada, K. 2000. "Some acoustic characteristics of Australian English /ai/ and Japanese /ai/ in native and non-native speech production." in M. Barlow (eds.) The Eighth Australian International Conference on Speech Science ad Technology. Canberra: Australian Speech Science and Technology Association. Pp. 56-61

Vance, T. J. 1987. An Introduction to Japanese Phonology. Albany: State University of New York Press.

Watanabe, T. 1998. "Japanese pitch and mood." Nihongakuho, Osaka University 17: 97-110.

Wells, J. C. 1982. Accents of English: an Introduction. Cambridge: Cambridge University Press.

Wolf, J. J. 1972. "Efficient acoustic parameters for speaker recognition." Joumal of Acoustical Society of America 51: 2044-2055.

Woods, A., P. Fletcher, and A. Hughes. 1986. Statistics in Language Studies. Cambridge: Cambridge University Press

Yamane, H. 1973. "Speaker identification in modified voice." Reports of National Research Institution of Police Science 26:255-258

Young, M. A., and R. A. Campbell. 1967. "Effects of context on talker identification." Journal of Acoustical Society of America 42: 1250-1254. 